\title{
Untersuchungen zur enantioselektiven Totalsynthese von Parnafungin C
}

\author{
Dissertation \\ zur Erlangung des mathematisch-naturwissenschaftlichen Doktorgrades \\ „Doctor rerum naturalium“ \\ der Georg-August-Universität Göttingen \\ im Promotionsprogramm der \\ Georg-August University School of Science (GAUSS)
}

vorgelegt von

Sven Heidemann

aus Burg

Göttingen, 2016 

Betreuungsausschuss:

Prof. Dr. Dr. h.c. Lutz F. Tietze

Institut für Organische und Biomolekulare Chemie

Georg-August-Universität Göttingen

Prof. Dr. Ulf Diederichsen

Institut für Organische und Biomolekulare Chemie

Georg-August-Universität Göttingen

Mitglieder der Prüfungskommission:

Referent: Prof. Dr. Dr. h.c. Lutz F. Tietze

IOBC, Georg-August-Universität Göttingen

Koreferent: Prof. Dr. Ulf Diederichsen

IOBC, Georg-August-Universität Göttingen

Weitere Mitglieder der Prüfungskommission:

Prof. Dr. Hartmut Laatsch

IOBC, Georg-August-Universität Göttingen

Dr. Alexander Breder

IOBC, Georg-August-Universität Göttingen

Prof. Dr. Manuel Alcarazo

IOBC, Georg-August-Universität Göttingen

Dr. Shoubhik Das

IOBC, Georg-August-Universität Göttingen

Tag der mündlichen Prüfung:

04. August 2016 

Die vorliegende Arbeit wurde in der Zeit von Juni 2011 bis Juni 2015 unter der Leitung von Herrn Prof. Dr. Dr. h.c. Lutz F. Tietze am Institut für Organische und Biomolekulare Chemie der Georg-August-Universität Göttingen angefertigt.

Mein besonderer Dank gilt Herrn Prof. Dr. Dr. h.c. Lutz F. Tietze für die interessante Themenstellung, die Bereitstellung der hervorragenden Arbeitsbedingungen, seine Unterstützung sowie für die zahlreichen Diskussionnen und Anregungen und seinem steten Interesse am Fortgang dieser Arbeit. 



\section{Meiner Familie}



"To succeed, planning alone is insufficient. One must improvise as well." Isaac Asimov 



\section{Inhaltsverzeichnis}

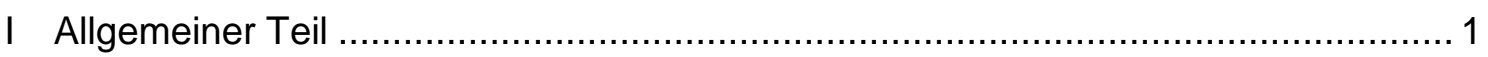

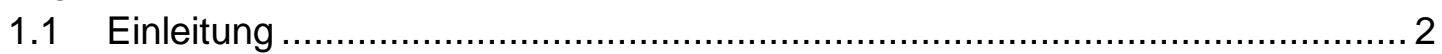

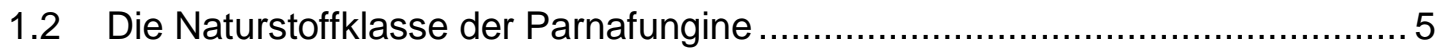

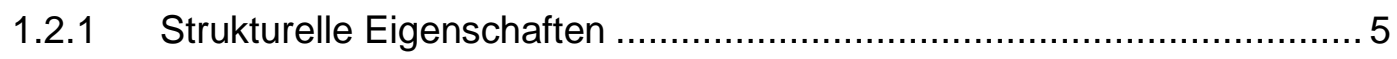

1.2.2 Eigenschaften und Wirkungsweise................................................ 8

1.2.3 Stand gegenwärtiger Forschungen ............................................ 10

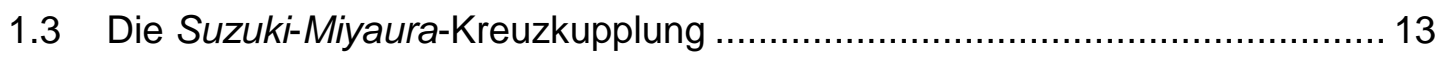

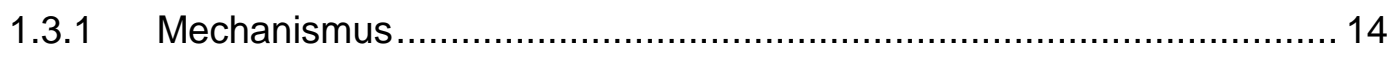

1.3.2 Synthese der Borverbindungen .................................................. 18

1.3.3 Anwendungen in der organischen Synthese ...................................20

1.4 Die Wacker-Oxidation ................................................................... 23

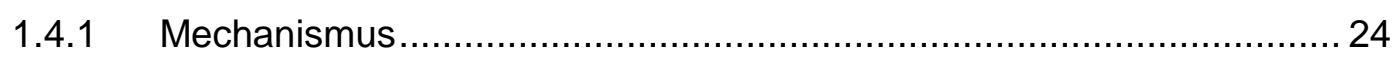

1.4.2 Anwendungen in der organischen Synthese .................................... 25

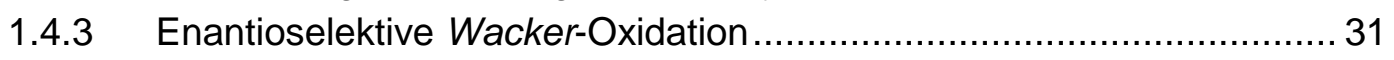

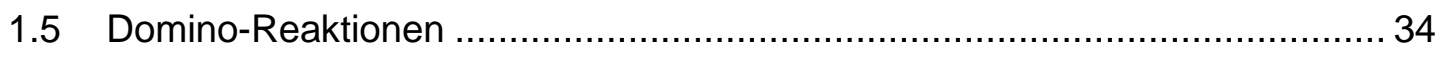

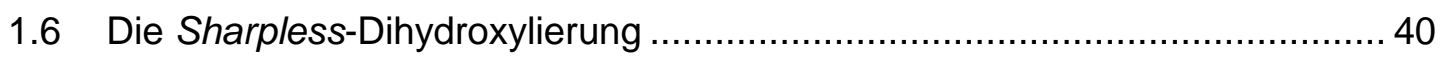

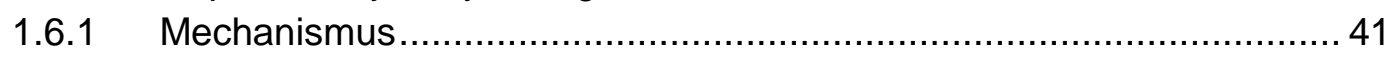

1.6.2 Anwendungen in der organischen Synthese .................................. 44

1.7 Zielsetzung und Planung der Arbeit.................................................... 47

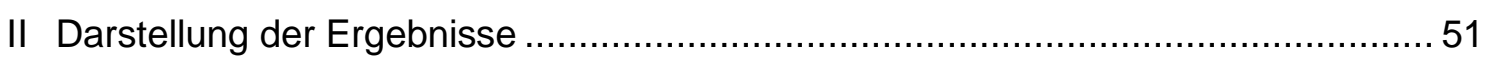

2.1 Optimierung der Synthesesequenz zum Aufbau des Biarylfragments ...........52

2.1.1 Synthese des Arylbromids 133 ................................................. 52

2.1.2 Miyaura-Borylierung und Suzuki-Miyaura-Kreuzkupplung zur Synthese des Biaryls 135..................................................... 54

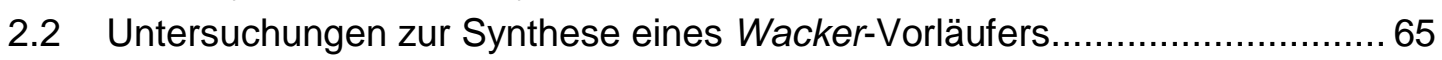

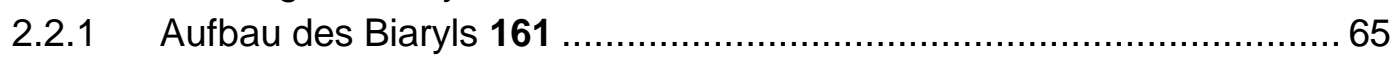

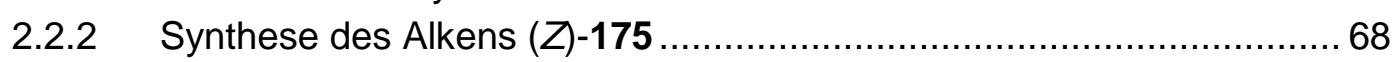

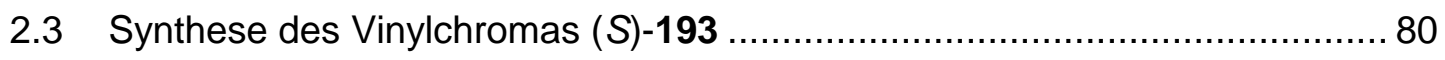

2.3.1 Untersuchungen zur Diversifizierung der Schutzgruppen ..................... 80

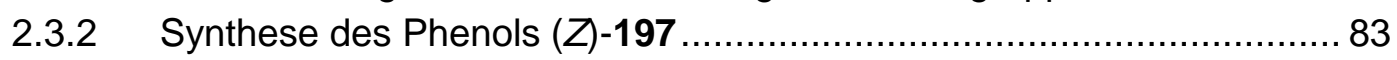

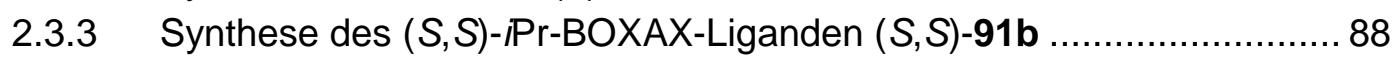

2.3.4 Wacker-Zyklisierung zum Aufbau des Vinylchromans (S)-207 ............. 91

2.4 Synthese des Chromans syn-266 und des Biaryls $(Z)-272 \ldots \ldots \ldots \ldots \ldots \ldots \ldots . . . \ldots 3$

2.4.1 Untersuchungen zum Aufbau des Ketons 217 ................................ 93

2.4.2 Synthese des Methylesters syn-266 ........................................... 101

2.4.3 Diskussion der spektroskopischen Daten des Aldehyds syn-265 ....... 110

2.4.4 Synthese des Biaryls (Z)-272 …............................................ 114

2.4.5 Diskussion der spektroskopischen Daten des Biaryls (Z)-272 ........... 116

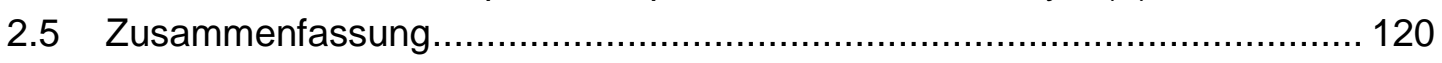

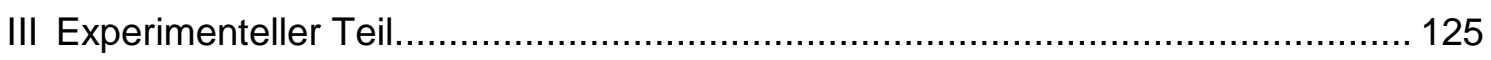

3.1 Allgemeine Methoden .................................................................... 126 


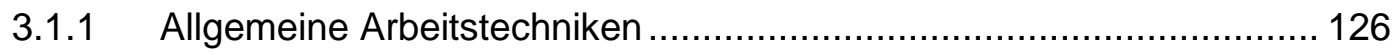

3.1.2 Lösungsmittel und Reagenzien .................................................. 126

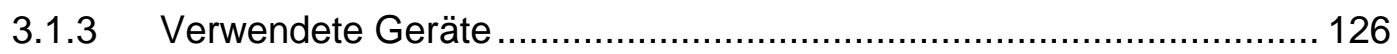

3.1.4 Chromatographische Methoden .............................................. 128

3.2 Synthese verwendeter Reagenzien ...................................................... 129

3.2.1 N,N-Di-iso-propyl-O-tert-butylisoharnstoff (146) ............................ 129

3.2.2 3-Chlor-2-nitrobenzoesäuremethylester (11) .............................. 130

3.2.3 2-Benzyloxyessigsäuremethylester (170) .................................. 131

3.2.4 1-Benzyloxy-3-triphenylphosphoranylidenpropan-2-on (171) ............. 133

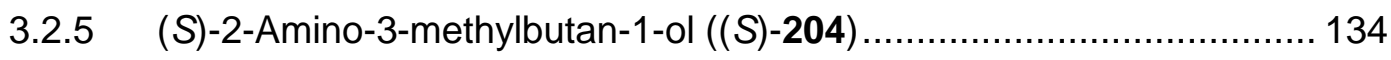

3.2.6 1-Brom-2-(dibrommethyl)naphthalin (199) ...................................... 135

3.2.7 1-Brom-2-naphthaldehyd (200) ................................................. 136

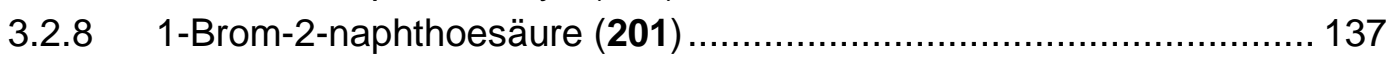

3.2.9 (S)-2-(1-Bromnaphthalin-2-yl)-4-iso-propyl-4,5-dihydrooxazol

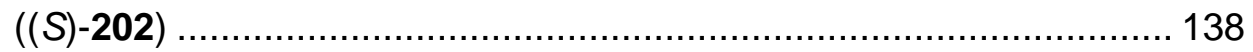

3.2.10 (S)-2,2'-Bis ((S)-4-iso-propyl-4,5-dihydrooxazol-2-yl)-1,1'-binaph-thalin

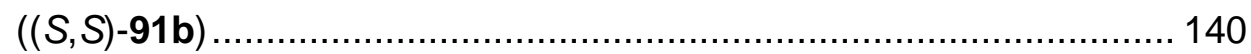

3.2.11 1-Hydroxy-1-oxo-1-benzo[d][1,2]iodoxol-3(1H)-on (275) ................... 141

3.2.12 1,1,1-Triacetoxy-1,1-dihydro-1,2-benziodoxol-3(1H)-on (276) ............ 142

3.2.13 1-Nitro-2-selenocyanatbenzol (254) .......................................... 143

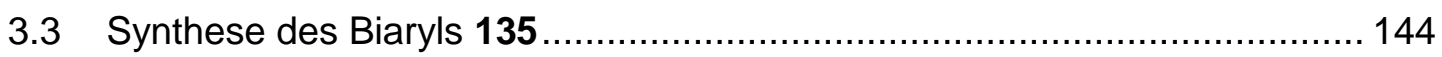

3.3.1 1,3-Dimethoxy-5-methylbenzol (143) ............................................ 144

3.3.2 2,6-Dimethoxy-4-methylbenzoesäure (144) .................................. 145

3.3.3 2,6-Dimethoxy-4-methylbenzoesäure-tert-butylester (147) ................ 146

3.3.4 3-Brom-2,6-dimethoxy-4-methylbenzoesäure-tert-butylester (148).... 147

3.3.5 3-Brom-4-brommethyl-2,6-dimethoxybenzoesäure-tert-butylester

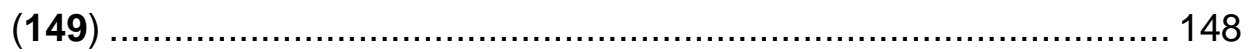

3.3.6 3-Brom-2,6-dimethoxy-4-(((4-methoxybenzyl)oxy)methyl)benzoe-

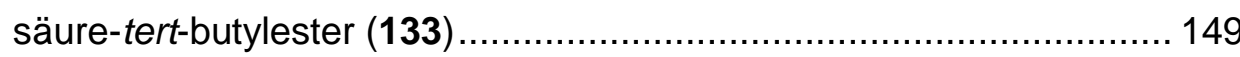

3.3.7 2,6-Dimethoxy-4-((4-methoxybenzyl)oxy)methyl)-3-(4,4,5,5-tetramethyl-1,3,2-dioxaborolan-2-yl)benzoesäure-tert-butylester (134) ..... 150

3.3.8 3-tert-Butyl-3'-methyl-2,4-dimethoxy-6-((4-methoxybenzyl)oxy)methyl-2'-nitro-[1,1'-biphenyl]-3,3'-dicarboxylat (135) ..................... 151

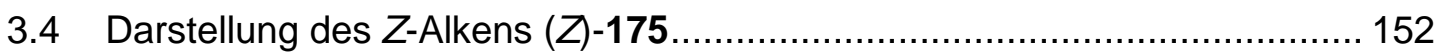

3.4.1 3,5-Dimethoxybenzoesäuremethylester (154) ............................. 152

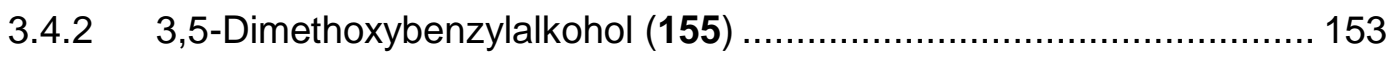

3.4.3 1-(((tert-Butyldimethylsilyl)oxy)methyl)-3,5-dimethoxybenzol (163) .... 154

3.4.4 4-(((tert-Butyldimethylsilyl)oxy)methyl)-2,6-dimethoxybenzaldehyd (164)

3.4.5 (E)-1-Benzyloxy-4-(4-(((tert-butyldimethylsilyl)oxy)methyl)-2,6-di-

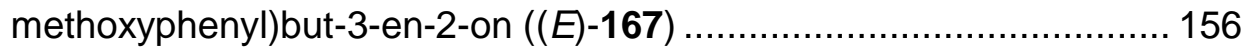

3.4.6 1-Benzyloxy-4-(4-(((tert-butyldimethylsilyl)oxy)methyl)-2,6-dimethoxyphenyl)butan-2-on (168)

3.4.7 1-Benzyloxy-4-(4-(((tert-butyldimethylsilyl)oxy)methyl)-3-iod-2,6dimethoxyphenyl)butan-2-on (172)...... 
3.4.8 1-Benzyloxy-4-(4-((tert-butyldimethylsilyl)oxy)methyl)-2,6-dimethoxy-3-(4,4,5,5-tetramethyl-1,3,2-dioxaborolan-2-yl)phenyl)butan-2-on (173)

3.4.9 3'-(4-Benzyloxy-3-oxobutyl)-6'-(((tert-butyldimethylsilyl)oxy)-methyl)2',4'-dimethoxy-2-nitro-[1,1'-biphenyl]-3-carbonsäure-methylester (174)

3.4.10 (Z)-3'-(3-((Benzyloxy)methyl)pent-3-en-1-yl)-6'-(((tert-butyldimethylsilyl)oxy)methyl)-2',4'-dimethoxy-2-nitro-[1,1'-biphenyl]-3carbonsäuremethylester ((Z)-175)

3.5 Synthese des Vinylchromans (S)-207

3.5.1 1-(((tert-Butyldiphenylsilyl)oxy)methyl)-3,5-dimethoxybenzol (188) .... 165

3.5.2 4-(((tert-Butyldiphenylsilyl)oxy)methyl)-2,6-dimethoxybenzaldehyd (189)

3.5.3 4-(((tert-Butyldiphenylsilyl)oxy)methyl)-2-hydroxy-6-methoxybenzaldehyd (190)

3.5.4 5-(((tert-Butyldiphenylsilyl)oxy)methyl)-2-formyl-3-methoxyphenylpivalat (191)

3.5.5 (E)-2-(4-Benzyloxy-3-oxobut-1-en-1-yl)-5-(((tert-butyldiphenylsilyl)oxy)methyl)-3-methoxyphenylpivalat $((E)-192)$

3.5.6 2-(4-Benzyloxy-3-oxobutyl)-5-((tert-butyldiphenylsilyl)oxy)-methyl)-3methoxyphenylpivalat (193)

3.5.7 2-(4-Benzyloxy-3-oxobutyl)-5-((tert-butyldiphenylsilyl)oxy)-methyl)-6iod-3-methoxyphenylpivalat (195)

3.5.8 (Z)-2-(3-((Benzyloxy)methyl)pent-3-en-1-yl)-5-(((tert-butyldiphenylsilyl)oxy)methyl)-3-methoxyphenol ((Z)-197)

3.5.9 (S)-2-((Benzyloxy)methyl)-7-(((tert-butyldiphenylsilyl)oxy)methyl)-5methoxy-2-vinylchroman ((S)-207)

3.6 Synthese des Chromans syn-266 und des Biaryls (Z)-272

3.6.1 4-Hydroxymethyl-2,6-dimethoxybenzaldehyd (158)

3.6.2 (E)-1-Benzyloxy-4-(4-hydroxymethyl-2,6-dimethoxyphenyl)but-3-en-2on $((E)-214)$.

3.6.3 (E)-1-Benzyloxy-4-(2,6-dimethoxy-4-((methoxymethoxy)methyl)phenyl)but-3-en-2-on $((E)-243)$

3.6.4 1-Benzyloxy-4-(2,6-dimethoxy-4-((methoxymethoxy)methyl)phenyl)butan-2-on (244a).

3.6.5 2-(3-((Benzyloxy)methyl)but-3-en-1-yl)-1,3-dimethoxy-5-((methoxymethoxy)methyl)benzol (245)

3.6.6 2-(3-((Benzyloxy)methyl)but-3-en-1-yl)-3-methoxy-5-((methoxymethoxy)methyl)phenol (246).

3.6.7 (S)-2-(2-((Benzyloxy)methyl)-5-methoxy-7-((methoxymethoxy)methyl)chroman-2-yl)essigsäuremethylester ((S)-250).

3.6.8 (S)-2-(2-((Benzyloxy)methyl)-5-methoxy-7-((methoxymethoxy)methyl)chroman-2-yl)ethan-1-ol ((S)-251)

3.6.9 (S)-2-((Benzyloxy)methyl)-5-methoxy-7-((methoxymethoxy)-methyl)2-vinylchroman $((S)-252)$ 
3.6.10 (E/Z)-2-(3-((Benzyloxy)methyl)pent-3-en-1-yl)-1,3-dimethoxy-5-

((methoxymethoxy)methyl)benzol ((E/Z)-255)

3.6.11 (E/Z)-2-(3-((Benzyloxy)methyl)pent-3-en-1-yl)-3-methoxy-5-

((methoxymethoxy)methyl)phenol $((E / Z)-256)$

3.6.12 (S)-2-((Benzyloxy)methyl)-5-methoxy-7-((methoxymethoxy)-methyl)2-vinylchroman $((S)-252)$....

3.6.13 (2'R)-1-(2-((Benzyloxy)methyl)-5-methoxy-7-((methoxymethoxy)methyl)chroman-2-yl)ethan-1,2-diol (anti/syn-257) .... 198

3.6.14 (2'R)-1-(2-(Benzyloxy)methyl-5-methoxy-7-((methoxymethoxy)methyl)chroman-2-yl)-1,2-bis((tert-butyldimethylsilyl)oxy)ethan (anti/syn-263)

3.6.15 (2'R)-2-(2-((Benzyloxy)methyl)-5-methoxy-7-((methoxymethoxy)methyl)chroman-2-yl)-2-((tert-butyldimethylsilyl)oxy)ethan-1-ol (anti/syn-264)

3.6.16 (2R,2'R)-2-(2-(Benzyloxy)methyl)-5-methoxy-7-((methoxymethoxy)methyl)chroman-2-yl)-2-((tert-butyldimethylsilyl)oxy)acetaldehyd (syn-265)

3.6.17 (4S,2'R)-(E)-4-(2-(Benzyloxy)methyl)-5-methoxy-7-((methoxymethoxy)methyl)chroman-2-yl)-4-((tert-butyldimethylsilyl)oxy)but-2ensäuremethylester (syn-266)

3.6.18 1-Benzyloxy-4-(3-iod-2,6-dimethoxy-4-((methoxymethoxy)methyl)phenyl)butan-2-on (269) 208

3.6.19 1-Benzyloxy-4-(2,6-dimethoxy-4-((methoxymethoxy)methyl)-3(4,4,5,5-tetramethyl-1,3,2-dioxaborolan-2-yl)phenyl)butan-2-on (270) 209

3.6.20 3'-(4-Benzyloxy-3-oxobutyl)-2',4'-dimethoxy-6'-((methoxymethoxy)methyl)-2-nitro-[1,1'-biphenyl]-3-carbonsäuremethylester (271)

3.6.21 (Z)-3'-(3-((Benzyloxy)methyl)pent-3-en-1-yl)-2',4'-dimethoxy-6'((methoxymethoxy)methyl)-2-nitro-[1,1'-biphenyl]-3-carbonsäuremethylester ((Z)-272)

IV Anhang.

4.1 Abkürzungsverzeichnis .............................................................. 216

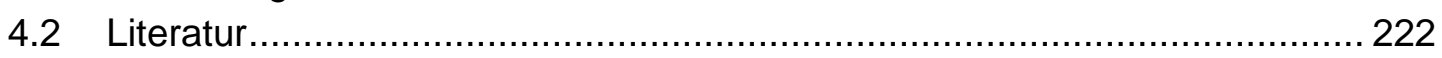

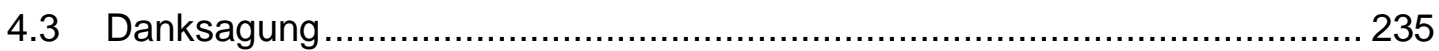

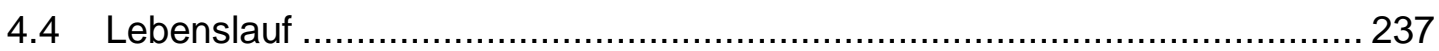


I Allgemeiner Teil 


\subsection{Einleitung}

Praktisch jeder Mensch wird in seinem Leben mindestens einmal mit einer Pilzerkrankung konfrontiert. So sind derzeitig geschätzte $1.2 \mathrm{Mrd}_{.}{ }^{[1]}$ Menschen weltweit von einer Erkrankung betroffen, die aus einem Befall mit pathogenen Pilzen resultierte. Größtenteils handelt es sich hierbei um gut therapierbare Infektionen der Haut und der Schleimhäute. Dahingegen leiden rund ein Viertel der Betroffenen unter einer schwerwiegenderen Form dieser Infektionskrankheit, von denen wiederum 25 Mio. einem hohen Risiko ausgesetzt sind, ihr Augenlicht oder sogar ihr Leben zu verlieren. Vor allem invasive und chronische Mykosen tragen aufgrund ihrer schlechten Diagnostizier- sowie Therapierbarkeit ihren Teil zu den jährlich 1.5 bis 2 Mio. Toten infolge einer Pilzinfektion bei. Damit liegt die Zahl der durch pathogene Pilze verursachten Todesfälle höher, als z. B. die der Malaria- oder Tuberkulose-bedingten. Verantwortlich hierfür zeichnet hauptsächlich die hohe Mortalität systemischer Mykosen der Fungi der Gattungen: Aspergillus, Candida, Cryptococcus und Pneumocystis. Im Falle einer Candidiasis beträgt die Mortalität zwischen 30 und $55 \%$ bei einer ansteigenden Zahl der jährlichen Infektionen in den letzten Jahren. Die Sterberate einer invasiven Aspergillose liegt mit 30-50\% auf nahezu dem gleichen hohen Niveau und beträgt $>99 \%$ ohne Therapie. ${ }^{[2]}$ Insbesondere Patienten mit einer Immunschwäche, wie sie z. B. im Zuge einer Infektion mit HIV oder durch Suppression des Immunsystems infolge einer Transplantation respektive Krebstherapie auftritt, aber auch Unfallpatienten auf Intensivstationen, bei denen durch invasive Maßnahmen (z. B. Katheter) den Pilzen der Zugang zum Blutkreislauf geöffnet wird, sind einem erhöhten Risiko ausgesetzt, diesen Krankheiten anheimzufallen. ${ }^{[3]}$

Das spärliche Arsenal an Antimykotika steuert hierbei einen signifikanten Anteil an der schlechten Therapierbarkeit und der damit einhergehenden hohen Mortalität systemischer Mykosen bei. Bis in die späten 1980er war das makrozyklische Polyen Amphotericin B, dem bereits 1956 seine fungizide Wirkung nachgewiesen wurde, praktisch der einzige zur medikamentösen Therapie eingesetzte Wirkstoff. ${ }^{[4]}$ Auch heutzutage findet Amphotericin B sowie dessen aktuellere Lipidformulierungen vorrangig noch als Reserverantimykotikum Verwendung. Zwischen 1990 und den frühen 2000er Jahren wurde das Spektrum wirksamer Medikamente um Vertreter der Wirkstoffklassen der Triazole (wichtige Arzneistoffe: Voriconazol, ${ }^{[5]}$ Fluconazol und Itraconazol) und der Echinocandine (wichtige Arzneistoffe: Caspofungin, ${ }^{[6]}$ Micafungin ${ }^{[7]}$ und Anidulafungin $\left.{ }^{[8]}\right)$ erweitert. Allen genannten Präparaten gemein ist die Erhöhung der Permeabilität der Pilzzellmembran bzw. -wand zur Auslösung des Zelltodes. Dies wird durch die Inhibition entweder des für die Stabilität der Zellmembran essentiellen Ergosterols bzw. dessen Synthese oder der Produktion des für die Zellwand substanziellen $\beta-1,3-$ Glukans erreicht. 
Aufgrund der geringen Vielfalt zur Therapie eingesetzter wirksamer Medikamente und deren mittlerweile langer Nutzung im klinischen Alltag wird die Behandlung invasiver Pilzerkrankungen zusehends durch eine voranschreitende Resistenzentwicklung pathogener Fungi erschwert. Im Falle der Triazole spitzt sich diese negative Entwicklung noch durch deren kommerzielle und landwirtschaftliche Nutzung weiter zu. ${ }^{\left[{ }^{9}\right]}$ Beispielsweise verzeichnet die Spezies Candida glabrata, die zweithäufigste isolierte Candida Art in Europa, einen hohen Grad an Resistenz gegenüber den Triazolen Fluconazol und Voriconazol sowie mittlerweile auch gegenüber Echinocandine. Zur Behandlung solch resistenter Pilze verbleibt somit nur noch intravenös appliziertes Amphotericin B, welches jedoch häufig toxisch wirkt. ${ }^{[10]}$

Aktuell befinden sich gerade einmal vier neuentwickelte Präparate in klinischen Studien, von denen indes nur zwei einen neuartigen Wirkmechanismus aufweisen. Hinzukommen noch einige wenige weitere Wirkstoffe, die sich in der präklinischen Entwicklung befinden, wodurch die spärlich befüllte Entwicklungspipeline wenig Hoffnung auf eine baldige Verbesserung des Status quo erlaubt. Die Entwicklung neuer Antimykotika mit einem breitem Wirkspektrum und verschiedenen neuartigen sowie spezifischen Wirkmechanismen stellt somit nicht nur eine Notwendigkeit, sondern auch eine Chance dar. ${ }^{[11]}$ In diesem Zusammenhang nimmt die Totalsynthese von Naturstoffen eine besondere Rolle ein. Natürlich vorkommende Wirkstoffe dienen oftmals als Leitmotive für Medikamente, können aber häufig nur in geringen Mengen aus natürlichen Quellen isoliert werden, sodass eine synthetische Darstellung des Wirkstoffes erstrebenswert ist. ${ }^{[12]}$ Des Weiteren besteht über den synthetischen Zugang zu einem Wirkstoff die Möglichkeit der Derivatisierung und somit zur Anpassung bestimmter Eigenschaften, um etwaige Nebenwirkungen zu verringern oder die biologische Aktivität zu erhöhen. Im Falle chiraler Wirkstoffe ist die Entwicklung eines enantioselektiven Zugangs zur Vermeidung potentieller Nebenwirkungen des unerwünschten Enantiomers von Bedeutung. Als Beispiel sei an dieser Stelle das Lokalanästhetikum Bupivacain (Carbostesin ${ }^{\circledR}$ ) aufgeführt, das zunächst über mehrere Jahrzehnte ausschließlich als Racemat eingesetzt wurde. Dieses kann schwere Nebenwirkungen, die bis zum Herzstillstand führen können, auslösen, sollte es durch z. B. eine versehentliche intravenöse Applikation in den systemischen Kreislauf gelangen. Die Nebenwirkungen resultieren dabei aus der Blockade kardialer $\mathrm{Na}^{+}$-Kanäle. $(R)$-Bupivacain blockiert diese Kanäle stärker und länger als das (S)-Enantiomer, sodass zur Verbesserung der Sicherheit bei gleichbleibender lokalanästhetischer Wirkung das (S)-Enantiomer Levobupivacain (Chirocain ${ }^{\circledR}$ ) 2004 neu eingeführt wurde. ${ }^{[13]}$

Die vorliegende Arbeit beschäftigt sich mit der Untersuchung einer enantioselektiven Darstellung des Antimykotikums Parnafungin C. Im Kontext der Naturstoffsynthese soll 
hierbei die Anwendbarkeit der enantioselektiven Palladium-katalysierten WackerZyklisierung bzw. Domino-Wacker/Carbonylierung/Methoxylierung zum Aufbau der Tetrahydroxanthenon-Grundstruktur demonstriert werden. 


\subsection{Die Naturstoffklasse der Parnafungine}

Parnafungine bilden eine neue Klasse von natürlich vorkommenden Wirkstoffen und wurden erstmalig im Jahr 2008 beschrieben. Eine Merck-Gruppe isolierte aus Fermentationsextrakten aus Spanien stammender Flechten, die von parasitären Schimmelpilzstämmen befallen waren, ein Gemisch aus Parnafungin A (1) und B (2), auf welches die Forscher durch eine erhöhte antimykotische Wirkung im Candida albicans Fitness Test (CaFT) aufmerksam wurden. ${ }^{[14]}$ Noch im gleichen Jahr konnten zwei weitere Derivate (Parnafungin C (3) und D (4)) durch dieselbe Gruppe identifiziert werden (Abbildung 1). ${ }^{[15]}$ Die Parnafungin-produzierenden Pilzstämme ähnelten dem Fungus Fusarium larvarum Fuckel und gehören damit der Abteilung der Schlauchpilze (Ascomycota), genauer der Ordnung der Krustenkugelpilzartigen (Hypocreales) an. Des Weiteren zeigte sich, dass neben einer Vielzahl an Pilzstämmen aus mindestens sechs Abstammungslinien des Fusarium larvarum Komplexes auch die parasitären Fungi Trichonectria rectipila und Cladobotryum pinarense, die ebenfalls zur Ordnung der Hypocreales gehören, dazu fähig sind, Parnafungine als Sekundärmetaboliten zu produzieren. ${ }^{[16]}$ Als Wirte dieser Pilzstämme dienen neben Flechten vor allem Schild- und Blattläuse sowie Pflanzen und Pflanzenstreu.

\subsubsection{Strukturelle Eigenschaften ${ }^{[14,15]}$}

Strukturell weisen die Parnafungine ein in natürlich vorkommenden Wirkstoffen häufig auftretendes Tetrahydroxanthenon-Gerüst und einen unter Naturstoffen bisher einzigartigen Isoxazolidinon-Ring (Ring F) auf. Wie oben bereits erwähnt, sind aktuell vier Vertreter dieser neuen Naturstoffklasse bekannt: die Konstitutionsisomere Parnafungin A (1) und B (2), das 7-O-methylierte Parnafungin C (3) sowie das ebenfalls 7-O-methylierte und an der $\beta$-Seite des A-Rings epoxidierte Parnafungin D (4).

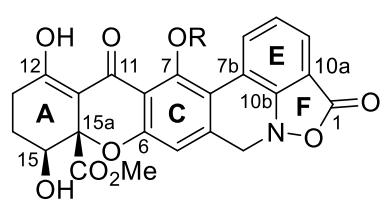

Parnafungin $A(1): R=H$ Parnafungin $C(3): R=M e$

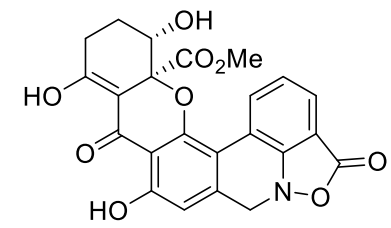

Parnafungin B (2)

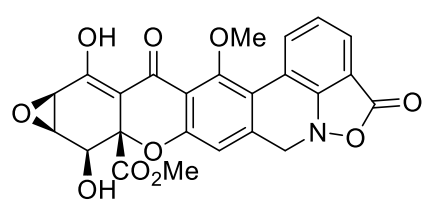

Parnafungin D (4)

Abbildung 1: Struktur der natürlich vorkommenden Parnafungine.

Jedes dieser vier Moleküle weißt mindestens die beiden stereogenen Zentren an C-15 sowie dem quartären Kohlenstoff C-15a auf. Die beiden Konstitutionsisomere Parnafungin 
A (1) („lineare“ Topologie) und Parnafungin B (2) („gekrümmte“ Topologie) liegen in einem thermodynamischen Gleichgewicht vor und wandeln sich in Lösung bereits bei Raumtemperatur ineinander um. Die Geschwindigkeit, mit der dieser Prozess abläuft, ist abhängig vom vorliegenden $\mathrm{pH}-$ Wert. So verlangsamt sich die Umwandlung im sauren Milieu, während sie unter neutralen oder basischen Bedingungen schneller erfolgt. Der diesem Vorgang zugrunde liegende Mechanismus besteht aus dem aufeinanderfolgenden Ablauf einer retro-Michael-Ringöffnung und anschließender Michael-Addition. Im ersten Schritt führt die Öffnung des B-Rings zur Freisetzung zweier phenolischer Hydroxygruppen (an C-6 und C-7), die durch eine Rotation um die Einfachbindung zwischen den Kohlenstoffatomen C-6a und C-11 für eine anschließende Michael-Addition an das Enon des A-Rings gleichermaßen zugänglich sind. Eine Michael-Addition der Hydroxyfunktionalität an C-6 mit dem Enon (5a) hat hierbei die Ausbildung des „linearen“ Isomers Parnafungin A (1) zur Folge. Konkurrierend hierzu resultiert eine Michael-Addition der Hydroxygruppe an C-7 mit dem Enon (5b) im "gekrümmten“ Isomer Parnafungin B (2) (Abbildung 2).

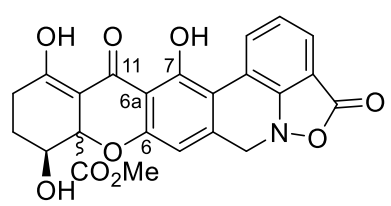

Parnafungin $\mathrm{A} 1$ (1a, syn- $\left.\mathrm{CO}_{2} \mathrm{Me}\right)$ Parnafungin $\mathrm{A} 2\left(\mathbf{1 b}\right.$, anti- $\left.\mathrm{CO}_{2} \mathrm{Me}\right)$

$$
\|
$$

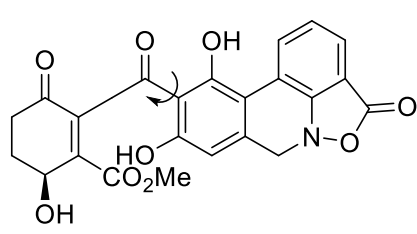

$5 \mathbf{a}$

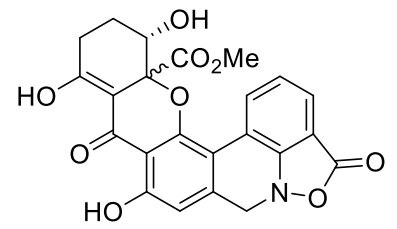

Parnafungin $\mathrm{B} 1\left(2 \mathrm{a}\right.$, syn- $\left.\mathrm{CO}_{2} \mathrm{Me}\right)$ Parnafungin $\mathrm{B} 2\left(\mathbf{2 b}\right.$, anti- $\left.\mathrm{CO}_{2} \mathrm{Me}\right)$
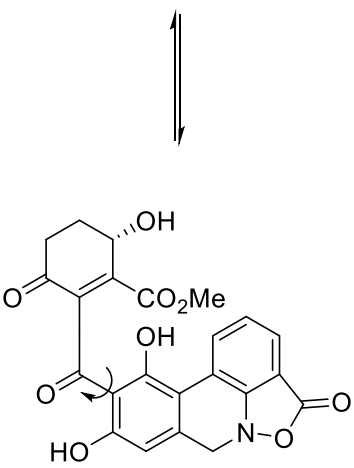

$5 \mathbf{b}$

Abbildung 2: Mechanismus der Umwandlung der beiden Konstitutionsisomere Parnafungin A (1) und B (2).

Während der Michael-Addition kann der Angriff der Hydroxyfunktionen an das Enon von beiden Seiten erfolgen, was eine Epimerisierung am quartären Kohlenstoffatom C-15a zur Folge hat und somit zu einem thermodynamisch kontrollierten Gleichgewicht zweier diastereomerer Formen führt. Hierbei ist die $S$-Konfiguration favorisiert, was sich in einem Verhältnis von 4:1:5:1 $\mathbf{1} \mathbf{a} / \mathbf{1} \mathbf{b} / \mathbf{2} \mathbf{a} / \mathbf{2} \mathbf{b}$ einer sich im Gleichgewicht befindenden DMSO- 
Lösung aller vier Isomere widerspiegelt. Bei den beiden 7-O-methylierten Vertretern Parnafungin $C(3)$ und $D(4)$ ist die generelle Abfolge aus retro-Michael-Ringöffnung und darauffolgender Michael-Addition zwar ebenfalls beobachtbar, jedoch durch die Methylierung der Hydroxygruppe an C-7 auf die Epimerisierung des Stereozentrums am Kohlenstoffatom C-15a sowie das Vorliegen beider Moleküle in der "linearen“ Topologie limitiert. Mittels NMR-Experimenten und kristallographischer Untersuchungen konnte neben der Konfiguration des quartären Kohlenstoffs zusätzlich die absolute Konfiguration der Hydroxyfunktionalität an C-15 eindeutig als S-konfiguriert bestimmt werden. Dabei nimmt die Hydroxygruppe im jeweiligen Hauptdiastereomer $(S, S)$ die äquatoriale Position in der Halbsesselkonformation des A-Rings ein. Die Methylestergruppe am quartären Kohlenstoff C-15a nimmt indes immer die jeweils axiale Position ein. Somit stehen im jeweiligen Hauptdiastereomer beide Substituenten syn zueinander, während sie im Mindermengendiastereomer eine anti-Anordnung zueinander einnehmen.

Aufgrund der sehr labilen N-O-Bindung des Isoxazolidinon-Strukturelements wird diese unter Ringöffnung leicht zu den entsprechenden Phenanthridinen gespalten (Abbildung $3)$.

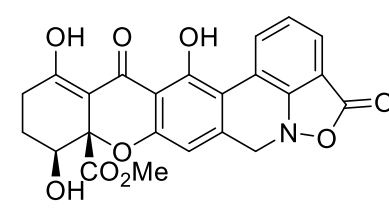

Parnafungin A (1)

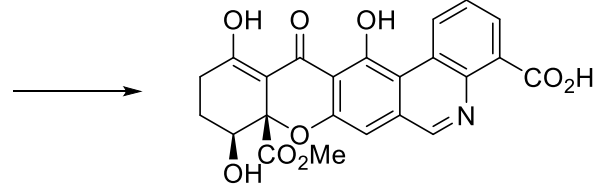

6

Abbildung 3: Spaltung von Parnafungin A (1) zum Phenanthridin 6.

Der Mechanismus der Ringöffnung konnte bisher noch nicht eindeutig aufgeklärt werden. Denkbar wäre die Eliminierung eines der beiden benzylischen Protonen an C-4 bei gleichzeitigem N-O-Bindungsbruch oder die Hydrolyse des Isoxazolidinons mit anschließender Eliminierung eines Moleküls Wasser. Die Spaltung zum Phenanthridin erfolgt bereits bei Raumtemperatur und in Abhängigkeit vom pH-Wert. Neutrale und basische Medien führen zu einer beschleunigten Öffnung des Isoxazolidinons $(<1 \mathrm{~h})$, während unter sauren Bedingungen eine Verlangsamung der Spaltung zu verzeichnen ist $(\mathrm{pH}=3,10-20 \mathrm{~h}){ }^{[14]}$

Bisher konnten noch keine Erkenntnisse zur Biosynthese der Parnafungine gewonnen werden. Die Biosynthese der Tetrahydroxanthenon-Einheit könnte hingegen analog des Aufbaus dieses Strukturelements in den Ergochromen der Secalonsäuren durch eine Reihe von Polyketid-Kondensationen und Zyklisierungen erfolgen. ${ }^{[17]}$ Der Aufbau des zusätzlichen Ringsystems und vor allem des Isoxazolidinon-Rings könnte via oxidativer Kupplung von Blennolid C (7) mit Anthranilsäure (8) und anschließender Oxidation der 
benzylischen Methylgruppe sowie des Anilins des substituierten Tetrahydroxanthenons 9 ablaufen (Abbildung 4). ${ }^{[18]}$

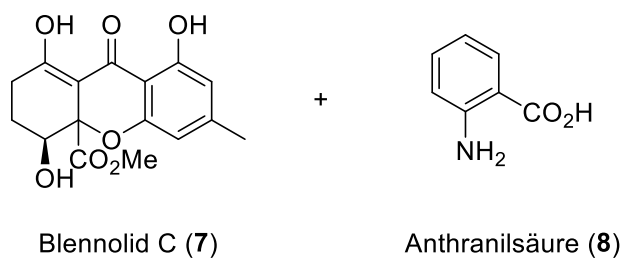

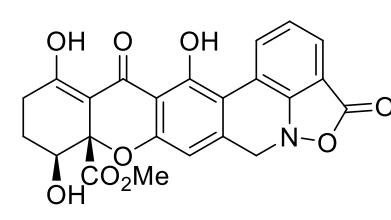

Parnafungin A (1)

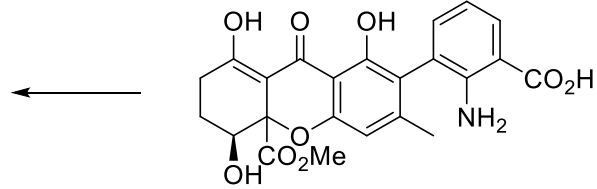

9

Abbildung 4: Mögliche Biosynthese von Parnafungin A (1).

\subsubsection{Eigenschaften und Wirkungsweise ${ }^{[19]}$}

Tabelle 1: Inhibierung verschiedener Candida-Arten durch Parnafungin A-D (1-4).

\begin{tabular}{cccc}
\hline \multirow{2}{*}{ Fungi } & $\mathbf{1 / 2}$ & $\mathbf{3}$ & $\mathbf{4}$ \\
\cline { 2 - 4 } MIC in $\mu \mathrm{g} / \mathrm{ml}$ \\
\hline C. albicans & 0.008 & 2 & 0.016 \\
C. glabrata & 1.25 & $>10$ & 5 \\
C. parapsilosis & 0.6 & $>10$ & 2.5 \\
C. lusitaniae & 0.3 & 0.16 & 0.16 \\
C. krusei & 0.008 & 0.08 & 0.016 \\
C. tropicallis & 2.5 & 2.5 & 0.6 \\
\hline
\end{tabular}

Parnafungine weisen ein breites Spektrum an antifungaler Aktivität gegenüber klinisch relevanten pathogenen Pilzen auf. Dabei zeigen Parnafungin C (3) und D (4) im Allgemeinen eine geringere Potenz gegenüber den in vitro getesteten CandidaArten verglichen mit Parnafungin A (1) und B (2) (Tabelle 1). ${ }^{[15]}$ Die Wirksamkeit der Mischung von Parnafungin A (1) und B (2) gegenüber dem Fungi Candida albicans ist mit $0.008 \mu \mathrm{g} / \mathrm{ml}$ vergleichbar mit der des kommerziell eingesetzten Antimykotikums Caspofungin $\left(\mathrm{MIC}=0.01 \mu \mathrm{g} / \mathrm{ml}\right.$ gegenüber $C$. albicans). ${ }^{[14]}$ Neben verschiedenen Candida-Spezies wird der ebenfalls klinisch relevante Pilz Aspergillus fumigatus durch Parnafungine inhibiert. Die beobachteten Phenanthridin-Derivate, die durch Spaltung des Isoxazolidinon-Rings aus den Parnafunginen hervorgehen, zeigen indes keine inhibierende Wirkung, sodass die Intaktheit dieses Strukturmerkmals elementar für die antifungale Aktivität der Parnafungine ist. Diese Eigenschaft könnte den Parnafunginproduzierenden Pilzen bei der Kolonisation ihrer Wirte hilfreich sein. So verschafft die 
Sekretion von Parnafungin womöglich den Pilzen einen entscheidenden Vorteil gegenüber konkurrierenden Stämmen und könnte gleichzeitig die Interaktion mit dem Wirt vereinfachen. Während der initialen Wachstumsphase vieler Fungi sezernieren diese organische Säuren zur Reduktion des pH-Wertes ihrer Umgebung, was der Stabilität von Parnafungin zugutekommen würde. Bei Erreichen des stationären Wachstums erfolgt sukzessiv eine Alkalisierung des umgebenden Mediums und damit einhergehend die Inaktivierung des Parnafungins, bevor eine für den Pilz toxische Konzentration erreicht wird. ${ }^{[16]}$

Aufgrund des Tetrahydroxanthenon-Grundgerüsts der Parnafungine wäre neben den guten fungiziden Eigenschaften auch ein breites Spektrum an antimikrobieller Aktivität, wie es z. B. die Secalonsäuren aufweisen, naheliegend. Allerdings konnte den Parnafunginen weder eine hemmende Wirkung gegenüber Staphylococcus aureus (grampositiv) noch gegenüber Escherichia coli (gramnegativ) nachgewiesen werden. Des Weiteren weisen die CaFT-Profile von Parnafunginen und Secalonsäuren keine Ähnlichkeiten auf, sodass die spezifische antifungale Wirkung der Parnafungine auf ihrer strukturellen Gesamtheit beruht.

Der Wirkmechanismus der Parnafungine besteht in der Inhibierung der fungalen Polyadenosin-Polymerase (PAP) und verleiht der Stoffklasse ihren Namen ( $\underline{P}$ oly $(\underline{A}) \underline{R N A}$ fungin). Das Enzym ist eine Kernkomponente des eukaryotischen mRNA-Spaltungs- und Polyadenylierungskomplexes und fügt dem 3'-Ende der mRNA einen Poly(A)-Schwanz an, was eine erhöhte Stabilität der mRNA aufgrund eines erschwerten Abbaus sowie eine verbesserte Effizienz der Translation zur Folge hat. Die Inhibierung erfolgt durch eine spezifische und reversible Bindung des Parnafungins an PAP. Zusätzlich konnte mittels Affinitäts-Massenspektrometrie nachgewiesen werden, dass eine Bindung von Parnafungin A (1) im Vergleich zu Parnafungin B (2) an PAP stark präferiert ist und dieses somit die höhere Aktivität aufweist. Die Aktivitätsunterschiede zu Parnafungin $C(3)$ und $D$ (4) lassen sich auf die strukturellen Modifikationen und der damit einhergehenden geringen Änderung in der Bindung an das Enzym erklären. Des Weiteren legen die Ergebnisse der Affinitätsexperimente den Schluss nahe, dass Parnafungin A (1) direkt durch die entsprechenden Fungi produziert wird, während Parnafungin B (2) nur durch Isomerisierung zugänglich ist. Diese Hypothese wird zusätzlich durch die beiden Derivate Parnafungin C (3) und D (4) untermauert, die direkte Analoga des „linearen“ Parnafungins A (1) darstellen und aus diesem via Methylierung und Oxidation zugänglich sind. ${ }^{[20]}$ Parnafungine hemmen neben der fungalen auch die humane Poly(A)-Polymerase, zeigen aber keine generelle Fähigkeit zur Inhibierung von DNA-Polymerasen. Trotz des gleichwertigen Potentials zur Inhibierung von sowohl fungaler als auch humaner Poly(A)Polymerasen traten in in vivo Tests in einem Mausmodell mit systemischer Candidiasis 
bei potenter antimykotischer Wirkung keine beobachtbaren toxischen Nebenwirkungen auf.

Die Stoffklasse der Parnafungine besitzt großes Potential aufgrund ihres breiten Spektrums an antifungaler Aktivität und des Enzym-spezifischen Wirkmechanismus als Antimykotikum Verwendung zu finden. Jedoch sollten Wirksamkeit, chemische Stabilität und vor allem Spezifität, in Anbetracht der äquipotenten Inhibierung der humanen $\operatorname{Poly}(A)$ Polymerase und damit einhergehenden etwaigen Nebenwirkungen, durch die Entwicklung von Parnafungin-Analoga gesteigert werden. In diesem Zusammenhang liefern die Parnafungin-Derivate C (3) und D (4) erste Erkenntnisse, welche Modifikationen bei gleichzeitigem Erhalt der antimykotischen Wirkung möglich sind.

\subsubsection{Stand gegenwärtiger Forschungen}

Die Naturstoffklasse der Parnafungine ist bisher noch sehr spärlich beschrieben. Zwar konnte bereits die Struktur aufgeklärt, eine antifungale Wirksamkeit nachgewiesen und das molekulare Ziel des Wirkmechanismus identifiziert werden, jedoch befindet sich die Forschung auf den Gebieten der Biosynthese und vor allem der Totalsynthese des Wirkstoffs noch am Anfang.

Erste Ergebnisse stammen von Snider et al. aus dem Jahr 2009 und beschäftigen sich mit dem Aufbau eines labilen tetrazyklischen Isoxazolo[4,3,2-de]phenanthridinon-Gerüsts 14, wie es in den Parnafunginen vorkommt. Dieses Strukturelement konnte in einer vierstufigen Synthese mit einer Gesamtausbeute von 39\% dargestellt werden (Abbildung 5). ${ }^{[18]}$ Ausgehend von der literaturbekannten Boronsäure 10 sowie dem ebenfalls bekannten Chloraren 11 konnte das Biaryl 12 mittels einer Suzuki-Miyaura-Kreuzkupplung unter Verwendung des Buchwald-Liganden SPhos (15) erhalten werden. Anschließende Reduktion der Nitrogruppe zum Amin mithilfe von Zink überführte das Biaryl 12 in das Benzisoxazolon 13. Mesylierung der Hydroxygruppe und darauffolgende intramolekulare $\mathrm{S}_{\mathrm{N} 2}$-Reaktion lieferte die Zielverbindung 14. 
<smiles>COc1cc(CO)c(Br)c(OC)c1</smiles><smiles>COc1cc(CO)c(-c2cccc3c(=O)o[nH]c23)c(OC)c1</smiles>

13
$\mathrm{Zn}, \mathrm{NH}_{4} \mathrm{Cl}$

$\mathrm{THF} / \mathrm{MeOH} / \mathrm{H}_{2} \mathrm{O}$ Ultraschall $\mathrm{RT}, 25 \mathrm{~min}$<smiles>COc1cc(CO)c(-c2cccc(C(C)=O)c2[N+](=O)[O-])c(OC)c1</smiles>

12

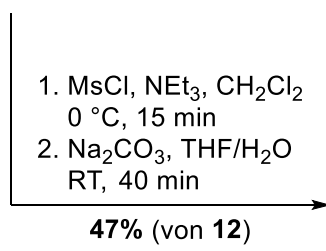

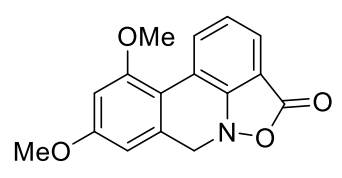

14

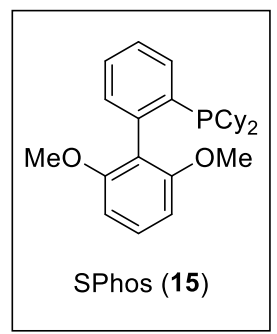

Abbildung 5: Synthese des tetrazyklischen Isoxazolo[4,3,2-de]phenanthridinon-Gerüsts (14) nach Snider.

Ebenfalls aus der Forschungsgruppe Snider stammen die Synthesen hexazyklischer Modellverbindungen der Parnafungine A (24a) und C (24b). Diese konnten in acht Stufen mit einer Gesamtausbeute von 13\% (24a) bzw. 8\% (24b) synthetisiert werden (Abbildung 6). [21] Das Xanthon 18 konnte aus Salicylsäure (16) und Orcinol (17) mittels Methansulfonsäure auf Aluminium aufgebaut werden und wurde anschließend in drei Stufen zum Phenol 19 oxidiert. lodierung mit lod und Periodsäure führte zum Aryliodid 20a sowie nach darauffolgender Methylierung der phenolischen Hydroxyfunktion zum O-methylierten Derivat 20b. Suzuki-Miyaura-Kreuzkupplung mit dem Boronsäureester 21 lieferte die Phenole 22a und 22b. Anschließend überführte die bereits beschriebene Reaktionssequenz zum Aufbau des Isoxazolo[4,3,2-de]phenanthridinon-Motivs die Phenole 22a/22b in die hexazyklischen Modellverbindungen 24a und 24b. 
<smiles>O=C(O)c1ccccc1O</smiles>

Salicylsäure (16)<smiles>Cc1cc(O)cc(O)c1</smiles>

Orcinol (17)

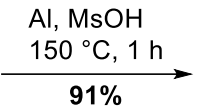

$91 \%$<smiles>Cc1cc(O)c2c(=O)c3ccccc3oc2c1</smiles>

18

$\mathbf{5 0 \%}$ (3 Stufen)

$\mathrm{I}_{2}, \mathrm{H}_{5} \mathrm{IO}_{6}$

EtOH/THF/ $/ \mathrm{H}_{2} \mathrm{O}$

RT, $40 \mathrm{~h}$
$\overbrace{\mathrm{NO}_{2} \mathrm{Me}}$

$21 \mathrm{Mel}, \mathrm{K}_{2} \mathrm{CO}_{3}$ DMF, RT, $12 \mathrm{~h} \longrightarrow 20 \mathrm{~b}: \mathrm{R}=\operatorname{Me}(\mathbf{6 9 \%})$

$\mathrm{Pd}(\mathrm{OAc})_{2}$, SPhos $\mathrm{K}_{3} \mathrm{PO}_{4}, \mathrm{THF} / \mathrm{H}_{2} \mathrm{O}$ $80{ }^{\circ} \mathrm{C}, 1 \mathrm{~h}$

22a: $\mathrm{R}=\mathrm{H}(\mathbf{5 8} \%)$

22b: $R=M e(53 \%)$

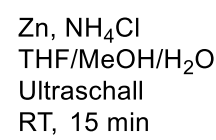

Ultraschall RT, $15 \mathrm{~min}$ $0^{\circ} \mathrm{C}, 1 \mathrm{~h}, \mathrm{CH}_{2} \mathrm{Cl}_{2}$ 2. $\mathrm{Na}_{2} \mathrm{CO}_{3}, \mathrm{THF} / \mathrm{H}_{2} \mathrm{O}$ $\mathrm{RT}, 1 \mathrm{~h}$

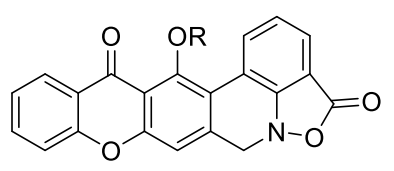

24a: $\mathrm{R}=\mathrm{H}(46 \%$ von $22 \mathrm{a})$

24b: $R=$ Me (42\% von 22b)<smiles>[R]Oc1c(-c2cccc3c(=O)o[nH]c23)c(CO)cc2oc3ccccc3c(=O)c12</smiles>

23a: $\mathrm{R}=\mathrm{H}$

23b: $R=M e$<smiles>O=c1c2ccccc2oc2cc(CO)cc(O)c12</smiles>

19
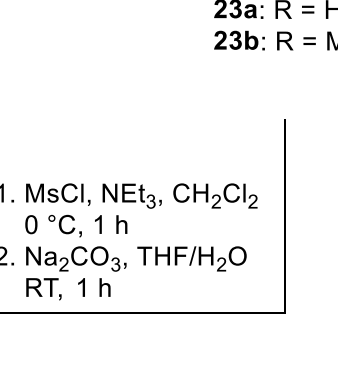

Abbildung 6: Synthese hexazyklischer Modellverbindungen der Parnafungine A (24a) und C (24b) nach Snider. 


\subsection{Die Suzuki-Miyaura-Kreuzkupplung}

Die Entwicklung Übergangsmetall-katalysierter Reaktionen zur Bildung neuer C-CBindungen führte im letzten Viertel des 20. Jahrhunderts zu einer Fülle neuer Möglichkeiten in der organischen Synthese und machen sie aufgrund der Tolerierung funktioneller Gruppen sensibler Verbindungen heutzutage zu einem unverzichtbaren Werkzeug. Vor allem Palladium-katalysierte Kreuzkupplungsreaktionen finden insbesondere in der Naturstoffsynthese verbreitet Anwendung ${ }^{[2]}$ und unter innen ragt noch einmal die Suzuki-Miyaura-Kreuzkupplung (im weiteren Verlauf mit "SMC“ abgekürzt) als die mit Abstand am häufigsten publizierte Kreuzkupplungsreaktion hervor. ${ }^{[23,24,25]}$ Ihre Relevanz in der modernen organischen Synthese spiegelt sich auch in der Verleihung des Nobelpreises 2010 an Richard F. Heck, Ei-ishi Negishi und Akira Suzuki wider. ${ }^{[26]}$

In der SMC erfolgt der Aufbau neuer C-C-Bindungen durch die Palladium-katalysierte Kupplung von Bororganylen (26) mit organischen Elektrophilen (25), wie z. B. Alkyl- oder Arylhalogeniden bzw. -triflaten unter basischer Reaktionsführung (Abbildung 7).

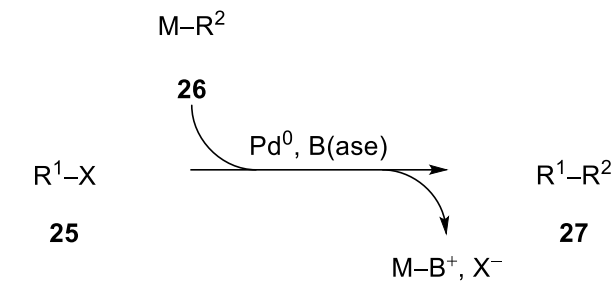

$\mathrm{R}^{1}, \mathrm{R}^{2}=$ Aryl, Vinyl, Allyl, Benzyl, Alkyl
$\mathrm{X}=\mathrm{I}, \mathrm{Br}, \mathrm{OTf}, \mathrm{Cl}$
$\mathrm{M}=\mathrm{BR}_{2}^{\prime}, \mathrm{B}\left(\mathrm{OR}^{\prime}\right)_{2}, \mathrm{~B}(\mathrm{OH})_{2}$
$\mathrm{~B}$ (ase) $=\mathrm{OH}^{-}, \mathrm{F}^{-}$

Abbildung 7: Die Suzuki-Miyaura-Kreuzkupplung.

Die SMC bietet hierbei viele Vorteile gegenüber anderen Kreuzkupplungsreaktionen. So sind die verwendeten Bororganyle meist kommerziell erhältlich oder synthetisch leicht zugänglich und beständig gegenüber Wärme, Luft und Feuchtigkeit. Des Weiteren sind Bororganyle nicht toxisch und aufgrund ihrer Eigenschaften gut zu handhaben. ${ }^{[27,28]}$ Ebenfalls vorteilhaft gestaltet sich die leichte Abtrennbarkeit der atoxischen anorganischen borhaltigen Nebenprodukte. Infolge der geringen Nukleophilie der Organoborverbindungen sowie der milden Reaktionsbedingungen wird eine Vielzahl funktioneller Gruppen in der SMC toleriert. Durch die fortschreitende Entwicklung der Liganden stehen mittlerweile Katalysatorsysteme zur Verfügung, die in der Lage sind, bereits bei Raumtemperatur und mit geringen Katalysatorbeladungen selbst unreaktive Chlorarene umzusetzen oder sterisch anspruchsvolle Biaryle aufzubauen $\left(F u,{ }^{[29,30,31]}\right.$ 
Herrmann und Beller, ${ }^{[32]}$ Buchwald $\left.{ }^{[33]}\right)$. Ferner ist die SMC nicht nur auf die $\mathrm{C}\left(\mathrm{sp}^{2}\right)-\mathrm{C}\left(\mathrm{sp}^{2}\right)$ Bindungsbildung beschränkt, mittels der B-Alkyl-SMC ist ebenfalls der Aufbau von $C\left(s p^{2}\right)-C\left(s p^{3}\right)$ - sowie $C\left(s p^{3}\right)-C\left(s p^{3}\right)$-Bindungen realisierbar. ${ }^{[34]}$ Einzig die basischen Reaktionsbedingungen können als ein Nachteil der SMC angesehen werden.

Aufgrund der genannten Vorteile ist die SMC zu einem bedeutenden Werkzeug der medizinischen Chemie sowie in der Synthese von Pharmazeutika und Feinchemikalien, auch im industriellen Maßstab, geworden und bietet sich vor allem bei der Darstellung von Biarylen oder substituierten aromatischen Gerüsten als eine sehr effiziente Methode an. ${ }^{[35]}$

\subsubsection{Mechanismus}

Der Katalysezyklus der SMC gleicht im Allgemeinen denen anderer Kreuzkupplungsreaktionen und besteht aus der Abfolge der drei Elementarschritte: oxidative Addition an $\mathrm{Pd}^{0}$ (I), Transmetallierung (II), reduktive Eliminierung (III) (Abbildung 8).

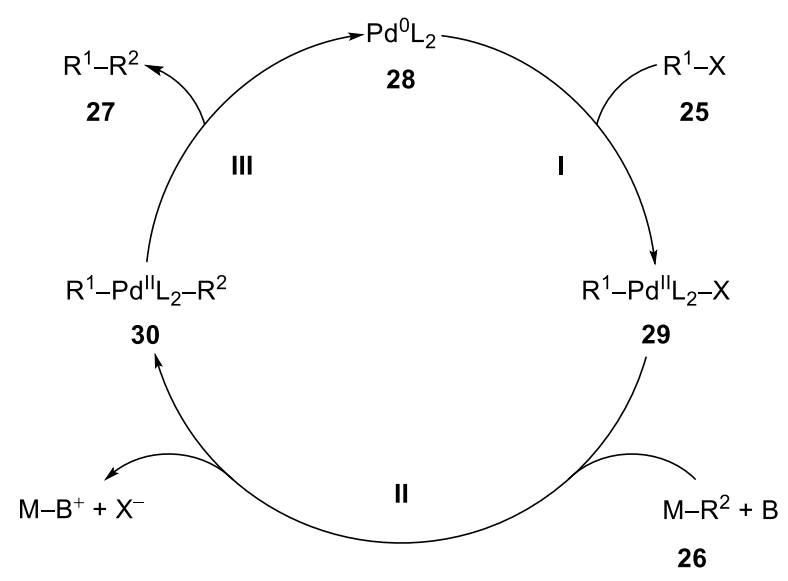

Abbildung 8: Allgemeiner Katalysezyklus der SMC.

Das Vorliegen koordinativ ungesättigten Palladiums als katalytisch aktive Spezies in der Kreuzkupplungsreaktion gilt als entscheidendes Kriterium, um eine hohe Reaktivität zu generieren. Um dies zu gewährleisten, ist entweder die Existenz eines disubstituierten 14-Elektronen- $\mathrm{Pd}^{0}-$ Komplexes $\left(\mathrm{Pd}^{0} \mathrm{~L}_{2}(\mathbf{2 8})\right)^{[36]}$ oder bei Verwendung sterisch anspruchsvoller Liganden (z. B. SPhos) einer monosubstituierten 12-Elektronen-Pd ${ }^{0}$-Spezies $\left(\mathrm{Pd}^{0} \mathrm{~L}\right)$ denkbar. ${ }^{[30,31,37]}$ Im zweiten Fall wird die Reaktivität obendrein durch den Elektronenmangel der $\mathrm{Pd}^{0}$-Spezies erhöht. Die Generierung der katalytisch aktiven Spezies kann zum einen durch die Zugabe eines $\mathrm{Pd}^{0}$-Präkatalysators (z. B. $\mathrm{Pd}\left(\mathrm{PPh}_{3}\right)_{4}$ ) unter anschließender Abspaltung von Liganden und zum anderen durch in situ Reduktion einer

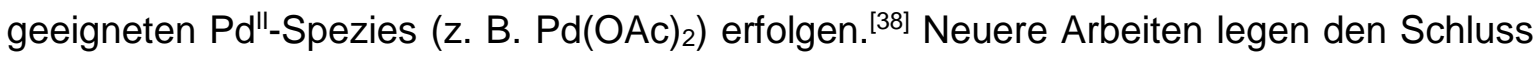


nahe, dass auch dreifach koordinierte, anionische Palladiumkomplexe entsprechende katalytische Eigenschaften aufweisen können. ${ }^{[39]}$

\subsubsection{Oxidative Addition}

Die oxidative Addition von organischen Elektrophilen 25 an den $\mathrm{Pd}^{0}-$ Komplex 28/32 unter Ausbildung der $\sigma-\mathrm{R}^{1}-\mathrm{Pd}^{\prime \prime}$-Spezies $\mathbf{3 1 / 3 3}$ stellt den ersten Schritt im Katalysezyklus dar. Hierfür werden verschiedene Mechanismen diskutiert. In Abhängigkeit des organischen Rests (Alkyl oder Aryl) des verwendeten Elektrophils 25 kann dieser Elementarschritt entweder über eine einstufige Insertion des Palladiums in die $\mathrm{R}^{1}-\mathrm{X}$-Bindung oder über eine zweistufige $S_{N} 2$-artige ionische Reaktion ablaufen. Für Arylhalogenide, die häufig in SMCs zum Aufbau von Biarylen zum Einsatz kommen, wird eine konzertierte Wechselwirkung zwischen der reaktiven $\mathrm{Pd}^{0}$-Spezies 28/32 und dem Arylhalogenid $\mathrm{R}^{1}-\mathrm{X}$ 25 in einem Dreizentren-Übergangszustand 31/33 angenommen. Bei der Verwendung monodentater Liganden kann der ursprünglich gebildete cis-Komplex cis-29/34 anschließend aufgrund der destabilisierenden Interaktion gegenüberliegender Phosphorund Aryl-Liganden zum thermodynamisch stabileren trans-Komplex trans-29 isomerisieren. Mehrzähnige Liganden unterdrücken infolge ihrer strukturell bedingten Unfähigkeit zur Umlagerung die Isomerisierung (Abbildung 9).

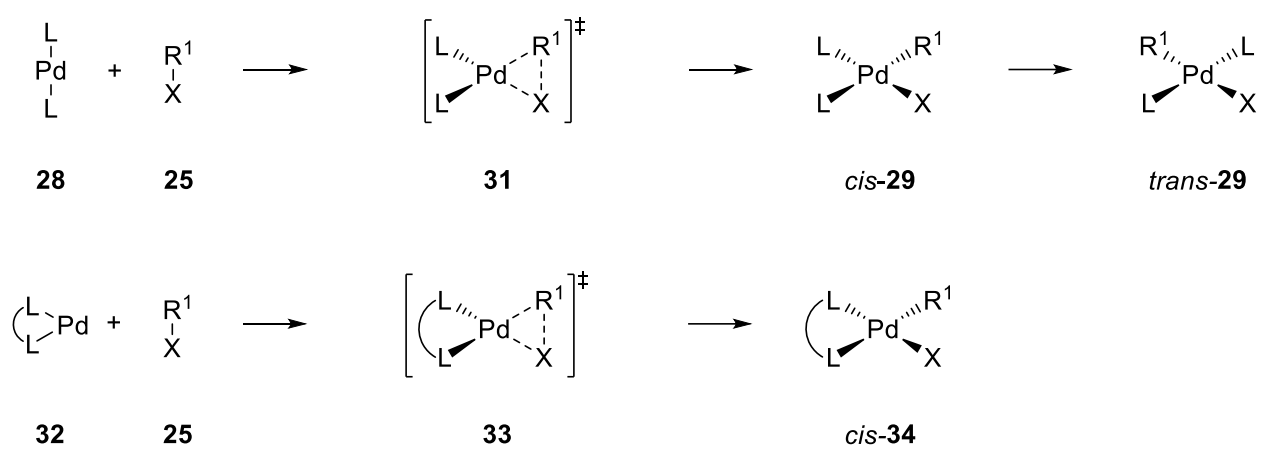

Abbildung 9: Mechanismus der oxidativen Addition.

Die Reaktivität der Elektrophile 25 bezüglich der oxidativen Addition nimmt in der Reihenfolge $\mathrm{I}>\mathrm{Br} \approx \mathrm{OTf}>>\mathrm{Cl}$ angesichts der zunehmenden Stabilität der $\mathrm{R}^{1}-\mathrm{X}$-Bindung ab. Die Geschwindigkeit des Ablaufs der oxidativen Addition für aromatische Substrate hängt außerdem von etwaigen Substituenten und infolgedessen von einer veränderten Elektronendichte am Aromaten ab. So bewirken elektronenziehende Substituenten eine beschleunigte oxidative Addition, während elektronenschiebende zu einer Verlangsamung führen. Zusätzlich kann die oxidative Addition durch den Einsatz elektronenreicher Liganden (z. B. $\mathrm{P} t \mathrm{Bu}_{3}$ ) und der damit einhergehenden Erhöhung der Elektronendichte am Palladium beschleunigt werden. 


\subsubsection{Transmetallierung}

Im Gegensatz zu den beiden anderen Schritten des Katalysezyklus wird der Mechanismus der Transmetallierung der SMC noch nicht vollständig verstanden. Verglichen mit anderen Kreuzkupplungsreaktionen ist der Einsatz einer Base für die Palladium-katalysierte C-C-Bindungsknüpfung zwischen einem Bororganyl und einem organischen Elektrophil erforderlich, was jedoch auch eine stark beschleunigte Transmetallierung zur Folge hat. In diesem Zusammenhang werden hauptsächlich zwei Reaktionspfade in der Literatur diskutiert, die sich in dem Zeitpunkt des Eingreifens der Base in den Elementarschritt unterscheiden. ${ }^{[24,25]}$ Zum einen könnte ein Austausch des Halogenids im Pd"-Komplex 29 durch ein Hydroxid- oder Fluorid-lon zur Ausbildung des Komplexes 35 führen, der infolge seiner Basizität und der Oxo- bzw. Fluorophilie des Boratoms direkt mit dem Bororganyl 26 reagieren kann. Zum anderen könnte ein quartärer anionischer at-Komplex $\mathbf{3 6}$ aus der Reaktion der Base mit dem Bororganyl $\mathbf{2 6}$ in situ generiert werden, dessen organischer Rest aufgrund der gesteigerten Nukleophilie leichter während der Transmetallierung übertragen werden kann (Abbildung 10).

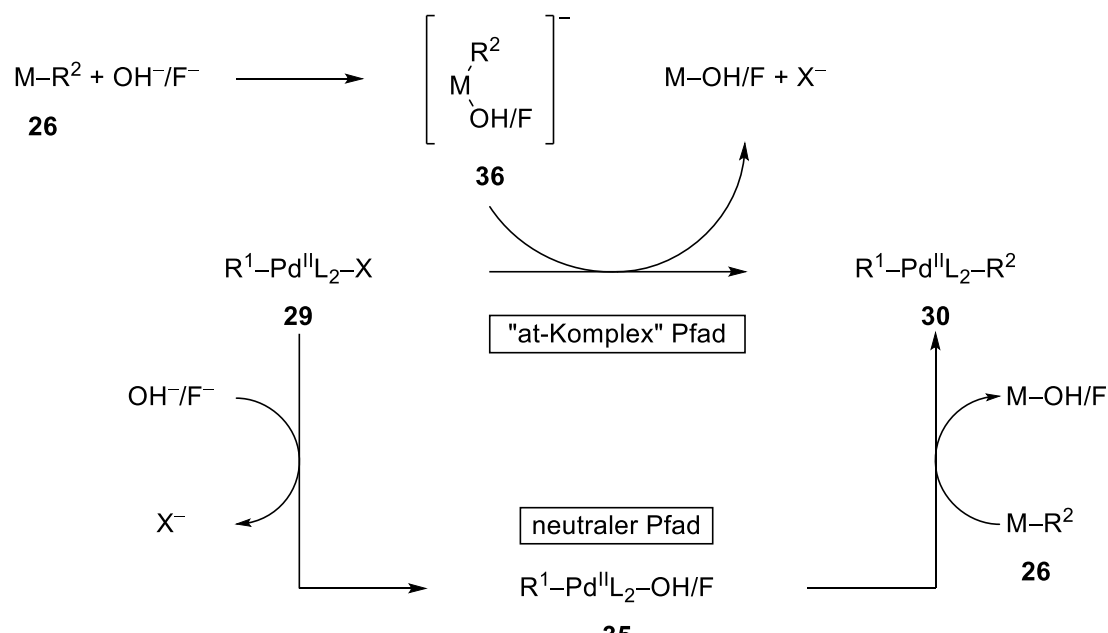

35

Abbildung 10: Hauptsächlich diskutierte mechanistische Abläufe der Transmetallierung.

Welcher dieser beiden Pfade beschritten wird, wurde bisher von den Substituenten am Bor sowie der verwendeten Base abhängig gemacht. Neuere Erkenntnisse auf Basis kinetischer Untersuchungen unter Verwendung von Zyklovoltametrie sowie NMRExperimenten deuten indes daraufhin, dass die Transmetallierung der SMC über die Ausbildung des Pd"-Komplexes 35 verläuft. ${ }^{[40-42,43]}$ So konnte nachgewiesen werden, dass der Pd"-Komplex 35 aufgrund der Oxophilie des Bors eine Präkomplexierung des Bororganyls 26 bewirkt und dadurch eine höhere Reaktivität verglichen mit dem Pd"Komplex 29 aufweist. Des Weiteren konnte dem im Gleichgewicht mit dem Bororganyl 26 stehenden anionischen Borat 36 keine erhöhte Reaktivität infolge einer erhöhten 
Nukleophilie des organischen Rests attestiert werden. Durch die Beteiligung der HydroxidIonen sowohl an der Ausbildung der reaktiveren Pd"-Spezies 35 als auch an der konkurrierenden Gleichgewichtsreaktion zum unreaktiven Borat 36 bestimmt das Verhältnis der Konzentration der Hydroxid-Ionen zur Konzentration der organischen Borverbindung 26 die Geschwindigkeit, mit der die Transmetallierung verläuft. Somit agieren Hydroxid-lonen in der SMC nicht als typische Base, sondern treten als Ligand im Pd"-Komplex 35 in Erscheinung (Abbildung 11).

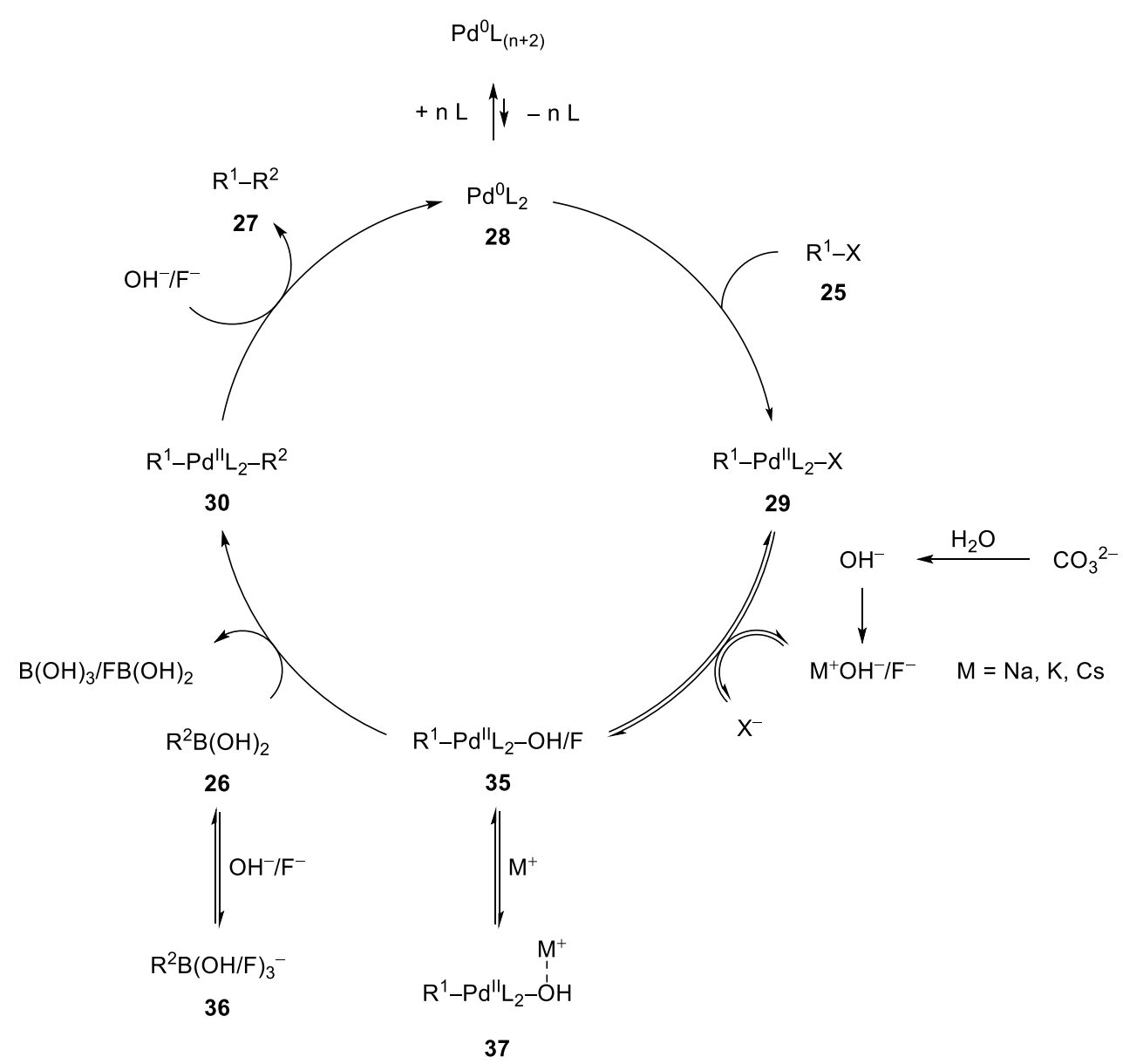

Abbildung 11: Postulierter Mechanismus der SMC nach Amatore und Jutand.

Zusätzlich konnten zwei weitere Faktoren identifiziert werden, die Einfluss auf die Geschwindigkeit der Transmetallierung nehmen. Beim Einsatz nicht-Hydroxid-basierter anorganischer Basen nehmen die Anionen nicht direkt an der Reaktion teil und die SMC verläuft bestenfalls sehr langsam. Die Verwendung von Wasser als zusätzliches Additiv und die damit einhergehende in situ Generierung von Hydroxid-lonen, die wiederum zur Bildung des reaktiveren Pd"-Komplexes 35 führt, bewirkt eine Beschleunigung der Reaktion. Den Kationen konnten sowohl positive als auch negative Effekte auf die Reaktionsgeschwindigkeit nachgewiesen werden. So verschieben $\mathrm{Ag}^{+}$und $\mathrm{Tl}^{+}$das Gleichgewicht zwischen den Pd"-Komplexen 29 und $\mathbf{3 5}$ auf die Seite des Letztgenannten, 
indem sie das nach dem Austausch durch ein Hydroxid-lon freigegebene Halogenid in Form eines unlöslichen Silber- oder Talliumsalzes binden und aus dem Gleichgewicht entfernen. Dadurch sollte sich die Konzentration der reaktiveren Pd"-Spezies 35 und infolgedessen auch die Geschwindigkeit der Transmetallierung erhöhen. Es konnte allerdings auch eine Verlangsamung der Reaktionsgeschwindigkeit durch die Komplexierung des Hydroxo-Liganden am Pd"-Komplex 37 durch bestimmte Kationen in Konkurrenz zur Präkomplexierung mit dem Bororganyl $\mathbf{2 6}$ beobachtet werden. Die Stärke dieses Effekts nimmt in der Reihenfolge $\mathrm{Na}^{+}>\mathrm{Cs}^{+}>\mathrm{K}^{+}>n \mathrm{Bu}_{4} \mathrm{~N}^{+}$ab.

\subsubsection{Reduktive Eliminierung}

Aus dem vorangegangenen Transmetallierungsschritt geht zunächst der trans-Pd"Komplex trans-30 hervor, dem eine gewisse Stabilität nachgewiesen wurde. Dieser experimentelle Befund wird zusätzlich durch DFT-Berechnungen gestützt. Damit die reduktive Eliminierung unter $\mathrm{C}-\mathrm{C}$-Bindungsbildung und anschließender Freisetzung des Kreuzkupplungsprodukts sowie der Regeneration der katalytisch aktiven $\mathrm{Pd}^{0}$-Spezies 28 erfolgen kann, ist initial eine endergonische und somit langsam ablaufende Isomerisierung zum cis-Komplex cis-30 notwendig. Hydroxid-Ionen können die reduktive Eliminierung dabei unter Ausbildung eines temporären fünffach koordinierten Pd"-Komplexes $\mathbf{3 8}$ katalysieren, wodurch eine Beschleunigung des Elementarschrittes unter Umgehung der langsamen trans/cis-Isomerisierung erreicht wird. ${ }^{[39-41,44]}$ Während der reduktiven Eliminierung erfolgt die Übertragung des organischen Restes $R^{2}$ auf das organische Elektrophil unter Retention der Stereochemie (Abbildung 12). ${ }^{[45,46]}$

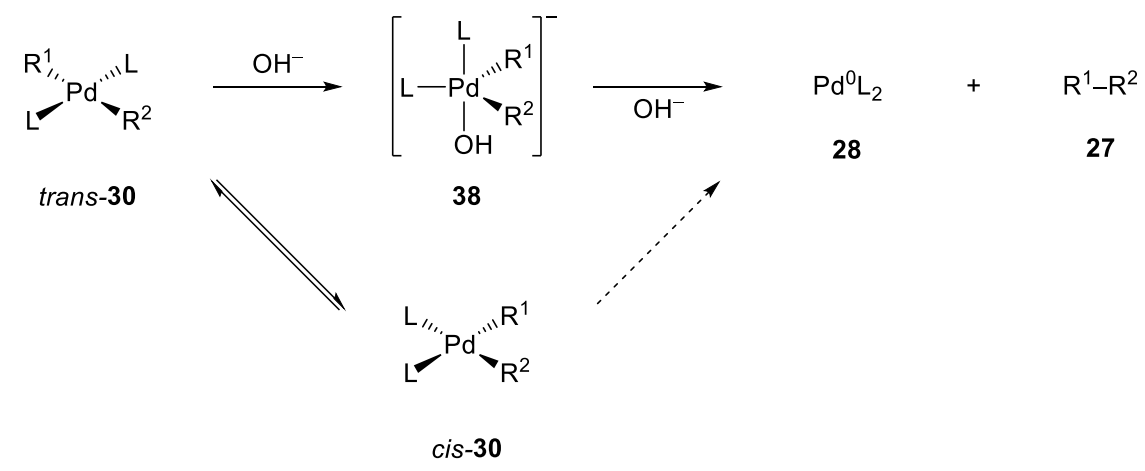

Abbildung 12: Mechanistischer Verlauf der reduktiven Eliminierung.

\subsubsection{Synthese der Borverbindungen}

Die benötigten Bororganyle sind auf vielfältige und meist einfache Weise synthetisch zugänglich. ${ }^{[25,27]}$ So können Organoborverbindungen $\mathbf{4 0}$ via Hydroborierung mit beispielsweise 9-BBn (41) oder Catecholboran (42) aus Alkinen und Alkenen 39 
synthetisiert werden. Die Hydroborierung verläuft dabei unter anti-MarkownikowRegioselektivität. Des Weiteren können aus organischen Halogeniden 43 erhaltene Grignard-Verbindungen $\mathbf{4 4}$ sowie mittels Lithium-Halogen-Austausch gewonnene Lithiumverbindungen 45 mit Trialkoxyboranen zu den jeweiligen Bororganylen 46 umgesetzt werden (Abbildung 13). Aufgrund der teils harschen Reaktionsbedingungen zur Herstellung der reaktiven Metallorganyle bietet dieses Verfahren jedoch nur eine reduzierte Toleranz gegenüber funktionellen Gruppen.

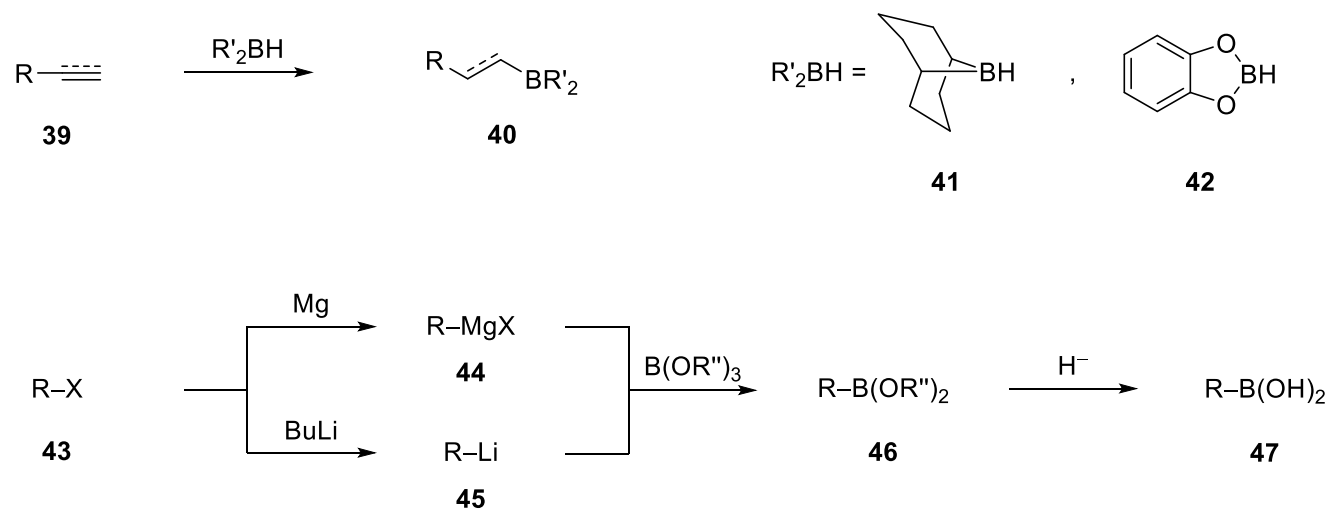

Abbildung 13: Synthesemethoden zur Darstellung von Bororganylen.

Eine weitere Möglichkeit der Synthese organischer Borverbindungen stellt die MiyauraBorylierung dar. Hierbei werden in einer Palladium-katalysierten Kreuzkupplungsreaktion Arylhalogenide oder -triflate $\mathbf{4 8}$ mit Tetraalkoxyboranen, wie z. B. Bis(pinakolato)diboran (50) oder Bis(catecholato)diboran respektive den wesentlich günstigeren monomeren Varianten z. B. Pinakolboran (49) zu den entsprechenden Boronsäureestern 51 umgesetzt (Abbildung 14). ${ }^{[4]}$

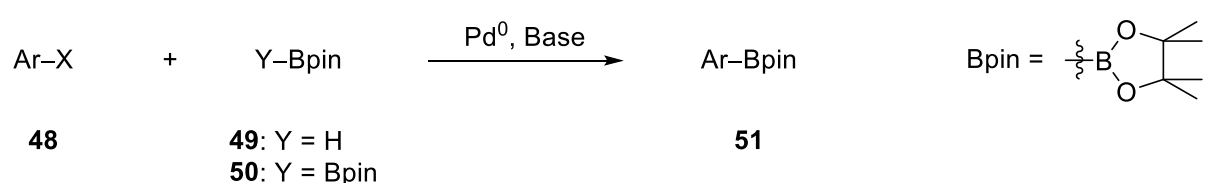

Abbildung 14: Miyaura-Borylierung.

Es wird angenommen, dass der Katalysezyklus analog zu dem der SMC verläuft. Zunächst erfolgt die oxidative Addition des Arylhalogenids an den $\mathrm{Pd}^{0}$-Katalysator unter Ausbildung der $\sigma$-Aryl-Pd"-Spezies. Darauffolgende Substitution des Halogenids durch die Lewis-Base $\mathrm{OR}^{-}$resultiert in einem reaktiveren $\mathrm{Pd}^{\prime \prime}-K o m p l e x$, der durch die Präkomplexierung des Borans eine effizientere Transmetallierung ermöglicht. Abschließende reduktive Eliminierung führt zur Freisetzung des Boronsäureesters und Regeneration der katalytisch aktiven Spezies. Die Vorteile dieser Darstellungsmethode lassen sich ebenso wie der Mechanismus von der SMC ableiten. Vor allem die milden Reaktionsbedingungen 
und die damit einhergehende Toleranz gegenüber einer Vielzahl funktioneller Gruppen eröffnen einen Weg zu funktionalisierten Bororganylen ohne zusätzliche Schützung und Entschützung. Aufgrund der sich ähnelnden Reaktionsbedingungen lassen sich asymmetrische Biaryle aus zwei verschiedenen Arylhalogeniden in einer zweistufigen Sequenz bestehend aus Miyaura-Borylierung und darauffolgender SMC in einem Eintopfverfahren realisieren.

Eine weitere Möglichkeit der Darstellung von Bororganylen stellt die Iridium-katalysierte C-H-Aktivierung von Benzolderivaten dar (Abbildung 15). ${ }^{[48]}$

$$
\begin{aligned}
& \mathrm{Ar}-\mathrm{H}+\mathrm{Y} \text {-Bpin } \longrightarrow \text { Ir } \mathrm{r}^{\mathrm{II}} \longrightarrow \quad \text { Ar-Bpin } \\
& 52 \quad 49: Y=H \quad 51
\end{aligned}
$$

Abbildung 15: Iridium-katalysierte Darstellung von Bororganylen.

\subsubsection{Anwendungen in der organischen Synthese}<smiles>COc1cc(OC)c2c(c1)OB(O)OC2COc1ccccc1</smiles>

53<smiles>COc1ccc([C@@H](NC(C)=O)C(C)(C)C)cc1I</smiles>

54
$\mathrm{Pd}\left(\mathrm{PPh}_{3}\right)_{4}, \mathrm{Na}_{2} \mathrm{CO}_{3}$

$\mathrm{PhMe} / \mathrm{MeOH} / \mathrm{H}_{2} \mathrm{O}$

$90{ }^{\circ} \mathrm{C}, 4 \mathrm{~h}$

$84 \%, 55 a / 55 b=2: 1$ 
In der Totalsynthese des Vancomycin-Aglykons (56) konnten Nicolaou und Mitarbeiter mittels einer intermolekularen atropselektiven SMC der Boronsäure $\mathbf{5 3}$ mit dem Aryliodid 54 das gewünschte Biaryl 55a in einer Gesamtausbeute von 84\% und einer Selektivität von 2:1 für das favorisierte Stereoisomer realisieren. Nach chromatographischer Trennung vom Mindermengenstereoisomer 55b wurde das Biaryl 55a in mehreren Schritten in das Vancomycin-Aglykon (56) überführt (Abbildung 16). ${ }^{[49]}$

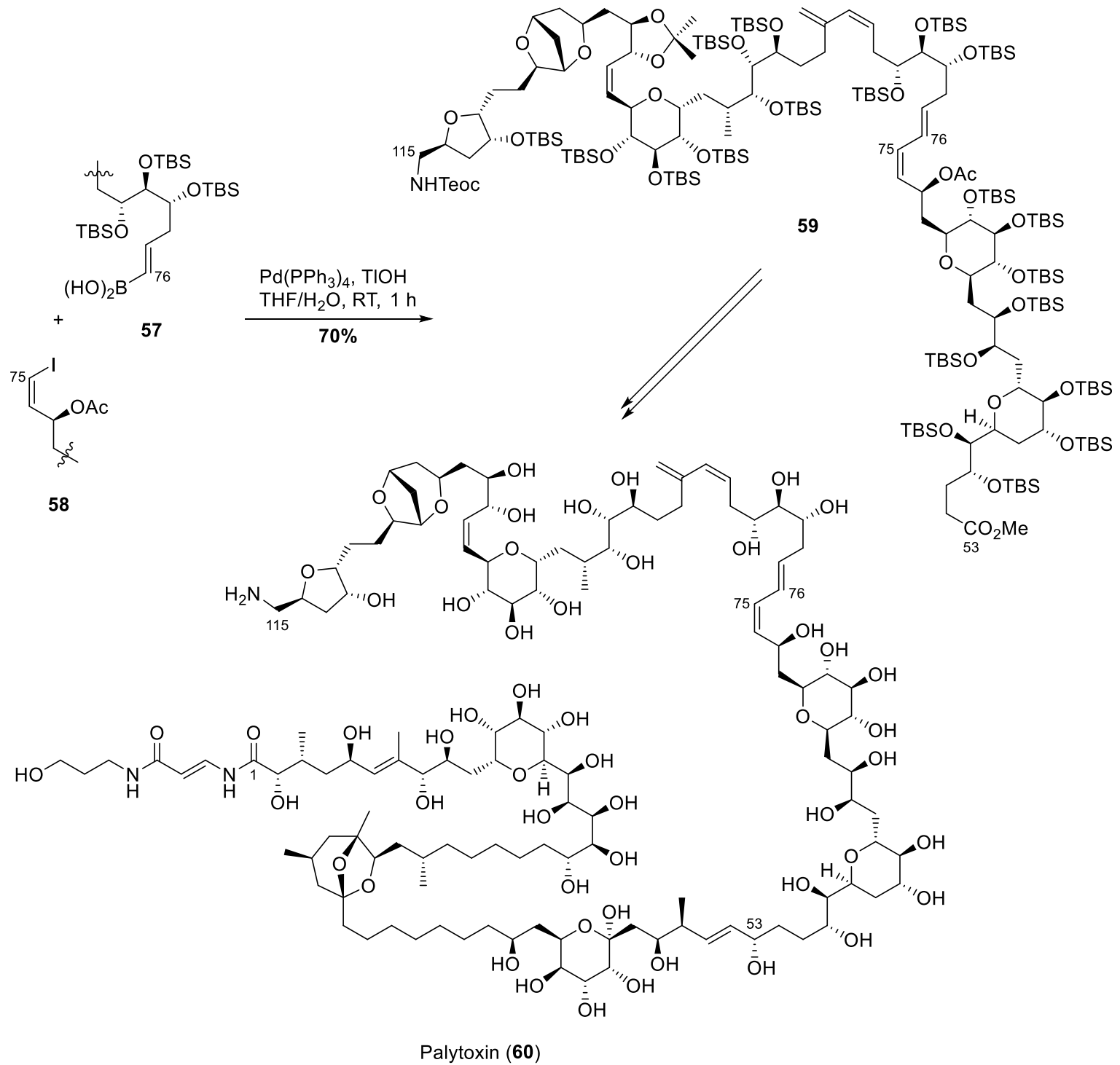

Abbildung 17: Synthese des Fragments 59 in der Totalsynthese des Palytoxin (60) durch Kishi.

Als eine der herausragendsten Arbeiten in der Geschichte der Totalsynthese gilt die durch Kishi et al. durchgeführte Synthese des marinen Naturstoffs Palytoxin (60). Mit 71 stereogenen Elementen (64 Stereozentren sowie sieben Doppelbindungen) besitzt es die höchste Anzahl dieser, unter allen bisher synthetisierten, nicht polymeren oder aus Aminosäuren aufgebauten Naturstoffen sowie das höchste Molekulargewicht in dieser Kategorie. In einem entscheidenden Schlüsselschritt wurden mittels einer SMC das 
Alkenyliodid 58 und die Boronsäure 57 zu dem Fragment 59 mit einer Ausbeute von $70 \%$ verknüpft (Abbildung 17). ${ }^{[0,51]}$ Besonderes Augenmerk gilt hierbei der verwendeten Base, Thalliumhydroxid und der Reaktionsführung bei Raumtemperatur. 


\subsection{Die Wacker-Oxidation}

Als Wacker- oder Wacker-Hoechst-Verfahren wird der von Smidt und Mitarbeitern am Consortium für elektrochemische Industrie, dem zentralen Forschungsableger der Wacker Chemie, 1959 entwickelte Prozess der $\mathrm{PdCl}_{2}-$ katalysierten aeroben oxidativen Kupplung von Ethylen und Wasser bezeichnet. Die Intention hinter dieser Entwicklung lag in der Schaffung eines großtechnischen Verfahrens zur Produktion von Acetaldehyd, einem bedeutenden Zwischenprodukt in der Synthese von Essigsäure sowie wichtigen $\mathrm{C}_{4^{-}}$ Verbindungen. ${ }^{[52]}$ Das Fundament der Wacker-Oxidation bereitete jedoch bereits Phillips im Jahre 1894 mit der Entdeckung der Oxidation von Ethylen in wässriger Lösung unter Einsatz stöchiometrischer Mengen von $\mathrm{PdCl}_{2} \cdot{ }^{[53]}$ Dem Makel der stöchiometrischen Verwendung des $\mathrm{PdCl}_{2}$ dieser Reaktion nahmen sich Smidt und Mitarbeiter gut 60 Jahre später an und reoxidierten das anfallende inaktive $\mathrm{Pd}^{0}$ mithilfe von $\mathrm{CuCl}_{2}$, das wiederum per Oxidation mit molekularem Sauerstoff regeneriert und damit katalytisch eingesetzt werden konnte, zur aktiven Pd"-Spezies. Die Leistung der Gruppe um Smidt liegt somit in der Umwandlung der von Phillips entdeckten Reaktion in einen hocheffizienten katalytisch geführten Prozess, bestehend aus drei Teilreaktionen, zur Oxidation von Ethylen zu Ethanal unter Verwendung von Luftsauerstoff begründet (Abbildung 18).

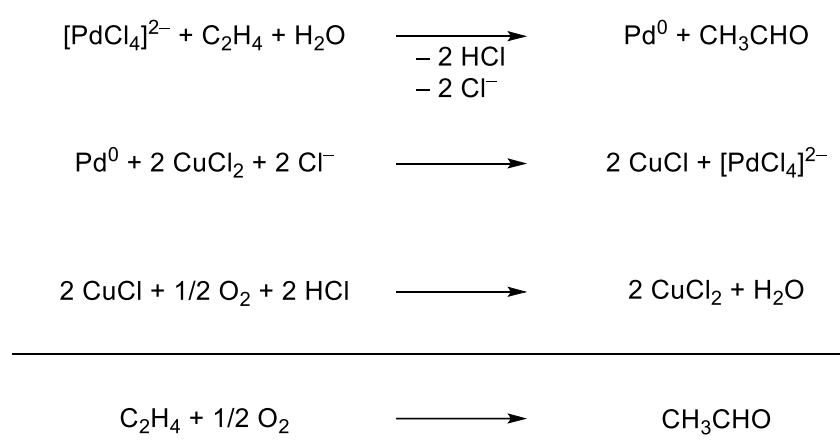

Abbildung 18: Wacker-Oxidation des Ethylen.

Des Weiteren konnten Smidt et al. die Herkunft des Carbonylsauerstoffs auf das Wasser zurückführen, weshalb angenommen wurde, dass der Pd"-katalysierte nukleophile Angriff des Wassers auf das Ethylen in einem Hydroxypalladierungsschritt erfolgt.

Die Relevanz des Wacker-Prozesses in der heutigen chemischen Industrie schwindet zusehends, infolge einer steigenden Produktion von Essigsäure durch die Carbonylierung von Methanol (Monsanto-Prozess) ${ }^{[54]}$ sowie der Herstellung von $\mathrm{C}_{4}$-Verbindungen durch die Hydroformylierung von Propylen (Oxosynthese). ${ }^{[55]}$ Nichtsdestotrotz stellt die WackerOxidation immer noch ein hochaktives Forschungsfeld dar, was sich in den zahlreichen Anwendungen in der organischen Synthese widerspiegelt. ${ }^{[56,57]}$ 


\subsubsection{Mechanismus}

Der Mechanismus der Wacker-Oxidation und insbesondere der Ablauf der Hydroxypalladierung werden seit ihrer Entdeckung kontrovers in der Literatur diskutiert. ${ }^{[58,59]}$ Hierbei wurden in Abhängigkeit der Reaktionsbedingungen experimentelle und theoretische Belege sowohl für einen intramolekularen syn-Angriff (I) eines koordinierten Moleküls Wasser respektive eines Hydroxo-Liganden als auch für einen intermolekularen anti-Angriff (II) eines exogenen Sauerstoffnukleophils erbracht (Abbildung 19). ${ }^{[60]}$

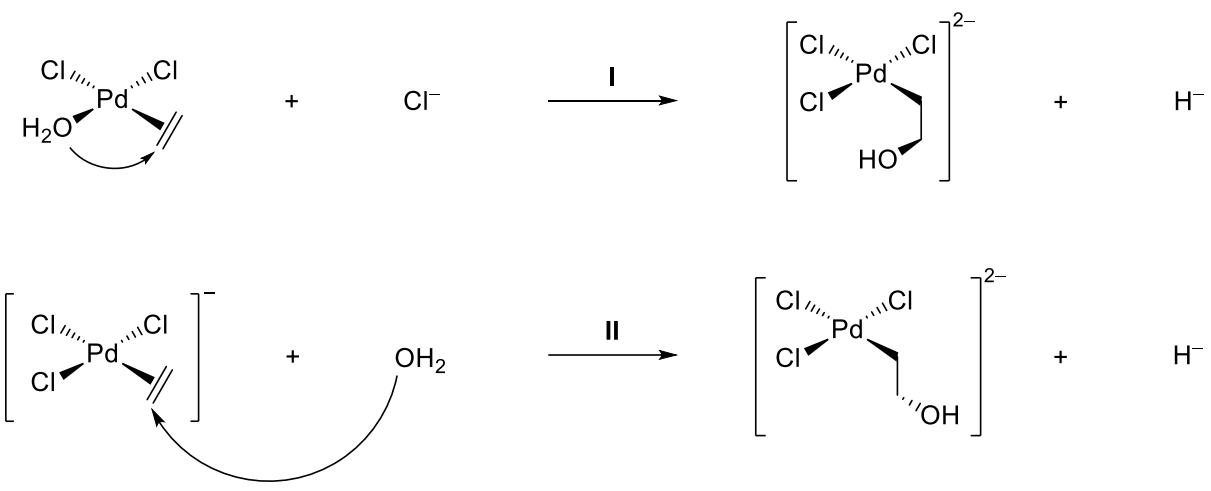

Abbildung 19: Stereochemische Reaktionsmöglichkeiten der Hydroxypalladierung.

Zur Klärung der Frage, welcher Reaktionspfad beschritten wird, wäre die Dokumentation der stereochemischen Information des Hydroxypalladierungsschrittes vonnöten. Diese Information geht indes während der Reaktion durch die Oxidation von Ethylen zum achiralen Acetaldehyd, mit seinem $\mathrm{sp}^{2}$-hybridisierten Carbonylkohlenstoff, verloren. Anhand der unzähligen kinetischen, stereochemischen und theoretischen Studien zu diesem Thema lassen sich allerdings gewisse Leitsätze definieren, unter welchen Bedingungen die Reaktion dem einen oder dem anderen Reaktionspfad folgt. Hohe Konzentrationen an $\mathrm{Cl}^{-}(>3 \mathrm{M})$ und $\mathrm{CuCl}_{2}$ (>2.5 M) führen sowohl zur Bildung von Acetaldehyd als auch Chlorhydrin über einen anti-Angriff des Sauerstoffnukleophils am Ethylen. Hingegen führen niedrige Konzentrationen an $\mathrm{Cl}^{-}$und $\mathrm{CuCl}_{2}(<1 \mathrm{M})$, wie sie in der industriellen Reaktionsführung vorgesehen sind, höchstwahrscheinlich zu einem intramolekularen syn-Angriff während der Hydroxypalladierung. ${ }^{[58,59]}$ Einen Katalysezyklus, zur Abhandlung des letztgenannten Falles, der auch als inner sphere Mechanismus bezeichnet wird, wurde von Goddard et al. vorgeschlagen (Abbildung 20). ${ }^{[61]}$ 


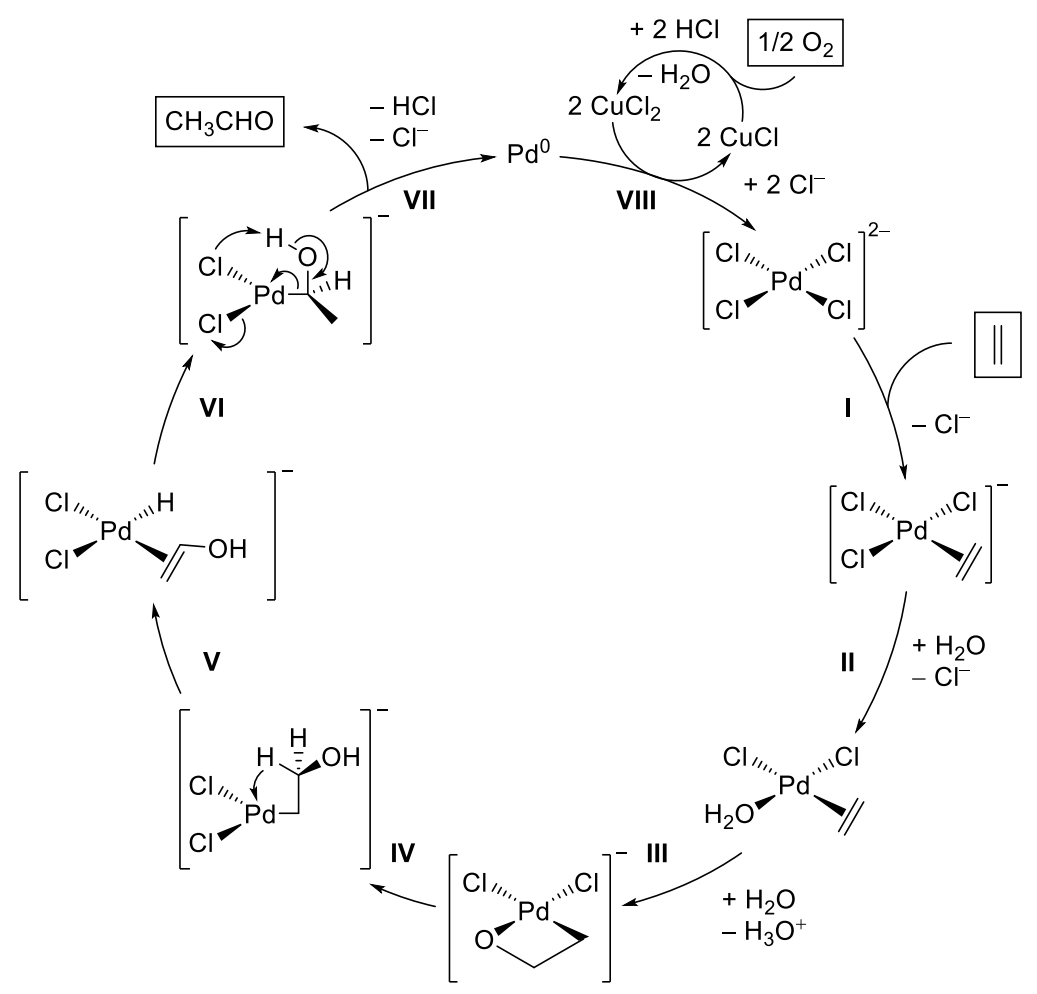

Abbildung 20: Inner sphere Katalysezyklus nach Goddard.

Den ersten Schritt im Katalysezyklus stellt die Koordination des Ethylens an $\left[\mathrm{PdCl}_{4}\right]^{2-}$ (I) und die damit einhergehende Ausbildung eines 16-Elektronen- $\pi-K o m p l e x e s$ dar. ${ }^{[62]}$ Der darauffolgende Ligandenaustausch eines Chlorid-lons durch ein Molekül Wasser (II) bereitet die Bühne für die intramolekulare syn-Hydroxypalladierung, die unter simultaner Deprotonierung durch ein weiteres Molekül Wasser einen viergliedrigen Palladazyklus (III) generiert. Rotation um $120^{\circ}$ um die C-C-Bindung (IV) ermöglicht in der Folge eine $\beta$-Hydrideliminierung (V), die wiederum eine Reinsertion in die Doppelbindung (VI) bedingt. Abschließende Chlorid-vermittelte reduktive Eliminierung setzt Ethanal als Produkt sowie eine $\mathrm{Pd}^{0}$-Spezies (VII) frei, die mithilfe des gekoppelten $\mathrm{CuCl}_{2} / \mathrm{O}_{2}$ Redoxsystems zum aktiven $\left[\mathrm{PdCl}_{4}\right]^{2-}$ reoxidiert wird (VIII).

Abgesehen von seiner Eigenschaft $\mathrm{Pd}^{0}$ zu oxidieren, ist die Rolle des $\mathrm{CuCl}_{2}$ nicht vollständig aufgeklärt. So konnten zum einen Hosokawa et al. die Ausbildung von Pd-CuBimetallkomplexen als aktive Spezies in der Wacker-Oxidation nachweisen ${ }^{[63-65]}$ und zum anderen neuere experimentelle ${ }^{[66]}$ sowie theoretische Studien ${ }^{[67]}$ einen anti-Angriff während der Hydroxypalladierung unter kupferfreier Reaktionsführung aufzeigen.

\subsubsection{Anwendungen in der organischen Synthese}

Ursprünglich als großtechnischer Prozess zur Gewinnung von Acetaldehyd entwickelt, ist die Wacker-Oxidation angesichts der Toleranz gegenüber Luft und Feuchtigkeit sowie 
einer Vielzahl funktioneller Gruppen von großer Bedeutung in der organischen Synthese. Die Wacker-Oxidation sieht prinzipiell die Verwendung katalytischer Mengen einer Palladiumquelle und optional eines Liganden in polaren organischen Lösungsmitteln, wie $\mathrm{DMF} / \mathrm{H}_{2} \mathrm{O}$, DMSO, 1,4-Dioxan oder Alkoholen vor. Als Oxidantien zur Regeneration der katalytisch aktiven $\mathrm{Pd}^{\prime \prime}$-Spezies kommen vorrangig Kombinationen von Sauerstoff mit Kupfersalzen, ${ }^{[68]} p$-Benzochinon, ${ }^{[69]} \mathrm{DMSO} /{ }^{6}{ }^{6}$ auerstoff ${ }^{[70]}$ und $\mathrm{AcOH} / \mathrm{tBuOOH}^{[71]}$ zum Einsatz. Im Gegensatz zu terminalen Olefinen, die fast ausschließlich zu den jeweiligen Methylketonen abreagieren, benötigt die regioselektive Oxidation interner Doppelbindungen das Vorhandensein einer dirigierenden Gruppe. ${ }^{[72]}$

In der enantioselektiven Totalsynthese des Antibiotikums Platencin (63) nutzten Nicolaou und Mitarbeiter eine Wacker-Oxidation zur Einführung der Ketofunktionalität am Kohlenstoff C-5 (Abbildung 21). ${ }^{[73]}$

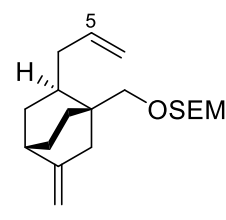

61

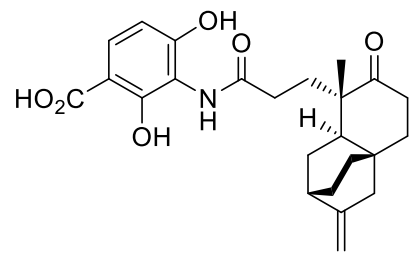

Platencin (63)

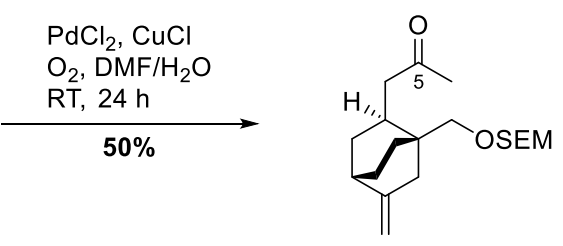

62

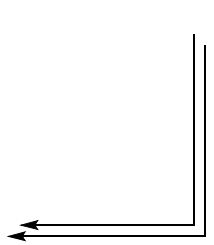

Abbildung 21: Wacker-Oxidation in der enantioselektiven Totalsynthese des Platencin (63) durch Nicoloau.

Die intramolekulare Wacker-Oxidation bietet zusätzlich eine nützliche Methode zur Synthese sauerstoff- und stickstoffhaltiger Heterozyklen. ${ }^{[74}$ Wie in der Wacker-Oxidation des Ethylens kann der intramolekulare Angriff des Sauerstoff- oder Stickstoffnukleophils auf das Alken entweder syn oder anti erfolgen. In Anbetracht des Aufbaus neuer stereogener Zentren, der häufig im Zuge von Oxy- oder Aminopalladierungen stattfindet, verwundert es nicht, dass sich diese Methode großer Beachtung in der chemischen Forschung der letzten Jahrzehnte erfreute. ${ }^{[56]}$ Nach derzeitiger Auffassung verläuft der Palladierungsschritt für das Gros dieser Reaktionen über einen syn-Angriff. Der stereochemische Ausgang der Zyklisierung kann jedoch bereits durch geringe Änderungen der Reaktionsbedingungen oder der Substratstruktur beeinflusst werden. 
Zum effizienten Aufbau des Tetrahydrofuran-Rings $\mathrm{C}$ in der Totalsynthese des Garsubellin A (66) griffen Shibasaki et al. auf eine Wacker-Zyklisierung zurück. Nach dem Entfernen der Acetonid-Schutzgruppe führte der Palladium-vermittelte Angriff der freigesetzten sekundären Hydroxygruppe an C-18 zur Ausbildung des C-Rings (Abbildung 22). ${ }^{[75]}$

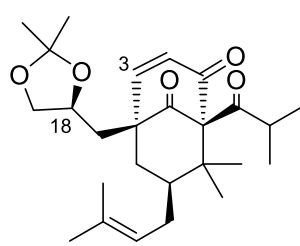

64
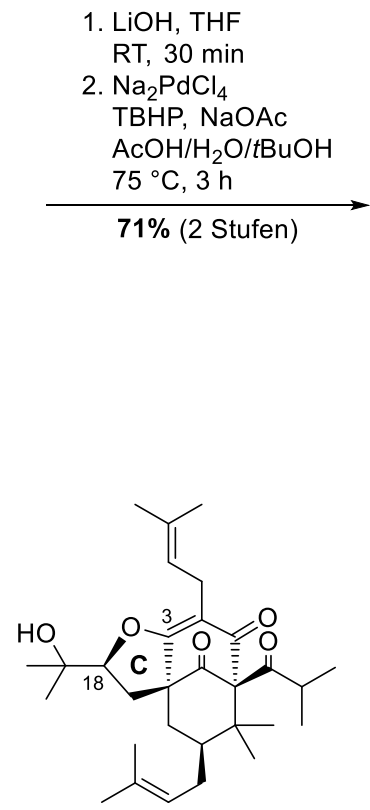

Garsubellin A (66)

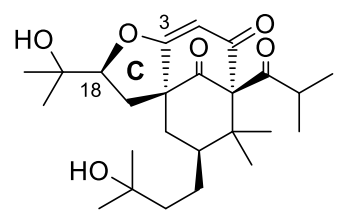

65

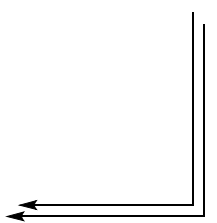

Abbildung 22: Wacker-Zyklisierung zum Aufbau des Tetrahydrofuran-Rings $C$ in der racemischen Totalsynthese des Garsubellin A (66) durch Shibasaki.

Ein weiteres vielbeachtetes Charakteristikum der intramolekularen Nukleopalladierung stellt die Partizipationsmöglichkeit des gebildeten $\sigma$-Pd"-Intermediats 68 in einer Vielzahl sich anschließender Reaktionen dar, was diese Reaktion vor allem im Hinblick auf die Verwendung in Domino-Sequenzen interessant macht. Die $\beta$-Hydrideleminierung unter Ausbildung einer C-C-Doppelbindung repräsentiert hierbei die einfachste Möglichkeit (Abbildung 23, a), die allerdings durch fehlende $\beta$-ständige Wasserstoffatome oder Unterdrückung der erforderlichen syn-coplanaren Anordnung von Palladium und $\beta$-ständigem Wasserstoffatom durch die dem Substrat inhärente Sterik blockiert werden kann. In diesem Fall kann die $\sigma$-Pd"-Spezies 68 über Kohlenstoff-Heteroatom oder Kohlenstoff-Kohlenstoff bindungsbildende Reaktionen terminiert werden. Unter anderem erhält man Zugriff auf verschiedene Methoden zur Diheterofunktionalisierung, wie z. B. Amino-Acetoxylierungen, ${ }^{[76]}$ Dihydroxylierungen ${ }^{[77]}$ oder Diaminierungen (Abbildung 23, b), ${ }^{[78]}$ die über eine Abfolge von Oxidation der $\mathrm{Pd}^{\prime \prime}$ - zur $\mathrm{Pd}^{\mathrm{IV}}$-Spezies, folgendem interoder intramolekularem Angriff eines Heteroatom-Nukleophils und abschließender reduktiver Eliminierung von $\mathrm{Pd}^{\prime \prime}$ zugänglich sind. 


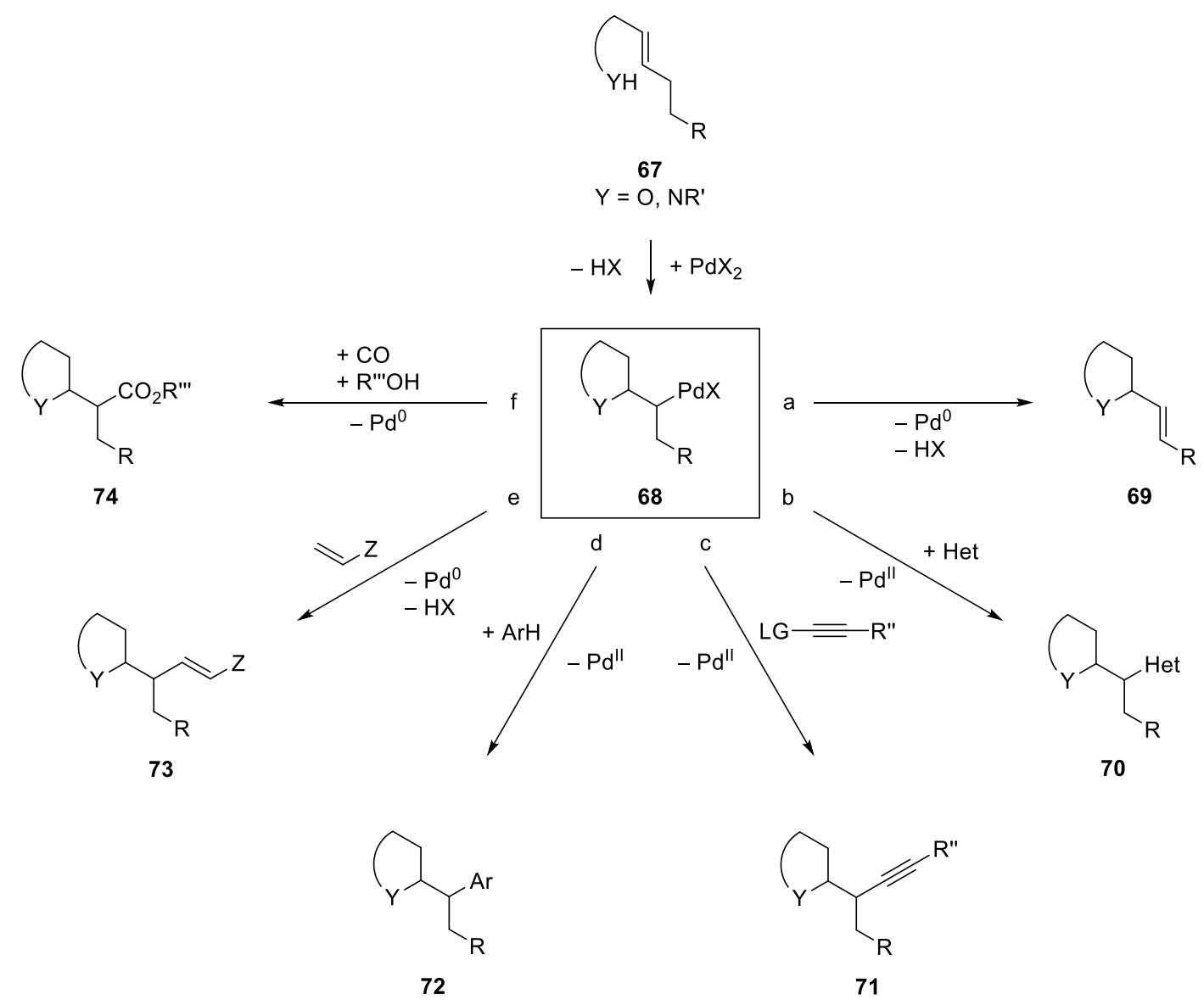

Abbildung 23: Möglichkeiten der Umsetzungen der $\sigma$-Alkyl-Pd"l-Spezies 68.

Kohlenstoff-Kohlenstoff bindungsbildende Reaktionen, die einer Nukleopalladierung nachfolgen können, sind beispielsweise Alkinylierungen (Abbildung 23, c), ${ }^{[79]}$ Arylierungen $^{\left[{ }^{80]}\right.}$ (Abbildung 23, d) oder Indolierungen. ${ }^{[81]}$

Eine weitere Möglichkeit stellt die Insertion der $\sigma$-Alkyl-Pd"-Zwischenstufe 68 in Olefine mit darauffolgender $\beta$-Hydrideliminierung (Abbildung 23, e) dar, ${ }^{[82]}$ wie sie im Arbeitskreis Tietze in Form einer Domino-Wacker/Heck-Reaktion in der enantioselektiven Totalsynthese des Vitamin E genutzt wurde. ${ }^{[83]}$

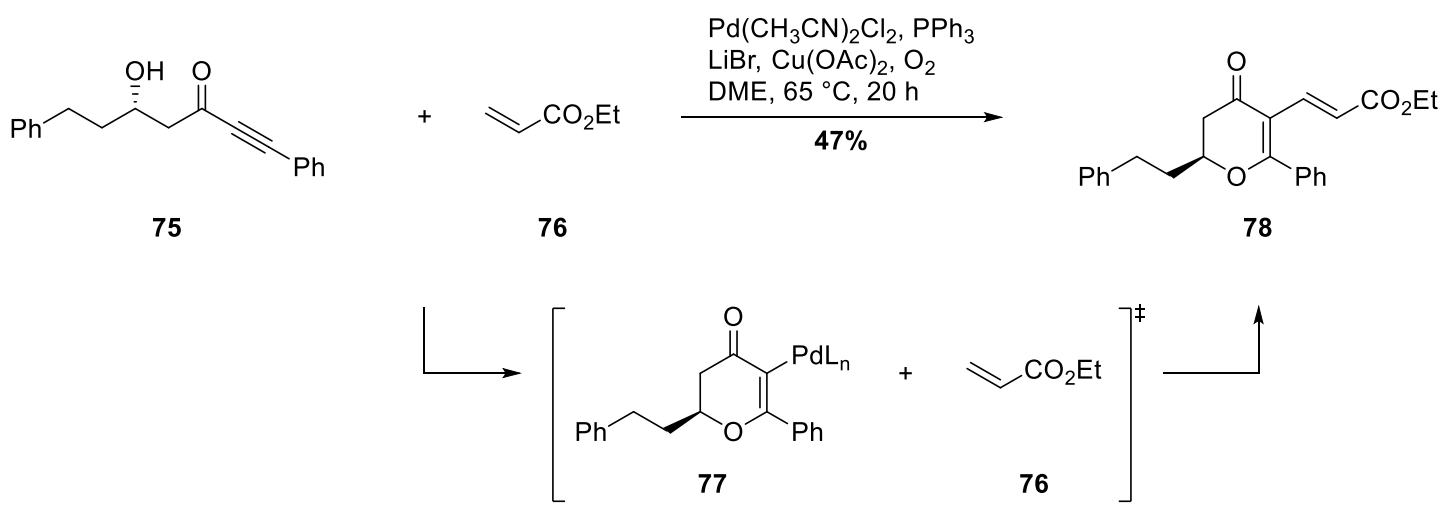

Abbildung 24: Domino-Wacker/Heck-Reaktion zum Aufbau des Dihydropyranons 78 durch Gouverneur. 
Des Weiteren fand diese Methodik unter anderem in der Synthese des Dihydropyranons 78 aus dem $\beta$-Hydroxyinon 75 und Ethylacrylat 76 mit einer moderaten Ausbeute von $47 \%$ durch Gouverneur et al. Verwendung (Abbildung 24). ${ }^{[84]}$

In Gegenwart von CO kann aus der Pd"-Spezies 68 via Carbonylierung ein AcylpalladiumIntermediat generiert werden, das unter nachfolgender Alkoholyse den Zugang zu Estern eröffnet (Abbildung 23, f). ${ }^{[85]}$

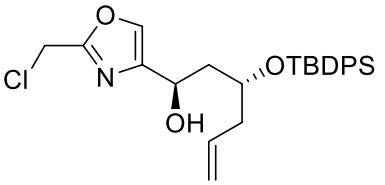

79

$\mathrm{Pd}\left(\mathrm{CH}_{3} \mathrm{CN}\right)_{2} \mathrm{Cl}_{2}$ $\mathrm{CO}, p$-Benzochinon $\mathrm{MeOH} / \mathrm{CH}_{3} \mathrm{CN}$

RT, $24 \mathrm{~h}$

$58 \%$

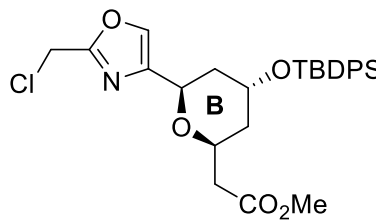

80

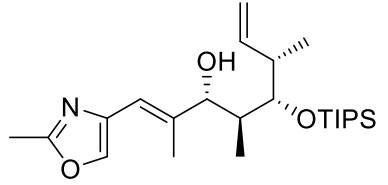

81

$\mathrm{Pd}(\mathrm{OAc})_{2}, \mathrm{CO}$

$\mathrm{MeOH} / \mathrm{CH}_{3} \mathrm{CN}$

RT, $44 \mathrm{~h}$

$86 \%$

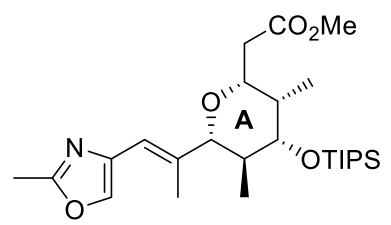

82
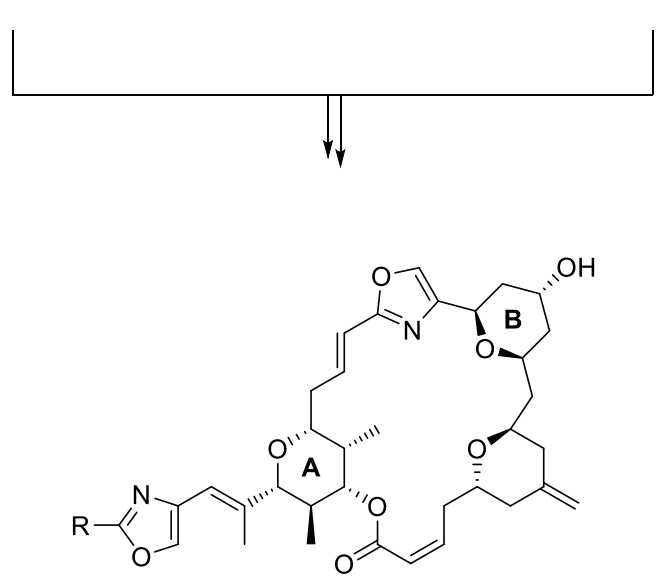

Phorboxazol A (83)

Abbildung 25: Totalsynthese des Phorboxazol A (83) durch White.

In der Totalsynthese des marinen Makrolids Phorboxazol A (83) griffen White und Mitarbeiter dieses Konzept zum Aufbau der beiden Tetrahydropyran-Ringe A und B durch den Einsatz zweier Domino-Wacker/Carbonylierung/Methoxylierungen auf. Das Tetrahydropyran B konnte in moderaten 58\% Ausbeute und hoher Diastereoselektivität unter katalytischer Reaktionsführung dargestellt werden, wohingegen die Synthese des A-Rings 
zwar in guten $86 \%$ Ausbeute resultierte, jedoch eine stöchiometrische Verwendung des $\mathrm{Pd}(\mathrm{OAc})_{2}$ vonnöten war (Abbildung 25). ${ }^{[86]}$

Dieses Reaktionsschema ist nicht nur auf eine intermolekular terminierende Alkoholyse limitiert, sondern kann, sofern eine geeignete zweite Hydroxyfunktionalität im Molekül vorhanden ist, ebenfalls intramolekular ablaufen. Dadurch werden bizyklische Lactone, vor allem verknüpfte Tetrahydropyran- und Tetrahydrofuran-y-Lactone, zugänglich. ${ }^{[87]}$
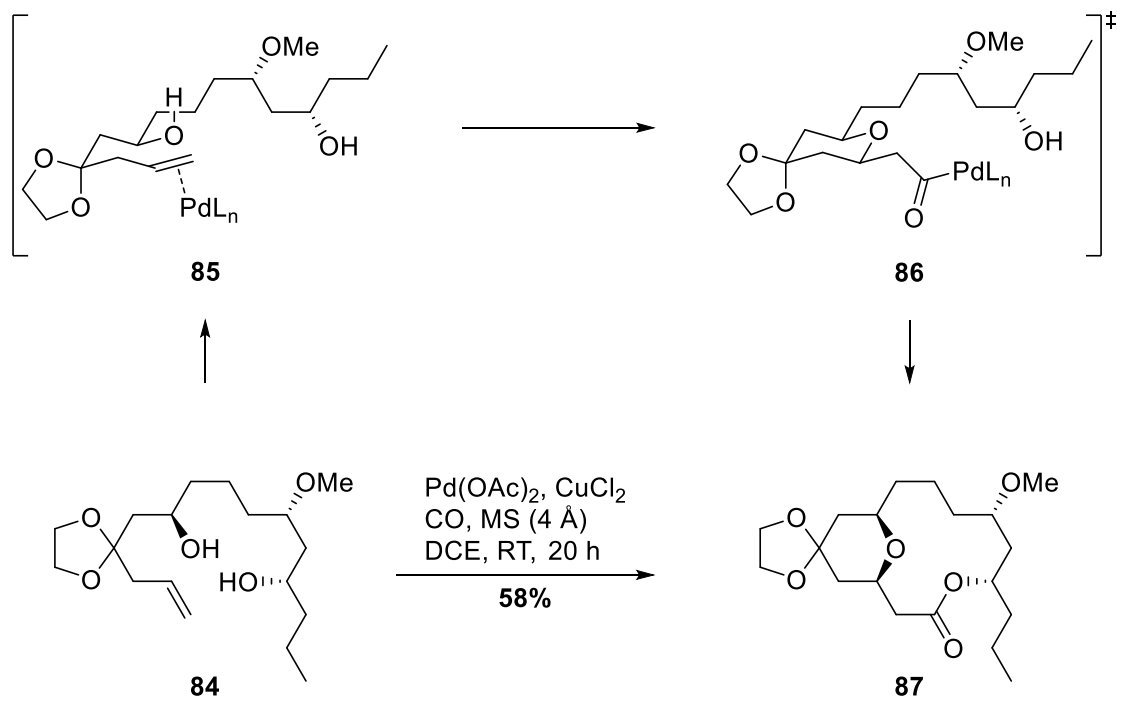

86
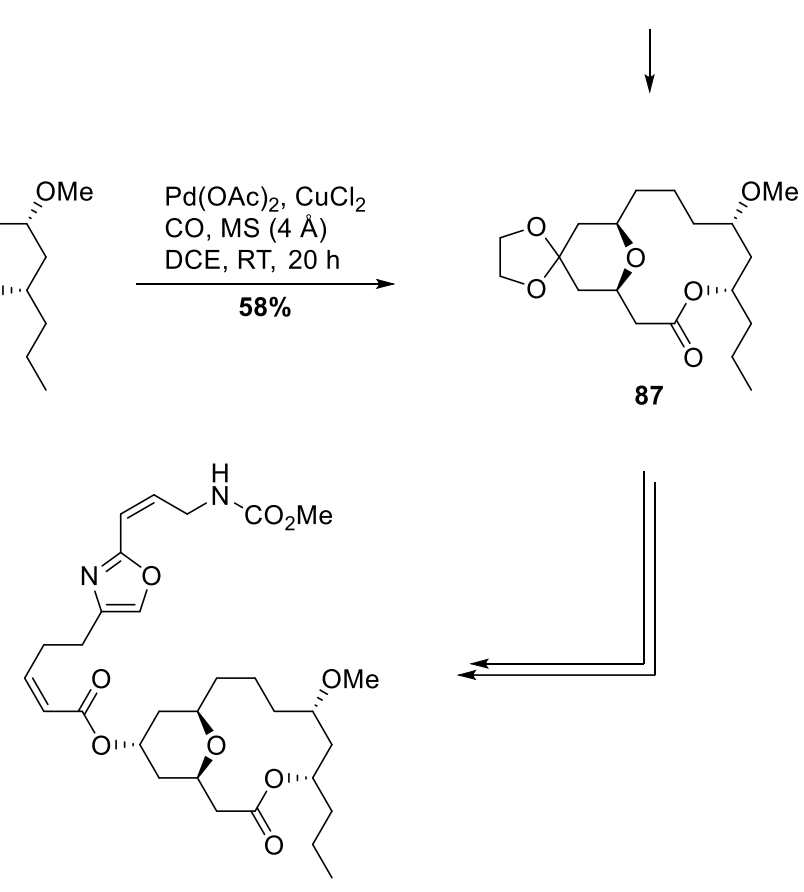

9-Demethylneopeltolid (88)

Abbildung 26: Domino-Wacker/Carbonylierung/Makrolactonisierung in der Totalsynthese von 9-Demethylneopeltolid (88) durch Dai.

Die von Dai et al. in der Totalsynthese des 9-Demethylneopeltolid (88) verwendete intramolekulare Domino-Wacker/Carbonylierung/Makrolactonisierung nimmt diesen Ansatz zum Aufbau sowohl des Tetrahydropyran-Rings als auch des elfgliedrigen Makrolactons in moderaten 58\% Ausbeute auf (Abbildung 26). ${ }^{[88]}$ 


\subsubsection{Enantioselektive Wacker-Oxidation}

Im Vergleich zur umfassenden Forschung auf dem Gebiet der Wacker-Oxidation sind Arbeiten auf dem Gebiet der enantioselektiven Wacker-Reaktion eher rudimentär. ${ }^{[56]}$ Dass chirale Phosphin-Liganden, wie sie im Allgemeinen in asymmetrischen $\mathrm{Pd}^{0}$-katalysierten Reaktionen zum Einsatz kommen, nicht in Wacker-Reaktionen verwendet werden können, stellt hierfür einen Grund dar. Dies lässt sich zum einen auf die oxidativen Reaktionsbedingungen der Wacker-Oxidation zurückführen, die in der Regel ungeeignet für den Einsatz Phosphin-basierter Liganden sind und zum anderen auf die $\sigma$-DonorFähigkeiten der Phosphine, die eine Verringerung des elektrophilen Charakters des Palladiums bewirken können. Eine weitere Limitierung stellt die durch mechanistische Studien ermittelte geringe Differenz der Aktivierungsenergien des enantiobestimmenden syn- und anti-Nukleopalladierungsschrittes dar, wodurch keiner der beiden Reaktionspfade ausgeschlossen werden kann.

Erste nennenswerte Erfolge auf diesem Gebiet verzeichneten Hosokawa und Murahashi im Jahr 1978 mit der enantioselektiven Pd"-katalysierten Zyklisierung von orthoAllylphenolen unter Verwendung des chiralen Liganden $\beta$-Pinen, der einen Enantiomerenüberschuss von bis zu 29\% ermöglichte. ${ }^{[63,64,89]}$ Mit dem Einsatz neuartiger Binaphthylbasierter Bisoxazolin-Liganden (BOXAX) (S,S)-91a/91b gelang Uozumi und Hayashi 1997 die erste hoch enantioselektive Reaktionsführung in der Wacker-Reaktion zur Zyklisierung tetrasubstituierter ortho-Allyl- und Homoallylphenole 89 zu Dihydrobenzofuranen 90b und Chromanen 90a mit Enantioselektivitäten von bis zu 97\% ee (Abbildung 27). ${ }^{[90,91]}$

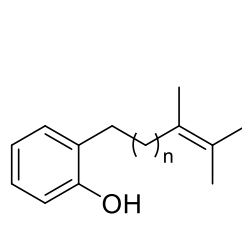

89

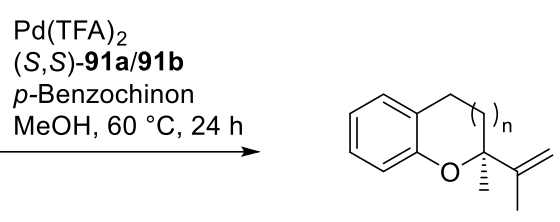

90a: $\mathrm{n}=1,(S, S)-91 \mathrm{a}, \mathbf{6 1 \%}, \mathbf{9 7 \%}$ ee 90b: $\mathrm{n}=0,(S, S)-91 \mathrm{a}, \mathbf{5 9 \%}, \mathbf{9 4 \%}$ ee 90b: $\mathrm{n}=0,(S, S)-91 \mathrm{~b}, \mathbf{7 5 \%}, \mathbf{9 6 \%}$ ee

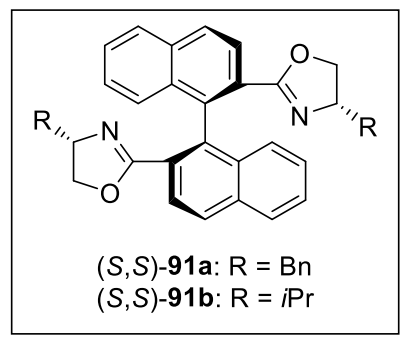

$(S, S)-91 \mathrm{a}: \mathrm{R}=\mathrm{Bn}$ $(S, S)-91 \mathbf{b}: \mathrm{R}=i \mathrm{Pr}$

Abbildung 27: Enantioselektive Wacker-Oxidation von ortho-Allyl- und Homoallylphenolen 89 durch Uozumi und Hayashi.

Im Arbeitskreis Tietze finden ebenjene BOXAX-Liganden Anwendung, um in hohen Enantioselektivitäten chirale Chromane via Domino-WackerlHeck- oder Domino-Wackerl Carbonylierung/Methoxylierung-Reaktionen zu synthetisieren. ${ }^{\text {[83,92,93] }}$

Achsenchirale Biphenyl-Liganden mit einem Tetraoxazolin-Rückgrat bilden eine weitere Ligandenklasse, fähig Enantioselektivität zu induzieren und wurden von Zhang und 
Mitarbeitern in der Zyklisierung von tri- und tetrasubstituierten ortho-Allyl- und Homoallylphenolen eingesetzt. ${ }^{[94]}$

Eine weitere Innovation stellt die Verwendung molekularen Sauerstoffs als Oxidans ${ }^{[95]}$ und die damit einhergehende aerobe Reaktionsführung in der durch Stoltz et al. entwickelten enantioselektiven Wacker-Zyklisierung von ortho-Allylphenol 92 zum Dihydrobenzofuran 93 dar. Als Ligand wurde hierbei auf den $C_{1}$-symmetrischen Naturstoff (-)-Spartein (94) zurückgegriffen, was jedoch neben den erhaltenen moderaten Ausbeuten der Reaktion zusätzlich den Nachteil des anspruchsvollen Zugangs zu enantiomerenreinem (+)-Spartein (ent-94) aufweist. Dem gegenüber stehen zum einen die erhaltenen hohen ee-Werte und zum anderen die ökologisch vorteilhaften Reaktionsbedingungen (Abbildung 28). ${ }^{[96]}$

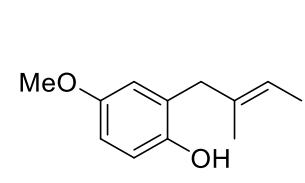

92

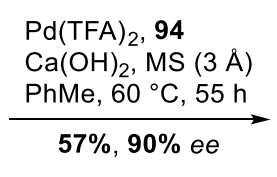

$57 \%, 90 \%$ ee

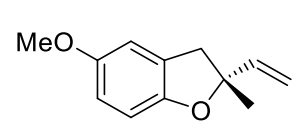

93

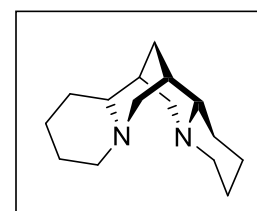

(-)-Spartein (94)

Abbildung 28: Enantioselektive Wacker-Oxidation des ortho-Allylphenols 92 durch Stoltz.

Die oxidativen Reaktionsbedingungen der Wacker-Reaktion sowie die Neigung primärer und sekundärer aliphatischer Alkohole zur Oxidation unter ebenjenen Konditionen erfordert die sorgfältige Komposition des Katalysatorsystems, um diese interessante Stoffklasse als Nukleophile zur Funktionalisierung von Alkenen nutzbar zu machen.

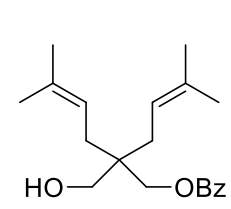

95

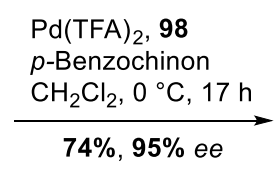

$74 \%, 95 \%$ ee

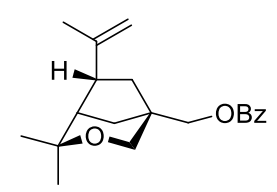

97

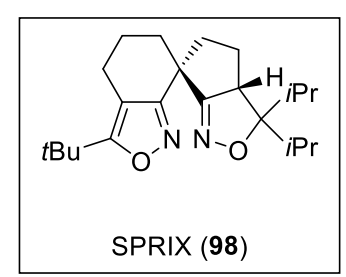

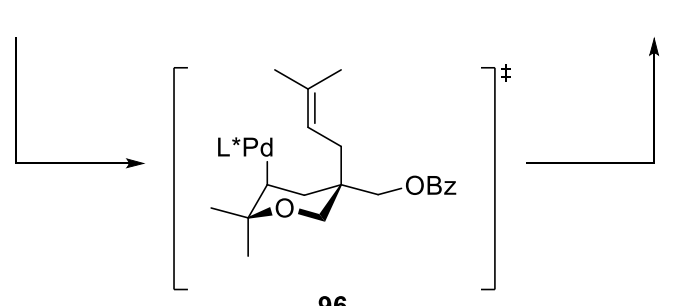

96

Abbildung 29: Enantioselektive Synthese des Bizyklus 97 durch Sasai.

Die erste Publikation über eine erfolgreiche Umsetzung aliphatischer Alkohole in einer enantioselektiven Wacker-Reaktion entstammt der Forschungsgruppe Sasai. Der Oxypalladierung des einfach geschützten Diols 95 unter Verwendung des neuartigen 
Spiro(isoxazol-isoxazolin)-Liganden (SPRIX) (98) zum Pd"-Intermediat 96 folgte eine Insertion in die Alkenyl-Seitenkette und die Bildung des Bizyklus 97 in 74\% Ausbeute und 95\% ee (Abbildung 29). ${ }^{[97]}$ 


\subsection{Domino-Reaktionen}

Dem stetig steigenden Bewusstsein über die Endlichkeit natürlich vorkommender Ressourcen und für den Schutz der Umwelt muss sich auch die organische Synthesechemie stellen und nimmt sich dieser Herausforderung durch die Entwicklung innovativer Konzepte an. Exemplarisch für diese Bestrebungen steht das von Tietze eingeführte Konzept der Domino-Reaktionen. ${ }^{[98]}$ Eine Domino-Reaktion ist definiert als eine Synthesesequenz von „zwei oder mehr nacheinander ablaufende[n] Transformationen, in denen unter Bindungsknüpfung oder Fragmentierung die jeweils nachfolgende Reaktion an den im vorhergehenden Schritt gebildeten Funktionalitäten erfolg[t] ${ }^{\text {“ }}{ }^{[99]}$ Die durch diese Definition etablierten Kriterien werden von den ebenfalls in der Literatur anzutreffenden Begrifflichkeiten Tandem- und Kaskaden-Reaktion nur unzureichend erfüllt. ${ }^{[100]}$ Im Gegensatz zu Domino-Reaktionen handelt es sich bei der Tandem-Reaktion nicht um einen zwingend zeitaufgelösten Prozess, weswegen auch zeitgleich, jedoch lokal unabhängig voneinander ablaufende Reaktionen in einem Substrat unter diese Kategorie fallen. Der Terminus Kaskaden-Reaktion hingegen ist unter anderem aufgrund der Beschreibung zahlreicher anderer chemischer Prozesse (z. B. in der Photo- oder Biochemie) nicht zweckmäßig. Domino-Reaktionen werden entsprechend der Reaktionsart der jeweiligen Einzelschritte oder gemäß der in den Einzelreaktionen gebildeten reaktiven Spezies klassifiziert. Zur Typisierung haben sich folgende Kategorien etabliert: kationisch, anionisch, radikalisch, perizyklisch, photochemisch, Übergangsmetall-induziert, oxidativ/reduktiv oder enzymatisch. Gehören die einzelnen Reaktionsschritte einer Domino-Reaktion ein und derselben Klasse an, so spricht man in diesem Fall von einer Homo-Domino-Reaktion. Liegen andererseits unterschiedliche Reaktionstypen vor, handelt es sich um eine Hetero-Domino-Reaktion. Tendenziell gleichen sich die beiden ersten Transformationen einer Domino-Reaktion bezüglich ihres Reaktionstyps und die zweiten und nachfolgenden Schritte verlaufen zumeist intramolekular.

Domino-Reaktionen bieten eine Fülle positiver Aspekte, so werden in der Regel hohe Ausbeuten sowie Chemo-, Regio- und Stereoselektivitäten erzielt. Ein weiteres Merkmal ist die signifikante Erhöhung der Komplexität der erhaltenen Produkte, die durch das Zusammenfassen mehrerer bindungsbildender Einzelreaktionen in einer einzigen Domino-Reaktion ermöglicht wird. Dies und der Verzicht auf die Isolierung einzelner Reaktionsintermediate und die damit einhergehende Reduzierung der zeit- und arbeitsintensiven Aufarbeitungs- und Aufreinigungsschritte stellt einen entscheidenden ökologischen und ökonomischen Vorteil dar, was Einsparungen beim Einsatz von Chemikalien (v. a. Lösungsmittel), der Erzeugung von Abfall und dem Verbrauch von Energie zur Folge hat. All diese positiven Attribute machen Domino-Reaktionen zu einem 
nützlichen Werkzeug, nicht nur im Laboreinsatz sondern ebenso in der chemischen Industrie. ${ }^{[101]}$

Beim Konzept der Domino-Reaktionen handelt es sich nicht um ein rein künstliches Konstrukt, was offenkundig wird, wenn man einen Blick auf die zahlreichen Beispiele wirft, die die Natur zu bieten hat. So sind Domino-Reaktionen unter anderem in der Biosynthese von Alkaloiden, Terpenen oder Steroiden anzutreffen, wie das Beispiel der Biosynthese des Lanosterin (100) ausgehend von (S)-2,3-Oxidosqualen (99) unter Ausbildung von vier neuen Bindungen sowie sechs stereogenen Zentren eindrucksvoll verdeutlicht (Abbildung 30). ${ }^{[102]}$

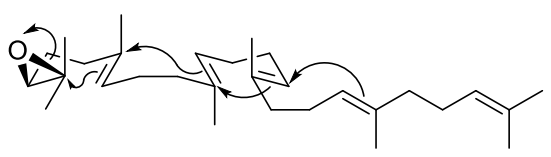

(S)-2,3-Oxidosqualen (99)

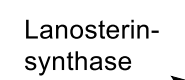

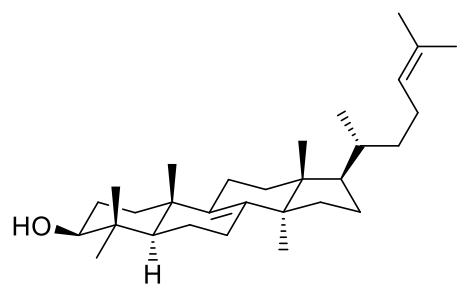

Lanosterin $(\mathbf{1 0 0})$

Abbildung 30: Enzymatische Zyklisierung von (S)-2,3-Oxidosqualen (99) zu Lanosterin (100).

Der Aufbau steroidaler und terpenoidaler Strukturen ist in der organischen Synthese aufgrund ihres häufigen Auftretens in einer Vielzahl natürlich vorkommender Wirkstoffe von großer Bedeutung, dient aber auch als Benchmark zur Erprobung neuartiger Synthesestrategien. Ein Konzept, das sich in diesem Zusammenhang bewährte, ist die von MacMillan entwickelte enantioselektive Organo-SOMO-Katalyse. Im Gegensatz zu der bereits oftmals erprobten kationischen Polyen-Zyklisierung wird hierbei auf einen radikalischen Ansatz gesetzt, sodass nach Kondensation des Imidazolidinon-Katalysators 105 mit dem ungesättigten Aldehyd 101 und anschließender Oxidation mit $\mathrm{Cu}(\mathrm{OTf})_{2}$ eine Reihe radikalischer 6-endo-trig-Zyklisierungen die Generierung des pentazyklischen Aldehyds 104 zur Folge hat. Vier neue Bindungen sowie sieben stereogene Zentren wurden im Zuge dieser Domino-Sequenz mit einer Ausbeute von 56\% gebildet, bei einem Katalysator-induzierten Enantiomerenüberschuss von 93\% und dem Erhalt eines einzigen Diastereomers (Abbildung 31). ${ }^{[103]}$ 


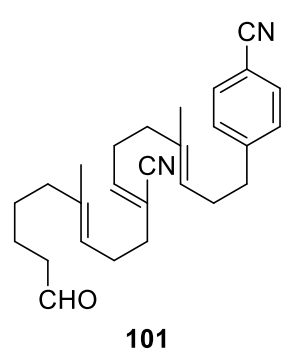

101

$$
105 \mid \begin{aligned}
& -\mathrm{H}_{2} \mathrm{O} \\
& -\mathrm{e}^{-}
\end{aligned}
$$

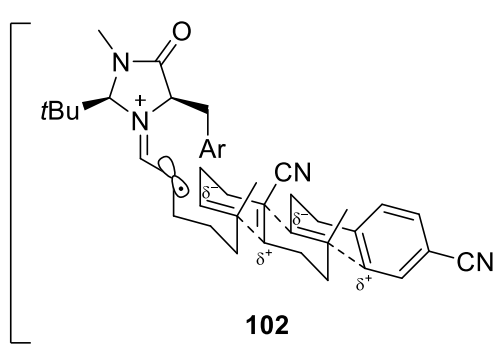

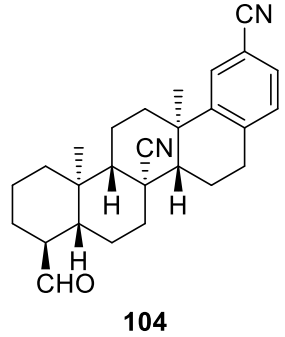

$\left\{\begin{array}{l}-105 \\ -\mathrm{H}^{+} \\ -\mathrm{e}^{-}\end{array}\right.$

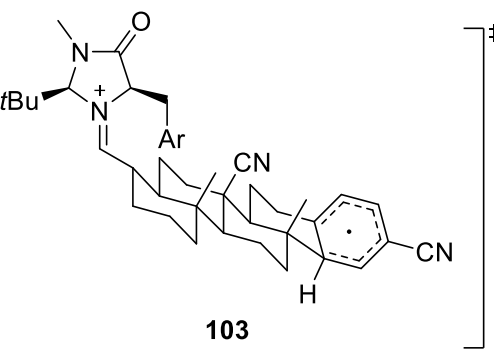

Abbildung 31: Enantioselektive Organo-SOMO-Katalyse zum Aufbau des pentazyklischen Aldehyds 104 durch MacMillan.

Die Shimalactone A (110a) und B (110b) wurden 2005 aus dem marinen Fungus Emericella variecolor isoliert und weisen eine höchst interessante Struktur in Form eines Oxabicyclo[2.2.1] heptans auf, das über eine dreifach substituierte Doppelbindung mit einer Bicyclo[4.2.0]oktadien-Untereinheit verbunden ist. ${ }^{[104]}$ Beide Shimalactone konnten 2007 in einer biomimetischen Synthese durch Trauner et al. erfolgreich präpariert werden.

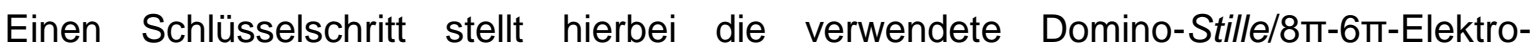
zyklisierung dar, die zum finalen Aufbau der Shimalactone A (110a) und B (110b) aus dem Vinylstannan 106 sowie dem lodtrien 107 in 66\% Ausbeute und in einem Diastereomerenverhältnis von 5:1 (110a:110b) führte. Die Kupplung der beiden Vorstufen 106 und 107 zur $(E, E, Z, Z, E)$-Polyen-Zwischenstufe 108 unter modifizierten StilleLiebeskind Reaktionsbedingungen resultiert in einer spontanen $8 \pi-6 \pi$-Elektrozyklisierungssequenz, bei der der $6 \pi-S c h r i t t$ mit hoher Diastereoselektivität erfolgt und somit nur die beiden Diastereomere Shimalacton A (110a) und B (110b) erhalten werden. Weiterhin erwähnenswert ist, dass die gesamte Synthese schutzgruppenfrei durchgeführt wurde (Abbildung 32). ${ }^{[105]}$ 


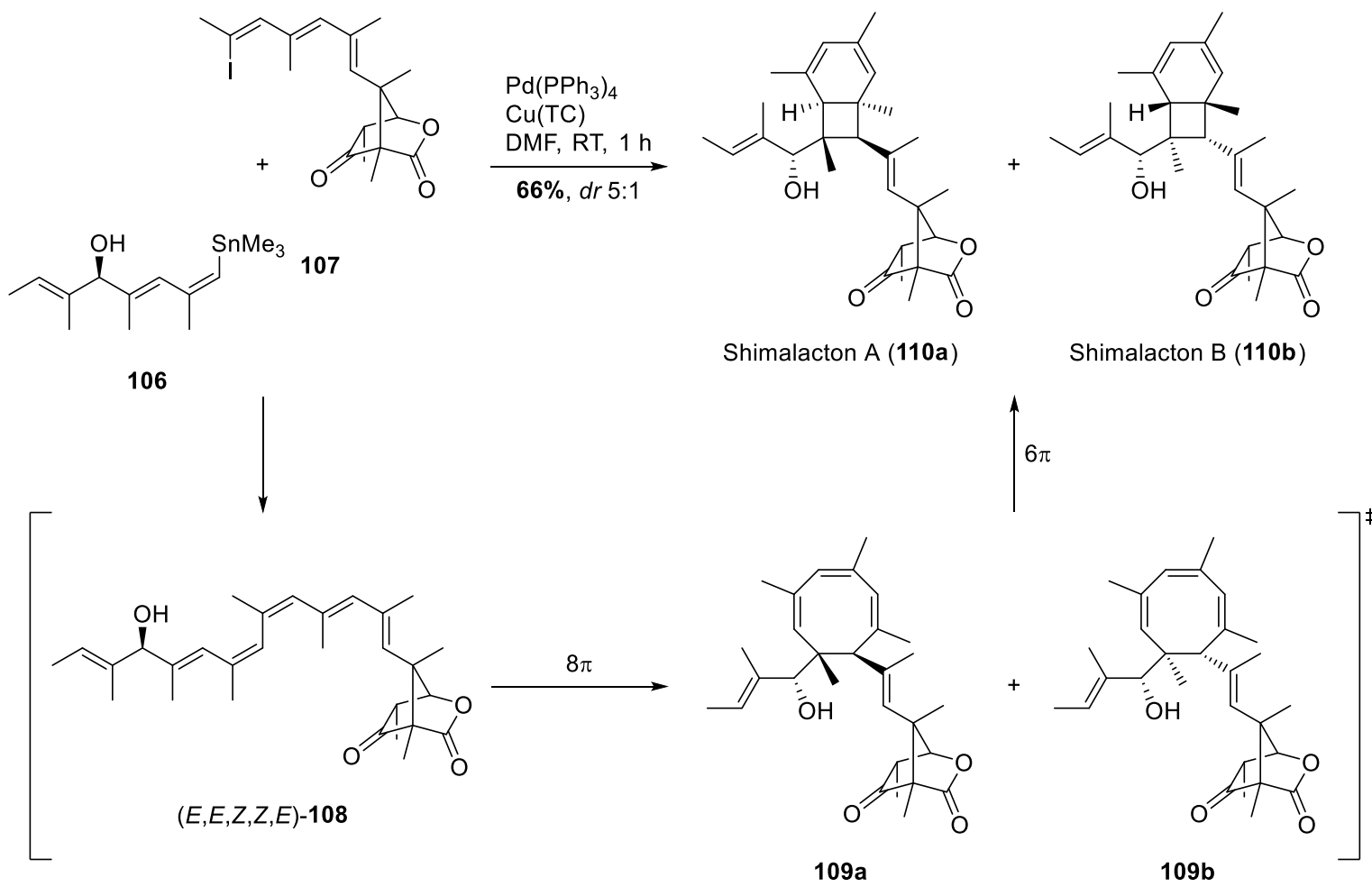

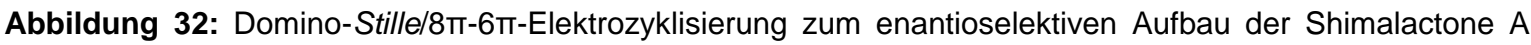
(110a) und B (110b) durch Trauner.

Der Neuraminidase-Inhibitor (-)-Oseltamivir 115 (Tamiflu ${ }^{\circledR}$ ) bildet einen der Hauptbestandteile der medikamentösen Therapie und Prophylaxe von sowohl Influenza A als auch Influenza B. Um dieser Relevanz gerecht zu werden, sollte die Synthese so kostengünstig, schnell und praktikabel wie möglich sein. Hayashi und Mitarbeiter realisierten dieses Unterfangen in neun Stufen, die sich auf drei Eintopfreaktionen aufteilen und in einer Gesamtausbeute von 57\% ablaufen. Die erste der drei Eintopfreaktionen beinhaltet unter anderem eine Domino-Michael/Horner-Wadsworth-EmmonsReaktion. Hierbei addiert zunächst in einer intermolekularen Reaktion das Nitroalkan 111 an das Vinylphosphonat 112, wodurch es im generierten Phosphonat $113 \mathrm{zu}$ einem intramolekularen Angriff auf die Formylfunktionalität unter Ausbildung des Cyclohexens 114 kommt. Des Weiteren findet das Domino-Konzept in Form einer Domino-CurtiusUmlagerung/Amid-Bildung ein zweites Mal Anwendung in der Synthese. Dieser bereits sehr effektive enantioselektive Zugang zum (-)-Oseltamivir 115 konnte durch dieselbe Gruppe weiter verbessert werden, indem die Reaktionssequenz auf nur noch zwei Eintopfreaktionen komprimiert wurde. Neben einer leicht gestiegenen Gesamtausbeute von $60 \%$ konnte zusätzlich noch eine Reaktionsführung unter Aussparung des einzigen säulenchromatographischen Aufreinigsschrittes etabliert werden (Abbildung 33). ${ }^{[06]}$ 

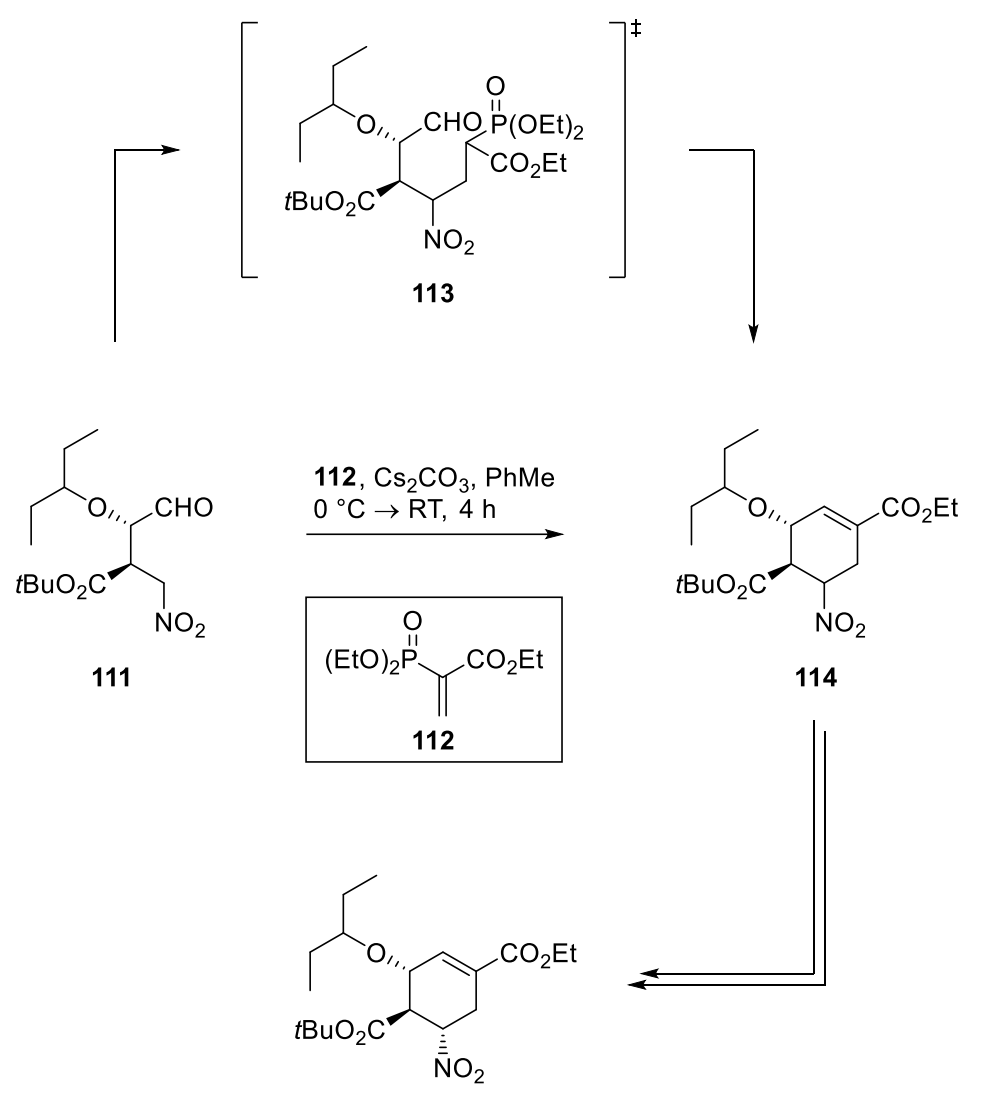

(-)-Oseltamivir (115)

Abbildung 33: Enantioselektive Totalsynthese von (-)-Oseltamivir (115) unter Verwendung eines DominoAnsatzes durch Hayashi.

Ein aktuelles Beispiel auf dem Gebiet der Domino-Reaktionen aus der Forschungsgruppe Tietze bildet die Synthese dimerer tetrasubstituierter Alkene mit helicaler Chiralität mittels einer vier- oder sechsfachen Tandem-Domino-Reaktion. Substrat 121, mit anti angeordneten tetrasubstituierten Alkenfunktionalitäten, wird durch zwei unabhängig voneinander ablaufende Domino-Carbopalladierung/C-H-Aktivierungen aus dem Alkadiin 118 in 94\% Ausbeute generiert. Die Sonogashira-Reaktion zum Aufbau des DominoVorläufers 118 aus dem Diin 116 kann ebenfalls in den Tandem-Domino-Prozess integriert werden, wodurch die dimere Zielverbindung 121 direkt aus dem Diin 116 in einer Ausbeute von $56 \%$ synthetisiert werden kann. Dimere Strukturen mit einer syn-Anordnung der tetrasubstituierten Alkene konnten ebenfalls erfolgreich hergestellt werden (Abbildung 34). ${ }^{[107]}$ 


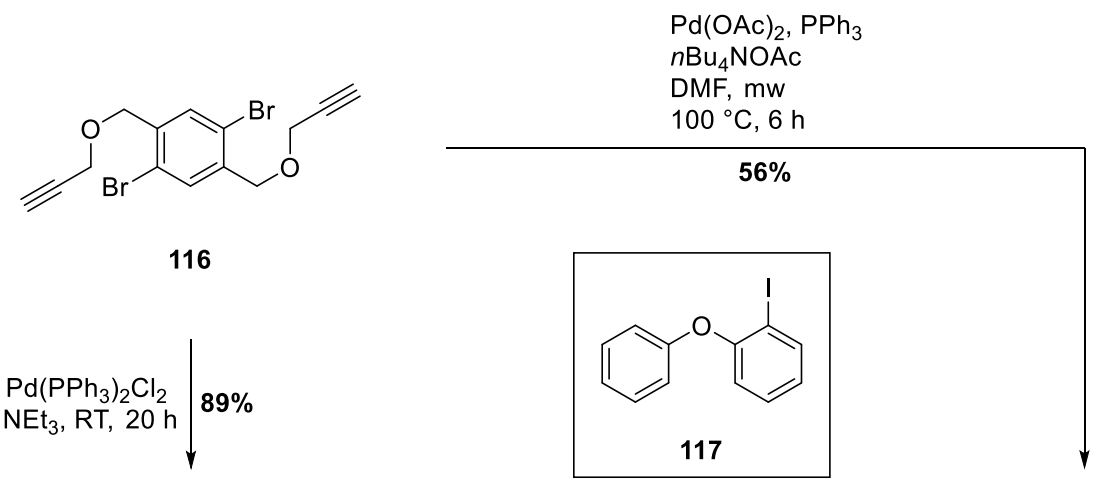

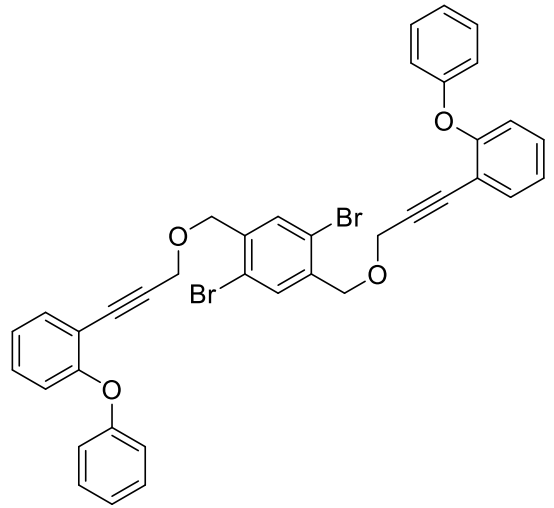

118

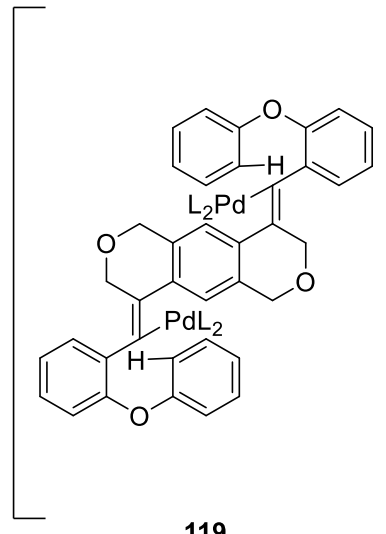

119
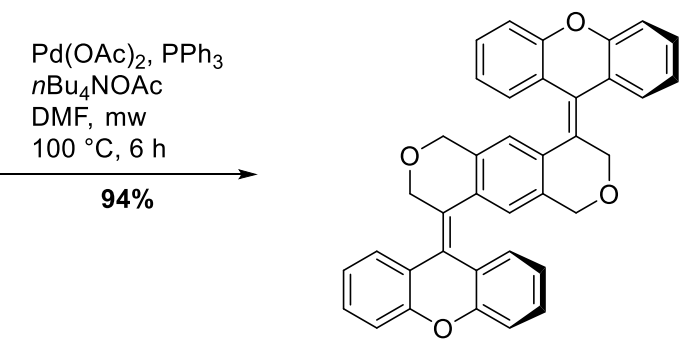

121

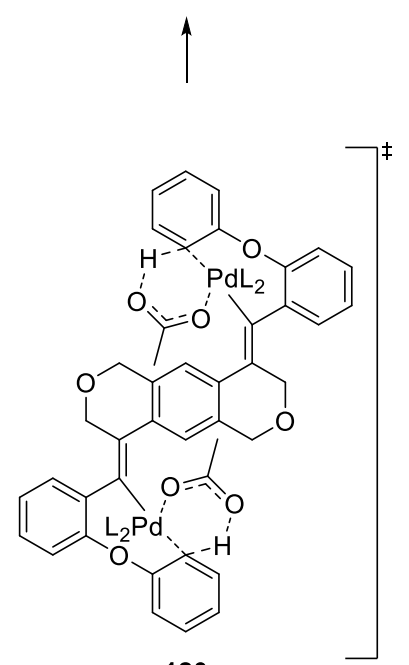

120

Abbildung 34: Vier- oder sechsfache Tandem-Domino-Reaktionen zum Aufbau dimerer tetrasubstituierter Alkene 121 mit helicaler Chiralität nach Tietze. 


\subsection{Die Sharpless-Dihydroxylierung}

Die von Sharpless et al. entwickelte $\mathrm{OsO}_{4}$-katalysierte, asymmetrische Dihydroxylierung ermöglicht die Überführung einer Vielzahl prochiraler Olefine in chirale 1,2-Diole und zeichnet sich durch ihre einfache Handhabung und hohe Enantioselektivität sowie katalytische Produktivität aus, weshalb sie zu einem bedeutenden Werkzeug in der Synthesechemie avancierte. ${ }^{[108-110]}$ Standardmäßig kommen hierbei 0.2 mol\% eines Os ${ }^{\mathrm{VI}}$ Salzes, 1 mol\% des jeweiligen Liganden sowie stöchiometrische Mengen der Base $\mathrm{K}_{2} \mathrm{CO}_{3}$ und des Oxidationsmittels $\mathrm{K}_{3}\left[\mathrm{Fe}(\mathrm{CN})_{6}\right]$ entweder in einem zweiphasigen Lösungsmittelsystem $\left(\mathrm{tBuOH} / \mathrm{H}_{2} \mathrm{O}\right)$ oder unter Verwendung von $\mathrm{NMO}$ in einer homogenen Lösung zum Einsatz. Als Liganden wurden vor allem die Phthalazin-verbrückten dimeren Dihydrochinin (DHQ) (122) und Dihydrochinidin (DHQD) (123) dank der durch sie induzierten hohen Enantioselektivitäten und des breiten Substratspektrums etabliert. ${ }^{[111,112]}$ Zuträglich der großen Akzeptanz der Methode ist ebenfalls die kommerzielle Verfügbarkeit vorgefertigter Mischungen bestehend aus $\mathrm{K}_{2} \mathrm{OsO}_{4} \cdot 2 \mathrm{H}_{2} \mathrm{O}$, (DHQ) ${ }_{2} \mathrm{PHAL}$ oder (DHQD) ${ }_{2} \mathrm{PHAL}, \mathrm{K}_{2} \mathrm{CO}_{3}$ und $\mathrm{K}_{3}\left[\mathrm{Fe}(\mathrm{CN})_{6}\right]$, die unter der Bezeichnung $A D$-mix- $\alpha$ respektive $A D$-mix- $\beta$ vermarktet werden (Abbildung 35).

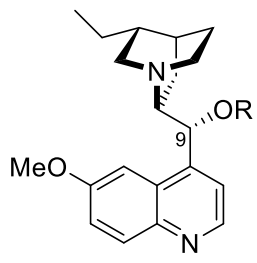

$\mathrm{DHQ}$
Dihydrochinin $(\mathrm{R}=\mathrm{H})$

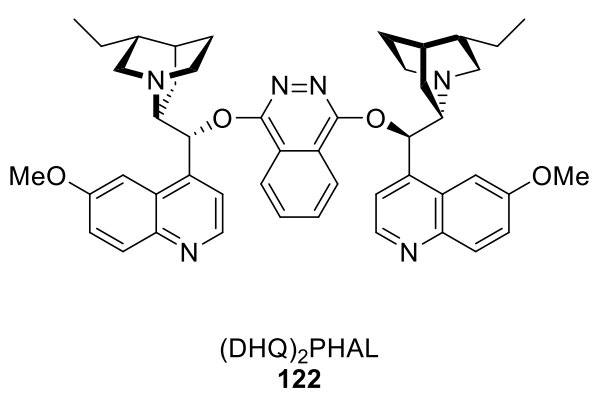

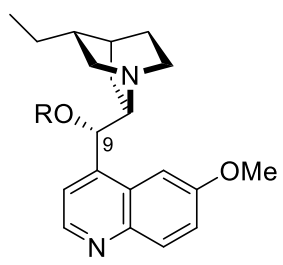

DHQD
Dihydrochinidin $(\mathrm{R}=\mathrm{H})$

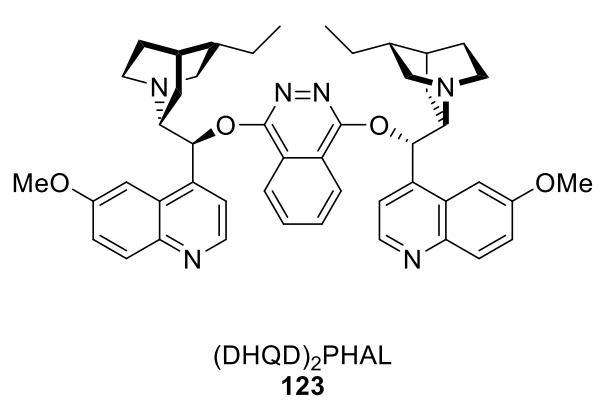

Abbildung 35: Phthalazin-verbrückte dimere Dihydrochinin- (DHQ) ${ }_{2} \mathrm{PHAL}$ (122) und DihydrochinidinLiganden (DHQD) $)_{2} \mathrm{PHAL}$ (123). 


\subsubsection{Mechanismus}

Erste Arbeiten von Sharpless und Mitarbeitern zur Reduzierung des hochtoxischen und teuren $\mathrm{OsO}_{4}$ auf katalytische Mengen für die Dihydroxylierung bauten auf einem System aus Cinchona-Alkaloiden als Liganden und stöchiometrische Mengen des Oxidationsmittels $\mathrm{NMO}$ in einem homogenen Aceton/ $\mathrm{H}_{2} \mathrm{O}$-Gemisch auf. ${ }^{[108]}$ Dieses frühe System lieferte zwar nur geringe ee-Werte von bis zu $8 \%$, erlaubte jedoch bereits erste wichtige Erkenntnisse über den Mechanismus des katalytischen Prozesses (Abbildung 36). ${ }^{[113]}$
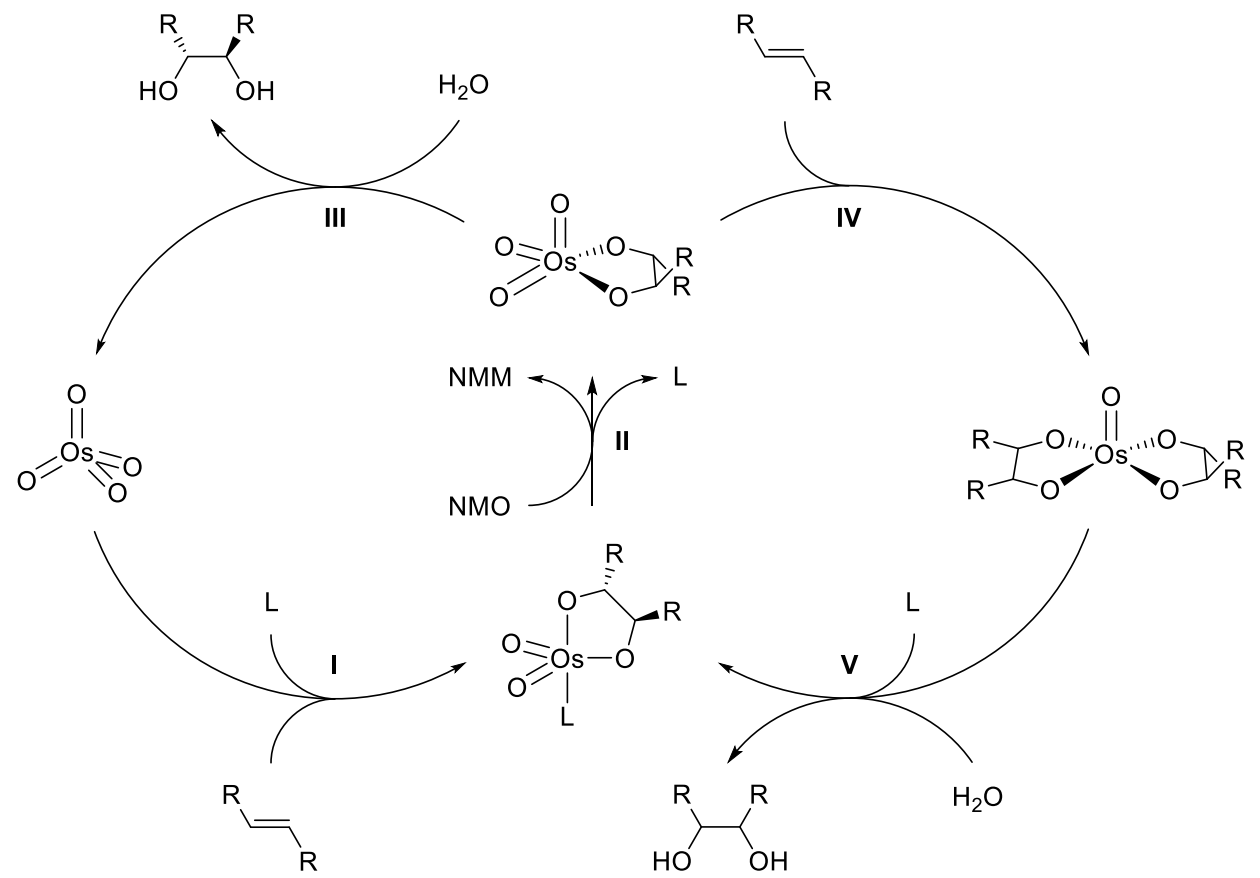

Abbildung 36: Mechanismus der Sharpless-Dihydroxylierung in einem einphasigen Lösungsmittelsystem mit NMO als Oxidationsmittel.

Dieser beinhaltet zwei katalytische Zyklen, die in mechanistischen Studien identifiziert wurden und unter den genannten Reaktionsbedingungen beide aktiv sind. Die Erzeugung einer Os ${ }^{\mathrm{VI}}$-Glycolat-Spezies mit definierter Stereoinformation infolge der Osmylierung des Olefins durch $\mathrm{OsO}_{4}$ (I) markiert den Ausgangspunkt der Sharpless-Dihydroxylierung. Nachfolgend führen die Oxidation mit NMO und die Freisetzung des Liganden zur

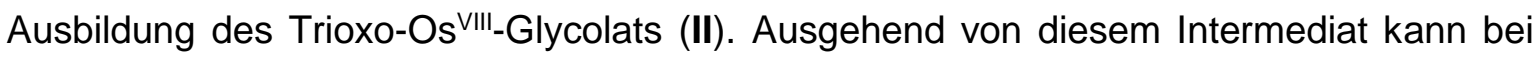
einphasiger Reaktionsführung einer von zwei Reaktionspfaden beschritten werden, was in den beiden beobachteten konkurrierenden Katalysezyklen resultiert. Vorteilhaft, weil mit hoher Stereoselektivität verbunden, ist die Hydrolyse des Trioxo-Os VIII-Glycolats unter Freisetzung des enantiomerenangereicherten Diols sowie der Regeneration des $\mathrm{OsO}_{4}$ (III) und der damit einhergehenden Komplettierung des ersten katalytischen Kreisprozesses. Bedingt durch die Möglichkeit der Bindung eines zweiten Moleküls des Olefins an das 


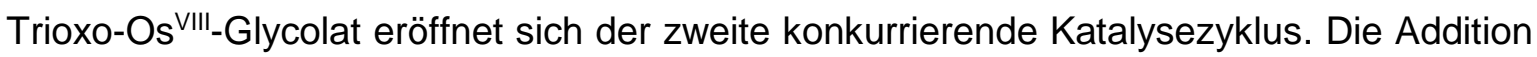
des zweiten Olefins findet durch den im vorherigen Reaktionsschritt eliminierten Liganden in diesem Fall jedoch ohne asymmetrische Induktion (IV) statt, wodurch in der darauffolgenden Hydrolyse das Diol aus dem Bisglycolatester mit stark verminderter Enantioselektivität abgespalten (V) und somit die Stereoselektivität des Gesamtprozesses negativ beeinflusst wird.

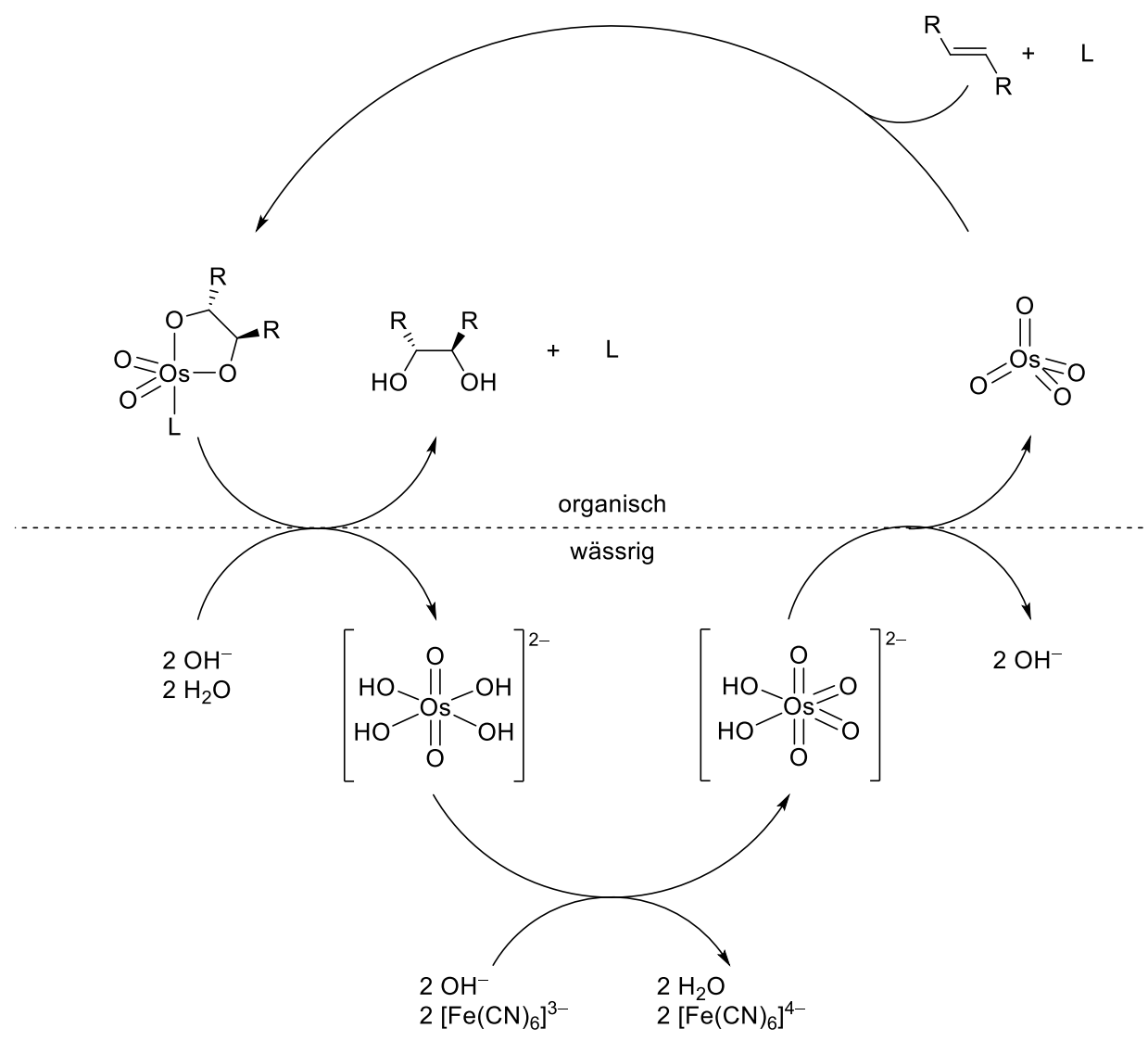

Abbildung 37: Mechanismus der Sharpless-Dihydroxylierung in einem zweiphasigen Lösungsmittelsystem und $\mathrm{K}_{3}\left[\mathrm{Fe}(\mathrm{CN})_{6}\right]$ als Reoxidans.

Durch die Verwendung eines zweiphasigen Lösungsmittelsystems und dem wasserlöslichen Oxidationsmittel $\mathrm{K}_{3}\left[\mathrm{Fe}(\mathrm{CN})_{6}\right]$ kann der nachteilige zweite Zyklus allerdings vollständig unterdrückt werden. Hierbei erfolgt zunächst erneut die Osmylierung des Olefins und damit die Ausbildung des hydrophoben Monoglycolatesters. Aufgrund des Fehlens eines Oxidationsmittels in der organischen Phase findet daraufhin die hydrolytische Spaltung des Osmatesters statt, wobei sowohl das freigesetzte Diol als auch der Ligand in der organischen Phase verbleiben, während die Os ${ }^{\mathrm{VI}}$-Spezies in die wässrige Phase übertritt und dort $\mathrm{zu} \mathrm{OsO}_{4}$ reoxidiert wird (Abbildung 37). ${ }^{[14]}$

Trotz unzähliger mechanistischer Studien und zahlreicher daraus gewonnener Erkenntnisse besteht noch immer Uneinigkeit über dem der Osmylierung zugrunde 
liegenden Mechanismus. Eine stufenweise Abfolge von [2+2]-Addition unter Ausbildung des Osmaoxetans 124 und Umlagerung zum Monoglycolatester 126, wie von Sharpless et al. postuliert, ${ }^{[15]}$ konkurriert mit der von Corey et al. vorgeschlagenen direkten [3+2]Cycloaddition des $\mathrm{OsO}_{4}$ und des Olefins über das Intermediat 125 zum Monoglycolatester 126. ${ }^{[116]}$ Letzteres wird nach dem derzeitigen mechanistischen Verständnis des Prozesses favorisiert ${ }^{[17]}$ und durch quantenmechanische Berechnungen, die für die [3+2]-Addition im Vergleich zur [2+2]-Addition eine um ca. $30 \mathrm{kcal} / \mathrm{mol}$ geringere Aktivierungsenergie aufzeigen, ${ }^{[118]}$ gestützt (Abbildung 38 ).

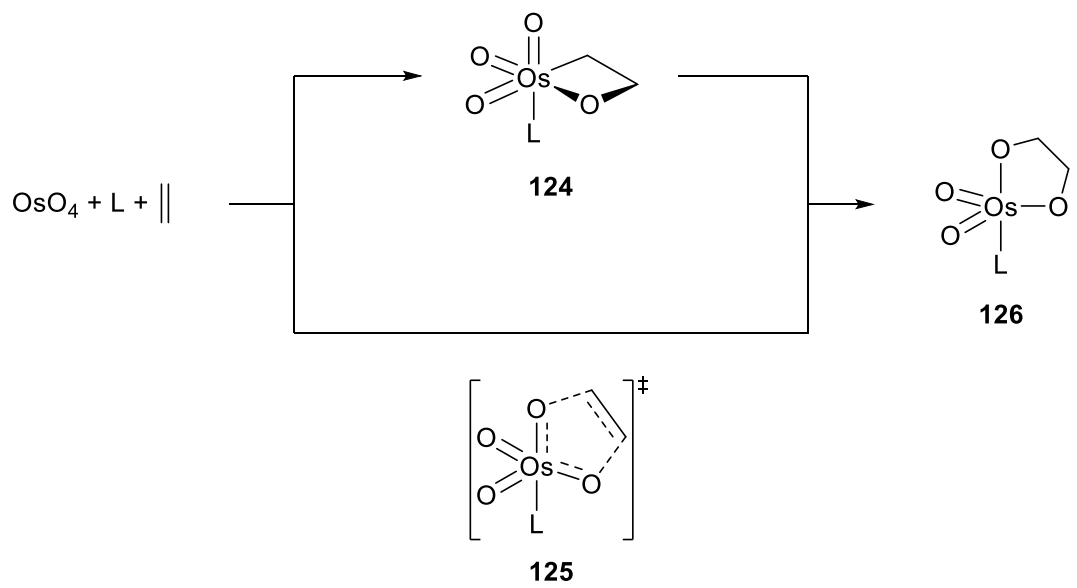

Abbildung 38: Ausbildung des Monoglycolatesters 126 durch eine stufenweise [2+2]-Addition/Umlagerung oder durch eine konzertierte [3+2]-Addition.

Klarheit besteht hingegen über den geschwindigkeitsbestimmenden Schritt der SharplessDihydroxylierung, der als die hydrolytische Spaltung des Monoglycolatesters 126 identifiziert wurde. Dieser kann durch die Zugabe von Methansulfonamid, vor allem bei Reaktionen mit geringer Umsatzrate aufgrund einer limitierten Hydrolyse stark beschleunigt werden (um das bis zu 50-fache). Die durch das Additiv erzielte Wirkung wird auch als "Sulfonamid-Effekt“ bezeichnet und erlaubt sogar die Umsetzung tetrasubstituierter Alkene. ${ }^{[109,110]}$

Die in der Sharpless-Dihydroxylierung zur Anwendung kommenden Cinchona-Alkaloide verfügen über eine herausragende asymmetrische Induktion, die zum Teil auf einen durch den Liganden hervorgerufenen beschleunigenden Effekt des Reaktionsschrittes zurückzuführen ist. Die Bindung des Chinuclidin-Stickstoffs am $\mathrm{OsO}_{4}$ führt hierbei zu einer stark erhöhten Geschwindigkeit, mit der die Enantioselektivität bestimmende Ausbildung des Monoglycolatesters 126 verläuft. Darüber hinaus verlieren durch diesen Effekt alle in der Osmylierung beschreitbaren nicht beschleunigten Reaktionswege stark an Bedeutung. Vor allem die Liganden (DHQ) 2 PHAL und (DHQD) $)_{2} \mathrm{PHAL}$, deren CinchonaAlkaloid-Einheiten beide am jeweiligen 9-O über ein Phthalazin miteinander verbrückt sind 
und somit eine Enzym-ähnliche Bindungstasche aufweisen, induzieren sehr hohe Enantioselektivitäten. ${ }^{[109,110]}$

Zur Prognose der Stereoselektivität der Dihydroxylierung kann das von Sharpless et al. vorgeschlagene mnemonic device konsultiert werden. Der südöstliche (SE) sowie der nordwestliche (NW) Quadrant in diesem Schema werden hierbei durch den sterischen Einfluss des Liganden abgeschirmt, wohingegen der nordöstliche (NE) Quadrant als Eintrittspunkt für das Olefin fungiert und der südwestliche (SW) Quadrant für flache, aromatische oder große aliphatische Substituenten verbleibt. Die Positionierung des Olefins erfolgt damit in einer Art und Weise, die den kleinsten Substituenten, im Regelfall ein Proton, im südöstlichen und den sperrigsten Substituenten im südwestlichen Quadranten anordnet. Der Angriff auf das Olefin findet im Falle von AD-mix- $\alpha$ von der Unterseite statt, während er mit AD-mix- $\beta$ von der Oberseite her verläuft (Abbildung 39). ${ }^{[110,111]}$

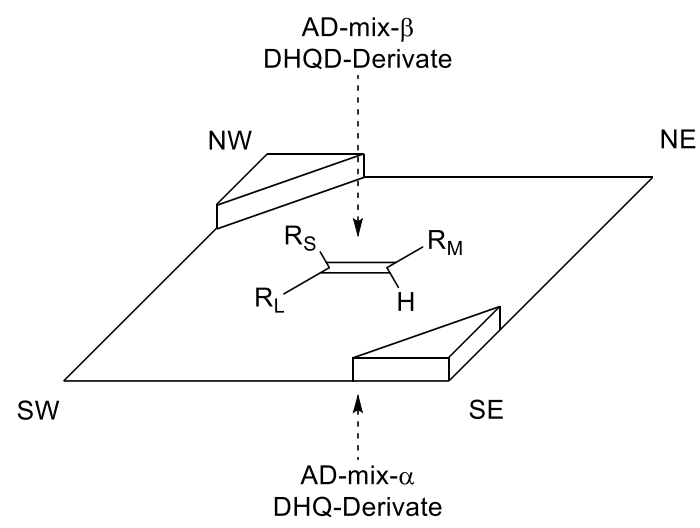

Abbildung 39: Mnemonic device zur Vorhersage der Stereoselektivität.

\subsubsection{Anwendungen in der organischen Synthese}

Die Vorteile der Sharpless-Dihydroxylierung, namentlich das breite Substratspektrum, die milden Reaktionsbedingungen sowie die erzielten hohen Ausbeuten und ausgezeichneten Stereoselektivitäten, machen ebenjene zu einem vielgenutzten Werkzeug in der Synthese zahlreicher komplexer Moleküle.

In der enantioselektiven Totalsynthese von (+)-Zaragoesäure C (129) griffen Armstrong und Mitarbeiter auf eine doppelte Sharpless-Dihydroxylierung zum Aufbau vier benachbarter Stereozentren zurück. Das ursprüngliche Vorhaben, dies in einer Eintopfreaktion mittels $A D$-mix- $\beta$ durchzuführen, scheiterte dabei an einer zu geringen Ausbeute sowie Stereoselektivität. Durch die Separierung in zwei aufeinanderfolgenden Dihydroxylierungen, in denen zunächst das Dien 127 mit Super-AD-mix- $\beta$ in einem Zweiphasen- 
system aus $t \mathrm{BuOH} / \mathrm{H}_{2} \mathrm{O}$ ins Triol überführt wurde, das seinerseits mittels katalytischer Mengen $\mathrm{OsO}_{4}$ und (DHQD) ${ }_{2} \mathrm{PHAL}$ in einem homogenen Aceton $/ \mathrm{H}_{2} \mathrm{O}$-Gemisch zum Pentaol 128 umgesetzt wurde, konnten sowohl die Ausbeute auf moderate 45\% sowie die Enantio- und Diastereoselektivität auf gute 76\% ee respektive $d r=9: 1$ verbessert werden (Abbildung 40). ${ }^{[119]}$

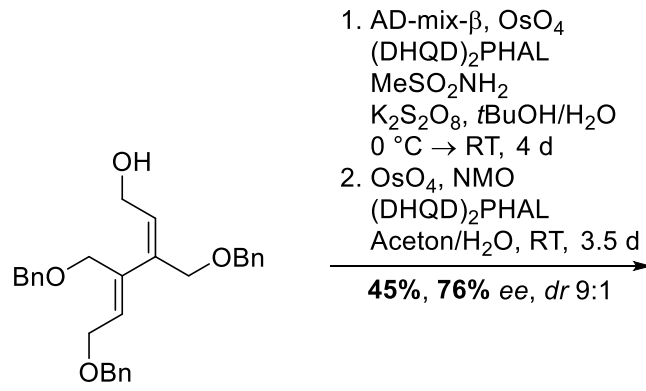

127

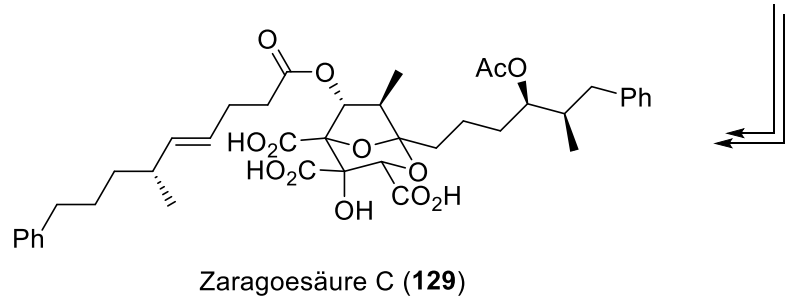<smiles>OCC(O)COCC(O)C(O)(CO)C(O)CO</smiles>

128

Abbildung 40: Zweifache Sharpless-Dihydroxylierung zur enantioselektiven Totalsynthese von Zaragoesäure C (129) durch Armstrong.

Mithilfe der Sharpless-Dihydroxylierung war es Shair und Mitarbeitern möglich in ihrer enantioselektiven Synthese von (+)-Cephalostatin 1 (132) neben der Hydroxygruppe an C-16 vor allem die angrenzende Hydroxyfunktionalität an C-17 mit vollständiger Diastereoselektivität und in hoher Ausbeute ausgehend vom Olefin 130 in das Substrat zu inkorporieren. Hierbei verblieben sowohl die Acetal- als auch die Silylschutzgruppen aufgrund der milden Reaktionsbedingungen während der Transformation unbeeinflusst (Abbildung 41). ${ }^{[120]}$ 


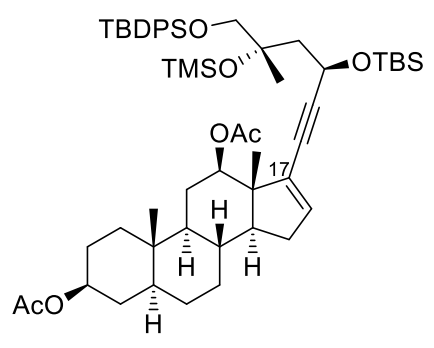

130

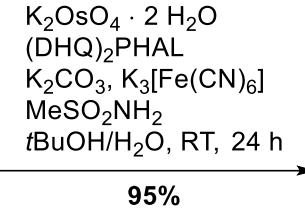

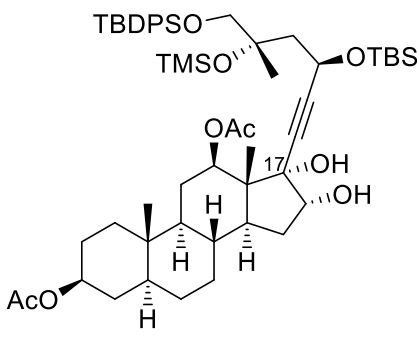

131
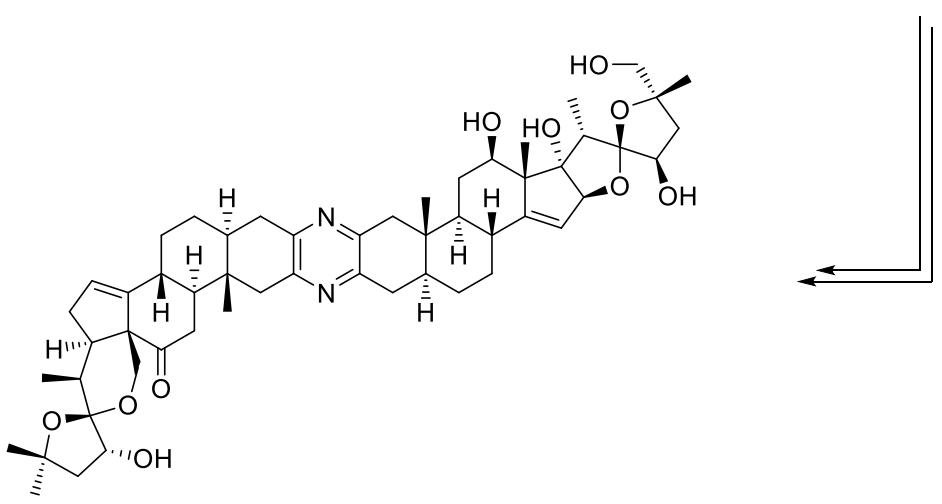

(+)-Cephalostatin 1 (132)

Abbildung 41: Sharpless-Dihydroxylierung in der enantioselektiven Totalsynthese von (+)-Cephalostatin 1 (132) durch Shair. 


\subsection{Zielsetzung und Planung der Arbeit}

Im Jahr 2008 gelang es einer Forschungsgruppe der Merck \& Co., Inc. erstmals, Vertreter der neuen Naturstoffklasse der Parnafungine zu identifizieren, zu isolieren und zu beschreiben. ${ }^{[14]}$ Die vorwiegend von Schimmelpilzen des Fusarium larvarum Komplexes sezernierten Sekundärmetabolite zeichnen sich durch zwei interessante Eigenschaften aus. Zum einen sind sie biologisch aktiv gegenüber einem breiten Spektrum vor allem klinisch relevanter pathogener Pilze und zum anderen besitzen sie in Form eines Isoxazolidinon-Ringes ein unter Naturstoffen bisher einzigartiges Strukturelement. In Anbetracht der schlechten Therapierbarkeit und hohen Mortalität systemischer Mykosen, des spärlichen Arsenals wirksamer Antimykotika sowie der dürftig befültten Entwicklungspipeline neuer Wirkstoffe und der zunehmenden Resistenzentwicklung pathogener Fungi stellen Parnafungine mit ihrem neuartigen Enzym-spezifischen Wirkmechanismus, neben der aus chemischer Sicht reizvollen Struktur, ein attraktives Ziel für eine Darstellung auf synthetischem Wege dar.

Somit bestand das Bestreben der vorliegenden Arbeit darin, einen enantioselektiven Zugang zu dieser neuen Klasse von Wirkstoffen zu erlangen. Parnafungin C (3) erschien von den vier bisher bekannten Vertretern der Stoffklasse aus synthetischer Sicht als sinnvollstes Ziel. Im Vergleich zu Parnafungin A (1) und B (2) weist es keine Tendenz zur Konstitutionsisomerie auf und ist gleichzeitig strukturell weniger komplex, als das um einen zusätzlichen Oxiran-Ring erweiterte Parnafungin D (4) (siehe S. 5, Abbildung 1).

Wirft man einen Blick in die Literatur bzgl. der synthetischen Darstellung der Parnafungine, stößt man seit ihrer Entdeckung nur auf zwei Arbeiten aus der Forschungsgruppe Snider. Dabei handelt es sich um Publikationen zur Synthese des labilen Isoxazolo[4,3,2-de]phenanthridinon-Motivs in verschiedenen Modellverbindungen (siehe S. 10 ff.). ${ }^{[18,21]}$ Diese, im Arbeitskreis Tietze bereits in der Masterarbeit von Björn Göricke auf ihre Validität überprüfte Methode,$^{[121]}$ sollte ebenfalls am Ende der geplanten Synthese zum Aufbau des Isoxazolidinon-Ringes Anwendung finden. Des Weiteren konnte in ersten Untersuchungen zur enantioselektiven Totalsynthese von Parnafungin $C$ (3) im Rahmen meiner vorangegangenen Diplomarbeit bereits das hochsubstituierte Biaryl 135 synthetisiert werden, ${ }^{[122]}$ das alle Funktionalitäten besitzt, um zum einen die bereits erwähnte Synthesesequenz von Snider zu inkorporieren und zum anderen das Tetrahydroxanthenon-Grundgerüst in enantioselektiver Art und Weise nach den im Arbeitskreis Tietze entwickelten Methoden aufbauen zu können (Abbildung 42). ${ }^{\text {[93,123-126] }}$ Jedoch litt die achtstufige Synthese dieser Verbindung unter einer eher moderaten Ausbeute der abschließenden beiden Stufen. Für die Miyaura-Borylierung und die Suzuki- 
Miyaura-Kreuzkupplung konnten Ausbeuten von 50\% respektive 19\% erzielt werden, sodass in beiden Fällen Optimierungsbedarf bestand.

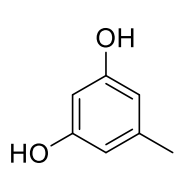

Orcinol (17)

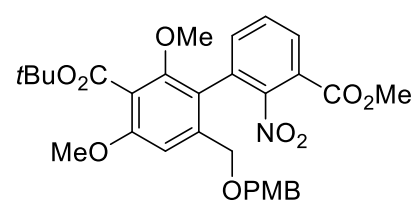

135

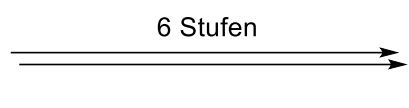

4:11 (1:1), $\mathrm{Pd}(\mathrm{OAc})_{2}$

SPhos, $\mathrm{K}_{3} \mathrm{PO}_{4}$

1,4-Dioxan $/ \mathrm{H}_{2} \mathrm{O}(100: 1)$

$45^{\circ} \mathrm{C}, 3 \mathrm{~d}$

$19 \%(43 \%$ brsm $)$

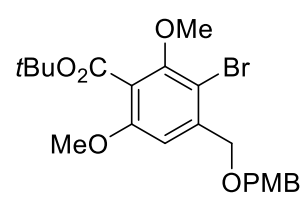

133

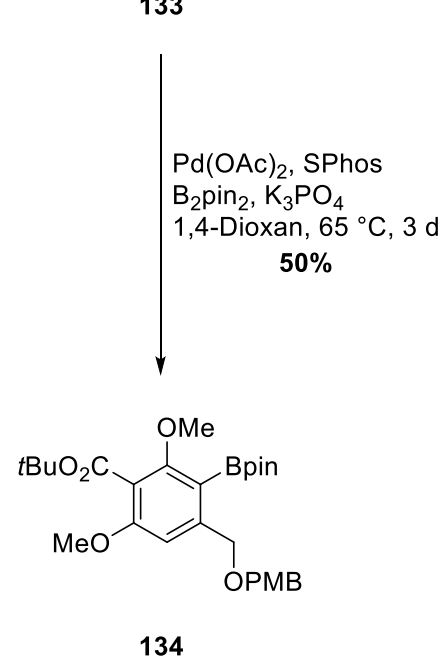

Abbildung 42: Synthese des Biaryls 135.

Die Synthese des Tetrahydroxanthenon-Motivs sollte vor allem in Analogie zu den Arbeiten von Ling Ma ${ }^{[124,125,127]}$ und Stefan Jackenkrol/ ${ }^{[23,126,128]}$ mit etwaigen Modifikationen, die den früh in der Synthese eingeführten zusätzlichen Funktionalitäten geschuldet waren, erfolgen. Basierend auf den genannten Methoden und den bereits gesammelten Erkenntnissen auf diesem Gebiet ergab sich aus der retrosynthetischen Analyse von Parnafungin C (3) folgende Planung für dessen enantioselektive Darstellung (Abbildung 43).

Aufgrund der Labilität des Isoxazolidinon-Ringes sollte der Aufbau dieses Strukturelements mithilfe der von Snider entwickelten Methode am Ende der Synthese erfolgen, sodass sich Parnafungin C (3) auf die Nitroverbindung 136 zurückführen lässt. Diese wiederum ist durch eine intramolekulare Acylierung sowie Ent- und Umschützung aus dem Chromanon 137 zugänglich. Ausgehend vom Vinylchroman 138 lässt sich das Chromanon 137 mittels einer Sharpless-Dihydroxylierung, diverser Modifikationen funktioneller Gruppen unter Einbeziehen verschiedener Schutzgruppenstrategien, Seitenkettenverlängerung und benzylischer Oxidation synthetisieren. Die enantioselektive Darstellung des Vinylchromans 138 und damit der Schlüsselschritt der Synthese soll über eine enantioselektive Palladium-katalysierte Wacker-Zyklisierung aus der phenolischen Vorstufe 139 realisiert werden. Seitenkettenverlängerung und selektive Entschützung der 
sterisch weniger stark gehinderten Methoxygruppe am Aldehyd 140 resultiert in dem Phenol 139. Der Aldehyd 140 lässt sich auf die Arylhalogenide 141 und 11 zurückführen, von denen 141 per Miyaura-Borylierung in das entsprechende Pinakolboran überführt und anschließend mittels einer Suzuki-Miyaura-Kreuzkupplung mit dem verbleibenden Arylhalogenid 11 gekoppelt werden soll. Das Arylhalogenid 140 lässt sich über verschiedene Funktionalisierungen aus dem kommerziell erhältlichen Orcinol (17) aufbauen, während das Arylhalogenid $\mathbf{1 1}$ per Methylierung aus der ebenfalls kommerziell erhältlichen 3-Chlor-2-nitrobenzoesäure (142) zugänglich ist.

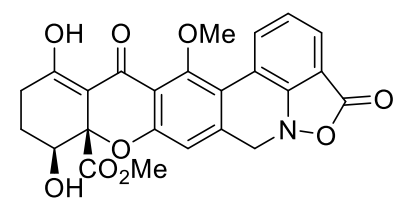

Parnafungin C (3)<smiles>C=C[C@]1(COc2ccccc2)CCc2c(cc(CO[O+])c(-c3cccc(C(C)=O)c3OC)c2[N+](=O)[O-])O1</smiles>

138<smiles>[C]=C</smiles><smiles>C=C=CCc1cc(O)c(CC/C(=C/C)COc2ccccc2)c(OC)c1-c1cccc(C(=O)OC)c1[N+](=O)[O-]</smiles>

139<smiles>COc1c2c(cc(CO[Na-])c1[N+](=O)[O-])O[C@@]1(C(C)OC)C(=C(O)CC[C@@H]1O)C2=O</smiles>

136<smiles>[C]=C</smiles>

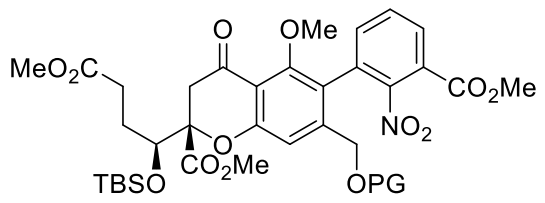

137<smiles>COc1cc(CO[Pb])c(-c2cccc(C(OC)OC)c2[N+](=O)[O-])c(OC)c1C=O</smiles>

140<smiles>Cc1cc(O)cc(O)c1</smiles>

Orcinol (17)<smiles>C=C</smiles><smiles>[X]c1c(CO[Pb])cc(OC)c(C=O)c1OC</smiles>

141

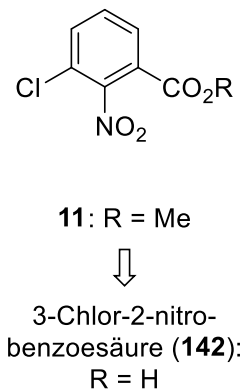

Abbildung 43: Retrosynthetische Analyse des Parnafungin C (3). 
Des Weiteren sollte der für die enantioselektive Darstellung des Chromans 138 benötigte $(S, S)$-Bn-BOXAX-Ligand $(S, S)$-91a in Anlehnung an Arbeiten von Meyers hergestellt werden. ${ }^{[91,129-133]}$

Zusammenfassend ergeben sich somit folgende Aufgabenstellungen:

- Synthese des enantiomerenreinen (S,S)-Bn-BOXAX-Liganden (S,S)-91a

- Optimierung der Miyaura-Borylierung und Suzuki-Miyaura-Kreuzkupplung zum Aufbau des Biaryls 135

- Synthese des Alkenylphenols 139

- Durchführung der enantioselektiven Palladium-katalysierten Wacker-Zyklisierung zum Erhalt des Chromans 138

- Funktionalisierung des Chromans 138 mittels einer Sharpless-Dihydroxylierung

- Abschluss der Synthese der Tetrahydroxanthenon-Grundstruktur

- Aufbau des Isoxazolidinon-Ringes nach Snider und Beendigung der enantioselektiven Totalsynthese des Parnafungin C (3) 
II Darstellung der Ergebnisse 


\subsection{Optimierung der Synthesesequenz zum Aufbau des Biarylfragments}

Wie bereits erwähnt, sollte das Biaryl 135 als Ausgangspunkt für die weitere Synthese des Parnafungin C (3) fungieren. In Anbetracht der bereits benötigten acht Syntheseschritte für dessen Darstellung und der folgenden komplexen sowie aufwendigen Synthesesequenz sollten sowohl die Miyaura-Borylierung als auch die Suzuki-MiyauraKreuzkupplung hinsichtlich der erzielten Ausbeuten optimiert werden, um eine effiziente und praktikable Synthese zu ermöglichen. Zu diesem Zweck wurde zunächst das erforderliche Arylbromid 133, entsprechend der in der Diplomarbeit entwickelten Synthese, dargestellt.

\subsubsection{Synthese des Arylbromids $133^{[122]}$}

Den Beginn der Synthese bildete die Methylierung der beiden phenolischen Hydroxyfunktionalitäten am kommerziell erhältlichen Orcinol (17) unter basischer Reaktionsführung mittels Dimethylsulfat in 86\% Ausbeute. Der erhaltene Dimethylether 143 wurde daraufhin unter Verwendung von $n \mathrm{BuLi}$ ortho-lithiiert und durch die Zugabe von Trockeneis mit einer Ausbeute von 69\% in die entsprechende Carbonsäure 144 überführt.

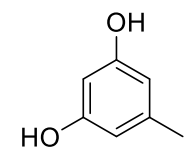

Orcinol (17)

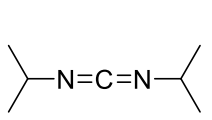

145

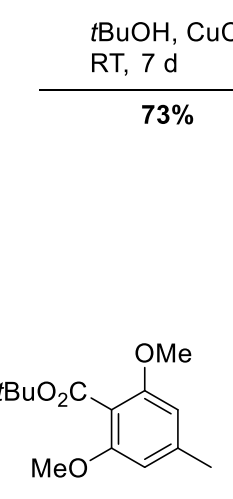

147
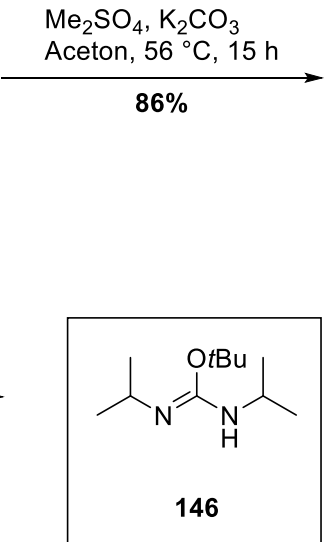

146, $\mathrm{NH}_{4} \mathrm{Cl}$

$\mathrm{CH}_{2} \mathrm{Cl}_{2} / \mathrm{tBuOH}(1: 1)$ $\mathrm{RT}, 2 \mathrm{~h}$

quant.<smiles>COc1cc(C)cc(OC)c1</smiles>

143
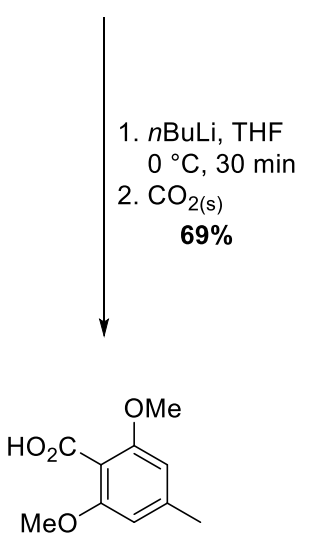

144

Abbildung 44: Darstellung des tert-Butylesters 147.

Die anschließende Übertragung des tert-Butylfragments des literaturbekannten Isoharnstoffs 146, der durch die Umsetzung des kommerziell erhältlichen 
$N, N$-Di-iso-propylcarbodiimids $\mathbf{1 4 5}$ mit $t \mathrm{BuOH}$ unter Einsatz einer katalytischen Menge $\mathrm{CuCl}$ in $73 \%$ Ausbeute zugänglich war, auf die Carbonsäure 144 erfolgte hierbei quantitativ (Abbildung 44). ${ }^{[134]}$

Die eingeführte tert-Butylesterfunktion diente hierbei als maskierte Aldehydgruppe, um eine Wohl-Ziegler-Reaktion zur Einführung des Brom-Substituenten in benzylischer Position durchführen zu können. Wie sich im Rahmen der Diplomarbeit zeigte, tendierte die freie Aldehydfunktion in einem analogen Substrat zur Oxidation zum Carbonsäurebromid unter den typischen Bedingungen der Wohl-Ziegler-Bromierung, wodurch nach wässriger Aufarbeitung die Carbonsäure erhalten wurde. Die Schützung der Aldehydfunktion als zyklisches Acetal stellte sich durch die anschließende Öffnung des Acetals zum Bromalkylester in der Wohl-Ziegler-Reaktion ebenfalls als ertraglos heraus. Ferner würde mit der später erfolgenden Suzuki-Miyaura-Kreuzkupplung eine weitere Estergruppe in Form eines Methylesters in das Substrat eingeführt, sodass sich an dieser Stelle für die Maskierung des Aldehyds als tert-Butylester entschieden wurde, um diesen nach der Suzuki-Miyaura-Kreuzkupplung selektiv zu entschützen, die freigesetzte Säure in Gegenwart des Methylesters zum Aldehyd zu reduzieren und mit der Synthese an ebenjener Aldehydfunktion fortzusetzen.

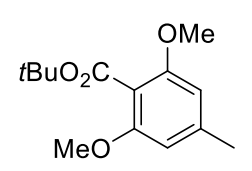

147

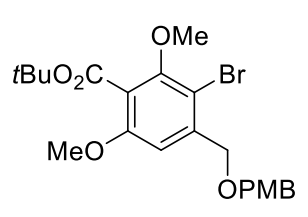

133
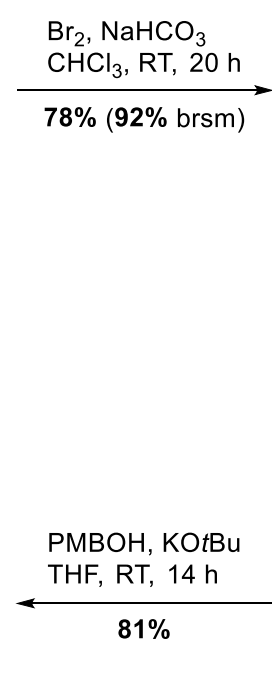
konnte in einer Ausbeute von 69\% realisiert werden. Entscheidend für den Ablauf der Reaktion ist der Einsatz von Benzol als Lösungsmittel, weil weder in $\mathrm{CCl}_{4}$ noch in $\mathrm{CH}_{2} \mathrm{Cl}_{2}$ die benzylische Bromierung beobachtet werden konnte. Abschließende basenvermittelte nukleophile Substitution des neu eingeführten Brom-Substituenten durch $\mathrm{PMBOH}$ resultierte in der Darstellung des für die folgenden Optimierungsuntersuchungen benötigten PMB-geschützten Alkohols 133 in 81\% Ausbeute (Abbildung 45).

\subsubsection{Miyaura-Borylierung und Suzuki-Miyaura-Kreuzkupplung zur Synthese des Biaryls 135}

Ausgehend vom Arylbromid 133 konnten bereits während der Diplomarbeit erste Methoden zur erfolgreichen Durchführung sowohl der Borylierung zum Pinakolboronsäureester 134 als auch der Suzuki-Miyaura-Kreuzkupplung zum Biaryl 135 etabliert werden (Abbildung 46). ${ }^{[122]}$

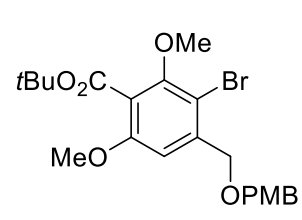

133

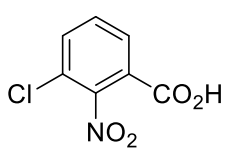

3-Chlor-2-nitrobenzoesäure (142)
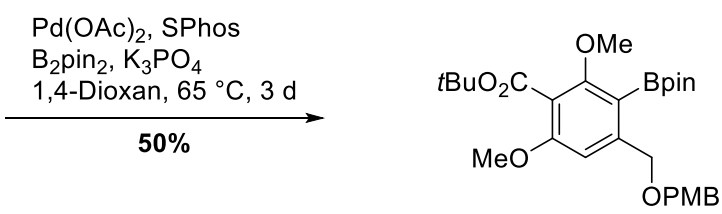

134

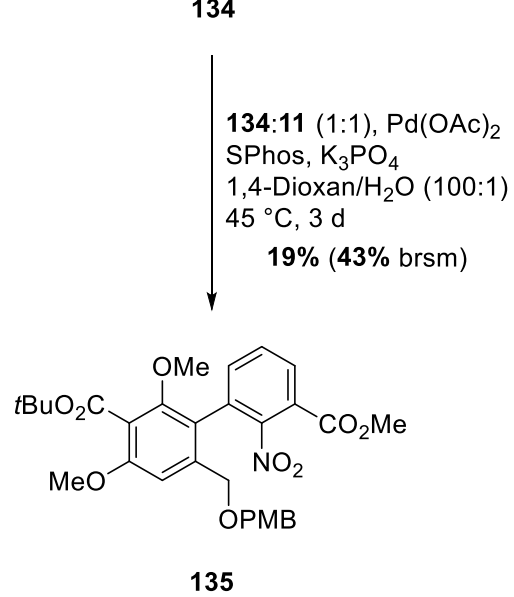

Abbildung 46: Miyaura-Borylierung und Suzuki-Miyaura-Kreuzkupplung zum Aufbau des Biaryls 135.

Zum Erhalt des Boronsäureesters 134 erwies sich eine Miyaura-Borylierung unter Verwendung von $\mathrm{Pd}(\mathrm{OAc})_{2}$ (5 mol\%), dem Buchwald-Liganden SPhos (12.5 mol\%), der Base $\mathrm{K}_{3} \mathrm{PO}_{4}$ (3.00 Äq.) und dem dimeren Boronsäureester $\mathrm{B}_{2}$ pin 2 (3.00 Äq.) in 1,4-Dioxan bei $65^{\circ} \mathrm{C}$ als durchführbar. Für die erzielten $50 \%$ Ausbeute war allerdings eine Reaktionszeit von drei Tagen erforderlich. Unter den zu diesem Zeitpunkt getesteten Reaktionsbedingungen, die aus diversen Kombinationen der Palladiumquellen $\operatorname{Pd}(\mathrm{OAc})_{2}$ und $\mathrm{Pd}_{2} \mathrm{dba}_{3}$ mit den Liganden SPhos sowie XPhos oder dem kommerziell erhältlichen 
Katalysatorsystem $\mathrm{Pd}(\mathrm{dppf}) \mathrm{Cl}_{2}$ unter Verwendung der Basen $\mathrm{K}_{3} \mathrm{PO}_{4}$ und KOAc in DMSO sowie vor allem 1,4-Dioxan bei unterschiedlichen Temperaturen und optionaler Nutzung von Mikrowellenstrahlung bestanden, stellte dies das erreichbare Optimum dar. Ein synthetisch simplerer Zugang über einen Lithium-Halogen-Austausch mit anschließender Umsetzung der in situ generierten Organolithium-Verbindung mit einem geeigneten Boronsäureester wurde ebenfalls untersucht, lieferte den gewünschten Boronsäureester 134 im besten Fall jedoch nur in massenspektrometrisch nachweisbaren Spuren. Dieses Verfahren sowie eine Miyaura-Borylierung wurden zusätzlich mit dem Arylchlorid 11, das aus der kommerziell erhältlichen 3-Chlor-2-nitrobenzoesäure (142) durch eine basenvermittelte Methylierung mit $\mathrm{Mel}$ in 96\% Ausbeute zugänglich war, durchgeführt. Beide Umsetzungen waren jedoch nicht erfolgreich.

Dabei wäre die Borylierung des Arylchlorids insofern erstrebenswert, dass der Reaktionsschritt der Borylierung im Hauptsynthesepfad entfallen würde, was diesen um ebenjene Stufe verkürzen und die Gesamtausbeute verbessern würde. Des Weiteren hätte man in der nachfolgenden Suzuki-Miyaura-Kreuzkupplung das borylierte Analogon des Arylchlorids 11, welches in diesem Fall leicht in größeren Mengen hätte präpariert werden können, im Überschuss verwenden und damit unter Umständen noch bessere Ausbeuten erzielen können. Vorweggreifend sollte in diesem Zusammenhang erwähnt werden, dass der Forschungsgruppe Snider, wie in der Publikation zu den hexazyklischen Parnafungin Modellsystemen beschrieben, die Borylierung des Nitrobenzoesäuremethylesters 151 gelang (Abbildung 47). ${ }^{[21]}$

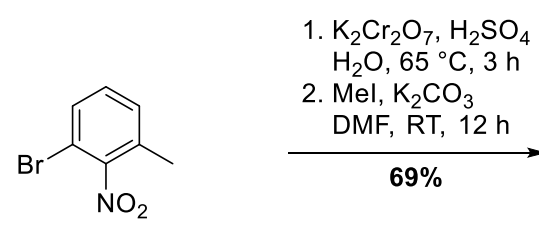

150

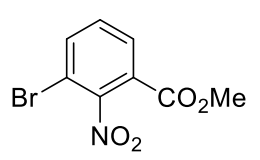

151
1. iPrOBpin, $n$ BuLi $\mathrm{PhMe} / \mathrm{THF}(4: 1)$

$-97,1 \mathrm{~h}$

2. $-78^{\circ} \mathrm{C}, 1 \mathrm{~h}$

$\rightarrow-20^{\circ} \mathrm{C}, \mathrm{AcOH}$ $52 \%$

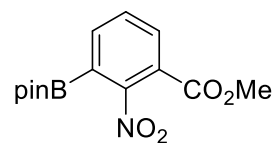

21

Abbildung 47: Borylierung des Arylbromids 151 nach Snider.

$\mathrm{Zu}$ diesem Zeitpunkt konnte indes bereits eine eigene reproduzierbare und auf das jeweilige Substrat anpassbare Methode etabliert werden, die im Regelfall vergleichbare oder leicht bessere Ergebnisse lieferte und deren Optimierungsprozess im Folgenden beschrieben ist.

In Anbetracht der nahezu identischen Reaktionsstruktur der Miyaura-Borylierung verglichen mit der Suzuki-Miyaura-Kreuzkupplung wurden zunächst die gefundenen Reaktionsbedingungen der Borylierung auf die Kreuzkupplungsreaktion übertragen. Initial konnte dabei keine Reaktion beobachtet werden und erst durch das Hinzufügen von 
Wasser konnte das Biaryl 135 erhalten werden. $\mathrm{Pd}(\mathrm{OAc})_{2}$ (5 mol\%), SPhos (12.5 mol\%), $\mathrm{K}_{3} \mathrm{PO}_{4}$ (3.00 Äq.) in 1,4-Dioxan/Wasser (100:1) ermöglichten die Kupplung des Bororganyls 134 mit dem Chloraren 11 im Verhältnis 1:1 in einer Ausbeute von 19\% nach einer Reaktionszeit von drei Tagen. Zusätzlich konnten $24 \%$ des eingesetzten Bororganyls 134 zurückgewonnen werden.

Um die unter Umständen langwierige Suche nach einem reaktiveren Katalysatorsystem oder einer komplett neuen Reaktionsmethode für die Borylierung und die Kreuzkupplung, wobei eine Borylierungsmethode für das Arylchlorid 11 unter den weiter oben genannten Gesichtspunkten wünschenswert wäre, zu vermeiden, wurden die bereits etablierten Methoden erneut aufgegriffen und versucht diese hinsichtlich der erzielten Ausbeuten und der Reaktionsführung (dreitägige Reaktionszeit) effizienter zu gestalten. Aufgrund der erwiesenen Übertragbarkeit der Reaktionsbedingungen der Miyaura-Borylierung auf die Suzuki-Miyaura-Kreuzkupplung erfolgte ein Großteil der Optimierungsversuche in der erstgenannten Reaktion und die Verbesserungen wurden anschließend auf letztgenannte angewandt (Abbildung 48).

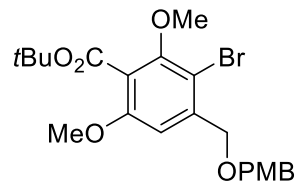

133

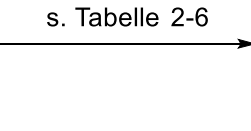

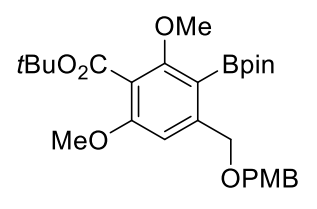

134

Abbildung 48: Miyaura-Borylierung des Arylbromids 133.

So wurde zunächst die eingesetzte Base sowie das verwendete Lösungsmittel auf etwaiges Steigerungspotenzial untersucht. Hierbei wurde die Auswahl auf häufig für diese Reaktion genutzte Vertreter beschränkt. Als Basen kamen die Fluoride CsF und KF, $\mathrm{Cs}_{2} \mathrm{CO}_{3}, \mathrm{KOAc}, \mathrm{K}_{3} \mathrm{PO}_{4}$ und $\mathrm{NaOH}$ zum Einsatz. Im Falle der Lösungsmittel reichte das Spektrum vom unpolaren Toluol, über die Ether 1,4-Dioxan, DME und THF, bis zu sehr polaren Solventien in Form von DMF und $n \mathrm{BuOH}$. Zusätzlich wurde in diesem Rahmen die Verwendung von Pinakolboran als Bororganyl untersucht, wodurch eine höhere Atomökonomie im Vergleich zum bisher verwendeten dimeren $B_{2}$ pin $n_{2}$ erreicht werden würde. In diesem Falle erfolgte der Einsatz von $\mathrm{NEt}_{3}$ als Base (Tabelle 2). Alle Reaktionen wurden zunächst in dem jeweiligen reinen Lösungsmittel bei Raumtemperatur durchgeführt und regelmäßig via Dünnschichtchromatographie kontrolliert. Mit Ausnahme der Reaktionen, die in $n \mathrm{BuOH}$ durchgeführt wurden, konnte im Allgemeinen kein Umsatz nach ca. 2-4 h nachgewiesen werden, weshalb jeweils eine katalytische Menge Wasser hinzugegeben wurde. Die Zugabe von Wasser resultierte in den aufgeführten Ausbeuten 
in einer verkürzten Reaktionszeit von insgesamt einem Tag bei gleichzeitig reduzierter Reaktionstemperatur.

Tabelle 2: Ermittlung einer optimalen Base-Lösungsmittel-Kombination für die Miyaura-Borylierung.

\begin{tabular}{c|ccccccc}
\hline LM Base & $\mathrm{CsF}$ & $\mathrm{KF}$ & $\mathrm{Cs}_{2} \mathrm{CO}_{3}$ & $\mathrm{KOAc}$ & $\mathrm{K}_{3} \mathrm{PO}_{4}$ & $\mathrm{NaOH}$ & $\mathrm{NEt}_{3}{ }^{[\mathrm{c}]}$ \\
\hline 1,4-Dioxan & $31 \%$ & $25 \%$ & $55 \%$ & Spuren & $50 \%$ & $29 \%$ & - \\
Toluol & Spuren & - & Spuren & - & Spuren & Spuren & - \\
DMF & - & $20 \%$ & $24 \%$ & Spuren & $30 \%$ & $18 \%$ & - \\
DME & $33 \%$ & $31 \%$ & $41 \%$ & Spuren & $42 \%$ & Spuren & - \\
THF & $51 \%$ & $45 \%$ & $52 \%$ & - & $47 \%$ & $20 \%$ & - \\
$n$ BuOH $^{[\mathrm{b}]}$ & $21 \%$ & $19 \%$ & $17 \%$ & Spuren & $34 \%$ & $15 \%$ & - \\
\hline
\end{tabular}

Reaktionsbedingungen: $\mathrm{Pd}(\mathrm{OAc})_{2}$ (5 mol\%), SPhos (12.5 mol\%), B2pin2 (2.50 Äq.), Base (2.50 Äq.), $\mathrm{LM} / \mathrm{H}_{2} \mathrm{O}(100: 1), \mathrm{RT}, 1$ d. [a]

[a] die Zugabe der katalytischen Menge Wasser erfolgte nach 2-4 h, nachdem im Regelfall keine Reaktion per DC-Kontrolle nachgewiesen werden konnte; [b] die Reaktion verlief ohne die Zugabe von Wasser; [c] HBpin (1.50 Äq.) anstelle von B $\mathrm{B}_{2} \mathrm{pin}_{2}$

Von den getesteten Lösungsmitteln erwies sich Toluol als ungeeignet für diese spezifische Umsetzung des Arylbromids 133 zum Pinakolboronsäureester 134. Die polaren Vertreter DMF und $n \mathrm{BuOH}$ lieferten schlechtere Ergebnisse als die eingesetzten Ether und sind gerade wegen ihres hohen Siedepunktes unter praktischen Gesichtspunkten weniger gut geeignet. Ergo konnten mit den drei Ethern 1,4-Dioxan, DME und THF die besten Ausbeuten bei identischen Reaktionsverläufen erzielt werden. Bedenkt man die verringerte Reaktionstemperatur, erwies sich THF als das praktikabelste Lösungsmittel, allerdings stellen die beiden anderen Ether gute Alternativen dar, sollte ein höherer Temperaturbereich für eine erfolgreiche Durchführung der Reaktion vonnöten sein.

Ähnlich dem Toluol bei den Lösungsmitteln zeigte sich mit KOAc ebenfalls eine der untersuchten Basen als ungeeignet in dieser Miyaura-Borylierung, sodass das gewünschte Produkt bestenfalls massenspektrometrisch nachweisbar war. Aus den verbliebenen fünf Basen kristallisierten sich $\mathrm{Cs}_{2} \mathrm{CO}_{3}$ und $\mathrm{K}_{3} \mathrm{PO}_{4}$ als die Vertreter heraus, mit denen in der Regel über das untersuchte Lösungsmittelspektrum gesehen, die besten Ausbeuten erzielt werden konnten. Kam THF als Lösungsmittel zum Einsatz, wurden jedoch auch mit den beiden Fluoriden CsF und KF ähnlich gute Ausbeuten erreicht. Dennoch stellen $\mathrm{Cs}_{2} \mathrm{CO}_{3}$ und $\mathrm{K}_{3} \mathrm{PO}_{4} \mathrm{im}$ Allgemeinen die für diese Reaktion optimalen Basen dar. Mit der Erstgenannten erfolgte dabei ein schnellerer Ablauf der Reaktion zum Preis einer verstärkten Bildung von Nebenprodukten, wohingegen $\mathrm{K}_{3} \mathrm{PO}_{4}$ zwar einen langsameren, dafür aber saubereren Reaktionsverlauf zur Folge hatte. Keine Reaktion über alle untersuchten Lösungsmittel hinweg konnte mit der Variante HBpin in 
Kombination mit $\mathrm{NEt}_{3}$ beobachtet werden, sodass die Reaktion in Bezug auf das Transmetallierungsagens nicht atomökonomischer gestaltet werden konnte.

Somit bestätigte das Screening der Basen und Lösungsmittel die ehedem verwendete Base $\mathrm{K}_{3} \mathrm{PO}_{4}$ als Ideallösung und ergänzte diese um eine reaktivere Alternative in Form von $\mathrm{Cs}_{2} \mathrm{CO}_{3}$. Das vormals eingesetzte 1,4-Dioxan lieferte zwar äquivalente Ausbeuten verglichen mit THF, trotzdem ist 1,4-Dioxan aus rein praktischen Gründen weniger gut geeignet. Den größten Einfluss auf die Reaktion konnte indes der Zugabe einer katalytischen Menge an Wasser bei der Verwendung aprotischer Lösungsmittel bescheinigt werden, wodurch eine verkürzte Reaktionsführung bei Raumtemperatur ermöglicht wurde. ${ }^{[42,45,135]}$ Nichtsdestotrotz resultierten diese Ergebnisse noch nicht in einer signifikanten Steigerung der Ausbeute, sodass nach weiteren Faktoren gesucht wurde, die sich positiv auf die Reaktion auswirken könnten.

Zunächst wurde untersucht, welchen Einfluss die Menge des Bororganyls $\mathrm{B}_{2} \mathrm{pin}_{2}$ sowie der Base auf die Reaktion ausübt. Es wurden sowohl $\mathrm{K}_{3} \mathrm{PO}_{4}$ als auch $\mathrm{Cs}_{2} \mathrm{CO}_{3} \mathrm{im}$ Lösungsmittel THF in diesem Zusammenhang getestet.

Tabelle 3: Untersuchungen zur Ermittlung der optimalen Basen- und $\mathrm{B}_{2} \mathrm{pin}_{2}$-Äquivalente.

\begin{tabular}{ccccc}
\hline Eintrag & Äquivalente $\mathrm{B}_{2}$ pin & Base & Äquivalente Base & Ausbeute \\
\hline 1 & 2.00 & $\mathrm{~K}_{3} \mathrm{PO}_{4}$ & 2.00 & $74 \%$ \\
2 & 2.00 & $\mathrm{Cs}_{2} \mathrm{CO}_{3}$ & 2.00 & $87 \%$ \\
3 & 1.00 & $\mathrm{~K}_{3} \mathrm{PO}_{4}$ & 1.00 & $44 \%$ \\
4 & 1.00 & $\mathrm{Cs}_{2} \mathrm{CO}_{3}$ & 1.00 & $59 \%$ \\
\hline
\end{tabular}

Reaktionsbedingungen: $\mathrm{Pd}(\mathrm{OAc})_{2}$ (5 mol\%), SPhos (12.5 mol\%), B $\mathrm{B}_{2} \mathrm{pin}_{2}$, Base, THF/H $\mathrm{H}_{2} \mathrm{O}$ (100:1), RT, $1 \mathrm{~d}$.

Eine leichte Verringerung von den vormals verwendeten 2.50 Äquivalenten auf nunmehr 2.00 Äquivalente an $\mathrm{B}_{2} \mathrm{pin}_{2}$ sowie an der jeweiligen Base führte zu einer signifikanten Erhöhung der Ausbeute (Tabelle 3, Eintrag 1 und 2). Bei weiterer Reduktion der Menge auf jeweils 1.00 Äquivalente wurde dieser Effekt jedoch wieder ins Negative verkehrt (Tabelle 3, Eintrag 3 und 4). In beiden Fällen konnten mit $\mathrm{Cs}_{2} \mathrm{CO}_{3}$ leicht bessere Ausbeuten als mit $\mathrm{K}_{3} \mathrm{PO}_{4}$ erzielt werden, was einen weiteren Beleg für die höhere Reaktivität des $\mathrm{Cs}_{2} \mathrm{CO}_{3}$ darstellt. Für die beobachtete Steigerung der Ausbeute bei leicht reduzierter Menge an eingesetztem Bororganyl und Base können verschiedene Faktoren verantwortlich sein. So könnten die 2.00 Äquivalente der Base in einem nahezu idealen Verhältnis zur hinzugegebenen katalytischen Menge an Wasser stehen, wodurch dieses zu einem beträchtlichen Teil zu Hydroxid-Ionen umgesetzt werden sollte, die wiederum zur Ausbildung eines reaktiveren Hydroxo-Pd"-Komplexes führen und die Reaktion beschleunigen sollten. Weiterhin könnte auch das Verhältnis der freien, sich in Lösung 
befindlichen Kationen zu dieser Hydroxo-Pd"-Spezies günstig sein und somit eine Präkomplexierung und infolgedessen eine Beeinträchtigung des Transmetallierungsschrittes minimal ausfallen. Des Weiteren könnte die Relation zwischen dem Bororganyl und den gebildeten Hydroxid-Ionen und damit eine verminderte Ausbildung eines unreaktiven Borats der Reaktion zuträglich sein. Darüber, welche Faktoren letztendlich zur gestiegenen Ausbeute tatsächlich beitrugen, kann allerdings nur gemutmaßt werden. Eindeutiger stellte sich dies hingegen im Falle der weiteren Reduktion der Quantität des $\mathrm{B}_{2} \mathrm{pin}_{2}$ und der Base auf 1.00 Äquivalente dar. Hierbei konnte eine Aggregation der Base bedingt durch ein unvorteilhaftes Verhältnis zum Wasser registriert werden. Aufgrund dessen ist anzunehmen, dass weit weniger freie Hydroxid-lonen der Reaktion zur Verfügung standen, um diese zu beschleunigen. Die zuvor genannten Faktoren können allerdings auch unter diesen Umständen nicht ausgeschlossen werden und ein gewisser Effekt auf den Ausgang der Reaktion ist innen sicherlich auch auf die eine oder andere Weise zuzuschreiben. Zusammengefasst führte eine leichte Reduktion der eingesetzten Äquivalente an $\mathrm{B}_{2}$ pin ${ }_{2}$ und Base zu einer Verbesserung der Ausbeute. Zusätzlich hat der verringerte Chemikalieneinsatz und der damit einhergehende verminderte anfallende chemische Abfall einen positiven Effekt.

Des Weiteren wurden verschiedene Additive der Reaktion hinzugegeben, um den Reaktionsverlauf möglichst positiv zu beeinflussen. Bei den Additiven handelte es sich um die Silbersalze ${ }^{[51,136]} \mathrm{AgOAc}$ und $\mathrm{Ag}_{2} \mathrm{CO}_{3}$ sowie um $\mathrm{LiCl},{ }^{[137]}$ deren Ausbeute-steigernde Wirkung in verschiedenen Kreuzkupplungsreaktionen hinlänglich bekannt ist. Weiterhin

Tabelle 4: Untersuchungen zur Auswirkung diverser Additive. kamen bereits die optimierten

\begin{tabular}{|c|c|c|c|}
\hline Eintrag & Base & Additiv & Ausbeute \\
\hline 1 & $\mathrm{~K}_{3} \mathrm{PO}_{4}$ & $\mathrm{AgOAc}$ & Spuren \\
\hline 2 & $\mathrm{Cs}_{2} \mathrm{CO}_{3}$ & $\mathrm{Ag}_{2} \mathrm{CO}_{3}$ & $28 \%$ \\
\hline 3 & $\mathrm{~K}_{3} \mathrm{PO}_{4}$ & $\mathrm{LiCl}$ & $37 \%$ \\
\hline
\end{tabular}
Mengen an eingesetztem $\mathrm{B}_{2} \mathrm{pin}_{2}$ sowie an der Base zum Tragen. Als Base wurde vorrangig $\mathrm{K}_{3} \mathrm{PO}_{4}$ verwendet, jedoch im Falle von $\mathrm{Ag}_{2} \mathrm{CO}_{3}$ aufgrund desselben Anions auf $\mathrm{Cs}_{2} \mathrm{CO}_{3}$ zurückgegriffen, um die Zahl verschiedener lonen in der Reaktion zu begrenzen. Weder mit den Silbersalzen noch mit $\mathrm{LiCl}$ konnte ein Anstieg der Ausbeute verzeichnet werden. Ihr Effekt war eher gegenteiliger Natur und führte zu einer drastischen Verschlechterung der Ausbeute (Tabelle 4). Vor allem AgOAc erwies sich als kontraproduktiv und das borylierte Reaktionsprodukt konnte nur in Spuren massenspektrometrisch nachgewiesen werden (Tabelle 4, Eintrag 1). Bedenkt man die schlechten Ergebnisse, die mit KOAc als Base erzielt wurden, liegt die Vermutung nahe, dass das Acetat-Anion in dieser Umsetzung hinderlich ist. Hohe Konzentrationen an Acetat-Ionen könnten zum einen den aus $\mathrm{Pd}(\mathrm{OAc})_{2}$ und SPhos in situ 
generierten monosubstituierten 12-Elektronen- $\mathrm{Pd}^{0}$-Komplex durch Besetzung der freien Bindungsstellen desaktivieren oder sogar bereits dessen Ausbildung durch Abfangen des Phosphin-Liganden unterbinden, zum anderen aber auch das Transmetallierungsagens zu unreaktiven Boraten umsetzen und dadurch die Reaktion inhibieren.

Parallel zu den vorherigen beiden Screenings wurde die Auswirkung einer Verdopplung der Katalysatorbeladung auf $10 \mathrm{~mol} \% \mathrm{Pd}(\mathrm{OAc})_{2}$ und $25 \mathrm{~mol} \%$ SPhos untersucht, weswegen diese Testreihe noch mit der

Tabelle 5: Erbegnisse der Verdopplung der Katalysatorbeladung.

\begin{tabular}{|c|c|c|}
\hline Eintrag & Base & Ausbeute \\
\hline 1 & $\mathrm{~K}_{3} \mathrm{PO}_{4}$ & $92 \%$ \\
\hline 2 & $\mathrm{Cs}_{2} \mathrm{CO}_{3}$ & $80 \%$ \\
\hline \multicolumn{3}{|c|}{ 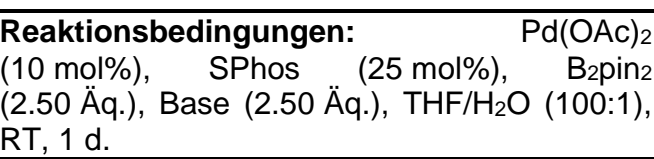 } \\
\hline
\end{tabular}
unoptimierten Menge an $\mathrm{B}_{2} \mathrm{pin}_{2}$ und Base erfolgte. Wie zu erwarten war, resultierte eine Verdopplung der Katalysatorbeladung in einer Erhöhung der Ausbeute (Tabelle 5). Aus der Kombination aller bis dato erhaltenen Erkenntnisse ergaben sich die folgenden optimierten Reaktionsbedingungen für die Miyaura-Borylierung zum Erhalt des Boronsäureesters 134: $\mathrm{Pd}(\mathrm{OAc})_{2}$ (10 mol\%), SPhos (25 mol\%), B 2 $_{2}$ in $_{2}$ (2.00 Äq.), $\mathrm{K}_{3} \mathrm{PO}_{4} / \mathrm{Cs}_{2} \mathrm{CO}_{3}$ (2.00 Äq.), THF/H $/ \mathrm{H}_{2} \mathrm{O}(100: 1), \mathrm{RT}$, 1d. Wider Erwarten fielen die erhaltenen Ausbeuten unter diesen Bedingungen zunächst nur moderat aus (63-75\%) und variierten zudem stark in Abhängigkeit zur Ansatzgröße. Dieses Verhalten wurde auf die bereits in der Untersuchung der einzusetzenden Menge an $\mathrm{B}_{2} \mathrm{pin}_{2}$ und Base beobachteten Faktoren zurückgeführt, sodass der Wasseranteil von einem Volumen-basierten auf einen Stoffmengen-basierten Ansatz umgestellt und somit in ein direktes Verhältnis zur Base und dem Borylierungsagens gesetzt wurde.

Ausgehend von 1.00 Äquivalent Wasser stieg die erzielte Ausbeute mit steigender Stoffmenge an Wasser an (Tabelle 6, Eintrag 1 bis 3), bis bei 4.00 Äquivalenten ein

Tabelle 6: Untersuchungen zur Bestimmung der optimalen $\mathrm{H}_{2} \mathrm{O}$-Menge.

\begin{tabular}{ccc}
\hline Eintrag & Äquivalente $\mathrm{H}_{2} \mathrm{O}$ & Ausbeute \\
\hline 1 & 1.00 & $36 \%$ \\
2 & 2.00 & $61 \%$ \\
3 & 3.00 & $76 \%$ \\
4 & 4.00 & $81 \%$ \\
5 & 5.00 & $79 \%$ \\
\hline
\end{tabular}

Reaktionsbedingungen: $\mathrm{Pd}(\mathrm{OAc})_{2}$ (10 mol\%), SPhos (25 mol\%), B B $_{2}$ in2 (2.00 Äq.), $\mathrm{K}_{3} \mathrm{PO}_{4}$ (2.00 Äq.), $\mathrm{H}_{2} \mathrm{O}$, THF, RT, $1 \mathrm{~d}$. Maximum erreicht wurde (Tabelle 6, Eintrag 4) und bei weiterer Erhöhung bereits ein leichter Rückgang zu verzeichnen war (Tabelle 6, Eintrag 5). Zusätzlich konnten erste Anzeichen einer beginnenden Aggregation der Base bei 5.00 Äquivalenten an Wasser registriert werden. Erweitert um diesen Wert ergaben sich die neuen optimierten Reaktionsparameter zu $\mathrm{Pd}(\mathrm{OAc})_{2}$ (10 mol\%), SPhos (25 mol\%), B pin $_{2}$ (2.00 Äq.), $\mathrm{K}_{3} \mathrm{PO}_{4}$ (2.00 Äq.), $\mathrm{H}_{2} \mathrm{O}$ (4.00 Äq.), THF, 
RT, $1 \mathrm{~d}$. Mit dieser Methode konnte die Reaktion mit einer Ausbeute von 81\% durchgeführt werden und zudem waren die Ergebnisse weitgehend unabhängig von der Ansatzgröße (Abbildung 49). Interessant wäre in dieser Hinsicht sicherlich noch eine Untersuchung des Verhältnisses zwischen Transmetallierungsagens und Base gewesen, jedoch wurde darauf, auch aufgrund der bereits erzielten guten Ergebnisse, in diesem Rahmen verzichtet. Im nächsten Schritt wurde überprüft, inwiefern sich die optimierte Methode der Miyaura-Borylierung auf die Suzuki-Miyaura-Kreuzkupplung des Chlorarens 11 mit dem Boronsäureester 134 übertragen ließ. Dies wurde anhand einer kurzen Versuchsreihe bestehend aus den bereits in der Miyaura-Borylierung getesteten Basen in THF evaluiert. Dabei muss erwähnt werden, dass diese Untersuchung zeitlich noch vor der Umstellung des erforderlichen Wasseranteils erfolgte, sodass in diesem Fall noch auf $\mathrm{THF} / \mathrm{H}_{2} \mathrm{O}(100: 1)$ zurückgegriffen wurde.

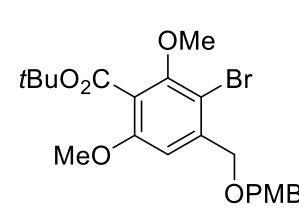

133
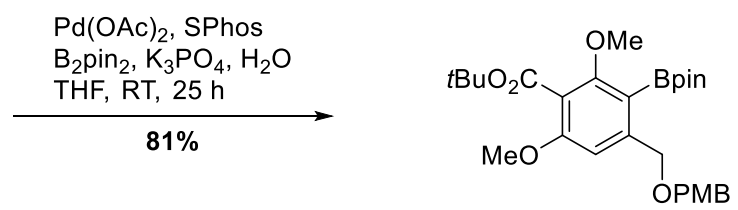

134

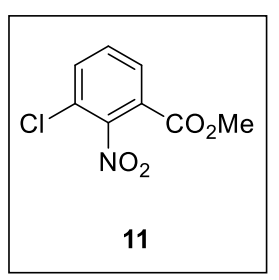

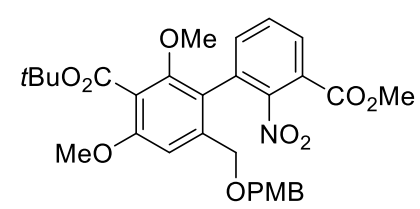

135

Abbildung 49: Suzuki-Miyaura-Kreuzkupplung zum Erhalt des Biaryls 135.

Trotz der leichten Einschränkung bzgl. des Wasseranteils, erbrachte die Untersuchung den Beweis der generellen Anwendbarkeit der Methode auf die Suzuki-MiyauraKreuzkupplung bei einer gleichzeitig signifikanten Steigerung der Ausbeute im Vergleich zu den vormals erreichten 19\%. Des Weiteren waren die gleichen Tendenzen wie im Falle der Miyaura-Borylierung zu beobachten, weswegen im Lösungsmittel THF neben den Basen $\mathrm{K}_{3} \mathrm{PO}_{4}$ und $\mathrm{Cs}_{2} \mathrm{CO}_{3}$ (Tabelle 7, Eintrag 3 und 5) auch die beiden Fluoride CsF und KF (Tabelle 7, Eintrag 1 und 2) gute Ausbeuten ermöglichten, während mit KOAc und $\mathrm{NaOH}$ (Tabelle 7, Eintrag 4 und 6) so gut wie kein Umsatz in der Reaktion verzeichnet und das Produkt nur massenspektrometrisch nachgewiesen werden konnte. Aufgrund der marginalen Unterschiede der Ausbeuten, die bei der Verwendung von $\mathrm{CsF}, \mathrm{KF}, \mathrm{Cs}_{2} \mathrm{CO}_{3}$ und $\mathrm{K}_{3} \mathrm{PO}_{4}$ erzielt wurden, wurde wie schon in der Miyaura-Borylierung vorrangig auf die letztgenannte Base zurückgegriffen. Die daraus resultierenden nahezu identischen 
Tabelle 7: Untersuchung zur Übertragbarkeit der optimierten Borylierungsparameter auf die Suzuki-Miyaura-Kreuzkupplung.

\begin{tabular}{ccc}
\hline Eintrag & Base & Ausbeute \\
\hline 1 & $\mathrm{CsF}$ & $73 \%$ \\
2 & $\mathrm{KF}$ & $67 \%$ \\
3 & $\mathrm{Cs}_{2} \mathrm{CO}_{3}$ & $62 \%$ \\
4 & $\mathrm{KOAc}$ & Spuren \\
5 & $\mathrm{~K}_{3} \mathrm{PO}{ }_{4}$ & $65 \%$ \\
6 & $\mathrm{NaOH}$ & Spuren \\
\hline Reaktionsbedingungen: & $\mathbf{1 3 4 : 1 1}(1: 1)$, \\
$\mathrm{Pd}(\mathrm{OAc})_{2}(10 \mathrm{~mol} \%), \mathrm{SPhos}(25 \mathrm{~mol} \%)$, Base \\
$\left(2.00\right.$ Äq.) , THF/H $/ \mathrm{H}_{2} \mathrm{O}(100: 1), \mathrm{RT}, \mathbf{2 d}$. \\
\hline
\end{tabular}

Reaktionsbedingungen der Miyaura-Borylierung und der Suzuki-Miyaura-Kreuzkupplung würden zusätzlich die Möglichkeit einer Eintopfsynthese beider Reaktionsschritte eröffnen.

Nach der Anpassung des Wasseranteils konnte mittels $\mathrm{Pd}(\mathrm{OAc})_{2}$ (10 mol\%), SPhos (25 mol\%), $\mathrm{K}_{3} \mathrm{PO}_{4}$ (2.00 Äq.), $\mathrm{H}_{2} \mathrm{O}$ (4.00 Äq.), THF, RT, $1 \mathrm{~d}$ aus einem 1:1-Verhältnis des Boronsäureesters 134 und des Chlorarens 11 das Biaryl 135 in 53\% Ausbeute erhalten werden. Dieser reproduzierbare Wert lag zwar deutlich über der ehemals erzielten Ausbeute von 19\%, jedoch unter den erreichten Ausbeuten der Testversuche und ebenfalls niedriger als bei der Miyaura-Borylierung. Die Ursachen hierfür sind vielfältiger Natur, so erfolgt unter anderem die oxidative Addition in diesem Fall im Vergleich zur vorangegangenen Miyaura-Borylierung nicht an einem Arylbromid, sondern an einem unreaktiveren Chloraren. Weiterhin dürfte während der Kupplung auch die Sterik Einfluss auf den Ausgang der Reaktion genommen haben, bedenkt man, dass im gebildeten Produkt drei der vier Positionen ortho zur Biarylachse durch teils sterisch anspruchsvolle Substituenten besetzt sind. Des Weiteren lagen im Vergleich zur Miyaura-Borylierung das Bororganyl, die Base und das Wasser nicht mehr im Verhältnis 1:1:2 sondern in einer 1:2:4 Relation zueinander vor, wodurch möglicherweise ein signifikanter Anteil des Boronsäureesters 134 zum unreaktiven BoratAnalogon umgesetzt und somit quasi aus der Reaktion entfernt wurde. In der Retrospektive wäre möglicherweise eine erneute Manipulation dieses Verhältnisses gewinnbringend im Hinblick auf eine weitere Steigerung der Ausbeute gewesen. Unter Umständen hätte aber auch eine simple Anhebung der Reaktionstemperatur ausgereicht um dies zu realisieren.

Die erzielten Verbesserungen waren soweit zufriedenstellend, sodass zunächst die Fortführung der Synthese priorisiert wurde. Dementsprechend bestand der nächste Schritt darin, den tert-Butylester selektiv in Gegenwart des Methylesters zu entschützen. Laut Literatur sollte die Entschützung des tert-Butylesters bei gleichzeitigem Erhalt der Methylestereinheit dabei unter moderat sauren Reaktionsbedingungen gelingen (Tabelle 8). ${ }^{[138]}$ 


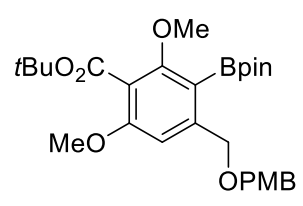

134
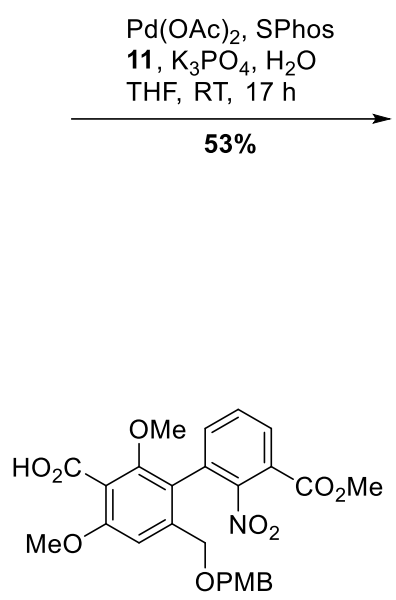

s. Tabelle 8

152

Tabelle 8: Untersuchungen zur Entschützung der tert-Butylesterfunktionalität.

\begin{tabular}{|c|c|c|}
\hline Eintrag & Reaktionsbedingung & Ausbeute \\
\hline 1 & TFA (1.00 Äq.), $\mathrm{CH}_{2} \mathrm{Cl}_{2}, \mathrm{RT}, 1 \mathrm{~h} \rightarrow \mathrm{TFA}$ (3.00 Äq.), RT, $3 \mathrm{~h}$ & Spuren \\
\hline 2 & Montmorillonit $\mathrm{KSF}, \mathrm{CH}_{3} \mathrm{CN}, 82^{\circ} \mathrm{C}, 1.5 \mathrm{~h}$ & Zersetzung \\
\hline 3 & $\begin{array}{l}\text { Montmorillonit } \mathrm{KSF}, \mathrm{CH}_{3} \mathrm{CN}, \mathrm{RT} \rightarrow 82^{\circ} \mathrm{C} \text { über } 5 \mathrm{~h} \rightarrow 82^{\circ} \mathrm{C} \text {, } \\
1 \mathrm{~h}\end{array}$ & Spuren \\
\hline 4 & $\begin{array}{l}\text { Montmorillonit } \mathrm{K} 10, \mathrm{CH}_{3} \mathrm{CN}, \mathrm{RT} \rightarrow 82^{\circ} \mathrm{C} \text { über } 5 \mathrm{~h} \rightarrow 82^{\circ} \mathrm{C} \text {, } \\
2.5 \mathrm{~h}\end{array}$ & Spuren \\
\hline 5 & Montmorillonit $\mathrm{K} 10, \mathrm{CH}_{3} \mathrm{CN}, 45^{\circ} \mathrm{C}, 4 \mathrm{~d}$ & Spuren \\
\hline 6 & $\mathrm{H}_{2} \mathrm{SO}_{4}$ (0.50 Äq.), $\mathrm{CH}_{2} \mathrm{Cl}_{2}, 0^{\circ} \mathrm{C} \rightarrow \mathrm{RT}, 2 \mathrm{~h}$ & Zersetzung \\
\hline 7 & $\begin{array}{l}\text { TBSOTf (1.50 Äq.), 2,6-Lutidin (2.00 Äq.), } \mathrm{CH}_{2} \mathrm{Cl}_{2}, 0^{\circ} \mathrm{C}, 1 \mathrm{~h} \rightarrow \\
\mathrm{RT}, 1.5 \mathrm{~h} \rightarrow \operatorname{TBSOTf}(0.50 \text { Äq.) } \mathrm{RT}, 3 \mathrm{~d}\end{array}$ & $\begin{array}{c}\text { keine } \\
\text { Reaktion }\end{array}$ \\
\hline
\end{tabular}

Die Zugabe äquimolarer Mengen TFA war nicht ausreichend um eine Entschützung zu induzieren, weshalb das Hinzufügen weiterer Mengen an TFA vonnöten war, um eine Reaktion zu bewirken. Massenspektrometrisch konnte die entschützte Carbonsäure 152 in Spuren nachgewiesen, jedoch nicht isoliert werden. Vielmehr wurde primär eine Entschützung des PMB-geschützten benzylischen Alkohols beobachtet, sodass vorrangig der freie benzylische Alkohol, sowie zum Teil ein Substrat mit beiden entschützten Funktionalitäten und eben in Spuren die entschützte Carbonsäure 152 erhalten bzw. identifiziert wurden (Tabelle 8, Eintrag 1). Ein Wechsel auf den sauren Ionentauscher Montmorillonit KSF lieferte zunächst die beiden unerwünschten Nebenprodukte (Tabelle 8, Eintrag 2). Nach der Absenkung der Reaktionstemperatur auf Raumtemperatur konnte anfänglich keine Umsetzung verzeichnet werden, sodass die Temperatur schrittweise langsam erhöht wurde. Letztendlich konnten aber ebenfalls nur die Ergebnisse, die bereits mit TFA erzielt wurden, wiederholt werden (Tabelle 8, Eintrag 3). Auch die Verwendung des weniger aciden Montmorillonit K10 brachte keine Änderung der Reaktionsergebnisse, wobei weiterhin die PMB-Schutzgruppe bevorzugt abgespalten 
wurde (Tabelle 8, Eintrag 4 und 5). Ähnliche Ergebnisse wurden auch bei Gebrauch geringer Mengen an konz. $\mathrm{H}_{2} \mathrm{SO}_{4}$ erhalten (Tabelle 8, Eintrag 6). Infolge der anscheinend höheren Labilität der PMB-Schutzgruppe im Vergleich zum tert-Butylester wurde von einer sauren Hydrolyse abgesehen und zunächst versucht den tert-Butylester in einen TBSEster zu überführen, welcher anschließend mit einer geeigneten Fluoridquelle unter Freigabe der Carbonsäure 152 gespalten werden sollte. Der Versuch einer Umesterung mit TBSOTf und 2,6-Lutidin blieb allerdings erfolglos und ein Umsatz während der Reaktion aus (Tabelle 8, Eintrag 7). Neben der Labilität der PMB-Schutzgruppe zeichnete sicherlich auch der hohe sterische Anspruch des tert-Butylesters an sich als auch in der Kombination mit den beiden ortho-ständigen Methoxysubstituenten verantwortlich für das Scheitern einer selektiven Ent- bzw. Umschützung. Somit war ein Fortgang der Synthese mit diesem Substrat bedingt durch die fehlende Möglichkeit der Entschützung des tert-Butylesters 135 und demzufolge eine Demaskierung der nötigen Aldehydfunktion nicht zu realisieren. 


\subsection{Untersuchungen zur Synthese eines Wacker-Vorläufers}

Um dem Aufbau des tert-Butylesterfragments aus dem Weg zugehen, sollte die Funktionalisierung der benzylischen Position, die die Maskierung der Aldehydfunktion überhaupt erst nötig machte, anderweitig erfolgen. Mit dem Wechsel des Startmoleküls vom Orcinol (17) hin zum kommerziell erhältlichen 3,5-Dimethoxybenzylalkohol (155), der bereits über die erforderliche Hydroxygruppe in benzylischer Position verfügte, konnte dieses Problem relativ einfach umgangen werden.

\subsubsection{Aufbau des Biaryls 161}

Der gestiegene Kostenfaktor, der durch den Wechsel auf das stärker funktionalisierte Startmaterial entstand, konnte durch die Überführung der erheblich günstigeren kommerziell erhältlichen 3,5-Dihydroxybenzoesäure (153) in den Benzylalkohol 155 nach einer literaturbekannten Methode vermindert werden (Abbildung 50). ${ }^{[139]}$<smiles>O=C(O)c1cc(O)cc(O)c1</smiles>

153
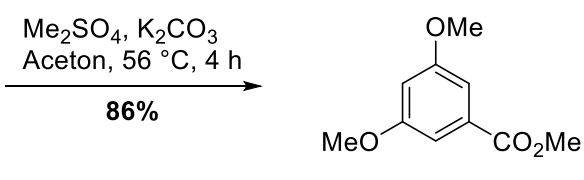

154
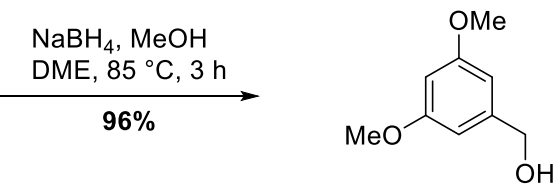

155

Abbildung 50: Darstellung des 3,5-Dimethoxybenzylalkohol (155).

Methylierung der beiden Hydroxygruppen sowie der Carboxylgruppe unter basischer Reaktionsführung mit $\mathrm{Me}_{2} \mathrm{SO}_{4}$ lieferte den Methylester 154 in 86\% Ausbeute, der seinerseits mittels $\mathrm{NaBH}_{4}$ nahezu quantitativ zum Benzylalkohol 155 reduziert werden konnte. Der freie Alkohol 155 wurde nach erfolgter Deprotonierung mit KOtBu per $\mathrm{PMBBr}$ in ebenfalls 96\% Ausbeute zum PMB-geschützten benzylischen Alkohol 156 umgesetzt.

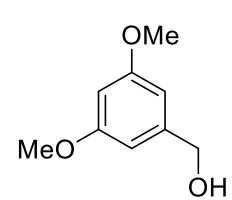

155
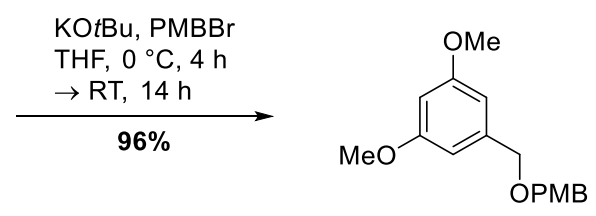

156

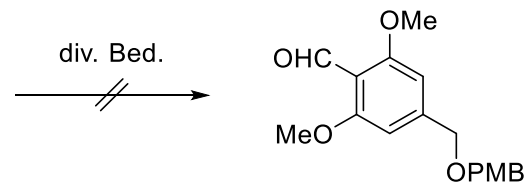

157

Abbildung 51: Versuch der ortho-Formylierung am geschützten Benzylalkohol 156.

Die folgende regioselektive Darstellung des Aldehyds 157 über eine Sequenz aus orthoLithiierung und anschließender Formylierung blieb jedoch trotz einer Vielzahl verschiedener Versuche erfolglos (Abbildung 51). Hierbei wurde sich an einer literatur- 
bekannten Methode zur Formylierung des dimethylierten Orcinol-Analogons $\mathbf{1 4 3}$ orientiert. ${ }^{[140,141]}$ Mittels der Zugabe von $n B u L i ~\left(1.20\right.$ Äq.) und TMEDA (2.00 Äq.) bei $0{ }^{\circ} \mathrm{C}$ zum in $\mathrm{Et}_{2} \mathrm{O}$ gelösten Edukt 156 und anschließendem Erwärmen auf $35^{\circ} \mathrm{C}$ sollte das lithiierte Intermediat gebildet werden, welches durch das Hinzufügen eines Überschusses an DMF bei $0^{\circ} \mathrm{C}$, darauffolgendem Erwärmen auf Raumtemperatur und abschließender wässriger Aufarbeitung den entsprechenden Aldehyd 157 ergeben sollte. Trotz Variation der verwendeten Base (neben nBuLi kamen sBuLi, tBuLi, LiHMDS, LDA sowie NaH zum Einsatz), Wechsel des Lösungsmittels ( $\mathrm{Et}_{2} \mathrm{O}$ und THF) sowie Änderung der Zugabe- und Reaktionstemperatur von $-78^{\circ} \mathrm{C}$ über $0{ }^{\circ} \mathrm{C}$ und Raumtemperatur bis hin zu $35^{\circ} \mathrm{C}$ konnte größtenteils nur das Startmaterial reisoliert werden. Verantwortlich für dieses Ergebnis könnten die sehr aciden benzylischen Protonen des Ausgangsmaterials sein, die von der lithiierten Zwischenstufe nach erfolgter Ausbildung direkt abstrahiert werden und somit eine Reprotonierung zur Folge haben könnten. Aufgrund der Annahme, dass die deprotonierte ungeschützte benzylische Hydroxyfunktion die Acidität der benachbarten benzylischen Protonen durch eine Umkehr des induktiven Effektes (von -l im Falle der Hydroxygruppe $z u+l$ für den Alkoholat-Sauerstoff) unter Umständen leicht verringern und somit eine Lithiierung ermöglichen könnte, wurde die Formylierung des Benzylalkohols 155 und die daran erst anschließende Schützung der Hydroxygruppe in Erwägung gezogen.

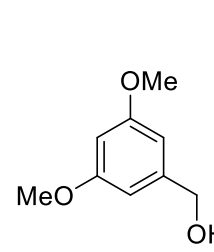

155

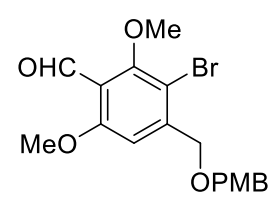

159

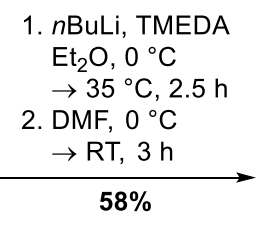

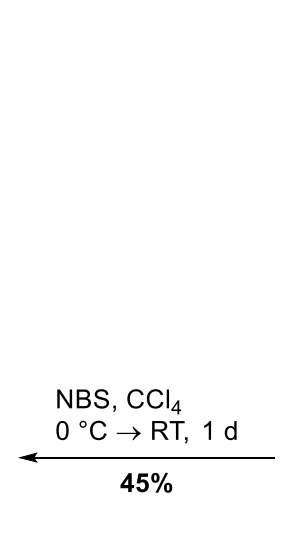

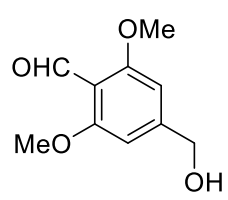

158

1. KOtBu, THF

$-78^{\circ} \mathrm{C}, 1 \mathrm{~h}$

2. $\mathrm{PMBBr},-78^{\circ} \mathrm{C}$

$\rightarrow \mathrm{RT}, 12 \mathrm{~h}$

$31 \%$

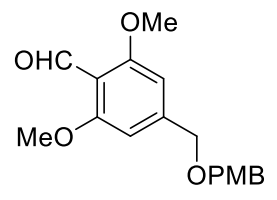

157

Abbildung 52: Synthese des Arylbromids 159. 
Zur Durchführung der ortho-Formylierung wurde wieder auf die zuvor verwendete Methode zurückgegriffen und nach einiger Reaktionsoptimierung konnte der Aldehyd 158 nach Lithiierung mit einem Überschuss an $n B u L i$ und TMEDA sowie anschließendem Abfangen des in situ generierten lithiierten Intermediats mit DMF mit einer Ausbeute von $58 \%$ gewonnen werden. Anzumerken ist hierbei, dass die Ausbeute von 58\% nur im niedrigen Milligramm-Maßstab erreicht werden konnte, während sie im MultigrammMaßstab auf $11 \%$ einbrach. Der Ausgang der Reaktion zeigte dabei eine starke Abhängigkeit von der Qualität des nBuLi, der Trockenheit des TMEDAs sowie des Lösungsmittels und vor allem von der Reaktionstemperatur. Des Weiteren neigten größere Ansätze zum Ausfallen der lithiierten Spezies während der Zugabe des nBuLi, wodurch eine Durchmischung der Reaktionslösung nicht mehr gewährleistet werden konnte. Nach dem Erhalt des Benzaldehyds 158 wurde die freie Hydroxyfunktion durch eine baseninduzierte nukleophile Substitution am $\mathrm{PMBBr}$ geschützt. Die Reaktion litt jedoch ebenfalls unter der Acidität der benzylischen Protonen, die teilweise bereits vom verwendeten $\mathrm{KOtBu}$ abstrahiert wurden, weswegen der PMB-geschützte Alkohol 157 anfänglich in 12\% Ausbeute und nach Anpassung der eingesetzten Basenmenge sowie der Reaktionstemperatur mit maximal 31\% Ausbeute erhalten werden konnte. Im nachfolgenden Schritt wurde der Brom-Substituent am aromatischen Kern des PMBgeschützten Benzylalkohols 157 mittels NBS in einer Ausbeute von 45\% eingeführt (Abbildung 52).

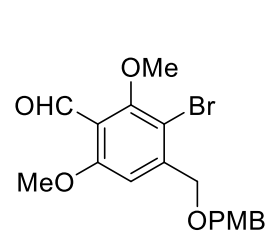

159
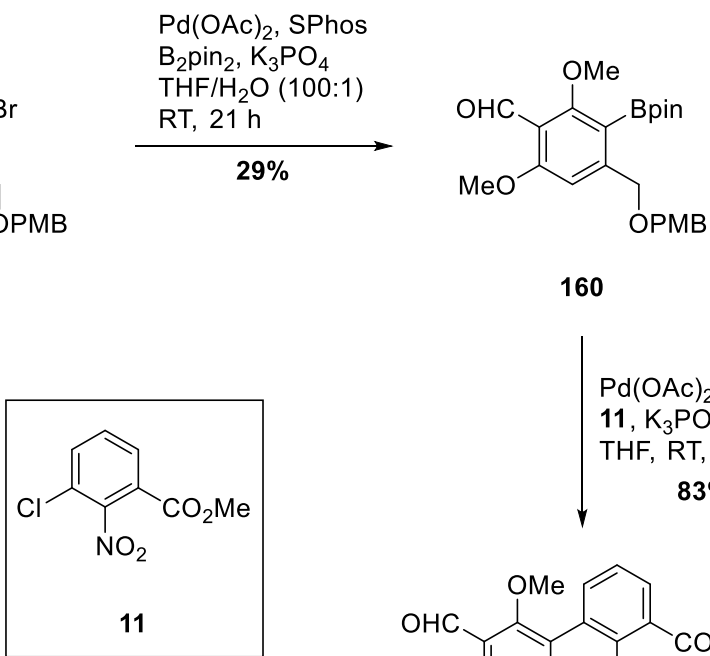

160 $\mathrm{Pd}(\mathrm{OAc})_{2}$, SPhos $11, \mathrm{~K}_{3} \mathrm{PO}_{4}, \mathrm{H}_{2} \mathrm{O}$ THF, RT, $21 \mathrm{~h}$

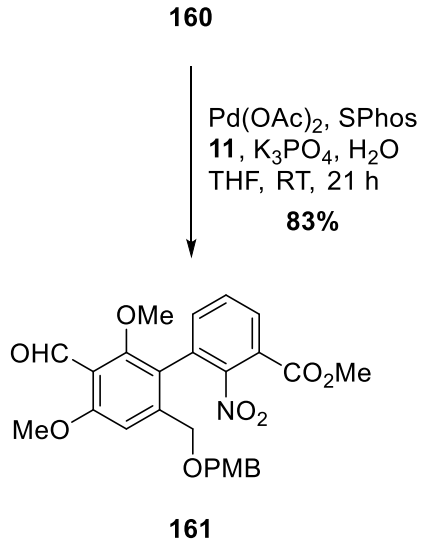

Abbildung 53: Aufbau des Biaryls 161.

Das Arylbromid 159 wiederum wurde unter nicht ganz optimalen Reaktionsbedingungen (Volumen-basierter Wasseranteil) in 29\% Ausbeute zum Pinakolboronsäurester 160 
boryliert. Die anschließende Suzuki-Miyaura-Kreuzkupplung des Arylchlorids $\mathbf{1 1}$ mit dem Boronsäuresster 160 konnte mithilfe optimaler Reaktionsparameter mit einer Ausbeute von $83 \%$ durchgeführt und somit das Biaryl 161 erhalten werden (Abbildung 53). Die erzielten Ergebnisse der letzten beiden Reaktionen sollten aufgrund der geringen Ansatzgröße von 13 respektive $4 \mathrm{mg}$ jedoch nicht als vollständig repräsentativ verstanden werden. In Anbetracht der Widrigkeiten, die die Formylierung sowie die darauffolgende Einführung der PMB-Schutzgruppe in das Substrat mit sich brachten und in unpraktikablen Ausbeuten vor allem in großen Ansätzen resultierte, stellten alle weiteren Reaktionen primär eine Machbarkeitsstudie dar, um etwaige Komplikationen mit der Aldehydfunktion in diesen Reaktionen zu identifizieren. Daraufhin wurde versucht, durch einen Wechsel der Schutzgruppe zumindest den Zweiten der beiden mit niedrigen Ausbeuten verlaufenden Reaktionsschritte in dieser Hinsicht zu verbessern.

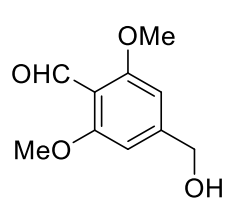

158

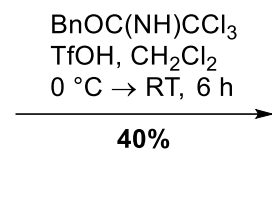

Abbildung 54: Benzyl-Schützung des Alkohols 158.

Zur Schützung der Hydroxyfunktion sollte die Benzyl-Schutzgruppe in das Substrat inkorporiert werden, zumal dies unter anderem auch bei saurer Reaktionsführung erfolgen kann, wodurch die Acidität der benzylischen Protonen keine Nebenreaktion zur Folge haben sollte. In mehreren Versuchen zur Einführung der Benzyl-Schutzgruppe konnte maximal eine Ausbeute von 40\% erreicht werden, wodurch sich diese Methode als ungeeignet erwies, eine deutliche Verbesserung herbeizuführen (Abbildung 54).

\subsubsection{Synthese des Alkens (Z)-175}

Die Formylierung des ungeschützten Benzylalkohols 158 zeigte, dass die Erhöhung der Elektronendichte am Alkoholat-Sauerstoff im Zuge der Deprotonierung die Acidität der benzylischen Protonen bedingt durch die elektronenschiebende Wirkung des Alkoholats weit genug absenkt, um dadurch verursachte Nebenreaktionen zu verringern und die Reaktion generell zu ermöglichen. Die TBS-Schutzgruppe sollte aufgrund ihres elektropositiven Charakters einen ähnlichen Effekt induzieren und infolge ihrer räumlichen Ausdehnung eine sterische Barriere gegen eine mögliche Abstraktion der Protonen aufbauen. Somit sollte zunächst eine TBS-Schützung des freien Alkohols 155 erfolgen, bevor die ortho-Formylierung am Substrat Anwendung finden sollte. 


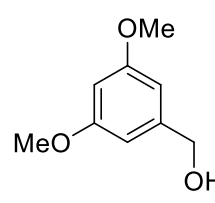

155

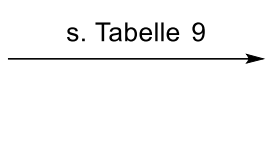

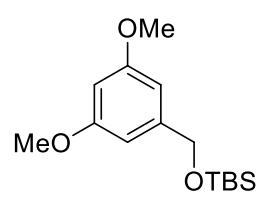

163

Tabelle 9: TBS-Schützung des Benzylalkohols 155.

\begin{tabular}{ccc}
\hline Eintrag & Reaktionsbedingungen & Ausbeute \\
\hline 1 & TBSCl (1.20 Äq.), Imidazol (1.20 Äq.), $\mathrm{CH}_{2} \mathrm{Cl}_{2}, \mathrm{RT}, 1 \mathrm{~d}$ & $65 \%$ \\
2 & $\mathrm{TBSCl}$ (2.00 Äq.), Imidazol (2.00 Äq.), $\mathrm{CH}_{2} \mathrm{Cl}_{2}, \mathrm{RT}, 1 \mathrm{~d}$ & $97 \%$ \\
\hline
\end{tabular}

Die TBS-Schützung lieferte bereits im ersten Versuch mit einem leichten Überschuss an TBSCI und Imidazol eine Ausbeute von moderaten 65\% (Tabelle 9, Eintrag 1) und konnte durch eine Anhebung der eingesetzten Äquivalente an diesen Reagenzien auf 97\% Ausbeute gesteigert werden (Tabelle 9, Eintrag 2).

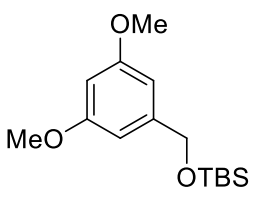

163

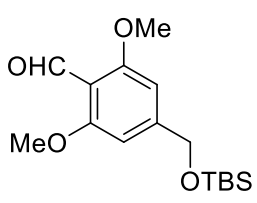

164

Tabelle 10: Ortho-Formylierung am Aryl 163.

\begin{tabular}{|c|c|c|}
\hline Eintrag & Reaktionsbedingungen & Ausbeute \\
\hline 1 & $\begin{array}{l}\text { 1. } n \text { BuLi (1.50 Äq.), TMEDA (3.00 Äq.), } \mathrm{Et}_{2} \mathrm{O}, 0^{\circ} \mathrm{C} \rightarrow 40^{\circ} \mathrm{C} \text {, } \\
90 \min \\
\text { 2. } \mathrm{DMF}\left(5.00 \text { Äq.), } 0^{\circ} \mathrm{C} \rightarrow \mathrm{RT}, 90 \mathrm{~min}\right.\end{array}$ & $71 \%$ \\
\hline 2 & $\begin{array}{l}\text { 1. } n \text { BuLi (1.75 Äq.), TMEDA ( } 3.50 \text { Äq.), } \mathrm{Et}_{2} \mathrm{O}, 0^{\circ} \mathrm{C} \rightarrow 40^{\circ} \mathrm{C} \text {, } \\
2 \mathrm{~h} \\
\text { 2. DMF (5.00 Äq.), } 0^{\circ} \mathrm{C} \rightarrow \mathrm{RT}, 2 \mathrm{~h}\end{array}$ & $41 \%$ \\
\hline
\end{tabular}

Ein erster Test der Formylierung fiel ebenfalls vielversprechend aus und lieferte bei einem unvollständigen Umsatz des Startmaterials eine Ausbeute von guten 71\% (Tabelle 10, Eintrag 1). Für eine möglichst komplette Umsetzung des Ausgangsmaterials wurde die Menge an eingesetztem nBuLi um 0.25 Äquivalente erhöht. Im damit durchgeführten Multigramm-Maßstab nahm die Ausbeute allerdings auf 41\% ab (Tabelle 10, Eintrag 2), was auf die bereits im Rahmen der Formylierung des freien Alkohols 155 angesprochenen Faktoren zurückzuführen ist. Nichtsdestotrotz hatte die Sequenz aus TBS-Schützung und anschließender ortho-Formylierung eine deutliche Steigerung der Ausbeute im Vergleich zu den zuvor durchgeführten Versuchen zur Folge. 


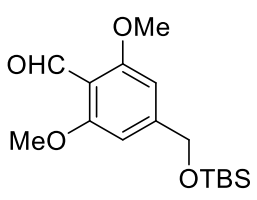

164
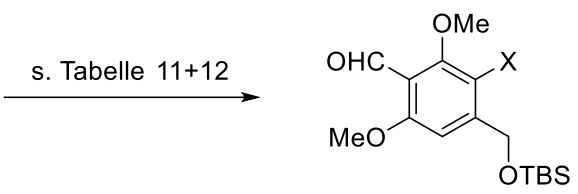

165: $\mathrm{X}=\mathrm{Br}$

166: $X=1$

Tabelle 11: Untersuchungen zur Bromierung des Benzaldehyds 164.

\begin{tabular}{clc}
\hline Eintrag & \multicolumn{1}{c}{ Reaktionsbedingungen } & Ausbeute \\
\hline 1 & $\mathrm{NBS}\left(1.00 \mathrm{Äq}\right.$.), $\mathrm{CCl}_{4}, \mathrm{RT}, 1 \mathrm{~d}$ & $19 \%$ \\
2 & $\mathrm{NBS}\left(1.25 \mathrm{Äq}\right.$ ), $\mathrm{CCl}_{4}, \mathrm{RT}, 1 \mathrm{~d}$ & $32 \%$ \\
3 & $\mathrm{NBS}\left(1.50\right.$ Äq.), $\mathrm{CCl}_{4}, \mathrm{RT}, 1 \mathrm{~d}$ & $28 \%$ \\
4 & $\mathrm{NBS}\left(1.00\right.$ Äq.), $\mathrm{CCl}_{4}, 0^{\circ} \mathrm{C}, 2 \mathrm{~h} \rightarrow \mathrm{RT}, 3 \mathrm{~d}$ & $25 \%$ \\
5 & $\mathrm{Br}_{2}$ (1.00 Äq.), $\mathrm{NaHCO}_{3}(1.20 \mathrm{Äq}),. \mathrm{CHCl}_{3}, 0^{\circ} \mathrm{C}, 1 \mathrm{~h}$ & $32 \%$ \\
\hline
\end{tabular}

Die anschließende Funktionalisierung des aromatischen Kerns zur Vorbereitung auf die Miyaura-Borylierung sollte mittels einer Bromierung erfolgen. Hierzu kamen die zuvor bereits erprobten Methoden unter Verwendung von NBS (Tabelle 11, Eintrag 1 bis 4) sowie der Kombination von elementarem Brom mit $\mathrm{NaHCO}_{3}$ zum Einsatz (Tabelle 11, Eintrag 5). ${ }^{[122]}$ Sowohl die Bromierung mit NBS und deren Variationen mit gesteigertem NBS-Einsatz oder verringerter Zugabetemperatur und gleichzeitig verlängerter Reaktionszeit als auch die Bromierung mit $\mathrm{Br}_{2}$ und $\mathrm{NaHCO}_{3}$ ermöglichten Ausbeuten im Bereich von $30 \%$. Ursächlich dafür zeichnete die vorrangig verlaufende Entschützung der TBSSchutzgruppe durch freie Bromid-lonen in der Reaktionslösung.

Tabelle 12: Untersuchungen zur lodierung des Benzaldehyds 164.

\begin{tabular}{|c|c|c|}
\hline Eintrag & Reaktionsbedingungen & Ausbeute \\
\hline 1 & $\mathrm{I}_{2}$ (1.00 Äq.), $\mathrm{Ag}_{3} \mathrm{PO}_{4}$ (1.10 Äq.), $\mathrm{CHCl}_{3}, \mathrm{RT}, 1 \mathrm{~d}$ & $28 \%$ \\
\hline 2 & $\mathrm{I}_{2}$ (1.00 Äq.), $\mathrm{Ag}_{3} \mathrm{PO}_{4}$ (0.37 Äq.), $\mathrm{CHCl}_{3}, \mathrm{RT}, 1 \mathrm{~d}$ & $45 \%$ \\
\hline 3 & $\mathrm{I}_{2}$ (1.25 Äq.), $\mathrm{Ag}_{3} \mathrm{PO}_{4}$ (0.46 Äq.), $\mathrm{CHCl}_{3}, \mathrm{RT}, 1 \mathrm{~d}$ & $57 \%$ \\
\hline 4 & $\mathrm{I}_{2}$ (1.50 Äq.), $\mathrm{Ag}_{3} \mathrm{PO}_{4}$ (0.55 Äq.), $\mathrm{CHCl}_{3}, \mathrm{RT}, 1 \mathrm{~d}$ & $65 \%$ \\
\hline 5 & $\mathrm{I}_{2}$ (1.75 Äq.), $\mathrm{Ag}_{3} \mathrm{PO}_{4}$ (0.64 Äq.), $\mathrm{CHCl}_{3}, \mathrm{RT}, 1 \mathrm{~d}$ & $59 \%$ \\
\hline 6 & $\mathrm{I}_{2}$ (2.00 Äq.), $\mathrm{Ag}_{3} \mathrm{PO}_{4}$ (0.73 Äq.), $\mathrm{CHCl}_{3}, \mathrm{RT}, 1 \mathrm{~d}$ & $49 \%$ \\
\hline
\end{tabular}

Wegen der Labilität der TBS-Schutzgruppe unter den Bedingungen der getesteten Bromierungsmethoden wurde auf das unreaktivere Halogen lod gewechselt. Mithilfe der Kombination von $\mathrm{I}_{2}$ und $\mathrm{Ag}_{3} \mathrm{PO}_{4}$ konnte die gewünschte lodierung bei gleichzeitiger Unterdrückung der TBS-Entschützung realisiert werden, jedoch wurde dies mit dem Auftreten einer anderen Nebenreaktion erkauft. Hervorgerufen durch die Koordination der 
Silber-Ionen am Carbonylsauerstoff fand eine Substitution der Carbonylgruppe durch lod am Aromaten statt, weswegen die Ausbeute nicht über 28\% hinauskam (Tabelle 12, Eintrag 1). Die Anpassung der Menge an eingesetztem $\mathrm{Ag}_{3} \mathrm{PO}_{4}$ basierend auf einem 1:1.1 Verhältnis zwischem $\mathrm{I}_{2}$ und $\mathrm{Ag}^{+}$, das Vorlösen des $\mathrm{I}_{2}$ und die Zugabe des $\mathrm{Ag}_{3} \mathrm{PO}_{4}$ erst nach erfolgter $\mathrm{I}_{2}$-Zugabe führten zu einer Minimierung der beobachteten Nebenreaktion. Tests der benötigten Reagenzienmenge ergaben eine maximal erreichbare Ausbeute von $65 \%$ bei der Verwendung von 1.50 Äquivalenten $\mathrm{I}_{2}$ und 0.55 Äquivalenten $\mathrm{Ag}_{3} \mathrm{PO}_{4}$ (Tabelle 12, Eintrag 4). Trotzdem erschien es sinnvoller, zunächst die Verlängerung der Seitenkette an der Aldehydfunktion durchzuführen und daran anschließend den aromatischen Kern zu iodieren, um diese Nebenreaktion komplett zu unterbinden und eine höhere Gesamtausbeute zu erzielen.

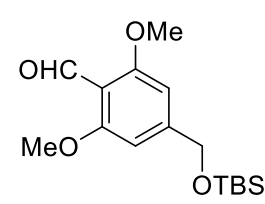

164
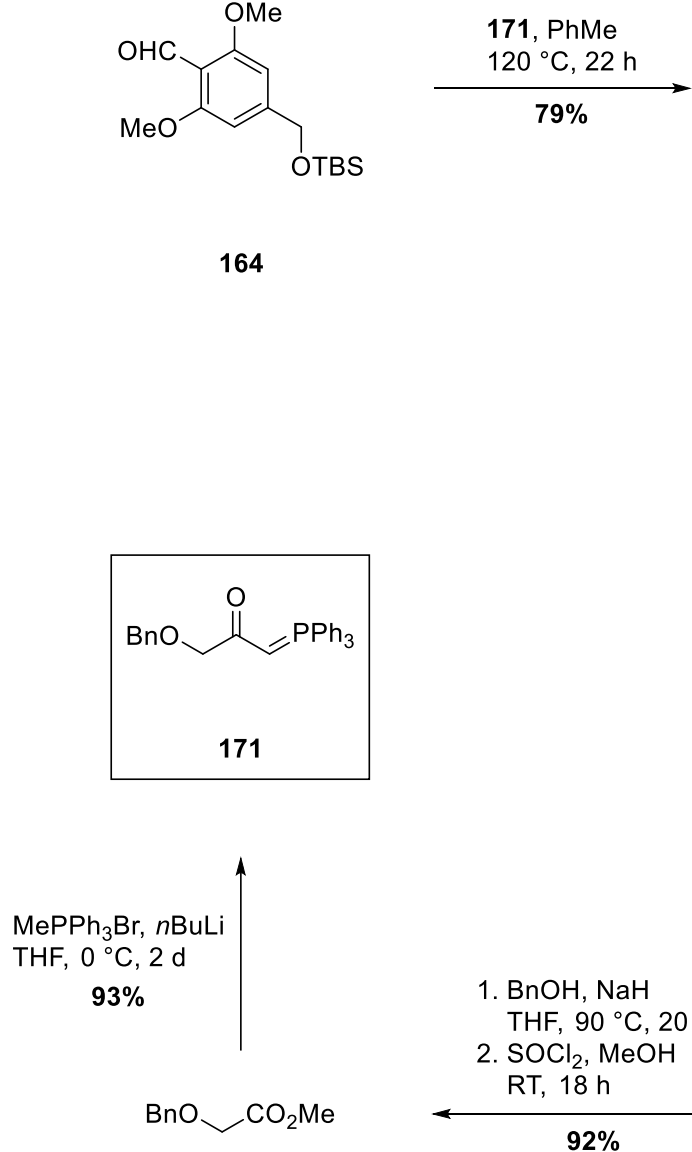

170

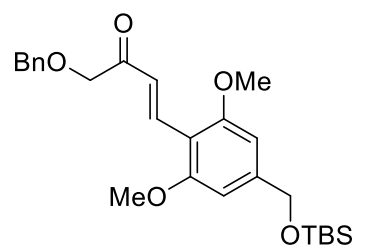

(E)-167
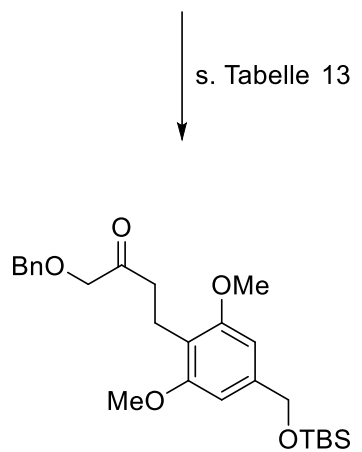

168

$$
\mathrm{Br} \sim \mathrm{CO}_{2} \mathrm{H}
$$

$\alpha$-Bromessigsäure (169)

Abbildung 55: Darstellung des P-Ylids 171 und anschließende Verwendung in der Synthese des Ketons 168.

Das zum Aufbau der Seitenkette verwendete Phosphor-Ylid 171 wurde nach einer im Arbeitskreis Tietze bewährten Methode in zwei Schritten aus der kommerziell erhältlichen a-Bromessigsäure (169) synthetisiert. ${ }^{[142]}$ Nukleophile Substitution des Brom- 
Substituenten durch das aus $\mathrm{BnOH}$ mittels $\mathrm{NaH}$ in situ generierte Alkoholat und anschließende Veresterung der Benzyl-geschützten Glycolsäure via $\mathrm{SOCl}_{2}$ und $\mathrm{MeOH}$ lieferten den Methylester 170 in 92\% Ausbeute. Aus der folgenden Umsetzung mit $\mathrm{MePPh}_{3} \mathrm{Br}$ und $n \mathrm{BuLi}$ konnte das P-Ylid 171 in einer Ausbeute von 93\% gewonnen werden. Die Synthese des $\alpha, \beta$-ungesättigten Ketons $(E)-167$ erfolgte mittels einer WittigReaktion des Aldehyds 164 mit dem zuvor hergestellten P-Ylid 171 in 79\% Ausbeute (Abbildung 55).

Tabelle 13: Reduktion des Alkens (E)-167.

\begin{tabular}{|c|c|c|}
\hline Eintrag & Reaktionsbedingungen & Ausbeute \\
\hline 1 & $\begin{array}{l}\text { 1. } \mathrm{PtO}_{2}(5 \mathrm{~mol} \%), \mathrm{H}_{2}(1 \mathrm{~atm}), \mathrm{EtOH}, \mathrm{RT}, 3 \mathrm{~h} \\
\text { 2. } \mathrm{IBX}_{2} \mathrm{CH}_{3} \mathrm{CN}, 80^{\circ} \mathrm{C}, 4 \mathrm{~h}\end{array}$ & $55 \%$ \\
\hline 2 & $\begin{array}{l}\text { 1. } \mathrm{PtO}_{2}(5 \mathrm{~mol} \%), \mathrm{H}_{2}(1 \mathrm{~atm}), \mathrm{EtOH}, \mathrm{RT}, 3 \mathrm{~h} \\
\text { 2. IBX, } \mathrm{CH} \mathrm{H}_{3} \mathrm{CN}, 80^{\circ} \mathrm{C}, 4 \mathrm{~h} \\
\text { 3. TBSCl (2.00 Äq.), Imidazol (2.00 Äq.), } \mathrm{CH}_{2} \mathrm{Cl}_{2}, \mathrm{RT}, 19 \mathrm{~h}\end{array}$ & $51 \%$ \\
\hline
\end{tabular}

Die Doppelbindung sollte in der Folge reduziert werden. Hierfür wurde ebenfalls auf eine im Arbeitskreis Tietze häufig verwendete Methode zurückgegriffen und diese Umsetzung mithilfe von katalytischen Mengen $\mathrm{PtO}_{2}$ und $\mathrm{H}_{2}$ vollzogen. ${ }^{[143]}$ Die während der Reduktion der Doppelbindung teilweise auftretende Überreduktion, von der die Carbonylgruppe betroffen ist und zur Ausbildung des analogen Alkohols führt, wird dabei durch die nachfolgend angeschlossene Reoxidation des Alkohols mittels IBX aufgefangen. ${ }^{[144]}$ In einem ersten Testversuch konnte mit dieser Methode eine Ausbeute von moderaten 55\% erhalten werden (Tabelle 13, Eintrag 1). Die geringe Ausbeute wurde auf eine mutmaßlich zu starke Überreduktion und infolgedessen zu geringen Menge an verwendetem IBX zur Reoxidation zurückgeführt. In der Wiederholung der Reaktion im großen Maßstab mit einer angehobenen Menge an eingesetztem IBX zeigte sich jedoch, dass zwar wie zu erwarten war, ein gewisser Anteil des eingesetzten Ketons (E)-167 zum korrespondierenden Alkohol überreduziert wurde, zusätzlich allerdings ein erheblicher Teil durch IBX TBS-entschützt wurde. Das zusätzlich hinzugefügte IBX hatte zu diesem Zeitpunkt daher eine Verringerung der Ausbeute auf nunmehr 20\% zur Folge. Ein signifikanter Anteil des erhaltenen Produktgemisches konnte aber durch erneute TBSSchützung zurückgewonnen und somit das gewünschte Keton 168 in einer Gesamtausbeute von $51 \%$ erhalten werden (Tabelle 13, Eintrag 2).

Die darauffolgende lodierung am aromatischen Kern des Ketons 168 mittels $I_{2}$ und $\mathrm{Ag}_{3} \mathrm{PO}_{4}$ konnte mit einer Ausbeute von 95\% durchgeführt und somit wie angenommen, die zuvor beobachtete Nebenreaktion vollständig umgangen werden (Abbildung 56). 


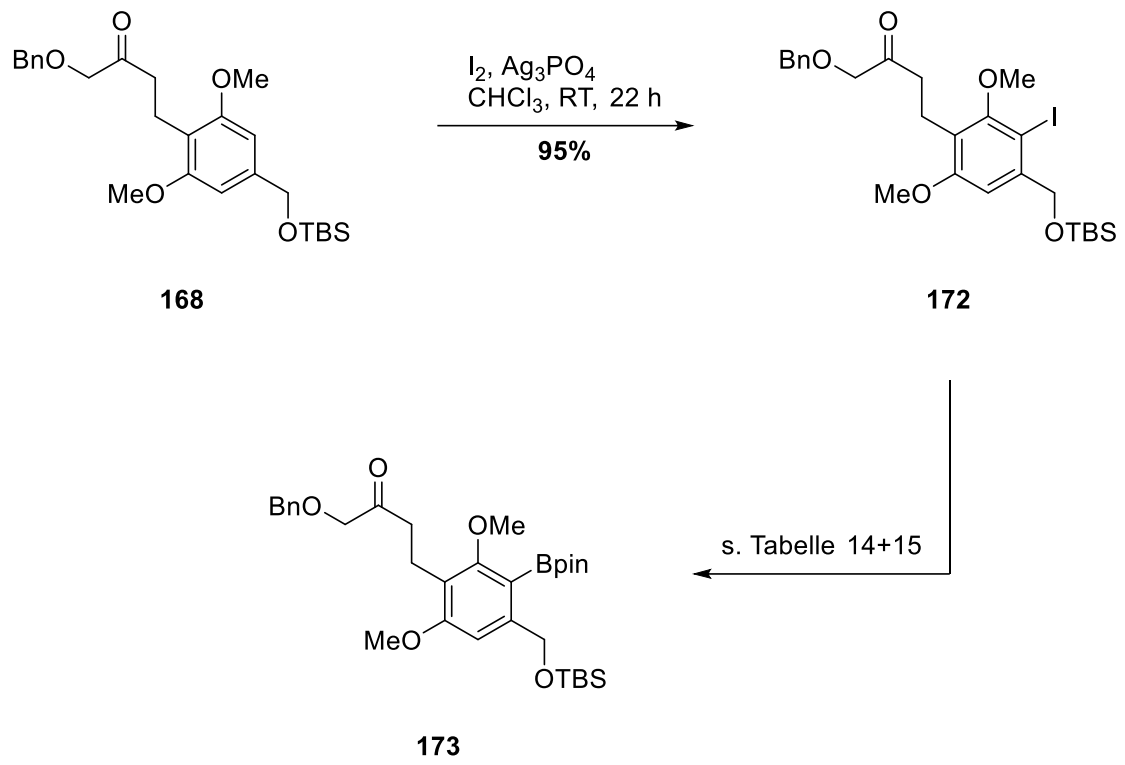

Abbildung 56: Synthese des Boronsäureesters 173.

Die Anwendung der optimierten Reaktionsparameter auf das Aryliodid 172 in der anschließenden Miyaura-Borylierung führte zu einer Ausbeute von 28\% (Tabelle 14, Eintrag 1). Die geringe Ausbeute ließ sich auf die veränderte Elektronendichte am

Tabelle 14: Miyaura-Borylierung des Aryliodids 172.

\begin{tabular}{|c|c|c|}
\hline Eintrag & Temperatur & Ausbeute \\
\hline 1 & RT & $28 \%$ \\
\hline 2 & $40^{\circ} \mathrm{C}$ & $19 \%$ \\
\hline 3 & $60^{\circ} \mathrm{C}$ & $56 \%$ \\
\hline 4 & $50^{\circ} \mathrm{C} \rightarrow 60^{\circ} \mathrm{C}$ & Spuren ${ }^{[a]}$ \\
\hline \multicolumn{3}{|c|}{ 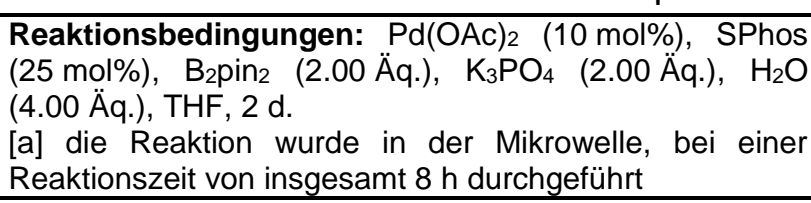 } \\
\hline
\end{tabular}

Aromaten zurückführen. Im Vergleich zum tert-Butylester 133 an dem die Methode optimiert wurde, fehlte dem Aryliodid 172 die elektronenziehende Esterfunktion in meta-Position, weswegen in diesem Falle kein Ausgleich zu den beiden elektronenschiebenden Methoxygruppen in ortho- und para-Position vorhanden war. Die daraus resultierende höhere

Elektronendichte am aromatischen Kern bewirkte eine Reduktion der Geschwindigkeit, mit der die oxidative Addition verlief. Daher wurde zunächst versucht, die oxidative Addition durch eine Erhöhung der Reaktionstemperatur wieder zu beschleunigen und somit eine Steigerung der Ausbeute zu erreichen. Nachdem eine Erhöhung der Temperatur auf $40{ }^{\circ} \mathrm{C}$ keine relevante Änderung mit sich brachte (Tabelle 14, Eintrag 2), ließ eine weitere Anhebung der Temperatur auf $60{ }^{\circ} \mathrm{C}$ die Ausbeute auf signifikant verbesserte $56 \%$ anwachsen (Tabelle 14, Eintrag 3). Die Verwendung von Mikrowellenstrahlung zur Temperaturerzeugung hatte indes keine positiven Auswirkungen auf die Umsetzung zur Folge (Tabelle 14, Eintrag 4). 
Tabelle 15: Untersuchungen zur Optimierung der Miyaura-Borylierung am Aryliodid 172.

\begin{tabular}{clc}
\hline Eintrag & \multicolumn{1}{c}{ Katalysatorsystem } & Ausbeute \\
\hline 1 & $(\mathrm{cod}) \mathrm{Pd}\left(\mathrm{CH}_{2} \mathrm{TMS}\right)_{2}(10 \mathrm{~mol} \%)$, SPhos $(25 \mathrm{~mol} \%)$ & Spuren \\
2 & $\mathrm{Pd}(\mathrm{OAc})_{2}(10 \mathrm{~mol} \%), \mathrm{PCy}_{3}(20 \mathrm{~mol} \%)$ & $13 \%$ \\
3 & $\mathrm{Pd}(\mathrm{OAc})_{2}(10 \mathrm{~mol} \%), \mathrm{PtBu}_{3}(20 \mathrm{~mol} \%)$ & - \\
4 & $\mathrm{Pd}(\mathrm{OAc})_{2}(10 \mathrm{~mol} \%), n \mathrm{BuPA}_{2}(20 \mathrm{~mol} \%)$ & $23 \%$ \\
5 & $\mathrm{Pd}(\mathrm{OAc})_{2}(10 \mathrm{~mol} \%), \mathrm{PCy}_{3}(20 \mathrm{~mol} \%)$ & $14 \%$ \\
\hline
\end{tabular}

Reaktionsbedingungen: Katalysatorsystem, $\mathrm{B}_{2} \mathrm{pin}_{2}$ (2.00 Äq.), $\mathrm{K}_{3} \mathrm{PO}_{4}$ (2.00 Äq.), $\mathrm{H}_{2} \mathrm{O}$ (4.00 Äq.), THF, $2 \mathrm{~d}$. [a] Base: KF

Des Weiteren wurde auch ein Wechsel des Katalysatorsystems auf einen möglichen Ausbeute-steigernden Effekt hin untersucht. Es konnte jedoch weder mit der reaktiveren Palladiumquelle (cod) Pd $\left(\mathrm{CH}_{2} \mathrm{TMS}\right)_{2}$ (Tabelle 15, Eintrag 1) noch mit den Fu-Liganden $\mathrm{PCy}_{3}$ und $\mathrm{PtBu}_{3}$ (Tabelle 15, Eintrag 2, 3 und 5) sowie dem von Beller entwickeltem cataCXium $^{\circledR} \mathrm{A}\left(n \mathrm{BuPAd}_{2}\right)$ (Tabelle 15, Eintrag 4) eine Verbesserung erzielt werden. Nachdem bis dato nur die Erhöhung der Reaktionstemperatur sich positiv auf den Ausgang der Reaktion auswirkte, wurde letzlich noch die Kombination von erhöhter Temperatur und der Base $\mathrm{Cs}_{2} \mathrm{CO}_{3}$, die sich bereits in einem vorrangegangenen Screening als reaktivere Alternative $\mathrm{zu} \mathrm{K}_{3} \mathrm{PO}_{4}$ etablierte (siehe Tabelle 2), getestet. Mit dieser zuletzt vorgenommenen Anpassung konnte die Ausbeute der Miyaura-Borylierung wieder auf gute $81 \%$ angehoben werden (Abbildung 57).

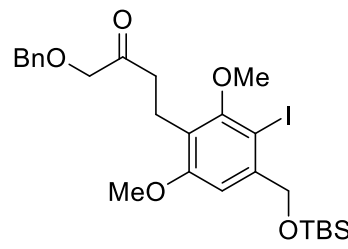

172
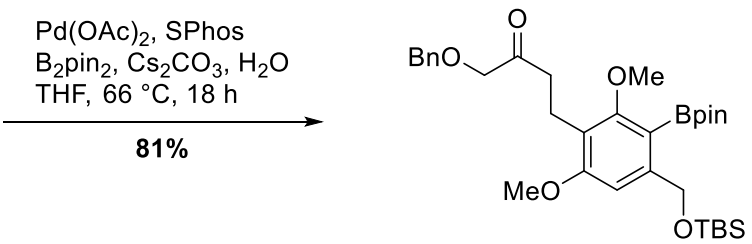

173

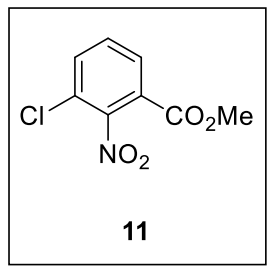

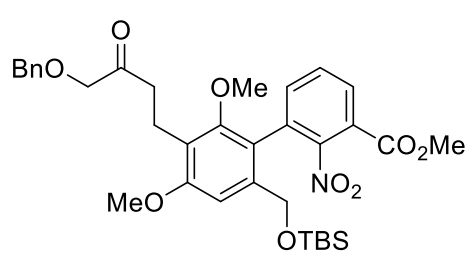

174

Abbildung 57: Synthese des Biaryls 174. 
Tabelle 16: Suzuki-Miyaura-Kreuzkupplung zum Aufbau des Biaryls 174.

\begin{tabular}{cccc}
\hline Eintrag & Base & Temperatur & Ausbeute \\
\hline 1 & $\mathrm{~K}_{3} \mathrm{PO}_{4}$ & $\mathrm{RT}$ & $34 \%$ \\
2 & $\mathrm{Cs}_{2} \mathrm{CO}_{3}$ & $\mathrm{RT}$ & $29 \%$ \\
3 & $\mathrm{Cs}_{2} \mathrm{CO}_{3}$ & $66{ }^{\circ} \mathrm{C}$ & $72 \%$ \\
\hline
\end{tabular}

Reaktionsbedingungen: 173:11 (1:1), $\mathrm{Pd}(\mathrm{OAc})_{2}(10 \mathrm{~mol} \%)$, SPhos (25 mol\%), Base (2.00 Äq.), $\mathrm{H}_{2} \mathrm{O}$ (4.00 Äq.), THF, 1 d.

In der anschließenden Suzuki-Miyaura-Kreuzkupplung wurde zunächst ein Testversuch unter den bisherigen Standardbedingungen bei Raumtemperatur mit den beiden Basen $\mathrm{K}_{3} \mathrm{PO}_{4}$ und $\mathrm{Cs}_{2} \mathrm{CO}_{3}$ durchgeführt. Wie schon zuvor bei der Miyaura-Borylierung beobachtet, fiel auch die Ausbeute in der Suzuki-Miyaura-Kreuzkupplung signifikant ab (Tabelle 16, Eintrag 1 und 2). Hinsichtlich der getesteten Basen ergaben sich keine relevanten Unterschiede, sodass für die Suzuki-Miyaura-Kreuzkupplung ebenfalls auf $\mathrm{Cs}_{2} \mathrm{CO}_{3}$ als Base zurückgegriffen wurde, um die Möglichkeit einer Eintopfsynthese zu erhalten. Die zusätzliche Anhebung der Reaktionstemperatur resultierte, wie zu erwarten war, in einer stark verbesserten Ausbeute von $72 \%$ respektive im Gramm-Maßstab reproduzierten $70 \%$ (Tabelle 16, Eintrag 3).

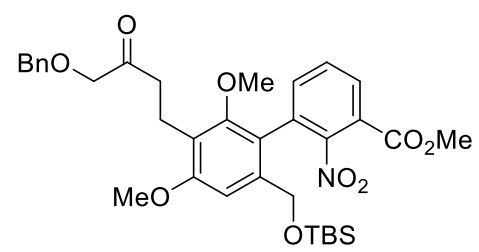

174
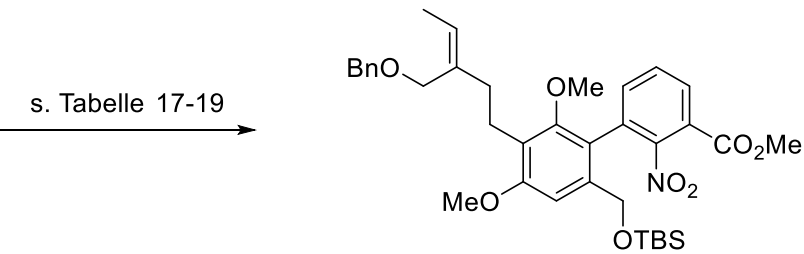

(Z)-175

Tabelle 17: Untersuchungen zur diastereoselektiven Darstellung des Alkens (Z)-175.

\begin{tabular}{|c|c|c|}
\hline Eintrag & Reaktionsbedingungen & Ausbeute \\
\hline 1 & $\begin{array}{l}\text { 1. [EtPPh }]_{3}{ }^{+} \mathrm{Br}^{-}\left(3.00 \text { Äq.), } n \text { BuLi }\left(2.80 \text { Äq.), THF, } 0^{\circ} \mathrm{C} \rightarrow \mathrm{RT} \text {, }\right.\right. \\
30 \mathrm{~min} \\
\text { 2. } 174 \text { (1.00 Äq.), THF, } 0^{\circ} \mathrm{C} \rightarrow \mathrm{RT}, 1 \mathrm{~d}\end{array}$ & $\begin{array}{c}40 \% \\
E / Z 1: 3.7\end{array}$ \\
\hline 2 & $\begin{array}{l}\text { 1. }\left[\mathrm{EtPPh}_{3}\right]^{+} \mathrm{Br}^{-} \text {(1.40 Äq.), KHMDS (1.30 Äq.), THF, } 0^{\circ} \mathrm{C} \rightarrow \\
\mathrm{RT}, 45 \mathrm{~min} \\
\text { 2. } 174 \text { (1.00 Äq.), THF, }-78^{\circ} \mathrm{C} \rightarrow \mathrm{RT}, 1 \mathrm{~d}\end{array}$ & - \\
\hline 3 & $\begin{array}{l}\text { 1. }\left[\mathrm{EtPPh}_{3}\right]^{+} \mathrm{Br}^{-}(1.40 \mathrm{Äq} .), \mathrm{KHMDS} \text { (1.30 Äq.), THF/HMPA } \\
(9: 1), 0^{\circ} \mathrm{C} \rightarrow \mathrm{RT}, 45 \mathrm{~min} \\
\text { 2. } 174(1.00 \mathrm{Äq} .), \mathrm{THF},-78^{\circ} \mathrm{C} \rightarrow \mathrm{RT}, 1 \mathrm{~d}\end{array}$ & - \\
\hline 4 & $\begin{array}{l}\text { 1. [EtPPh }]^{+} \mathrm{Br}^{-}\left(1.40 \text { Äq.), } \mathrm{KH} \text { (1.30 Äq.), THF, } 0^{\circ} \mathrm{C} \rightarrow \mathrm{RT} \text {, }\right. \\
45 \mathrm{~min} \\
\text { 2. } 174 \text { (1.00 Äq.), THF, }-78^{\circ} \mathrm{C} \rightarrow \mathrm{RT}, 1 \mathrm{~d}\end{array}$ & - \\
\hline 5 & $\begin{array}{l}\text { 1. [EtPPh }]_{3}{ }^{+} \mathrm{Br}^{-} \text {(3.00 Äq.), NaH (2.80 Äq.), THF, } 0^{\circ} \mathrm{C} \rightarrow \mathrm{RT} \text {, } \\
45 \mathrm{~min} \\
\text { 2. } 174 \text { (1.00 Äq.), THF, }-78^{\circ} \mathrm{C} \rightarrow \mathrm{RT}, 1 \mathrm{~d}\end{array}$ & - \\
\hline
\end{tabular}


Im nächsten Schritt sollte das Keton 174 in einer Wittig-Reaktion mit [EtPPh $]^{+} \mathrm{Br}^{-}$zum Olefin (Z)-175 umgesetzt werden. Hierfür wurde zunächst auf eine im Arbeitskreis Tietze für analoge Substrate etablierte Methode zurückgegriffen. ${ }^{[125,127]}$ Nach erfolgter Generierung des reaktiven $\mathrm{P}$-Ylids in situ aus $\left[\mathrm{EtPPh}_{3}\right]^{+} \mathrm{Br}^{-}$mittels $n \mathrm{BuLi}$ und anschließender Zugabe des Ketons 174 wurde das Olefin (E/Z)-175 in einer Ausbeute von $40 \%$ und einem Diastereomerenverhältnis $E / Z=1: 3.7$ erhalten (Tabelle 17, Eintrag 1). Aus Arbeiten von Trost et al. zur enantioselektiven Synthese von Chromanen sowie vorrangegangenen Arbeiten aus dem Arbeitskreis Tietze war jedoch bereits bekannt, dass in der später folgenden enantioselektiven Wacker-Zyklisierung bei vergleichbaren Ausbeuten Z-konfigurierte Alkene signifikant bessere Enantioselektivitäten zur Folge haben, verglichen mit ihren E-konfigurierten Analoga. ${ }^{[141]}$ Neben der moderaten Ausbeute wurde deshalb auch versucht die E/Z-Selektivität günstiger zu gestalten. Bekanntlich führen sowohl das Vorhandensein von Lithium-Ionen als auch hohe Temperaturen zur bevorzugten Ausbildung E-konfigurierter Alkene in der Wittig-Reaktion, weswegen zum einen auf Lithium-Basen verzichtet und zum anderen die Reaktion kinetisch kontrolliert bei niedrigen Temperaturen durchgeführt werden sollte. Der Gebrauch der starken Basen KHMDS, KH sowie NaH zur Ylid-Bildung und die anschließende Zugabe des Startmaterials bei $-78^{\circ} \mathrm{C}$ blieben allerdings zunächst erfolglos (Tabelle 17, Eintrag 2 bis 4). Selbst mit dem Additiv HMPA konnte kein Umsatz in der Wittig-Reaktion verzeichnet werden (Tabelle 17, Eintrag 5).

Aufgrund der gänzlich ausgebliebenen Reaktion wurde vermutet, dass die Ausbildung des P-Ylids, wenn überhaupt, nur unzureichend erfolgte. Daher wurde nach Möglichkeiten gesucht, Einfluss auf diesen Teil der Reaktion zu nehmen. Der Anteil des gebildeten

Tabelle 18: Untersuchungen zur P-Ylid-Bildung.

\begin{tabular}{ccc}
\hline Eintrag & Base & Ylid-Bildung \\
\hline 1 & KHMDS & kaum Umsatz \\
2 & KOtBu & kaum Umsatz \\
3 & $\mathrm{NaH}$ & guter Umsatz \\
\hline
\end{tabular}

Reaktionsbedingungen: [EtPPh 3$]^{+} \mathrm{Br}^{-}$(3.00 Äq.), Base (2.80 Äq.), THF, $0^{\circ} \mathrm{C} \rightarrow \mathrm{RT}, 45 \mathrm{~min} \rightarrow$ $66^{\circ} \mathrm{C}, 3 \mathrm{~h}$.
P-Ylids korrelierte dabei mit dem Farbverlauf der Suspension von weiß über gelb und orange nach rot, weswegen eine Verfolgung sowie Bewertung des Umsatzes rein visuell erfolgen konnte. Eine Deprotonierung des Ylid-Vorläufers erfolgte im Temperaturbereich von $0^{\circ} \mathrm{C}$ bis Raumtemperatur mit keiner der drei getesteten Basen und erst die Erhöhung der Temperatur auf $66^{\circ} \mathrm{C}$ führte im Falle von $\mathrm{NaH}$ (Tabelle 18, Eintrag 3) zu einem deutlichen Farbumschlag der Suspension. Zur Evaluierung der Beobachtungen wurde diese Methode in einer Versuchsreaktion getestet. Nach erfolgter Zugabe von $\mathrm{NaH}$ (2.80 Äq.) zu einer Suspension von [EtPPh $]^{+} \mathrm{Br}^{-}$ (3.00 Äq.) in THF bei Raumtemperatur und Erwärmen auf $66^{\circ} \mathrm{C}$ für $2 \mathrm{~h}$, führte die Zugabe des Startmaterials bei $0^{\circ} \mathrm{C}$ zur Ylid-Suspension und anschließendem Rühren bei 
Raumtemperatur für $1 \mathrm{~d}$ zu einer Ausbeute von $40 \%$ bei vollständiger Z-Selektivität der Reaktion.

Tabelle 19: Weitere Untersuchungen zur P-Ylid-Bildung.

\begin{tabular}{cccc}
\hline Eintrag & Äquivalente [EtPPh $]^{+} \mathrm{Br}^{-}$ & Äquivalente $\mathrm{NaH}$ & Ylid-Bildung \\
\hline 1 & 1.50 & 1.35 & kein Umsatz \\
2 & 3.00 & 2.70 & kaum Umsatz \\
3 & 5.00 & 4.50 & moderater Umsatz \\
4 & 10.0 & 9.00 & guter Umsatz \\
\hline
\end{tabular}

Reaktionsbedingungen: $\left[\mathrm{EtPPh}_{3}\right]^{+} \mathrm{Br}, \mathrm{NaH}, \mathrm{THF}, \mathrm{RT} \rightarrow 66^{\circ} \mathrm{C}, 5 \mathrm{~h}$.

Hinsichtlich der Stereoselektivität erwies sich ein Wechsel der Base von $n \mathrm{BuLi}$ zu NaH bereits als vorteilhaft, jedoch verblieb die Ausbeute bei moderaten $40 \%$, weswegen weitere Modifikationen in Betracht gezogen wurden, um diese zu steigern. Hierzu wurde wiederum die Ylid-Bildung weiteren Tests unterworfen, in der Annahme, dass eine Erhöhung der vorhandenen Menge des aktiven P-Ylid einen stärkeren Umsatz des Startmaterials und damit einen Anstieg der Ausbeute zur Folge hat. Daher wurden Änderungen an der Äquivalenteanzahl an eingesetztem [EtPPh $]^{+} \mathrm{Br}^{-}$und $\mathrm{NaH}$ auf ihre Auswirkungen auf die P-Ylid-Bildung untersucht. Den Erwartungen entsprechend konnte mit einem verringerten Reagenzieneinsatz kein Umsatz beobachtet werden (Tabelle 19, Eintrag 1), während dieser mit steigendem Materialeinsatz ebenfalls zunahm (Tabelle 19, Eintrag 2 bis 4). Die P-Ylid-Bildung wurde daraufhin in einer Testreaktion mit $\left[\mathrm{EtPPh}_{3}\right]^{+} \mathrm{Br}^{-}$ (10.0 Äq.) und $\mathrm{NaH}$ (9.00 Äq.) in THF nach erfolgter Zugabe bei Raumtemperatur bei $66{ }^{\circ} \mathrm{C}$ für $1 \mathrm{~h}$ durchgeführt. Die anschließende Zugabe des Ketons 174 erfolgte bei stark verminderten $-78^{\circ} \mathrm{C}$, um zum einen die Z-Selektivität zusätzlich sicherstellen und zum anderen die doch recht harschen Reaktionsbedingungen etwas abzumildern. Nach einer Reaktionszeit von $15 \mathrm{~min}$ bei $-78^{\circ} \mathrm{C}$ konnte das Z-Olefin (Z)-175 in leicht verbesserten $53 \%$ Ausbeute isoliert werden. Im Gramm-Maßstab wurde aber wiederum eine Verringerung der Ausbeute auf $32 \%$ verzeichnet. 


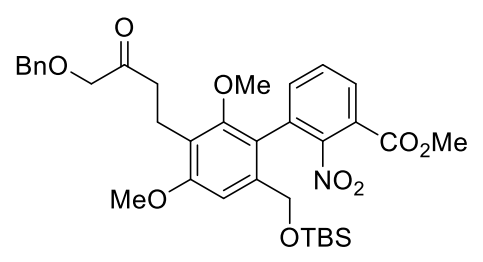

174

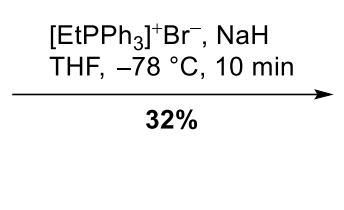

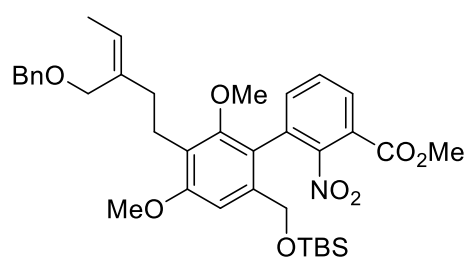

$(Z)-175$
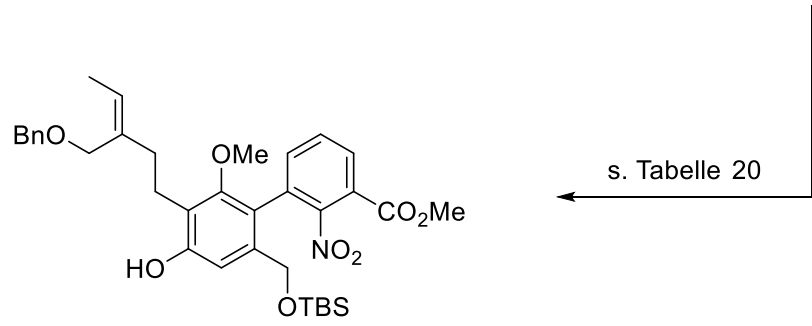

(Z)-176

Tabelle 20: Versuch der selektiven mono-Demethylierung des Alkens (Z)-175.

\begin{tabular}{clc}
\hline Eintrag & \multicolumn{1}{c}{ Reaktionsbedingungen } & Ausbeute \\
\hline 1 & $B$-Br-9-BBN (1.00 Äq.), $\mathrm{CH}_{2} \mathrm{Cl}_{2}, \mathrm{RT}, 1 \mathrm{~d}$ & TBS-Entschützung \\
2 & $B$-I-9-BBN (1.00 Äq.), $\mathrm{CH}_{2} \mathrm{Cl}_{2}, \mathrm{RT}, 3 \mathrm{~d}$ & TBS-Entschützung \\
3 & NaSEt (2.00 Äq.), DMF, $120^{\circ} \mathrm{C}, 3 \mathrm{~d}$ & Spuren \\
4 & L-Selektrid (4.00 Äq.), THF, $66^{\circ} \mathrm{C}, 2 \mathrm{~d}$ & Zersetzung \\
\hline
\end{tabular}

Im nachfolgenden Schritt sollte die sterisch weniger stark gehinderte Methoxygruppe entschützt und somit das Phenol (Z)-176 als Vorläufer der enantioselektiven WackerZyklisierung aus dem Z-Alken (Z)-175 erhalten werden. Die Verwendung der sterisch anspruchsvollen 9-BBN-basierten Halogenide $B$-Br-9-BBN und $B$-I-9-BBN führte jedoch selektiv zur Entschützung der TBS-Schutzgruppe (Tabelle 20, Eintrag 1 und 2). NaSEt lieferte massenspektrometrisch nachweisbare Spuren einer mono-demethylierten Spezies (Tabelle 20, Eintrag 3). Eine Isolation aus dem Rohprodukt gelang allerdings nicht. Neben der Entschützung einer der beiden Methoxygruppen könnte auch eine Umesterung des Methylesters zum Thioester stattgefunden haben, der wiederum in der wässrigen Aufarbeitung zur Carbonsäure hätte hydrolysieren können. Diese Möglichkeit würde zumindest teilweise die Schwierigkeiten der Isolierung der beobachteten Spezies erklären. Des Weiteren legen Publikationen zur Entschützung aromatischer Methylether mittels NaSEt den Schluss nahe, dass die sterisch vermeintlich stärker gehinderte Methoxygruppe bedingt durch den sterischen Einfluss aus der aromatischen Ebene herausgedreht wird und damit einem nukleophilen Angriff des Ethanthiolats stärker ausgesetzt wäre. ${ }^{[145]}$ Übertragen auf das Biaryl (Z)-176 hätte dies die Entschützung der falschen Methoxygruppe zur Folge. Ein weiterer Versuch der selektiven Entschützung 
mithilfe von L-Selektrid führte zur Zersetzung des Edukts (Tabelle 20, Eintrag 4). In Anbetracht der Schwierigkeiten, die bei der selektiven Entschützung der Methoxygruppe auftraten, wurden neue Ansätze zum Aufbau des Zyklisierungsvorläufers ins Auge gefasst. 


\subsection{Synthese des Vinylchromas (S)-193}

Aufgrund der aufgetretenen Problematik, die sich während der selektiven Entschützung der sterisch weniger stark gehinderten Methoxygruppe ergab, rückte eine Diversifizierung der Schutzgruppen der beiden phenolischen Hydroxygruppen in den Fokus. Zur Emulation des Substituentenmusters des angestrebten Naturstoffs Parnafungin C (3) sollte eine der beiden Hydroxygruppen weiterhin geschützt in Form eines Methylethers vorliegen. Um eine einfache sowie selektive Entschützung und gleichzeitig eine Differenzierung der beiden nicht mehr äquivalenten aromatischen Positionen für die Halogenierung zu gewährleisten, sollte die sterisch anspruchsvolle Pivaloyl-Schutzgruppe zur Schützung der zweiten Hydroxygruppe in das Substrat inkorporiert werden.

\subsubsection{Untersuchungen zur Diversifizierung der Schutzgruppen}

Ausgehend von der 3,5-Dihydroxybenzoesäure (153) wurde zunächst die Carboxylfunktionalität säurekatalysiert zum Methylester in nahezu quantitativer Weise verseift. Die darauffolgende basenvermittelte Piv-Schützung einer der beiden äquivalenten Hydroxyfunktionen mittels PivCl verlief jedoch recht unspezifisch, sodass neben dem einfach geschützten Phenol 178, das in 37\% Ausbeute erhalten wurde sowie $12 \%$ an reisoliertem Startmaterial, ein erheblicher Anteil zum doppelt geschütztem Methylester 179 umgesetzt wurde.<smiles>O=C(O)c1cc(O)cc(O)c1</smiles>

153
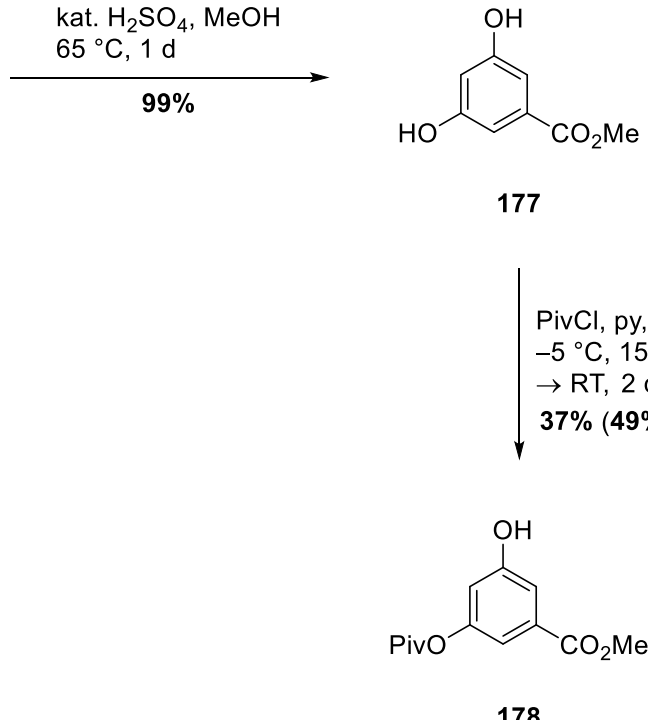

178
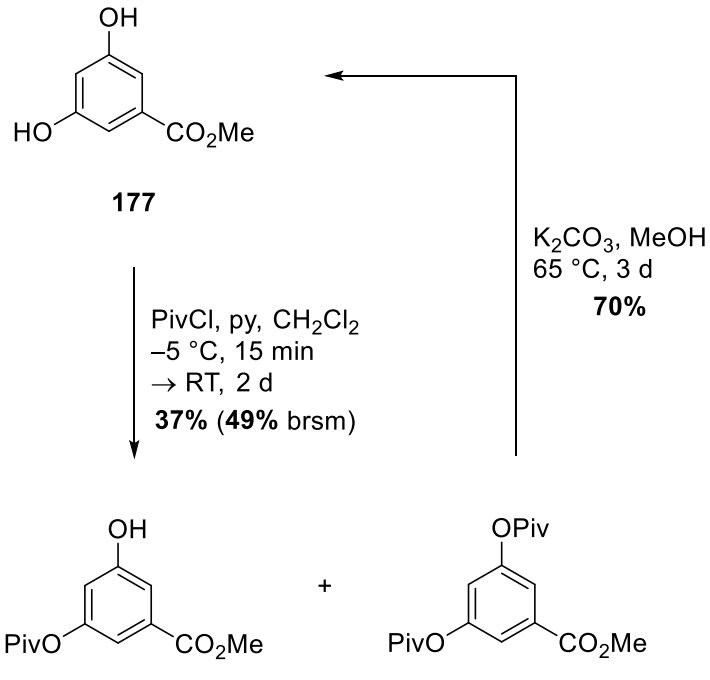

179

Abbildung 58: Synthese des Pivaloylesters 178 und Validierung einer geeigneten Entschützungsmethode.

Das doppelt Piv-geschützte Nebenprodukt 179 diente zur Evaluierung einer geeigneten Entschützungsmethode. Mithilfe von $\mathrm{K}_{2} \mathrm{CO}_{3}$ in $\mathrm{MeOH}$ konnte das doppelt Piv-geschützte 
Phenol 179 unter Erhalt der Methylesterfunktionalität in 70\% Ausbeute wieder in das Phenol 177 überführt werden (Abbildung 58).

Der Erhalt der Methylesterfunktion während der Hydrolyse des Pivaloylesters 179 ist insofern von Bedeutung, dass diese Funktionalität infolge der Suzuki-MiyauraKreuzkupplung dem Substrat hinzugefügt wird und nicht hydrolysiert werden darf.<smiles>COC(=O)c1cc(O)cc(O[Na])c1</smiles>

178

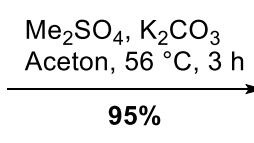

$95 \%$

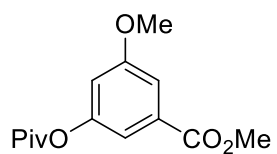

180

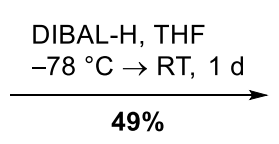

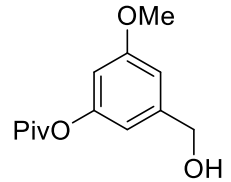

181

Abbildung 59: Darstellung des Benzylalkohols 181.

Daraufhin erfolgte die Methylierung der verbliebenen freien Hydroxygruppe mittels $\mathrm{Me}_{2} \mathrm{SO}_{4}$ unter basischen Reaktionsbedingungen in 95\% Ausbeute. In der Folge sollte der Methylester 180 zum benzylischen Alkohol 181 reduziert werden. Verschiedene Methoden unter Nutzung von $\mathrm{NaBH}_{4}$, L-Selektrid oder DIBAL-H resultierten im Falle von DIBAL-H in einer Ausbeute von maximal 49\% im kleinen Testmaßstab. Bei der Übertragung auf einen Multigramm-Maßstab konnte jedoch nur die Zersetzung des Startmaterials verzeichnet werden (Abbildung 59).<smiles>COc1cc(CO)cc(OCCCCCCCCCCCOc2ccccc2)c1</smiles>

181
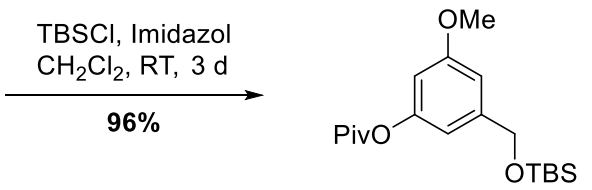

182

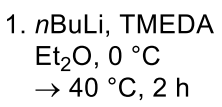

1. $n$ BuLi, TMEDA

$\mathrm{Et}_{2} \mathrm{O}, 0^{\circ} \mathrm{C}$

$\rightarrow 40^{\circ} \mathrm{C}, 2 \mathrm{~h}$

2. DMF, $0{ }^{\circ} \mathrm{C}$

$\rightarrow \mathrm{RT}, 1 \mathrm{~h}$

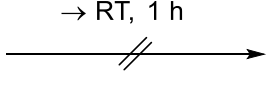

183

Abbildung 60: Versuch der Synthese des Benzaldehyds 183.

Anschließende Schützung der freien benzylischen Hydroxyfunktionalität mithilfe von TBSCI und Imidazol konnte in 96\% Ausbeute durchgeführt werden. Eine Formylierung nach der standardmäßig angewandten Methode gelang am komplett geschützten Substrat 182 nicht (Abbildung 60).

Aufgrund der problematischen Reduktion der Esterfunktion des aromatischen Diesters 180 zum benzylischen Alkohol 181 sowie der Labilität des komplett geschützten Substrats 182 unter den harschen Bedingungen der Formylierung sollten zum einen diese Schritte in der Synthesesequenz vorgezogen werden und zum anderen die Durchführung der Formylierung durch die Einführung eines Brom-Substituenten in entsprechender Position und anschließendem Lithium-Halogen-Austausch unterstützt werden. 


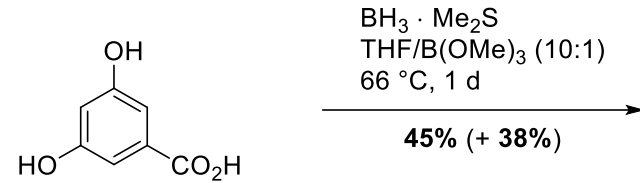

153<smiles>OCc1cc(O)cc(O)c1</smiles>

184

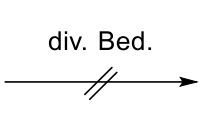<smiles>OCc1cc(O)c(Br)c(O)c1</smiles>

185

Abbildung 61: Versuch der Darstellung des Benzaldehyds 185.

Die Reduktion der 3,5-Dihydroxybenzoesäure (153) via $\mathrm{BH}_{3} \cdot \mathrm{Me}_{2} \mathrm{~S}$ erfolgte mit einer Ausbeute von $45 \%$. Weitere $38 \%$ Ausbeute am benzylischen Alkohol 184, deren vollständige Aufreinigung jedoch nicht bewerkstelligt werden konnte, wurden zusätzlich erhalten. In der anschließenden Bromierung konnte das gewünschte Arylbromid 185 nur in massenspektrometrisch nachgewiesenen Spuren bzw. in einer nicht aufreinigbaren Form erhalten werden (Abbildung 61). Somit sollten die Bromierung und die Reduktion der Carbonsäure zum benzylischen Alkohol in ihrer Abfolge getauscht werden, um diese Problematik möglicherweise zu umgehen.<smiles>O=C(O)c1cc(O)cc(O)c1</smiles>

153<smiles>[13CH3]OCc1cc(O)c(Br)c(O)c1</smiles>

187

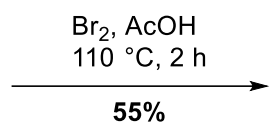

TBSCl, $\mathrm{NEt}_{3}$ DMAP, $\mathrm{CH}_{2} \mathrm{Cl}_{2}$ $0{ }^{\circ} \mathrm{C}, 30 \mathrm{~min}$ $\rightarrow$ RT, 90 min

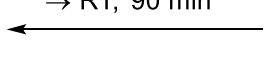<smiles>O=C(O)c1cc(O)c(Br)c(O)c1</smiles>

186 $\mathrm{BH}_{3} \cdot \mathrm{Me}_{2} \mathrm{~S}$ $\mathrm{THF} / \mathrm{B}(\mathrm{OMe})_{3}(10: 1)$ $66^{\circ} \mathrm{C}, 1 \mathrm{~d}$ $65 \%$<smiles>OCc1cc(O)c(Br)c(O)c1</smiles>

185

Abbildung 62: Synthese des TBS-Ethers 187.

Die Bromierung der 3,5-Dihydroxybenzoesäure (153) mit elementarem Brom in Eisessig verlief in einer Ausbeute von 55\%. Die nachfolgende Reduktion der Carbonsäure 186 wurde wiederum mit $\mathrm{BH}_{3} \cdot \mathrm{Me}_{2} \mathrm{~S}$ durchgeführt und lieferte den Benzylalkohol 185 in $65 \%$ Ausbeute. Eine TBS-Schützung der benzylischen Hydroxygruppe mittels $\mathrm{TBSCl}, \mathrm{NEt}_{3}$ und katalytischen Mengen DMAP erfolgte dabei jedoch in einer unselektiven Art und Weise. Die Auftrennung des daraus resultierten Produktgemisches diverser TBS-geschützter Substrate war nicht realisierbar (Abbildung 62). 


\subsubsection{Synthese des Phenols (Z)-197}

In Anbetracht der Schwierigkeiten mit denen eine schrittweise durchgeführte selektive Schützung der einzelnen funktionellen Gruppen einherging, sollte das geplante Schutzgruppenmuster über den Zwischenschritt einer zusätzlichen Entschützung eingestellt werden.

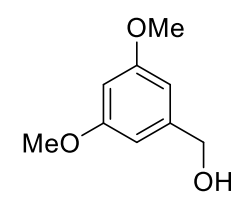

155

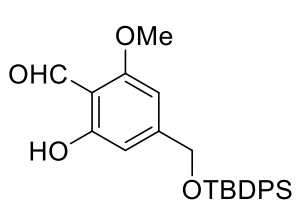

190

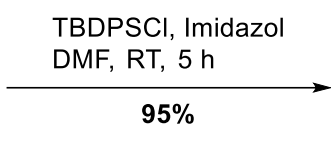

$\mathrm{Nal}, \mathrm{AlCl}_{3}$

$\mathrm{CH}_{2} \mathrm{Cl}_{2} / \mathrm{CH}_{3} \mathrm{CN}(2: 1)$

$0{ }^{\circ} \mathrm{C}, 1 \mathrm{~h}$

$55 \%$

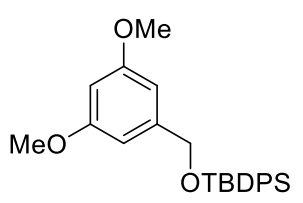

188
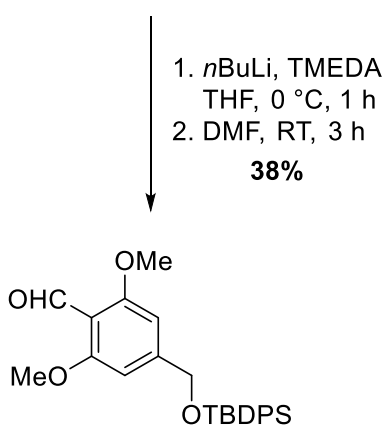

189

Abbildung 63: Synthese des Phenols 190.

Zu Beginn wurde der Benzylalkohol 155 via TBDPSCI und Imidazol in einer Ausbeute von 93\% TBDPS-geschützt. Daran anschließend erfolgte die regioselektive Formylierung des TBDPS-geschützten Benzylalkohols 188 in 38\% Ausbeute nach einer leicht modifizierten Variante der bisher verwendeten Methode. Zur Erzeugung des geplanten Schutzgruppenmusters fand im nachfolgenden Schritt mittels $\mathrm{AlCl}_{3}$ und Nal selektiv die Entschützung einer der beiden phenolischen Methylether am Aldehyd 189 in 55\% Ausbeute statt (Abbildung 63).

Nach vollzogener Entschützung wurde die freigesetzte Hydroxyfunktionalität am Phenol 190 wiederum Piv-geschützt. Nachdem ein initialer Versuch der Schützung keinen Umsatz des Phenols 190 generierte (Tabelle 21, Eintrag 1), konnte durch eine Anhebung der Äquivalente an PivCl auf 2.80, der Zugabe katalytischer Mengen DMAP sowie der Erhöhung der Reaktionstemperatur der Pivaloylester 191 in einer Ausbeute von 70\% erhalten werden (Tabelle 21, Eintrag 2). 


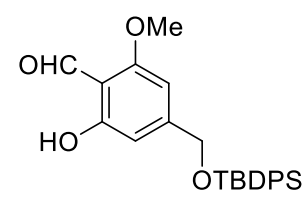

190 s. Tabelle 21

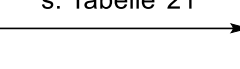

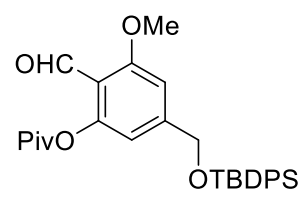

191

Tabelle 21: Untersuchungen zur Piv-Schützung des Phenols 190.

\begin{tabular}{|c|c|c|}
\hline Eintrag & Reaktionsbedingungen & Ausbeute \\
\hline 1 & PivCl (1.10 Äq.), py (3.00 Äq.), $\mathrm{CH}_{2} \mathrm{Cl}_{2},-78^{\circ} \mathrm{C} \rightarrow \mathrm{RT}, 7 \mathrm{~d}$ & - \\
\hline 2 & $\begin{array}{l}\text { PivCl (2.80 Äq.), py (3.00 Äq.), DMAP (10 mol\%), } \mathrm{CH}_{2} \mathrm{Cl}_{2} \text {, } \\
40^{\circ} \mathrm{C}, 5 \mathrm{~h} \rightarrow \mathrm{RT}, 3 \mathrm{~d}\end{array}$ & $70 \%[\mathrm{a}]$ \\
\hline
\end{tabular}

[a] $46 \%$ im Multigramm-Maßstab

Der erneut eingetretene Einbruch der Ausbeute auf 46\% im Multigramm-Maßstab soll an dieser Stelle ebenfalls nicht unerwähnt bleiben. Im Anschluss wurde der Aldehyd 191 mit dem P-Ylid 171 in einer Wittig-Reaktion mit einer Ausbeute von 85\% zum Alken $(E)-192$ umgesetzt (Abbildung 64).

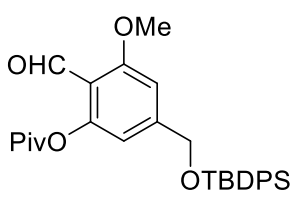

191

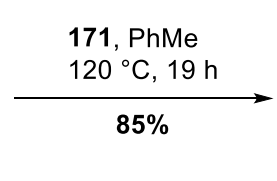

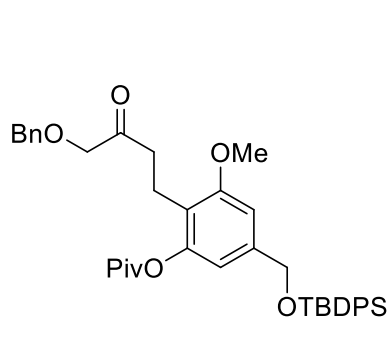

193

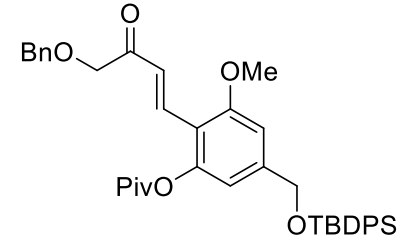

$(E)-192$

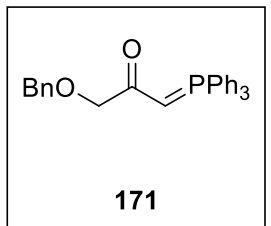

Abbildung 64: Darstellung des Ketons 193.

Für die nachfolgende Hydrierung der entstandenen Doppelbindung wurde wieder auf $\mathrm{PtO}_{2}$ und $\mathrm{H}_{2}$ zurückgegriffen, jedoch zunächst auf eine direkt angeschlossene Oxidation des erwarteten Mindermengenanteils an überreduziertem Alkohol verzichtet. Unter Verwendung dieser Methode konnte keine Umsetzung des Startmaterials nach einer Reaktionszeit von $2 \mathrm{~h}$ dünnschichtchromatographisch nachgewiesen werden (Tabelle 22, Eintrag 1). Im Zuge der Verlängerung der Reaktionszeit auf $1 \mathrm{~d}$ stieg ebenfalls der Anteil 
an umgesetztem Startmaterial auf ein registrierbares Niveau an, befand sich aber geschätzt bei <25\% (Tabelle 22 ,Eintrag 2).

Des Weiteren konnte das Alkan 193 bereits massenspektrometrisch validiert werden. Aufgrund der schleppend verlaufenden Reaktion wurde sowohl die Katalysatorbeladung

Tabelle 22: Untersuchungen zur Reduktion des Alkens (E)-192.

\begin{tabular}{ccccc}
\hline Eintrag & Äquivalente $\mathrm{PtO}_{2}$ & $\mathrm{H}_{2}$-Druck & Zeit & Umsatz \\
\hline 1 & $5 \mathrm{~mol} \%$ & $1 \mathrm{~atm}$ & $2 \mathrm{~h}$ & $<5 \%$ \\
2 & $5 \mathrm{~mol} \%$ & $1 \mathrm{~atm}$ & $1 \mathrm{~d}$ & $<25 \%$ \\
3 & $20 \mathrm{~mol} \%$ & $4 \mathrm{~atm}$ & $1 \mathrm{~d}$ & Multispot \\
\hline
\end{tabular}

Reaktionsbedingungen: $\mathrm{PtO}_{2}, \mathrm{H}_{2}, \mathrm{EtOH}, \mathrm{RT}$.

dukte dünnschichtchromatographisch registriert, deren Separierung nicht realisiert werden konnte (Tabelle 22, Eintrag 3). Hinsichtlich des langsamen und unsauberen Reaktionsverlaufs, der mit $\mathrm{PtO}_{2}$ erhalten wurde, wurde ein Wechsel des Reaktionssystems ins Auge gefasst.

Tabelle 23: Weitere Untersuchungen zur Hydrierung der Doppelbindung.

\begin{tabular}{|c|c|c|}
\hline Eintrag & Reaktionsbedingungen & Ausbeute \\
\hline 1 & {$\left[\mathrm{Ph}_{3} \mathrm{PCuH}\right]_{6}$ (0.37 Äq.), PhMe, RT, 2 h } & $\begin{array}{c}12 \% \\
(73 \% \text { brsm }\end{array}$ \\
\hline 2 & {$\left[\mathrm{Ph}_{3} \mathrm{PCuH}\right]_{6}(0.37 \mathrm{Äq}),. \mathrm{PhMe}, 111^{\circ} \mathrm{C}, 3 \mathrm{~d}$} & $39 \%$ \\
\hline 3 & Noyori-Kat. (10 mol\%), $\mathrm{PrOH} / \mathrm{CH}_{3} \mathrm{CN}(10: 1), \mathrm{RT}, 3 \mathrm{~d}$ & Spuren \\
\hline 4 & $\mathrm{PtO}_{2}(5 \mathrm{~mol} \%), \mathrm{H}_{2}$ (1 atm), AcOH (10.0 Äq.), EtOH, RT, $3 \mathrm{~d}$ & $42 \%$ \\
\hline
\end{tabular}

Der für seine selektive Hydrierung der Doppelbindung $\alpha, \beta$-ungesättigter Carbonylverbindungen bekannte hexamere CuH-Komplex Stryker's Reagenz ${ }^{[146]}$ lieferte das Alkan 193 in einer Ausbeute von 12\% nach einer Reaktionszeit von $2 \mathrm{~h}$ bereits bei Raumtemperatur. Zusätzlich konnten $61 \%$ des eingesetzten Startmaterials wiedergewonnen werden (Tabelle 23, Eintrag 1). Infolge der Anhebung der Reaktionstemperatur und eine Verlängerung der Reaktionszeit konnte die Ausbeute auf 39\% gesteigert werden (Tabelle 23, Eintrag 2). Zum Vergleich wurden zur Hydrierung außerdem Noyoris TransferHydrierung-Katalysator in einem $i \mathrm{PrOH} / \mathrm{CH}_{3} \mathrm{CN}$-Lösungsmittelgemisch (Tabelle 23, Eintrag 3) sowie ein um AcOH erweitertes System aus $\mathrm{PtO}_{2}$ und $\mathrm{H}_{2}$ getestet (Tabelle 23, Eintrag 4). Das erstgenannte System lieferte nur mutmaßlich massenspektrometrisch nachweisbare Spuren am gewünschten Alkan 193, während Letzteres, neben einer Vielzahl diverser Nebenprodukte, das Alken (E)-192 mit einer Ausbeute von 42\% zum entsprechenden Alkan 193 reduzierte. Aufgrund des saubereren Reaktionsverlaufs, der 
daraus resultierenden simpleren Aufreinigung und der geringen Ausbeuteunterschiede fiel die Wahl auf Stryker's Reagenz zur Reduktion der Doppelbindung.

Es wurden daraufhin Untersuchungen mit dem Ziel der Optimierung dieser Reaktion durchgeführt. Unter ökonomischen und ökologischen Gesichtspunkten wurde der katalytische Einsatz

Tabelle 24: Untersuchungen zur Optimierung der Reduktion der Doppelbindung.

\begin{tabular}{cccc}
\hline Eintrag & Äquivalente $\mathrm{PhSiH}_{3}$ & Temperatur & Ausbeute \\
\hline 1 & 1.50 & $100{ }^{\circ} \mathrm{C}$ & $19-28 \%$ \\
2 & 1.50 & $0^{\circ} \mathrm{C} \rightarrow \mathrm{RT}$ & $30 \%$ \\
3 & 1.00 & $0^{\circ} \mathrm{C} \rightarrow \mathrm{RT}$ & $41 \%$ \\
4 & 0.75 & $0^{\circ} \mathrm{C} \rightarrow \mathrm{RT}$ & $27 \%$ \\
\hline
\end{tabular}

des Stryker's Reagenz durch die Verwendung

Reaktionsbedingungen: $\left[\mathrm{Ph}_{3} \mathrm{PCuH}\right]_{6}(5 \mathrm{~mol} \%), \mathrm{PhSiH}_{3}, \mathrm{PhMe}, 1 \mathrm{~d}$. von $\mathrm{PhSiH}_{3}$ als Hydridquelle angestrebt. Hierbei konnten Ausbeuten von $19-28 \%$ erzielt werden (Tabelle 24, Eintrag 1). Die

Reduktion der Reaktionstemperatur hatte weder positiv noch negativ signifikante Auswirkungen auf den Ausgang der Reaktion zur Folge (Tabelle 24, Eintrag 2). Durch eine Verminderung der eingesetzten Menge an $\mathrm{PhSiH}_{3}$ auf 1.00 Äquivalente konnte eine leichte Steigerung der Ausbeute auf 41\% erreicht werden (Tabelle 24, Eintrag 3), während eine weitere Absenkung auf 0.75 Äquivalente wiederum eine Verringerung der Ausbeute auf $27 \%$ nach sich zog (Tabelle 24, Eintrag 4). Der Einsatz von Stryker's Reagenz erforderte außerdem den peniblen Ausschluss von $\mathrm{O}_{2}$, um die Aktivität des $\mathrm{CuH}$ Komplexes über eine ausreichend lange Zeitspanne zu gewährleisten. Nach der erfolgten Reduktion der Doppelbindung sollte der aromatische Kern des Alkans 193 in der verbliebenen freien Position ortho zur Methoxygruppe selektiv halogeniert werden.

Hierzu wurde zunächst auf die bereits auf einem analogen Substrat erfolgreich etablierte Methode unter Verwendung von $\mathrm{I}_{2}$ und $\mathrm{Ag}_{3} \mathrm{PO}_{4}$ zurückgegriffen. Trotz ähnlicher elektronischer Eigenschaften des Substrats blieb eine Umsetzung zum Aryliodid 194 vermutlich infolge des gestiegenen sterischen Anspruchs aus (Tabelle 25, Eintrag 1). Hingegen führte eine Bromierung mittels NBS ungeachtet der höheren Stabilität der TBDPS-Schutzgruppe hauptsächlich zur Abspaltung ebenjener Schutzgruppe unter diesen Reaktionsbedingungen (Tabelle 25, Eintrag 2). Somit wurde wiederum von einer Bromierung Abstand genommen und mittels $I_{2}$ und CAN erneut versucht, den aromatischen Kern zu funktionalisieren. Wider Erwarten erfolgte die lodierung trotz des größeren sterischen Anspruchs des Substrats selektiv in ortho-Position zum Piv-Ester in 57\% Ausbeute (Tabelle 25, Eintrag 3). Ein weiterer Versuch der lodierung mithilfe eines Überschusses an $\mathrm{Ag}_{3} \mathrm{PO}_{4}$ zur Aktivierung des $\mathrm{I}_{2}$ lieferte allerdings keinen Umsatz (Tabelle 25, Eintrag 4). 


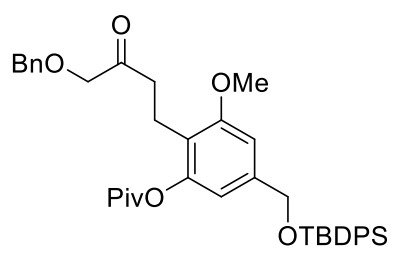

193
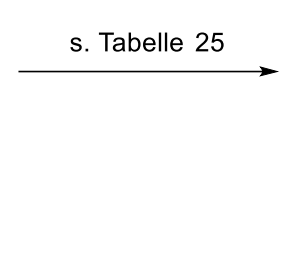

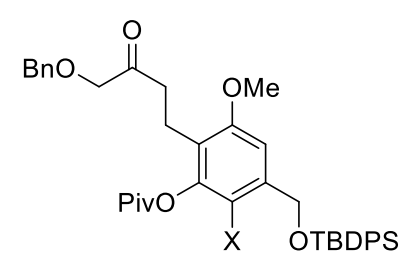

194: $\mathrm{X}=\mathrm{Br}$ 195: $X=1$

Tabelle 25: Untersuchungen zur Halogenierung des Aryls 193.

\begin{tabular}{clc}
\hline Eintrag & \multicolumn{1}{c}{ Reaktionsbedingungen } & Ausbeute \\
\hline 1 & $\mathrm{I}_{2}$ (1.00 Äq.), $\mathrm{Ag}_{3} \mathrm{PO}_{4}\left(0.37\right.$ Äq.), $\mathrm{CHCl}_{3}, \mathrm{RT}, 6 \mathrm{~d}$ & - \\
2 & $\mathrm{NBS}\left(1.00\right.$ Äq.), $\mathrm{CCl}_{4}, 77^{\circ} \mathrm{C}, 1 \mathrm{~d}$ & Zersetzung \\
3 & $\mathrm{I}_{2}$ (1.00 Äq.), $\mathrm{CAN}\left(1.00 \mathrm{Äq}\right.$ ) $, \mathrm{CH}_{3} \mathrm{CN}, \mathrm{RT}, 5 \mathrm{~d}$ & $57 \%$ \\
4 & $\mathrm{I}_{2}$ (1.00 Äq.), $\mathrm{Ag}_{3} \mathrm{PO}_{4}\left(1.10 \mathrm{Äq}\right.$.), $\mathrm{CHCl}_{3}, \mathrm{RT}, 1 \mathrm{~d}$ & - \\
\hline
\end{tabular}

In Anbetracht der Tatsache, dass von den untersuchten Methoden zur Halogenierung die Einzige, die einen Umsatz des Substrats erbrachte, mit der verkehrten Regioselektivität einherging, wurde die selektive Spaltung des Methylethers auf ihre Durchführbarkeit hin überprüft. Dadurch bestünde die Möglichkeit, das Aryliodid 195 zunächst zum korrespondierenden Biaryl aufzubauen, die Seitenkette via Wittig-Reaktion zu verlängern und abschließend die phenolische Hydroxygruppe aus dem Methylether freizusetzen, um so ein geeignetes Substrat für die enantioselektive Wacker-Zyklisierung zu erhalten. Zur Einstellung des Substituentenmusters des Parnafungin C (3) müsste daraufhin noch eine Umschützung des Piv-Esters zum Methylether erfolgen.

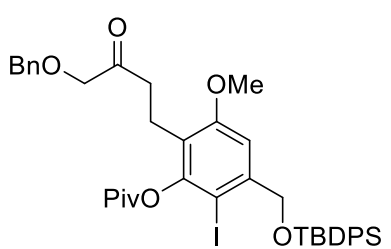

195

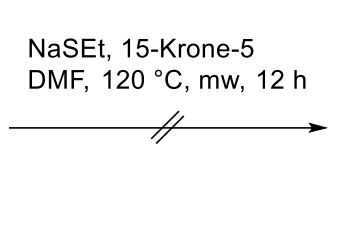

19

Abbildung 65: Versuch der Entschützung der Methoxygruppe am Substrat 195.

Jedoch konnte trotz der Zugabe des Kronenethers 15-Krone-5 zum Abfangen der $\mathrm{Na}^{+}$und der daraus resultierenden gesteigerten Reaktivität des Thioethanolats keine Entschützung verzeichnet werden (Abbildung 65). Damit konnte weder eine regioselektive lodierung noch eine selektive Entschützung der Methoxygruppe realisiert werden, weswegen die selektive Funktionalisierung des aromatischen Kerns und der daran anschließende Aufbau des Biarylelements zu einem geeigneten Zeitpunkt nach erfolgter WackerZyklisierung stattfinden sollte. 


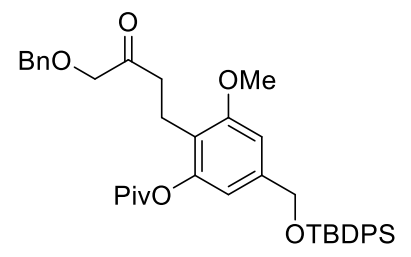

193
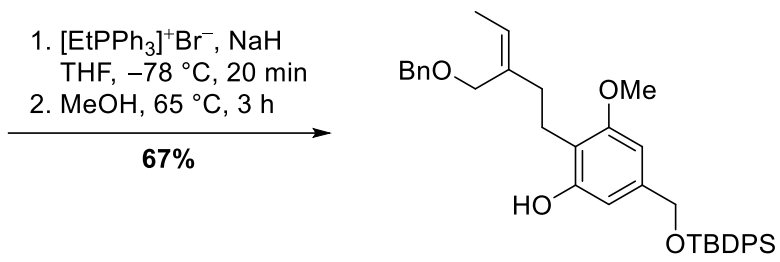

$(Z)-197$

Abbildung 66: Kombinierte Wittig-Reaktion und Entschützung der Piv-Schutzgruppe zum Erhalt des Z-Alkens (Z)-197.

Als Ausgangspunkt hierfür diente das Alkan 193, das unter den modifizierten Bedingungen der Wittig-Reaktion mit vollständiger Z-Selektivität zum entsprechenden Z-Alken umgesetzt und nach Entfernung des Lösungsmittels durch die Zugabe von $\mathrm{MeOH}$ zu den weiterhin vorherrschenden basischen Bedingungen zum Phenol (Z)-197 mit einer Ausbeute von $67 \%$ entschützt wurde (Abbildung 66).

\subsubsection{Synthese des (S,S)-iPr-BOXAX-Liganden (S,S)-91b}

Nach dem Erhalt des entschützten Phenols (Z)-197 als Startmaterial der enantioselektiven Wacker-Zyklisierung stand für deren Durchführung die Synthese des chiralen Liganden (S,S)-iPr-BOXAX $(S, S)-91 b$ an. Gegenüber dem ursprünglich für diese Umsetzung angedachten $(S, S)$-Bn-BOXAX-Liganden (S,S)-91a wies dieser in aktuelleren vorangegangenen Untersuchungen in der Arbeitsgruppe Tietze bei nahezu identischen Ausbeuten eine nochmals höhere Induktion der jeweils gewollten Stereoinformation auf. ${ }^{[128]}$ Die Synthese erfolgte hierbei entlang einem in der Arbeitsgruppe optimierten Protokoll, basierend auf einer literaturbekannten Vorschrift von Hayashi und Myers et al. ${ }^{[91,129-133]}$

Beginnend mit der benzylischen Dibromierung des kommerziell erhältlichen 1-Brom-2methylnaphthalen (198) wurde das Tribromid 199 in einer Wohl-Ziegler-Reaktion mittels NBS und dem Radikalstarter AIBN in $85 \%$ Ausbeute erhalten. ${ }^{[147]}$ Die Dibrommethylgruppe wurde anschließend in siedender verdünnter wässriger $\mathrm{HCO}_{2} \mathrm{H}$ in die Formylgruppe des Carbaldehyds 200 mit einer einer Ausbeute von 76\% überführt. ${ }^{[48]}$ Diese wurde wiederum direkt in einer Pinnick-Oxidation mittels dem Oxidationsmittel $\mathrm{NaClO}_{2}$, dem Scavenger 2-Methyl-2-buten sowie $\mathrm{NaH}_{2} \mathrm{PO}_{4} \cdot \mathrm{H}_{2} \mathrm{O}$ als Puffer zur entsprechenden Carbonsäure 201 mit einer Ausbeute von 93\% oxidiert (Abbildung 67). ${ }^{[149]}$ 


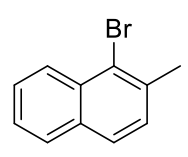

198

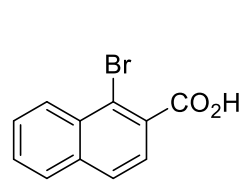

201

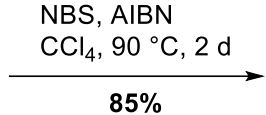

$\mathrm{NaClO}_{2}$ (aq.)

$\mathrm{NaH}_{2} \mathrm{PO}_{4} \cdot \mathrm{H}_{2} \mathrm{O}_{(\text {aq. }}$

2-Methyl-2-buten

Aceton, RT, $22 \mathrm{~h}$

$93 \%$<smiles>Brc1c(C(Br)Br)ccc2ccccc12</smiles>

199

$\mathrm{HCO}_{2} \mathrm{H}_{(\text {aq. }}$ $110{ }^{\circ} \mathrm{C}, 20 \mathrm{~h}$

$76 \%$

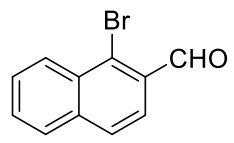

200

Abbildung 67: Synthese der Carbonsäure 201.

Parallel dazu wurde die L-Aminosäure (S)-Valin ((S)-203) mittels $\mathrm{LiAlH}_{4}$ mit einer Ausbeute von 66\% zum Alkohol (S)-Valinol ((S)-204) reduziert. ${ }^{[150]}$ Sowohl die Carbonsäure 201 als auch der chirale Alkohol (S)-204 wurden in einer vierstufigen Synthesesequenz zum Aufbau des Oxazolin-Grundgerüsts (S)-202 kombiniert. ${ }^{[151]}$

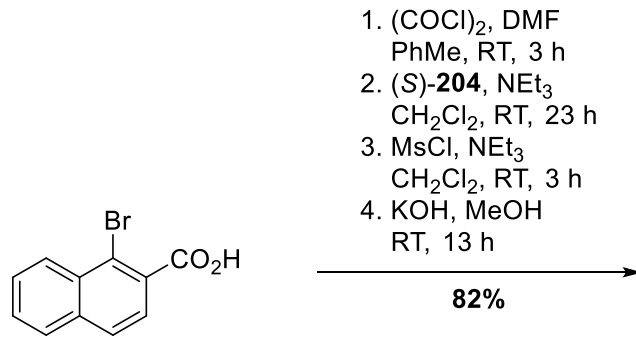

201
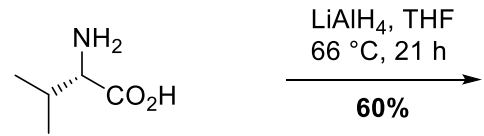

(S)-Valin ((S)-203)

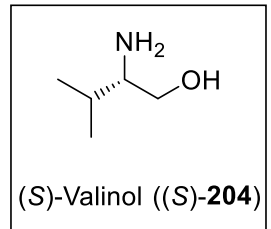

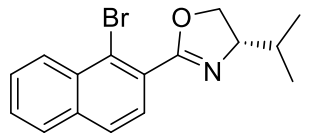

(S)-202

Cu, py $115{ }^{\circ} \mathrm{C}, 19 \mathrm{~h}$ $71 \%$

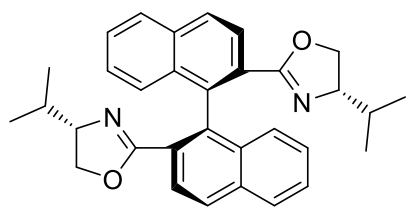

$(S, S)-i \operatorname{Pr}-B O X A X(S, S)-\mathbf{9 1 b}$

Abbildung 68: Darstellung des (S,S)-iPr-BOXAX-Liganden $(S, S)-91 b$. 
Hierzu fand zunächst eine in situ Aktivierung der Carbonsäure 201 mit $(\mathrm{COCl})_{2}$ und katalytischen Mengen DMF zum Säurechlorid statt, das nachfolgend basenvermittelt mit dem chiralen Aminoalkohol (S)-204 zum Amid umgesetzt wurde. Mesylierung der freien Hydroxygruppe mittels $\mathrm{MsCl}$ unter basischen Reaktionsbedingungen, gefolgt von einer baseninduzierten intramolekularen nukleophilen Substitution lieferte das (S)-Oxazolin (S)-202 in 82\% Ausbeute. Dabei ist erwähnenswert, dass keine Aufreinigungsschritte für die jeweiligen Intermediate anfallen, was sich zum einen positiv in der Gesamtausbeute niederschlägt und zum anderen in der simplen praktischen Durchführbarkeit widerspiegelt. Den abschließenden Schritt in der gesamten Synthesesequenz bildete eine hoch diastereoselektive Kupfer-vermittelte Ullmann-Kupplung. ${ }^{[129]}$ Zur Gewährleistung der Reproduzierbarkeit und kurzer Reaktionszeiten sind hierbei allerdings einige Vorleistungen zu erbringen. Zum einen wäre in diesem Zusammenhang die Aktivierung des Kupfers durch aufeinanderfolgendes Waschen mit $\mathrm{AcOH}, \mathrm{MeOH}$ und $\mathrm{Et}_{2} \mathrm{O}$ sowie abschließendes Trocknen im Hochvakuum zu nennen und zum anderen sollte das verwendete Pyridin frisch über $\mathrm{CaH}$ getrocknet und abdestilliert werden. Unter diesen Voraussetzungen konnte der (S,S)-iPr-BOXAX-Ligand $(S, S)-91 b$ in $71 \%$ Ausbeute erhalten werden (Abbildung 68).

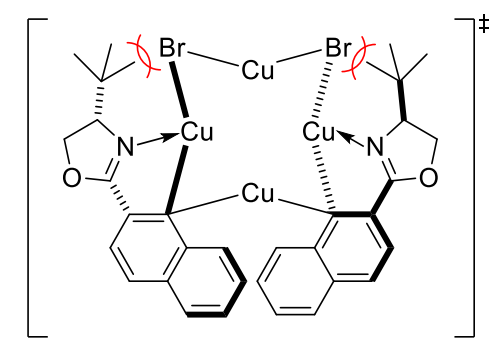

Abbildung 69: Postulierter Übergungszustand der Ullmann-Kupplung am Modell des (S,S)-tBu-BOXAXLiganden nach Andrus.

Der hohe Grad an Diastereoselektivität, mit der die Ausbildung des BOXAX-Liganden in der Ullmann-Kupplung einhergeht, rührt von der Ausbildung eines achtgliedrigen Übergangszustand bestehend aus einer Diaryl- $\mathrm{C}_{i p s o}-\mathrm{Cu}^{\prime}$-Spezies und $\mathrm{Cu}$ 'Br, wie er von Andrus für ein Modell der Ausbildung des $(S, S)$-tBu-BOXAX-Liganden postuliert wurde. ${ }^{[152]}$ Die Konfiguration der Biaryl-Achse ergibt sich dabei als Folge der Minimierung der sterischen Abstoßung zwischen den Oxazolin-Substituenten sowie der verbrückenden Bromid-Liganden (Abbildung 69). 


\subsubsection{Wacker-Zyklisierung zum Aufbau des Vinylchromans (S)-207}

Die Voraussetzungen der enantioselektiven Wacker-Zyklisierung wurden mit der Synthese des (S,S)-IPr-BOXAX-Liganden (S,S)-91b und dem Erhalt des Phenols (Z)-197 als Ausgangsmaterial beide erfüllt, sodass das Phenol (Z)-197 mittels $\operatorname{Pd}(\operatorname{TFA})_{2}$ und dem $(S, S)-\operatorname{Pr}$-BOXAX-Liganden $(S, S)$-91b bei einer Katalysatorbeladung von 10 mol\% sowie $p$-Benzochinon in einer Ausbeute von 15\% und einem Enantiomerenüberschuss von 96.6\% in das Vinylchroman (S)-207 überführt wurde. Zusätzlich konnten 7\% des Startmaterials reisoliert werden (Abbildung 70).

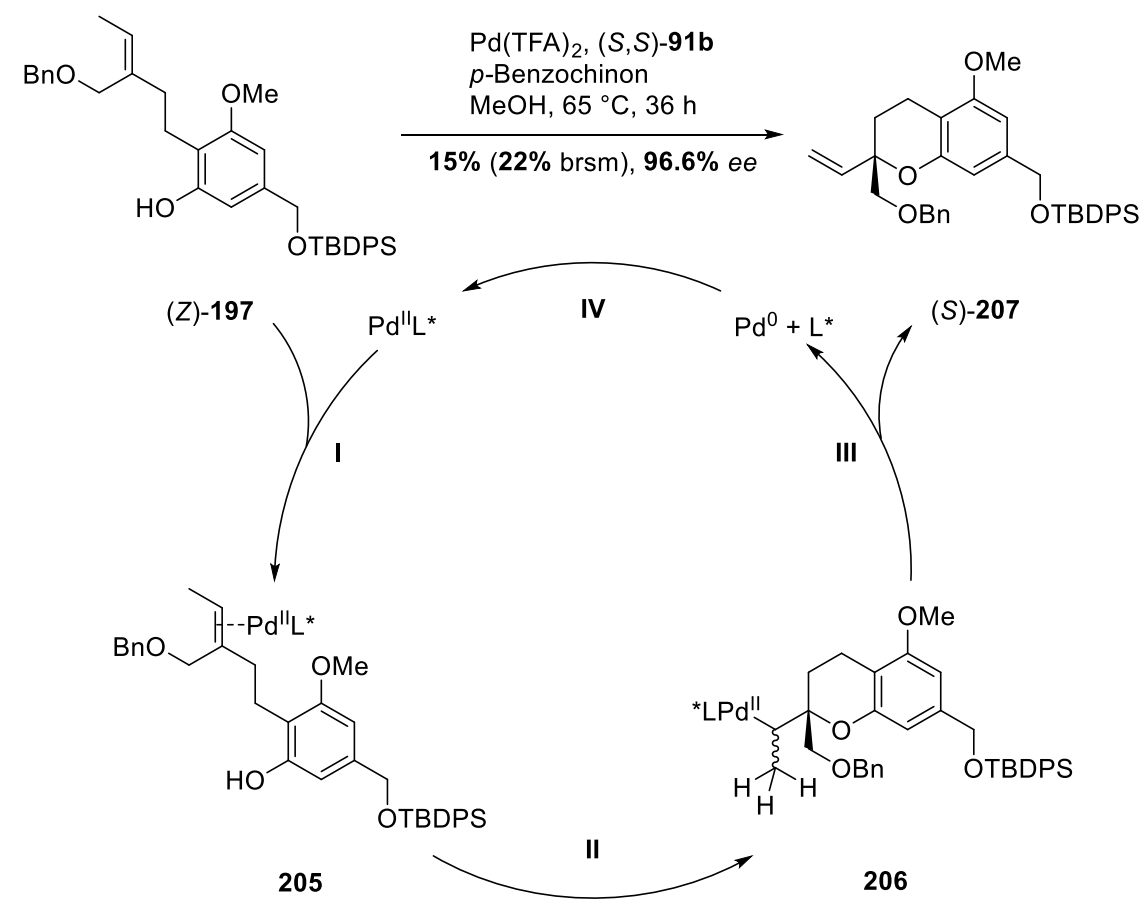

Abbildung 70: Enantioselektive Synthese des Vinylchromans (S)-193 und postulierter Mechanismus der Wacker-Zyklisierung.

Die enantioselektive Umsetzung des Phenols (Z)-197 zum Vinylchroman (S)-207 in der Wacker-Zyklisierung folgt dem nachfolgend dargestellten postulierten Mechanismus. Ausgehend vom Phenol (Z)-197 erfolgt zunächst eine enantiofaciale Koordination des in situ generierten chiralen $\mathrm{Pd}^{\prime \prime}$-BOXAX-Komplexes an der Z-konfigurierten dreifach substituierten Doppelbindung (I). Anschließend überführt eine intramolekulare Oxy-

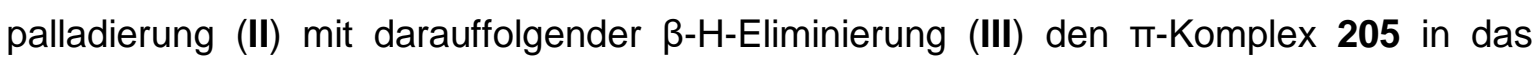
Vinylchroman (S)-207 unter Freisetzung einer $\mathrm{Pd}^{0}$-Spezies. Diese wird wiederum nachfolgend via $p$-Benzochinon zum aktiven Pd"-Ligand-Komplex reoxidiert (IV). Die Annahme $\operatorname{der}(S)$-Konfiguration des erhaltenen Vinylchromans (S)-207 basiert hierbei auf Vergleiche der optischen Rotation und NMR-spektroskopischer Daten verschiedener (S)-konfigurierter analoger Strukturen. 
Die Synthese des Vinylchromans (S)-207 konnte zwar auf dem beschriebenen Weg durchgeführt werden, allerdings fielen die Ausbeuten nicht zufriedenstellend aus. 


\subsection{Synthese des Chromans syn-266 und des Biaryls (Z)-272}

Nachdem sowohl eine selektive Entschützung einer der beiden Hydroxygruppen nach erfolgtem Aufbau der Biarylstruktur als auch eine selektive Schützung dieser beiden Gruppen aufgrund einer inadäquaten Regioselektivität während der darauffolgenden Halogenierung scheiterten, sollte der Aufbau des Biarylelements zu einem geeigneten Zeitpunkt in der Synthese nach Erhalt des Vinylchromans durchgeführt werden. Dieses Vorgehen würde zusätzlich zur Wacker-Zyklisierung auch die Verwendung der enantioselektiven Domino-Wacker/Carbonylierung/Methoxylierung zum Aufbau des Vinylchromans ermöglichen, auf die bisher unter Berücksichtigung möglicher Schwierigkeiten, die eine selektive Reduktion einer der beiden dann im Substrat befindlichen Methylester mit sich bringen würde, verzichtet wurde. Des Weiteren war vorgesehen, die aufgetretenen Schwierigkeiten der Reduktion der Doppelbindung des $\alpha, \beta$-ungesättigten Ketons $(E)-208$ zu adressieren.

\subsubsection{Untersuchungen zum Aufbau des Ketons 217}

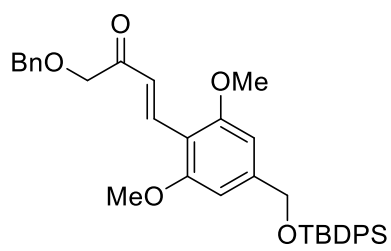

(E)-208

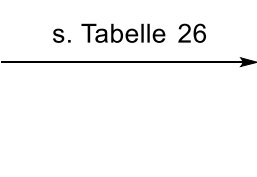

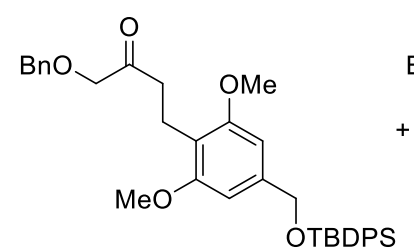

209

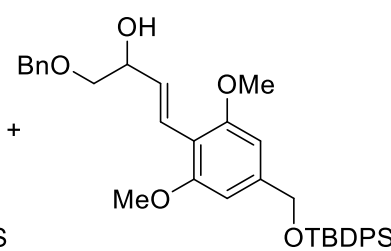

210

Tabelle 26: Untersuchungen zur Optimierung der Hydrierung der Doppelbindung.

\begin{tabular}{|c|c|c|}
\hline Eintrag & Reaktionsbedingungen & Ausbeute \\
\hline 1 & $\begin{array}{l}{\left[\mathrm{Ph}_{3} \mathrm{PCuH}\right]_{6}(1 \mathrm{~mol} \%), \mathrm{PhSiH}_{3}(1.00 \mathrm{Äq} .), \mathrm{PhMe}, 0^{\circ} \mathrm{C} \rightarrow \mathrm{RT},} \\
1 \mathrm{~d}\end{array}$ & $66 \%$ \\
\hline 2 & $\begin{array}{l}{\left[\mathrm{Ph}_{3} \mathrm{PCuH}\right]_{6}(1 \mathrm{~mol} \%), \mathrm{PMHS} \text { (3.00 Äq.), PhMe, } 0^{\circ} \mathrm{C} \rightarrow \mathrm{RT},} \\
1 \mathrm{~d}\end{array}$ & $62 \%$ \\
\hline 3 & $\begin{array}{l}{\left[\mathrm{Ph}_{3} \mathrm{PCuH}\right]_{6}(1 \mathrm{~mol} \%), \mathrm{PMHS} \text { (3.00 Äq.), PhMe, }-78^{\circ} \mathrm{C}, 4 \mathrm{~h}} \\
\rightarrow \mathrm{RT}, 2 \mathrm{~d}\end{array}$ & $<20 \%$ Umsatz \\
\hline 4 & $\begin{array}{l}\mathrm{Cu}(\mathrm{OAc})_{2}(1 \mathrm{~mol} \%), \operatorname{BDP} \quad(0.1 \mathrm{~mol} \%), \text { PMHS (2.00 Äq.), } \\
\text { PhMe, RT, } 1 \mathrm{~d}\end{array}$ & $<5 \%$ Umsatz \\
\hline 5 & $\begin{array}{l}\mathrm{Cu}(\mathrm{OAc})_{2}(10 \mathrm{~mol} \%), \text { BDP }(1 \mathrm{~mol} \%) \text {, PMHS (2.00 Äq.), } \\
\text { PhMe, RT, } 1 \mathrm{~d}\end{array}$ & $<20 \%$ Umsatz \\
\hline 6 & {$\left[\mathrm{Ph}_{3} \mathrm{PCuH}\right]_{6}$ (0.50 Äq.), PMHS (2.00 Äq.), PhMe, $0{ }^{\circ} \mathrm{C}, 1 \mathrm{~d}$} & $210 \uparrow$ \\
\hline 7 & {$\left[\mathrm{Ph}_{3} \mathrm{PCuH}\right]_{6}\left(0.50\right.$ Äq.), PMHS (2.00 Äq.), PhMe, $66^{\circ} \mathrm{C}, 1 \mathrm{~d}$} & Polymerisation \\
\hline
\end{tabular}

Die Untersuchungen zur Optimierung der Reduktion wurden am $\alpha, \beta$-ungesättigten Keton (E)-208 durchgeführt und fußten auf der katalytischen Nutzung von Stryker's Reagenz, 
mit der bereits in vorherigen Tests erste Erfahrungen gesammelt wurden. Durch den sorgfältigen Ausschluss von $\mathrm{O}_{2}$ infolge einer optimierten praktischen Durchführung der Reaktion konnte mit einer Katalysatorbeladung von 1 mol\% das Keton 209 in einer Ausbeute von $66 \%$ erhalten werden (Tabelle 26, Eintrag 1). Ein Wechsel der Hydridquelle von $\mathrm{PhSiH}_{3}$ hin zum weniger empfindlichen Polymer PMHS resultierte in einer nahezu identischen Ausbeute (Tabelle 26, Eintrag 2). Jedoch wurde entgegen der literaturgestützten Annahme der hohen Selektivität des Stryker's Reagenz im Reaktionsprozess die Reduktion des Ketons zum Allylalkohol 210 ebenfalls verzeichnet. Die Unterdrückung dieser Nebenreaktion konnte weder durch eine Verminderung der Temperatur (Tabelle 26, Eintrag 3) noch durch den Wechsel auf ein selektiveres Katalysatorsystem (Tabelle 26, Eintrag 4 und 5) bei gleichzeitigem Erhalt einer zufriedenstellenden Umsatzrate erzielt werden. Während die Erhöhung der Katalysatorbeladung und die damit einhergehende gesteigerte Umsatzrate in einer unselektiveren Hydrierung resultierte (Tabelle 26, Eintrag 6), wurde bei zusätzlicher Erhöhung der Reaktionstemperatur ein unlösliches Polymer erhalten (Tabelle 26, Eintrag 7).

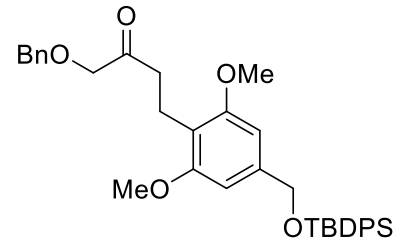

209

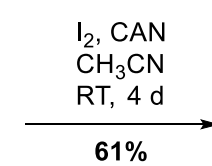

$61 \%$

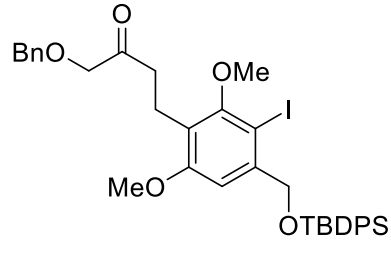

211
$\mathrm{EtPPh}_{3}{ }^{+} \mathrm{Br}^{-}, \mathrm{KHMDS}$

THF, $-78{ }^{\circ} \mathrm{C}, 20 \mathrm{~min}$ $72 \%$

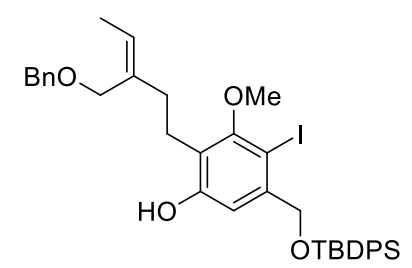

(Z)-213
NaSEt, 15-Krone-5

DMF, mw, $120^{\circ} \mathrm{C}, 12 \mathrm{~h}$

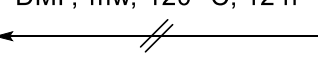

Abbildung 71: Untersuchung einer möglichen selektiven mono-Demethylierung am Aryliodid (Z)-212.

Des Weiteren wurde am Substrat 209 die Durchführbarkeit einer selektiven Entschützung nach erfolgter Halogenierung getestet. Nach lodierung mittels $I_{2}$ und CAN in $61 \%$ Ausbeute wurde das Keton 211 in der darauffolgenden Wittig-Reaktion in einer Ausbeute von $72 \%$ zum Z-Alken (Z)-212 umgesetzt. Die Wittig-Reaktion erforderte indes eine 
Modifizierung der Reaktionsbedingungen, um eine Zersetzung des iodierten Substrats 211, die allem Anschein nach als Folge einer reduktiven Dehalogenierung durch die Hydrid-basierte Base $\mathrm{NaH}$ eintrat, zu verhindern. Somit genügte bereits der Wechsel auf KHMDS als Base für einen erfolgreichen Ablauf der Reaktion. Die anschließende monoDemethylierung des Aryliodids (Z)-212 mithilfe von NaSEt konnte zwar beobachtet werden, ging jedoch mit einer gleichzeitig ablaufenden reduktiven Dehalogenierung einher (Abbildung 71).

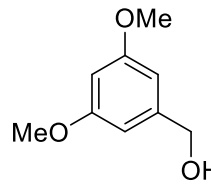

155
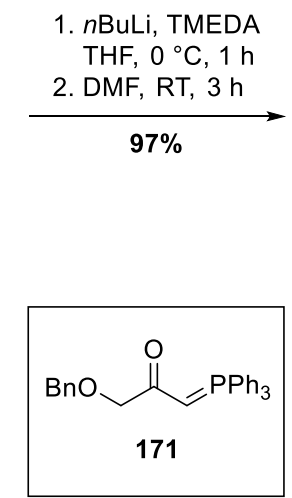<smiles>COc1cc(CO)cc(OC)c1C=O</smiles>

158

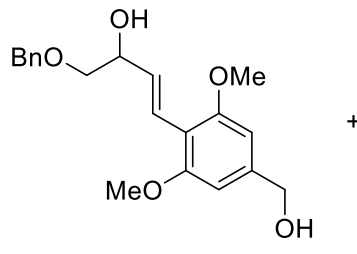

216<smiles>COc1cc(CO)cc(OC)c1CCC(=O)COc1ccccc1</smiles>

215

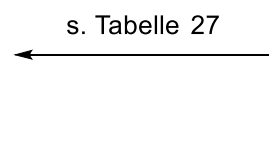

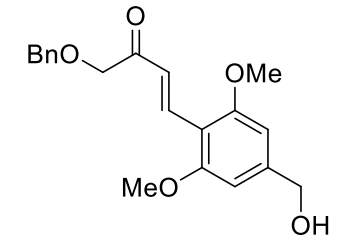

$(E)-\mathbf{2 1 4}$

Tabelle 27: Untersuchungen zur Reduktion der Doppelbindung am Benzylalkohol $(E)-214$.

\begin{tabular}{|c|c|c|}
\hline Eintrag & Reaktionsbedingungen & Ausbeute \\
\hline 1 & {$\left[\mathrm{Ph}_{3} \mathrm{PCuH}\right]_{6}(1 \mathrm{~mol} \%), \mathrm{PMHS}$ (3.00 Äq.), PhMe, $0^{\circ} \mathrm{C}, 1 \mathrm{~d}$} & - \\
\hline 2 & {$\left[\mathrm{Ph}_{3} \mathrm{PCuH}\right]_{6}(1 \mathrm{~mol} \%), \mathrm{PMHS}$ (3.00 Äq.), THF, $0^{\circ} \mathrm{C}, 1 \mathrm{~d}$} & $216 \uparrow$ \\
\hline 3 & {$\left[\mathrm{Ph}_{3} \mathrm{PCuH}\right]_{6}(1 \mathrm{~mol} \%), \mathrm{PMHS}$ (3.00 Äq.), THF, $66^{\circ} \mathrm{C}, 1 \mathrm{~d}$} & 215:216 2:1 \\
\hline 4 & {$\left[\mathrm{Ph}_{3} \mathrm{PCuH}\right]_{6}$ (0.50 Äq.), PMHS (2.50 Äq.), THF, RT, 2 d } & Polymerisation \\
\hline
\end{tabular}

Parallel zu den Hydrierungsuntersuchungen am geschützten Benzylalkohol (E)-208 (Tabelle 26) wurden in diesem Zusammenhang weitere Tests am ungeschützten Analogon (E)-214 durchgeführt. Für dessen Synthese fand ausgehend vom Benzylalkohol 155 zunächst eine ortho-Formylierung nach einer aus der Arbeitsgruppe Bräse entliehenen Vorschrift statt, ${ }^{[153]}$ die sich nur geringfügig von den zuvor getesteten Methoden unterschied, allerdings Maßstab-unabhängige reproduzierbare Ergebnisse lieferte, sofern die Qualität des nBuLi gewährleistet werden konnte. Die Formylierung gelang hiermit in einer Ausbeute von 97\% und der erhaltene Aldehyd 158 wurde im 
Anschluss mit dem P-Ylid 171 zum Alken $(E)$-214 umgesetzt. Die Wittig-Reaktion lieferte das $\alpha, \beta$-ungesättigte Keton $(E)$-214 in Reinform allerdings nur mit einer Ausbeute von $21 \%$ sowie weitere $30 \%$ in Form einer mit Triphenylphosphanoxid verunreinigten Fraktion. In der nachfolgenden Hydrierung wurde nach dem löslichkeitsbedingten Wechsel des Solvents eine erhöhte Ausbildung des durch Reduktion der Carbonylfunktion erhaltenen korrespondierenden Alkohols 216 verzeichnet (Tabelle 27, Eintrag 2 und 3). In Analogie zum geschützten Benzylalkohol $(E)$-208 trat auch in diesem Fall bei einer Erhöhung der Katalysatorbeladung eine Polymerisation zu einem unlöslichen glasigen Feststoff auf (Tabelle 27, Eintrag 4).

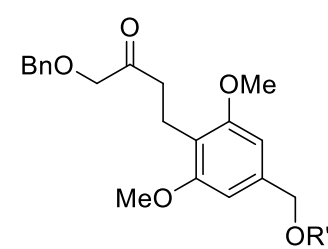

217

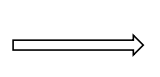

$\mathrm{R}^{\prime}$

$\mathrm{R}=\mathrm{Br}, \mathrm{I}, \mathrm{Bpin}$

$R^{\prime}=H, P G$

Abbildung 72: Retrosynthetische Analyse des Ketons 217.

Im Hinblick auf die weiterhin bestehenden Probleme (moderate Ausbeuten, Reduktion der Carbonylgruppe) mit der die Reduktion der Doppelbindung einherging, wurde eine weitere Modifikation der Synthesestrategie in diesem Punkt angedacht. Eine Suzuki-MiyauraKreuzkupplung der Fragmente vom Typ 218 und 219 würde zum einen die Reduktion der Doppelbindung obsolet machen und zum anderen ein höheres $\mathrm{Maß}$ an Flexibilität in Bezug auf eine Schützung der benzylischen Hydroxyfunktion gewähren. Des Weiteren wäre diese Strategie mit einer Einsparung von Syntheseschritten verbunden (Abbildung 72).

Die Synthese des aromatischen Bausteins 219 erfolgte ausgehend vom kommerziell erhältlichen Arylbromid 220. Methylierung mit $\mathrm{Me}_{2} \mathrm{SO}_{4}$ unter basischer Reaktionsführung erzeugte den Methylester 221 in einer Ausbeute von 89\%. Dieser wurde wiederum mittels DIBAL-H in 84\% Ausbeute zum Benzylalkohol 222 reduziert. Somit konnte innerhalb zweier Reaktionsschritte mit jeweils guten Ausbeuten ein notfalls flexibel schützbares Substrat für die Kreuzkupplung synthetisiert werden. 


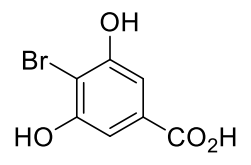

220

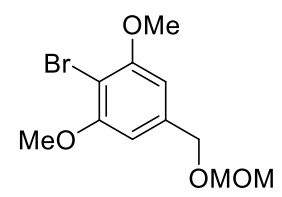

223

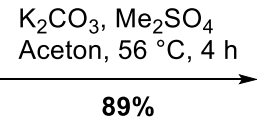

MOMCl, iPrNEt 2

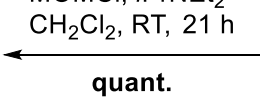

quant.<smiles>COc1cc(OC)c(Br)c(OC)c1</smiles>

221
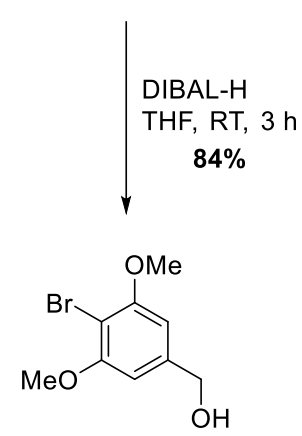

222
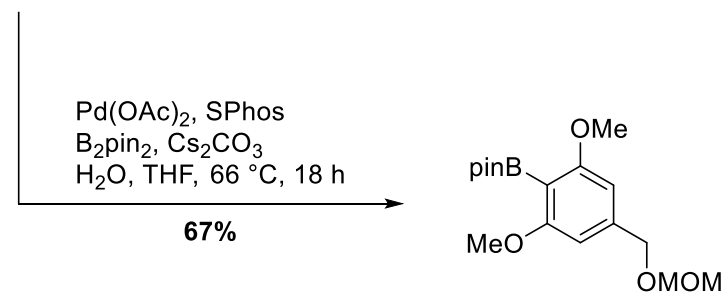

224

Abbildung 73: Synthese des Arylbromids 222 sowie des Boronsäureesters 224.

Zusätzlich wurde ein Teil des Arylbromids 222 zur weiteren Steigerung der Flexibilität zum Arylpinakolboronsäureester 224 umgesetzt. Hierzu wurde der Benzylalkohol 222 zunächst basenvermittelt via $\mathrm{MOMCl}$ quantitativ in den entsprechenden Ether 223 überführt und anschließend unter den optimierten Bedingungen der Miyaura-Borylierung in 67\% Ausbeute boryliert (Abbildung 73).

Die Synthese des Alkylfragments 218 erfolgte über eine Umsetzung des kommerziell erhältlichen Säurechlorids $\mathbf{2 2 5}$ zum Weinrebamid 226 unter Einsatz von N,O-Dimethylhydroxylamin-Hydrochlorid im basischen Milieu in einer Ausbeute von 98\%. Die nachfolgende Grignard-Reaktion mit Vinylmagnesiumbromid konnte trotz verschiedener getesteter Variationen einer literaturbekannten Methode in maximal 14\% Ausbeute reproduziert werden. ${ }^{[154]}$ Ein effizienter Zugang zum $\alpha, \beta$-ungesättigten Keton 227 durch ebenjene Grignard-Reaktion aus dem Methylester 170 scheiterte ebenfalls (Abbildung 74). 


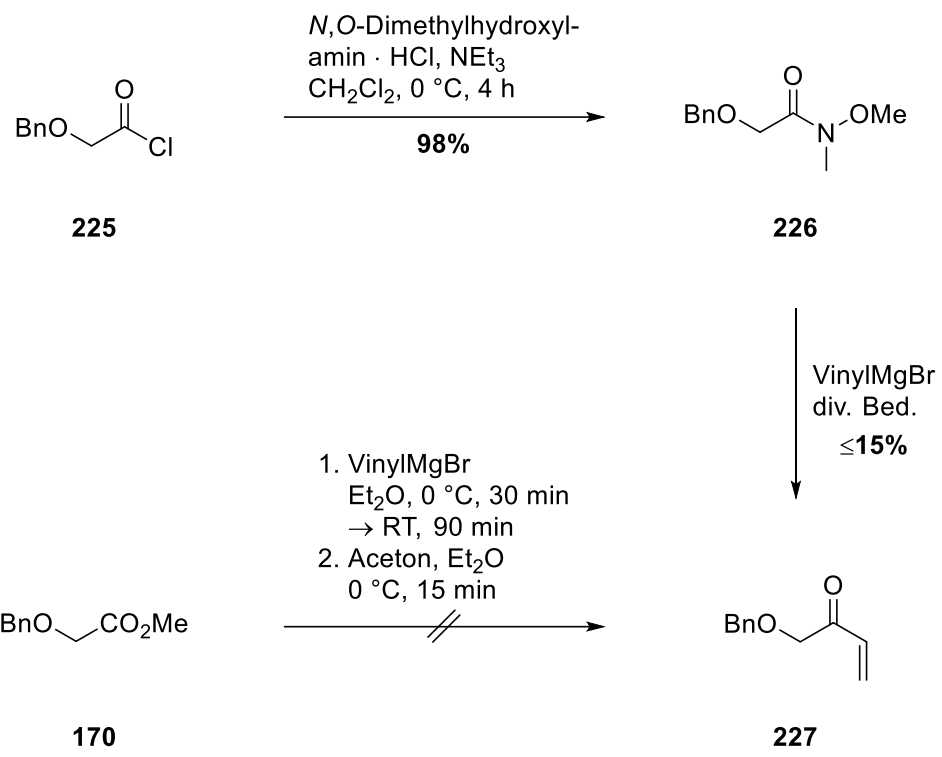

Abbildung 74: Darstellung des $\alpha, \beta$-ungesättigten Ketons 227.

Als problematisch in diesem Zusammenhang stellte sich die Neigung des $\alpha, \beta$-ungesättigten Ketons 227 zur Polymerisation heraus. Nichtsdestotrotz konnte eine regioselektive Borylierung zum Pinakolboronsäureester 228 mit dem Katalysator 229 in $21 \%$ Ausbeute durchgeführt werden (Abbildung 75). ${ }^{[155]}$

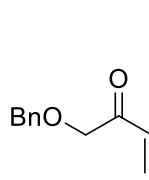

227

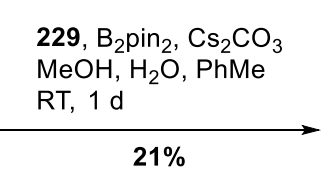

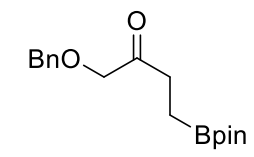

228

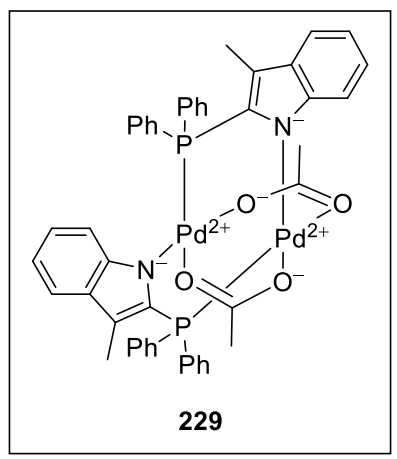

Abbildung 75: Borylierung des $\alpha, \beta$-ungesättigten Ketons 227.

Aufgrund der Labilität des $\alpha, \beta$-ungesättigten Ketons 227 entpuppte sich dieser Synthesepfad zum Aufbau des Alkylfragments 218 allerdings als nicht zielführend. Daher wurde ein Aufbau des Alkylfragments 218 über eine Oxygenierung des Alkens 230 mittels $m C P B A$ zum Epoxid 231 in quantitativer Weise mit darauffolgender Lewis-Säurekatalysierter regioselektiver Öffnung des Oxiran-Rings durch $\mathrm{BnOH}$ verfolgt. Die Ringöffnung erfolgte jedoch nicht mit der erhofften Selektivität, wodurch der sekundäre Alkohol 232 nur mit einer Ausbeute von 18\% erhalten wurde (Abbildung 76). 


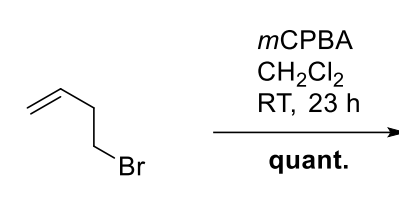

230

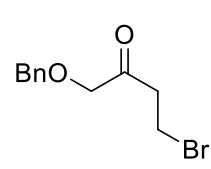

233

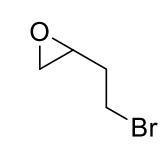

231

$\mathrm{BnOH}, \mathrm{BF}_{3} \cdot \mathrm{OEt}_{2}$

$\mathrm{CH}_{2} \mathrm{Cl}_{2}, \mathrm{RT}, 17 \mathrm{~h}$

$18 \%$

Abbildung 76: Synthese der Alkylbromide 232 und 233.

Der Alkohol 232 wiederum konnte via DMP zum Keton 233 in 70\% Ausbeute oxidiert werden und sowohl der Alkohol 232 als auch das Keton 233 sollten daraufhin Cu'katalysiert zu den entsprechenden Pinakolboronsäureestern 234 und 235 umgesetzt werden.

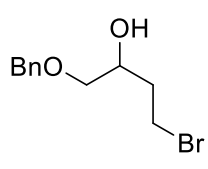

232

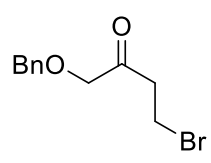

233

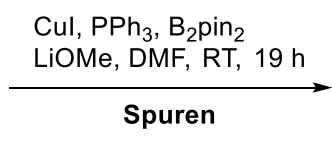

Cul, $\mathrm{PPh}_{3}, \mathrm{~B}_{2} \mathrm{pin}_{2}$

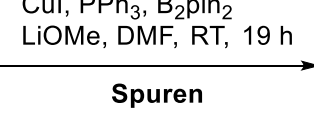

Spuren

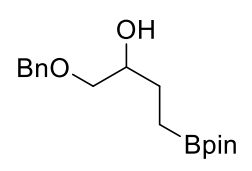

234

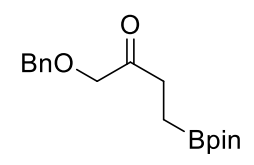

235

Abbildung 77: Umsetzung der Alkylbromide 232 und 233 zu den korrespondierenden Boronsäureestern 234 und 235

Die beiden Bororganyle 234 und 235 konnten jedoch nicht in signifikanten Mengen isoliert, sondern nur massenspektrometrisch nachgewiesen werden (Abbildung 77). Ebenso scheiterte der Versuch der Borylierung des Alkohols 232 über einen intermediären Lithium-Halogen-Austausch (Abbildung 78). 


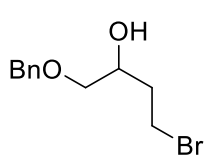

232

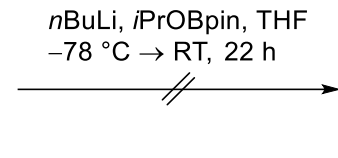

nBuLi, IPrOBpin, THF

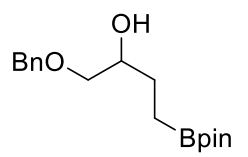

234

Abbildung 78: Versuch der Borylierung des Alkylbromids 232.

Infolge des flexiblen Zugangs zu den beiden Fragmenten wurde ein Versuch der Kupplung des Alkylbromids $\mathbf{2 3 2}$ mit dem Arylpinakolboronsäureester 224 unternommen. Bei Anwendung der optimierten Reaktionsparameter der Suzuki-Miyaura-Kreuzkupplung wurde jedoch keine Produktbildung beobachtet (Abbildung 79).<smiles>OC(CBr)CCBr</smiles>

232<smiles>COCc1cc(OC)c([18OH])c(OC)c1</smiles>

224

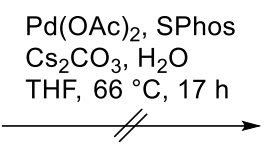<smiles>COCc1cc(OC)c(CCC(O)COc2ccccc2)c(OC)c1</smiles>

236

Abbildung 79: Suzuki-Miyaura-Kreuzkupplung der beiden Fragmente 224 und 232.

Des Weiteren wurde die Hydroborierung eines entsprechend geschützten Vinylalkohols untersucht.

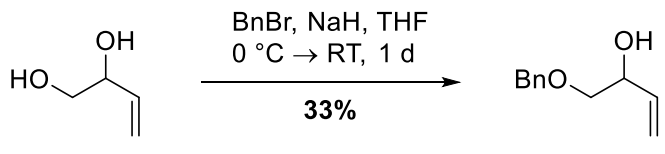

237

238

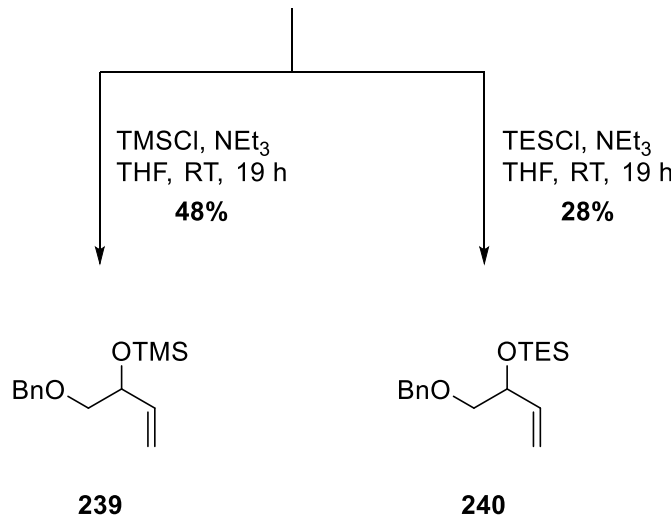

Abbildung 80: Synthese der Alkene 239 und 240.

Die mono-Benzylierung der primären Hydroxyfunktion des kommerziell erhältlichen Alkendiol 237 zum sekundären Alkohol 238 verlief erwartungsgemäß mit 33\% Ausbeute und nur geringer Selektivität. Daraufhin erfolgte eine Silylierung der verbliebenen freien 
Hydroxygruppe mittels TMSCI und TESCI zu den entsprechenden Silylethern 239 und 240 in $48 \%$ respektive $28 \%$ Ausbeute (Abbildung 80 ).

Sowohl die TMS- als auch die TES-Schutzgruppe sollten hierbei nur temporär im Substrat verbleiben und in Folge der basischen Bedingungen in der Reaktionsführung der SuzukiMiyaura-Kreuzkupplung nach erfolgter in situ Hydroborierung direkt wieder entfernt werden.

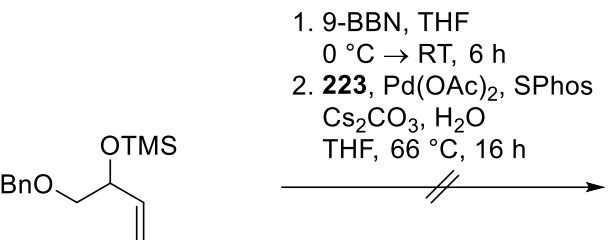

239

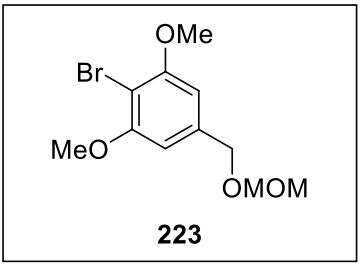

9-BBN, THF

$0{ }^{\circ} \mathrm{C} \rightarrow \mathrm{RT}, 6 \mathrm{~h}$

$\mathrm{Cs}_{2} \mathrm{CO}_{3}, \mathrm{H}_{2} \mathrm{O}$

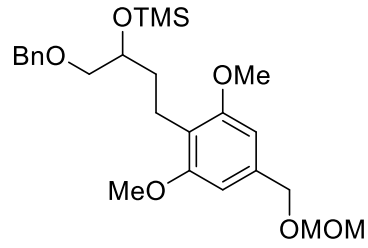

241

1. 9-BBN, THF

$0^{\circ} \mathrm{C} \rightarrow \mathrm{RT}, 6 \mathrm{~h}$

2. 223, $\mathrm{Pd}(\mathrm{OAC})_{2}$, SPhos

$\mathrm{Cs}_{2} \mathrm{CO}_{3}, \mathrm{H}_{2} \mathrm{O}$

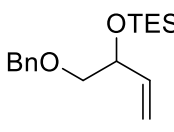

$\mathrm{THF}, 66^{\circ} \mathrm{C}, 16 \mathrm{~h}$

240

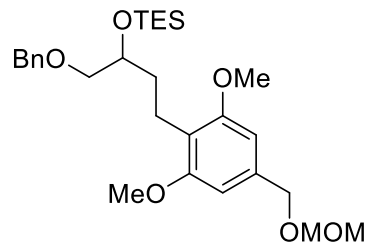

242

Abbildung 81: In situ Hydroborierung und darauffolgende Suzuki-Miyaura-Kreuzkupplung der Alkene 239 und 240.

Es konnte allerdings mit keinem der beiden Substrate die in situ Hydroborierung mittels 9-BBN und darauffolgender Suzuki-Miyaura-Kreuzkupplung unter den bisherigen Standardbedingungen erfolgreich gestaltet werden (Abbildung 81). Konzeptionell würde die Palladium-katalysierte Kupplung eines Alkyl- und eines Arylfragments Vorteile in Bezug auf eine erhöhte Flexibilität der Schutzgruppenstrategie vor allem der benzylischen Hydroxygruppe sowie eine verkürzte und elegantere Syntheseroute bieten. Zur Realisierung hätten indes noch grundlegende Probleme gelöst werden müssen, die nicht mehr im zeitlichen Rahmen dieser Arbeit verfolgt werden konnten.

\subsubsection{Synthese des Methylesters syn-266}

Nachdem eine Verbesserung in der Darstellung des Ketons 217 in den beschriebenen Untersuchungen nicht erreicht werden konnte, wurde im Folgenden auf Arbeiten von 
Judith Hierold im Rahmen ihrer Untersuchungen zur Synthese von Parnafungin-Derivaten zurückgegriffen. ${ }^{[156]}$ Durch die Verwendung der MOM-Schutzgruppe zur Schützung der benzylischen Hydroxygruppe sollten sowohl die Hydrierung der Doppelbindung des analogen $\alpha, \beta$-ungesättigten Ketons als auch die selektive Entschützung einer der beiden Methoxygruppen zum Erhalt des Vorläufersubstrats der Wacker-Zyklisierung zufriedenstellend bewerkstelligt werden können.

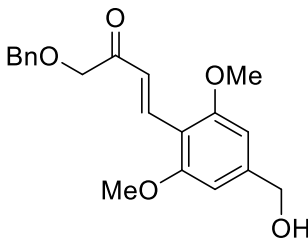

$(E)-\mathbf{2 1 4}$

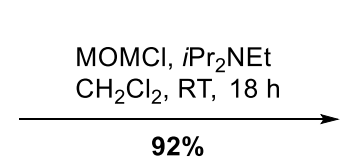

$92 \%$

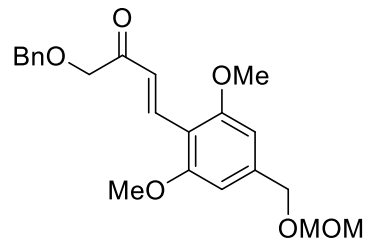

$(E)-\mathbf{2 4 3}$

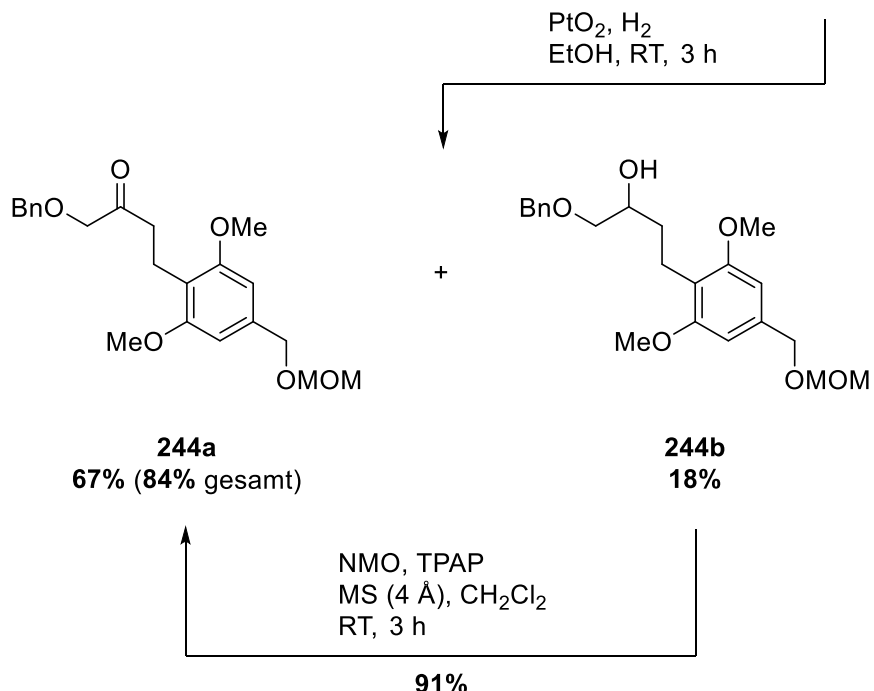

Abbildung 82: Darstellung des Ketons 244a.

Für die angedachte Synthese diente das Keton $(E)$-214 als Ausgangssubstrat, das aus einer Wittig-Reaktion des Aldehyds 158 mit dem P-Ylid 171 resultierte (siehe S. 95, Tabelle 27). Die darauffolgende baseninduzierte Schützung der freien benzylischen Hydroxygruppe mittels $\mathrm{MOMCl}$ lieferte den MOM-Ether $(E)-243$ in einer Ausbeute von 92\%. Hierbei war auch der Einsatz der mit Triphenylphosphanoxid verunreinigten Fraktion des Benzylalkohols $(E)$-214 bedenkenlos möglich, weshalb auf eine allzu aufwendige und zeitintensive Abtrennung dieser Verunreinigung verzichtet werden kann. In der anschließenden Reduktion der Doppelbindung wurde erneut auf $\mathrm{PtO}_{2}$ und $\mathrm{H}_{2}$ zurückgegriffen und das $\alpha, \beta$-ungesättigte Keton $(E)$-243 mit einer Ausbeute von $67 \%$ in das Keton 244a überführt. Zwar verlief die Umsetzung des MOM-geschützten Substrats (E)-243 unter diesen Bedingungen verhältnismäßig unproblematisch, jedoch sollte zur Vermeidung einer verstärkten Bildung verschiedener Nebenprodukte die Reaktion 
dünnschichtchromatographisch kontrolliert werden. Zur einfachen Bestimmung des Reaktionsendes konnte zudem die nahezu vollständige Entfärbung der zuvor schwarzen Suspension herangezogen werden. Neben dem Keton 244a wurden zusätzlich 18\% des überreduzierten Alkohols 244b erhalten, der separat mittels katalytischer Mengen TPAP und NMO in 91\% Ausbeute zum Keton 244a aufoxidiert werden konnte. Auf diese Weise ließ sich die Gesamtausbeute am Keton 244a auf 84\% steigern (Abbildung 82).

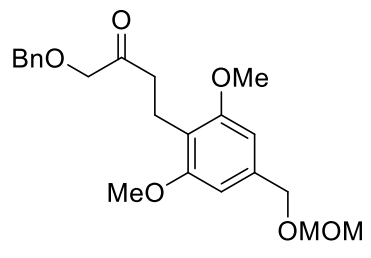

244a

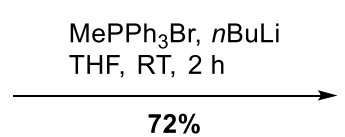

$72 \%$

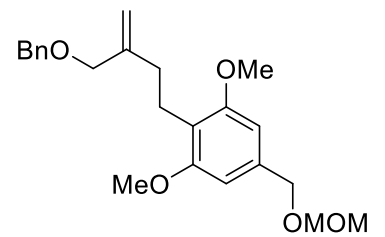

245

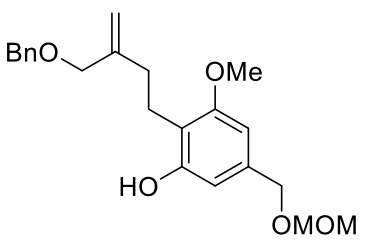

NaSEt, 15-Krone-5

$\mathrm{DMF}, \mathrm{mw}, 120^{\circ} \mathrm{C}, 12 \mathrm{~h}$

$83 \%$

Abbildung 83: Synthese des Domino-Vorläufers 246.

Eine nachfolgende Wittig-Reaktion mit $\mathrm{MePPh}_{3} \mathrm{Br}$ sowie $n \mathrm{BuLi}$ zur Erzeugung des aktiven Li-Ylids lieferte das Olefin 245 in 72\% Ausbeute, das in der Folge dank der Stabilität der

Tabelle 28: Untersuchungen zur enantioselektiven Domino-Wacker/ Carbonylierung/Methoxylierung.

\begin{tabular}{cccc}
\hline Eintrag & BOXAX & Ausbeute & ee \\
\hline 1 & $(S, S)-\operatorname{Bn}(S, S)-91 \mathrm{a}$ & $56 \%$ & $91.8 \%$ \\
2 & $(S, S)-i \operatorname{Pr}(S, S)-91 \mathrm{~b}$ & $53 \%$ & $99.2 \%$ \\
\hline
\end{tabular}

Reaktionsbedingungen: Pd(TFA)2 (5 mol\%), BOXAX (20 mol\%), p-Benzochinon (4.00 Äq.), $\mathrm{CO}(1 \mathrm{~atm}), \mathrm{MeOH}, 40^{\circ} \mathrm{C}, 2 \mathrm{~d}$.
MOM-Schutzgruppe unter Verwendung von NaSEt und dem Kronenether 15-Krone-5 in der Mikrowelle in einer Ausbeute von $83 \%$ mono-demethyliert werden konnte (Abbildung 83). Das erhaltene Phenol 246 wurde daraufhin in einer enantioselektiven Domino-Wackerl Carbonylierung/Methoxylierung unter Verwendung von $\mathrm{Pd}(\mathrm{TFA})_{2}$ (5 mol\%), BOXAXLigand (S,S)-91a/b (20 mol\%), p-Benzochinon (4.00 Äq.), CO (1 atm) in $\mathrm{MeOH}$ bei $40{ }^{\circ} \mathrm{C}$ und einer Reaktionszeit von $2 \mathrm{~d}$ umgesetzt. Beide BOXAX-Liganden lieferten mit 56 bzw. $53 \%$ nahezu identische Ausbeuten, allerdings konnte mit dem (S,S)-IPr-BOXAX-Liganden $(S, S)-91 b$ mit 99.2\% ee eine erheblich höhere Enantioselektivität erhalten werden (Tabelle 28, Eintrag 2). Nachfolgend ist der postulierte mechanistische Verlauf der 
Domino-Sequenz für die Umsetzung zum chiralen Chroman (S)-250 illustriert (Abbildung 84).

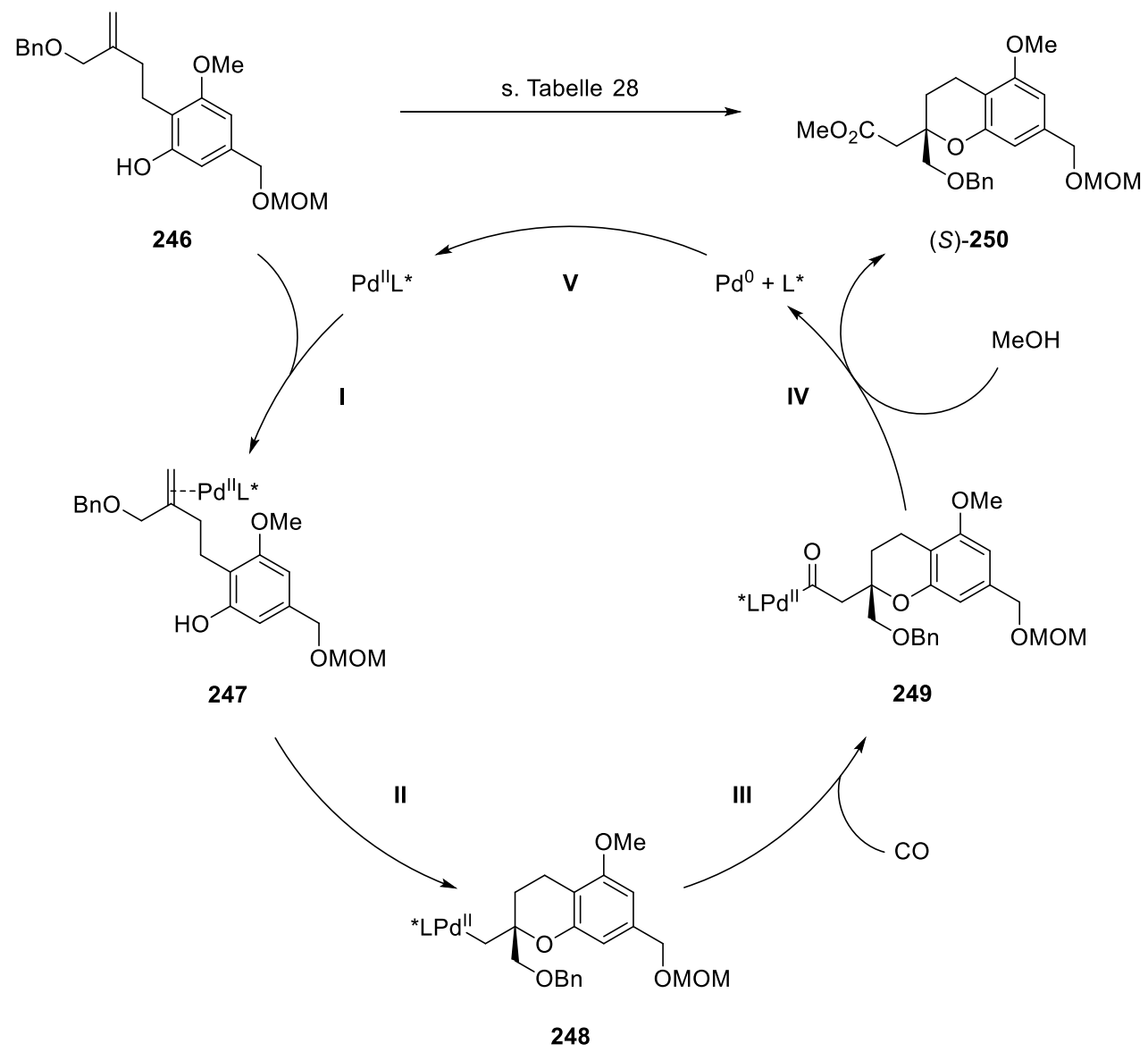

Abbildung 84: Postulierter Mechanismus der enantioselektiven Domino-Wacker/Carbonylierung/Methoxylierung.

Ausgehend vom Alkenylphenol 246 findet zunächst eine enantiofaciale Koordination des chiralen Pd"-Ligand-Komplexes, bestehend aus $\mathrm{Pd}(\mathrm{TFA})_{2}$ und dem chiralen $(S, S)$-Pr-BOXAX-Liganden (S,S)-91b statt (I). Anschließend erfolgt der intramolekulare Angriff der phenolischen Hydroxygruppe auf die m-Olefin-Pd"-Spezies 247 (II). Aufgrund der Chlorid-freien Reaktionsbedingungen sollte dieser Angriff nach Hayashi über eine syn-Oxypalladierung verlaufen. Die infolge der Zyklisierung entstandene $\sigma$-Alkyl-Pd"Spezies 248 kann angesichts fehlender $\beta$-ständiger Wasserstoffatome indes keine reduktive Eliminierung durchlaufen und insertiert dadurch in das in die Reaktionslösung eingeführte Kohlenstoffmonoxid (III). Darauffolgende Alkoholyse des Acyl-Pd"Intermediats 249 setzt sowohl das Domino-Produkt (S)-250 als auch den chiralen $(S, S)$-IPr-BOXAX-Liganden (S,S)-91b und $\mathrm{Pd}^{0}$ frei (IV), das abschließend via p-Benzochinon zum aktiven $\mathrm{Pd}^{\prime \prime}$-Ligand-Komplex reoxidiert wird (V). 


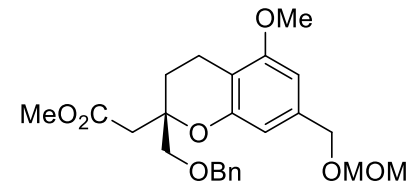

(S)-250

1. $t \mathrm{BuNO}_{2}, \mathrm{BF}_{3} \cdot \mathrm{OEt}_{2}$ $\mathrm{CH}_{2} \mathrm{Cl}_{2},-12{ }^{\circ} \mathrm{C}$

$\rightarrow 0{ }^{\circ} \mathrm{C}, 75 \mathrm{~min}$

2. $\mathrm{KSeCN}, \mathrm{H}_{2} \mathrm{O}$

RT, 30 min<smiles>Nc1ccccc1[N+](=O)[O-]</smiles>

o-Nitroanilin (253)

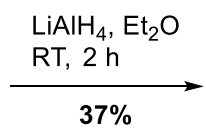

(S)-251<smiles>COCc1cc(OC)c2c(c1)OC(CO)(CCO)CC2</smiles>

254, $\mathrm{PnBu}_{3}$ THF, $0{ }^{\circ} \mathrm{C}, 4 \mathrm{~h}$

2. $m \mathrm{CPBA}, i \mathrm{Pr}_{2} \mathrm{NH}$ $\mathrm{Na}_{2} \mathrm{HPO}_{4} \cdot 2 \mathrm{H}_{2} \mathrm{O}$ $\mathrm{CH}_{2} \mathrm{Cl}_{2}, \mathrm{RT}, 15 \mathrm{~h}$ $66 \%$<smiles>C=C[C@]1(COCc2ccccc2)CCc2c(OC)cc(COC)cc2O1</smiles>

(S)-252

Abbildung 85: Überführung des Methylesters (S)-250 in das Vinylchroman (S)-252.

Zur Überführung des erhaltenen Methylesters (S)-250 in das Vinylchroman (S)-252 wurde dieser zunächst mittels $\mathrm{LiAlH}_{4}$ zum korrespondierenden Alkohol (S)-251 in 37\% Ausbeute reduziert. Abschließende Elminierung der Hydroxyfunktion nach Grieco unter Verwendung des Selenocyanats 254, $\mathrm{PnBu}_{3}$ und mCPBA führte zum Vinylchroman (S)-252 in 66\% Ausbeute. ${ }^{[157]}$ Das hierfür benötigte Selenocyanat 254 konnte in einer zweistufigen Synthesesequenz aus dem kommerziell erhältlichen o-Nitroanilin (253) mit einer Ausbeute von $69 \%$ hergestellt werden (Abbildung 85 ). ${ }^{[158]}$<smiles>COCc1cc(OC)c(CCC(=O)COc2ccccc2)c(OC)c1</smiles>

244a

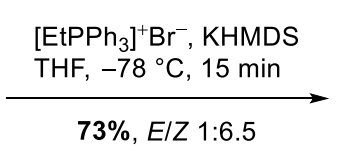

$73 \%, E / Z 1: 6.5$

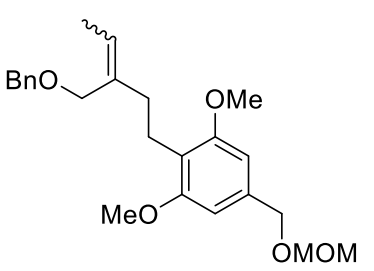

(E/Z)-255<smiles>C/C=C(/CCc1c(O)cc(COC)cc1OC)COc1ccccc1</smiles>

NaSEt, 15-Krone-5

DMF, mw, $120^{\circ} \mathrm{C}, 12 \mathrm{~h}$

$88 \%$

(E/Z)-256

Abbildung 86: Darstellung des Wacker-Vorläufers (E/Z)-256. 
Des Weiteren sollte ebenfalls die enantioselektive Wacker-Zyklisierung zum direkten Erhalt des chiralen Vinylchromans (S)-252 auf das entsprechende MOM-geschützte Derivat angewendet werden. Hierzu wurde zunächst das Keton 244a unter den optimierten Bedingungen der Wittig-Reaktion mit einer Ausbeute von 73\% zum Alken (E/Z)-255 umgesetzt. Im Gegensatz zu vorherigen Umsetzungen dieser Art konnte in diesem Fall keine vollständige Z-Selektivität, jedoch ein weiterhin annehmbares Verhältnis $E / Z$ von 1:6.5 erreicht werden. Daraufhin führten vergleichbare Reaktionsbedingungen, wie sie vorher am terminalen Olefin 245 angewandt worden waren, unter monoDemethylierung zum Phenol (E/Z)-256 in 88\% Ausbeute (Abbildung 86).

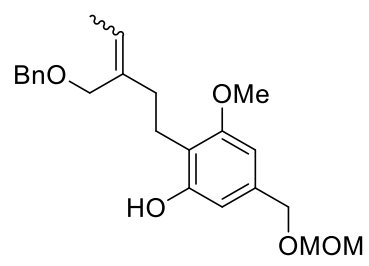

$(E / Z)-256$

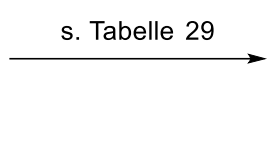

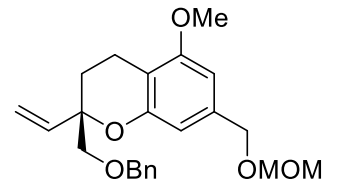

(S)-252

Tabelle 29: Untersuchungen zur enantioselektiven Wacker- In der nachfolgenden Zyklisierung.

Wacker-Zyklisierung kamen wiederum sowohl der

\begin{tabular}{cccc}
\hline Eintrag & BOXAX & Ausbeute & ee \\
\hline 1 & $(S, S)-\operatorname{Bn}(S, S)-91 \mathbf{a}$ & $62 \%$ & $69.7 \%$ \\
2 & $(S, S)-i \operatorname{Pr}(S, S)-91 \mathbf{b}$ & $75 \%$ & $98.8 \%$ \\
\hline
\end{tabular}

Reaktionsbedingungen: Pd(TFA)2 (10 mol\%), BOXAX (20 mol\%), $p$-Benzochinon (4.00 Äq.), $\mathrm{MeOH}, 40^{\circ} \mathrm{C}, 2 \mathrm{~d}$. $(S, S)-\mathrm{Bn}-$ als auch der $(S, S)$-IPr-BOXAX-Ligand $(S, S)-\mathbf{9 1 a / b}$ zum Einsatz. Hierbei wurde jedoch im

Vergleich zur Domino-Sequenz für den $(S, S)$-Bn-BOXAX-Liganden $(S, S)$-91a ein starker Abfall der Enantioselektivität bei einer nur unwesentlich besseren Ausbeute beobachtet (Tabelle 29, Eintrag 1). Hingegen konnte mit dem (S,S)-iPr-BOXAX-Liganden (S,S)-91b wiederum ein exzellenter Enantiomerenüberschuss bei einer siginifikant verbesserten Ausbeute erzielt werden (Tabelle 29, Eintrag 2). In vorangegangenen Arbeiten im Arbeitskreis Tietze konnten mit dem Domino-Ansatz auf analogen Substraten im Regelfall höhere Enantioselektivitäten im Vergleich zur Wacker-Zyklisierung erzielt werden. ${ }^{[93,126-}$ 128,159] Dieses Verhalten spiegelt sich im Vergleich der beiden Reaktionen unter Verwendung des $(S, S)$-Bn-BOXAX-Liganden (S,S)-91a wider. Mit Blick auf die überlegene Stereoinduktion des $(S, S)$-iPr-BOXAX-Liganden $(S, S)-91$ b entfällt dieser Vorteil der Domino-Sequenz, weswegen in diesem Fall die höhere Ausbeute und der kürzere Synthesepfad ausschlaggebend für die direkte Synthese des chiralen Vinylchromans (S)-252 über eine enantioselektive Wacker-Zyklisierung waren. 


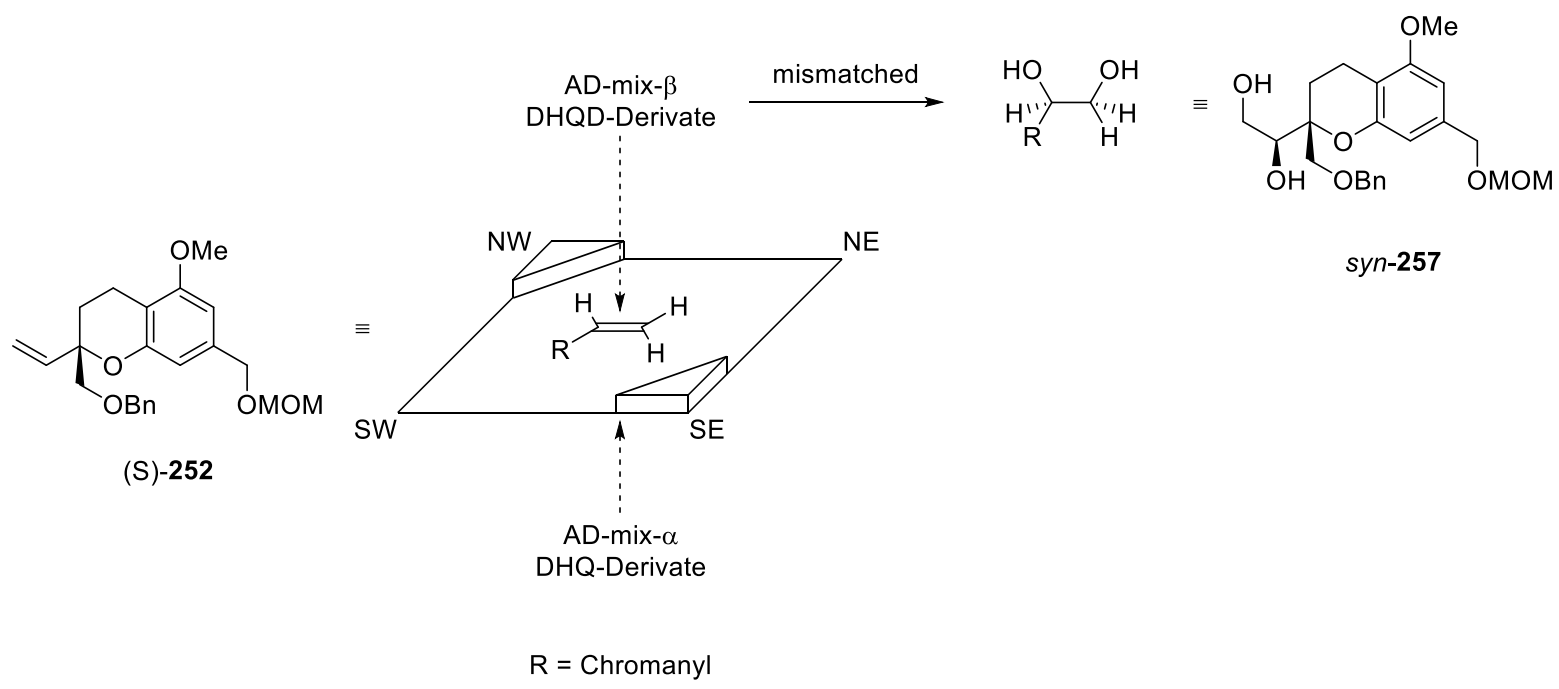

Abbildung 87: Voraussage der Stereoinduktion in der Sharpless-Dihydroxylierung des Vinylchromans (S)-252.

Mit dem Aufbau des ersten der beiden in Parnafungin C (3) lokalisierten Stereozentren im Zuge der enantioselektiven Wacker-Zyklisierung respektive der Domino-Wackerl Carbonylierung/Methoxylierung sollte im nächsten Schritt das zweite Stereozentrum selektiv in das Molekül inkorporiert werden. Für diesen Zweck stellt die SharplessDihydroxylierung das Mittel der Wahl dar und sollte die diastereoselektive Funktionalisierung des Vinylchromans (S)-252 ermöglichen. Die syn-Anordnung der beiden (S)-konfigurierten Stereozentren im Parnafungin C (3) spiegelt allerdings nach Sharpless's mnemonic device den mismachted-Fall der Dihydroxylierung wider (Abbildung 87), sodass zur Maximierung der Diastereoselektivität in der Umsetzung des Vinylchromans (S)-252 zum Diol antilsyn-257 zunächst verschiedene Dihydrochinidinbasierte Liganden getestet wurden.

Für die Sharpless-Dihydroxylierung wurde neben den unterschiedlichen (DHQD)Liganden auf ein System aus $\mathrm{K}_{2} \mathrm{OsO}_{4} \cdot 2 \mathrm{H}_{2} \mathrm{O}$ (5 mol\%), $\mathrm{K}_{3}\left[\mathrm{Fe}(\mathrm{CN})_{6}\right]\left(6.00 \mathrm{Äq}\right.$.), $\mathrm{K}_{2} \mathrm{CO}_{3}$ (6.00 Äq.) und $\mathrm{MeSO}_{2} \mathrm{NH}_{2}$ (1.00 Äq.) in einem $1: 1$ Gemisch aus $t \mathrm{BuOH} / \mathrm{H}_{2} \mathrm{O}$ bei Raumtemperatur gesetzt. Von den untersuchten Liganden wurden mit dem monomeren Liganden (DHQD)CLB (260) sowie den beiden dimeren Liganden (DHQD) ${ }_{2}$ PYR (261) und $(\mathrm{DHQD})_{2} \mathrm{PHAL}$ (123) mit anti/syn-Verhältnissen von 1:1.5 respektive 1:2.3 und 1:2.6 die geringsten Diastereoselektivitäten erzielt. Die erreichten Ausbeuten bewegten sich dabei in einem Bereich von $70-86 \%$ (Tabelle 30, Eintrag 4 bis 6). Etwas besser schnitten der Anthrachinon-verbrückte dimere Ligand (DHQD) ${ }_{2} \mathrm{AQN}$ (262) und der monomere Vertreter (DHQD)MEQ (259) mit einer induzierten Diastereoselektivität von 1:3.6 und 1:3.5 ab, wobei mit letztgenanntem mit $88 \%$ die höchste Ausbeute erreicht werden konnte (Tabelle 30, Eintrag 2 und 3). Die optimale Kombination aus einer Ausbeute von 75\% und einer 
Diastereoselektivität von 1:4.3 konnte mit dem monomeren Phenanthrylether-basierten Liganden (DHQD)PHN (258) erwirkt werden (Tabelle 30; Eintrag 1).

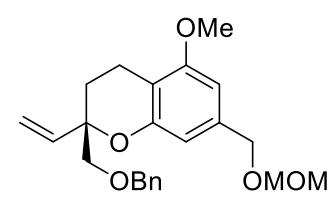

(S)-252 s. Tabelle 30<smiles>Cc1cc(O[Ga])nc2ccccc12</smiles>

(DHQD)MEQ (259)

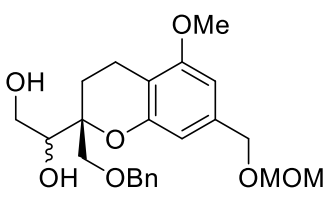

antilsyn-257<smiles>Oc1cc2ccccc2c2ccccc12</smiles>

(DHQD)PHN (258)<smiles>Oc1nnc(O[Na])c2ccccc12</smiles>

(DHQD) $)_{2}$ PHAL (123)<smiles>O=COc1nc(-c2ccccc2)nc(O[O+])c1-c1ccccc1</smiles>

(DHQD) ${ }_{2}$ PYR (261)<smiles>Cc1ccc(C(=O)OOc2ccccc2)cc1</smiles>

(DHQD)CLB (260)<smiles>O=COc1ccc(O)c2c1C(=O)c1ccccc1C2=O</smiles>

$(\mathrm{DHQD})_{2} \mathrm{AQN}(\mathbf{2 6 2})$
Tabelle 30: Untersuchungen zur Sharpless-Dihydroxylierung.

\begin{tabular}{|c|c|c|c|}
\hline Eintrag & Ligand & Ausbeute & $d r_{\text {anti/syn }}$ \\
\hline 1 & (DHQD)PHN & $75 \%$ & $1: 4.3$ \\
\hline 2 & $(\mathrm{DHQD})_{2} \mathrm{AQN}$ & $60 \%$ & $1: 3.6$ \\
\hline 3 & (DHQD)MEQ & $88 \%$ & $1: 3.5$ \\
\hline 4 & (DHQD)CLB & $86 \%$ & $1: 1.5$ \\
\hline 5 & $(\mathrm{DHQD})_{2} \mathrm{PYR}$ & $70 \%$ & $1: 2.3$ \\
\hline 6 & $(\mathrm{DHQD})_{2} \mathrm{PHAL}$ & $76 \%$ & $1: 2.6$ \\
\hline \multicolumn{4}{|c|}{ 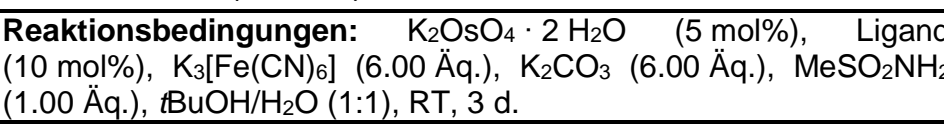 } \\
\hline
\end{tabular}

Zur Einordnung des erzielten Diastereomerenverhältnisses sollte neben der notwendigen Überwindung der diastereofacialen Präferenz des Olefins durch den chiralen Liganden, die im vorliegenden mismatchedFall bewerkstelligt werden muss, auch die hinlänglich bekannten Schwierigkeiten, mit denen eine Sharpless-Dihydroxylierung terminaler Olefine mit allylischem Heteroatom einhergeht, ${ }^{[110,160]}$ Erwähnung finden. Des Weiteren legen Berechnungen von Denmark et al. für die Dihydroxylierung allylischer Olefine ein hohes $\mathrm{Maß}$ an Freiheit bzgl. der Rotation der Vinylgruppe und damit eine Vielzahl verschiedener reaktiver Konformationen, die eine Koordination mit dem Osmium-Ligand-Komplex 
eingehen können, nahe, die in der Folge eine Verminderung der Diastereoselektivität nach sich ziehen.
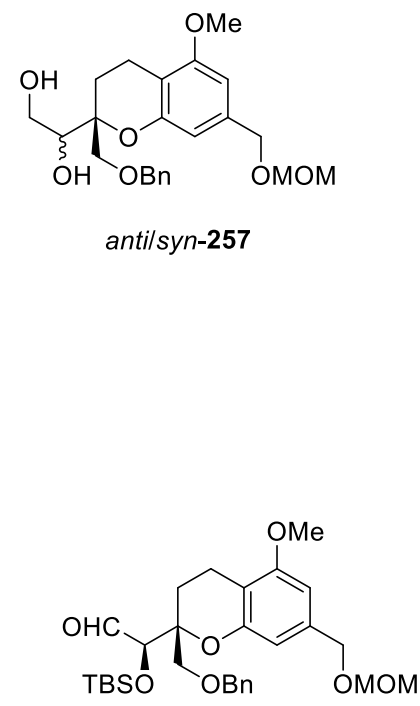

syn-265
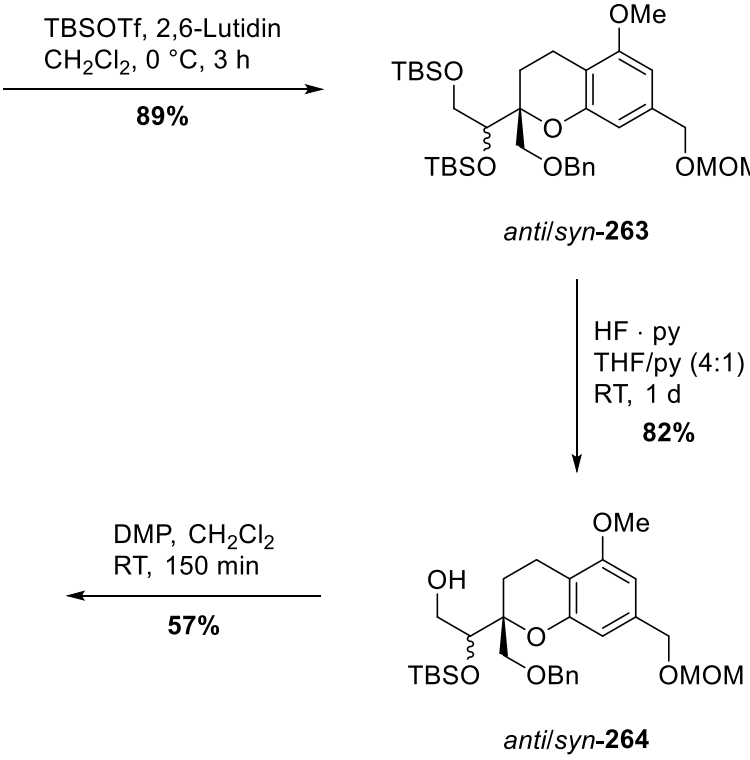
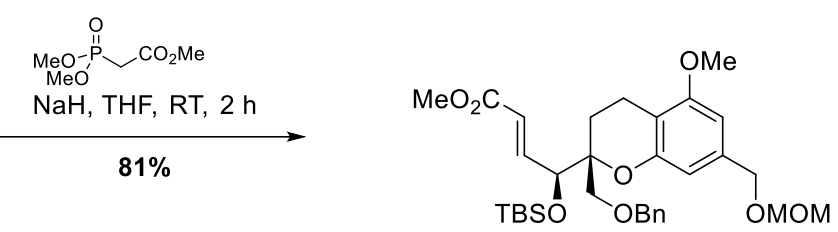

syn-266

Abbildung 88: Darstellung des $\alpha, \beta$-ungesättigten Methylesters syn-266.

Das Diol antilsyn-257 wurde anschließend baseninduziert mittels TBSOTf in 89\% Ausbeute vollständig TBS-geschützt ${ }^{[161]}$ und im darauffolgenden Schritt die primäre Hydroxygruppe des TBS-geschützten Diols antilsyn-263 mithilfe von HF - py mit einer Ausbeute von $84 \%$ wieder freigesetzt. ${ }^{[162]}$ Der primäre Alkohol anti/syn-264 wiederum wurde unter Verwendung von DMP mit einer Ausbeute von 89\% zum korrespondierenden Aldehyd syn-265 aufoxidiert. Auf dieser Stufe konnte das Diastereomerengemisch via Säulenchromatographie an Kieselgel getrennt und somit eine zeit- und lösungsmittelintensive HPLC-Trennung vermieden werden. Eine Horner-Wadsworth-Emmons-Reaktion mit dem in situ erzeugten $\mathrm{Na}$-Ylid des Trimethylphosphonoacetats überführte den enantiomerenreinen Aldehyd syn-265 in 81\% Ausbeute in den $\alpha, \beta$-ungesättigten Methylester syn-266 (Abbildung 88). 


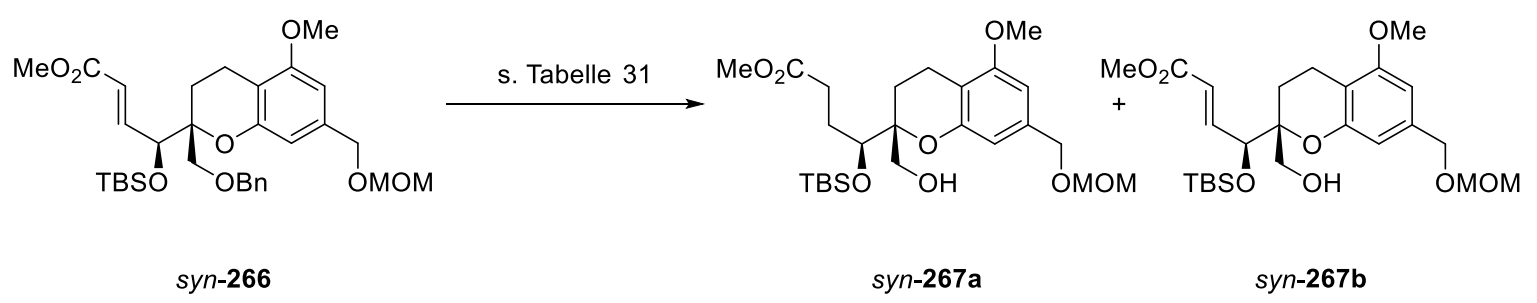

Tabelle 31: Untersuchungen zur Reduktion des $\alpha, \beta$-ungesättigten Methylesters syn-266.

\begin{tabular}{clc}
\hline Eintrag & \multicolumn{1}{c}{ Reaktionsbedingungen } & Ausbeute \\
\hline 1 & {$\left[\mathrm{PPh}_{3} \mathrm{CuH}\right]_{6}(1 \mathrm{~mol} \%), \mathrm{PhSiH}_{3}$ (1.00 Äq.), PhMe, RT, } & $<10 \%$ Umsatz \\
& $21 \mathrm{~h}$ & $53 \%$ \\
2 & $\mathrm{Pd} / \mathrm{C}(10 \mathrm{~mol} \%), \mathrm{H}_{2}$ (3.5 bar), MeOH, RT, 3 d & $(\mathbf{2 6 7 a : 2 6 7 b ~ 1 : 1 )}$ \\
\hline
\end{tabular}

Nachfolgend sollte die Doppelbindung des $\alpha, \beta$-ungesättigten Methylesters syn-266 reduziert werden. Zunächst wurde auf eine katalytische Nutzung von Stryker's Reagenz zurückgegriffen, die an einem analogen Substrat, dem die zur hydrierenden Doppelbindung angrenzende TBS-geschützte Hydroxyfunktionalität fehlte, gute Ausbeuten generierte. Die zusätzliche TBS-Schutzgruppe im Molekül verhinderte aller Voraussicht nach aufgrund ihres sterischen Einflusses jedoch einen zufriedenstellenden Umsatz des Startmaterials, der nach $21 \mathrm{~h}$ dünnschichtchromatographisch kontrolliert bei unter 10\% verweilte (Tabelle 31, Eintrag 1). Eine Umsetzung mit Pd/C und einem Überdruck an $\mathrm{H}_{2}$ führte zum Erhalt eines per Säulenchromatorgraphie nicht zu separierenden 1:1 Gemisches der beiden Benzyl-entschützten Alkohole syn-267a/b in 53\% Ausbeute (Tabelle 31, Eintrag 2). Eine weitere Untersuchung der Reduktion der Doppelbindung des $\alpha, \beta$-ungesättigten Methylesters syn-266 konnte im Rahmen dieser Arbeit bedingt durch einen Mangel an Material nicht mehr erfolgen.

\subsubsection{Diskussion der spektroskopischen Daten des Aldehyds syn-265}

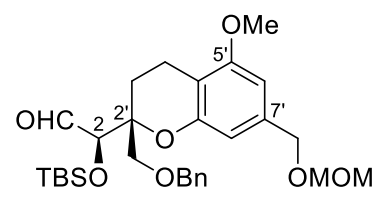

syn-265

Im Folgenden sollen einige ausgewählte analytische Daten des Aldehyds syn-265 diskutiert werden, dessen bei Raumtemperatur in deuteriertem Chloroform aufgenommene ${ }^{1} \mathrm{H}$ - und ${ }^{13} \mathrm{C}-N M R$-Spektren in den Abbildung 89 und Abbildung 90 dargestellt sind. Neben den unterschiedlichen Intensitäten der Signale im ${ }^{13} \mathrm{C}-\mathrm{NMR}$, mit 
denen vor allem quartäre Kohlenstoffatome identifiziert werden konnten, basierte eine genauere Zuordnung der Signale, sofern dies durch eindeutige Korrelationen belegbar war, auf der Auswertung entsprechender zweidimensionaler NMR-Spektren (COSY, HSQC, HMBC, NOESY).
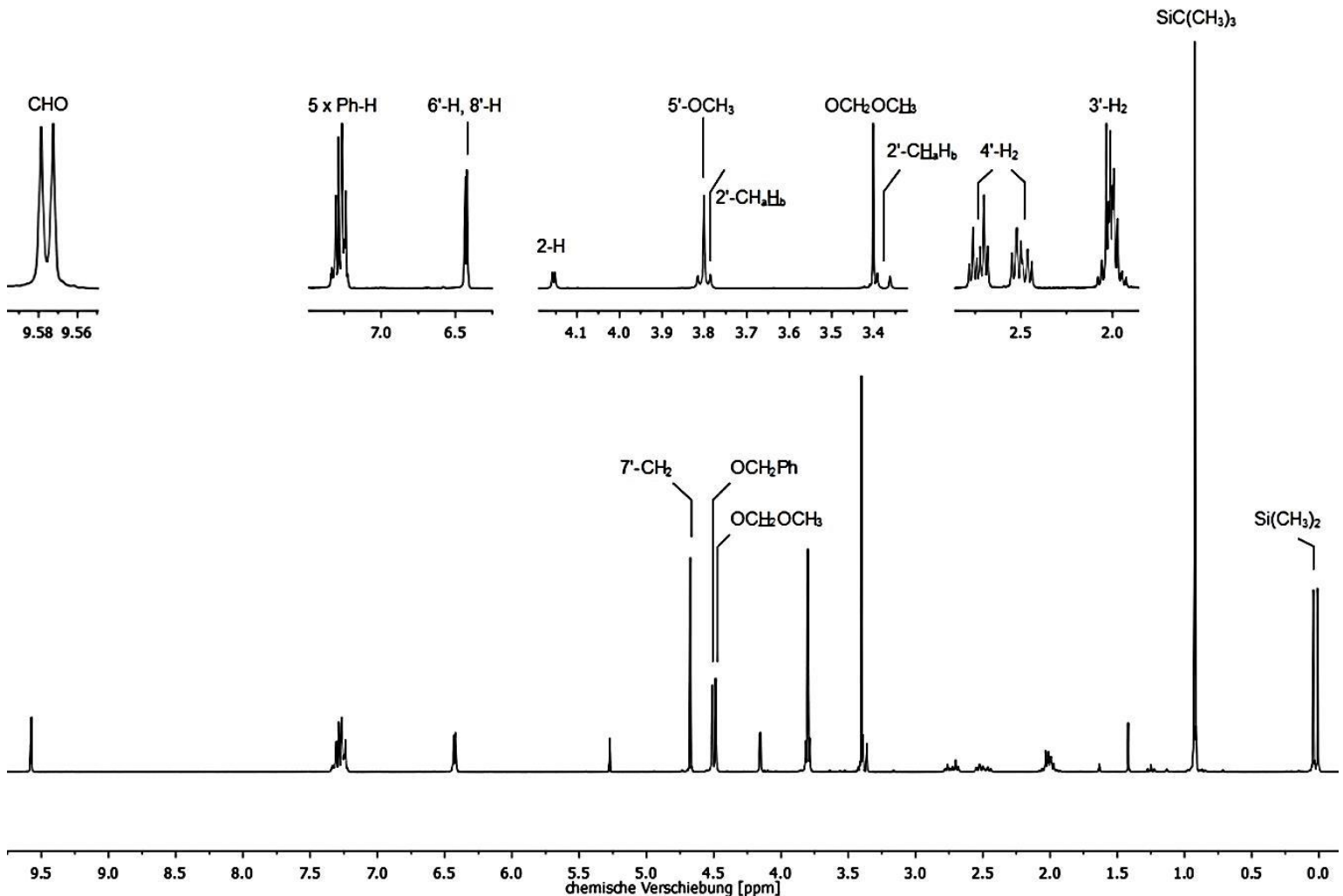

Abbildung 89: ${ }^{1} \mathrm{H}-\mathrm{NMR}$-Spektrum des Aldehyds syn-265.

Am stärksten hochfeldverschoben im ${ }^{1} \mathrm{H}$-NMR-Spektrum zeigen sich die beiden Methylgruppen der TBS-Schutzgruppe, welche jeweils in Form eines Singuletts bei $\delta=$ $0.01 \mathrm{ppm}$ und $\delta=0.04 \mathrm{ppm}$ resonieren. Die neun Wasserstoffatome des tert-Butylfragments dieser Schutzgruppe folgen bei $\delta=0.92 \mathrm{ppm}$ und treten ebenfalls als Singulett in Erscheinung. Eine Auflösung des Kopplungsmusters der beiden Wasserstoffatome an C-3' war indes nicht möglich, sodass deren Signale als Multiplett im Bereich von $\delta=1.96-2.05$ ppm wieder zu finden sind. Ebenso konnte dem Wasserstoffatom 4'- $\mathrm{H}_{\mathrm{a}}$ keine eindeutige Multiplizität zugewiesen werden, es liegt als Multiplett im Bereich von $\delta=$ 2.43-2.56 ppm vor. Leicht tieffeldverschoben bei $\delta=2.73 \mathrm{ppm}$ konnte hingegen das zweite Wasserstoffatom am Kohlenstoffatom C-4' als Dublett von Tripletts identifiziert werden. Neben der geminalen Kopplungskonstante von $J=17.6 \mathrm{~Hz}$ zum Wasserstoffatom 4'- $\mathrm{H}_{\mathrm{a}}$ ist weiterhin auch die vicinale Kopplungskonstante von $J=6.2 \mathrm{~Hz}$ zur benachbarten Methyleneinheit zu finden. Die beiden diastereotopen Wasserstoffatome der Methylengruppe am Kohlenstoffatom C-2' resonieren bei $\delta=3.38 \mathrm{ppm}$ und $\delta=3.80 \mathrm{ppm}$ jeweils 
als Dublett mit einer gemeinsamen geminalen Kopplungskonstante von $J=9.0 \mathrm{~Hz}$. Zwischen diesen beiden Signalen ist außerdem das Methylfragment der MOMSchutzgruppe zu finden, die bei $\delta=3.40$ ppm in Form eines Singuletts liegt. Des Weiteren fällt in das Dublett des Wasserstoffatoms 2 ' $-\mathrm{CH}_{\mathrm{a}} \underline{\mathrm{H}}_{\mathrm{b}}$ bei $\delta=3.80 \mathrm{ppm}$ das Signal der Methoxygruppe am Kohlenstoffatom C-5', das ebenfalls bei dieser chemischen Verschiebung als Singulett wieder zu finden ist. Weiter tieffeldverschoben folgt bei einer Resonanzfrequenz von $\delta=4.15 \mathrm{ppm}$ das Wasserstoffatom $2-\mathrm{H}$, welches zu einem Dublett mit einer Kopplungskonstante von $J=1.8 \mathrm{~Hz}$ aufspaltet. Daran schließen sich die Methyleneinheiten der MOM- sowie der Benzyl-Schutzgruppe und die Methylengruppe am Kohlenstoffatom C-7' mit den chemischen Verschiebungen $\delta=4.49 \mathrm{ppm}, \delta=4.51 \mathrm{ppm}$ und $\delta=4.67 \mathrm{ppm}$ an. Die drei Singuletts sind im Vergleich zu den beiden Methyleneinheiten des Dihydropyran-Rings hierbei aufgrund des elektronenziehenden Sauerstoffatoms weiter Richtung Tieffeld verschoben. Im aromatischen Bereich des Spektrums finden sich zunächst die beiden Wasserstoffatome 6'- $\mathrm{H}$ und $8^{\prime}-\mathrm{H}$ bei $\delta=6.42 \mathrm{ppm}$ und $\delta=$ 6.43 ppm wieder, die jeweils als Dubletts mit einer gemeinsamen Kopplungskonstante von $J=1.4 / 1.5 \mathrm{~Hz}$ resonieren. Die Signale der phenolischen Wasserstoffatome der Benzylschutzgruppe liegen als nicht differenzierbares Multiplett im Bereich von $\delta=7.21-$ 7.35 ppm vor. Am weitesten ins Tieffeld verschoben ist erwartungsgemäß das AldehydWasserstoffatom. Es liegt als Dublett mit einer Kopplungskonstante von $J=1.9 \mathrm{~Hz}$ bei einer chemischen Verschiebung von $\delta=9.58 \mathrm{ppm}$.

Das ${ }^{13} \mathrm{C}$-NMR-Spektrum weist für die Methylgruppen der TBS-Schutzgruppe ein Signal im negativen Bereich bei $\delta=-4.9 \mathrm{ppm}$ auf. Die weiteren Signale der Kohlenstoffatome der TBS-Schutzgruppe lassen sich ebenfalls im Hochfeldbereich des Spektrums, bei $\delta=$ $18.2 \mathrm{ppm}$ für das quartäre Kohlenstoffatom des tert-Butylfragments und bei $\delta=25.7 \mathrm{ppm}$ für die Methylgruppen dieses Fragments finden. Dazwischen befinden sich noch die Methylengruppen des Dihydropyran-Rings bei $\delta=15.7$ ppm für das Kohlenstoffatom C-4' und bei $\delta=23.6$ ppm für C-3'. Es folgen die Methoxygruppe der MOM-Schutzgruppe bei $\delta=55.3 \mathrm{ppm}$ sowie die am Kohlenstoffatom C-5' positionierte Methoxygruppe bei $\delta=$ 55.4 ppm. Weiterhin im aliphatischen Bereich verortet, jedoch infolge der Nachbarschaft zu einem elektronenziehenden Sauerstoffatom weiter tieffeldverschoben, schließen sich die Methyleneinheiten am Kohlenstoffatom C-2' bei $\delta=68.4 \mathrm{ppm}$, an C-7' bei $\delta=$ $69.1 \mathrm{ppm}$ und die Methyleneinheit der Benzyl-Schutzgruppe bei $\delta=73.6 \mathrm{ppm}$ an. Die Signale mit den chemischen Verschiebungen von $\delta=79.4$ ppm und $\delta=79.7$ ppm lassen sich dem Kohlenstoffatom C-2 respektive dem quartären Kohlenstoffatom C-2' zuordnen. Das durch die Nähe zu zwei Sauerstoffatomen am weitesten tieffeldverschobene aliphatische Signal bei $\delta=95.9$ ppm kann dem Methylenkohlenstoffatom der MOMSchutzgruppe zugewiesen werden. 


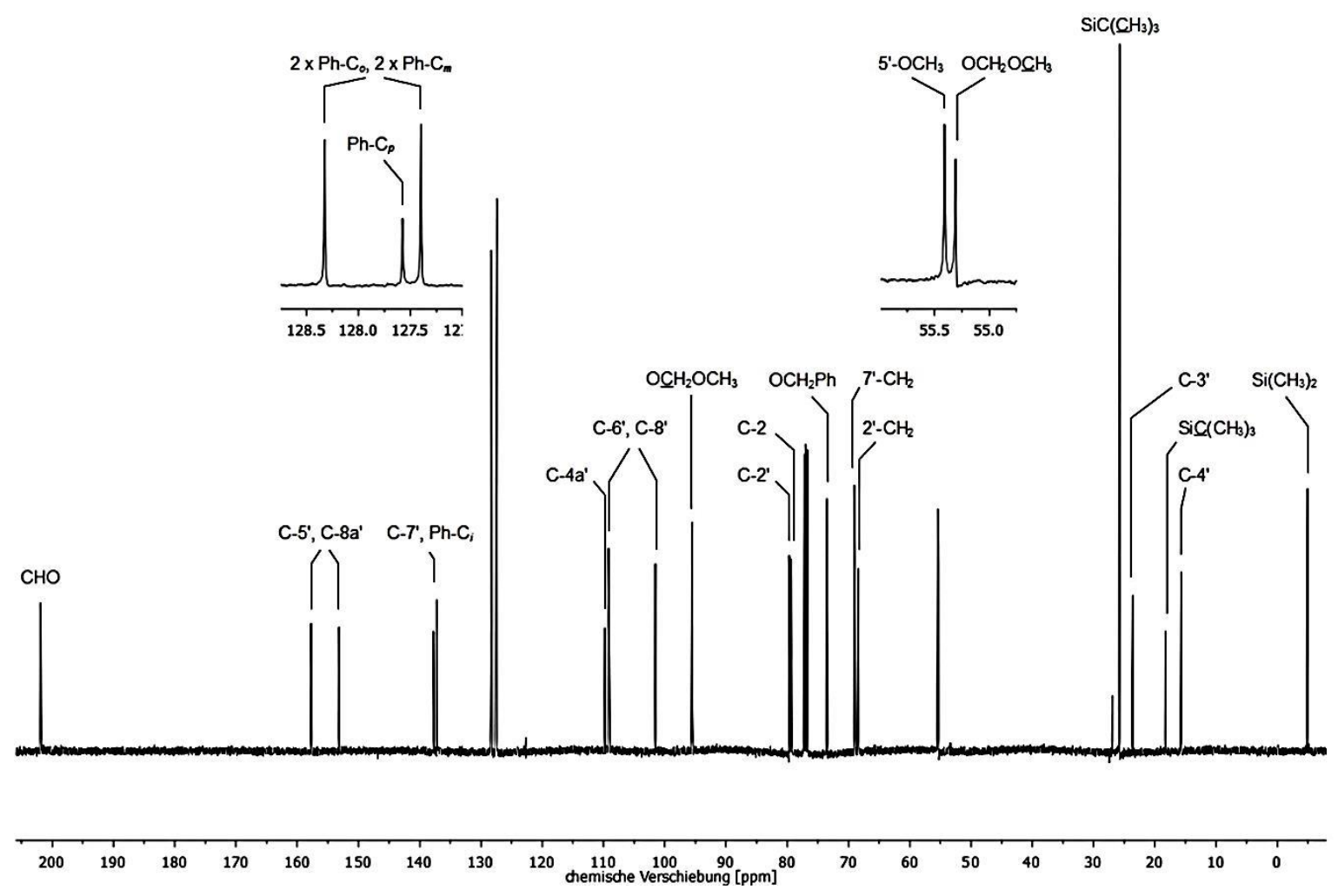

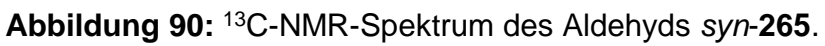

Die Signale mit den chemischen Verschiebungen von $\delta=101.5 \mathrm{ppm}$ und $\delta=109.2 \mathrm{ppm}$ sind den Resonanzen der Kohlenstoffatome C-6' und C-8' zu zuordnen, können aber nicht eindeutig unterschieden werden. Das quartäre Kohlenstoffatom C-4a' ist bei $\delta=$ $109.8 \mathrm{ppm}$ zu finden und durch die weit geringere Intensität vom Signal bei $\delta=109.2 \mathrm{ppm}$ zu differenzieren. Weiter in Richtung Tieffeld verschoben, folgen mit chemischen Verschiebungen von $\delta=127.4 \mathrm{ppm}, \delta=127.6 \mathrm{ppm}$ und $\delta=128.3 \mathrm{ppm}$ die Signale der aromatischen Kohlenstoffatome der Benzyl-Schutzgruppe $\mathrm{Ph}-\mathrm{C}_{o}, \mathrm{Ph}-\mathrm{C}_{m}$ und $\mathrm{Ph}-\mathrm{C}_{p}$. Aus dieser Reihe lässt sich einzig dem Kohlenstoffatom $\mathrm{Ph}-\mathrm{C}_{p}$ das Signal bei $\delta=127.6 \mathrm{ppm}$ zuweisen. Die beiden quartären Kohlenstoffatome C-7' und $\mathrm{Ph}^{\prime} \mathrm{C}_{i}$ resonieren bei $\delta=$ 137.2 ppm und $\delta=137.7 \mathrm{ppm}$, können aber nicht weiter aufgelöst werden. Gleichermaßen verhält es sich mit den Signalen bei $\delta=153.2 \mathrm{ppm}$ und $\delta=157.8 \mathrm{ppm}$, die auf die Resonanzen der quartären Kohlenstoffatome C-5' und C-8a' zurückzuführen sind. Erwartungsgemäß am weitesten im Tieffeld lokalisiert, findet sich der AldehydKohlenstoffatom bei $\delta=201.9$ ppm wieder.

Zusätzlich zur NMR-Spektroskopie lieferte auch die ESI-Massenspektrometrie einen eindeutigen Nachweis der Verbindung. Zum einen konnten den Konjuganten $[\mathrm{M}+\mathrm{H}]^{+}$und $[\mathrm{M}+\mathrm{Na}]^{+}$die Masse-zu-Ladung-Verhältnisse von $\mathrm{m} / \mathrm{z}=531.3$ respektive $553.3 \mathrm{im}$ positiven lonisierungsmodus zugeordnet werden und zum anderen dem Masse-zuLadung-Verhältnis von $\mathrm{m} / \mathrm{z}=529.3$ eindeutig das deprotonierte Anion des Moleküls 
$\left[\mathrm{M}-\mathrm{H}^{-}\right.$im negativen lonisierungsmodus zugewiesen werden. Des Weiteren konnte durch ESI-HRMS die Summenformel von $\mathrm{C}_{29} \mathrm{H}_{42} \mathrm{O}_{7} \mathrm{Si}$ bestätigt werden. So stimmen neben den kalkulierten und gefundenen exakten Massen des $[\mathrm{M}+\mathrm{Na}]^{+}$-Addukts sowie des $[\mathrm{M}-\mathrm{H}]^{-}$-Anions, diese Werte auch sehr gut für das $[\mathrm{M}+\mathrm{H}]^{+}$-Konjugat (berechnet: $\mathrm{m} / \mathrm{z}=$ 531.2773, gefunden: $\mathrm{m} / \mathrm{z}=531.2764)$ überein .

Das IR-Spektrum der Verbindung weist die für den Aldehyd erwartete charakteristische $\mathrm{C}=\mathrm{O}-$ Valenzschwingung bei $\tilde{v}=1732 \mathrm{~cm}^{-1}$ auf. Weitere ausgeprägte Banden werden durch die Ringschwingung des Aromaten bei $\tilde{v}=1588 \mathrm{~cm}^{-1}$ und den $\mathrm{CH}_{3}$-Deformationsschwingungen bei $\tilde{v}=1378 \mathrm{~cm}^{-1}$ und $\tilde{v}=1359 \mathrm{~cm}^{-1}$ hervorgerufen. Daneben steuern auch die $\mathrm{C}-\mathrm{O}-\mathrm{C}-$ Valenzschwingungen der verschiedenen Ether Resonanzen bei $\tilde{v}=$ $1252 \mathrm{~cm}^{-1}, \tilde{v}=1212 \mathrm{~cm}^{-1}, \tilde{v}=1147 \mathrm{~cm}^{-1}$ und $\tilde{v}=1047 \mathrm{~cm}^{-1}$ bei. Die Absorptionsbande bei $\tilde{v}=1102 \mathrm{~cm}^{-1}$ kann der C-O-Valenzschwingung der TBS-geschützten sekundären Alkoholfunktion zugeordnet werden.

\subsubsection{Synthese des Biaryls (Z)-272}

Aufgrund der Stabilität der MOM-Schutzgruppe während der mono-Demethylierung der Olefine 246 und (E/Z)-256 wurde eine Entschützung nach dem Aufbau des Biaryls erneut in Augenschein genommen.

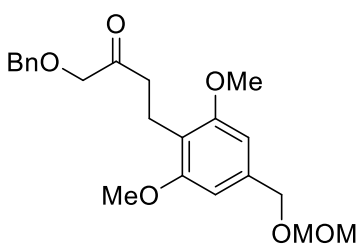

244a
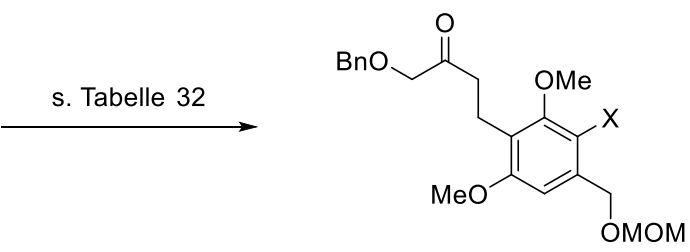

268: $X=\mathrm{Br}$

269: $X=1$

Tabelle 32: Untersuchungen zur Halogenierung des Ketons 244a.

\begin{tabular}{|c|c|c|}
\hline Eintrag & Reaktionsbedingungen & Ausbeute \\
\hline 1 & NBS (1.00 Äq.), $\mathrm{CCl}_{4}, \mathrm{RT}, 2 \mathrm{~d}$ & $4 \%$ \\
\hline 2 & $\mathrm{Br}_{2}$ (1.00 Äq.), $\mathrm{NaHCO}_{3}$ (1.20 Äq.), $\mathrm{CHCl}_{3}, \mathrm{RT}, 21 \mathrm{~h}$ & Zersetzung \\
\hline 3 & $\mathrm{I}_{2}$ (1.00 Äq.), $\mathrm{Ag}_{3} \mathrm{PO}_{4}$ (0.37 Äq.), $\mathrm{CHCl}_{3}, \mathrm{RT}, 2 \mathrm{~d}$ & $65 \%$ \\
\hline
\end{tabular}

Hierzu sollte zunächst das Keton 244a am aromatischen Kern halogeniert werden. Eine Bromierung mittels NBS oder elementarem Brom lieferte das Arylbromid in marginalen $4 \%$ Ausbeute (Tabelle 32, Eintrag 1) oder führte zur Zersetzung des Startmaterials 
(Tabelle 32, Eintrag 2). Eine lodierung mit $\mathrm{I}_{2}$ und $\mathrm{Ag}_{3} \mathrm{PO}_{4}$ erwies sich als die geeignetere Methode und führte zum Aryliodid 269 in 65\% Ausbeute (Tabelle 32, Eintrag 3).

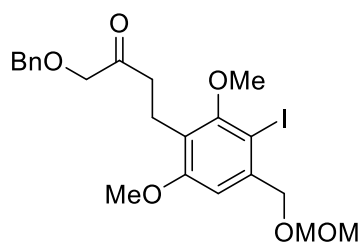

269
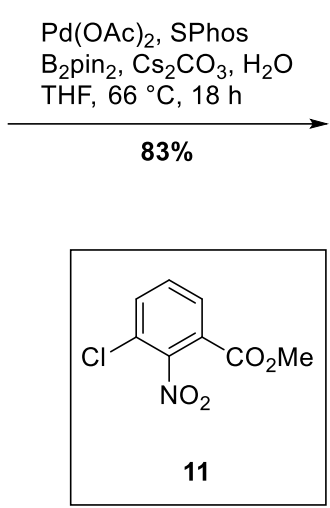

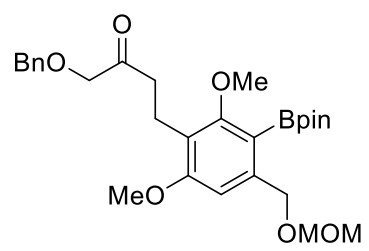

270 $\mathrm{Pd}(\mathrm{OAc})_{2}$, SPhos 11, $\mathrm{Cs}_{2} \mathrm{CO}_{3}, \mathrm{H}_{2} \mathrm{O}$ THF, $66^{\circ} \mathrm{C}, 20 \mathrm{~h}$ $62 \%$

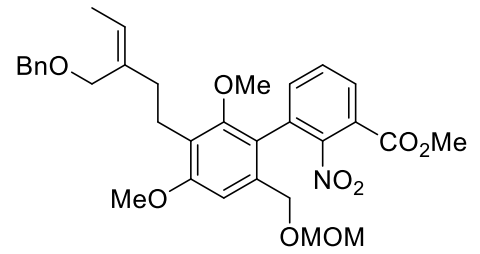

(Z)-272

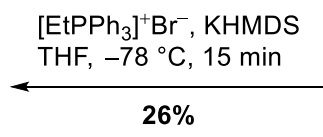

$26 \%$

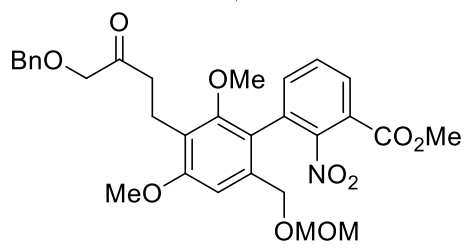

271

Abbildung 91: Synthese des (Z)-Olefins (Z)-272.

Dieses wurde in der bekannten Synthesesequenz aus Miyaura-Borylierung und darauffolgender Suzuki-Miyaura-Kreuzkupplung unter den optimierten Reaktionsparametern mit Ausbeuten von 83\% und 62\% in das Biaryl 271 überführt. Wittig-Reaktion des Ketons 271 lieferte in 26\% Ausbeute das Z-Olefin (Z)-272, das im Anschluss den Reaktionsbedingungen der mono-Demethylierung unterworfen werden sollte (Abbildung 91).

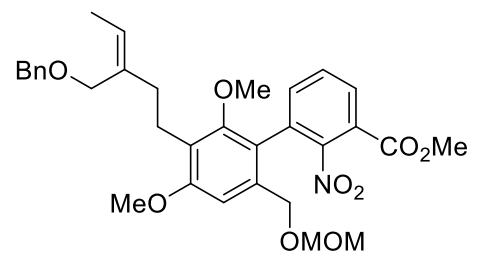

(Z)-272

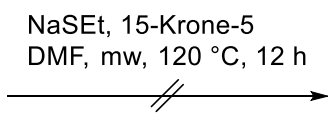

Abbildung 92: Versuch der mono-Demethylierung des Biaryls (Z)-272.

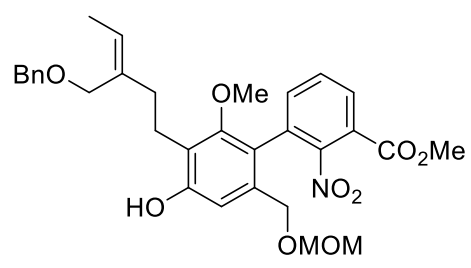

(Z)-273

Nach nahezu vollständigem Umsatz des Startmaterials wurde analog zu vorangegangenen Untersuchungen eine einfach demethylierte Spezies massenspektrometrisch nach erfolgter wässriger Aufarbeitung nachgewiesen. Diese Spezies ließ sich jedoch nach säulenchromatographischer Aufreinigung an Kieselgel wiederum nicht isolieren (Abbildung 92). Die aufgenommenen spektroskopischen Daten der einzigen isolierbaren 
Fraktion erlaubten allerdings aufgrund der geringen erhaltenen Substanzmenge und der ungenügenden Reinheit keine Strukturbestimmung. Es wird jedoch vermutet, dass primär der Methylester gespalten wurde. Sicher ist, dass die Benzyl- sowie die MOMSchutzgruppe im Produkt erhalten sind. Somit stellt die Methylesterfunktionalität, die in der Suzuki-Miyaura-Kreuzkupplung in das Substrat eingebracht wurde, den limitierenden Faktor für einen Aufbau der Biarylstruktur vor der Wacker-Zyklisierung bzw. der DominoWacker/Carbonylierung/Methoxylierung dar.

\subsubsection{Diskussion der spektroskopischen Daten des Biaryls (Z)-272}

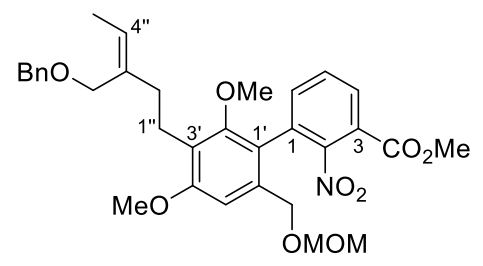

(Z)-272

Im Folgenden sollen einige ausgewählte analytische Daten des Biaryls (Z)-272 diskutiert werden, dessen bei Raumtemperatur in deuteriertem Chloroform aufgenommene ${ }^{1} \mathrm{H}$ - und ${ }^{13} \mathrm{C}$-NMR-Spektren in den Abbildung 93 und Abbildung 94 dargestellt sind. Neben den unterschiedlichen Intensitäten der Signale im ${ }^{13} \mathrm{C}-\mathrm{NMR}$, mit denen vorallem quartäre Kohlenstoffatome identifiziert werden konnten, basierte eine genauere Zuordnung der Signale, sofern dies durch eindeutige Korrelationen belegbar war, auf der Auswertung entsprechender zweidimensionaler NMR-Spektren (COSY, HSQC, HMBC, NOESY).

Im aliphatischen Bereich des Spektrums treten zunächst die Signale der Seitenkette bei $\delta=1.62 \mathrm{ppm}\left(5^{\prime \prime}-\mathrm{H}_{3}\right), \quad \delta=2.33 \mathrm{ppm}\left(2{ }^{\prime \prime}-\mathrm{H}_{2}\right)$ und $\delta=2.69-2.78 \mathrm{ppm}\left(1 "-\mathrm{H}_{2}\right)$ in Erscheinung. Das Signal der Methylgruppe spaltet dabei in ein Dublett mit einer Kopplungskonstante von $J=6.9 \mathrm{~Hz}$ auf, während die Methylenwasserstoffatome an den Kohlenstoffatomen C-2" und C-1" als Multipletts vorliegen, deren Multiplizitäten nicht eindeutig aufgelöst werden können. Bei einer chemischen Verschiebung von $\delta=$ 3.24 ppm resonieren die Wasserstoffatome der Methoxygruppe am Kohlenstoffatom C-2' in Form eines Dubletts mit einer Kopplungskonstante von $J=0.3 \mathrm{~Hz}$. Weiter Richtung Tieffeld verschoben folgen vier Singuletts bei $\delta=3.37 \mathrm{ppm}, \delta=3.81 \mathrm{ppm}, \delta=3.89 \mathrm{ppm}$ und $\delta=4.09 \mathrm{ppm}$ die dem Methoxyfragment der MOM-Schutzgruppe, der Methoxygruppe am Kohlenstoffatom C-4', dem Methylester und der Methyleneinheit am Kohlenstoffatom C-3" zugeordnet werden können. Daran reihen sich die Signale der diastereotopen Wasserstoffatome der Methylengruppe am Kohlenstoffatom C-6' bei chemischen 
Verschiebungen von $\delta=4.17 \mathrm{ppm}$ und $\delta=4.31 \mathrm{ppm}$ ein, die jeweils als Dublett mit einer geminalen Kopplungskonstante von $J=12.1 / 0 \mathrm{~Hz}$ und einem charakteristischen Dacheffekt vorliegen. Ein analoges Aufspaltungsmuster zeigen die Signale der ebenfalls diastereotopen Methylenwasserstoffatome der MOM-Schutzgruppe, die bei $\delta=4.50 \mathrm{ppm}$ und $\delta=4.55 \mathrm{ppm}$ als Dubletts mit einer gemeinsamen Kopplungskonstanten von $J=$ $6.5 \mathrm{~Hz}$ resonieren. In das weiter hochfeldverschobene Dublett der beiden Signale fällt zusätzlich das Singulett bei $\delta=4.47 \mathrm{ppm}$, das die Resonanzfrequenz der Methylenwasserstoffatome der Benzyl-Schutzgruppe abbildet. Das Wasserstoffatom 4"-H der dreifach substituierten Doppelbindung findet sich als Quartett mit einer Kopplungskonstante von $J=6.8 \mathrm{~Hz}$ bei $\delta=5.47$ ppm wieder.
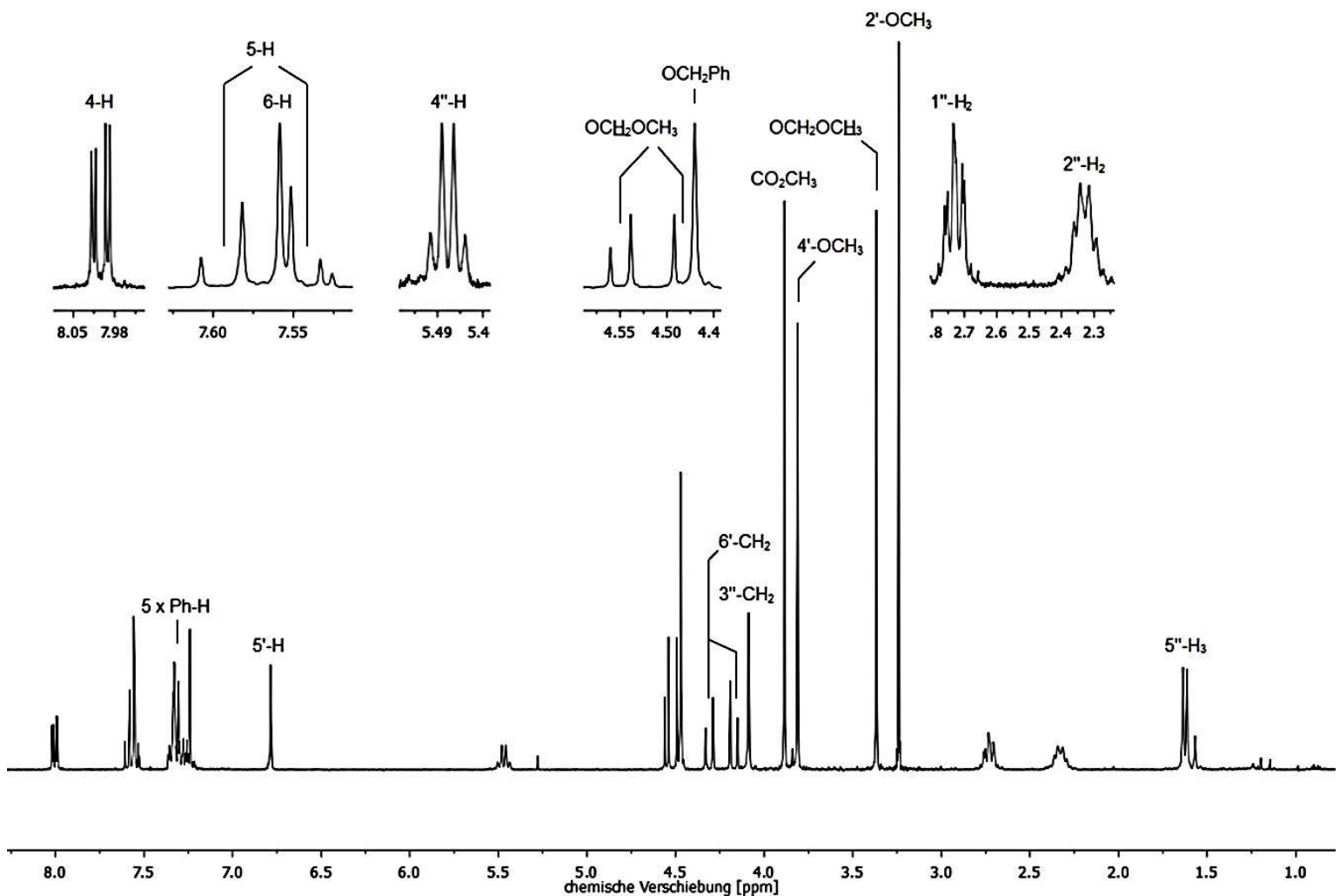

Abbildung 93: ${ }^{1} \mathrm{H}-\mathrm{NMR}-$ Spektrum des Biaryls (Z)-272.

Im aromatischen Bereich des Spektrums liegt am weitesten hochfeldverschoben das Signal des Wasserstoffatoms 5'-H des elektronenreichen Aromaten als Singulett bei einer chemischen Verschiebung von $\delta=6.79 \mathrm{ppm}$ vor. Im Bereich von $\delta=7.21-7.37 \mathrm{ppm}$ folgen die phenolischen Wasserstoffatome der Benzyl-Schutzgruppe in Form eines nicht näher auflösbaren Multipletts. Den Abschluss bilden die Wasserstoffatome des elektronenarmen Aromaten bei $\delta=7.54 \mathrm{ppm}(6-\mathrm{H}), \delta=7.55-7.61 \mathrm{ppm}(5-\mathrm{H})$ und $\delta=$ 8.00 ppm (4-H). Die Signale der Wasserstoffatome 6-H und 5-H fallen hierbei ineinander, weswegen die Multiplizität für das Erstgenannte als Dublett mit einer Kopplungskonstante 
von $J=5.5 \mathrm{~Hz}$ bestimmt werden konnte, dies jedoch für das Letztgenannte nicht eindeutig möglich war und somit als Multiplett verbleibt. Das Wasserstoffatom 4-H hingegen liegt in Form eines Dublett von Dubletts mit Kopplungskonstanten von $J=7.1 \mathrm{~Hz}$ und $J=2.2 \mathrm{~Hz}$ vor.
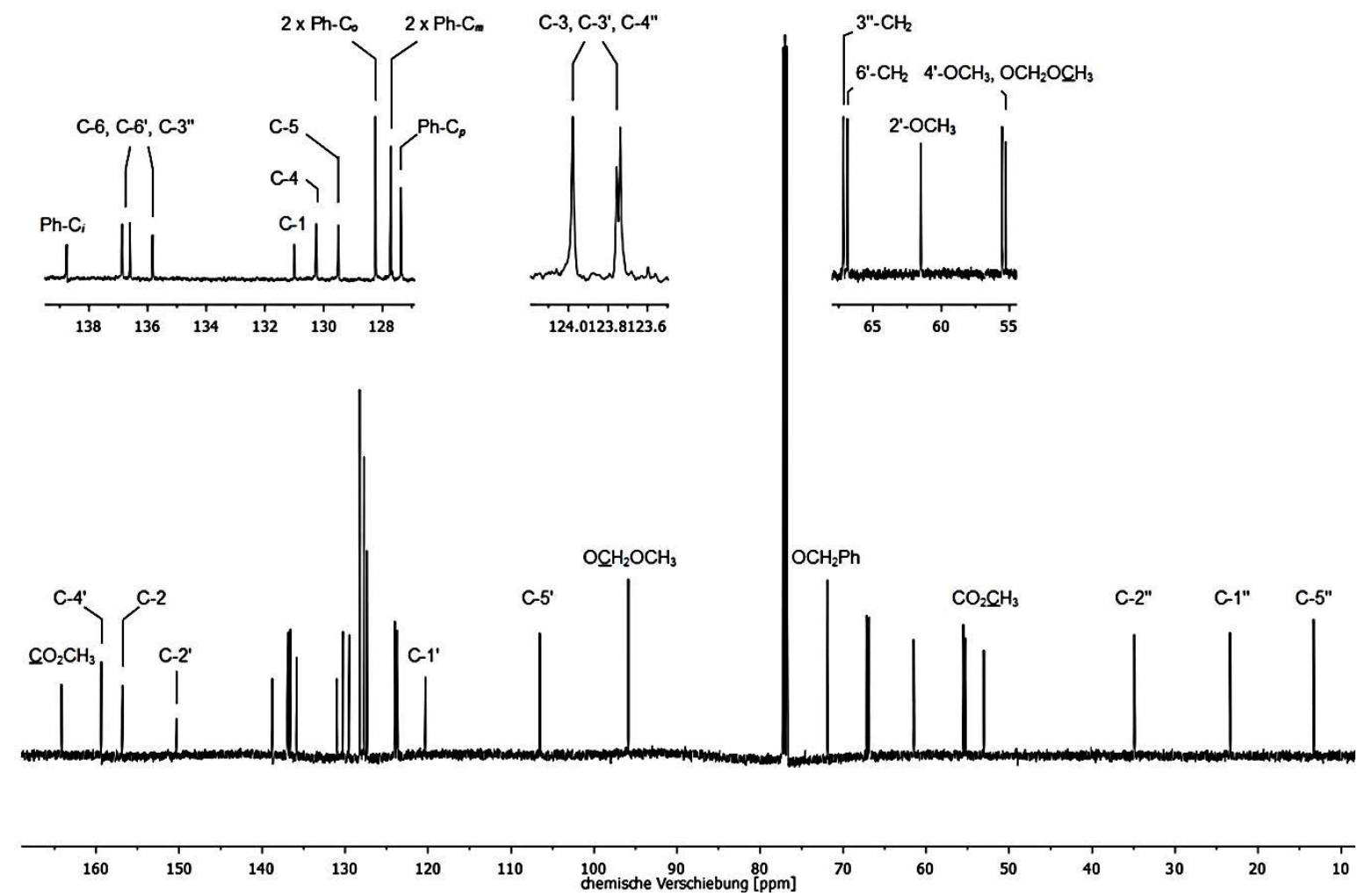

Abbildung 94: ${ }^{13} \mathrm{C}-\mathrm{NMR}$-Spektrum des Biaryls (Z)-272.

Wie bereits im ${ }^{1} \mathrm{H}-\mathrm{NMR}$-Spektrum finden sich ebenfalls im ${ }^{13} \mathrm{C}-\mathrm{NMR}$-Spektrum am weitesten hochfeldverschoben die Signale der Kohlenstoffatome der Seitenkette bei $\delta=$ $13.3 \mathrm{ppm}$ (C-5"), $\delta=23.4 \mathrm{ppm}$ (C-1") und $\delta=34.9 \mathrm{ppm}$ (C-2”) wieder. Analog dazu resonieren weiter tieffeldverschoben die Methylgruppe des Methylesters bei $\delta=53.1 \mathrm{ppm}$, die Methoxygruppe am Kohlenstoffatom C-4' sowie das Methoxyfragment der MOMSchutzgruppe bei $\delta=55.3 \mathrm{ppm}$ und $\delta=55.5 \mathrm{ppm}$, die nicht weiter aufgelöst werden konnten und die Methoxygruppe am Kohlenstoffatom C-2' bei $\delta=61.5 \mathrm{ppm}$. Es folgen weiter in Richtung Tieffeld verschoben die Methylengruppen am Kohlenstoffatom C-3" bei $\delta=66.9$ ppm, am Kohlenstoffatom C- 6 ' bei $\delta=67.2$ ppm sowie die Methyleneinheiten der Benzyl-Schutzgruppe bei $\delta=71.9 \mathrm{ppm}$ und der MOM-Schutzgruppe bei $\delta=95.9 \mathrm{ppm}$. Im aromatischen Bereich lassen sich den Signalen bei $\delta=106.5 \mathrm{ppm}$ und $\delta=120.3 \mathrm{ppm}$ die Resonanzen der Kohlenstoffatome C-5' respektive C-1' zuordnen. Eine genaue Zuordnung der Signale mit den chemischen Verschiebungen von $\delta=123.7 \mathrm{ppm}, \delta=$ $123.8 \mathrm{ppm}$ und $\delta=124.0 \mathrm{ppm}$ war indes nicht möglich und konnte nur auf die beiden 
aromatischen Kohlenstoffatome C-3 und C-3' sowie das Doppelbindungskohlenstoffatom C-4" eingegrenzt werden. Weiter tieffeldverschoben schließen sich die phenolischen Kohlenstoffatome der Benzyl-Schutzgruppe $\mathrm{Ph}-\mathrm{C}_{p}$ bei $\delta=127.4 \mathrm{ppm}, \mathrm{Ph}-\mathrm{C}_{m}$ bei $\delta=$ $127.7 \mathrm{ppm}$ und $\mathrm{Ph}-\mathrm{C}_{o}$ bei $\delta=128.2 \mathrm{ppm}$ an. Die Kohlenstoffatome des elektronenarmen Aromaten C-5, C-4 und C-1 resonieren bei chemischen Verschiebungen von $\delta=$ $129.5 \mathrm{ppm}, \delta=130.3 \mathrm{ppm}$ und $\delta=131.0 \mathrm{ppm}$. Die Resonanzsignale bei $\delta=135.8 \mathrm{ppm}$, $\delta=136.6 \mathrm{ppm}$ und $\delta=136.9 \mathrm{ppm}$ können den beiden aromatischen Kohlenstoffatomen C-6 und C-6' sowie dem zweiten Doppelbindungskohlenstoffatom C-3" zugewiesen aber nicht weiter aufgelöst werden. Im Gegensatz dazu konnten die Signale bei $\delta=138.8 \mathrm{ppm}$ $\left(\mathrm{Ph}-\mathrm{C}_{i}\right), \delta=150.3$ ppm (C-2), $\delta=156.8$ ppm (C-2') und $\delta=159.4$ ppm (C-4') eindeutig den jeweiligen Kohlenstoffatomen zugeordnet werden. Am weitesten tieffeldverschoben resoniert erwartungsgemäß das Carboxylkohlenstoffatom bei einer chemischen Verschiebung von $\delta=164.2 \mathrm{ppm}$.

Neben den Daten der NMR-Spektroskopie bestätigte auch das ESI-Spektrum durch das Vorhandensein der Peaks für das $[\mathrm{M}+\mathrm{Na}]^{+}$-Addukt bei $\mathrm{m} / \mathrm{z}=602.2$ sowie für das Konjugat $[2 \times \mathrm{M}+\mathrm{Na}]^{+}$bei $\mathrm{m} / z=1181.5$ die Verbindung. Des Weiteren konnte die Summenformel $\mathrm{C}_{32} \mathrm{H}_{37} \mathrm{NO}_{9}$ durch die gute Übereinstimmung des per ESI-HRMS bestimmten Masse-zu-Ladung-Verhältnisses von $\mathrm{m} / \mathrm{z}=602.2365$ für das $[\mathrm{M}+\mathrm{Na}]^{+-}$ Addukt mit dem kalkulierten Wert von $\mathrm{m} / \mathrm{z}=602.2361$ belegt werden.

Im IR-Spektrum zeigen vor allem der Methylester sowie die Nitrogruppe charakteristische Absorptionsbanden. Die $\mathrm{C}=\mathrm{O}-$ Valenzschwingungen des Methylesters lässt sich bei $\tilde{v}=$ $1732 \mathrm{~cm}^{-1}$ wiederfinden, während die $\mathrm{NO}_{2}$-Valenzschwingungen Banden bei $\tilde{v}=$ $1542 \mathrm{~cm}^{-1}$ und $\tilde{v}=1287 \mathrm{~cm}^{-1}$ hervorrufen. Weitere ausgeprägte Absorptionsbanden steuern die Ringschwingung des aromatischen Systems bei $\tilde{v}=1600 \mathrm{~cm}^{-1}$ und die $\mathrm{CH}_{3}$ Deformationsschwingung bei $\tilde{v}=1368 \mathrm{~cm}^{-1}$ bei. Zusätzlich verursachen die C-O-CValenzschwingungen der diversen Ethersubstituenten Resonanzen bei $\tilde{v}=1148 \mathrm{~cm}^{-1}, \tilde{v}=$ $1098 \mathrm{~cm}^{-1}, \tilde{v}=1066 \mathrm{~cm}^{-1}$ und $\tilde{v}=1035 \mathrm{~cm}^{-1}$. 


\subsection{Zusammenfassung}

Das Ziel dieser Arbeit bestand in der ersten enantioselektiven Synthese eines Parnafungins. Es handelt sich hierbei um eine Naturstoffklasse, die 2008 entdeckt und die sich einerseits durch eine strukturelle Novität in Form des Isoxazolidinon-Ringes und anderseits durch ihre Enzym-spezifische biologische Aktivität gegenüber einem breiten Spektrum an klinisch relevanten pathogenen Fungi auszeichnet. Als Syntheseziel war Parnafungin C (3) ausgewählt worden, das gegenüber den meisten anderen Parnafunginen eine höhere Stabilität aufweist (Abbildung 95).

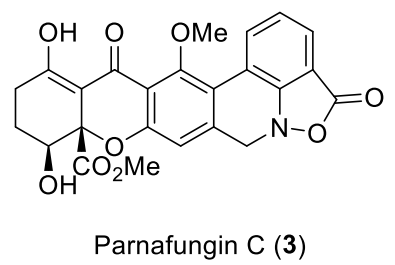

Abbildung 95: Syntheseziel Parnafungin C (3).

Die ursprüngliche retrosynthetische Analyse sah zunächst den Aufbau der Biarylstruktur mittels einer Suzuki-Miyaura-Kreuzkupplung vor. Anschließend sollten die strukturellen Voraussetzungen zur Durchführung einer enantioselektiven Palladium-katalysierten Wacker-Zyklisierung als Schlüsselschritt zur Inkorporierung des Stereozentrums am quartären Kohlenstoffatom geschaffen werden. Das zweite stereogene Zentrum sollte im Anschluss an die Wacker-Zyklisierung durch eine diastereoselektive SharplessDihydroxylierung in das Molekül eingeführt werden. Für den Abschluss der Synthese war die Zyklisierung der verbliebenen Ringe durch eine intramolekulare Acylierung sowie der von der Forschungsgruppe Snider entwickelten Methode zum Aufbau des labilen Isoxazolo[4,3,2-de]phenanthridinon-Motivs vorgesehen (siehe S. 49, Abbildung 43).

Im Verlauf der Arbeiten zu diesem Thema wurden diverse Modifikationen an der Syntheseplanung erforderlich, nachdem zunächst eine bereits in der vorangegangenen Diplomarbeit zu diesem Themengebiet hergestellte mögliche Vorstufe nicht entschützt werden konnte (siehe Kap. 2.1), eine regioselektive Entschützung der sterisch weniger gehinderten Methoxygruppe an einem weiter fortgeschrittenen Biaryl nicht durchführbar war (siehe Kap. 2.2) und an einem selektiv geschütztem Substrat eine lodierung zur Vorbereitung der Suzuki-Miyaura-Kreuzkupplung mit einer inkorrekten Regioselektivität einherging (siehe Kap. 2.3). Aufgrund dieser Beobachtungen wurde die Darstellung der Tetrahydroxanthenon-Grundstruktur vorgezogen, die zu einem geeigneten Punkt in der Synthese um den zusätzlichen Aromaten erweitert werden sollte. 
<smiles>O=C(O)c1cc(O)cc(O)c1</smiles>

153

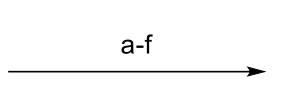<smiles>COCc1cc(OC)c(CCC(=O)COc2ccccc2)c(OC)c1</smiles>

244a

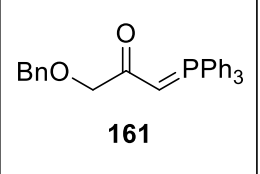

Abbildung 96: Synthese des Ketons 244a: a) $\mathrm{Me}_{2} \mathrm{SO}_{4}, \mathrm{~K}_{2} \mathrm{CO}_{3}$, Aceton, $56{ }^{\circ} \mathrm{C}, 4 \mathrm{~h}, 86 \%$; b) $\mathrm{NaBH}_{4}, \mathrm{MeOH}$ DME, $85^{\circ} \mathrm{C}, 3 \mathrm{~h}, 96 \%$; c) 1. nBuLi, TMEDA, THF, $0{ }^{\circ} \mathrm{C}, 1$ h, 2. DMF, RT, 3 h, 97\%; d) 171, PhMe, $120^{\circ} \mathrm{C}, 2 \mathrm{~d}$, 20\% (+31\%); e) MOMCl, $\mathrm{Pr}_{2} \mathrm{NEt}, \mathrm{CH}_{2} \mathrm{Cl}_{2}, \mathrm{RT}, 18 \mathrm{~h}, 92 \%$; f) 1. $\mathrm{PtO}_{2}, \mathrm{H}_{2}, \mathrm{EtOH}, \mathrm{RT}, 3 \mathrm{~h}, 2 . \mathrm{NMO}, \mathrm{TPAP}, \mathrm{MS}$ (4 ̊̊), $\mathrm{CH}_{2} \mathrm{Cl}_{2}, \mathrm{RT}, 3 \mathrm{~h}, 84 \%$.

Ausgehend von der kommerziell erhältlichen 3,5-Dihydroxybenzoesäure (153) wurde das Keton 244a als Ausgangspunkt für weitere Funktionalisierungen in sechs Stufen mit einer Ausbeute von $32 \%$ synthetisiert (Abbildung 96).<smiles>COCc1cc(OC)c(CCC(=O)COc2ccccc2)c(OC)c1</smiles>

244a<smiles>CC=C(C)CCc1c(O)cc(COC)cc1OC</smiles>

$(E / Z)-256$

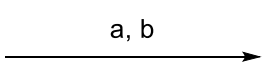<smiles>C=C(CCc1c(O)cc(COC)cc1OC)COCc1ccccc1</smiles>

246

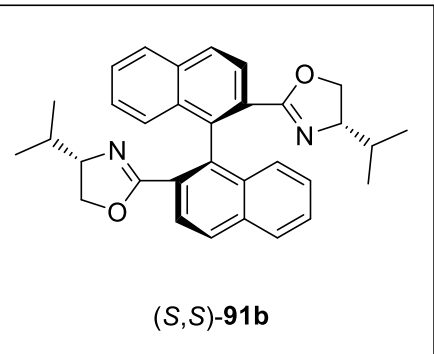

$\mathrm{h}$<smiles>C=C[C@]1(COCc2ccccc2)CCc2c(OC)cc(COC)cc2O1</smiles>

(S)-252

Abbildung 97: Darstellung des chiralen Vinylchromans (S)-252 via Domino-Wacker/Carbonylierung/Methoxylierung (a-e) oder Wacker-Zyklisierung (f-h): a) MePPh 3 Br, nBuLi, THF, RT, 2 h, 72\%; b) NaSEt, 15-Krone-5, DMF, mw, $120^{\circ} \mathrm{C}, 12 \mathrm{~h}, 83 \%$; c) Pd(TFA)2, (S,S)-iPr-BOXAX (S,S)-91b, $p$-Benzochinon, $\mathrm{CO}, \mathrm{MeOH}, 40^{\circ} \mathrm{C}$, 2 d, 53\%, 99.0\% ee; d) $\mathrm{LiAlH}_{4}, \mathrm{Et}_{2} \mathrm{O}, \mathrm{RT}, 2 \mathrm{~h}, 37 \%$; e) 1. 254, $\mathrm{PnBu}$, THF, $0{ }^{\circ} \mathrm{C}, 4 \mathrm{~h}, 2 . m \mathrm{CPBA}, \mathrm{Pr}_{2} \mathrm{NH}$, $\mathrm{Na}_{2} \mathrm{HPO}_{4} \cdot 2 \mathrm{H}_{2} \mathrm{O}, \mathrm{CH}_{2} \mathrm{Cl}_{2}, \mathrm{RT}, 15 \mathrm{~h}, 66 \%$; f) [EtPPh $]^{+} \mathrm{Br}$, KHMDS, THF, -78 ${ }^{\circ} \mathrm{C}, 15 \mathrm{~min}, 73 \%, E / Z$ 1:6.5; g) NaSEt, 15-Krone-5, DMF, mw, $120^{\circ} \mathrm{C}, 12$ h, 88\%; h) Pd(TFA)2, (S,S)-iPr-BOXAX (S,S)-91b, p-Benzochinon, $\mathrm{MeOH}, 40^{\circ} \mathrm{C}, 2 \mathrm{~d}, 75 \%, 98.4 \%$ ee. 
Durch die Umstellungen in der Syntheseroute konnte der Zugang zum chiralen Vinylchroman (S)-252 über zwei verschiedene Synthesepfade realisiert werden. Eine Domino-Wacker/Carbonylierung/Methoxylierung des terminalen Alkens 246 lieferte das Vinylchroman (S)-252 ausgehend vom Keton 244a in insgesamt fünf Stufen mit einer Ausbeute von $8 \%$ und einem Enantiomerenüberschuss von $99.0 \%$. Als eine etwas direktere Alternative stellte sich die Synthese des Vinylchromans (S)-252 über eine Wacker-Zyklisierung des internen Olefins (E/Z)-256 dar. Innerhalb von drei Stufen gelang der Zugang zum Vinylchroman (S)-252 in 48\% Ausbeute und minimal schlechteren $98.4 \%$ ee (Abbildung 97).

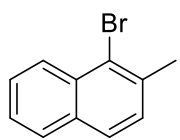

198

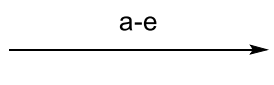
$\overbrace{}^{(S)} \mathrm{OH}$

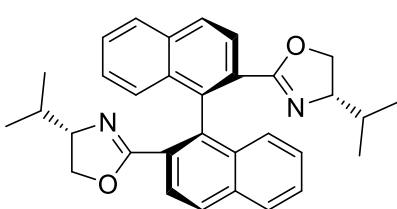

$(S, S)-91 b$

(S)-204

Abbildung 98: Synthese des (S,S)-iPr-BOXAX-Liganden (S,S)-91b: a) NBS, AIBN, $\mathrm{CCl}_{4}, 90{ }^{\circ} \mathrm{C}, 2 \mathrm{~d}, 85 \%$; b) $\mathrm{HCO}_{2} \mathrm{H}_{(\text {aq.) }}, 110{ }^{\circ} \mathrm{C}, 20$ h, 76\%; c) $\mathrm{NaClO}_{2 \text { (aq.) }}, \mathrm{NaH}_{2} \mathrm{PO}_{4} \cdot \mathrm{H}_{2} \mathrm{O}_{\text {(aq.), }}$ 2-Methyl-2-buten, Aceton, RT, $22 \mathrm{~h}, 93 \%$; d) 1. $(\mathrm{COCl})_{2}$, DMF, PhMe, RT, 3 h, 2. (S)-204, NEt $\mathrm{N}_{3} \mathrm{CH}_{2} \mathrm{Cl}_{2}, \mathrm{RT}, 23 \mathrm{~h}, 3 . \mathrm{MsCl}, \mathrm{NEt}_{3}, \mathrm{CH}_{2} \mathrm{Cl}_{2}, \mathrm{RT}, 3 \mathrm{~h}, 4$. $\mathrm{KOH}$, $\mathrm{MeOH}, \mathrm{RT}, 13 \mathrm{~h}, 82 \%$; e) $\mathrm{Cu}, \mathrm{py}, 115^{\circ} \mathrm{C}, 19$ h, $71 \%$.

Der für die Induktion der Stereoselektivität in der Wacker-Zyklisierung und der DominoWacker/Carbonylierung/Methoxylierung erforderliche (S,S)-IPr-BOXAX-Ligand (S,S)-91b konnte ausgehend vom kommerziell erhältlichen 1-Brom-2-methylnaphthalen (198) in sechs Stufen mit einer Ausbeute von 35\% nach einem optimierten Protokoll, basierend auf einer literaturbekannten Vorschrift von Hayashi und Myers et al., dargestellt werden (Abbildung 98).

Eine Sharpless-Dihydroxylierung des Vinylchromans (S)-252 zum Diol anti/syn-257 mit $\mathrm{K}_{2} \mathrm{OsO}_{4} \cdot 2 \mathrm{H}_{2} \mathrm{O}$ (5 mol\%), (DHQD)PHN (10 mol\%), $\mathrm{K}_{3}\left[\mathrm{Fe}(\mathrm{CN})_{6}\right] \quad(6.00 \mathrm{Äq}),. \mathrm{K}_{2} \mathrm{CO}_{3}$ (6.00 Äq.) und $\mathrm{MeSO}_{2} \mathrm{NH}_{2}$ (1.00 Äq.) in einem $1: 1$ Gemisch aus $t \mathrm{BuOH} / \mathrm{H}_{2} \mathrm{O}$ bei Raumtemperatur ermöglichte bei einer Reaktionszeit von $3 \mathrm{~d}$ die Inkorporation des zweiten Stereozentrums in das Molekül mit einem Diasteromerenverhältnis für den vorliegenden mismatched-Fall von antilsyn 1:4.3. Die Trennung der beiden Diastereomere erfolgte auf der Stufe des Aldehyds per Säulenchromatographie an Kieselgel, wodurch auf eine HPLC-Aufreinigung verzichtet werden konnte. Somit konnte der $\alpha, \beta$-ungesättigte 
Methylester syn-266 in einer fünfstufigen Synthese in 25\% Ausbeute aus dem Vinylchroman (S)-252 erhalten werden (Abbildung 99).<smiles>C=C[C@]1(COc2ccccc2)CCc2c(OC)cc(COC)cc2O1</smiles>

(S)-252
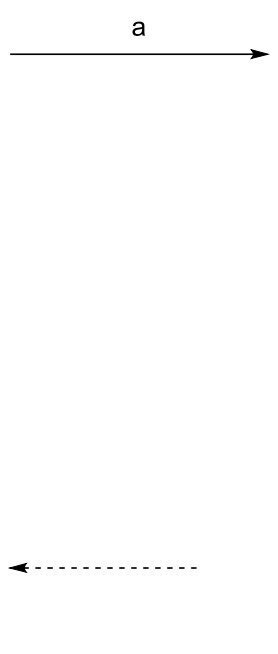<smiles>COCc1cc(OC)c2c(c1)O[C@@](COc1ccccc1)([C@H](O)CO)CC2</smiles>

antilsyn-257 syn-266

Parnafungin C (3)<smiles>COCc1cc(OC)c2c(c1)O[C@](COCc1ccccc1)(C(/C=C/C(C)(C)C)OC(C)(C)C)CC2</smiles><smiles>COCc1cc(OC)c2c(c1)O[C@](COCc1ccccc1)(C(/C=C/C(C)(C)C)OC(C)(C)C)CC2</smiles>

Abbildung 99: Synthese des $\alpha, \beta$-ungesättigten Methylesters syn-266: a) $\mathrm{K}_{2} \mathrm{OsO}_{4} \cdot 2 \mathrm{H}_{2} \mathrm{O}$, (DHQD)PHN, $\mathrm{K}_{2} \mathrm{CO}_{3}, \mathrm{~K}_{3}\left[\mathrm{Fe}(\mathrm{CN})_{6}\right], \mathrm{MeSO}_{2} \mathrm{NH}_{2}, \mathrm{tBuOH} / \mathrm{H}_{2} \mathrm{O}$ (1:1), RT, $3 \mathrm{~d}, 74 \%$, drantisyn 1:4.3; b) TBSOTf, 2,6-Lutidin, $\mathrm{CH}_{2} \mathrm{Cl}_{2}, 0{ }^{\circ} \mathrm{C}, 3 \mathrm{~h}, 89 \%$; c) HF · py, THF/py (4:1), RT, $26 \mathrm{~h}, 82 \%$; d) DMP, $\mathrm{CH}_{2} \mathrm{Cl}_{2}, \mathrm{RT}, 150 \mathrm{~min}, 57 \%$; e) $(\mathrm{MeO})_{2} \mathrm{P}(\mathrm{O}) \mathrm{CH}_{2} \mathrm{CO}_{2} \mathrm{Me}, \mathrm{NaH}, \mathrm{THF}, \mathrm{RT}, 2 \mathrm{~h}, 81 \%$.

Eine weitere Fortführung der Synthese des Parnafungin C (3) ausgehend vom Methylester syn-266 mit der korrekten absoluten und relativen Konfiguration aller stereogenen Zentren, wie sie im Parnafungin C (3) vorliegt, war aus Zeitgründen infolge der vorangegangenen zahlreichen negativen Untersuchungen nicht mehr möglich.<smiles>COCc1cc(OC)c(CCC(=O)COc2ccccc2)c(OC)c1</smiles>

244a
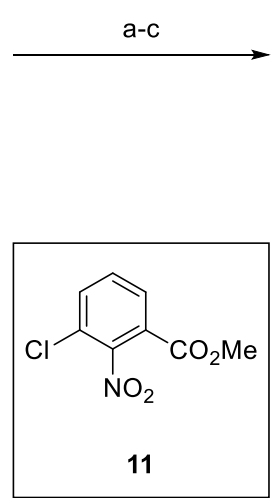

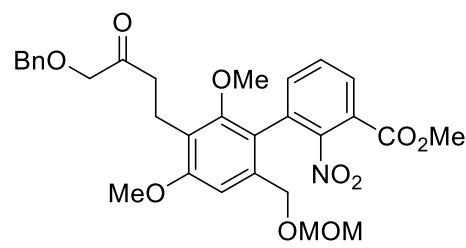

271

Abbildung 100: Synthese des Biaryls 271: a) $\mathrm{I}_{2}, \mathrm{Ag}_{3} \mathrm{PO}_{4}, \mathrm{CHCl}_{3}, \mathrm{RT}, 2$ d. 65\%; b) $\mathrm{Pd}(\mathrm{OAc})_{2}$, SPhos, B $\mathrm{B}_{2}$ pin 2 , $\mathrm{Cs}_{2} \mathrm{CO}_{3}, \mathrm{H}_{2} \mathrm{O}$, THF, $66^{\circ} \mathrm{C}, 18 \mathrm{~h}, 83 \%$; c) 11, $\mathrm{Pd}(\mathrm{OAc})_{2}$, SPhos, $\mathrm{Cs}_{2} \mathrm{CO}_{3}, \mathrm{H}_{2} \mathrm{O}, \mathrm{THF}, 66^{\circ} \mathrm{C}, 20 \mathrm{~h}, 62 \%$. 
Als Beispiel für den besonderen Schwierigkeitsgrad der Arbeiten zum Parnafungin C (3) sei die Entwicklung einer Syntheseroute zum Erhalt des Biaryls 271 genannt. Diese Verbindung mit der erforderlichen Biarylkomponente ließ sich aus dem Keton 244a nach erfolgter lodierung durch eine Miyaura-Borylierung und Suzuki-Miyaura-Kreuzkupplung sehr elegant mit 33\% Ausbeute herstellen (Abbildung 100). Nachfolgend wurde eine Wittig-Reaktion zum korrespondierenden internen Olefin (Z)-272 erfolgreich durchgeführt, jedoch war eine darauffolgende selektive mono-Demethylierung nicht zu realisieren.

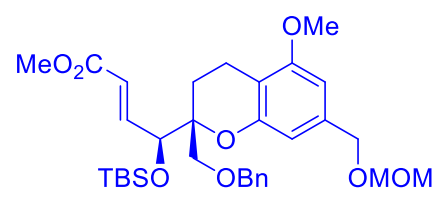

syn-266

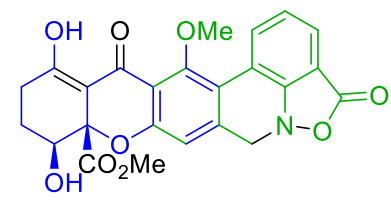

Parnafungin C (3)

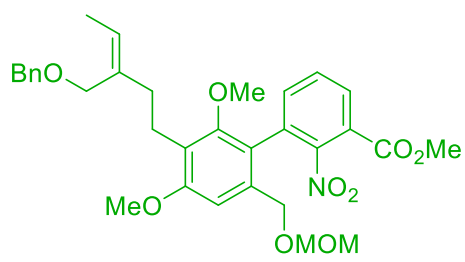

(Z)-272

Abbildung 101: Synthetisierte Fragmente des Parnafungin C (3).

Im Rahmen dieser Arbeit konnten letztendlich eine Methode zum effektiven Aufbau der Biarylstruktur (grün) des Isoxazolo[4,3,2-de]phenanthridinon-Motivs im Parnafungin C (3), etabliert und ein weit fortgeschrittenes chirales Chroman (blau), das beide im Parnafungin C (3) befindliche stereogene Zentren besaß, synthetisiert werden. Eine Kombination der beiden Strukturelemente war indes nicht mehr möglich (Abbildung 101). 


\section{Experimenteller Teil}




\subsection{Allgemeine Methoden}

\subsubsection{Allgemeine Arbeitstechniken}

Sämtliche Umsetzungen wurden, soweit Arbeiten unter Ausschluss von Wasser und/oder Sauerstoff erforderlich war, in ausgeheizten Glasapparaturen unter einem leichten ArgonÜberdruck durchgeführt. Reagenzien wurden, sofern es sich um Flüssigkeiten handelte, via Spritze oder Transferkanüle und im Falle von Feststoffen im Argon-Gegenstrom hinzugefügt.

\subsubsection{Lösungsmittel und Reagenzien}

Alle verwendeten Lösungsmittel wurden über Molekularsieb (3 $\AA$ ) getrocknet, welches zuvor in einem Vakuumtrockenschrank Vacutherm 6025 der Firma Heraeus Instruments bei $100{ }^{\circ} \mathrm{C}$ in vacuo aktiviert wurde. Das Entgasen von Solventien geschah entweder mittels Hindurchleiten eines Argon-Gasstroms über einen längeren Zeitraum oder bei sehr sauerstoffempfindlichen Reaktionen per dreimaligem Freeze-Pump-Thaw Zyklus. Alle kommerziell erhältlichen Reagenzien wurden, falls nicht anders erwähnt, ohne weitere Aufreinigung verwendet. Zur Gewährleistung der Qualität von wasser- oder sauerstoffempfindlichen Reagenzien wurden diese in einer Trockenbox des Modells LABmaster sp der Firma MBraun gelagert und abgewogen.

\subsubsection{Verwendete Geräte}

Kältetechnik: Die Reaktionsführung bei konstant tiefen Temperaturen über einen längeren Zeitraum erfolgte unter Einsatz von Kryostaten. Hierbei kamen die Modelle Haake EK90, Haake EK101 und Julabo FT902 mit EtOH/PrOH als Kühlmittel zum Einsatz. Des Weiteren wurden gängige Kältemischungen wie Eis/ $\mathrm{H}_{2} \mathrm{O}$ oder Trockeneis/Aceton zur temporären Kühlung verwendet.

Reaktionsmikrowelle: Mikrowellenreaktionen wurden in einem Reaktor vom Typ Initiator der Firma Biotage ${ }^{\circledR}$ durchgeführt (absorption level: very high, pre-stirring: $60 \mathrm{~s}$, fixed hold time: on).

Schmelzpunkte: Die Bestimmung der Schmelzpunkte erfolgte mithilfe einer vollautomatischen Schmelzpunktbestimmungsapparatur MPA120 (EZ-Melt) der Firma Stanford Research Systems. 
Drehwerte: Drehwerte wurden mit einem Polarimeter P-2000 der Firma Jasco ermittelt.

UV/Vis-Spektren: Die Aufnahme der UV/Vis-Spektren erfolgte mit dem Modell V-630 der Firma Jasco. Die Messung erfolgte im Wellenlängenbereich von 190-600 nm.

IR-Spektren: Infrarotspektren wurden mit dem Modell FT/IR-4100 der Firma Jasco aufgenommen. Alle Substanzen wurden direkt auf eine ATR-Einheit aufgetragen (neat).

'H-NMR-Spektren: ${ }^{1} \mathrm{H}-N M R-S p e k t r e n$ wurden mit den Geräten Mercury 300 (300 MHz), Unity $300(300 \mathrm{MHz})$, Inova $500(500 \mathrm{MHz})$ und Inova $600(600 \mathrm{MHz})$ der Firma Varian sowie dem Gerät Avance 300 (300 MHz) der Firma Bruker von in deuterierten Solventien gelösten Proben aufgenommen. Die chemische Verschiebung $\delta$ ist in ppm angegeben und auf das Signal des jeweiligen Lösungsmittels referenziert. Die Multiplizitäten von Signalen erster Ordnung wurden wie folgt bezeichnet: s (Singulett), sbr (breites Singulett), d (Dublett), t (Triplett), q (Quartett), quint. (Quintett), dd (Dublett von Dubletts), dt (Dublett von Tripletts) usw. Signale, die durch Überlagerung oder Anteile höherer Ordnung nicht interpretierbar waren, wurden mit $\mathrm{m}$ (Multiplett) bzw. $\mathrm{m}_{\mathrm{c}}$ (symmetrisches, zentriertes Multiplett) bezeichnet. Zur Bezeichnung aromatischer Wasserstoff- oder Kohlenstoffatome wurden folgende Abkürzungen verwendet: $i$ (ipso), o (ortho), m (meta), $p$ (para). Die Kopplungskonstanten $J$ sind in $\operatorname{Hertz}(\mathrm{Hz})$ angegeben und beziehen sich, sofern nicht anders angegeben auf ${ }^{1} \mathrm{H}-{ }^{1} \mathrm{H}$-Kopplungen.

${ }^{13} \mathrm{C}$-NMR-Spektren: Die ${ }^{1} \mathrm{H}$-breitbandentkoppelten ${ }^{13} \mathrm{C}-\mathrm{NMR}$-Spektren wurden mit den Modellen Mercury 300 (75 MHz), Unity 300 (75 MHz) und Inova 500 (126 MHz) der Firma Varian aufgenommen. Die chemische Verschiebung $\delta$ ist in ppm angegeben und auf das Signal des jeweiligen Lösungsmittels referenziert. Die Substitutionsmuster der Signale wurden ggf. durch APT-Pulsfolgen ermittelt.

Massenspektren: Zur Aufnahme der El-Spektren diente ein doppelfokussierendes Sektorfeld-Massenspektrometer MAT 95 der Firma Finnigan. Als Ionisierungsmethode kam dabei die Elektronenstoß-Ionisation (EI, $70 \mathrm{eV}$ ) zum Einsatz. ESI-Spektren wurden mit dem Time-of-Flight Massenspektrometer micrOTOF der Firma Bruker aufgenommen. Die Messung der ESI-HRMS-Spektren erfolgte an dem 7-Tesla-Fourier-Transform-IonCyclotron-Resonance (FTICR)-Massenspektrometer Apex IV der Firma Bruker sowie am micrOTOF. Angegeben sind die Quotienten aus Masse zu Ladung sowie in Klammern die 
relativen Intensitäten bezogen auf den Basispeak $(I=100)$. Zur Aufnahme und Auswertung der Spektren diente das Programm XMASS.

\subsubsection{Chromatographische Methoden}

Dünnschichtchromatographie (DC): Zur dünnschichtchromatographischen Reaktionskontrolle wurden DC-Fertigfolien Kieselgel $60 \mathrm{~F}_{254}$ der Firma Merck verwendet. Angegeben sind $R_{\mathrm{f}}$-Werte (Laufhöhe der Substanz relativ zur Laufmittelfront). Neben der UV-Detektion (254 nm, $365 \mathrm{~nm})$ diente eine Molybdatophosphorsäure-Lösung $(5 \mathrm{~g}$ Molybdatophosphorsäure, $100 \mathrm{ml} \mathrm{EtOH)} \mathrm{als} \mathrm{Anfärbereagenz.}$

Säulenchromatographie (SC): Säulenchromatographische Trennungen wurden unter erhöhtem Druck (Flashchromatographie, $0.1-0.8$ bar) mit Kieselgel Geduran ${ }^{\circledR} \operatorname{Si60}(\varnothing=$ 40-63 $\mu \mathrm{m}$ ) der Firma Merck oder via dem Mitteldruckchromatographiesystem Isolera ${ }^{\mathrm{TM}}$ One der Firma Biotage ${ }^{\circledR}$ durchgeführt. Im letztgenannten Fall wurden entweder selbstgepackte Säulen, gefüllt mit obigem Kieselgel, oder Fertigsäulen SNAP KP-Sil $(10 \mathrm{~g}, 25 \mathrm{~g}, 50 \mathrm{~g}, 100 \mathrm{~g})$ der Firma Biotage ${ }^{\circledR}$ verwendet. Die in technischer Qualität vorliegenden Lösungsmittel wurden vor der Verwendung destilliert.

Analytische HPLC: Analytische Trennungen wurden auf einer HPLC-Anlage der Firma Jasco, ausgestattet mit einer Lösungsmittelpumpe PU-2080, einer Mischkammer LG-1590-04, einem Multiwellenlängendetektor MD-2010 Plus und der Steuerung LC-Net II/ADC vorgenommen. Für die Injektion wurde ein automatischer Probenwechsler (Autosampler AS-2055) derselben Firma verwendet. Zur Bedienung, Datenerfassung und -auswertung wurde das Programm ChromNAV der Firma Jasco eingesetzt. Für die analytischen Messungen wurden die Säulen CHIRALPAK ${ }^{\circledR} \mid \mathrm{A}(4.6 \times 250 \mathrm{~mm}, 5 \mu \mathrm{m}$, Daicel Chemical Industries Ltd.) sowie CHIRALPAK ${ }^{\circledR} \mathrm{IB}(4.6 \times 250 \mathrm{~mm}, 5 \mu \mathrm{m}$, Daicel Chemical Industries $L t d$.) mit chiraler stationärer Phase verwendet. Die verwendeten Lösungsmittel waren von HPLC-Qualität. Alle Proben wurden membranfiltriert mit Polytetrafluorethylen(PTFE)-Filtern der Firma Roth $(\varnothing=25 \mathrm{~mm}, 0.2 \mu \mathrm{m})$ bzw. $V W R(\varnothing=25 \mathrm{~mm}, 0.2 \mu \mathrm{m})$. 


\subsection{Synthese verwendeter Reagenzien}

\subsubsection{N,N'Di-iso-propyl-O-tert-butylisoharnstoff (146)}

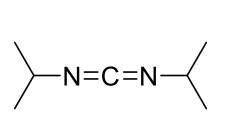

145
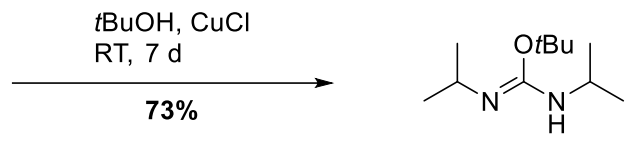

146

N,N'Di-iso-propylcarbodiimid (145) $(50.0 \mathrm{ml}, 40.5 \mathrm{~g}, 321 \mathrm{mmol}, 1.00 \mathrm{Äq}$.) wurde bei Raumtemperatur und unter Lichtausschluss mit tBuOH $(35.1 \mathrm{ml}, 27.4 \mathrm{~g}, 369 \mathrm{mmol}$, 1.15 Äq.) sowie $\mathrm{CuCl}(318 \mathrm{mg}, 3.21 \mathrm{mmol}, 1 \mathrm{~mol} \%)$ versetzt und $7 \mathrm{~d}$ bei dieser Temperatur gerührt. Das Reaktionsgemisch wurde anschließend unter vermindertem Druck (1.0 mbar) fraktioniert destilliert und der Isoharnstoff 146 (47.2 g, 236 mmol, 73\%) als farbloses Öl erhalten.

Sdp.: $\vartheta\left[{ }^{\circ} \mathrm{C}\right]=39$ (1.0 mbar).

IR $(A T R): \tilde{v}\left[\mathrm{~cm}^{-1}\right]=2963,1656,1383,1363,1327,1253,1170,1122,953$.

${ }^{1} \mathrm{H}-\mathrm{NMR}\left(300 \mathrm{MHz}, \mathrm{CDCl}_{3}\right): \delta[\mathrm{ppm}]=1.01-1.08\left(\mathrm{~m}, 12 \mathrm{H}, 2 \times \mathrm{CH}\left(\mathrm{C}_{3}\right)_{2}\right), 1.44(\mathrm{~s}, 9 \mathrm{H}$, $\left.\mathrm{C}\left(\mathrm{CH}_{3}\right)_{3}\right), 3.06-3.17\left(\mathrm{~m}, 1 \mathrm{H}, \mathrm{NHC} \underline{H}\left(\mathrm{CH}_{3}\right)_{2}\right), 3.57-3.77\left(\mathrm{~m}, 1 \mathrm{H}, \mathrm{C}=\mathrm{NC} \underline{\mathrm{H}}\left(\mathrm{CH}_{3}\right)_{2}\right)$.

$\left.{ }^{13} \mathrm{C}-\mathrm{NMR}(75 \mathrm{MHz}, \mathrm{CDCl})_{3}\right): \delta[\mathrm{ppm}]=23.9\left(\mathrm{NHCH}\left(\underline{\mathrm{CH}}_{3}\right)_{2}\right), 24.4\left(\mathrm{C}=\mathrm{NCH}\left(\underline{\mathrm{CH}}_{3}\right)_{2}\right), \quad 28.5$, $29.1\left(\mathrm{C}\left(\underline{\mathrm{C}} \mathrm{H}_{3}\right)_{3}\right), \quad 43.6 \quad\left(\mathrm{NH} \underline{\mathrm{CH}}\left(\mathrm{CH}_{3}\right)_{2}\right), \quad 46.3 \quad\left(\mathrm{C}=\mathrm{N} \underline{\mathrm{CH}}\left(\mathrm{CH}_{3}\right)_{2}\right), \quad 78.2 \quad\left(\underline{\mathrm{C}}\left(\mathrm{CH}_{3}\right)_{3}\right), \quad 149.7$ $\left(\underline{\mathrm{COC}}\left(\mathrm{CH}_{3}\right)_{3}\right)$.

MS (ESI): $m / z(\%)=201.2(9)[\mathrm{M}+\mathrm{H}]^{+}$.

$\mathrm{C}_{11} \mathrm{H}_{24} \mathrm{~N}_{2} \mathrm{O}(200.32)$

ber.: 201.1961

gef.: $201.1967[\mathrm{M}+\mathrm{H}]^{+}(\mathrm{ESI}-\mathrm{HRMS})$. 


\subsubsection{3-Chlor-2-nitrobenzoesäuremethylester (11)}

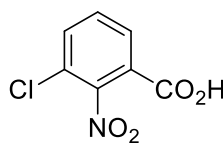

142

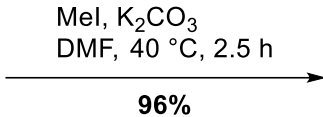

$96 \%$<smiles>COC(=O)c1cccc(Cl)c1[N+](=O)[O-]</smiles>

11

Mel (15.4 ml, $35.2 \mathrm{~g}, 248 \mathrm{mmol}, 2.00$ Äq.) wurde bei Raumtemperatur zu einer Lösung von 3-Chlor-2-nitrobenzoesäure (142) (25.0 g, 124 mmol, 1.00 Äq.) und $\mathrm{K}_{2} \mathrm{CO}_{3}$ (51.4 g, $372 \mathrm{mmol}, 3.00$ Äq.) in DMF (125 ml) gegeben. Das Reaktionsgemisch wurde $2.5 \mathrm{~h}$ bei $40{ }^{\circ} \mathrm{C}$ gerührt, anschließend die Reaktion durch Zugabe von $\mathrm{H}_{2} \mathrm{O}(150 \mathrm{ml})$ beendet und mit EtOAc $(250 \mathrm{ml})$ verdünnt. Die Phasen wurden getrennt und die org. Phase mit $\mathrm{H}_{2} \mathrm{O}$ $(100 \mathrm{ml})$ sowie ges. $\mathrm{NaCl}$-Lsg. $(100 \mathrm{ml})$ gewaschen. Nach Trocknung über $\mathrm{MgSO}_{4}$ und Entfernen des Lösungsmittels unter vermindertem Druck wurde der Methylester 11 $(25.8 \mathrm{~g}, 119 \mathrm{mmol}, 96 \%)$ analysenrein als weißer Feststoff isoliert.

DC: $R_{f}=0.51$ (Petrolether/EtOAc 7:3).

Smp.: $\vartheta\left[{ }^{\circ} \mathrm{C}\right]=106$.

UV $\left(\mathrm{CH}_{3} \mathrm{CN}\right): \lambda_{\max }[\mathrm{nm}](\lg \varepsilon)=203$ (4.610), 290 (3.063).

IR (ATR): $\tilde{v}\left[\mathrm{~cm}^{-1}\right]=3107,2955,1730,1542,1441,1369,1284,1214,1171,1105,1061$, $976,851,820,756,716,692$.

${ }^{1} \mathrm{H}-\mathrm{NMR}\left(300 \mathrm{MHz}, \mathrm{CDCl}_{3}\right): \delta[\mathrm{ppm}]=3.89\left(\mathrm{~s}, 3 \mathrm{H}, \mathrm{CO}_{2} \mathrm{CH}_{3}\right), 7.50(\mathrm{t}, J=8.0 \mathrm{~Hz}, 1 \mathrm{H}$, 5-H), 7.68 (dd, $J=8.1,1.3 \mathrm{~Hz}, 1 \mathrm{H}, 4-\mathrm{H}), 7.96$ (dd, $J=7.8,1.2 \mathrm{~Hz}, 1 \mathrm{H}, 6-\mathrm{H}$ ).

${ }^{13} \mathrm{C}-\mathrm{NMR}\left(75 \mathrm{MHz}, \mathrm{CDCl}_{3}\right): \delta[\mathrm{ppm}]=53.3\left(\mathrm{CO}_{2} \mathrm{CH}_{3}\right), 124.4(\mathrm{C}-1), 126.3$ (C-3), 129.6 (C-5), 130.6 (C-6), 134.7 (C-4), 148.4 (C-2), $162.5\left(\mathrm{CO}_{2} \mathrm{CH}_{3}\right)$.

MS (ESI): $m / z(\%)=238.0(72)[\mathrm{M}+\mathrm{Na}]^{+}, 453.0(100)[2 \times \mathrm{M}+\mathrm{Na}]^{+}$.

$\mathrm{C}_{8} \mathrm{H}_{6} \mathrm{CINO}_{4}$ (215.59) ber.: 237.9878 gef.: $237.9880[\mathrm{M}+\mathrm{Na}]^{+}(\mathrm{ESI}-\mathrm{HRMS})$. 


\subsubsection{2-Benzyloxyessigsäuremethylester (170)}

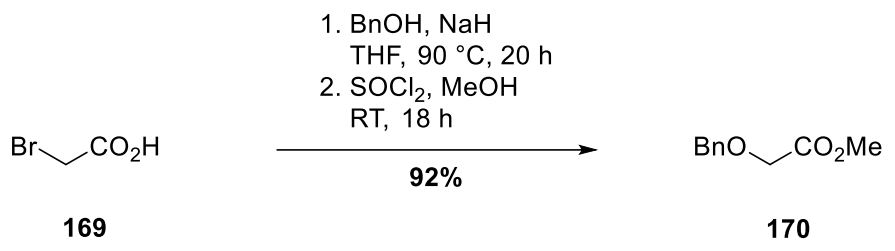

Zu einer Suspension von NaH (60\%ig in Mineralöl, $35.3 \mathrm{~g}, 882 \mathrm{mmol}, 3.50 \mathrm{Äq}$.) in THF $(1.2 \mathrm{l})$ wurde bei $0^{\circ} \mathrm{C}$ eine Lösung des Benzylalkohols $(41.7 \mathrm{ml}, 43.6 \mathrm{~g}, 403 \mathrm{mmol}$, 1.60 Äq.) in THF (150 ml) getropft und anschließend $1 \mathrm{~h}$ bei Raumtemperatur gerührt. Daraufhin wurde eine Lösung der Bromessigsäure (169) (35.0 g, 252 mmol, 1.00 Äq.) in THF (150 ml) bei $0{ }^{\circ} \mathrm{C}$ langsam zugetropft, das Reaktionsgemisch auf $90^{\circ} \mathrm{C}$ erhitzt und 20 h bei dieser Temperatur unter Rückfluss gerührt. Nach Abkühlen auf Raumtemperatur wurde die Reaktionslösung unter vermindertem Druck eingeengt, $\mathrm{H}_{2} \mathrm{O}(500 \mathrm{ml})$ und MTBE $(500 \mathrm{ml})$ hinzugegeben, die Phasen getrennt und die org. Phase $\mathrm{mit}_{2} \mathrm{H}(500 \mathrm{ml})$ extrahiert. Die vereinigten wässr. Phasen wurden mit MTBE $(2 \times 750 \mathrm{ml})$ gewaschen, mit konz. $\mathrm{HCl}(100 \mathrm{ml})$ versetzt und mit EtOAc $(4 \times 400 \mathrm{ml})$ extrahiert. Abschließend wurden die vereinigten EtOAc-Extrakte über $\mathrm{MgSO}_{4}$ getrocknet, das Lösungsmittel unter vermindertem Druck entfernt und die erhaltene Benzyloxyessigsäure im Hochvakuum getrocknet.

$\mathrm{SOCl}_{2}\left(22.0 \mathrm{ml}, 36.0 \mathrm{~g}, 302 \mathrm{mmol}, 1.20 \mathrm{Äq}\right.$.) wurde bei $0{ }^{\circ} \mathrm{C}$ langsam zu MeOH (180 ml) getropft und die resultierende Lösung $30 \mathrm{~min}$ bei dieser Temperatur gerührt. Anschließend wurde eine Lösung der Benzyloxyessigsäure in $\mathrm{MeOH}(70 \mathrm{ml})$ langsam zugetropft und die Reaktionslösung $18 \mathrm{~h}$ bei Raumtemperatur gerührt. Daraufhin wurde das Lösungsmittel unter vermindertem Druck entfernt, der Rückstand in $\mathrm{CH}_{2} \mathrm{Cl}_{2}$ (1.5 I) aufgenommen und mit ges. $\mathrm{NaHCO}_{3}$-Lsg. $(2 \times 400 \mathrm{ml})$ sowie $\mathrm{H}_{2} \mathrm{O}(2 \times 400 \mathrm{ml})$ gewaschen. Die org. Phase wurde über $\mathrm{MgSO}_{4}$ getrocknet, das Lösungsmittel unter vermindertem Druck entfernt und nach Säulenchromatographie an Kieselgel (Petrolether/MTBE 7:1) der Methylester 170 (42.0 g, $233 \mathrm{mmol}, 92 \%)$ als farbloses Öl erhalten.

DC: $R_{\mathrm{f}}=0.21$ (Petrolether/MTBE 6:1).

UV (MeOH): $\lambda_{\max }[\mathrm{nm}](\lg \varepsilon)=207$ (3.934), 252 (2.292), 257 (2.366), 263 (2.157).

IR (ATR): $\tilde{v}\left[\mathrm{~cm}^{-1}\right]=3032,2953,1755,1497,1455,1438,1393,1283,1211,1130,1029$, 908, 741, 699.

${ }^{1} \mathrm{H}-\mathrm{NMR}\left(300 \mathrm{MHz}, \mathrm{CDCl}_{3}\right): \delta[\mathrm{ppm}]=3.74\left(\mathrm{~s}, 3 \mathrm{H}, \mathrm{CO}_{2} \mathrm{CH}_{3}\right), 4.09\left(\mathrm{~s}, 2 \mathrm{H}, \mathrm{CH}_{2} \mathrm{OBn}\right), 4.62$ (s, $\left.2 \mathrm{H}, \mathrm{OCH}_{2} \mathrm{Ph}\right), 7.24-7.37(\mathrm{~m}, 5 \mathrm{H}, 5 \times \mathrm{Ph}-\mathrm{H})$. 
${ }^{13}$ C-NMR $\left(126 \mathrm{MHz}, \mathrm{CDCl}_{3}\right): \delta[\mathrm{ppm}]=51.7\left(\mathrm{CO}_{2} \underline{\mathrm{C}} \mathrm{H}_{3}\right), 67.0\left(\mathrm{CH}_{2} \mathrm{OBn}\right), 73.3\left(\mathrm{OCH}_{2} \mathrm{Ph}\right)$, $128.0\left(\mathrm{Ph}-\mathrm{C}_{p}\right), 128.0,128.4\left(2 \times \mathrm{Ph}-\mathrm{C}_{o}, 2 \times \mathrm{Ph}-\mathrm{C}_{m}\right), 137.0\left(\mathrm{Ph}-\mathrm{C}_{i}\right), 170.7\left(\mathrm{CO}_{2} \mathrm{CH}_{3}\right)$. MS (ESI): $m / z(\%)=198.1(16)\left[\mathrm{M}+\mathrm{NH}_{4}\right]^{+}, 203.1(71)[\mathrm{M}+\mathrm{Na}]^{+}, 219.0(100)[\mathrm{M}+\mathrm{K}]^{+}$. $\mathrm{C}_{10} \mathrm{H}_{12} \mathrm{O}_{3}(180.20)$ ber.: 198.1125

203.0679

219.0418

gef.: $198.1123\left[\mathrm{M}+\mathrm{NH}_{4}\right]^{+}$

$203.0683[\mathrm{M}+\mathrm{Na}]^{+}$

$219.0419[\mathrm{M}+\mathrm{K}]^{+}(\mathrm{ESI}-\mathrm{HRMS})$. 


\subsubsection{1-Benzyloxy-3-triphenylphosphoranylidenpropan-2-on (171)}

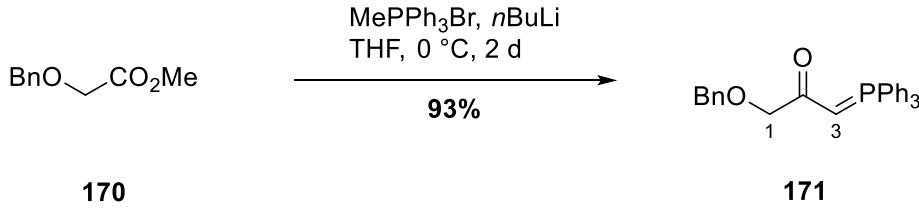

Zu einer Suspension von Methyltriphenylphosphoniumbromid (183 g, 512 mmol, 2.20 Äq.) in THF (1.4I) wurde bei $0^{\circ} \mathrm{C} n \mathrm{BuLi}(2.5 \mathrm{M}$ in $n$-Hexan, $205 \mathrm{ml}, 512 \mathrm{mmol}, 2.20$ Äq.) getropft und die resultierende Mischung $30 \mathrm{~min}$ bei dieser Temperatur gerührt. Anschließend wurde eine Lösung des Methylesters 170 (41.9 g, 233 mmol, 1.00 Äq.) in THF $(100 \mathrm{ml})$ bei $0^{\circ} \mathrm{C}$ zugetropft und $2 \mathrm{~d}$ bei dieser Temperatur gerührt. Das Lösungsmittel wurde unter vermindertem Druck entfernt, $\mathrm{H}_{2} \mathrm{O}(1.5 \mathrm{I})$ hinzugegeben und mit EtOAc $(3 \times 500 \mathrm{ml})$ extrahiert. Die wässr. Phase wurde mit $\mathrm{NaCl}$ gesättigt und erneut mit EtOAc $(500 \mathrm{ml})$ extrahiert. Daraufhin wurden die vereinigten org. Phasen über $\mathrm{MgSO}_{4}$ getrocknet, das Lösungsmittel unter vermindertem Druck entfernt und mittels säulenchromatographischer Reinigung an Kieselgel (EtOAc/MeOH 20:1) die Zielverbindung 171 (92.1 g, 217 mmol, 93\%) als leicht brauner Feststoff erhalten.

DC: $R_{\mathrm{f}}=0.48(\mathrm{EtOAc} / \mathrm{MeOH} 20: 1)$.

UV $\left(\mathrm{CH}_{3} \mathrm{CN}\right): \lambda_{\max }[\mathrm{nm}](\lg \varepsilon)=197$ (4.804), 292 (3.515).

IR (ATR): $\tilde{v}\left[\mathrm{~cm}^{-1}\right]=3047,2849,2822,1733,1573,1539,1479,1454,1437,1403,1368$, 1342, 1251, 1207, 1181, 1165, 1095, 1079, 1026, 1007, 992, 940, 920, 868, 750, 716, 692, 678, 667, 639, 594, 575, 543, 512.

${ }^{1} \mathrm{H}-\mathrm{NMR}\left(300 \mathrm{MHz}, \mathrm{CDCl}_{3}\right): \delta[\mathrm{ppm}]=4.02\left(\mathrm{~s}, 2 \mathrm{H}, 1-\mathrm{H}_{2}\right), 4.23\left(\mathrm{~d}, J_{\mathrm{PH}}=25.4 \mathrm{~Hz}, 1 \mathrm{H}\right.$, 3-H), 4.66 (s, $\left.2 \mathrm{H}, \mathrm{OCH}_{2} \mathrm{Ph}\right), 7.20-7.73(\mathrm{~m}, 20 \mathrm{H}, 20 \times \mathrm{Ph}-\mathrm{H})$.

${ }^{13} \mathrm{C}-N M R\left(126 \mathrm{MHz}, \mathrm{CDCl}_{3}\right): \delta[\mathrm{ppm}]=49.4\left(\mathrm{~d}, \mathrm{~J}_{\mathrm{PC}}=109.4 \mathrm{~Hz}, \mathrm{C}-3\right), 73.2\left(\mathrm{OCH}_{2} \mathrm{Ph}\right), 75.1$ $\left(\mathrm{d}, J_{\mathrm{PC}}=13.3 \mathrm{~Hz}, \mathrm{C}-1\right), 126.8\left(\mathrm{~d}, \mathrm{JPC}_{\mathrm{PC}}=90.9 \mathrm{~Hz}, 3 \times \mathrm{PPh}_{3}-\mathrm{C}_{i}\right), 127.4\left(\mathrm{Ph}-\mathrm{C}_{p}\right), 127.8,128.2$ $\left(2 \times \mathrm{Ph}_{-} \mathrm{C}_{0}, 2 \times \mathrm{Ph}-\mathrm{C}_{m}\right), 128.8\left(\mathrm{~d}, \mathrm{~J}_{\mathrm{PC}}=12.3 \mathrm{~Hz}, 6 \times \mathrm{PPh}_{3}-\mathrm{C}_{m}\right), 132.0\left(\mathrm{~d}, \mathrm{~J}_{\mathrm{PC}}=2.9 \mathrm{~Hz}, 3 \times\right.$ $\left.\mathrm{PPh}_{3}-\mathrm{C}_{p}\right), 133.1\left(\mathrm{~d}, \mathrm{~J}_{\mathrm{PC}}=10.3 \mathrm{~Hz}, 6 \times \mathrm{PPh}_{3}-\mathrm{C}_{o}\right), 138.5\left(\mathrm{Ph}-\mathrm{C}_{i}\right), 189.7\left(\mathrm{~d}, \mathrm{~J}_{\mathrm{PC}}=3.1 \mathrm{~Hz}\right.$, C-2).

MS (ESI): $m / z(\%)=425.2(100)[\mathrm{M}+\mathrm{H}]^{+}, 447.2(5)[\mathrm{M}+\mathrm{Na}]^{+}, 463.1(20)[\mathrm{M}+\mathrm{K}]^{+}$.

$\mathrm{C}_{28} \mathrm{H}_{25} \mathrm{O}_{2} \mathrm{P}(424.48)$

ber.: 425.1665

gef.: $425.1665[M+H]^{+}$(ESI-HRMS). 


\subsection{5 (S)-2-Amino-3-methylbutan-1-ol ((S)-204)}

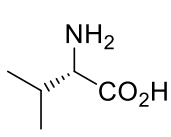

(S)-203

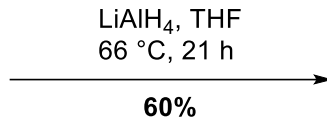

$60 \%$

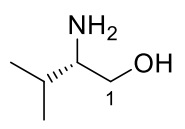

(S)-204

Zu einer Suspension von $\mathrm{LiAlH}_{4}(25.8 \mathrm{~g}, 683 \mathrm{mmol}, 2.00 \mathrm{Äq}$.) in THF (300 ml) wurde bei $0^{\circ} \mathrm{C}$ portionsweise L-Valin ((S)-203) $(40.0 \mathrm{~g}, 341 \mathrm{mmol}, 1.00 \mathrm{Äq}$.) gegeben und das Reaktionsgemisch anschließend $21 \mathrm{~h}$ bei $66^{\circ} \mathrm{C}$ unter Rückfluss gerührt. Die Reaktion wurde durch Zugabe von eiskaltem $\mathrm{H}_{2} \mathrm{O}(40 \mathrm{ml})$ bei $0^{\circ} \mathrm{C}$ beendet und der ausgefallene Feststoff abfiltriert. Der Feststoff wurde in $\mathrm{THF} / \mathrm{H}_{2} \mathrm{O}(4: 1,200 \mathrm{ml})$ suspendiert, 30 min bei Raumtemperatur gerührt und erneut abfiltriert. Der Vorgang wurde einmal wiederholt und die vereinigten Filtrate unter vermindertem Druck eingeengt. Der Rückstand wurde in $\mathrm{CHCl}_{3}(200 \mathrm{ml})$ aufgenommen und verbliebenes $\mathrm{H}_{2} \mathrm{O}$ mittels Wasserabscheider abgetrennt. Daraufhin wurde das Lösungsmittel unter vermindertem Druck entfernt und nach fraktionierter Destillation unter vermindertem Druck (17 mbar) L-Valinol ((S)-204) (21.3 g, $206 \mathrm{mmol}, 60 \%)$ als farbloser Feststoff isoliert.

Drehwert: $[\alpha]_{D}^{20}\left[{ }^{\circ}\right]=+23.3\left(c=0.50, \mathrm{CHCl}_{3}\right)$.

UV $\left(\mathrm{CH}_{3} \mathrm{CN}\right): \lambda_{\max }[\mathrm{nm}](\lg \varepsilon)=193(2.960)$.

IR (ATR): $\widetilde{v}\left[\mathrm{~cm}^{-1}\right]=2995,2945,2888,2835,2361,2332,1690,1585,1559,1513,1458$, 1440, 1417, 1397, 1353, 1242, 1202, 1177, 1162, 1115, 1066, 1033, 1014, 1000, 970, 904, 847, 824, 808, 759, 742, 724, 643, 587, 572, 554, 529, 503.

${ }^{1} \mathrm{H}-\mathrm{NMR}\left(300 \mathrm{MHz}, \mathrm{CDCl}_{3}\right): \delta[\mathrm{ppm}]=0.85\left(\mathrm{~d}, J=3.0 \mathrm{~Hz}, 3 \mathrm{H}, 3-\mathrm{CH}_{3}\right), 0.86(\mathrm{~d}, J=3.0 \mathrm{~Hz}$, $\left.3 \mathrm{H}, 4-\mathrm{H}_{3}\right), 1.47-1.56(\mathrm{~m}, 1 \mathrm{H}, 3-\mathrm{H}), 2.35\left(\mathrm{sbr}, 3 \mathrm{H}, 1-\mathrm{OH}, 2-\mathrm{NH}_{2}\right), 2.48-2.54(\mathrm{~m}, 1 \mathrm{H}, 2-\mathrm{H})$, 3.24 (dd, $J=12.0,9.0 \mathrm{~Hz}, 1 \mathrm{H}$ ), 3.57 (dd, $J=9.0,6.0 \mathrm{~Hz}, 1 \mathrm{H})\left(1-\mathrm{H}_{2}\right)$.

${ }^{13} \mathrm{C}-\mathrm{NMR}\left(126 \mathrm{MHz}, \mathrm{CDCl}_{3}\right): \delta[\mathrm{ppm}]=18.4,19.3\left(\mathrm{C}-4,3-\mathrm{CH}_{3}\right), 31.4$ (C-3), 58.4 (C-2), $64.5(\mathrm{C}-1)$.

MS (ESI): $m / z(\%)=104.1(100)[M+H]^{+}$.

$\mathrm{C}_{5} \mathrm{H}_{13} \mathrm{NO}(103.17)$

ber.: 104.1070

gef.: $104.1071[\mathrm{M}+\mathrm{H}]^{+}($ESI-HRMS). 


\subsubsection{1-Brom-2-(dibrommethyl)naphthalin (199)}

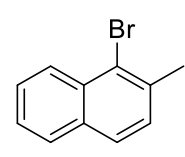

198

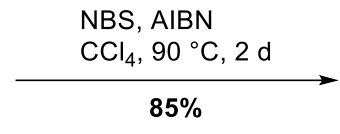

$85 \%$

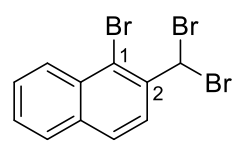

199

Zu einer Lösung von 1-Brom-2-methylnaphthalin (198) (90\%ig, 28.2 ml, 40.0 g, 163 mmol, 1.00 Äq.) in $\mathrm{CCl}_{4}(800 \mathrm{ml}$ ) wurden bei Raumtemperatur NBS (86.8 g, $488 \mathrm{mmol}, 3.00$ Äq.) und AIBN (5.36 g, $32.6 \mathrm{mmol}, 20 \mathrm{~mol} \%$ ) gegeben und die resultierende Suspension bei $90{ }^{\circ} \mathrm{C}$ unter Rückfluss gerührt. Es wurde weiteres AIBN (jeweils $2.68 \mathrm{~g}, 16.3 \mathrm{mmol}$, 10 mol\%) nach 19 und $27 \mathrm{~h}$ hinzugegeben und das Reaktionsgemisch insgesamt für $2 \mathrm{~d}$ bei $90^{\circ} \mathrm{C}$ unter Rückfluss gerührt. Nach Abkühlen auf Raumtemperatur wurde der Feststoff abfiltriert und mit $\mathrm{CCl}_{4}(400 \mathrm{ml})$ gewaschen. Das Filtrat wurde unter vermindertem Druck auf ca. $500 \mathrm{ml}$ eingeengt, mit ges. $\mathrm{NaHSO}_{3}$-Lsg. (400 ml) gewaschen, über $\mathrm{MgSO}_{4}$ getrocknet und das Lösungsmittel unter vermindertem Druck entfernt. Umkristallisation aus EtOH und darauffolgende säulenchromatographische Reinigung an Kieselgel (Petrolether) lieferte das Tribromid 199 (52.2 g, 138 mmol, 85\%) als farblosen Feststoff.

DC: $R_{\mathrm{f}}=0.48$ (Petrolether).

UV $\left(\mathrm{CH}_{3} \mathrm{CN}\right): \lambda_{\max }[\mathrm{nm}](\mathrm{lg} \varepsilon)=300$ (4.771), 312 (3.481), 335 (3.522).

IR $($ ATR $): \widetilde{v}\left[\mathrm{~cm}^{-1}\right]=2940,1908,1619,1595,1556,1501,1459,1382,1323,1301,1258$, 1218, 1206, 1141, 1033, 973, 958, 906, 863, 804, 770, 747, 734, 677, 665, 464, 596, 528, 515.

${ }^{1} \mathrm{H}-\mathrm{NMR}\left(300 \mathrm{MHz}, \mathrm{CDCl}_{3}\right): \delta[\mathrm{ppm}]=7.47\left(\mathrm{~s}, 1 \mathrm{H}, \mathrm{CHBr}_{2}\right), 7.53-7.66(\mathrm{~m}, 2 \mathrm{H}, 6-\mathrm{H}, 7-\mathrm{H})$, $7.82(\mathrm{~d}, J=7.2 \mathrm{~Hz}, 1 \mathrm{H}, 5-\mathrm{H}), 7.88(\mathrm{~d}, J=8.7 \mathrm{~Hz}, 1 \mathrm{H}, 4-\mathrm{H}), 8.05(\mathrm{~d}, J=8.7 \mathrm{~Hz}, 1 \mathrm{H}, 3-\mathrm{H})$, $8.29(\mathrm{~d}, \mathrm{~J}=8.4 \mathrm{~Hz}, 1 \mathrm{H}, 8-\mathrm{H})$.

${ }^{13} \mathrm{C}-N M R\left(126 \mathrm{MHz}, \mathrm{CDCl}_{3}\right): \delta[\mathrm{ppm}]=41.2\left(\mathrm{CHBr}_{2}\right), 119.5$ (C-1), 126.7 (C-3), 127.9 (C-6), 128.2, 128.3, 128.4 (C-5, C-7, C-8), 129.0 (C-4), 131.2 (C-8a), 134.6 (C-4a), 137.9 (C-2).

MS (El): $m / z(\%)=139.1(100)[\mathrm{M}-3 \times \mathrm{Br}]^{+}, 219.0(7)[\mathrm{M}-2 \times \mathrm{Br}]^{+}, 298.9(95)[\mathrm{M}-\mathrm{Br}]^{+}$, $379.8(5)[\mathrm{M}]^{+}$.

$\mathrm{C}_{11} \mathrm{H}_{7} \mathrm{Br}_{3}(378.89)$

ber.: 377.8078

gef.: $377.8071[\mathrm{M}]^{+}(\mathrm{El}-\mathrm{HRMS})$. 


\subsubsection{1-Brom-2-naphthaldehyd (200)}

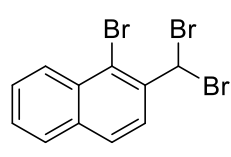

199

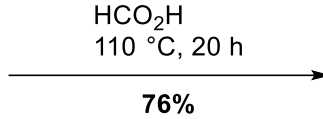

$76 \%$

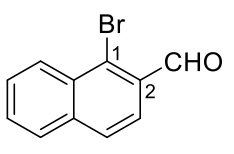

200

Eine Lösung des Tribromids 199 (52.0 g, 137 mmol, 1.00 Äq.) in Ameisensäure (88\%ig, $1.4 \mathrm{I})$ wurde $20 \mathrm{~h}$ bei $110^{\circ} \mathrm{C}$ unter Rückfluss gerührt. Nach Abkühlen auf Raumtemperatur wurde das Lösungsmittel unter vermindertem Druck entfernt, der Rückstand in $\mathrm{H}_{2} \mathrm{O}$ $(600 \mathrm{ml})$ aufgenommen und mit $\mathrm{CH}_{2} \mathrm{Cl}_{2}(3 \times 400 \mathrm{ml})$ extrahiert. Die vereinigten org. Phasen wurden über $\mathrm{MgSO}_{4}$ getrocknet, das Lösungsmittel unter vermindertem Druck entfernt und nach Umkristallisation aus $\mathrm{CH}_{2} \mathrm{Cl}_{2}$ der Aldehyd 200 (24.4 g, 104 mmol, 76\%) als leicht gelbe kristalline Nadeln isoliert.

DC: $R_{\mathrm{f}}=0.25$ (Petrolether/EtOAc 30:1).

UV $\left(\mathrm{CH}_{3} \mathrm{CN}\right): \lambda_{\max }[\mathrm{nm}](\lg \varepsilon)=213$ (4.266), 228 (4.193), 248 (4.568), 254 (4.653), 290 (3.956), 300 (3.879), 344 (3.363), 353 (3.362).

IR (ATR): $\widetilde{v}\left[\mathrm{~cm}^{-1}\right]=3057,1683,1454,1232,1215,969,887,869,810,751,538$.

${ }^{1} \mathrm{H}-\mathrm{NMR}\left(300 \mathrm{MHz}, \mathrm{CDCl}_{3}\right): \delta[\mathrm{ppm}]=7.61-7.72(\mathrm{~m}, 2 \mathrm{H}, 6-\mathrm{H}, 7-\mathrm{H}), 7.77-7.97(\mathrm{~m}, 3 \mathrm{H}$, 3-H, 4-H, 5-H), 8.43-8.56 (m, $1 \mathrm{H}, 8-\mathrm{H}), 10.66$ (s, $1 \mathrm{H}, \mathrm{CHO})$.

${ }^{13} \mathrm{C}-\mathrm{NMR}\left(126 \mathrm{MHz}, \mathrm{CDCl}_{3}\right): \delta[\mathrm{ppm}]=124.1$ (C-3), 128.1, 128.2, 128.3, 128.5 (C-4, C-5, C-7, C-8), 129.7 (C-6), 131.2, 131.3, 132.1 (C-1, C-4a, C-8a), 137.2 (C-2), 192.8 (CHO). MS $(\mathrm{ESI}): m / z(\%)=257.0(100)[\mathrm{M}+\mathrm{Na}]^{+}$.

$\mathrm{C}_{11} \mathrm{H}_{7} \mathrm{BrO}(235.08)$ ber.: 256.9572 gef.: $256.9573[\mathrm{M}+\mathrm{Na}]^{+}(\mathrm{ESI}-\mathrm{HRMS})$. 


\subsubsection{1-Brom-2-naphthoesäure (201)}

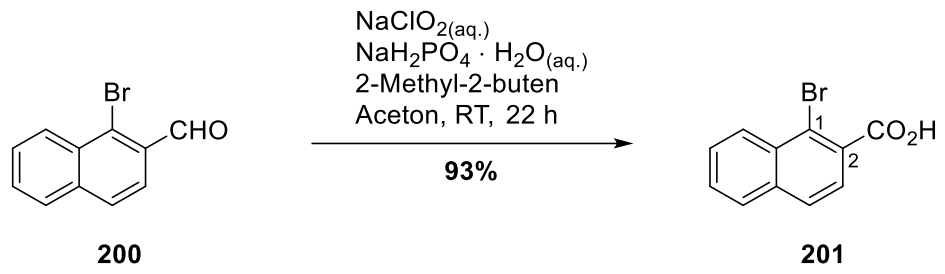

Zu einer Lösung des Aldehyds 200 (24.0 g, 102 mmol, 1.00 Äq.) und 2-Methyl-2-buten (80.8 ml, $53.5 \mathrm{~g}, 725 \mathrm{mmol}, 7.10 \mathrm{Äq}$.) in Aceton (1.5 I) wurde bei $0{ }^{\circ} \mathrm{C}$ eine Lösung von $\mathrm{NaClO}_{2}$ (80\%ig, $69.2 \mathrm{~g}, 613 \mathrm{mmol}, 6.00 \mathrm{Äq}$ ) und $\mathrm{NaH}_{2} \mathrm{PO}_{4} \cdot \mathrm{H}_{2} \mathrm{O}$ (98.6 g, $715 \mathrm{mmol}$, 7.00 Äq.) in $\mathrm{H}_{2} \mathrm{O}(750 \mathrm{ml})$ getropft und die Reaktionslösung $22 \mathrm{~h}$ bei Raumtemperatur gerührt. Anschließend wurde das Lösungsmittel unter vermindertem Druck entfernt, der Rückstand in verd. $\mathrm{HCl}(2 \mathrm{M}, 750 \mathrm{ml})$ aufgenommen, mit $\mathrm{Et}_{2} \mathrm{O}(3 \times 500 \mathrm{ml})$ extrahiert und die vereinigten org. Phasen über $\mathrm{MgSO}_{4}$ getrocknet. Nach Entfernen des Lösungsmittels unter vermindertem Druck und Umkristallisation aus EtOAc wurde die Carbonsäure 201 $(23.9 \mathrm{~g}, 95.1 \mathrm{mmol}, 93 \%)$ als leicht gelber Feststoff erhalten.

UV $\left(\mathrm{CH}_{3} \mathrm{CN}\right): \lambda_{\max }[\mathrm{nm}](\lg \varepsilon)=226$ (4.706), 285 (3.840), 326 (3.049).

IR (ATR): $\tilde{v}\left[\mathrm{~cm}^{-1}\right]=2764,2583,1690,1659,1619,1599,1548,1498,1460,1430,1395$, 1309, 1265, 1240, 1213, 1160, 1143, 1124, 1027, 954, 932, 883, 863, 822, 779, 755, 677, $663,600,564,537$.

${ }^{1} \mathrm{H}-\mathrm{NMR}\left(300 \mathrm{MHz}\right.$, Aceton- $\left.\mathrm{d}_{6}\right): \delta[\mathrm{ppm}]=7.61-7.79(\mathrm{~m}, 3 \mathrm{H}, 5-\mathrm{H}, 6-\mathrm{H}, 7-\mathrm{H}), 7.93-8.05$ (m, $2 \mathrm{H}, 3-\mathrm{H}, 4-\mathrm{H}), 8.39-8.43(\mathrm{~m}, 1 \mathrm{H}, 8-\mathrm{H})$.

${ }^{13} \mathrm{C}-N M R\left(126 \mathrm{MHz}\right.$, Aceton- $\left.\mathrm{d}_{6}\right): \delta[\mathrm{ppm}]=121.6(\mathrm{C}-1), 126.4(\mathrm{C}-3), 128.6,128.8,128.8$, 129.1, 129.2 (C-4, C-5, C-6, C-7, C-8), 132.7, 133.3 (C-4a, C-8a), 135.8 (C-2), 168.2 $\left(\mathrm{CO}_{2} \mathrm{H}\right)$.

MS (ESI): $m / z(\%)=251.0(90)[\mathrm{M}+\mathrm{H}]^{+}, 273.0(100)[\mathrm{M}+\mathrm{Na}]^{+}, 524.9(18)[2 \times \mathrm{M}+\mathrm{Na}]^{+}$.

$\mathrm{C}_{11} \mathrm{H}_{7} \mathrm{BrO}_{2}(251.08)$

ber.: 250.9702

272.9522

gef.: $250.9709[\mathrm{M}+\mathrm{H}]^{+}$

$272.9524[\mathrm{M}+\mathrm{Na}]^{+}(\mathrm{ESI}-\mathrm{HRMS})$. 


\subsection{9 (S)-2-(1-Bromnaphthalin-2-yl)-4-iso-propyl-4,5-dihydrooxazol}

$((S)-202)$

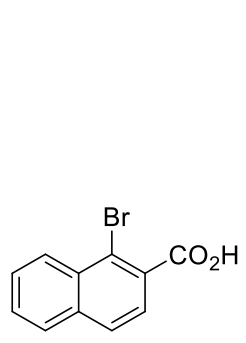

201

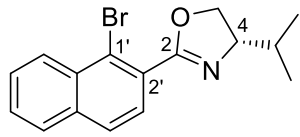

(S)-202

Oxalylchlorid (25.3 ml, $37.9 \mathrm{~g}, 299 \mathrm{mmol}, 2.00 \mathrm{Äq}$.) wurde bei $0{ }^{\circ} \mathrm{C}$ zu einer Suspension der Carbonsäure 201 (37.5 g, 149 mmol, 1.00 Äq.) und einer katalytischen Menge DMF $(1.16 \mathrm{ml}, 1.09 \mathrm{~g}, 14.9 \mathrm{mmol}, 10 \mathrm{~mol} \%)$ in Toluol $(300 \mathrm{ml})$ getropft und $3 \mathrm{~h}$ bei Raumtemperatur gerührt. Daraufhin wurde das Lösungsmittel unter vermindertem Druck entfernt und das Säurechlorid im Hochvakuum getrocknet.

Das Säurechlorid wurde in $\mathrm{CH}_{2} \mathrm{Cl}_{2}(250 \mathrm{ml})$ gelöst, bei $0{ }^{\circ} \mathrm{C}$ zu einer Lösung von L-Valinol ((S)-204) (16.9 g, 164 mmol, 1.10 Äq.) sowie $\mathrm{NEt}_{3}$ (43.1 ml, $31.3 \mathrm{~g}, 309$ mmol, 2.07 Äq.) in $\mathrm{CH}_{2} \mathrm{Cl}_{2}$ (1.5 I) getropft und $23 \mathrm{~h}$ bei Raumtemperatur gerührt. Anschließend wurde das Reaktionsgemisch mit verd. $\mathrm{HCl}(1 \mathrm{M}, 750 \mathrm{ml})$ sowie ges. NaCl-Lsg. (750 ml) gewaschen, die vereinigten wässr. Phasen mit EtOAc $(5 \times 500 \mathrm{ml})$ extrahiert, die vereinigten org. Phasen über $\mathrm{MgSO}_{4}$ getrocknet und das Lösungsmittel unter vermindertem Druck entfernt.

Das erhaltene Carbonsäureamid wurde in $\mathrm{CH}_{2} \mathrm{Cl}_{2}(2 \mathrm{I})$ gelöst, bei $0^{\circ} \mathrm{C} \mathrm{NEt}_{3}(62.5 \mathrm{ml}$, $45.3 \mathrm{~g}, \quad 448 \mathrm{mmol}$, 3.00 Äq.) sowie $\mathrm{MsCl}$ (17.3 ml, $25.7 \mathrm{~g}, 224 \mathrm{mmol}, 1.50$ Äq.) hinzugetropft, die resultierende Reaktionslösung $3 \mathrm{~h}$ bei Raumtemperatur gerührt und daraufhin das Lösungsmittel unter vermindertem Druck entfernt.

Das Mesylat wurde in $\mathrm{MeOH}$ (1.5 I) gelöst, $\mathrm{KOH}$ (85\%ig, $49.3 \mathrm{~g}, 747 \mathrm{mmol}, 5.00$ Äq.) bei Raumtemperatur hinzugegeben und das Reaktionsgemisch $13 \mathrm{~h}$ bei dieser Temperatur gerührt. Nach Entfernen des Lösungsmittels unter vermindertem Druck wurde der Rückstand in $\mathrm{H}_{2} \mathrm{O}(1.5 \mathrm{I})$ aufgenommen und mit EtOAc $(3 \times 1 \mathrm{I})$ extrahiert. Die vereinigten org. Phasen wurden über $\mathrm{MgSO}_{4}$ getrocknet, das Lösungsmittel unter vermindertem Druck entfernt und nach säulenchromatographischer Reinigung an Kieselgel (Petrolether/EtOAc 7:1) die Zielverbindung (S)-202 (39.0 g, 123 mmol, 82\%) als gelbes ÖI isoliert.

Drehwert: $[\alpha]_{D}^{20}\left[^{\circ}\right]=-51.9\left(c=0.50, \mathrm{CHCl}_{3}\right)$.

UV $\left(\mathrm{CH}_{3} \mathrm{CN}\right): \lambda_{\max }[\mathrm{nm}](\lg \varepsilon)=227$ (4.767), 286 (3.849), 322 (2.944). 
IR (ATR): $\widetilde{v}\left[\mathrm{~cm}^{-1}\right]=3058,2956,2926,2895,2871,1657,1596,1555,1498,1463,1425$, 1374, 1344, 1323, 1300, 1282, 1256, 1237, 1211, 1165, 1149, 1137, 1094, 1029, 974, 952, 925, 894, 864, 816, 770, 748, 662, 600, 530.

${ }^{1} \mathrm{H}-\mathrm{NMR}\left(300 \mathrm{MHz}, \mathrm{CDCl}_{3}\right): \delta[\mathrm{ppm}]=1.01(\mathrm{~d}, J=6.0 \mathrm{~Hz}, 3 \mathrm{H}), 1.08(\mathrm{~d}, J=6.0 \mathrm{~Hz}, 3 \mathrm{H})$ $\left(\mathrm{CH}\left(\mathrm{C}_{3}\right)_{2}\right), 1.88-2.03\left(\mathrm{~m}, 1 \mathrm{H}, \mathrm{C} \underline{\mathrm{H}}\left(\mathrm{CH}_{3}\right)_{2}\right), 4.17-4.26\left(\mathrm{~m}, 2 \mathrm{H}, 4-\mathrm{H}, 5-\mathrm{H}_{\mathrm{a}}\right), 4.44-4.53(\mathrm{~m}$, $\left.1 \mathrm{H}, 5-\mathrm{H}_{\mathrm{b}}\right), 7.52-7.63\left(\mathrm{~m}, 3 \mathrm{H}, 5^{\prime}-\mathrm{H}^{\prime}, 6^{\prime}-\mathrm{H}^{\prime}, 7^{\prime}-\mathrm{H}\right), 7.79-7.82\left(\mathrm{~m}, 2 \mathrm{H}, 3^{\prime}-\mathrm{H}, 4^{\prime}-\mathrm{H}\right), 8.40$ (d, J = $\left.9.0 \mathrm{~Hz}, 1 \mathrm{H}, 8^{\prime}-\mathrm{H}\right)$.

${ }^{13} \mathrm{C}-\mathrm{NMR}\left(126 \mathrm{MHz}, \mathrm{CDCl}_{3}\right): \delta[\mathrm{ppm}]=18.4,18.9 \quad\left(\mathrm{CH}\left(\underline{\mathrm{CH}}_{3}\right)_{2}\right), \quad 32.7 \quad\left(\underline{\mathrm{C}} \mathrm{H}\left(\mathrm{CH}_{3}\right)_{2}\right), \quad 70.5$ (C-5), 73.0 (C-4), 123.0 (C-1'), 126.7, 127.6, 127.6, 128.1, 128.2 (C-3', C-4', C-5', C-6', C-7', C-8'), 128.6 (C-8a'), 132.2 (C-4a'), 134.8 (C-2'), 163.7 (C-2).

MS (EI): $m / z(\%)=126.0(44)\left[\mathrm{C}_{10} \mathrm{H}_{6}\right]^{+}, 274.0(100)\left[\mathrm{M}-\mathrm{C}_{3} \mathrm{H}_{7}\right]^{+}, 317.0(13)[\mathrm{M}]^{+}$.

$\mathrm{C}_{16} \mathrm{H}_{16} \mathrm{BrNO}(318.21)$

ber.: 317.0415

gef.: $317.0417[\mathrm{M}]^{+}($El-HRMS). 


\subsubsection{0 (S)-2,2'-Bis((S)-4-iso-propyl-4,5-dihydrooxazol-2-yl)-1,1'-binaph- thalin $((S, S)-91 b)$}

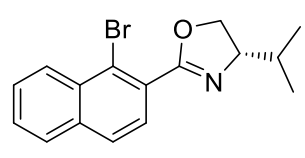

(S)-202

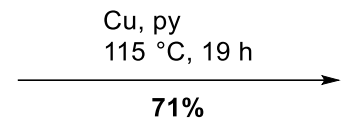

Zu einer Lösung des Bromarens (S)-202 (28.7 g, 90.4 mmol, 1.00 Äq.) in Pyridin (1 I, frisch destilliert über $\mathrm{CaH}$ ) wurde bei Raumtemperatur frisch aktiviertes Kupferpulver (86.1 g, $1.36 \mathrm{~mol}, 15.0$ Äq.) gegeben, die Reaktionslösung auf $115^{\circ} \mathrm{C}$ erwärmt und bei dieser Temperatur unter Rückfluss $19 \mathrm{~h}$ gerührt. Nach Abkühlen auf Raumtemperatur wurde das Lösungsmittel unter vermindertem Druck entfernt, der Rückstand in $\mathrm{CH}_{2} \mathrm{Cl}_{2}$ (1 I) aufgenommen, durch Celite ${ }^{\circledR}$ filtriert und mit $\mathrm{CH}_{2} \mathrm{Cl}_{2}(3 \times 500 \mathrm{ml})$ nachgewaschen. Das Filtrat wurde mit konz. $\mathrm{NH}_{3}(4 \times 1 \mathrm{I})$ gewaschen, bis die wässr. Phase nahezu farblos blieb, über $\mathrm{MgSO}_{4}$ getrocknet und das Lösungsmittel unter vermindertem Druck entfernt. Säulenchromatographie an Kieselgel (Petrolether/EtOAc 9:1) und Umkristallisation aus EtOAc lieferte den (S,S)-IPr-BOXAX-Liganden (S,S)-91b (15.4 g, $32.3 \mathrm{mmol}, 71 \%)$ in Form leicht gelber Kristalle.

Drehwert: $[\alpha]_{D}^{22}\left[^{\circ}\right]=-224.5\left(c=0.54, \mathrm{CHCl}_{3}\right)$.

UV $\left(\mathrm{CH}_{3} \mathrm{CN}\right): \lambda_{\max }[\mathrm{nm}](\lg \varepsilon)=229$ (4.753), 289 (4.036), 337 (3.150).

IR (ATR): $\tilde{v}\left[\mathrm{~cm}^{-1}\right]=3043,2953,2896,2867,2364,2335,1645,1597,1560,1505,1464$, $1425,1381,1363,1337,1316,1292,1273,1258,1230,1165,1147,1057,1027,1012$, 982, 948, 909, 857, 834, 822, 798, 758, 712, 690, 679, 571, 531, 522.

${ }^{1} \mathrm{H}-\mathrm{NMR}\left(300 \mathrm{MHz}, \mathrm{CDCl}_{3}\right): \delta[\mathrm{ppm}]=0.58\left(\mathrm{dd}, J=6.7,2.7 \mathrm{~Hz}, 12 \mathrm{H}, 2 \times \mathrm{CH}\left(\mathrm{CH}_{3}\right)_{2}\right), 1.28$ (dq, $\left.J=13.2,6.6 \mathrm{~Hz}, 2 \mathrm{H}, 2 \times \mathrm{CH}\left(\mathrm{CH}_{3}\right)_{2}\right), 3.54-3.59\left(\mathrm{~m}, 2 \mathrm{H}, 2 \times 4^{\prime}-\mathrm{H}\right), 3.60-3.73(\mathrm{~m}, 4 \mathrm{H}$, $\left.2 \times 5^{\prime}-\mathrm{H}_{2}\right), 7.17-7.21(\mathrm{~m}, 4 \mathrm{H}, 2 \times 6-\mathrm{H}, 2 \times 7-\mathrm{H}), 7.43$ (ddd, J=8.1, 5.4, $2.7 \mathrm{~Hz}, 2 \mathrm{H}, 2 \times$ $5-\mathrm{H}), 7.87(\mathrm{~d}, J=8.3 \mathrm{~Hz}, 2 \mathrm{H}, 2 \times 3-\mathrm{H}), 7.91(\mathrm{~d}, J=8.5 \mathrm{~Hz}, 2 \mathrm{H}, 2 \times 4-\mathrm{H}), 8.08(\mathrm{~d}, J=$ $8.6 \mathrm{~Hz}, 2 \mathrm{H}, 2 \times 8-\mathrm{H})$.

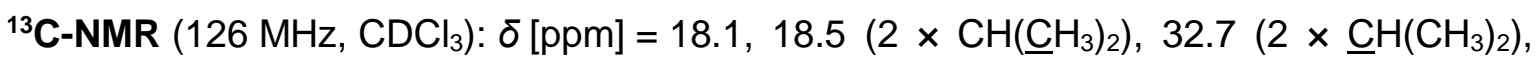
$70.0\left(2 \times C^{\prime}-5^{\prime}\right), 72.4\left(2 \times C^{\prime}-4^{\prime}\right), 125.8(2 \times C-8 a), 126.1,126.2,126.7,127.1,127.5,127.7$ $(2 \times \mathrm{C}-3,2 \times \mathrm{C}-4,2 \times \mathrm{C}-5,2 \times \mathrm{C}-6,2 \times \mathrm{C}-7,2 \times \mathrm{C}-8), 133.0,134.2(2 \times \mathrm{C}-2,2 \times \mathrm{C}-4 \mathrm{a})$, $\left.137.9(2 \times \mathrm{C}-1), 163.7(2 \times \mathrm{C}-2)^{\prime}\right)$.

MS (ESI): $m / z(\%)=477.3(100)[M+H]^{+}$.

$\mathrm{C}_{32} \mathrm{H}_{32} \mathrm{~N}_{2} \mathrm{O}_{2}$ (476.62)

ber.: 477.2537

gef.: $477.2540[\mathrm{M}+\mathrm{H}]^{+}$(ESI-HRMS). 


\subsubsection{1-Hydroxy-1-oxo-1-benzo[d][1,2]iodoxol-3(1H)-on (275)}

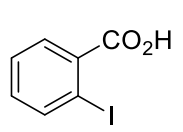

274

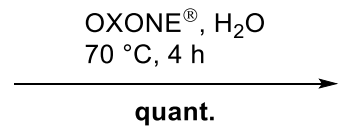

quant.

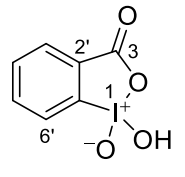

275

Zu einer Suspension von OXONE ${ }^{\circledR}\left(94.1 \mathrm{~g}, 306 \mathrm{mmol}, 3.50 \mathrm{Äq}\right.$.) in $\mathrm{H}_{2} \mathrm{O}$ (250 ml) wurde bei Raumtemperatur 2-lodbenzoesäure (274) (21.7 g. 87.5 mmol, 1.00 Äq.) gegeben und 10 min bei dieser Temperatur gerührt. Anschließend wurde das Reaktionsgemisch auf $70^{\circ} \mathrm{C}$ erwärmt und $4 \mathrm{~h}$ bei dieser Temperatur gerührt. Nach Abkühlen auf Raumtemperatur wurde die Reaktionslösung zunächst bei $5^{\circ} \mathrm{C}$ und anschließend bei $-28^{\circ} \mathrm{C}$ gelagert. Der ausgefallene Feststoff wurde abfiltriert, mit $\mathrm{H}_{2} \mathrm{O}(6 \times 40 \mathrm{ml})$ sowie Aceton $(2 \times 40 \mathrm{ml})$ gewaschen und abschließend im Hochvakuum getrocknet. IBX (275) (27.0 g, $96.5 \mathrm{mmol}$, quant.) wurde in Form eines weißen Feststoffs isoliert.

${ }^{1} \mathrm{H}-\mathrm{NMR}\left(500 \mathrm{MHz}, \mathrm{DMSO}-\mathrm{d}_{6}\right): \delta[\mathrm{ppm}]=7.84(\mathrm{td}, J=7.4,1.1 \mathrm{~Hz}, 1 \mathrm{H}, 4$ '-H), 7.99 (td, $J=$ 7.6, $1.5 \mathrm{~Hz}, 1 \mathrm{H}, 5^{\prime}-\mathrm{H}$ ), 8.04 (dd, J = 7.5, $\left.1.5 \mathrm{~Hz}, 1 \mathrm{H}, 3^{\prime}-\mathrm{H}\right), 8.13-8.16$ (m, $1 \mathrm{H}, 6$ ' $-\mathrm{H}$ ).

${ }^{13} \mathrm{C}-\mathrm{NMR}\left(126 \mathrm{MHz}, \mathrm{DMSO}-\mathrm{d}_{6}\right): \delta[\mathrm{ppm}]=124.9$ (C-4'), 130.0, 131.4, 132.9, 133.9 (C-1', C-2', C-3', C-6'), 146.6 (C-5'), 167.4 (C-3).

MS (ESI): $m / z(\%)=278.9(84)[\mathrm{M}-\mathrm{H}]^{-}$.

$\mathrm{C}_{7} \mathrm{H}_{5} \mathrm{IO}_{4}(280.02)$ ber.: 278.9160 gef.: $278.9166[\mathrm{M}-\mathrm{H}]^{-}(\mathrm{ESI}-\mathrm{HRMS})$. 


\subsubsection{1,1,1-Triacetoxy-1,1-dihydro-1,2-benziodoxol-3(1 H)-on (276)}

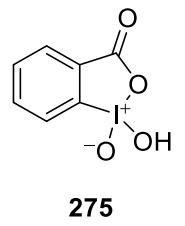

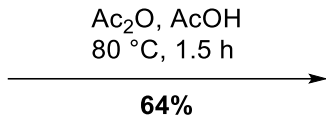

$64 \%$

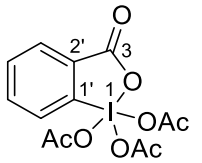

276

IBX (275) (26.5 g, 96.5 mmol, 1.00 Äq.) wurde bei Raumtemperatur zu einer Mischung von frisch destilliertem Essigsäureanhydrid ( $31.9 \mathrm{ml}, 34.5 \mathrm{~g}, 338 \mathrm{mmol}, 3.50$ Äq.) und konz. AcOH (27.1 ml, $28.4 \mathrm{~g}, 473 \mathrm{mmol}, 5.00$ Äq.) gegeben, über den Zeitraum von $1 \mathrm{~h}$ langsam auf $80^{\circ} \mathrm{C}$ erwärmt und $1.5 \mathrm{~h}$ bei dieser Temperatur unter Rückfluss gerührt. Daraufhin wurde die Reaktionslösung langsam auf $0^{\circ} \mathrm{C}$ abgekühlt, der ausgefallene Feststoff abfiltriert und mit $\mathrm{Et}_{2} \mathrm{O}(6 \times 20 \mathrm{ml})$ gewaschen. Nach Trocknung im Hochvakuum wurde DMP (276) (25.5 g, 60.2 mmol, 64\%) als weißer kristalliner Feststoff erhalten. $\mathrm{C}_{13} \mathrm{H}_{13} \mathrm{IO}_{8}(424.14)$. 


\subsubsection{1-Nitro-2-selenocyanatbenzol (254)}

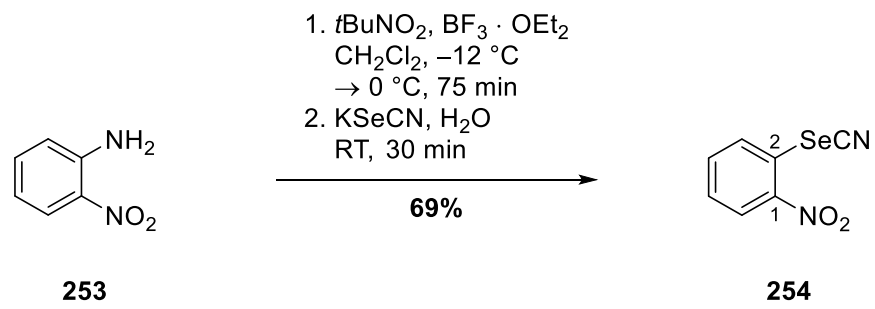

Eine Lösung von o-Nitroanilin (253) (400 mg, 2.90 mmol, 1.00 Äq.) in $\mathrm{CH}_{2} \mathrm{Cl}_{2}$ (4 ml) wurde bei $-12{ }^{\circ} \mathrm{C}$ mit $\mathrm{BF}_{3} \cdot \mathrm{OEt}_{2}(536 \mu \mathrm{l}, 617 \mathrm{mg}, 4.34 \mathrm{mmol}, 1.50 \mathrm{Äq}$ ) versetzt und die resultierende Lösung 45 min bei dieser Temperatur gerührt, ehe ebenfalls bei $-12^{\circ} \mathrm{C}$ eine Lösung von $\mathrm{tBuNO}_{2}$ (90\%ig, $459 \mu \mathrm{l}, 398 \mathrm{mg}, 3.48 \mathrm{mmol}, 1.20 \mathrm{Äq}$.) in $\mathrm{CH}_{2} \mathrm{Cl}_{2}$ (1 ml) innerhalb von $30 \mathrm{~min}$ hinzugetropft wurde. Die Reaktionslösung wurde $45 \mathrm{~min}$ bei $-12{ }^{\circ} \mathrm{C}$ und anschließend weitere $30 \mathrm{~min}$ bei $0^{\circ} \mathrm{C}$ gerührt. Die Reaktion wurde durch Zugabe von eiskaltem $n$-Pentan $(5 \mathrm{ml})$ beendet, der ausgefallene Feststoff abfiltriert, mit $\mathrm{Et}_{2} \mathrm{O}(5 \mathrm{ml})$ gewaschen und abschließend im Hochvakuum getrocknet.

$\mathrm{Zu}$ einer Lösung des Tetrafluorborats in $\mathrm{H}_{2} \mathrm{O}(15 \mathrm{ml})$ wurde bei $0^{\circ} \mathrm{C}$ eine Lösung von $\mathrm{KSeCN}$ (441 mg, $3.06 \mathrm{mmol}, 1.06$ Äq.) in $\mathrm{H}_{2} \mathrm{O}$ (5 ml) getropft und das resultierende Reaktionsgemisch bei dieser Temperatur 30 min gerührt. Der ausgefallene Feststoff wurde abfiltriert, mit $\mathrm{H}_{2} \mathrm{O}(25 \mathrm{ml})$ gewaschen und im Hochvakuum getrocknet. Säulenchromatographie an Kieselgel (Petrolether/EtOAc 9:1 $\rightarrow$ 4:1) und Umkristallisation aus $\mathrm{EtOH}$ lieferte die Zielverbindung 254 (456 mg, $2.01 \mathrm{mmol}, 69 \%)$ in Form gelber Kristalle.

DC: $R_{\mathrm{f}}=0.27($ Petrolether/EtOAc 8:1).

Smp.: $\vartheta\left[{ }^{\circ} \mathrm{C}\right]=142$.

${ }^{1} \mathrm{H}-\mathrm{NMR}\left(300 \mathrm{MHz}, \mathrm{CDCl}_{3}\right): \delta[\mathrm{ppm}]=7.59$ (ddd, $\left.J=8.4,7.3,1.2 \mathrm{~Hz}, 1 \mathrm{H}, 5-\mathrm{H}\right), 7.76$ (ddd, $J=8.0,7.3,1.5 \mathrm{~Hz}, 1 \mathrm{H}, 4-\mathrm{H}), 8.19$ (dd, $J=8.2,1.2 \mathrm{~Hz}, 1 \mathrm{H}, 3-\mathrm{H}), 8.42$ (dd, $J=8.3$, $1.5 \mathrm{~Hz}, 1 \mathrm{H}, 6-\mathrm{H})$.

${ }^{13} \mathrm{C}-\mathrm{NMR}\left(126 \mathrm{MHz}, \mathrm{CDCl}_{3}\right): \delta[\mathrm{ppm}]=104.3(\mathrm{SeCN}), 125.8$ (C-2), 126.5 (C-6), 129.0 (C-5), 131.0 (C-3), 136.1 (C-4), 144.5 (C-1).

MS (El): $m / z(\%)=201.9(41)[\mathrm{M}-\mathrm{CN}]^{+}, 228.0[\mathrm{M}]^{+}$.

$\mathrm{C}_{7} \mathrm{H}_{4} \mathrm{~N}_{2} \mathrm{O}_{2} \mathrm{Se}$ (227.08). 


\subsection{Synthese des Biaryls 135}

\subsubsection{1,3-Dimethoxy-5-methylbenzol (143)}

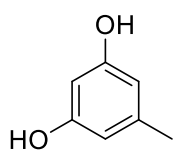

17

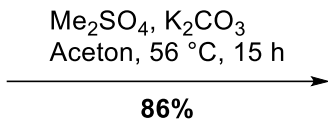

$86 \%$

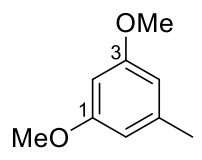

143

$\mathrm{Zu}$ einer Suspension von Orcinol-Monohydrat (17) $(2.00 \mathrm{~g}, 14.1 \mathrm{mmol}, 1.00$ Äq.) und $\mathrm{K}_{2} \mathrm{CO}_{3}$ (3.89 g, $28.1 \mathrm{mmol}, 2.00 \mathrm{Äq}$ ) in Aceton (30 ml) wurde bei Raumtemperatur $\mathrm{Me}_{2} \mathrm{SO}_{4}$ (3.07 ml, $4.08 \mathrm{~g}, 32.4 \mathrm{mmol}, 2.30$ Äq.) getropft. Die Reaktionslösung wurde $15 \mathrm{~h}$ bei $56^{\circ} \mathrm{C}$ unter Rückfluss gerührt, ehe konz. $\mathrm{NH}_{3}(2 \mathrm{ml})$ zugegeben und weitere $15 \mathrm{~min}$ bei dieser Temperatur gerührt wurde. Nach Abkühlen auf Raumtemperatur wurde der Feststoff abfiltriert und das Filtrat unter vermindertem Druck eingeengt. Der Rückstand wurde in $\mathrm{Et}_{2} \mathrm{O}(5 \mathrm{ml})$ aufgenommen, mit $\mathrm{H}_{2} \mathrm{O}(20 \mathrm{ml})$ gewaschen und die wässr. Phase mit $\mathrm{Et}_{2} \mathrm{O}(2 \times 5 \mathrm{ml})$ extrahiert. Die vereinigten org. Phasen wurden mit $\mathrm{H}_{2} \mathrm{O}(5 \mathrm{ml})$, verd. $\mathrm{NaOH}$-Lsg. $(3 \mathrm{M}, 2 \times 5 \mathrm{ml})$ sowie ges. $\mathrm{NaCl}$-Lsg. $(5 \mathrm{ml})$ gewaschen, über $\mathrm{MgSO}_{4}$ getrocknet und das Lösungsmittel unter vermindertem Druck entfernt. Nach Säulenchromatographie an Kieselgel (Petrolether/EtOAc 7:3) wurde 1,3-Dimethoxy-5methylbenzol (143) (1.85 g, $12.1 \mathrm{mmol}, 86 \%)$ als farblose Flüssigkeit erhalten.

DC: $R_{\mathrm{f}}=0.63$ (Petrolether/EtOAc 7:3).

Sdp.: $\vartheta\left[{ }^{\circ} \mathrm{C}\right]=98(16 \mathrm{mbar})$.

UV $\left(\mathrm{CH}_{3} \mathrm{CN}\right): \lambda_{\max }[\mathrm{nm}](\lg \varepsilon)=204$ (4.645), 273 (3.181), 280 (3.182).

IR (ATR): $\tilde{v}\left[\mathrm{~cm}^{-1}\right]=3059,2955,2838,1597,1461,1321,1295,1205,1151,1070,921$, $828,686$.

${ }^{1} \mathrm{H}-\mathrm{NMR}\left(300 \mathrm{MHz}, \mathrm{CDCl}_{3}\right): \delta[\mathrm{ppm}]=2.32\left(\mathrm{~d}, J=0.6 \mathrm{~Hz}, 3 \mathrm{H}, 5-\mathrm{CH}_{3}\right), 3.78(\mathrm{~s}, 6 \mathrm{H}, 2 \times$ $\mathrm{OCH}_{3}$ ), 6.30 (t, $\left.J=2.3,0.6 \mathrm{~Hz}, 1 \mathrm{H}, 2-\mathrm{H}\right), 6.35$ (dd, $\left.J=2.3,0.6 \mathrm{~Hz}, 2 \mathrm{H}, 4-\mathrm{H}, 6-\mathrm{H}\right)$.

${ }^{13} \mathrm{C}-N M R\left(126 \mathrm{MHz}, \mathrm{CDCl}_{3}\right): \delta[\mathrm{ppm}]=21.7\left(5-\mathrm{CH}_{3}\right), 55.1\left(2 \times \mathrm{OCH}_{3}\right), 97.5(\mathrm{C}-2), 107.0$ (C-4, C-6), 140.1 (C-5), 160.7 (C-1, C-3).

MS (ESI): $m / z(\%)=153.1(53)[\mathrm{M}+\mathrm{H}]^{+}, 175.1(100)[\mathrm{M}+\mathrm{Na}]^{+}$.

$\mathrm{C}_{9} \mathrm{H}_{12} \mathrm{O}_{2}$ (152.19)

ber.: 153.0910

175.0730

gef.: $153.0907[\mathrm{M}+\mathrm{H}]^{+}$

$175.0732[\mathrm{M}+\mathrm{Na}]^{+}(\mathrm{ESI}-\mathrm{HRMS})$. 


\subsubsection{2,6-Dimethoxy-4-methylbenzoesäure (144)}

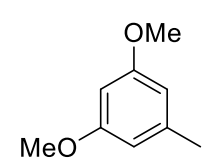

143

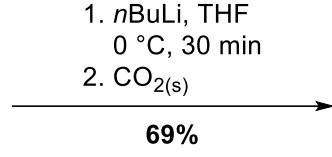

$69 \%$

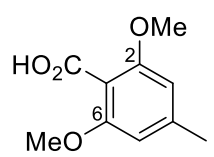

144

Zu einer Lösung von 1,3-Dimethoxy-5-methylbenzol (143) (500 mg, 3.29 mmol, 1.00 Äq.) in THF $(25 \mathrm{ml})$ wurde bei $0^{\circ} \mathrm{C}$ langsam $n B u L i ~(2.5 \mathrm{M}$ in $n$-Hexan, $1.58 \mathrm{ml}, 3.94 \mathrm{mmol}$, 1.20 Äq.) getropft und die resultierende Lösung $30 \mathrm{~min}$ bei dieser Temperatur gerührt. Die Reaktion wurde durch Zugabe von Trockeneis beendet, das Lösungsmittel unter vermindertem Druck entfernt und der Rückstand in $\mathrm{H}_{2} \mathrm{O}(20 \mathrm{ml})$ aufgenommen. Die wässr. Phase wurde mit $\mathrm{Et}_{2} \mathrm{O}(3 \times 10 \mathrm{ml})$ extrahiert, die vereinigten org. Phasen über $\mathrm{MgSO}_{4}$ getrocknet und das Lösungsmittel unter vermindertem Druck entfernt. Die Benzoesäure 144 (442 mg, 2.25 mmol, 69\%) wurde analysenrein als weißer Feststoff isoliert.

DC: $R_{\mathrm{f}}=0.43(\mathrm{EtOAc} / \mathrm{MeOH} 19: 1)$.

Smp.: $\vartheta\left[{ }^{\circ} \mathrm{C}\right]=181$.

UV $\left(\mathrm{CH}_{3} \mathrm{CN}\right): \lambda_{\max }[\mathrm{nm}](\mathrm{lg} \varepsilon)=206$ (4.467), 280 (3.205).

IR (ATR): $\widetilde{v}\left[\mathrm{~cm}^{-1}\right]=2998,2943,2711,2664,2565,1688,1608,1583,1467,1411,1333$, 1296, 1237, 1174, 1129, 1101, 969, 819, 794.

${ }^{1} \mathrm{H}-\mathrm{NMR}\left(300 \mathrm{MHz}, \mathrm{DMSO}-\mathrm{d}_{6}\right): \delta[\mathrm{ppm}]=2.31\left(\mathrm{~s}, 3 \mathrm{H}, 4-\mathrm{CH}_{3}\right), 3.73\left(\mathrm{~s}, 6 \mathrm{H}, 2 \times \mathrm{OCH}_{3}\right)$, $6.51(\mathrm{~s}, 2 \mathrm{H}, 3-\mathrm{H}, 5-\mathrm{H}), 12.48\left(\mathrm{sbr}, 1 \mathrm{H}, \mathrm{CO}_{2} \mathrm{H}\right)$.

${ }^{13} \mathrm{C}-\mathrm{NMR}\left(126 \mathrm{MHz}, \mathrm{DMSO}-\mathrm{d}_{6}\right): \delta[\mathrm{ppm}]=21.6\left(4-\mathrm{CH}_{3}\right), 55.6\left(2 \times \mathrm{OCH}_{3}\right), 104.8(\mathrm{C}-3$, C-5), 111.8 (C-1), 140.4 (C-4), 156.1 (C-2, C-6), $166.7\left(\mathrm{CO}_{2} \mathrm{H}\right)$.

MS (ESI): $m / z(\%)=197.1(8)[\mathrm{M}+\mathrm{H}]^{+}, 219.1(78)[\mathrm{M}+\mathrm{Na}]^{+}, 415.1(100)[2 \times \mathrm{M}+\mathrm{Na}]^{+}$; $195.1(96)[\mathrm{M}-\mathrm{H}]^{-}$.

$\mathrm{C}_{10} \mathrm{H}_{12} \mathrm{O}_{4}(196.20)$

ber.: 195.0663

197.0808

219.0628

gef.: $195.0666\left[\mathrm{M}-\mathrm{H}^{-}\right.$

$197.0808[\mathrm{M}+\mathrm{H}]^{+}$

$219.0629[\mathrm{M}+\mathrm{Na}]^{+}(\mathrm{ESI}-\mathrm{HRMS})$. 


\subsubsection{2,6-Dimethoxy-4-methylbenzoesäure-tert-butylester (147)}

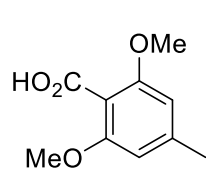

144

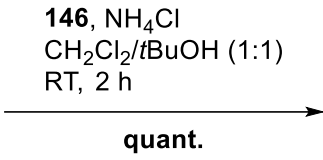

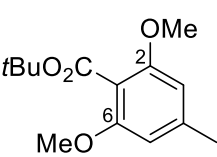

147

Die Benzoesäure 144 (50.0 mg, $255 \mu \mathrm{mol}, 1.00$ Äq.) wurde in $\mathrm{CH}_{2} \mathrm{Cl}_{2} / \mathrm{tBuOH}(1: 1,1 \mathrm{ml})$ gelöst und mit $\mathrm{NH}_{4} \mathrm{Cl}$ (81.8 mg, $1.53 \mathrm{mmol}, 6.00$ Äq.) versetzt. Daraufhin wurde der Isoharnstoff 146 (511 mg, $2.55 \mathrm{mmol}, 10.0$ Äq.) tropfenweise bei Raumtemperatur hinzugegeben und die Reaktionslösung $2 \mathrm{~h}$ bei dieser Temperatur gerührt, ehe die Reaktion durch Zugabe von $\mathrm{H}_{2} \mathrm{O}(3 \mathrm{ml})$ beendet wurde. Nach Trennung der Phasen wurde die wässr. Phase mit $\mathrm{CH}_{2} \mathrm{Cl}_{2}(2 \times 1 \mathrm{ml})$ extrahiert, die vereinigten org. Phasen über $\mathrm{MgSO}_{4}$ getrocknet und das Lösungsmittel unter vermindertem Druck entfernt. Säulenchromatographische Reinigung an Kieselgel (Petrolether/EtOAc 7:3) lieferte den tert-Butylester 147 (65.1 mg, 258 umol, quant.) als weißen Feststoff.

DC: $R_{\mathrm{f}}=0.61$ (Petrolether/EtOAc 7:3).

Smp.: $\vartheta\left[{ }^{\circ} \mathrm{C}\right]=75$.

UV $\left(\mathrm{CH}_{3} \mathrm{CN}\right): \lambda_{\max }[\mathrm{nm}](\mathrm{lg} \varepsilon)=206$ (4.550), 280 (3.167).

IR $(A T R): \tilde{v}\left[\mathrm{~cm}^{-1}\right]=2971,2938,2841,1726,1608,1584,1465,1451,1410,1390,1369$, 1329, 1282, 1239, 1160, 1122, 1082, 1038, 968, 846, 821, 767, 727.

${ }^{1} \mathrm{H}-\mathrm{NMR}\left(300 \mathrm{MHz}, \mathrm{CDCl}_{3}\right): \delta[\mathrm{ppm}]=1.54\left(\mathrm{~s}, 9 \mathrm{H}, \mathrm{C}(\mathrm{CH})_{3}\right), 2.30\left(\mathrm{~s}, 3 \mathrm{H}, 4-\mathrm{CH}_{3}\right), 3.77(\mathrm{~s}$, $\left.6 \mathrm{H}, 2 \times \mathrm{OCH}_{3}\right), 6.33(\mathrm{~s}, 2 \mathrm{H}, 3-\mathrm{H}, 5-\mathrm{H})$.

${ }^{13} \mathrm{C}-\mathrm{NMR}(126 \mathrm{MHz}, \mathrm{CDCl}): \delta[\mathrm{ppm}]=22.2\left(4-\mathrm{CH}_{3}\right), 28.2\left(\mathrm{C}\left(\underline{\mathrm{CH}}_{3}\right)_{3}\right), 55.9\left(2 \times \mathrm{OCH}_{3}\right)$, $81.4\left(\underline{\mathrm{C}}\left(\mathrm{CH}_{3}\right)_{3}\right), 104.9$ (C-3, C-5), 112.4 (C-1), 140.8 (C-4), 157.0 (C-2, C-6), 165.9 $\left(\underline{\mathrm{CO}_{2}} \mathrm{C}\left(\mathrm{CH}_{3}\right)_{3}\right)$.

MS (ESI): $m / z(\%)=275.1(17)[\mathrm{M}+\mathrm{Na}]^{+}, 527.3(100)[2 \times \mathrm{M}+\mathrm{Na}]^{+}$.

$\mathrm{C}_{14} \mathrm{H}_{20} \mathrm{O}_{4}$ (252.31)

ber.: 275.1254

gef.: $275.1258[\mathrm{M}+\mathrm{Na}]^{+}(\mathrm{ESI}-\mathrm{HRMS})$. 


\subsubsection{3-Brom-2,6-dimethoxy-4-methylbenzoesäure-tert-butylester (148)}

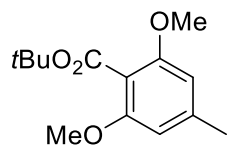

147

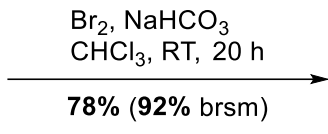

$8 \%(92 \%$ brsm $)$

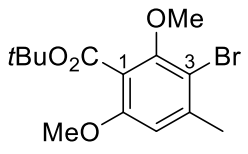

148

Zu einer Suspension des tert-Butylesters 147 (5.00 g, 19.8 mmol, 1.00 Äq.) und $\mathrm{NaHCO}_{3}$ (2.50 g, $29.7 \mathrm{mmol}, 1.50 \mathrm{Äq}$.) in $\mathrm{CHCl}_{3}(25 \mathrm{ml})$ wurde bei $0{ }^{\circ} \mathrm{C}$ langsam eine Lösung von Brom (0.76 ml, $2.37 \mathrm{~g}, 14.9 \mathrm{mmol}, 0.75 \mathrm{Äq}$ ) in $\mathrm{CHCl}_{3}(2 \mathrm{ml})$ getropft. Die resultierende Reaktionslösung wurde langsam auf Raumtemperatur erwärmt und $20 \mathrm{~h}$ bei dieser Temperatur gerührt. Der Feststoff wurde abfiltriert und mit $\mathrm{CH}_{2} \mathrm{Cl}_{2}(3 \times 20 \mathrm{ml})$ nachgewaschen. Das Filtrat wurde mit ges. $\mathrm{Na}_{2} \mathrm{~S}_{2} \mathrm{O}_{3}$-Lsg. $(30 \mathrm{ml})$ und ges. NaCl-Lsg. $(30 \mathrm{ml})$ gewaschen, über $\mathrm{MgSO}_{4}$ getrocknet und das Lösungsmittel unter vermindertem Druck entfernt. Nach säulenchromatographischer Reinigung an Kieselgel (Petrolether/EtOAc 7:3) konnte das Bromaren 148 (5.11 g, 15.4 mmol, 78\%, 92\% brsm) als weißer kristalliner Feststoff isoliert werden.

DC: $R_{\mathrm{f}}=0.62$ (Petrolether/EtOAc 7:3).

Smp.: $\vartheta\left[{ }^{\circ} \mathrm{C}\right]=62$.

UV $\left(\mathrm{CH}_{3} \mathrm{CN}\right): \lambda_{\max }[\mathrm{nm}](\lg \varepsilon)=205$ (4.597), 285 (3.240).

IR (ATR): $\tilde{v}\left[\mathrm{~cm}^{-1}\right]=2974,2943,2848,1718,1592,1561,1459,1366,1326,1282,1259$, 1215, 1160, 1106, 1041, 1012, 970, 920, 847, 795, 737, 677.

${ }^{1} \mathrm{H}-\mathrm{NMR}\left(300 \mathrm{MHz}, \mathrm{CDCl}_{3}\right): \delta[\mathrm{ppm}]=1.56\left(\mathrm{~s}, 9 \mathrm{H}, \mathrm{C}\left(\mathrm{CH}_{3}\right)_{3}\right), 2.38\left(\mathrm{~s}, 3 \mathrm{H}, 4-\mathrm{CH}_{3}\right), 3.78(\mathrm{~s}$, $\left.3 \mathrm{H}, 6-\mathrm{OCH}_{3}\right), 3.86$ (s, $\left.3 \mathrm{H}, 2-\mathrm{OCH}_{3}\right), 6.57$ (s, $\left.1 \mathrm{H}, 5-\mathrm{H}\right)$.

${ }^{13} \mathrm{C}-N M R\left(126 \mathrm{MHz}, \mathrm{CDCl}_{3}\right): \delta[\mathrm{ppm}]=23.7\left(4-\mathrm{CH}_{3}\right), 28.1\left(\mathrm{C}\left(\mathrm{CH}_{3}\right)_{3}\right), 56.1\left(6-\mathrm{OCH}_{3}\right), 61.9$ (2- $\left.\mathrm{OCH}_{3}\right), 82.2\left(\underline{\mathrm{C}}\left(\mathrm{CH}_{3}\right)_{3}\right), 109.4$ (C-5), 110.6 (C-3), 119.2 (C-1), 140.7 (C-4), 154.3 (C-2), 155.6 (C-6), $164.6\left(\underline{\mathrm{CO}_{2}} \mathrm{C}\left(\mathrm{CH}_{3}\right)_{3}\right)$.

MS (ESI): $m / z(\%)=353.0(17)[\mathrm{M}+\mathrm{Na}]^{+}, 685.1(100)[2 \times \mathrm{M}+\mathrm{Na}]^{+}$.

$\mathrm{C}_{14} \mathrm{H}_{19} \mathrm{BrO}_{4}(331.21)$

ber.: 353.0359

gef.: $353.0358[\mathrm{M}+\mathrm{Na}]^{+}(\mathrm{ESI}-\mathrm{HRMS})$. 


\subsubsection{3-Brom-4-brommethyl-2,6-dimethoxybenzoesäure-tert-butylester}

(149)

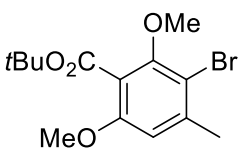

148

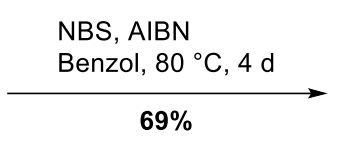

$69 \%$

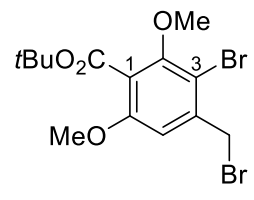

149

NBS (3.15 g, $17.7 \mathrm{mmol}, 1.20$ Äq.) und AIBN (363 mg, $2.21 \mathrm{mmol}, 15 \mathrm{~mol} \%$ ) wurden in Benzol gelöst, auf $80^{\circ} \mathrm{C}$ erwärmt, anschließend das Bromaren 148 (4.89 g, $14.8 \mathrm{mmol}$, 1.00 Äq.) hinzugegeben und die Reaktionslösung $4 \mathrm{~d}$ bei $80^{\circ} \mathrm{C}$ unter Rückfluss gerührt. Nach Abkühlen auf Raumtemperatur wurde der Feststoff abfiltriert, der Filterkuchen mit Benzol $(3 \times 25 \mathrm{ml})$ gewaschen und das Filtrat unter vermindertem Druck vom Lösungsmittel befreit. Säulenchromatographie an Kieselgel (Petrolether/EtOAc 7:3) lieferte das Dibromid 149 (4.20 g, 10.2 mmol, 69\%) als weißen Feststoff.

DC: $R_{\mathrm{f}}=0.68$ (Petrolether/EtOAc 7:3).

UV $\left(\mathrm{CH}_{3} \mathrm{CN}\right): \lambda_{\max }[\mathrm{nm}](\mathrm{lg} \varepsilon)=215$ (4.404), 300 (3.305).

IR (ATR): $\tilde{v}\left[\mathrm{~cm}^{-1}\right]=2975,2938,2846,1721,1591,1564,1450,1386,1366,1330,1277$, 1256, 1207, 1159, 1097, 1040, 979, 926, 884, 864, 843, 780, 739, 692, 665.

${ }^{1} \mathrm{H}-\mathrm{NMR}\left(300 \mathrm{MHz}, \mathrm{CDCl}_{3}\right): \delta[\mathrm{ppm}]=1.56\left(\mathrm{~s}, 9 \mathrm{H}, \mathrm{C}\left(\mathrm{CH}_{3}\right)_{3}\right), 3.81\left(\mathrm{~s}, 3 \mathrm{H}, 6-\mathrm{OCH}_{3}\right), 3.88$ (s, $\left.3 \mathrm{H}, 2-\mathrm{OCH}_{3}\right), 4.55$ (s, $\left.2 \mathrm{H}, \mathrm{CH}_{2} \mathrm{Br}\right), 6.77(\mathrm{~s}, 1 \mathrm{H}, 5-\mathrm{H})$.

${ }^{13} \mathrm{C}-N M R\left(126 \mathrm{MHz}, \mathrm{CDCl}_{3}\right): \delta[\mathrm{ppm}]=28.1\left(\mathrm{C}\left(\underline{\mathrm{C}} \mathrm{H}_{3}\right)_{3}\right), 33.4\left(\mathrm{CH}_{2} \mathrm{Br}\right), 56.3\left(6-\mathrm{OCH}_{3}\right), 62.1$ $\left(2-\mathrm{OCH}_{3}\right), 82.8\left(\underline{\mathrm{C}}\left(\mathrm{CH}_{3}\right)_{3}\right), 109.6(\mathrm{C}-5), 110.6(\mathrm{C}-3), 121.7$ (C-1), $139.2(\mathrm{C}-4), 154.8$ (C-2), 156.0 (C-6), $164.0\left(\underline{\mathrm{CO}}_{2} \mathrm{C}\left(\mathrm{CH}_{3}\right)_{3}\right)$.

MS (ESI): $m / z(\%)=432.9(13)[\mathrm{M}+\mathrm{Na}]^{+}, 842.9(100)[2 \times \mathrm{M}+\mathrm{Na}]^{+}$.

$\mathrm{C}_{14} \mathrm{H}_{18} \mathrm{Br}_{2} \mathrm{O}_{4}(410.10)$

ber.: 432.9444

gef.: $432.9442[\mathrm{M}+\mathrm{Na}]^{+}(\mathrm{ESI}-\mathrm{HRMS})$. 


\subsubsection{3-Brom-2,6-dimethoxy-4-(((4-methoxybenzyl)oxy)methyl)benzoe- säure-tert-butylester (133)}

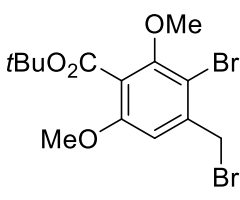

149

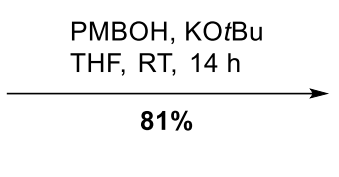

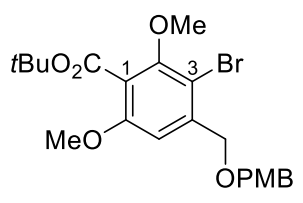

133

PMBOH (152 $\mu$ l, $168 \mathrm{mg}, 1.22 \mathrm{mmol}, 1.00$ Äq.) wurde in THF $(5 \mathrm{ml})$ gelöst und bei $0{ }^{\circ} \mathrm{C}$ mit KOtBu (205 mg, 1.83 mmol, 1.50 Äq.) versetzt. Das Reaktionsgemisch wurde 5 min bei dieser Temperatur gerührt, ehe das Dibromid 149 (500 mg, 1.22 mmol, 1.00 Äq.) ebenfalls bei $0^{\circ} \mathrm{C}$ hinzugegeben wurde. Die resultierende Lösung wurde langsam auf Raumtemperatur erwärmt und $14 \mathrm{~h}$ bei dieser Temperatur gerührt. Zur Beendigung der Reaktion wurde die Reaktionslösung in ges. $\mathrm{NH}_{4} \mathrm{Cl}$-Lsg. $(10 \mathrm{ml}$ ) überführt, die Phasen getrennt und die wässr. Phase mit $\mathrm{Et}_{2} \mathrm{O}(2 \times 5 \mathrm{ml})$ extrahiert. Die vereinigten org. Phasen wurden mit ges. $\mathrm{NaCl}$-Lsg. $(5 \mathrm{ml})$ gewaschen, über $\mathrm{MgSO}_{4}$ getrocknet und das Lösungsmittel unter vermindertem Druck entfernt. Nach säulenchromatographischer Reinigung an Kieselgel (Petrolether/EtOAc 7:3) wurde die Zielverbindung 133 (459 mg, $983 \mu \mathrm{mol}, 81 \%)$ als leicht gelber Feststoff erhalten.

DC: $R_{\mathrm{f}}=0.66$ (Petrolether/EtOAc 7:3).

UV $\left(\mathrm{CH}_{3} \mathrm{CN}\right): \lambda_{\max }[\mathrm{nm}](\lg \varepsilon)=197$ (4.768), 226 (4.334), 282 (3.527).

IR (ATR): $\tilde{v}\left[\mathrm{~cm}^{-1}\right]=2976,2948,2859,1722,1595,1566,1515,1456,1388,1248,1201$, 1169, 1095, 1027, 921, 846, 825, 739, 685.

${ }^{1} \mathrm{H}-\mathrm{NMR}\left(300 \mathrm{MHz}, \mathrm{CDCl}_{3}\right): \delta[\mathrm{ppm}]=1.57\left(\mathrm{~s}, 9 \mathrm{H}, \mathrm{C}\left(\mathrm{CH}_{3}\right)_{3}\right), 3.79$ (s, $\left.3 \mathrm{H}, \mathrm{PMB}_{-} \mathrm{OCH}_{3}\right)$, $3.81\left(\mathrm{~s}, 3 \mathrm{H}, 6-\mathrm{OCH}_{3}\right), 3.87\left(\mathrm{~s}, 3 \mathrm{H}, 2-\mathrm{OCH}_{3}\right), 4.55\left(\mathrm{~s}, 4 \mathrm{H}, 2 \times \mathrm{CH}_{2}\right), 6.88(\mathrm{~d}, J=8.7 \mathrm{~Hz}$, $\left.2 \mathrm{H}, 2 \times \mathrm{PMB}_{-} \mathrm{C}_{m}\right), 6.92$ (s, $\left.1 \mathrm{H}, 5-\mathrm{H}\right), 7.29$ (d, J=8.5 Hz, $2 \mathrm{H}, 2 \times \mathrm{PMB}^{-\mathrm{C}_{o}}$ ).

${ }^{13} \mathrm{C}-\mathrm{NMR}\left(126 \mathrm{MHz}, \mathrm{CDCl}_{3}\right): \delta[\mathrm{ppm}]=28.1\left(\mathrm{C}\left(\mathrm{CH}_{3}\right)_{3}\right), 55.2\left(\mathrm{PMB}-\mathrm{OCH}_{3}\right), 56.2\left(6-\mathrm{OCH}_{3}\right)$, $62.0\left(2-\mathrm{OCH}_{3}\right), 71.2\left(4-\mathrm{CH}_{2}\right), 72.5\left(\mathrm{PMB}-\mathrm{CH}_{2}\right), 82.3\left(\underline{\mathrm{C}}\left(\mathrm{CH}_{3}\right)_{3}\right), 107.2(\mathrm{C}-5), 107.8(\mathrm{C}-3)$,

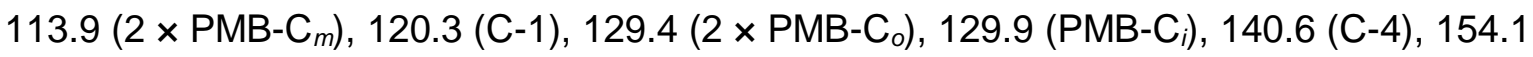
(C-2), 156.1 (C-6), $159.3\left(\mathrm{PMB}-\mathrm{C}_{p}\right), 164.5\left(\mathrm{CO}_{2} \mathrm{C}\left(\mathrm{CH}_{3}\right)_{3}\right)$. MS (ESI): $m / z(\%)=489.1(39)[\mathrm{M}+\mathrm{Na}]^{+}, 957.1(100)[2 \times \mathrm{M}+\mathrm{Na}]^{+}$.

$\mathrm{C}_{22} \mathrm{H}_{27} \mathrm{BrO}_{6}(467.36)$

ber.: 489.0883 gef.: $489.0877[\mathrm{M}+\mathrm{Na}]^{+}(\mathrm{ESI}-\mathrm{HRMS})$. 


\subsubsection{2,6-Dimethoxy-4-(((4-methoxybenzyl)oxy)methyl)-3-(4,4,5,5-tetra- methyl-1,3,2-dioxaborolan-2-yl)benzoesäure-tert-butylester (134)}

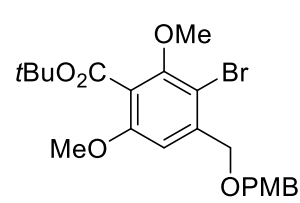

133

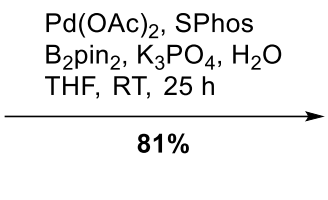
$\mathrm{Zu}$ einem Gemisch aus $\mathrm{Pd}(\mathrm{OAc})_{2}(4.80 \mathrm{mg}, 21.4 \mu \mathrm{mol}, 10 \mathrm{~mol} \%)$, SPhos $(22.0 \mathrm{mg}$,
$53.5 \mu \mathrm{mol}, 25 \mathrm{~mol} \%), \mathrm{B}_{2} \operatorname{pin}_{2}\left(109 \mathrm{mg}, 428 \mu \mathrm{mol}, 2.00 \mathrm{Äq}\right.$.) sowie $\mathrm{K}_{3} \mathrm{PO}_{4}(90.8 \mathrm{mg}$, $428 \mu \mathrm{mol}, 2.00 \mathrm{Äq}$.) wurde bei Raumtemperatur eine Lösung des Bromarens 133 (100 mg, $214 \mu \mathrm{mol}, 1.00$ Äq.) in sorgfältig entgastem THF (2 ml) und $\mathrm{H}_{2} \mathrm{O}(15.4 \mu \mathrm{l}$, $15.4 \mathrm{mg}, \quad 856 \mu \mathrm{mol}$, 4.00 Äq.) gegeben und $25 \mathrm{~h}$ bei dieser Temperatur gerührt. Anschließend wurde die Reaktionslösung durch Celite ${ }^{\circledR}$ filtriert, mit EtOAc $(3 \times 5 \mathrm{ml})$ nachgewaschen, das Lösungsmittel unter vermindertem Druck entfernt und nach Säulenchromatographie an Kieselgel (Petrolether/EtOAc 19:1 $\rightarrow$ 4:1) der Pinakolboronsäureester 134 (89.6 mg, 174 mol, 81\%) als farbloses Öl erhalten.

DC: $R_{\mathrm{f}}=0.71$ (Petrolether/EtOAc 7:3).

UV $\left(\mathrm{CH}_{3} \mathrm{CN}\right): \lambda_{\max }[\mathrm{nm}](\lg \varepsilon)=194$ (4.660), 276 (3.204).

IR (ATR): $\widetilde{v}\left[\mathrm{~cm}^{-1}\right]=2977,1723,1596,1455,1367,1309,1248,1103,1034,849$.

${ }^{1} \mathrm{H}-\mathrm{NMR}\left(600 \mathrm{MHz}, \mathrm{CDCl}_{3}\right): \delta[\mathrm{ppm}]=1.29\left(\mathrm{~s}, 12 \mathrm{H}, 4 \times \mathrm{CH}_{3}\right), 1.55\left(\mathrm{~s}, 9 \mathrm{H}, \mathrm{C}\left(\mathrm{CH}_{3}\right)_{3}\right), 3.78$

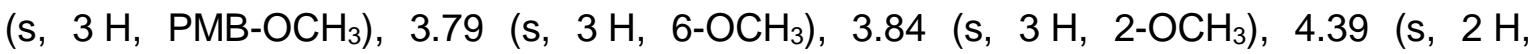
PMB- $\mathrm{CH}_{2}$ ), $4.56\left(\mathrm{~s}, 2 \mathrm{H}, 4-\mathrm{CH}_{2}\right), 6.71(\mathrm{~s}, 1 \mathrm{H}, 5-\mathrm{H}), 6.84\left(\mathrm{~d}, J=8.7 \mathrm{~Hz}, 2 \mathrm{H}, 2 \times \mathrm{PMB}^{-\mathrm{H}_{m}}\right)$, $7.25\left(\mathrm{~d}, J=8.6 \mathrm{~Hz}, 2 \mathrm{H}, 2 \times \mathrm{PMB}^{-} \mathrm{H}_{\circ}\right)$.

${ }^{13} \mathrm{C}-\mathrm{NMR}\left(126 \mathrm{MHz}, \mathrm{CDCl}_{3}\right): \delta[\mathrm{ppm}]=24.9\left(4 \times \mathrm{CH}_{3}\right), 28.2\left(\mathrm{C}\left(\underline{\mathrm{C}} \mathrm{H}_{3}\right)_{3}\right), 55.3\left(\mathrm{PMB}^{-\mathrm{OCH}_{3}}\right)$, $56.0\left(6-\mathrm{OCH}_{3}\right), 63.6\left(2-\mathrm{OCH}_{3}\right), 71.2\left(4-\mathrm{CH}_{2}\right), 71.4\left(\mathrm{PMB}-\mathrm{CH}_{2}\right), 81.7\left(\underline{\mathrm{C}}\left(\mathrm{CH}_{3}\right)_{3}\right), 83.5,83.6$ (C-4', C-5'), 106.1 (C-5), 113.7 (2 × PMB-C $m$ ), 115.1 (C-3), 118.7 (C-1), 129.6 (2 × PMB-C ${ }_{o}$ ), 130.3 (PMB-C $\left.)_{i}\right), 146.7$ (C-4), 158.3 (C-6), 159.2 (PMB-C ${ }_{p}$ ), 162.0 (C-2), 165.7 $\left(\underline{\mathrm{CO}_{2}} \mathrm{C}\left(\mathrm{CH}_{3}\right)_{3}\right)$.

MS (ESI): $m / z(\%)=515.3(5)[\mathrm{M}+\mathrm{H}]^{+}, 537.3(91)[\mathrm{M}+\mathrm{Na}]^{+}, 1051.5(100)[2 \times \mathrm{M}+\mathrm{Na}]^{+}$.

$\mathrm{C}_{28} \mathrm{H}_{39} \mathrm{BO}_{8}(514.42)$ ber.: 515.2816

537.2635

gef.: $515.2812[\mathrm{M}+\mathrm{H}]^{+}$

$537.2640[\mathrm{M}+\mathrm{Na}]^{+}(\mathrm{ESI}-\mathrm{HRMS})$. 


\subsubsection{3-tert-Butyl-3'-methyl-2,4-dimethoxy-6-(((4-methoxybenzyl)oxy)- methyl-2'-nitro-[1,1'-biphenyl]-3,3'-dicarboxylat (135)}

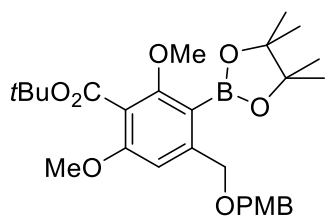

134

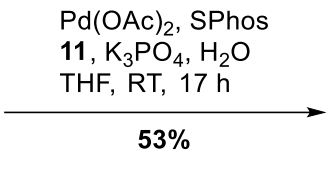

$53 \%$

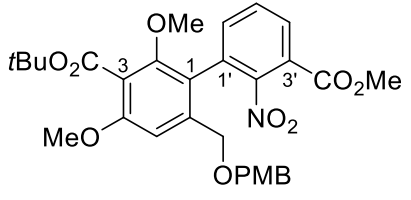

135

Zu einem Gemisch des Chlorarens 11 (41.9 mg, $194 \mu \mathrm{mol}, 1.00$ Äq.), Pd(OAc)2 (4.36 mg, $19.4 \mu \mathrm{mol}$, $10 \mathrm{~mol} \%)$, SPhos $(20.0 \mathrm{mg}, 48.6 \mu \mathrm{mol}, 25 \mathrm{~mol} \%)$ und $\mathrm{K}_{3} \mathrm{PO}_{4}(82.5 \mathrm{mg}$, $389 \mu \mathrm{mol}, 2.00$ Äq.) wurde bei Raumtemperatur eine Lösung des Pinakolboronsäureesters 134 (100 mg, $194 \mu \mathrm{mol}, 1.00$ Äq.) in sorgfältig entgastem THF (2 ml) und $\mathrm{H}_{2} \mathrm{O}$ (14.0 $\mu \mathrm{l}, 14.0 \mathrm{mg}, 776 \mu \mathrm{mol}, 4.00$ Äq.) gegeben und $17 \mathrm{~h}$ bei dieser Temperatur gerührt. Anschließend wurde die Reaktionslösung durch Celite ${ }^{\circledR}$ filtriert, mit EtOAc $(3 \times 5 \mathrm{ml})$ nachgewaschen, das Lösungsmittel unter vermindertem Druck entfernt und nach Säulenchromatographie an Kieselgel (Petrolether/EtOAc 4:1 $\rightarrow$ 2:1) das Biaryl 135 (59.0 mg, $104 \mu \mathrm{mol}, 53 \%$ ) als leicht gelbes Öl isoliert.

DC: $R_{\mathrm{f}}=0.44$ (Petrolether/EtOAc 7:3).

UV $\left(\mathrm{CH}_{3} \mathrm{CN}\right): \lambda_{\max }[\mathrm{nm}](\lg \varepsilon)=196$ (4.813).

IR $(A T R): ~ \tilde{v}\left[\mathrm{~cm}^{-1}\right]=2917,2360,1726,1602,1542,1366,1248,1088,1031,799$.

${ }^{1} \mathrm{H}-\mathrm{NMR}\left(600 \mathrm{MHz}, \mathrm{CDCl}_{3}\right): \delta[\mathrm{ppm}]=1.55\left(\mathrm{~s}, 9 \mathrm{H}, \mathrm{C}\left(\mathrm{CH}_{3}\right)_{3}\right), 3.55\left(\mathrm{~s}, 3 \mathrm{H}, 2-\mathrm{OCH}_{3}\right), 3.77$ (s, $3 \mathrm{H}, \mathrm{PMB}-\mathrm{OCH}_{3}$ ), 3.83 (s, $3 \mathrm{H}, 4-\mathrm{OCH}_{3}$ ), 3.89 (s, $\left.3 \mathrm{H}, \mathrm{CO}_{2} \mathrm{CH}_{3}\right), 4.11$ (d, J = $12.6 \mathrm{~Hz}$, $\left.1 \mathrm{H}, 6-\underline{C}_{a} \mathrm{H}_{\mathrm{b}}\right), 4.25\left(\mathrm{~d}, J=11.4 \mathrm{~Hz}, 1 \mathrm{H}, \mathrm{PMB}-\underline{C}_{\mathrm{a}} \mathrm{H}_{\mathrm{b}}\right), 4.26(\mathrm{~d}, J=12.6 \mathrm{~Hz}, 1 \mathrm{H}$, 6- $\mathrm{CH}_{\mathrm{a}} \underline{\mathrm{H}}_{\mathrm{b}}$ ), 4.30 (d, J = 11.4 Hz, $1 \mathrm{H}, \mathrm{PMB}-\mathrm{CH}_{a} \underline{\mathrm{H}}_{\mathrm{b}}$ ), 6.81 (d, J = 8.6 Hz, $2 \mathrm{H}, 2 \times \mathrm{PMB}^{-H_{m}}$ ), $6.84(\mathrm{~s}, 1 \mathrm{H}, 5-\mathrm{H}), 7.13\left(\mathrm{~d}, J=8.6 \mathrm{~Hz}, 2 \mathrm{H}, 2 \times \mathrm{PMB}^{-} \mathrm{H}_{\circ}\right), 7.46(\mathrm{dd}, J=7.7,1.4 \mathrm{~Hz}, 1 \mathrm{H}$, 6'-H), 7.53 (t, $J=7.7 \mathrm{~Hz}, 1 \mathrm{H}, 5^{\prime}-\mathrm{H}$ ), 7.99 (dd, $J=7.8,1.4 \mathrm{~Hz}, 1 \mathrm{H}, 4^{\prime}-\mathrm{H}$ ).

${ }^{13} \mathrm{C}-\mathrm{NMR}\left(126 \mathrm{MHz}, \mathrm{CDCl}_{3}\right): \delta[\mathrm{ppm}]=28.1\left(\mathrm{C}\left(\mathrm{CH}_{3}\right)_{3}\right), 53.1\left(\mathrm{CO}_{2} \underline{\mathrm{C}} \mathrm{H}_{3}\right)$, $55.2\left(\mathrm{PMB}^{-\mathrm{OCH}_{3}}\right)$, $56.1\left(4-\mathrm{OCH}_{3}\right), 61.8\left(2-\mathrm{OCH}_{3}\right), 69.3\left(6-\mathrm{CH}_{2}\right), 72.1\left(\mathrm{PMB}-\mathrm{CH}_{2}\right), 82.3\left(\underline{\mathrm{C}}\left(\mathrm{CH}_{3}\right)_{3}\right), 106.3$ (C-5), 113.7 (2 × PMB-C ${ }_{m}$ ), 118.4 (C-3), 119.7 (C-1), 123.6 (C-3'), 129.3 (2 × PMB-Co), 129.8, 129.9, 129.9 (C-1', C-5', PMB-Ci), 130.5 (C-4'), 137.0 (C-6'), 140.4 (C-6), 150.1 (C-2'), 155.3 (C-4), 158.0 (C-2), $159.2\left(\mathrm{PMB}-\mathrm{C}_{p}\right), 164.0\left(\underline{\mathrm{CO}}_{2} \mathrm{CH}_{3}\right), 165.1\left(\underline{\mathrm{CO}}{ }_{2} \mathrm{C}\left(\mathrm{CH}_{3}\right)_{3}\right)$. MS (ESI): $m / z(\%)=590.2(100)[\mathrm{M}+\mathrm{Na}]^{+}, 1157.4(95)[2 \times \mathrm{M}+\mathrm{Na}]^{+}$.

$\mathrm{C}_{30} \mathrm{H}_{33} \mathrm{NO}_{10}(567.59)$

ber.: 590.1997

gef.: $590.1989[\mathrm{M}+\mathrm{Na}]^{+}(\mathrm{ESI}-\mathrm{HRMS})$. 


\subsection{Darstellung des Z-Alkens (Z)-175}

\subsubsection{3,5-Dimethoxybenzoesäuremethylester (154)}<smiles>O=C(O)c1cc(O)cc(O)c1</smiles>

153

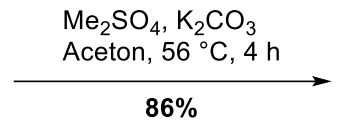

$86 \%$

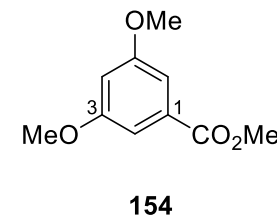

154

Zu einer Suspension aus 3,5-Dihydroxybenzoesäure (153) (25.0 g, 162 mmol, 1.00 Äq.) und $\mathrm{K}_{2} \mathrm{CO}_{3}(89.7 \mathrm{~g}, 649 \mathrm{mmol}, 4.00 \mathrm{Äq}$.) in Aceton (250 ml) wurde bei Raumtemperatur $\mathrm{Me}_{2} \mathrm{SO}_{4}$ (53.7 ml, $71.6 \mathrm{~g}, 568 \mathrm{mmol}, 3.55$ Äq.) gegeben, anschließend auf $56{ }^{\circ} \mathrm{C}$ erwärmt und $4 \mathrm{~h}$ bei dieser Temperatur unter Rückfluss gerührt. Nach Abkühlen auf Raumtemperatur wurde der Feststoff abfiltriert, mit Aceton $(2 \times 50 \mathrm{ml})$ nachgewaschen und das Filtrat unter vermindertem Druck eingeengt. Der Rückstand wurde in verd. $\mathrm{NH}_{3}$ (5\%ig, $125 \mathrm{ml}$ ) aufgenommen, $5 \mathrm{~min}$ bei Raumtemperatur gerührt und anschließend mit $\mathrm{Et}_{2} \mathrm{O}(125+2 \times 25 \mathrm{ml})$ extrahiert. Die vereinigten org. Phasen wurden mit verd. $\mathrm{HCl}$ (5\%ig, $100 \mathrm{ml}$ ) sowie ges. $\mathrm{NaHCO}_{3}$-Lsg. $(100 \mathrm{ml})$ gewaschen, über $\mathrm{MgSO}_{4}$ getrocknet und das Lösungsmittel unter vermindertem Druck entfernt. Nach Umkristallisation aus $\mathrm{MeOH}(40 \mathrm{ml})$ und $\mathrm{H}_{2} \mathrm{O}$ (25 ml, zur Induktion der Kristallisation), abschließendem Waschen mit eiskaltem $\mathrm{MeOH} / \mathrm{H}_{2} \mathrm{O}(2: 1,50 \mathrm{ml})$ sowie Trocknen im Hochvakuum wurde der Methylester 154 (27.4 g, 140 mmol, 86\%) als weißer Feststoff isoliert.

${ }^{1} \mathrm{H}-\mathrm{NMR}\left(300 \mathrm{MHz}, \mathrm{CDCl}_{3}\right): \delta[\mathrm{ppm}]=3.79\left(\mathrm{~s}, 6 \mathrm{H}, 2 \times \mathrm{OCH}_{3}\right), 3.87\left(\mathrm{~s}, 3 \mathrm{H}, \mathrm{CO}_{2} \mathrm{CH}_{3}\right)$, 6.60 (t, J=2.4 Hz, $1 \mathrm{H}, 4-\mathrm{H}), 7.15$ (d, J = 2.4 Hz, $2 \mathrm{H}, 2-\mathrm{H}, 6-\mathrm{H})$.

${ }^{13} \mathrm{C}-\mathrm{NMR}\left(126 \mathrm{MHz}, \mathrm{CDCl}_{3}\right): \delta[\mathrm{ppm}]=52.1\left(\mathrm{CO}_{2} \underline{\mathrm{CH}}_{3}\right), 55.5\left(2 \times \mathrm{OCH}_{3}\right), 105.7$ (C-4), 107.0 (C-2, C-6), 131.9 (C-1), 160.6 (C-3, C-5), $166.8\left(\underline{\mathrm{CO}_{2}} \mathrm{CH}_{3}\right)$.

MS (ESI): $m / z(\%)=197.1(100)[\mathrm{M}+\mathrm{H}]^{+}, 219.1(48)[\mathrm{M}+\mathrm{Na}]^{+}, 415.1(17)[2 \times \mathrm{M}+\mathrm{Na}]^{+}$.

$\mathbf{C}_{10} \mathrm{H}_{12} \mathbf{O}_{4}(196.20)$

ber.: 197.0808

219.0628

gef.: $197.0809[\mathrm{M}+\mathrm{H}]^{+}$

$219.0626[\mathrm{M}+\mathrm{Na}]^{+}(\mathrm{ESI}-\mathrm{HRMS})$. 


\subsubsection{3,5-Dimethoxybenzylalkohol (155)}

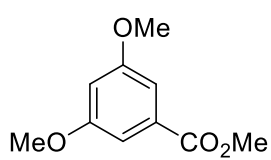

154

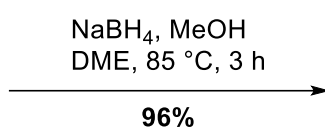

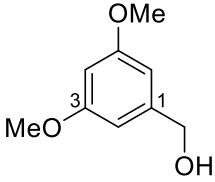

155

Zu einer Lösung des Methylesters 154 (27.3 g, 140 mmol, 1.00 Äq.) in DME (250 ml) wurde bei Raumtemperatur $\mathrm{NaBH}_{4}(26.5 \mathrm{~g}, 701 \mathrm{mmol}, 5.00$ Äq.) gegeben, das resultierende Reaktionsgemisch auf $85^{\circ} \mathrm{C}$ erwärmt und über einen Zeitraum von $2 \mathrm{~h}$ mit $\mathrm{MeOH}(125 \mathrm{ml})$ versetzt. Die Reaktionsmischung wurde eine weitere Stunde bei dieser Temperatur unter Rückfluss gerührt. Nach Abkühlen auf Raumtemperatur wurde das Lösungsmittel unter vermindertem Druck entfernt, der Rückstand in $\mathrm{Et}_{2} \mathrm{O}$ (100 ml) sowie $\mathrm{H}_{2} \mathrm{O}(150 \mathrm{ml})$ aufgenommen, die Phasen getrennt und die wässr. Phase mit $\mathrm{Et}_{2} \mathrm{O}(3 \times$ $50 \mathrm{ml}$ ) extrahiert. Die vereinigten org. Phasen wurden mit $\mathrm{H}_{2} \mathrm{O}(100 \mathrm{ml})$ und ges. $\mathrm{NaCl}$ Lsg. (100 ml) gewaschen, über $\mathrm{MgSO}_{4}$ getrocknet und das Lösungsmittel unter vermindertem Druck entfernt. Nach Trocknen im Hochvakuum wurde der Benzylalkohol 155 (22.5 g, $134 \mathrm{mmol}, 96 \%)$ als farbloses Öl erhalten, das während der Lagerung zu einem weißen Feststoff kristallisierte.

${ }^{1} \mathrm{H}-\mathrm{NMR}\left(300 \mathrm{MHz}, \mathrm{CDCl}_{3}\right): \delta[\mathrm{ppm}]=2.63(\mathrm{~s}, 1 \mathrm{H}, \mathrm{OH}), 3.73\left(\mathrm{~s}, 6 \mathrm{H}, 2 \times \mathrm{OCH}_{3}\right), 4.54(\mathrm{~s}$, $\left.2 \mathrm{H}, 1-\mathrm{CH}_{2}\right), 6.33(\mathrm{t}, J=2.3 \mathrm{~Hz}, 1 \mathrm{H}, 4-\mathrm{H}), 6.46(\mathrm{~d}, J=2.3 \mathrm{~Hz}, 2 \mathrm{H}, 2-\mathrm{H}, 6-\mathrm{H})$.

${ }^{13} \mathrm{C}-N M R\left(126 \mathrm{MHz}, \mathrm{CDCl}_{3}\right): \delta[\mathrm{ppm}]=55.2\left(2 \times \mathrm{OCH}_{3}\right), 64.9\left(1-\mathrm{CH}_{2}\right), 99.4(\mathrm{C}-4), 104.4$ (C-2, C-6), 143.4 (C-1), 160.7 (C-3, C-5).

MS (ESI): $m / z(\%)=169.1(97)[\mathrm{M}+\mathrm{H}]^{+}, 191.1(96)[\mathrm{M}+\mathrm{Na}]^{+}$.

$\mathrm{C}_{9} \mathrm{H}_{12} \mathrm{O}_{3}(168.19)$

ber.: 169.0859

191.0679

gef.: $169.0860[\mathrm{M}+\mathrm{H}]^{+}$

$191.0679[\mathrm{M}+\mathrm{Na}]^{+}(\mathrm{ESI}-\mathrm{HRMS})$. 


\subsubsection{1-(((tert-Butyldimethylsilyl)oxy)methyl)-3,5-dimethoxybenzol (163)}

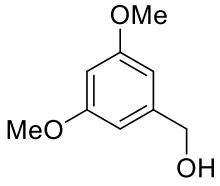

155

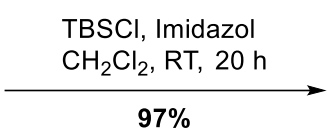

$97 \%$

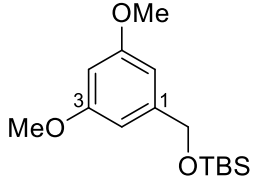

163

3,5-Dimethoxybenzylalkohol (155) (25.0 g, 149 mmol, 1.00 Äq.) wurde in $\mathrm{CH}_{2} \mathrm{Cl}_{2}$ (250 ml) gelöst, bei Raumtemperatur mit Imidazol (20.2 g, 297 mmol, 2.00 Äq.) sowie TBSCI (44.8 g, $297 \mathrm{mmol}, 2.00$ Äq.) versetzt und $20 \mathrm{~h}$ bei dieser Temperatur gerührt. Die Reaktion wurde durch Zugabe von ges. $\mathrm{NH}_{4} \mathrm{Cl}$-Lsg. $(200 \mathrm{ml})$ beendet, die Phasen getrennt und die wässr. Phase mit $\mathrm{CH}_{2} \mathrm{Cl}_{2}(2 \times 200 \mathrm{ml})$ extrahiert. Anschließend wurden die vereinigten org. Phasen über $\mathrm{MgSO}_{4}$ getrocknet, das Lösungsmittel unter vermindertem Druck entfernt und nach säulenchromatographischer Reinigung an Kieselgel (Petrolether/EtOAc 3:1) die Zielverbindung 163 (40.8 g, $144 \mathrm{mmol}$, 97\%) als farbloses Öl erhalten.

${ }^{1} \mathrm{H}-\mathrm{NMR}\left(300 \mathrm{MHz}, \mathrm{CDCl}_{3}\right): \delta[\mathrm{ppm}]=0.10\left(\mathrm{~d}, J=0.4 \mathrm{~Hz}, 6 \mathrm{H}, \mathrm{Si}\left(\mathrm{CH}_{3}\right)_{2}\right), 0.95(\mathrm{~d}, J=$ $\left.0.4 \mathrm{~Hz}, 9 \mathrm{H}, \mathrm{SiC}\left(\mathrm{CH}_{3}\right)_{3}\right), 3.78\left(\mathrm{~d}, J=0.4 \mathrm{~Hz}, 6 \mathrm{H}, 2 \times \mathrm{OCH}_{3}\right.$ ), 4.69 (quint., $J=0.7 \mathrm{~Hz}, 2 \mathrm{H}$, 1- $\left.\mathrm{CH}_{2}\right), 6.33-6.35(\mathrm{~m}, 1 \mathrm{H}, 4-\mathrm{H}), 6.50$ (dq, $\left.J=2.2,0.6 \mathrm{~Hz}, 2 \mathrm{H}, 2-\mathrm{H}, 6-\mathrm{H}\right)$.

${ }^{13} \mathrm{C}-\mathrm{NMR}\left(126 \mathrm{MHz}, \mathrm{CDCl}_{3}\right): \delta[\mathrm{ppm}]=-5.2\left(\mathrm{Si}\left(\mathrm{CH}_{3}\right)_{2}\right), 18.5\left(\mathrm{Si} \underline{\mathrm{C}}\left(\mathrm{CH}_{3}\right)_{3}\right), 26.0\left(\mathrm{SiC}\left(\underline{\mathrm{CH}}_{3}\right)_{3}\right)$, $55.2\left(2 \times \mathrm{OCH}_{3}\right), 64.8\left(1-\mathrm{CH}_{2}\right), 98.8$ (C-4), 103.6 (C-2, C-6), 143.9 (C-1), 160.6 (C-3, C-5). MS (ESI): $m / z(\%)=283.2(31)[\mathrm{M}+\mathrm{H}]^{+}, 305.2(100)[\mathrm{M}+\mathrm{Na}]^{+}, 587.4(16)[2 \times \mathrm{M}+\mathrm{Na}]^{+}$.

$\mathrm{C}_{15} \mathrm{H}_{26} \mathrm{O}_{3} \mathrm{Si}(282.46)$

ber.: 283.1724

305.1543

gef.: $283.1724[\mathrm{M}+\mathrm{H}]^{+}$

$305.1547[\mathrm{M}+\mathrm{Na}]^{+}(\mathrm{ESI}-\mathrm{HRMS})$. 


\subsubsection{4-(((tert-Butyldimethylsilyl)oxy)methyl)-2,6-dimethoxybenzaldehyd}

(164)

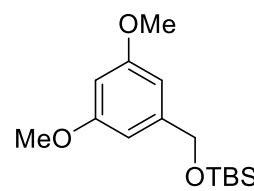

163

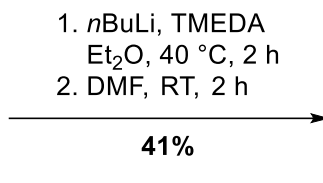

$\mathrm{Et}_{2} \mathrm{O}, 40^{\circ} \mathrm{C}, 2 \mathrm{~h}$

$41 \%$

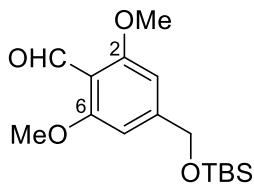

164

Zu einer Lösung des Silylethers 163 (40.0 g, $142 \mathrm{mmol}, 1.00$ Äq.) sowie frisch destilliertem TMEDA (74.8 ml, $57.6 \mathrm{~g}, 496 \mathrm{mmol}, 3.50 \mathrm{Äq}$.) in $\mathrm{Et}_{2} \mathrm{O}$ (400 ml) wurde bei $0{ }^{\circ} \mathrm{C} n \mathrm{BuLi}$ (2.5 M in $n$-Hexan, $99.1 \mathrm{ml}, 248 \mathrm{mmol}, 1.75 \mathrm{Äq}$.) getropft, die resultierende Lösung auf $40^{\circ} \mathrm{C}$ erwärmt und $2 \mathrm{~h}$ bei dieser Temperatur unter Rückfluss gerührt. Daraufhin wurde das Reaktionsgemisch auf $0^{\circ} \mathrm{C}$ gekühlt, DMF $(54.5 \mathrm{ml}, 51.8 \mathrm{~g}$, $708 \mathrm{mmol}, 5.00$ Äq.) hinzugetropft, auf Raumtemperatur erwärmt und weitere $2 \mathrm{~h}$ bei dieser Temperatur gerührt. Die Reaktion wurde durch vorsichtige Zugabe von $\mathrm{H}_{2} \mathrm{O}$ $(300 \mathrm{ml})$ beendet, die Phasen getrennt und die wässr. Phase mit $\mathrm{NaCl}$ gesättigt. Anschließend wurde die wässr. Phase mit $\mathrm{Et}_{2} \mathrm{O}(5 \times 400 \mathrm{ml})$ extrahiert, die vereinigten org. Phasen über $\mathrm{MgSO}_{4}$ getrocknet und das Lösungsmittel unter vermindertem Druck entfernt. Säulenchromatographische Reinigung an Kieselgel (Petrolether/EtOAc 3:1) lieferte den Aldehyd 164 (18.1 g, 58.2 mmol, 41\%) in Form eines leicht gelben Öls.

${ }^{1} \mathrm{H}-\mathrm{NMR}\left(300 \mathrm{MHz}, \mathrm{CDCl}_{3}\right): \delta[\mathrm{ppm}]=0.09\left(\mathrm{~s}, 6 \mathrm{H}, \mathrm{Si}\left(\mathrm{CH}_{3}\right)_{2}\right), 0.93\left(\mathrm{~s}, 9 \mathrm{H}, \mathrm{SiC}\left(\mathrm{CH}_{3}\right)_{3}\right)$, $3.85\left(\mathrm{~s}, 6 \mathrm{H}, 2 \times \mathrm{OCH}_{3}\right), 4.70$ (t, $\left.J=0.8 \mathrm{~Hz}, 2 \mathrm{H}, 4-\mathrm{CH}_{2}\right), 6.53(\mathrm{~d}, J=0.9 \mathrm{~Hz}, 2 \mathrm{H}, 3-\mathrm{H}$, 5-H), 10.42 (d, J = 0.4 Hz, $1 \mathrm{H}, \mathrm{CHO}$ ).

${ }^{13} \mathrm{C}-\mathrm{NMR}\left(126 \mathrm{MHz}, \mathrm{CDCl}_{3}\right): \delta[\mathrm{ppm}]=-5.3\left(\mathrm{Si}\left(\mathrm{CH}_{3}\right)_{2}\right), 18.4\left(\mathrm{Si} \underline{\mathrm{C}}\left(\mathrm{CH}_{3}\right)_{3}\right), 25.9\left(\mathrm{SiC}\left(\mathrm{CH}_{3}\right)_{3}\right)$, $55.9\left(2 \times \mathrm{OCH}_{3}\right), 64.6\left(4-\mathrm{CH}_{2}\right), 100.7$ (C-3, C-5), 112.8 (C-1), 150.7 (C-4), 162.1 (C-2, C-6), 188.8 (d, $J=3.9 \mathrm{~Hz}, \mathrm{CHO}$ ).

MS (ESI): $m / z(\%)=311.2(72)[\mathrm{M}+\mathrm{H}]^{+}, 333.2(17)[\mathrm{M}+\mathrm{Na}]^{+}, 643.4(100)[2 \times \mathrm{M}+\mathrm{Na}]^{+}$.

$\mathrm{C}_{16} \mathrm{H}_{26} \mathrm{O}_{4} \mathrm{Si}(310.47)$

ber.: 311.1673

333.1493

gef.: $311.1675[\mathrm{M}+\mathrm{H}]^{+}$

$333.1487[\mathrm{M}+\mathrm{Na}]^{+}(\mathrm{ESI}-\mathrm{HRMS})$. 


\subsection{5 (E)-1-Benzyloxy-4-(4-(((tert-butyldimethylsilyl)oxy)methyl)-2,6-di- methoxyphenyl)but-3-en-2-on ((E)-167)}

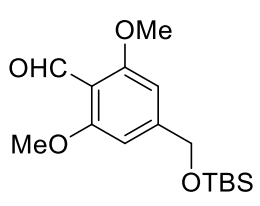

164

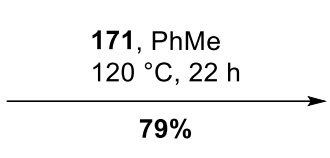
Eine Lösung des Aldehyds 164 (15.0 g, 48.3 mmol, 1.00 Äq.) und des P-Ylids 171 (26.7 g, $62.8 \mathrm{mmol}, 1.30$ Äq.) in Toluol (300 ml) wurde für $22 \mathrm{~h}$ bei $120^{\circ} \mathrm{C}$ unter Rückfluss gerührt. Nach Abkühlen auf Raumtemperatur wurde das Lösungsmittel unter vermindertem Druck entfernt und nach Säulenchromatographie an Kieselgel (Petrolether/EtOAc 4:1) das a,ß-ungesättigte Keton (E)-167 (17.4 g, $38.1 \mathrm{mmol}, 79 \%)$ als leicht gelbes Öl isoliert.

${ }^{1} \mathrm{H}-\mathrm{NMR}\left(300 \mathrm{MHz}, \mathrm{CDCl}_{3}\right): \delta[\mathrm{ppm}]=0.10\left(\mathrm{~d}, J=0.4 \mathrm{~Hz}, 6 \mathrm{H}, \mathrm{Si}\left(\mathrm{CH}_{3}\right)_{2}\right), 0.94(\mathrm{~d}, J=$ $\left.0.4 \mathrm{~Hz}, 9 \mathrm{H}, \mathrm{SiC}\left(\mathrm{CH}_{3}\right)_{3}\right), 3.84\left(\mathrm{~s}, 6 \mathrm{H}, 2 \times \mathrm{OCH}_{3}\right), 4.33\left(\mathrm{~s}, 2 \mathrm{H}, 1-\mathrm{H}_{2}\right), 4.64(\mathrm{~s}, 2 \mathrm{H}$, $\left.\mathrm{OCH}_{2} \mathrm{Ph}\right), 4.71\left(\mathrm{~s}, 2 \mathrm{H}, 4^{\prime}-\mathrm{CH}_{2}\right), 6.53(\mathrm{~s}, 2 \mathrm{H}, 3$ '- $\mathrm{H}, 5$ '- $\mathrm{H}), 7.24-7.43(\mathrm{~m}, 6 \mathrm{H}, 3-\mathrm{H}, 5 \times$ $\mathrm{Ph}-\mathrm{H}), 8.14$ (d, $J=16.4 \mathrm{~Hz}, 1 \mathrm{H}, 4-\mathrm{H})$.

${ }^{13} \mathrm{C}-N M R\left(126 \mathrm{MHz}, \mathrm{CDCl}_{3}\right): \delta[\mathrm{ppm}]=-5.2\left(\mathrm{Si}\left(\mathrm{CH}_{3}\right)_{2}\right), 18.4\left(\mathrm{Si} \underline{\mathrm{C}}\left(\mathrm{CH}_{3}\right)_{3}\right), 25.9\left(\mathrm{SiC}\left(\underline{\mathrm{CH}}_{3}\right)_{3}\right)$, $55.7\left(2 \times \mathrm{OCH}_{3}\right), 64.8\left(4^{\prime}-\mathrm{CH}_{2}\right), 73.2\left(\mathrm{OCH}_{2} \mathrm{Ph}\right), 74.6$ (C-1), $100.8(\mathrm{C}-3$ ', C-5'), 110.7 (C-1'), 123.8 (C-3), $127.7\left(\mathrm{Ph}-\mathrm{C}_{p}\right), 127.8,128.3$ (2 × $\left.\mathrm{Ph}-\mathrm{C}_{o}, 2 \times \mathrm{Ph}^{-\mathrm{C}_{m}}\right), 134.6(\mathrm{C}-4), 137.6$ $\left(\mathrm{Ph}-\mathrm{C}_{i}\right), 146.2$ (C-4'), 160.3 (C-2', C-6'), 198.5 (C-2).

MS (ESI): $m / z(\%)=457.3(42)[\mathrm{M}+\mathrm{H}]^{+}, 479.2(28)[\mathrm{M}+\mathrm{Na}]^{+}, 935.5(100)[2 \times \mathrm{M}+\mathrm{Na}]^{+}$; $455.2(52)[\mathrm{M}-\mathrm{H}]^{-}$.

$\mathrm{C}_{26} \mathrm{H}_{36} \mathrm{O}_{5} \mathrm{Si}(456.65)$

$$
\begin{aligned}
\text { ber.: } & 455.2259 \\
& 457.2405 \\
& 479.2224 \\
\text { gef.: } & 455.2256[\mathrm{M}-\mathrm{H}]^{-} \\
& 457.2394[\mathrm{M}+\mathrm{H}]^{+} \\
& 479.2215[\mathrm{M}+\mathrm{Na}]^{+}(\mathrm{ESI}-\mathrm{HRMS}) .
\end{aligned}
$$




\subsubsection{1-Benzyloxy-4-(4-((tert-butyldimethylsilyl)oxy)methyl)-2,6-di- methoxyphenyl)butan-2-on (168)}

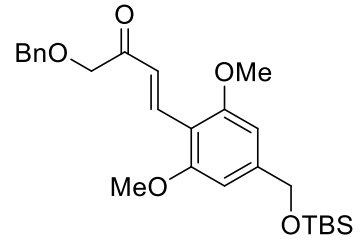

(E)-167

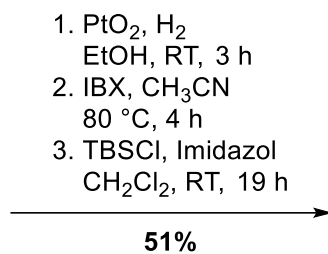

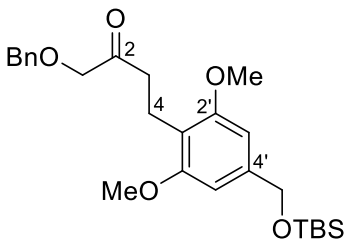

168

Durch eine Lösung des $\alpha, \beta$-ungesättigten Ketons $(E)-167$ (17.0 g, 37.2 mmol, 1.00 Äq.) und $\mathrm{PtO}_{2}$ (423 mg, $\left.1.86 \mathrm{mmol}, 5 \mathrm{~mol} \%\right)$ in $\mathrm{EtOH}(500 \mathrm{ml})$ wurde für $3 \mathrm{~h}$ bei Raumtemperatur $\mathrm{H}_{2}$ geleitet. Anschließend wurde der Katalysator mittels Filtration durch Celite $^{\circledR}$ abgetrennt, mit EtOAc $(300 \mathrm{ml})$ nachgewaschen und das Lösungsmittel unter vermindertem Druck entfernt.

Der Rückstand wurde in $\mathrm{CH}_{3} \mathrm{CN}(300 \mathrm{ml})$ aufgenommen, die resultierende Lösung bei Raumtemperatur mit IBX (275) (4.86 g, $17.4 \mathrm{mmol}, 0.47$ Äq.) versetzt, auf $80{ }^{\circ} \mathrm{C}$ erwärmt und $4 \mathrm{~h}$ bei dieser Temperatur unter Rückfluss gerührt. Nach Abkühlen auf Raumtemperatur wurde durch Celite ${ }^{\circledR}$ filtriert, mit EtOAc $(300 \mathrm{ml})$ nachgewaschen und das Lösungsmittel unter vermindertem Druck entfernt. Anschließend wurden das gewünschte Keton 168 und der korrespondierende TBS-entschützte Alkohol mittels Säulenchromatographie an Kieselgel (Petrolether/EtOAc 9:1 $\rightarrow$ 3:1) voneinander getrennt.

Der TBS-entschützte Benzyalkohol (8.96 g, 26.0 mmol, 1.00 Äq.) wurde in $\mathrm{CH}_{2} \mathrm{Cl}_{2}$ $(100 \mathrm{ml})$ gelöst, bei Raumtemperatur mit Imidazol (3.54 g, $52.0 \mathrm{mmol}, 2.00$ Äq.) sowie TBSCl (7.84 g, 52.0 mmol, 2.00 Äq.) versetzt und $19 \mathrm{~h}$ bei dieser Temperatur gerührt. Die Reaktion wurde durch Zugabe von ges. $\mathrm{NH}_{4} \mathrm{Cl}$-Lsg. $(100 \mathrm{ml})$ beendet, die Phasen getrennt und die wässr. Phase mit $\mathrm{CH}_{2} \mathrm{Cl}_{2}(2 \times 100 \mathrm{ml})$ extrahiert. Daraufhin wurden die vereinigten org. Phasen über $\mathrm{MgSO}_{4}$ getrocknet, das Lösungsmittel unter vermindertem Druck entfernt und nach säulenchromatographischer Reinigung an Kieselgel das Keton 168 (kombinierte Gesamtausbeute: 8.77 g, 19.1 mmol, 51\%) als leicht gelbes Öl erhalten.

${ }^{1} \mathrm{H}-\mathrm{NMR}\left(300 \mathrm{MHz}, \mathrm{CDCl}_{3}\right): \delta[\mathrm{ppm}]=0.09\left(\mathrm{~d}, J=0.6 \mathrm{~Hz}, 6 \mathrm{H}, \mathrm{Si}\left(\mathrm{CH}_{3}\right)_{2}\right), 0.93(\mathrm{~d}, J=$ $\left.0.7 \mathrm{~Hz}, 9 \mathrm{H}, \mathrm{SiC}\left(\mathrm{CH}_{3}\right)_{3}\right), 2.59\left(\mathrm{dd}, J=8.9,6.8 \mathrm{~Hz}, 2 \mathrm{H}, 3-\mathrm{H}_{2}\right), 2.88$ (dd, $J=8.9,6.8 \mathrm{~Hz}$, $\left.2 \mathrm{H}, 4-\mathrm{H}_{2}\right), 3.74\left(\mathrm{~d}, \mathrm{~J}=0.7 \mathrm{~Hz}, 6 \mathrm{H}, 2 \times \mathrm{OCH}_{3}\right), 4.06\left(\mathrm{~s}, 2 \mathrm{H}, 1-\mathrm{H}_{2}\right), 4.56$ (s, $2 \mathrm{H}, \mathrm{OCH}_{2} \mathrm{Ph}$ ), 4.68 (d, J = 0.8 Hz, 2 H, 4'-CH $), 6.49$ (s, 2 H, 3'-H, 5'-H), 7.23-7.35 (m, 5 H, $5 \times$ Ph-H).

$\left.{ }^{13} \mathrm{C}-\mathrm{NMR}(126 \mathrm{MHz}, \mathrm{CDCl})_{3}\right): \delta[\mathrm{ppm}]=-5.1\left(\mathrm{Si}\left(\mathrm{CH}_{3}\right)_{2}\right), 17.5(\mathrm{C}-4), 18.5\left(\mathrm{SiC}\left(\mathrm{CH}_{3}\right)_{3}\right), 26.0$ $\left(\mathrm{SiC}\left(\underline{\mathrm{CH}}_{3}\right)_{3}\right), 38.7(\mathrm{C}-3), 55.6\left(2 \times \mathrm{OCH}_{3}\right), 65.1\left(4^{\prime}-\mathrm{CH}_{2}\right), 73.3\left(\mathrm{OCH}_{2} \mathrm{Ph}\right), 74.9(\mathrm{C}-1), 101.1$ 


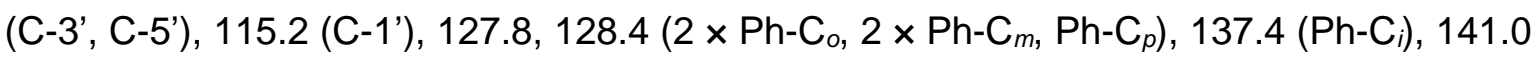
(C-4'), 157.9 (C-2', C-6'), 208.6 (C-2).

MS (ESI): $m / z(\%)=459.3(18)[\mathrm{M}+\mathrm{H}]^{+}, 476.3(31)\left[\mathrm{M}+\mathrm{NH}_{4}\right]^{+}, 481.3(53)[\mathrm{M}+\mathrm{Na}]^{+}$, $939.5(100)[2 \times \mathrm{M}+\mathrm{Na}]^{+}$.

$\mathrm{C}_{26} \mathrm{H}_{38} \mathrm{O}_{5} \mathrm{Si}$ (458.67)

ber.: 459.2561

481.2381

gef.: $459.2560[\mathrm{M}+\mathrm{H}]^{+}$

$481.2388[\mathrm{M}+\mathrm{Na}]^{+}(\mathrm{ESI}-\mathrm{HRMS})$. 


\subsubsection{1-Benzyloxy-4-(4-(((tert-butyldimethylsilyl)oxy)methyl)-3-iod-2,6- dimethoxyphenyl)butan-2-on (172)}

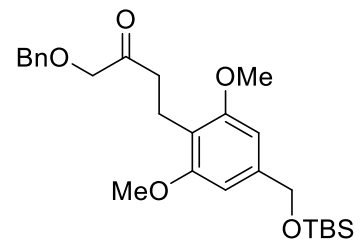

168

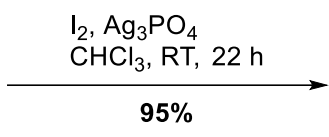

172

Zu einer Lösung des Ketons 168 (3.00 g, 6.54 mmol, 1.00 Äq.) in $\mathrm{CHCl}_{3}$ (50 ml) wurde bei Raumtemperatur eine Lösung von $\mathrm{I}_{2}\left(1.66 \mathrm{~g}, 6.54 \mathrm{mmol}, 1.00 \mathrm{Äq}\right.$.) in $\mathrm{CHCl}_{3}$ (50 ml) gegeben und nach erfolgter Zugabe die Reaktionslösung mit $\mathrm{Ag}_{3} \mathrm{PO}_{4}(1.04 \mathrm{~g}, 2.40 \mathrm{mmol}$, 0.37 Äq.) versetzt. Das Reaktionsgemisch wurde $22 \mathrm{~h}$ bei dieser Temperatur gerührt. Daraufhin wurde der Feststoff abfiltriert, mit $\mathrm{CH}_{2} \mathrm{Cl}_{2}(50 \mathrm{ml})$ nachgewaschen, das Filtrat mit ges. $\mathrm{Na}_{2} \mathrm{~S}_{2} \mathrm{O}_{3}$-Lsg. $(3 \times 50 \mathrm{ml})$ sowie ges. $\mathrm{NaCl}$-Lsg $(50 \mathrm{ml})$ gewaschen, die org. Phase über $\mathrm{MgSO}_{4}$ getrocknet und das Lösungsmittel unter vermindertem Druck entfernt. Säulenchromatographie an Kieselgel (Petrolether/EtOAc 7:1) lieferte das Aryliodid 172 (3.61 g, $6.18 \mathrm{mmol}, 95 \%)$ in Form eines leicht gelben Öls.

${ }^{1} \mathrm{H}-\mathrm{NMR}\left(300 \mathrm{MHz}, \mathrm{CDCl}_{3}\right): \delta[\mathrm{ppm}]=0.13\left(\mathrm{~d}, J=0.4 \mathrm{~Hz}, 6 \mathrm{H}, \mathrm{Si}\left(\mathrm{CH}_{3}\right)_{2}\right), 0.96(\mathrm{~d}, J=$ $\left.0.4 \mathrm{~Hz}, 9 \mathrm{H}, \mathrm{SiC}\left(\mathrm{CH}_{3}\right)_{3}\right), 2.68\left(\mathrm{dd}, J=9.1,6.6 \mathrm{~Hz}, 2 \mathrm{H}, 3-\mathrm{H}_{2}\right), 2.95$ (dd, $J=9.1,6.7 \mathrm{~Hz}$, $\left.2 \mathrm{H}, 4-\mathrm{H}_{2}\right), 3.73\left(\mathrm{~d}, J=0.4 \mathrm{~Hz}, 3 \mathrm{H}, 2^{\prime}-\mathrm{OCH}_{3}\right), 3.77$ (s, $\left.3 \mathrm{H}, 6{ }^{\prime}-\mathrm{OCH}_{3}\right), 4.06\left(\mathrm{~s}, 2 \mathrm{H}, 1-\mathrm{H}_{2}\right)$, 4.56 (s, $2 \mathrm{H}, \mathrm{OCH}_{2} \mathrm{Ph}$ ), 4.58 (d, J = 0.8 Hz, $\left.2 \mathrm{H}, 4^{\prime}-\mathrm{CH}_{2}\right), 6.96$ (s, $\left.1 \mathrm{H}, 5^{\prime}-\mathrm{H}\right), 7.23-7.35$ (m, $5 \mathrm{H}, 5 \times \mathrm{Ph}-\mathrm{H})$.

${ }^{13} \mathrm{C}-\mathrm{NMR}\left(126 \mathrm{MHz}, \mathrm{CDCl}_{3}\right): \delta[\mathrm{ppm}]=-5.3\left(\mathrm{Si}\left(\mathrm{CH}_{3}\right)_{2}\right), 18.3\left(\mathrm{SiC}\left(\mathrm{CH}_{3}\right)_{3}\right), 18.9(\mathrm{C}-4), 25.9$ $\left(\mathrm{SiC}\left(\underline{\mathrm{CH}}_{3}\right)_{3}\right), 38.8(\mathrm{C}-3), 55.5\left(6^{\prime}-\mathrm{OCH}_{3}\right), 61.3\left(2^{\prime}-\mathrm{OCH}_{3}\right), 69.5\left(4^{\prime}-\mathrm{CH}_{2}\right), 73.3\left(\mathrm{OCH}_{2} \mathrm{Ph}\right)$, 74.9 (C-1), 82.2 (C-3'), 106.1 (C-5'), 121.7 (C-1'), 127.9, 127.9, 128.5 (2 × Ph-Co, $2 \times$ Ph- $\left.\mathrm{C}_{m}, \mathrm{Ph}-\mathrm{C}_{p}\right), 137.2\left(\mathrm{Ph}-\mathrm{C}_{i}\right), 142.8$ (C-4'), 157.6 (C-2'), 158.9 (C-6'), 208.3 (C-2).

MS $(\mathrm{ESI}): m / z(\%)=602.2(70)\left[\mathrm{M}+\mathrm{NH}_{4}\right]^{+}, 607.1(28)[\mathrm{M}+\mathrm{Na}]^{+}, 1191.3(100)[2 \times \mathrm{M}+$ $\mathrm{Na}]^{+}$.

$\mathrm{C}_{26} \mathrm{H}_{37} \mathrm{IO}_{5} \mathrm{Si}$ (584.57)

ber.: 607.1347

gef.: $607.1346[\mathrm{M}+\mathrm{Na}]^{+}(\mathrm{ESI}-\mathrm{HRMS})$. 


\subsubsection{1-Benzyloxy-4-(4-(((tert-butyldimethylsilyl)oxy)methyl)-2,6-di- methoxy-3-(4,4,5,5-tetramethyl-1,3,2-dioxaborolan-2-yl)phenyl)- butan-2-on (173)}

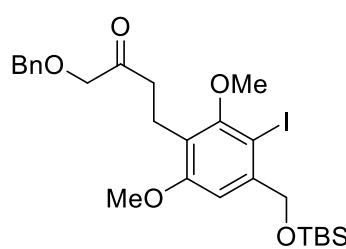

172
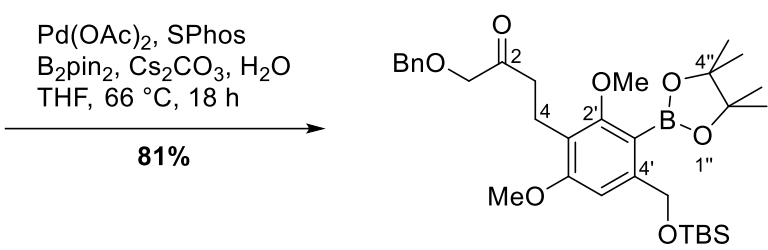

173

Zu einem Gemisch aus $\mathrm{Pd}(\mathrm{OAc})_{2}(38.4 \mathrm{mg}, 171 \mu \mathrm{mol}, 10 \mathrm{~mol} \%)$, SPhos (176 mg, $428 \mu \mathrm{mol}, 25 \mathrm{~mol} \%$ ), $\mathrm{B}_{2} \mathrm{pin}_{2}$ (869 mg, $3.42 \mathrm{mmol}, 2.00 \mathrm{Äq}$ ) sowie $\mathrm{Cs}_{2} \mathrm{CO}_{3}(1.11 \mathrm{~g}$, $3.42 \mathrm{mmol}, 2.00$ Äq.) wurde bei Raumtemperatur eine Lösung des Aryliodids 172 (1.00 g, $1.71 \mathrm{mmol}, 1.00$ Äq.) in sorgfältig entgastem THF (100 ml) und $\mathrm{H}_{2} \mathrm{O}(123 \mu \mathrm{l}, 123 \mathrm{mg}$, $6.84 \mathrm{mmol}, 4.00$ Äq.) gegeben, die resultierende Reaktionslösung auf $66^{\circ} \mathrm{C}$ erwärmt und $18 \mathrm{~h}$ bei dieser Temperatur unter Rückfluss gerührt. Anschließend wurde die Reaktionslösung auf Raumtemperatur abgekühlt, durch Celite ${ }^{\circledR}$ filtriert, mit EtOAc $(3 \times$ $50 \mathrm{ml}$ ) nachgewaschen, das Lösungsmittel unter vermindertem Druck entfernt und nach Säulenchromatographie an Kieselgel (Petrolether/EtOAc 7:1) der Pinakolboronsäureester 173 (814 mg, 1.39 mmol, 81\%) als leicht braunes Öl erhalten.

${ }^{1} \mathrm{H}-\mathrm{NMR}\left(300 \mathrm{MHz}, \mathrm{CDCl}_{3}\right): \delta[\mathrm{ppm}]=0.12\left(\mathrm{~d}, J=0.5 \mathrm{~Hz}, 6 \mathrm{H}, \mathrm{Si}\left(\mathrm{CH}_{3}\right)_{2}\right), 0.96(\mathrm{~d}, J=$ $\left.0.5 \mathrm{~Hz}, 9 \mathrm{H}, \mathrm{SiC}\left(\mathrm{CH}_{3}\right)_{3}\right), 1.24\left(\mathrm{~d}, J=0.6 \mathrm{~Hz}, 12 \mathrm{H}, 4 \times \mathrm{CH}_{3}\right), 2.67(\mathrm{dd}, J=9.1,6.6 \mathrm{~Hz}, 2 \mathrm{H}$, 3- $\mathrm{H}_{2}$ ), 2.94 (dd, $J=9.2,6.6 \mathrm{~Hz}, 2 \mathrm{H}, 4-\mathrm{H}_{2}$ ), 3.73 (d, $J=0.5 \mathrm{~Hz}, 3 \mathrm{H}, 2$ '- $\mathrm{OCH}_{3}$ ), 3.77 (s, $3 \mathrm{H}, 6$ ' $\left.-\mathrm{OCH}_{3}\right), 4.05\left(\mathrm{~s}, 2 \mathrm{H}, 1-\mathrm{H}_{2}\right), 4.56\left(\mathrm{~s}, 2 \mathrm{H}, \mathrm{OCH}_{2} \mathrm{Ph}\right), 4.58(\mathrm{~d}, J=0.8 \mathrm{~Hz}, 2 \mathrm{H}$, 4'- $\left.\mathrm{CH}_{2}\right), 6.95\left(\mathrm{~s}, 1 \mathrm{H}, 5^{\prime}-\mathrm{H}\right), 7.23-7.26(\mathrm{~m}, 5 \mathrm{H}, 5 \times \mathrm{Ph}-\mathrm{H})$.

${ }^{13} \mathrm{C}-\mathrm{NMR}\left(126 \mathrm{MHz}, \mathrm{CDCl}_{3}\right): \delta[\mathrm{ppm}]=-5.3\left(\mathrm{Si}\left(\mathrm{CH}_{3}\right)_{2}\right), 17.6(\mathrm{C}-4), 18.4\left(\mathrm{SiC}\left(\mathrm{CH}_{3}\right)_{3}\right), 24.6$ (4 × $\left.\mathrm{CH}_{3}\right), 25.6\left(\mathrm{SiC}\left(\underline{\mathrm{C}} \mathrm{H}_{3}\right)_{3}\right), 39.0(\mathrm{C}-3), 55.3\left(6^{\prime}-\mathrm{OCH}_{3}\right), 63.0\left(2^{\prime}-\mathrm{OCH}_{3}\right), 64.4\left(4^{\prime}-\mathrm{CH}_{2}\right)$, $73.3\left(\mathrm{OCH}_{2} \mathrm{Ph}\right), 74.8$ (C-1), 81.9 (C-3'), 83.4 (C-4", C-5”), 104.1 (C-5'), 119.7 (C-1'), 127.9 $\left(\mathrm{Ph}-\mathrm{C}_{p}\right), 127.9,128.4\left(2 \times \mathrm{Ph}^{-\mathrm{C}_{o}, 2} \times \mathrm{Ph} \mathrm{C}_{m}\right), 137.3\left(\mathrm{Ph}-\mathrm{C}_{i}\right), 147.2(\mathrm{C}-4$ ') 160.1 (C-2'), 163.5 (C-6'), 208.6 (C-2).

MS (ESI): $m / z(\%)=607.4(100)[\mathrm{M}+\mathrm{Na}]^{+}$.

$\mathrm{C}_{32} \mathrm{H}_{49} \mathrm{BO}_{7} \mathrm{Si}(584.63)$

ber.: 602.3685

607.3239

gef.: $602.3688\left[\mathrm{M}+\mathrm{NH}_{4}\right]^{+}$

$607.3245[\mathrm{M}+\mathrm{Na}]^{+}(\mathrm{ESI}-\mathrm{HRMS})$. 


\subsubsection{3'-(4-Benzyloxy-3-oxobutyl)-6'-((tert-butyldimethylsilyl)oxy)- methyl)-2',4'-dimethoxy-2-nitro-[1,1'-biphenyl]-3-carbonsäure- methylester (174)}

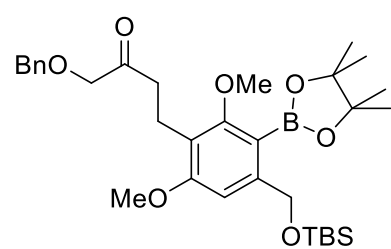

173

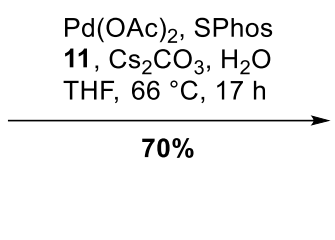

11, $\mathrm{Cs}_{2} \mathrm{CO}_{3}, \mathrm{H}_{2} \mathrm{O}$ $66^{\circ} \mathrm{C}, 17 \mathrm{r}$

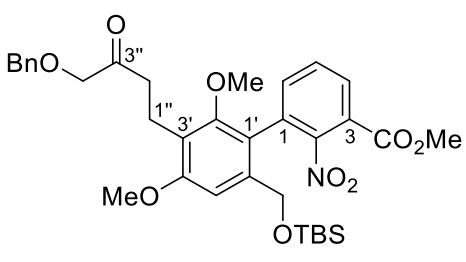

174

Zu einem Gemisch des Chlorarens 11 (1.48 g, 6.84 mmol, 1.00 Äq.), Pd(OAc) 2 (154 mg, $684 \mu \mathrm{mol}, 10 \mathrm{~mol} \%$ ), SPhos (702 mg, $1.71 \mathrm{mmol}, 25 \mathrm{~mol} \%$ ) und $\mathrm{Cs}_{2} \mathrm{CO}_{3}$ (4.46 g, $13.7 \mathrm{mmol}$, 2.00 Äq.) wurde bei Raumtemperatur eine Lösung des Pinakolboronsäureesters 173 (4.00 g, 6.84 mmol, 1.00 Äq.) in sorgfältig entgastem THF (200 ml) und $\mathrm{H}_{2} \mathrm{O}$ (493 $\mu \mathrm{l}, 493 \mathrm{mg}, 27.4 \mathrm{mmol}, 4.00$ Äq.) gegeben, die resultierende Reaktionslösung auf $66^{\circ} \mathrm{C}$ erwärmt und $17 \mathrm{~h}$ bei dieser Temperatur unter Rückfluss gerührt. Anschließend wurde die Reaktionslösung auf Raumtemperatur abgekühlt, durch Celite ${ }^{\circledR}$ filtriert, mit EtOAc $(3 \times 100 \mathrm{ml})$ nachgewaschen, das Lösungsmittel unter vermindertem Druck entfernt und nach Säulenchromatographie an Kieselgel (Petrolether/EtOAc 6:1 $\rightarrow$ 1:1) das Biaryl 174 (3.06 g, 4.79 mmol, 70\%) als oranges Öl isoliert.

${ }^{1} \mathrm{H}-\mathrm{NMR}\left(300 \mathrm{MHz}, \mathrm{CDCl}_{3}\right): \delta[\mathrm{ppm}]=-0.04(\mathrm{~s}, 3 \mathrm{H}),-0.01(\mathrm{~s}, 3 \mathrm{H})\left(\mathrm{Si}\left(\mathrm{CH}_{3}\right)_{2}\right), 0.87(\mathrm{~d}, J=$ $\left.0.5 \mathrm{~Hz}, 9 \mathrm{H}, \mathrm{SiC}\left(\mathrm{CH}_{3}\right)_{3}\right), 2.67$ (dd, $J=10.1,6.7 \mathrm{~Hz}, 2 \mathrm{H}, 2$ ''- $\left.\mathrm{H}_{2}\right), 2.79-2.97\left(\mathrm{~m}, 2 \mathrm{H}, 1\right.$ '”- $\mathrm{H}_{2}$ ), $3.36\left(\mathrm{~d}, J=0.5 \mathrm{~Hz}, 3 \mathrm{H}, 2\right.$ ' $\left.-\mathrm{OCH}_{3}\right), 3.80\left(\mathrm{~s}, 3 \mathrm{H}, 4{ }^{\prime}-\mathrm{OCH}_{3}\right), 3.89(\mathrm{~d}, J=0.5 \mathrm{~Hz}, 3 \mathrm{H}$, $\left.\mathrm{CO}_{2} \mathrm{CH}_{3}\right), 4.06\left(\mathrm{~d}, J=1.3 \mathrm{~Hz}, 2 \mathrm{H}, 4\right.$ "'- $\left.\mathrm{H}_{2}\right), 4.25(\mathrm{~d}, J=13.6 \mathrm{~Hz}, 1 \mathrm{H}), 4.42(\mathrm{~d}, J=13.5 \mathrm{~Hz}$, $1 \mathrm{H})\left(6^{\prime}-\mathrm{CH}_{2}\right), 4.57\left(\mathrm{~s}, 2 \mathrm{H}, \mathrm{OCH}_{2} \mathrm{Ph}\right), 6.88(\mathrm{~s}, 1 \mathrm{H}, 5$ '- $\mathrm{H}), 7.23-7.35(\mathrm{~m}, 5 \mathrm{H}, 5 \times \mathrm{Ph}-\mathrm{H})$, 7.51 (ddd, $J=7.7,1.6,0.5 \mathrm{~Hz}, 1 \mathrm{H}, 6-\mathrm{H}$ ), 7.58 (td, $J=7.7,0.5 \mathrm{~Hz}, 1 \mathrm{H}, 5-\mathrm{H}$ ), 8.02 (ddd, $J=7.6,1.6,0.5 \mathrm{~Hz}, 1 \mathrm{H}, 4-\mathrm{H})$.

$\left.{ }^{13} \mathrm{C}-\mathrm{NMR}(126 \mathrm{MHz}, \mathrm{CDCl})_{3}\right): \delta[\mathrm{ppm}]=-5.4\left(\mathrm{Si}\left(\mathrm{CH}_{3}\right)_{2}\right), 18.0\left(\mathrm{C}-1\right.$ '”), $18.2\left(\mathrm{SiC}\left(\mathrm{CH}_{3}\right)_{3}\right), 25.9$ $\left(\mathrm{SiC}\left(\underline{\mathrm{CH}}_{3}\right)_{3}\right), 38.6\left(\mathrm{C}-2^{\prime \prime}\right), 53.1\left(\mathrm{CO}_{2} \underline{\mathrm{C}} \mathrm{H}_{3}\right), 55.4\left(4^{\prime}-\mathrm{OCH}_{3}\right), 61.5\left(2^{\prime}-\mathrm{OCH}_{3}\right), 62.6\left(6^{\prime}-\mathrm{CH}_{2}\right)$, $73.3\left(\mathrm{OCH}_{2} \mathrm{Ph}\right.$ ), 74.9 (C-4'), 105.1 (C-5'), 118.7 (C-1'), 121.0 (C-3'), 123.8 (C-3), 127.9, 127.9, 128.5 (2 × Ph-Co, $\left.2 \times \mathrm{Ph}_{-} \mathrm{C}_{m}, \mathrm{Ph}-\mathrm{C}_{p}\right), 129.7$ (C-5), 130.4 (C-4), 130.7 (C-1), 136.7 (C-6), $137.3\left(\mathrm{Ph}-\mathrm{C}_{i}\right), 139.9$ (C-6'), 150.4 (C-2), 156.6 (C-2'), 159.1 (C-4'), $164.1\left(\mathrm{CO}_{2} \mathrm{CH}_{3}\right)$, 208.3 (C-3").

MS (ESI): $m / z(\%)=655.3(77)\left[\mathrm{M}+\mathrm{NH}_{4}\right]^{+}, 660.3(43)[\mathrm{M}+\mathrm{Na}]^{+}, 1297.6[2 \times \mathrm{M}+\mathrm{Na}]^{+}$.

$\mathrm{C}_{34} \mathrm{H}_{43} \mathrm{NO}_{9} \mathrm{Si}$ (637.80)

ber.: 638.2780 


$$
\begin{aligned}
\text { gef.: } & 638.2776[\mathrm{M}+\mathrm{H}]^{+} \\
& 655.3040\left[\mathrm{M}+\mathrm{NH}_{4}\right]^{+} \\
& 660.2591[\mathrm{M}+\mathrm{Na}]^{+}(\text {ESI-HRMS }) .
\end{aligned}
$$




\subsubsection{0 (Z)-3'-(3-((Benzyloxy)methyl)pent-3-en-1-yl)-6'-(((tert-butyl- dimethylsilyl)oxy)methyl)-2',4'-dimethoxy-2-nitro-[1,1'-biphenyl]-3- carbonsäuremethylester ((Z)-175)}

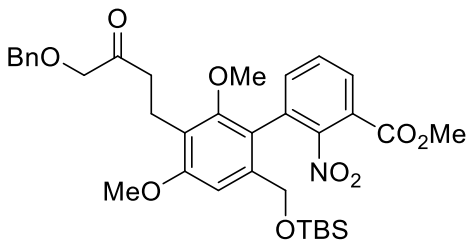

174

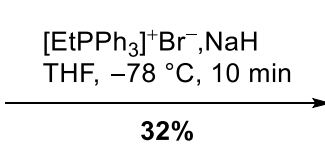

$32 \%$

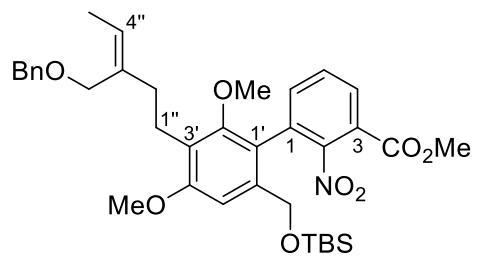

(Z)-175

Zu einer Suspension von Ethyltriphenylphosphoniumbromid (16.8 g, $45.3 \mathrm{mmol}, 10.0$ Äq.) in THF (50 ml) wurde $\mathrm{NaH}$ (60\%ig in Mineralöl, $1.63 \mathrm{~g}, 40.8 \mathrm{mmol}, 9.00$ Äq.) bei $0{ }^{\circ} \mathrm{C}$ gegeben, das resultierende Reaktionsgemisch auf $66^{\circ} \mathrm{C}$ erwärmt und $1 \mathrm{~h}$ bei dieser Temperatur unter Rückfluss gerührt. Anschließend wurde die Reaktionslösung auf $-78{ }^{\circ} \mathrm{C}$ gekühlt, eine Lösung des Biaryls $174(2.89 \mathrm{~g}, 4.53 \mathrm{mmol}, 1.00$ Äq.) in THF (150 ml) zugegeben und $10 \mathrm{~min}$ bei dieser Temperatur gerührt. Daraufhin wurde auf Raumtemperatur erwärmt, die Reaktion durch vorsichtige Zugabe von ges. $\mathrm{NH}_{4} \mathrm{Cl}$-Lsg. $(100 \mathrm{ml})$ sowie $\mathrm{H}_{2} \mathrm{O}(100 \mathrm{ml})$ beendet und die wässr. Phase mit MTBE $(3 \times 200 \mathrm{ml})$ extrahiert. Die vereinigten org. Phasen wurden über $\mathrm{MgSO}_{4}$ getrocknet, das Lösungsmittel unter vermindertem Druck entfernt und nach säulenchromatographischer Reinigung an Kieselgel (Petrolether/EtOAc 9:1) das Alken (Z)-175 (948 mg, 1.46 mmol, 32\%) als gelbes Öl isoliert.

${ }^{1} \mathrm{H}-\mathrm{NMR}\left(600 \mathrm{MHz}, \mathrm{CDCl}_{3}\right): \delta[\mathrm{ppm}]=-0.05(\mathrm{~s}, 3 \mathrm{H}),-0.05(\mathrm{~s}, 3 \mathrm{H})\left(\mathrm{Si}\left(\mathrm{CH}_{3}\right)_{2}\right), 0.87(\mathrm{~s}$, $\left.9 \mathrm{H}, \mathrm{SiC}\left(\mathrm{CH}_{3}\right)_{3}\right), 1.62$ (d, J = 6.9 Hz, 3 H, 5"'- $\left.\mathrm{H}_{3}\right), 2.28-2.39\left(\mathrm{~m}, 2 \mathrm{H}, 2\right.$ "'- $\left.\mathrm{H}_{2}\right), 2.72$ (qdd, $J=$ 12.7, 9.9, $6.0 \mathrm{~Hz}, 2 \mathrm{H}, 1$ ''- $\mathrm{H}_{2}$ ), 3.36 (s, $3 \mathrm{H}, 2^{\prime}-\mathrm{OCH}_{3}$ ), 3.80 (s, $\left.3 \mathrm{H}, 4^{\prime}-\mathrm{OCH}_{3}\right), 3.89$ (s, $3 \mathrm{H}$, $\left.\mathrm{CO}_{2} \mathrm{CH}_{3}\right), 4.09\left(\mathrm{~s}, 2 \mathrm{H}, 3\right.$ "'- $\left.\mathrm{CH}_{2}\right), 4.26(\mathrm{~d}, J=13.5 \mathrm{~Hz}, 1 \mathrm{H}), 4.44(\mathrm{~d}, J=13.5 \mathrm{~Hz}, 1 \mathrm{H})$ (6'- $\mathrm{CH}_{2}$ ), 4.47 (s, $2 \mathrm{H}, \mathrm{OCH}_{2} \mathrm{Ph}$ ), 5.46 (q, J = 6.8 Hz, $\left.1 \mathrm{H}, 4^{\prime \prime}-\mathrm{H}\right), 6.88$ (s, $\left.1 \mathrm{H}, 5^{\prime}-\mathrm{H}\right), 7.22-$ $7.26\left(\mathrm{~m}, 1 \mathrm{H}, \mathrm{Ph}-\mathrm{C}_{p}\right), 7.31\left(\mathrm{dd}, J=8.4,6.8 \mathrm{~Hz}, 2 \mathrm{H}, 2 \times \mathrm{Ph}^{-\mathrm{C}_{m}}\right), 7.33-7.36(\mathrm{~m}, 2 \mathrm{H}, 2 \times$ Ph-C ${ }_{0}$ ), 7.51 (dd, $\left.J=7.6,1.5 \mathrm{~Hz}, 1 \mathrm{H}, 6-\mathrm{H}\right), 7.57$ (t, $J=7.7 \mathrm{~Hz}, 1 \mathrm{H}, 5-\mathrm{H}$ ), 8.01 (dd, $J=$ 7.8, $1.5 \mathrm{~Hz}, 1 \mathrm{H}, 4-\mathrm{H})$.

${ }^{13} \mathrm{C}-\mathrm{NMR}\left(126 \mathrm{MHz}, \mathrm{CDCl}_{3}\right): \delta[\mathrm{ppm}]=-5.5, \quad-5.5 \quad\left(\mathrm{Si}\left(\mathrm{CH}_{3}\right)_{2}\right), \quad 13.3 \quad(\mathrm{C}-5$ "),$\quad 18.2$ (Sic $\left.\left(\mathrm{CH}_{3}\right)_{3}\right), 23.3$ (C-1"), $25.8\left(\mathrm{SiC}\left(\underline{\mathrm{CH}}_{3}\right)_{3}\right), 34.9$ (C-2"), $53.0\left(\mathrm{CO}_{2} \underline{\mathrm{C}} \mathrm{H}_{3}\right), 55.4\left(4^{\prime}-\mathrm{OCH}_{3}\right)$, $61.5\left(2^{\prime}-\mathrm{OCH}_{3}\right), 62.7\left(6^{\prime}-\mathrm{CH}_{2}\right), 66.8\left(3^{\prime \prime}-\mathrm{CH}_{2}\right), 71.8\left(\mathrm{OCH}_{2} \mathrm{Ph}\right), 105.0$ (C-5'), 118.6 (C-1'), 122.5 (C-3'), 123.7 (C-3), 123.9 (C-4"), $127.3\left(\mathrm{Ph}_{-} \mathrm{C}_{p}\right), 127.7$ (2 × Ph-C $\left.{ }_{m}\right), 128.2$ (2 × Ph-Co), 129.6 (C-5), 130.2 (C-4), 131.0 (C-1), 136.6 (C-3”), 136.8 (C-6), 138.8 (Ph-C $)$, 139.2 (C-6'), 150.4 (C-2), 156.6 (C-2'), 159.3 (C-4'), $164.1\left(\underline{\mathrm{CO}_{2}} \mathrm{CH}_{3}\right)$. 
MS (ESI): $m / z(\%)=667.3(100)\left[\mathrm{M}+\mathrm{NH}_{4}\right]^{+}, 672.3(73)[\mathrm{M}+\mathrm{Na}]^{+}, 1321.6(19)[2 \times \mathrm{M}+$ $\mathrm{Na}]^{+}$.

$\mathrm{C}_{36} \mathrm{H}_{47} \mathrm{NO}_{8} \mathrm{Si}(649.86)$

ber.: 672.2963

gef.: $672.2964[\mathrm{M}+\mathrm{Na}]^{+}($ESI-HRMS). 


\subsection{Synthese des Vinylchromans (S)-207}

\subsubsection{1-(((tert-Butyldiphenylsilyl)oxy)methyl)-3,5-dimethoxybenzol (188)}

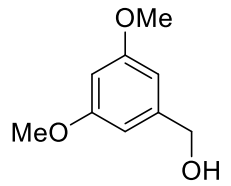

155

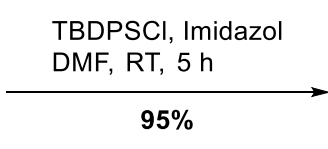

$95 \%$

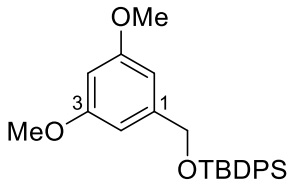

188

Zu einer Lösung des Benzylalkohols 155 (22.5 g, 134 mmol, 1.00 Äq.) in DMF (500 ml) wurden bei Raumtemperatur Imidazol (22.7 g, $333 \mathrm{mmol}, 2.50$ Äq.) sowie TBDPSCI (69.4 ml, 73.4 g, 267 mmol, 2.00 Äq.) gegeben und $5 \mathrm{~h}$ bei dieser Temperatur gerührt. Die Reaktion wurde durch Zugabe von $\mathrm{H}_{2} \mathrm{O}(250 \mathrm{ml})$ beendet, die Phasen getrennt und die wässr. Phase mit EtOAc $(3 \times 250 \mathrm{ml})$ extrahiert. Die vereinigten org. Phasen wurden mit $\mathrm{H}_{2} \mathrm{O}$ (250 ml) gewaschen, über $\mathrm{MgSO}_{4}$ getrocknet und das Lösungsmittel unter vermindertem Druck entfernt. Säulenchromatographische Reinigung an Kieselgel (Petrolether/EtOAc 9:1) lieferte die Zielverbindung 188 (51.7 g, 127 mmol, 95\%) in Form eines farblosen Öls.

${ }^{1} \mathrm{H}-\mathrm{NMR}\left(300 \mathrm{MHz}, \mathrm{CDCl}_{3}\right): \delta[\mathrm{ppm}]=1.15\left(\mathrm{~s}, 9 \mathrm{H}, \mathrm{SiC}\left(\mathrm{CH}_{3}\right)_{3}\right), 3.80\left(\mathrm{~s}, 6 \mathrm{H}, 2 \times \mathrm{OCH}_{3}\right)$, $4.78\left(\mathrm{~s}, 2 \mathrm{H}, 1-\mathrm{CH}_{2}\right), 6.40(\mathrm{dd}, J=2.7,2.1 \mathrm{~Hz}, 1 \mathrm{H}, 4-\mathrm{H}), 6.58(\mathrm{dt}, J=2.3,0.8 \mathrm{~Hz}, 2 \mathrm{H}$, 2-H, 6-H), 7.36-7.50 (m, $\left.6 \mathrm{H}, 4 \times \mathrm{Ph}^{-\mathrm{H}_{m}, 2} \times \mathrm{Ph}-\mathrm{H}_{p}\right), 7.71-7.78\left(\mathrm{~m}, 4 \mathrm{H}, 4 \times \mathrm{Ph}-\mathrm{H}_{o}\right)$.

${ }^{13} \mathrm{C}-N M R\left(126 \mathrm{MHz}, \mathrm{CDCl}_{3}\right): \delta[\mathrm{ppm}]=19.3 \quad\left(\mathrm{Si} \underline{\mathrm{C}}\left(\mathrm{CH}_{3}\right)_{3}\right), 26.8 \quad\left(\mathrm{SiC}\left(\underline{\mathrm{C}} \mathrm{H}_{3}\right)_{3}\right), 55.2(2 \times$ $\left.\mathrm{OCH}_{3}\right), 65.4$ (1- $\left.\mathrm{CH}_{2}\right), 99.0$ (C-4), 103.6 (C-2, C-6), 127.7, 129.7, 133.4, 135.5 (12 × Ph-C), 143.6 (C-1), 160.7 (C-3, C-5).

MS (ESI): $m / z(\%)=407.2(100)[\mathrm{M}+\mathrm{H}]^{+}, 429.2(87)[\mathrm{M}+\mathrm{Na}]^{+}, 835.4(48)[2 \times \mathrm{M}+\mathrm{Na}]^{+}$.

$\mathrm{C}_{25} \mathrm{H}_{30} \mathrm{O}_{3} \mathrm{Si}(406.60)$

ber.: 407.2037

429.1856

gef.: $407.2033[\mathrm{M}+\mathrm{H}]^{+}$

429.1852 [M + Na $]^{+}(\mathrm{ESI}-\mathrm{HRMS})$. 


\subsubsection{4-(((tert-Butyldiphenylsilyl)oxy)methyl)-2,6-dimethoxybenzaldehyd}

(189)

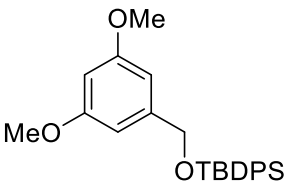

188

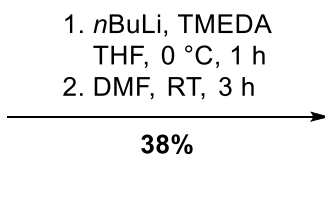

$\mathrm{THF}, 0{ }^{\circ} \mathrm{C}, 1 \mathrm{~h}$

$38 \%$

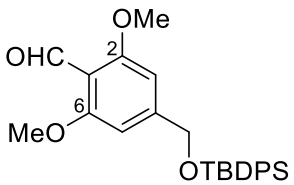

189

Eine Lösung des Silylethers 188 (51.7 g, 127 mmol, 1.00 Äq.) sowie frisch destilliertem TMEDA $\left(48.0 \mathrm{ml}, 37.2 \mathrm{~g}, 320 \mathrm{mmol}, 2.50\right.$ Äq.) in THF $(500 \mathrm{ml})$ wurde bei $0{ }^{\circ} \mathrm{C}$ tropfenweise mit $n$ BuLi (2.5 M in $n$-Hexan, $127 \mathrm{ml}, 320 \mathrm{mmol}, 2.50$ Äq.) versetzt und $1 \mathrm{~h}$ bei dieser Temperatur gerührt. Anschließend wurde ebenfalls bei $0^{\circ} \mathrm{C}$ DMF $(29.6 \mathrm{ml}$, $27.9 \mathrm{~g}, 382 \mathrm{mmol}, 3.00$ Äq.) hinzugegeben, auf Raumtemperatur erwärmt und weitere $3 \mathrm{~h}$ bei dieser Temperatur gerührt. Die Reaktion wurde durch Zugabe von ges. $\mathrm{NH}_{4} \mathrm{Cl}$-Lsg. $(200 \mathrm{ml})$ beendet, die Phasen getrennt und die wässr. Phase mit $\mathrm{Et}_{2} \mathrm{O}(3 \times 200 \mathrm{ml})$ extrahiert. Die vereinigten org. Phasen wurden mit ges. NaCl-Lsg. (300 ml) gewaschen, über $\mathrm{MgSO}_{4}$ getrocknet und das Lösungsmittel unter vermindertem Druck entfernt. Nach Säulenchromatographie an Kieselgel (Petrolether/EtOAc 4:1) wurde der Aldehyd 189 (21.2 g, $48.8 \mathrm{mmol}, 38 \%)$ als gelbes Öl erhalten.

${ }^{1} \mathrm{H}-\mathrm{NMR}\left(300 \mathrm{MHz}, \mathrm{CDCl}_{3}\right): \delta[\mathrm{ppm}]=1.12\left(\mathrm{~s}, 9 \mathrm{H}, \mathrm{SiC}\left(\mathrm{CH}_{3}\right)_{3}\right), 3.84\left(\mathrm{~s}, 6 \mathrm{H}, 2 \times \mathrm{OCH}_{3}\right)$, 4.77 (t, $\left.J=0.8 \mathrm{~Hz}, 2 \mathrm{H}, 4-\mathrm{CH}_{2}\right), 6.57$ (d, $\left.J=0.9 \mathrm{~Hz}, 2 \mathrm{H}, 3-\mathrm{H}, 5-\mathrm{H}\right), 7.33-7.47(\mathrm{~m}, 6 \mathrm{H}, 4 \times$ $\left.\mathrm{Ph}-\mathrm{H}_{m}, 2 \times \mathrm{Ph}-\mathrm{H}_{p}\right), 7.63-7.72\left(\mathrm{~m}, 4 \mathrm{H}, 4 \times \mathrm{Ph}-\mathrm{H}_{o}\right), 10.46(\mathrm{~s}, 1 \mathrm{H}, \mathrm{CHO})$.

${ }^{13} \mathrm{C}-\mathrm{NMR}\left(126 \mathrm{MHz}, \mathrm{CDCl}_{3}\right): \delta[\mathrm{ppm}]=19.3 \quad\left(\mathrm{Si} \underline{\mathrm{C}}\left(\mathrm{CH}_{3}\right)_{3}\right), 26.7 \quad\left(\mathrm{SiC}\left(\underline{\mathrm{C}} \mathrm{H}_{3}\right)_{3}\right), \quad 55.9(2 \times$ $\left.\mathrm{OCH}_{3}\right), 65.3\left(4-\mathrm{CH}_{2}\right), 100.9$ (C-3, C-5), $112.9(\mathrm{C}-1), 127.8,129.9,133.0,135.4$ (12 × $\mathrm{Ph}-\mathrm{C}), 150.3$ (C-4), 162.2 (C-2, C-6), 188.9 (CHO).

MS (ESI): $m / z(\%)=435.2(78)[\mathrm{M}+\mathrm{H}]^{+}, 891.3(42)[2 \times \mathrm{M}+\mathrm{Na}]^{+}$.

$\mathrm{C}_{26} \mathrm{H}_{30} \mathrm{O}_{4} \mathrm{Si}(434.61)$

ber.: 435.1986

457.1806

gef.: $435.1988[\mathrm{M}+\mathrm{H}]^{+}$

$457.1799[\mathrm{M}+\mathrm{Na}]^{+}(\mathrm{ESI}-\mathrm{HRMS})$. 


\subsubsection{4-(((tert-Butyldiphenylsilyl)oxy)methyl)-2-hydroxy-6-methoxy- benzaldehyd (190)}

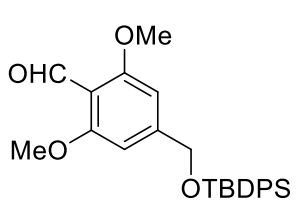

189

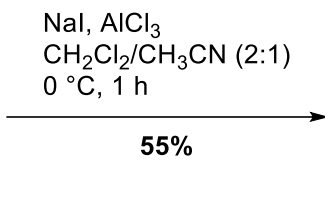

$\mathrm{CH}_{2} \mathrm{Cl}_{2} / \mathrm{CH}_{3}$

$0{ }^{\circ} \mathrm{C}, 1 \mathrm{~h}$<smiles>COc1cc(CO[18O][Na])cc(O)c1C=O</smiles>

190

Zu einer Lösung des Aldehyds 189 (21.2 g, 48.7 mmol, 1.00 Äq.) in $\mathrm{CH}_{2} \mathrm{Cl}_{2} / \mathrm{CH}_{3} \mathrm{CN}$ (2:1, $120 \mathrm{ml}$ ) wurde bei $0^{\circ} \mathrm{C}$ zunächst portionsweise $\mathrm{AlCl}_{3}$ (19.5 g, $146 \mathrm{mmol}, 3.00 \mathrm{Äq}$.) und daraufhin Nal (21.9 g, $146 \mathrm{mmol}, 3.00$ Äq.) gegeben. Das resultierende Reaktionsgemisch wurde $1 \mathrm{~h}$ bei $0^{\circ} \mathrm{C}$ gerührt, ehe die Reaktion durch Zugabe von ges. $\mathrm{NH}_{4} \mathrm{Cl}$-Lsg. $(100 \mathrm{ml})$ beendet wurde. Die Phasen wurden getrennt, die wässr. Phase mit EtOAc $(3 \times$ $50 \mathrm{ml}$ ) extrahiert, die vereinigten org. Phasen über $\mathrm{MgSO}_{4}$ getrocknet und das Lösungsmittel unter vermindertem Druck entfernt. Abschließende Säulenchromatographie an Kieselgel lieferte das Phenol $190(11.3 \mathrm{~g}, 27.0 \mathrm{mmol}, 55 \%)$ als farbloses Öl, das während der Lagerung zu einem leicht gelben Feststoff erstarrte.

${ }^{1} \mathrm{H}-\mathrm{NMR}\left(300 \mathrm{MHz}, \mathrm{CDCl}_{3}\right): \delta[\mathrm{ppm}]=1.11\left(\mathrm{~s}, 9 \mathrm{H}, \mathrm{SiC}\left(\mathrm{CH}_{3}\right)_{3}\right), 3.84\left(\mathrm{~s}, 3 \mathrm{H}, \mathrm{OCH}_{3}\right), 4.71$ (t, $\left.J=0.9 \mathrm{~Hz}, 2 \mathrm{H}, 4-\mathrm{CH}_{2}\right), 6.41(\mathrm{~d}, J=1.2 \mathrm{~Hz}, 1 \mathrm{H}, 3-\mathrm{H}), 6.53(\mathrm{dt}, J=1.6,0.8 \mathrm{~Hz}, 1 \mathrm{H}$, 5-H), 7.34-7.47 (m, $\left.6 \mathrm{H}, 4 \times \mathrm{Ph}-\mathrm{H}_{m}, 2 \times \mathrm{Ph}^{-\mathrm{H}_{p}}\right), 7.64-7.71\left(\mathrm{~m}, 4 \mathrm{H}, 4 \times \mathrm{Ph}-\mathrm{H}_{o}\right), 10.27$ (d, $J=0.6 \mathrm{~Hz}, 1 \mathrm{H}, \mathrm{CHO}), 12.03(\mathrm{~d}, J=0.4 \mathrm{~Hz}, 1 \mathrm{H}, \mathrm{OH})$.

${ }^{13} \mathrm{C}-N M R\left(126 \mathrm{MHz}, \mathrm{CDCl}_{3}\right): \delta[\mathrm{ppm}]=19.3\left(\mathrm{SiC}\left(\mathrm{CH}_{3}\right)_{3}\right), 26.8\left(\mathrm{SiC}\left(\underline{\mathrm{C}} \mathrm{H}_{3}\right)_{3}\right), 55.7\left(\mathrm{OCH}_{3}\right)$, $65.2\left(4-\mathrm{CH}_{2}\right), 98.2$ (C-3), 106.4 (C-5), 109.7 (C-1), 127.8, 129.9, 133.0, 135.5 (12 × Ph-C), 153.2 (C-4), 162.4 (C-6), 163.7 (C-2), 193.7 (CHO).

MS (ESI): $m / z(\%)=421.2(100)[\mathrm{M}+\mathrm{H}]^{+}, 443.2(68)[\mathrm{M}+\mathrm{Na}]^{+}, 863.3(90)[2 \times \mathrm{M}+\mathrm{Na}]^{+}$.

$\mathrm{C}_{25} \mathrm{H}_{28} \mathrm{O}_{4} \mathrm{Si}(420.58)$

ber.: 421.1830

443.1649

gef.: $421.1831[\mathrm{M}+\mathrm{H}]^{+}$

$443.1653[\mathrm{M}+\mathrm{Na}]^{+}(\mathrm{ESI}-\mathrm{HRMS})$. 


\subsubsection{5-(((tert-Butyldiphenylsilyl)oxy)methyl)-2-formyl-3-methoxyphenyl- pivalat (191)}

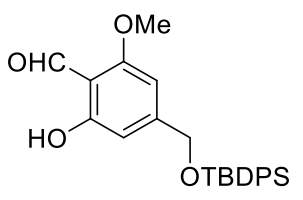

190

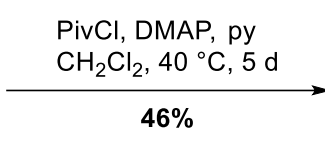

$46 \%$

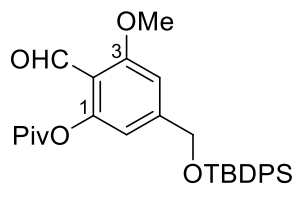

191

Das Phenol 190 (9.50 g, $22.6 \mathrm{mmol}, 1.00 \mathrm{Äq}$.) wurde in $\mathrm{CH}_{2} \mathrm{Cl}_{2}$ (300 ml) gelöst und nacheinander bei Raumtemperatur eine katalytische Menge DMAP (276 mg, $2.26 \mathrm{mmol}$, 10 mol\%), Pyridin (4.92 ml, 4.82 g, 67.8 mmol, 3.00 Äq.) sowie PivCl (7.79 ml, 7.63 g, $63.2 \mathrm{mmol}, 2.80$ Äq.) hinzugegeben. Die resultierende Reaktionslösung wurde auf $40{ }^{\circ} \mathrm{C}$ erwärmt und $5 \mathrm{~d}$ bei dieser Temperatur unter Rückfluss gerührt. Nach Abkühlen auf Raumtemperatur wurde mit ges. $\mathrm{NaHCO}_{3}$-Lsg. (200 ml) sowie ges. NaCl-Lsg. (200 ml) gewaschen, über $\mathrm{MgSO}_{4}$ getrocknet und das Lösungsmittel unter vermindertem Druck entfernt. Säulenchromatographie an Kieselgel (Petrolether/EtOAc 9:1) lieferte die Zielverbindung 191 (5.28 g, 10.5 mmol, 46\%) in Form eines leicht gelben Feststoffs.

${ }^{1} \mathrm{H}-\mathrm{NMR}\left(300 \mathrm{MHz}, \mathrm{CDCl}_{3}\right): \delta[\mathrm{ppm}]=1.11 \quad\left(\mathrm{~s}, \quad 9 \mathrm{H}, \quad \mathrm{SiC}\left(\mathrm{CH}_{3}\right)_{3}\right), \quad 1.38 \quad(\mathrm{~s}, \quad 9 \mathrm{H}$, $\left.\mathrm{C}(\mathrm{O}) \mathrm{C}\left(\mathrm{CH}_{3}\right)_{3}\right), 3.88\left(\mathrm{~s}, 3 \mathrm{H}, \mathrm{OCH}_{3}\right), 4.76\left(\mathrm{t}, J=0.9 \mathrm{~Hz}, 2 \mathrm{H}, 5-\mathrm{CH}_{2}\right), 6.52$ (dd, $J=1.4$, $0.7 \mathrm{~Hz}, 1 \mathrm{H}, 4-\mathrm{H}), 6.96(\mathrm{~d}, J=1.2 \mathrm{~Hz}, 1 \mathrm{H}, 6-\mathrm{H}), 7.34-7.47\left(\mathrm{~m}, 6 \mathrm{H}, 4 \times \mathrm{Ph}_{-} \mathrm{H}_{m}, 2 \times\right.$ $\left.\mathrm{Ph}-\mathrm{H}_{p}\right), 7.63-7.69\left(\mathrm{~m}, 4 \mathrm{H}, 4 \times \mathrm{Ph}-\mathrm{H}_{o}\right), 10.35(\mathrm{~d}, J=0.6 \mathrm{~Hz}, 1 \mathrm{H}, \mathrm{CHO})$.

${ }^{13} \mathrm{C}-\mathrm{NMR}\left(126 \mathrm{MHz}, \mathrm{CDCl}_{3}\right): \delta[\mathrm{ppm}]=19.3 \quad\left(\mathrm{SiC}\left(\mathrm{CH}_{3}\right)_{3}\right), \quad 26.8 \quad\left(\mathrm{SiC}\left(\underline{\mathrm{C}} \mathrm{H}_{3}\right)_{3}\right), \quad 27.1$ $\left(\mathrm{C}(\mathrm{O}) \mathrm{C}\left(\underline{\mathrm{C}} \mathrm{H}_{3}\right)_{3}\right), 39.1\left(\mathrm{C}(\mathrm{O}) \underline{\mathrm{C}}\left(\mathrm{CH}_{3}\right)_{3}\right), 56.0\left(\mathrm{OCH}_{3}\right), 64.8\left(5-\mathrm{CH}_{2}\right), 106.1$ (C-4), $112.5(\mathrm{C}-6)$, 116.3 (C-2), 127.8, 129.9, 132.9, 135.5 (12 × Ph-C), 149.9 (C-5), 151.3 (C-1), 162.9 (C-3), $175.6\left(\underline{\mathrm{C}}(\mathrm{O}) \mathrm{C}\left(\mathrm{CH}_{3}\right)_{3}\right), 187.7(\mathrm{CHO})$.

MS (ESI): $m / z(\%)=505.2(100)[\mathrm{M}+\mathrm{H}]^{+}, 519.3(31)\left[\mathrm{M}+\mathrm{NH}_{4}\right]^{+}, 527.2(35)[\mathrm{M}+\mathrm{Na}]^{+}$, $1031.5(60)[2 \times \mathrm{M}+\mathrm{Na}]^{+}$.

$\mathrm{C}_{30} \mathrm{H}_{36} \mathrm{O}_{5} \mathrm{Si}(504.70)$

ber.: 505.2405

527.2224

gef.: $505.2406[\mathrm{M}+\mathrm{H}]^{+}$

$527.2217[\mathrm{M}+\mathrm{Na}]^{+}(\mathrm{ESI}-\mathrm{HRMS})$. 


\subsection{5 (E)-2-(4-Benzyloxy-3-oxobut-1-en-1-yl)-5-(((tert-butyldiphenylsilyl)-} oxy)methyl)-3-methoxyphenylpivalat $((E)-192)$

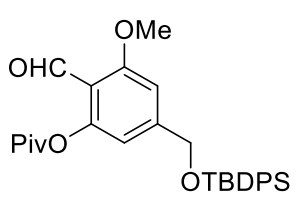

191

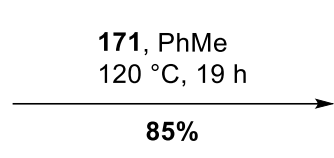

$(E)-192$

Eine Lösung des Aldehyds 191 (5.00 g, 9.91 mmol, 1.00 Äq.) und des P-Ylids 171 (6.31 g, $14.9 \mathrm{mmol}, 1.30$ Äq.) in Toluol (100 ml) wurde $19 \mathrm{~h}$ bei $120^{\circ} \mathrm{C}$ unter Rückfluss gerührt. Nach Abkühlen auf Raumtemperatur wurde das Lösungsmittel unter vermindertem Druck entfernt und nach Säulenchromatographie an Kieselgel (Petrolether/EtOAc 9:1) das a,ß-ungesättigte Keton (E)-192 (5.48 g, 8.43 mmol, 85\%) als leicht gelbes Öl isoliert.

${ }^{1} \mathrm{H}-\mathrm{NMR}\left(300 \mathrm{MHz}, \mathrm{CDCl}_{3}\right): \delta[\mathrm{ppm}]=1.08\left(\mathrm{~s}, 9 \mathrm{H}, \mathrm{SiC}\left(\mathrm{CH}_{3}\right)_{3}\right), 1.35(\mathrm{~d}, J=0.8 \mathrm{~Hz}, 9 \mathrm{H}$, $\left.\mathrm{C}(\mathrm{O}) \mathrm{C}\left(\mathrm{CH}_{3}\right)_{3}\right), 3.83\left(\mathrm{~s}, 3 \mathrm{H}, \mathrm{OCH}_{3}\right), 4.27\left(\mathrm{~s}, 2 \mathrm{H}, 4^{\prime}-\mathrm{H}_{2}\right), 4.62\left(\mathrm{~s}, 2 \mathrm{H}, \mathrm{OCH}_{2} \mathrm{Ph}\right), 4.74$ (s, $\left.2 \mathrm{H}, 5-\mathrm{CH}_{2}\right), 6.55(\mathrm{~d}, J=1.3 \mathrm{~Hz}, 1 \mathrm{H}, 4-\mathrm{H}), 6.87(\mathrm{~s}, 1 \mathrm{H}, 6-\mathrm{H}), 7.21-7.45(\mathrm{~m}, 12 \mathrm{H}, 2$ '- $\mathrm{H}$, $\left.4 \times \mathrm{Ph}-\mathrm{H}_{m}, 2 \times \mathrm{Ph}-\mathrm{H}_{p}, 5 \times \mathrm{OCH}_{2} \mathrm{Ph}-\underline{\mathrm{H}}\right), 7.61-7.68\left(\mathrm{~m}, 4 \mathrm{H}, 4 \times \mathrm{Ph}-\mathrm{H}_{o}\right), 7.73(\mathrm{~d}, J=$ 16.3 Hz, $\left.1 \mathrm{H}, 1^{\prime}-\mathrm{H}\right)$.

${ }^{13} \mathrm{C}-\mathrm{NMR}\left(126 \mathrm{MHz}, \mathrm{CDCl}_{3}\right): \delta[\mathrm{ppm}]=19.3 \quad\left(\mathrm{SiC}\left(\mathrm{CH}_{3}\right)_{3}\right), \quad 26.8 \quad\left(\mathrm{SiC}\left(\underline{\mathrm{C}} \mathrm{H}_{3}\right)_{3}\right), \quad 27.1$ $\left(\mathrm{C}(\mathrm{O}) \mathrm{C}\left(\underline{\mathrm{C}} \mathrm{H}_{3}\right)_{3}\right), 39.3\left(\mathrm{C}(\mathrm{O}) \underline{\mathrm{C}}\left(\mathrm{CH}_{3}\right)_{3}\right), 55.7\left(\mathrm{OCH}_{3}\right), 64.9\left(5-\mathrm{CH}_{2}\right), 73.3\left(\mathrm{OCH}_{2} \mathrm{Ph}\right), 74.6$ (C-4'), 105.8 (C-4), 112.2 (C-6), 115.2 (C-2), 125.3 (C-2'), 127.8, 127.9, 127.9, 128.4, 129.8, 133.0, 133.4, 135.5, 137.4 (C-1', $18 \times$ Ph-C), 145.3 (C-5), 151.5 (C-1), 160.2 (C-3), $176.7\left(\underline{\mathrm{C}}(\mathrm{O}) \mathrm{C}\left(\mathrm{CH}_{3}\right)_{3}\right), 197.8\left(\mathrm{C}-3^{\prime}\right)$.

MS (ESI): $m / z(\%)=651.3(20)[\mathrm{M}+\mathrm{H}]^{+}, 668.3(10)\left[\mathrm{M}+\mathrm{NH}_{4}\right]^{+}, 673.3(8)[\mathrm{M}+\mathrm{Na}]^{+}$.

$\mathrm{C}_{40} \mathrm{H}_{46} \mathrm{O}_{6} \mathrm{Si}(650.89)$

ber.: 651.3136

673.2956

gef.: $651.3136[\mathrm{M}+\mathrm{H}]^{+}$

$673.2952[\mathrm{M}+\mathrm{Na}]^{+}(\mathrm{ESI}-\mathrm{HRMS})$. 


\subsubsection{2-(4-Benzyloxy-3-oxobutyl)-5-(((tert-butyldiphenylsilyl)oxy)- methyl)-3-methoxyphenylpivalat (193)}

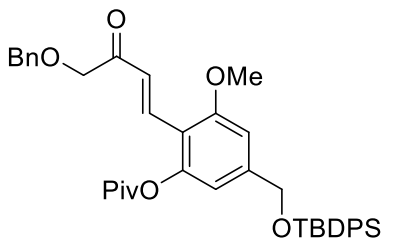

(E)-192

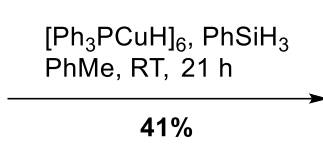

$41 \%$

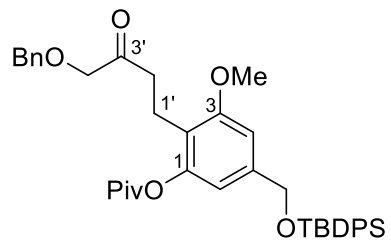

193

Eine Lösung des $\alpha, \beta$-ungesättigten Ketons $(E)$-192 (100 mg, $154 \mu \mathrm{mol}, 1.00$ Äq.) in Toluol $(1 \mathrm{ml})$ wurde bei $0^{\circ} \mathrm{C} z u$ einer Lösung von Stryker's Reagenz (15.8 mg, $7.68 \mu \mathrm{mol}$, $5 \mathrm{~mol} \%$ ) sowie $\mathrm{PhSiH}_{3}(19.0 \mu \mathrm{l}, 16.6 \mathrm{mg}, 154 \mu \mathrm{l}, 1.00 \mathrm{Äq}$ ) in Toluol (1 ml) gegeben, langsam auf Raumtemperatur erwärmt und $21 \mathrm{~h}$ bei dieser Temperatur gerührt. Die Reaktion wurde durch Hindurchleiten von $\mathrm{O}_{2}$ beendet, die Lösung durch Celite ${ }^{\circledR}$ filtriert, mit EtOAc $(3 \times 5 \mathrm{ml})$ nachgewaschen und das Lösungsmittel unter vermindertem Druck entfernt. Säulenchromatographie an Kieselgel (Petrolether/EtOAc 7:1) lieferte das Keton 193 (41.4 mg, 63.5 umol, 41\%) als leicht gelbes Öl.

${ }^{1} \mathrm{H}-\mathrm{NMR}\left(300 \mathrm{MHz}, \mathrm{CDCl}_{3}\right): \delta[\mathrm{ppm}]=1.08 \quad\left(\mathrm{~s}, \quad 9 \mathrm{H}, \quad \mathrm{SiC}\left(\mathrm{CH}_{3}\right)_{3}\right), \quad 1.32 \quad(\mathrm{~s}, \quad 9 \mathrm{H}$, $\left.\mathrm{C}(\mathrm{O}) \mathrm{C}\left(\mathrm{CH}_{3}\right)_{3}\right), 2.58-2.67\left(\mathrm{~m}, 2 \mathrm{H}, 2^{\prime}-\mathrm{H}_{2}\right), 2.71-2.79\left(\mathrm{~m}, 2 \mathrm{H}, 1^{\prime}-\mathrm{H}_{2}\right), 3.74\left(\mathrm{~s}, 3 \mathrm{H}, \mathrm{OCH}_{3}\right)$, 4.03 (s, $2 \mathrm{H}, 4$ '- $\mathrm{H}_{2}$ ), 4.56 (s, $2 \mathrm{H}, \mathrm{OCH}_{2} \mathrm{Ph}$ ), 4.71 (t, $\left.J=0.8 \mathrm{~Hz}, 2 \mathrm{H}, 5-\mathrm{CH}_{2}\right), 6.51$ (dd, J = 1.5, 0.7 Hz, $1 \mathrm{H}, 4-\mathrm{H}), 6.77(\mathrm{~d}, J=1.4 \mathrm{~Hz}, 1 \mathrm{H}, 6-\mathrm{H}), 7.25-7.43\left(\mathrm{~m}, 11 \mathrm{H}, 4 \times \mathrm{Ph}_{\mathrm{H}} \mathrm{H}_{m}, 2 \times\right.$ $\left.\mathrm{Ph}-\mathrm{H}_{p}, 5 \times \mathrm{OCH}_{2} \mathrm{Ph}-\underline{\mathrm{H}}\right), 7.64-7.69\left(\mathrm{~m}, 4 \mathrm{H}, 4 \times \mathrm{Ph}-\mathrm{H}_{0}\right)$.

${ }^{13} \mathrm{C}-\mathrm{NMR}\left(126 \mathrm{MHz}, \mathrm{CDCl}_{3}\right): \delta[\mathrm{ppm}]=17.8\left(\mathrm{C}-1^{\prime}\right), 19.3\left(\mathrm{SiC}\left(\mathrm{CH}_{3}\right)_{3}\right), 26.8\left(\mathrm{SiC}\left(\underline{\mathrm{C}} \mathrm{H}_{3}\right)_{3}\right)$, $27.2\left(\mathrm{C}(\mathrm{O}) \mathrm{C}\left(\underline{\mathrm{C}} \mathrm{H}_{3}\right)_{3}\right), 38.1\left(\mathrm{C}-2^{\prime}\right), 39.1\left(\mathrm{C}(\mathrm{O}) \underline{\mathrm{C}}\left(\mathrm{CH}_{3}\right)_{3}\right), 55.5\left(\mathrm{OCH}_{3}\right), 65.1\left(5-\mathrm{CH}_{2}\right), 73.3$ $\left(\mathrm{OCH}_{2} \mathrm{Ph}\right.$ ), 74.8 (C-4'), 105.5 (C-4), 111.8 (C-6), 120.2 (C-2), 127.7, 127.9, 127.9, 128.5, 129.7, 133.3, 135.5, 137.3 (18 × Ph-C), 140.8 (C-5), 149.6 (C-1), 158.3 (C-3), 177.1 $\left(\underline{\mathrm{C}}(\mathrm{O}) \mathrm{C}\left(\mathrm{CH}_{3}\right)_{3}\right), 208.0\left(\mathrm{C}-3^{\prime}\right)$.

MS (ESI): $m / z(\%)=670.3(100)\left[\mathrm{M}+\mathrm{NH}_{4}\right]^{+}, 675.3(21)[\mathrm{M}+\mathrm{Na}]^{+}, 1327.6(13)[2 \times \mathrm{M}+$ $\mathrm{Na}]^{+} ; 651.3(17)[\mathrm{M}-\mathrm{H}]^{-}$.

$\mathrm{C}_{40} \mathrm{H}_{48} \mathrm{O}_{6} \mathrm{Si}(652.90)$

ber.: 651.3147

670.3558

675.3112

gef.: $651.3142[\mathrm{M}-\mathrm{H}]^{-}$

$670.3568\left[\mathrm{M}+\mathrm{NH}_{4}\right]^{+}$

$675.3115[\mathrm{M}+\mathrm{Na}]^{+}(\mathrm{ESI}-\mathrm{HRMS})$. 


\subsubsection{2-(4-Benzyloxy-3-oxobutyl)-5-(((tert-butyldiphenylsilyl)oxy)- methyl)-6-iod-3-methoxyphenylpivalat (195)}

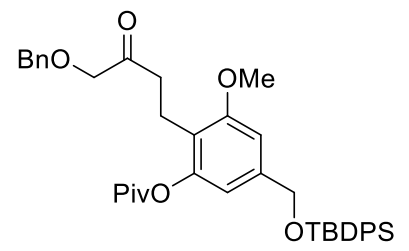

193

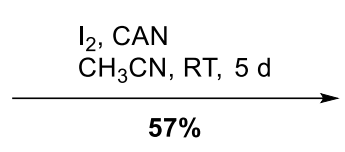

$57 \%$

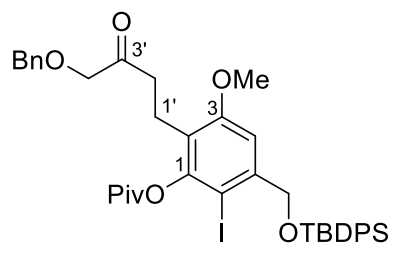

195

Zu einer Lösung des Ketons 193 (25.0 mg, $38.3 \mu$ mol, 1.00 Äq.) in $\mathrm{CH}_{3} \mathrm{CN}$ (1 ml) wurde bei Raumtemperatur $\mathrm{I}_{2}(9.73 \mathrm{mg}, 38.3 \mu \mathrm{mol}, 1.00$ Äq.) sowie CAN (21.7 mg, $38.3 \mu \mathrm{mol}$, 1.00 Äq.) gegeben und das resultierende Reaktionsgemisch $5 \mathrm{~d}$ bei dieser Temperatur gerührt. Daraufhin wurde der Feststoff abfiltriert, mit $\mathrm{CH}_{2} \mathrm{Cl}_{2}(5 \mathrm{ml})$ nachgewaschen, das Filtrat mit ges. $\mathrm{Na}_{2} \mathrm{~S}_{2} \mathrm{O}_{3}$-Lsg. $(3 \times 3 \mathrm{ml})$ sowie ges. $\mathrm{NaCl}$-Lsg $(5 \mathrm{ml})$ gewaschen, die org. Phase über $\mathrm{MgSO}_{4}$ getrocknet und das Lösungsmittel unter vermindertem Druck entfernt. Säulenchromatographie an Kieselgel (Petrolether/EtOAc 9:1) lieferte das Aryliodid 195 (17.0 mg, $21.8 \mu \mathrm{mol}, 57 \%$ ) in Form eines leicht gelben Öls.

${ }^{1} \mathrm{H}-\mathrm{NMR}\left(600 \mathrm{MHz}, \mathrm{CDCl}_{3}\right): \delta[\mathrm{ppm}]=1.11 \quad\left(\mathrm{~s}, \quad 9 \mathrm{H}, \quad \mathrm{SiC}\left(\mathrm{CH}_{3}\right)_{3}\right), \quad 1.38 \quad(\mathrm{~s}, \quad 9 \mathrm{H}$, $\left.\mathrm{C}(\mathrm{O}) \mathrm{C}\left(\mathrm{CH}_{3}\right)_{3}\right), 2.52\left(\mathrm{dd}, J=19.2,9.7 \mathrm{~Hz}, 1 \mathrm{H}, 2^{\prime}-\mathrm{H}_{\mathrm{a}}\right), 2.63-2.76\left(\mathrm{~m}, 2 \mathrm{H}, 1^{\prime}-\mathrm{H}_{\mathrm{a}}, 2^{\prime}-\mathrm{H}_{\mathrm{b}}\right), 2.86$ (t, $\left.J=13.0 \mathrm{~Hz}, 1 \mathrm{H}, 1^{\prime}-\mathrm{H}_{\mathrm{b}}\right), 3.79\left(\mathrm{~s}, 3 \mathrm{H}, \mathrm{OCH}_{3}\right), 4.03$ (d, J = 0.9 Hz, $\left.2 \mathrm{H}, 4^{\prime}-\mathrm{H}_{2}\right), 4.56$ (s, $2 \mathrm{H}, \mathrm{OCH}_{2} \mathrm{Ph}$ ), $4.66\left(\mathrm{~s}, 2 \mathrm{H}, 5-\mathrm{CH}_{2}\right), 5.63(\mathrm{~d}, J=1.0 \mathrm{~Hz}, 1 \mathrm{H}, 4-\mathrm{H}), 7.23-7.44(\mathrm{~m}, 11 \mathrm{H}$, $\left.4 \times \mathrm{Ph}-\mathrm{H}_{m}, 2 \times \mathrm{Ph}-\mathrm{H}_{p}, 5 \times \mathrm{OCH}_{2} \mathrm{Ph}-\underline{\mathrm{H}}\right), 7.65-7.69\left(\mathrm{~m}, 4 \mathrm{H}, 4 \times \mathrm{Ph}-\mathrm{H}_{o}\right)$.

${ }^{13} \mathrm{C}-N M R\left(126 \mathrm{MHz}, \mathrm{CDCl}_{3}\right): \delta[\mathrm{ppm}]=19.1\left(\mathrm{C}-1^{\prime}\right), 19.3\left(\mathrm{SiC}\left(\mathrm{CH}_{3}\right)_{3}\right), 26.8\left(\mathrm{SiC}\left(\underline{\mathrm{CH}}_{3}\right)_{3}\right)$, $27.4\left(\mathrm{C}(\mathrm{O}) \mathrm{C}\left(\underline{\mathrm{C}} \mathrm{H}_{3}\right)_{3}\right), 37.8\left(\mathrm{C}-2^{\prime}\right), 39.4\left(\mathrm{C}(\mathrm{O}) \underline{\mathrm{C}}\left(\mathrm{CH}_{3}\right)_{3}\right), 55.6\left(\mathrm{OCH}_{3}\right), 70.0\left(5-\mathrm{CH}_{2}\right), 73.3$ $\left(\mathrm{OCH}_{2} \mathrm{Ph}\right), 74.8$ (C-4'), 81.5 (C-6), 107.6 (C-4), 121.5 (C-2), 127.8, 127.8, 127.9, 128.5, 129.8, 133.0, 133.0, 135.4, 137.2 (18 × Ph-C), 142.7 (C-5), 149.2 (C-1), 158.6 (C-3), $175.9\left(\underline{\mathrm{C}}(\mathrm{O}) \mathrm{C}\left(\mathrm{CH}_{3}\right)_{3}\right), 207.8\left(\mathrm{C}-3^{\prime}\right)$.

$\mathrm{C}_{40} \mathrm{H}_{47} \mathrm{IO}_{6} \mathrm{Si}(778.80)$ 


\subsection{8 (Z)-2-(3-((Benzyloxy)methyl)pent-3-en-1-yl)-5-(((tert-butyldiphenyl- silyl)oxy)methyl)-3-methoxyphenol ((Z)-197)}

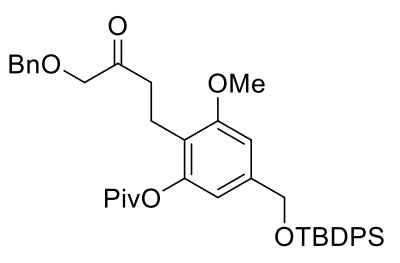

193

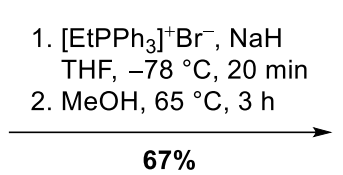

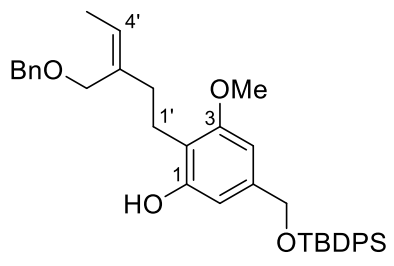

(Z)-197

Zu einer Suspension von Ethyltriphenylphosphoniumbromid (194 mg, $521 \mu \mathrm{mol}, 10.0$ Äq.) in THF (0.5 ml) wurde bei $0^{\circ} \mathrm{C} \mathrm{NaH} \mathrm{(60 \% ig} \mathrm{in} \mathrm{Mineralöl,} 18.8 \mathrm{mg}, 469 \mu \mathrm{mol}, 9.00 \mathrm{Äq}$.) gegeben, das resultierende Reaktionsgemisch auf $66^{\circ} \mathrm{C}$ erwärmt und $1 \mathrm{~h}$ bei dieser Temperatur unter Rückfluss gerührt. Anschließend wurde die Reaktionslösung auf $-78^{\circ} \mathrm{C}$ gekühlt, eine Lösung des Ketons $193(30.0 \mathrm{mg}, 52.1 \mu \mathrm{mol}, 1.00$ Äq.) in THF (1.5 ml) zugegeben und 20 min bei dieser Temperatur gerührt. Die Reaktionslösung wurde auf Raumtemperatur erwärmt, das Lösungsmittel unter vermindertem Druck entfernt, der Rückstand in $\mathrm{MeOH}(1 \mathrm{ml})$ aufgenommen, das Reaktionsgemisch auf $65^{\circ} \mathrm{C}$ erwärmt und $3 \mathrm{~h}$ bei dieser Temperatur unter Rückfluss gerührt. Nach Abkühlen auf Raumtemperatur wurde das Lösungsmittel unter vermindertem Druck entfernt, der Rückstand in EtOAc $(5 \mathrm{ml})$ aufgenommen und mit Pufferlösung $(\mathrm{pH}=6,5 \mathrm{ml})$ versetzt. Die Phasen wurden getrennt und die wässr. Phase mit EtOAc $(3 \times 5 \mathrm{ml})$ extrahiert. Die vereinigten org. Phasen wurden mit $\mathrm{H}_{2} \mathrm{O}(10 \mathrm{ml})$ gewaschen, über $\mathrm{MgSO}_{4}$ getrocknet und das Lösungsmittel unter vermindertem Druck entfernt. Abschließende säulenchromatographische Reinigung an Kieselgel (Petrolether/EtOAc 9:1) lieferte das Phenol (Z)-197 (17.8 mg, $306 \mu \mathrm{mol}, 67 \%$ ) in Form eines gelben Öls.

${ }^{1} \mathrm{H}-\mathrm{NMR}\left(300 \mathrm{MHz}, \mathrm{CDCl}_{3}\right): \delta[\mathrm{ppm}]=1.09\left(\mathrm{~s}, 9 \mathrm{H}, \mathrm{SiC}\left(\mathrm{CH}_{3}\right)_{3}\right), 1.52(\mathrm{~d}, J=6.9 \mathrm{~Hz}, 3 \mathrm{H}$, 5'- $\mathrm{H}_{3}$ ), $2.28\left(\mathrm{t}, J=7.1 \mathrm{~Hz}, 2 \mathrm{H}, 2^{\prime}-\mathrm{H}_{2}\right.$ ), $2.76\left(\mathrm{t}, J=7.0 \mathrm{~Hz}, 2 \mathrm{H}, 1^{\prime}-\mathrm{H}_{2}\right.$ ), 3.75 (s, $3 \mathrm{H}, \mathrm{OCH}_{3}$ ), 4.10 (s, $\left.2 \mathrm{H}, 3^{\prime}-\mathrm{CH}_{2}\right), 4.64\left(\mathrm{~s}, 2 \mathrm{H}, \mathrm{OCH}_{2} \mathrm{Ph}\right), 4.70\left(\mathrm{~s}, 2 \mathrm{H}, 5-\mathrm{CH}_{2}\right), 5.24(\mathrm{q}, J=6.9 \mathrm{~Hz}, 1 \mathrm{H}$, 4'-H), $6.47(\mathrm{~d}, J=1.4 \mathrm{~Hz}, 1 \mathrm{H}, 6-\mathrm{H}), 6.52(\mathrm{~d}, J=1.4 \mathrm{~Hz}, 1 \mathrm{H}, 4-\mathrm{H}), 7.27-7.46(\mathrm{~m}, 11 \mathrm{H}$, $\left.4 \times \mathrm{Ph}-\mathrm{H}_{m}, 2 \times \mathrm{Ph}-\mathrm{H}_{p}, 5 \times \mathrm{OCH}_{2} \mathrm{Ph}-\underline{\mathrm{H}}\right), 7.67-7.73\left(\mathrm{~m}, 4 \mathrm{H}, 4 \times \mathrm{Ph}-\mathrm{H}_{o}\right)$.

${ }^{13} \mathrm{C}-\mathrm{NMR}\left(126 \mathrm{MHz}, \mathrm{CDCl}_{3}\right): \delta[\mathrm{ppm}]=13.4\left(\mathrm{C}-5^{\prime}\right), 19.3\left(\mathrm{SiC}\left(\mathrm{CH}_{3}\right)_{3}\right), 22.9\left(\mathrm{C}-1^{\prime}\right), 26.9$ $\left(\mathrm{SiC}\left(\mathrm{CH}_{3}\right)_{3}\right), 36.6\left(\mathrm{C}-2^{\prime}\right), 55.5\left(\mathrm{OCH}_{3}\right), 65.5\left(5-\mathrm{CH}_{2}\right), 68.5\left(3^{\prime}-\mathrm{CH}_{2}\right), 73.0\left(\mathrm{OCH}_{2} \mathrm{Ph}\right), 100.2$ (C-4), 106.7 (C-6), 114.2 (C-2), 125.8 (C-4'), 127.7, 127.9, 128.2, 128.5, 129.6, 133.6, 134.6, 135.6, 137.5 (C-3', $18 \times \mathrm{Ph}-\mathrm{C}), 140.3$ (C-5), 155.5 (C-1), 158.1 (C-3).

MS $(E S I): m / z(\%)=581.3(31)\left[\mathrm{M}+\mathrm{H}^{+}, 598.3(77)\left[\mathrm{M}+\mathrm{NH}_{4}\right]^{+}, 603.3(100)[\mathrm{M}+\mathrm{Na}]^{+}\right.$. 
598.3347

603.2901

gef.: $581.3086[\mathrm{M}+\mathrm{H}]^{+}$

$598.3356\left[\mathrm{M}+\mathrm{NH}_{4}\right]^{+}$

$603.2917[\mathrm{M}+\mathrm{Na}]^{+}(\mathrm{ESI}-\mathrm{HRMS})$. 


\subsection{9 (S)-2-((Benzyloxy)methyl)-7-(((tert-butyldiphenylsilyl)oxy)methyl)- 5-methoxy-2-vinylchroman ((S)-207)}

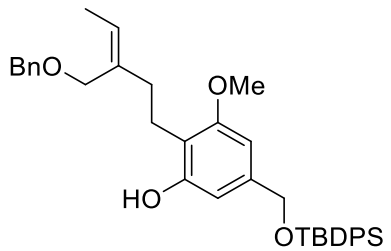

(Z)-197
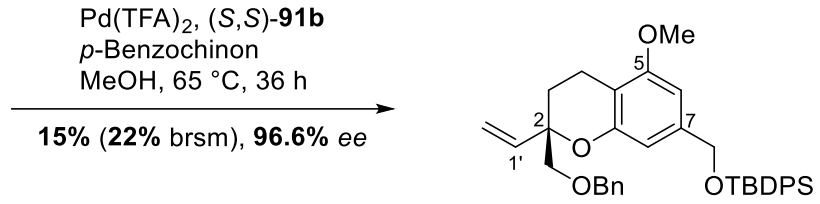

(S)-207

Eine Suspension von $\mathrm{Pd}(\mathrm{TFA})_{2}(1.91 \mathrm{mg}, 5.75 \mu \mathrm{mol}, 10 \mathrm{~mol} \%)$ und $(S, S)$-iPr-BOXAXLigand (S,S)-91b (2.74 mg, $5.75 \mu \mathrm{mol}, 10 \mathrm{~mol} \%)$ in $\mathrm{MeOH}(1 \mathrm{ml})$ wurde $30 \mathrm{~min}$ bei Raumtemperatur gerührt. Anschließend wurde eine Lösung des Phenols (Z)-197 (33.4 mg, $57.5 \mu \mathrm{mol}, 1.00 \mathrm{Äq}$.) in $\mathrm{MeOH}$ ( $1 \mathrm{ml}$ ) sowie $p$-Benzochinon (24.9 mg, $230 \mu \mathrm{mol}$, 4.00 Äq.) hinzugegeben, die resultierende Reaktionslösung auf $65^{\circ} \mathrm{C}$ erwärmt und $36 \mathrm{~h}$ bei dieser Temperatur unter Rückfluss gerührt. Nach Abkühlen auf Raumtemperatur wurde das Lösungsmittel unter vermindertem Druck entfernt, der Rückstand in EtOAc (5 ml) aufgenommen und mit verd. $\mathrm{HCl}(1 \mathrm{M}, 5 \mathrm{ml})$, verd. $\mathrm{NaOH}$-Lsg. (1 M, $3 \times 5 \mathrm{ml})$ sowie ges. NaCl-Lsg. (5 ml) gewaschen. Trocknen über $\mathrm{MgSO}_{4}$, Entfernen des Lösungsmittels unter vermindertem Druck und Säulenchromatographie an Kieselgel (Petrolether/EtOAc 19:1 $\rightarrow$ 9:1) lieferte das Vinylchroman (S)-207 (4.90 mg, $8.47 \mu \mathrm{mol}, 15 \%, 22 \%$ brsm, $96.6 \%$ ee) als farbloses Öl.

HPLC (analytisch): Säule: $\quad$ CHIRALPAK ${ }^{\circledR} \mathrm{IA}, 4.6 \times 250 \mathrm{~mm}, 5 \mu \mathrm{m}$

Eluent: $\quad n$-Hexan/ $\mathbb{P r O H}$ 99.3:0.7

Fluß: $\quad 0.8 \mathrm{ml} / \mathrm{min}$

$\lambda$ : $\quad 207 \mathrm{~nm}$

$t_{\mathrm{R}}: \quad 8.9 \min (R)-207,1.8 \%$

$9.7 \min (S)-207,98.2 \%, 96.6 \%$ ee.

${ }^{1} \mathrm{H}-\mathrm{NMR}\left(300 \mathrm{MHz}, \mathrm{CDCl}_{3}\right): \delta[\mathrm{ppm}]=1.08\left(\mathrm{~s}, 9 \mathrm{H}, \mathrm{SiC}\left(\mathrm{CH}_{3}\right)_{3}\right), 1.85-2.06\left(\mathrm{~m}, 2 \mathrm{H}, 3-\mathrm{H}_{2}\right)$, 2.40 (ddd, $J=17.0,10.7,6.3 \mathrm{~Hz}, 1 \mathrm{H}), 2.64-2.74(\mathrm{~m}, 1 \mathrm{H})\left(4-\mathrm{H}_{2}\right), 3.49-3.59(\mathrm{~m}, 2 \mathrm{H}$, 2- $\mathrm{CH}_{2}$ ), $3.74\left(\mathrm{~s}, 3 \mathrm{H}, \mathrm{OCH}_{3}\right.$ ), $4.61\left(\mathrm{~d}, J=2.6 \mathrm{~Hz}, 2 \mathrm{H}, \mathrm{OCH}_{2} \mathrm{Ph}\right), 4.68\left(\mathrm{~s}, 2 \mathrm{H}, 7-\mathrm{CH}_{2}\right), 5.15$ (dd, $\left.J=10.9,1.4 \mathrm{~Hz}, 1 \mathrm{H}, 2^{\prime}-\mathrm{H}_{c i s}\right), 5.23$ (dd, $J=17.4,1.4 \mathrm{~Hz}, 1 \mathrm{H}, 2$ '- $\mathrm{H}_{\text {trans }}$ ), 5.83 (dd, $J=$ 17.3, $\left.10.8 \mathrm{~Hz}, 1 \mathrm{H}, 1^{\prime}-\mathrm{H}\right), 6.42(\mathrm{~s}, 1 \mathrm{H}), 6.54$ (d, J = 1.3 Hz, 1 H) (6-H, 8-H), 7.24-7.44 (m, $11 \mathrm{H}, 4 \times \mathrm{Ph}-\mathrm{H}_{m}, 2 \times \mathrm{Ph}-\mathrm{H}_{p}, 5 \times \mathrm{OCH}_{2} \mathrm{Ph}-\underline{\mathrm{H}}$ ), 7.69 (dquint., $J=6.7,2.2 \mathrm{~Hz}, 4 \mathrm{H}, 4 \times$ $\left.\mathrm{Ph}-\mathrm{H}_{0}\right)$.

$\left.{ }^{13} \mathrm{C}-\mathrm{NMR}(126 \mathrm{MHz}, \mathrm{CDCl})_{3}\right): \delta[\mathrm{ppm}]=16.2(\mathrm{C}-4), 19.3 \quad\left(\mathrm{SiC}\left(\mathrm{CH}_{3}\right)_{3}\right), 26.5$ (C-3), 26.9 $\left(\mathrm{SiC}\left(\mathrm{CH}_{3}\right)_{3}\right), 55.3\left(\mathrm{OCH}_{3}\right), 65.5\left(7-\mathrm{CH}_{2}\right), 73.6\left(\mathrm{OCH}_{2} \mathrm{Ph}\right), 75.5\left(2-\mathrm{CH}_{2}\right), 78.8(\mathrm{C}-2), 99.6$, 
107.1, 109.1 (C-4a, C-6, C-8), 116.2 (C-2'), 127.5, 127.6, 127.7, 128.3, 129.6, 133.6, 135.6, 137.9, 138.3, 140.4 (C-7, C-1', $18 \times \mathrm{Ph}-\mathrm{C}), 154.2,157.4$ (C-5, C-8a).

MS (ESI): $m / z(\%)=579.9(33)[\mathrm{M}+\mathrm{H}]^{+}, 601.3(82)[\mathrm{M}+\mathrm{Na}]^{+}, 1179.6(15)[2 \times \mathrm{M}+\mathrm{Na}]^{+}$.

$\mathrm{C}_{37} \mathrm{H}_{42} \mathrm{O}_{4} \mathrm{Si}(578.82)$

ber.: 579.2925

601.2745

gef.: $579.2918[\mathrm{M}+\mathrm{H}]^{+}$

$601.2750[\mathrm{M}+\mathrm{Na}]^{+}(\mathrm{ESI}-\mathrm{HRMS})$. 


\subsection{Synthese des Chromans syn-266 und des Biaryls (Z)-272}

\subsubsection{4-Hydroxymethyl-2,6-dimethoxybenzaldehyd (158)}

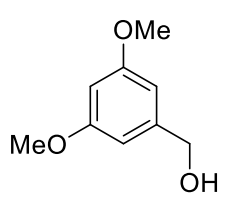

155

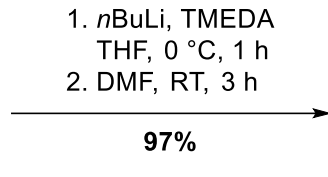

$97 \%$

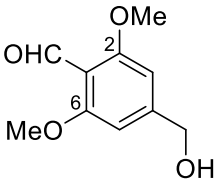

158

Eine Lösung des Benzylalkohols 155 (21.0 g, 125 mmol, 1.00 Äq.) und frisch destilliertem TMEDA (46.8 ml, $36.3 \mathrm{~g}, 312 \mathrm{mmol}, 2.50$ Äq.) in THF $(500 \mathrm{ml})$ wurde bei $0{ }^{\circ} \mathrm{C}$ tropfenweise mit $n \mathrm{BuLi}$ ( $2.5 \mathrm{M}$ in $n$-Hexan, $125 \mathrm{ml}, 312 \mathrm{mmol}, 2.50$ Äq.) versetzt und $1 \mathrm{~h}$ bei dieser Temperatur gerührt. Anschließend wurde ebenfalls bei $0^{\circ} \mathrm{C}$ langsam DMF (19.3 ml, $18.3 \mathrm{~g}, 250 \mathrm{mmol}, 2.00$ Äq.) zugetropft, die resultierende Reaktionslösung auf Raumtemperatur erwärmt und $3 \mathrm{~h}$ bei dieser Temperatur gerührt. Die Reaktion wurde durch Zugabe von ges. NaCl-Lsg. (200 ml) beendet, die Phasen getrennt und die wässr. Phase mit $\mathrm{Et}_{2} \mathrm{O}(3 \times 250 \mathrm{ml})$ extrahiert. Die vereinigten org. Phasen wurden über $\mathrm{MgSO}_{4}$ getrocknet, das Lösungsmittel unter vermindertem Druck entfernt und nach Säulenchromatographie an Kieselgel $\left(\mathrm{CH}_{2} \mathrm{Cl}_{2} / \mathrm{EtOAc} 1: 1\right)$ der Aldehyd 158 (23.8 g, $121 \mathrm{mmol}, 97 \%$ ) als gelber Feststoff erhalten.

${ }^{1} \mathrm{H}-\mathrm{NMR}\left(300 \mathrm{MHz}, \mathrm{CDCl}_{3}\right): \delta[\mathrm{ppm}]=3.82\left(\mathrm{~s}, 6 \mathrm{H}, 2 \times \mathrm{OCH}_{3}\right), 4.62-4.68\left(\mathrm{~m}, 2 \mathrm{H}, 4-\mathrm{CH}_{2}\right)$, 6.51 (d, $J=0.8 \mathrm{~Hz}, 2 \mathrm{H}, 3-\mathrm{H}, 5-\mathrm{H}), 10.33$ (s, $1 \mathrm{H}, \mathrm{CHO}$ ).

${ }^{13} \mathrm{C}-\mathrm{NMR}\left(126 \mathrm{MHz}, \mathrm{CDCl}_{3}\right): \delta[\mathrm{ppm}]=55.9\left(2 \times \mathrm{OCH}_{3}\right), 64.6\left(4-\mathrm{CH}_{2}\right), 101.4(\mathrm{C}-3, \mathrm{C}-5)$, 112.8 (C-1), 150.7 (C-4), 162.7 (C-2, C-6), 189.3 (q, J = $1.5 \mathrm{~Hz}, \mathrm{CHO}$ ).

MS (ESI): $m / z(\%)=197.1(22)[\mathrm{M}+\mathrm{H}]^{+}, 219.1(18)[\mathrm{M}+\mathrm{Na}]^{+} ; 195.1(100)[\mathrm{M}-\mathrm{H}]^{-}$.

$\mathbf{C}_{10} \mathrm{H}_{12} \mathrm{O}_{4}(196.20)$

ber.: 195.0663

197.0808

219.0628

gef.: $195.0664[\mathrm{M}-\mathrm{H}]^{-}$

$197.0810[\mathrm{M}+\mathrm{H}]^{+}$

$219.0633[\mathrm{M}+\mathrm{Na}]^{+}(\mathrm{ESI}-\mathrm{HRMS})$. 


\subsection{2 (E)-1-Benzyloxy-4-(4-hydroxymethyl-2,6-dimethoxyphenyl)but-3- en-2-on $((E)-214)$}

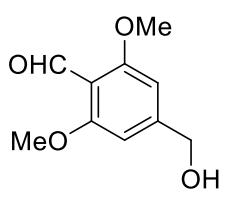

158

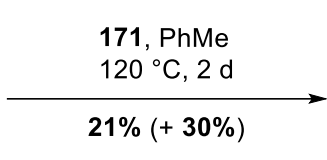

$(E)-\mathbf{2 1 4}$

Eine Lösung des Aldehyds 158 (23.8 g, 121 mmol, 1.00 Äq.) und des P-Ylids 171 (66.9 g, $158 \mathrm{mmol}, 1.30 \mathrm{Äq}$.) in Toluol (500 ml) wurde bei $120^{\circ} \mathrm{C}$ für $2 \mathrm{~d}$ unter Rückfluss gerührt. Nach Abkühlen auf Raumtemperatur wurde das Lösungsmittel unter vermindertem Druck entfernt und nach zweifacher Säulenchromatographie an Kieselgel (Petrolether/EtOAc 1:3 und $\mathrm{Et}_{2} \mathrm{O}$ ) sowie Umkristallisation aus $\mathrm{Et}_{2} \mathrm{O}$ das $\alpha, \beta$-ungesättigte Keton $(E)$-214 (8.75 g, $25.6 \mathrm{mmol}, 21 \%$ ) als leicht gelber Feststoff sowie eine mit Triphenylphosphanoxid (10.5 g) verunreinigte Fraktion des $\alpha, \beta$-ungesättigten Ketons $(E)-214(12.3 \mathrm{~g}, 35.9 \mathrm{mmol}, 30 \%)$ isoliert.

${ }^{1} \mathrm{H}-\mathrm{NMR}\left(300 \mathrm{MHz}, \mathrm{CDCl}_{3}\right): \delta[\mathrm{ppm}]=3.81\left(\mathrm{~s}, 6 \mathrm{H}, 2 \times \mathrm{OCH}_{3}\right), 4.30\left(\mathrm{~s}, 2 \mathrm{H}, 1-\mathrm{H}_{2}\right), 4.62(\mathrm{~s}$, $\left.2 \mathrm{H}, 4^{\prime}-\mathrm{CH}_{2}\right), 4.64\left(\mathrm{~s}, 2 \mathrm{H}, \mathrm{OCH}_{2} \mathrm{Ph}\right), 6.52\left(\mathrm{~s}, 2 \mathrm{H}, 3^{\prime}-\mathrm{H}, 5^{\prime}-\mathrm{H}\right), 7.23-7.41(\mathrm{~m}, 6 \mathrm{H}, 3-\mathrm{H}, 5 \times$ $\mathrm{Ph}-\mathrm{H}), 8.08$ (d, J=16.4 Hz, $1 \mathrm{H}, 4-\mathrm{H})$.

${ }^{13} \mathrm{C}-N M R\left(126 \mathrm{MHz}, \mathrm{CDCl}_{3}\right): \delta[\mathrm{ppm}]=55.7\left(2 \times \mathrm{OCH}_{3}\right), 64.9\left(4^{\prime}-\mathrm{CH}_{2}\right), 73.2\left(\mathrm{OCH}_{2} \mathrm{Ph}\right)$, 74.4 (C-1), 101.6 (C-3', C-5'), 111.0 (C-1'), 123.8 (C-3), $127.8\left(\mathrm{Ph}^{-\mathrm{C}_{p}}\right), 127.8,128.3$ (2 × Ph-C $\left.{ }_{o}, 2 \times \mathrm{Ph}_{-} \mathrm{C}_{m}\right), 134.6$ (C-4), $137.5\left(\mathrm{Ph}_{-} \mathrm{C}_{i}\right), 145.8$ (C-4'), 160.5 (C-2', C-6'), 198.8 (CHO).

MS (ESI): $m / z(\%)=343.2(9)[\mathrm{M}+\mathrm{H}]^{+}, 365.1(15)[\mathrm{M}+\mathrm{Na}]^{+}, 381.1(100)[\mathrm{M}+\mathrm{K}]^{+}, 707.3$ (20) $[2 \times \mathrm{M}+\mathrm{Na}]^{+}, 723.3(21)\left[2 \times \mathrm{M}+\mathrm{K}^{+} ; 341.1(100)[\mathrm{M}-\mathrm{H}]^{-}, 683.3(25)[2 \times \mathrm{M}-\mathrm{H}]^{-}\right.$.

$\mathrm{C}_{20} \mathrm{H}_{22} \mathrm{O}_{5}$ (342.39)

ber.: 341.1394

343.1540

365.1359

381.1099

gef.: $341.1385[\mathrm{M}-\mathrm{H}]^{-}$

$343.1541[\mathrm{M}+\mathrm{H}]^{+}$

$365.1363[\mathrm{M}+\mathrm{Na}]^{+}$

$381.1100[\mathrm{M}+\mathrm{K}]^{+}(\mathrm{ESI}-\mathrm{HRMS})$. 


\subsection{3 (E)-1-Benzyloxy-4-(2,6-dimethoxy-4-((methoxymethoxy)methyl)- phenyl)but-3-en-2-on $((E)-243)$}

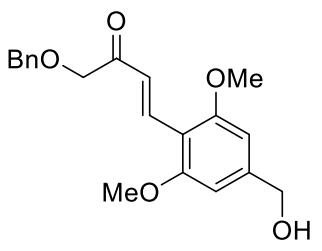

(E)-214

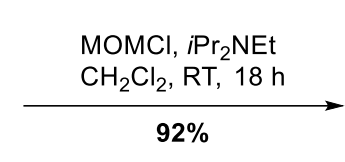

Eine Lösung des Benzylalkohols $(E)-214(8.50 \mathrm{~g}, 24.8 \mathrm{mmol}, 1.00$ Äq.) und Hünig-Base (6.49 ml, $4.81 \mathrm{~g}, \quad 37.2 \mathrm{mmol}, 1.50$ Äq.) in $\mathrm{CH}_{2} \mathrm{Cl}_{2}$ (100 ml) wurde $30 \mathrm{~min}$ bei Raumtemperatur gerührt, daraufhin auf $0^{\circ} \mathrm{C}$ gekühlt und bei dieser Temperatur langsam mit $\mathrm{MOMCl}$ (80\%ig, $3.54 \mathrm{ml}, 3.75 \mathrm{~g}, 37.2 \mathrm{mmol}, 1.50 \mathrm{Äq}$ ) versetzt. Die resultierende Reaktionslösung wurde auf Raumtemperatur erwärmt und $18 \mathrm{~h}$ bei dieser Temperatur gerührt. Anschließend wurde die Reaktion durch Zugabe von ges. NaCl-Lsg. (100 ml) beendet, die Phasen getrennt und die wässr. Phase mit EtOAc $(3 \times 100 \mathrm{ml})$ extrahiert. Die vereinigten org. Phasen wurden über $\mathrm{MgSO}_{4}$ getrocknet, das Lösungsmittel unter vermindertem Druck entfernt und nach säulenchromatographischer Reinigung an Kieselgel (Petrolether/EtOAc 2:1 $\rightarrow$ 1:1) das Keton $(E)-243(8.84 \mathrm{~g}, 22.9 \mathrm{mmol}, 92 \%)$ in Form eines leicht gelben Öls erhalten.

UV $(\mathrm{MeOH}): \lambda_{\max }[\mathrm{nm}](\mathrm{lg} \varepsilon)=326$ (4.288).

IR (ATR): $\widetilde{v}\left[\mathrm{~cm}^{-1}\right]=3088,3062,3030,3000,2937,2881,2842,1737,1694,1676,1589$, 1567, 1496, 1484, 1456, 1419, 1376, 1329, 1287, 1234, 1201, 1179, 1148, 1115, 1049, 1000, 984, 917, 824, 739, 698, 639, 604, 581, 537, 505.

${ }^{1} \mathrm{H}-\mathrm{NMR}\left(300 \mathrm{MHz}, \mathrm{CDCl}_{3}\right): \delta[\mathrm{ppm}]=3.40\left(\mathrm{~s}, 3 \mathrm{H}, \mathrm{OCH}_{2} \mathrm{OCH}_{3}\right), 3.85\left(\mathrm{~s}, 6 \mathrm{H}, 2\right.$ '- $\mathrm{OCH}_{3}$, 6'- $\left.-\mathrm{OCH}_{3}\right), 4.32\left(\mathrm{~s}, 2 \mathrm{H}, 1-\mathrm{H}_{2}\right), 4.56\left(\mathrm{~d}, J=0.6 \mathrm{~Hz}, 2 \mathrm{H}, \mathrm{OCH}_{2} \mathrm{OCH}_{3}\right), 4.63(\mathrm{~s}, 2 \mathrm{H}$, $\left.\mathrm{OCH}_{2} \mathrm{Ph}\right), 4.70\left(\mathrm{~s}, 2 \mathrm{H}, 4^{\prime}-\mathrm{CH}_{2}\right), 6.53(\mathrm{~s}, 2 \mathrm{H}, 3$ '- $\mathrm{H}, 5$ '- $\mathrm{H}), 7.24-7.42(\mathrm{~m}, 6 \mathrm{H}, 3-\mathrm{H}, 5 \times$ $\mathrm{Ph}-\mathrm{H}), 8.13$ (d, $J=16.4 \mathrm{~Hz}, 1 \mathrm{H}, 4-\mathrm{H})$.

${ }^{13} \mathrm{C}-\mathrm{NMR}\left(126 \mathrm{MHz}, \mathrm{CDCl}_{3}\right): \delta[\mathrm{ppm}]=55.4\left(\mathrm{OCH}_{2} \mathrm{OCH}_{3}\right), 55.7\left(2^{\prime}-\mathrm{OCH}_{3}, 6\right.$ '- $\left.\mathrm{OCH}_{3}\right), 69.0$ (4'- $\left.\mathrm{CH}_{2}\right), 73.1\left(\mathrm{OCH}_{2} \mathrm{Ph}\right), 74.5(\mathrm{C}-1), 95.8\left(\mathrm{O}^{\mathrm{C}} \mathrm{H}_{2} \mathrm{OCH}_{3}\right), 102.6(\mathrm{C}-3$ ', C-5'), 111.4 (C-1'), $124.2(\mathrm{C}-3), 127.7\left(\mathrm{Ph}-\mathrm{C}_{p}\right), 127.8,128.3\left(2 \times \mathrm{Ph} \mathrm{C}_{o}, 2 \times \mathrm{Ph}-\mathrm{C}_{m}\right), 134.3(\mathrm{C}-4), 137.6$ (Ph-Ci), 142.4 (C-4'), 160.4 (C-2', C-6'), 198.6 (C-2).

MS (ESI): $m / z(\%)=387.2(44)[\mathrm{M}+\mathrm{H}]^{+}, 409.2(13)[\mathrm{M}+\mathrm{Na}]^{+}, 425.2(100)[\mathrm{M}+\mathrm{K}]^{+}, 795.4$ (44) $[2 \times \mathrm{M}+\mathrm{Na}]^{+}, 811.3(54)[2 \times \mathrm{M}+\mathrm{K}]^{+}$.

$\mathrm{C}_{22} \mathrm{H}_{26} \mathrm{O}_{6}$ (386.44)

ber.: 387.1802

409.1622 
425.1361

gef.: $387.1803[\mathrm{M}+\mathrm{H}]^{+}$

$409.1614[\mathrm{M}+\mathrm{Na}]^{+}$

$425.1369[\mathrm{M}+\mathrm{K}]^{+}$(ESI-HRMS). 


\subsubsection{1-Benzyloxy-4-(2,6-dimethoxy-4-((methoxymethoxy)methyl)- phenyl)butan-2-on (244a)}

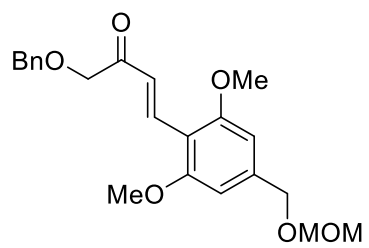

(E)-243

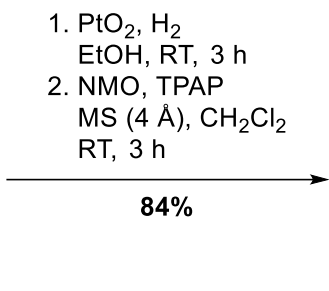

NMO, TPAP

$\mathrm{MS}(4 \AA), \mathrm{CH}_{2} \mathrm{Cl}_{2}$

$84 \%$

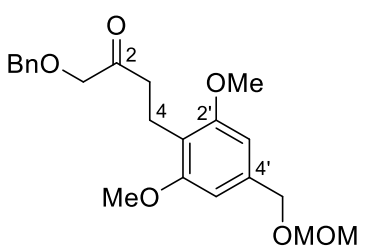

244a

Das $\alpha, \beta$-ungesättigte Keton ( $E$ )-243 (22.0 g, 56.9 mmol, 1.00 Äq.) wurde in EtOH (500 ml) gelöst, bei Raumtemperatur mit $\mathrm{PtO}_{2}(646 \mathrm{mg}, 2.85 \mathrm{mmol}, 5 \mathrm{~mol} \%)$ versetzt und unter einer $\mathrm{H}_{2}$-Atmosphäre (1 atm) $3 \mathrm{~h}$ (bis zum Aufklaren der Lösung) bei dieser Temperatur gerührt. Anschließend wurde der Katalysator mittels Filtration durch Celite ${ }^{\circledR}$ abgetrennt, mit EtOAc $(3 \times 250 \mathrm{ml})$ nachgespült und das Lösungsmittel unter vermindertem Druck entfernt. Daraufhin wurden das gewünschte Keton 244a sowie der korrespondierende überreduzierte Alkohol mittels Säulenchromatographie an Kieselgel (Petrolether/EtOAc $4: 1 \rightarrow 3: 1)$ voneinander getrennt.

Eine Lösung des überreduzierten Alkohols $\left(4.00 \mathrm{~g}, 10.2 \mathrm{mmol}, 1.00 \mathrm{Äq}\right.$.) in $\mathrm{CH}_{2} \mathrm{Cl}_{2}$ (50 ml) wurde bei Raumtemperatur nacheinander mit Molekularsieb (4 $\mathrm{A})$, einer katalytischen Menge TPAP (180 mg, $512 \mu \mathrm{mol}, 5 \mathrm{~mol} \%$ ) sowie NMO (1.80 g, $15.4 \mathrm{mmol}, 1.50$ Äq.) versetzt und $3 \mathrm{~h}$ bei dieser Temperatur gerührt. Anschließend wurde durch Celite ${ }^{\circledR}$ filtriert, mit EtOAc $(3 \times 50 \mathrm{ml})$ nachgewaschen und das Lösungsmittel unter vermindertem Druck entfernt. Säulenchromatographie an Kieselgel (Petrolether/EtOAc 2:1 $\rightarrow 1: 1$ ) lieferte das Keton 244a (kombinierte Gesamtausbeute: $18.5 \mathrm{~g}, 47.7 \mathrm{mmol}, 84 \%$ ) in Form eines farblosen Öls.

UV $\left(\mathrm{CH}_{3} \mathrm{CN}\right): \lambda_{\max }[\mathrm{nm}](\lg \varepsilon)=206$ (4.714), 235 (3.904), 274 (3.258).

IR (ATR): $\tilde{v}\left[\mathrm{~cm}^{-1}\right]=3088,3063,3029,2995,2935,2882,2839,1716,1608,1588,1497$, 1455, 1420, 1402, 1376, 1334, 1313, 1289, 1234, 1210, 1183, 1147, 1118, 1102, 1076, 1053, 1039, 1001, 983, 917, 824, 739, 698, 605, 580, 540, 526, 507.

${ }^{1} \mathrm{H}-\mathrm{NMR}\left(300 \mathrm{MHz}, \mathrm{CDCl}_{3}\right): \delta[\mathrm{ppm}]=2.56-2.64\left(\mathrm{~m}, 2 \mathrm{H}, 3-\mathrm{H}_{2}\right), 2.91(\mathrm{dd}, J=8.8,6.8 \mathrm{~Hz}$, $\left.2 \mathrm{H}, 4-\mathrm{H}_{2}\right), 3.41\left(\mathrm{~s}, 3 \mathrm{H}, \mathrm{OCH}_{2} \mathrm{OCH}_{3}\right), 3.77$ (s, $\left.6 \mathrm{H}, 2^{\prime}-\mathrm{OCH}_{3}, 6^{\prime}-\mathrm{OCH}_{3}\right), 4.07$ (s, $\left.2 \mathrm{H}, 1-\mathrm{H}_{2}\right)$, $4.53\left(\mathrm{~d}, J=0.6 \mathrm{~Hz}, 2 \mathrm{H}, \mathrm{OCH}_{2} \mathrm{Ph}\right), 4.56$ (s, $\left.2 \mathrm{H}, \mathrm{OC}_{2} \mathrm{OCH}_{3}\right), 4.70$ (s, $\left.2 \mathrm{H}, 4^{\prime}-\mathrm{CH}_{2}\right), 6.51$ (s, 2 H, 3'-H, 5'-H), 7.25-7.35 (m, 5 H, $5 \times \mathrm{Ph}-\mathrm{H})$.

$\left.{ }^{13} \mathrm{C}-N M R\left(126 \mathrm{MHz}, \mathrm{CDCl}_{3}\right): \delta[\mathrm{ppm}]=17.3(\mathrm{C}-4), 38.4(\mathrm{C}-3), 55.3\left(\mathrm{OCH}_{2} \mathrm{O}_{\mathrm{CH}}\right)_{3}\right), 55.5$ (2'- $\mathrm{OCH}_{3}, 6$ '- $\left.-\mathrm{OCH}_{3}\right), 69.4\left(4^{\prime}-\mathrm{CH}_{2}\right), 73.2\left(\mathrm{OCH}_{2} \mathrm{Ph}\right), 74.8(\mathrm{C}-1), 95.6\left(\mathrm{O}^{\mathrm{C}} \mathrm{H}_{2} \mathrm{OCH}_{3}\right), 103.1$ 
(C-3', C-5'), 116.2 (C-1'), 127.8, 127.8, 128.4 (2 × Ph-C $\left.{ }_{o}, 2 \times \mathrm{Ph}^{\prime} \mathrm{C}_{m}, \mathrm{Ph}-\mathrm{C}_{p}\right), 137.2,137.4$ (C-4', Ph- $\left.\mathrm{C}_{i}\right), 158.1$ (C-2', C-6'), 208.5 (C-2).

MS (ESI): $m / z(\%)=411.2(76)[\mathrm{M}+\mathrm{Na}]^{+}, 427.2(100)[\mathrm{M}+\mathrm{K}]^{+}, 799.4(9)[2 \times \mathrm{M}+\mathrm{Na}]^{+}$.

$\mathrm{C}_{22} \mathrm{H}_{28} \mathrm{O}_{6}(388.46)$ ber.: 411.1778

427.1517

gef.: $411.1785[\mathrm{M}+\mathrm{Na}]^{+}$

$427.1524[\mathrm{M}+\mathrm{K}]^{+}(\mathrm{ESI}-\mathrm{HRMS})$. 


\subsubsection{2-(3-((Benzyloxy)methyl)but-3-en-1-yl)-1,3-dimethoxy-5-((methoxy- methoxy)methyl)benzol (245)}

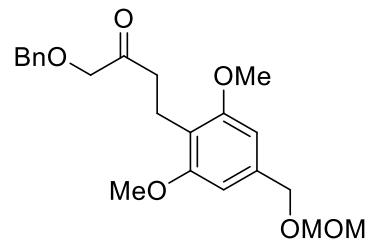

$244 a$

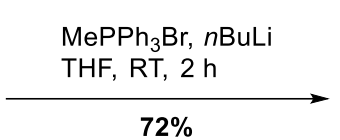

$72 \%$

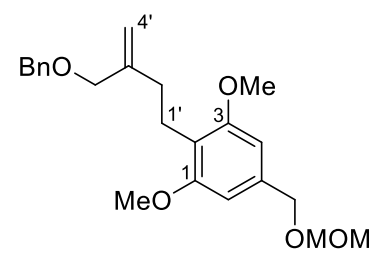

245

$\mathrm{Zu}$ einer Suspension von Methyltriphenylphosphoniumbromid $(2.76 \mathrm{~g}, 7.72 \mathrm{mmol}$, 3.00 Äq.) in THF (15 ml) wurde bei $0^{\circ} \mathrm{C}$ langsam $n B u L i ~(2.5 \mathrm{M}$ in $n$-Hexan, $2.88 \mathrm{ml}$, $7.21 \mathrm{mmol}, 2.80 \mathrm{Äq}$.) getropft, $30 \mathrm{~min}$ bei dieser Temperatur und anschließend weitere 30 min bei Raumtemperatur gerührt. Daraufhin wurde die Reaktionslösung auf $0{ }^{\circ} \mathrm{C}$ gekühlt, eine Lösung des Ketons 244a $(1.00 \mathrm{~g}, 2.57 \mathrm{mmol}, 1.00$ Äq.) in THF (5 ml) langsam zugetropft, auf Raumtemperatur erwärmt und $2 \mathrm{~h}$ bei dieser Temperatur gerührt. Die Reaktion wurde durch Zugabe von ges. $\mathrm{NH}_{4} \mathrm{Cl}$-Lsg. $(20 \mathrm{ml}), \mathrm{H}_{2} \mathrm{O}(10 \mathrm{ml})$ sowie $\mathrm{CH}_{2} \mathrm{Cl}_{2}$ $(20 \mathrm{ml})$ beendet, die Phasen getrennt und die wässr. Phase mit $\mathrm{CH}_{2} \mathrm{Cl}_{2}(3 \times 15 \mathrm{ml})$ extrahiert. Die vereinigten org. Phasen wurden über $\mathrm{MgSO}_{4}$ getrocknet, das Lösungsmittel unter vermindertem Druck entfernt und nach Säulenchromatographie an Kieselgel (Petrolether/EtOAc 7:1 $\rightarrow$ 4:1) das Alken 245 (719 mg, $1.86 \mathrm{mmol}, 72 \%)$ als leicht gelbes Öl erhalten

UV $\left(\mathrm{CH}_{3} \mathrm{CN}\right): \lambda_{\max }[\mathrm{nm}](\lg \varepsilon)=194$ (5.320), 206 (4.785), 273 (3.229).

IR (ATR): $\widetilde{v}\left[\mathrm{~cm}^{-1}\right]=3064,3029,2993,2935,2881,2838,2785,1650,1608,1588,1497$, 1454, 1420, 1377, 1363, 1335, 1313, 1235, 1211, 1184, 1162, 1147, 1116, 1102, 1074, 1055, 1040, 1000, 985, 947, 917, 825, 737, 697, 609, 581, 536, 526.

${ }^{1} \mathrm{H}-\mathrm{NMR}\left(300 \mathrm{MHz}, \mathrm{CDCl}_{3}\right): \delta[\mathrm{ppm}]=2.19-2.27\left(\mathrm{~m}, 2 \mathrm{H}, 2{ }^{\prime}-\mathrm{H}_{2}\right), 2.75-2.83(\mathrm{~m}, 2 \mathrm{H}$, 1'- $\mathrm{H}_{2}$ ), 3.42 (s, $3 \mathrm{H}, \mathrm{OCH}_{2} \mathrm{OCH}_{3}$ ), $3.78\left(\mathrm{~s}, 6 \mathrm{H}, 1-\mathrm{OCH}_{3}, 3-\mathrm{OCH}_{3}\right), 4.03$ (dd, J=1.3, $0.7 \mathrm{~Hz}$, $2 \mathrm{H}, 3^{\prime}-\mathrm{CH}_{2}$ ), 4.50 (d, J = 0.5 Hz, $2 \mathrm{H}, \mathrm{OCH}_{2} \mathrm{Ph}$ ), 4.54 (d, J = 0.6 Hz, $2 \mathrm{H}, \mathrm{OC}_{2} \mathrm{OCH}_{3}$ ), $4.71\left(\mathrm{~s}, 2 \mathrm{H}, 5-\mathrm{CH}_{2}\right), 4.94(\mathrm{qd}, J=1.4,0.7 \mathrm{~Hz}, 1 \mathrm{H}), 5.04(\mathrm{qd}, J=1.4,0.7 \mathrm{~Hz}, 1 \mathrm{H})\left(4^{\prime}-\mathrm{H}_{2}\right)$, 6.52 (s, $2 \mathrm{H}, 4-\mathrm{H}, 6-\mathrm{H}), 7.22-7.39(\mathrm{~m}, 5 \mathrm{H}, 5 \times \mathrm{Ph}-\mathrm{H})$.

${ }^{13} \mathrm{C}-\mathrm{NMR}\left(126 \mathrm{MHz}, \mathrm{CDCl}_{3}\right): \delta[\mathrm{ppm}]=21.4\left(\mathrm{C}-1^{\prime}\right), 32.4\left(\mathrm{C}-2{ }^{\prime}\right), 55.4\left(\mathrm{OCH}_{2} \mathrm{OCH}_{3}\right), 55.6$ (1- $\left.\mathrm{OCH}_{3}, 3-\mathrm{OCH}_{3}\right), 69.6\left(5-\mathrm{CH}_{2}\right), 71.9\left(\mathrm{OCH}_{2} \mathrm{Ph}\right), 73.2\left(3^{\prime}-\mathrm{CH}_{2}\right), 95.6\left(\mathrm{OCH}_{2} \mathrm{OCH}_{3}\right), 103.2$ (C-4, C-6), 111.1 (C-4'), 117.9 (C-2), $127.4\left(\mathrm{Ph}^{\prime} \mathrm{C}_{p}\right), 127.6,128.3$ (2 × Ph-Co, $\left.2 \times \mathrm{Ph}-\mathrm{C}_{m}\right)$, 136.7 (C-5), $138.7\left(\mathrm{Ph}^{-\mathrm{C}_{i}}\right), 146.5$ (C-3'), 158.2 (C-1, C-3).

MS (ESI): $m / z(\%)=409.2(54)[\mathrm{M}+\mathrm{Na}]^{+}, 425.2(100)[\mathrm{M}+\mathrm{K}]^{+}$.

$\mathrm{C}_{23} \mathrm{H}_{30} \mathrm{O}_{5}$ (386.49)

ber.: 409.1985 
425.1725

gef.: $409.1981[\mathrm{M}+\mathrm{Na}]^{+}$

$425.1724[\mathrm{M}+\mathrm{K}]^{+}$(ESI-HRMS). 


\subsubsection{2-(3-((Benzyloxy)methyl)but-3-en-1-yl)-3-methoxy-5-((methoxy- methoxy)methyl)phenol (246)}

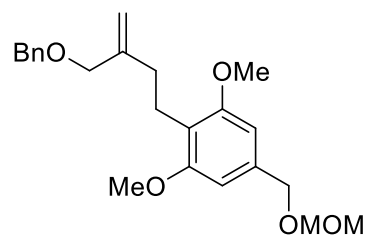

245

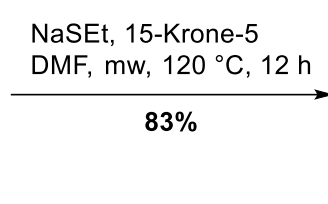

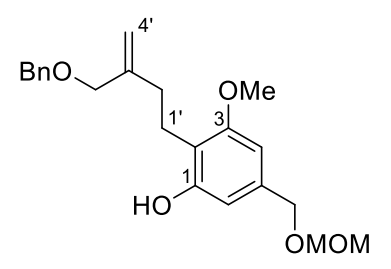

246

Eine Lösung des Alkens 245 (700 mg, 1.81 mmol, 1.00 Äq.), NaSEt (90\%ig, 508 mg, $5.43 \mathrm{mmol}, 3.00$ Äq.) sowie 15-Krone-5 (717 $\mu \mathrm{l}, 798 \mathrm{mg}, 3.62 \mathrm{mmol}, 2.00$ Äq.) in DMF $(10 \mathrm{ml})$ wurde $12 \mathrm{~h}$ bei $120^{\circ} \mathrm{C}$ in der Mikrowelle gerührt. Nach Abkühlen auf Raumtemperatur wurde $\mathrm{H}_{2} \mathrm{O}(10 \mathrm{ml})$ zugegeben und mit EtOAc $(3 \times 10 \mathrm{ml})$ extrahiert. Die vereinigten org. Phasen wurden mit $\mathrm{H}_{2} \mathrm{O}(20 \mathrm{ml})$ sowie ges. $\mathrm{NaCl}$-Lsg. $(2 \times 20 \mathrm{ml})$ gewaschen, über $\mathrm{MgSO}_{4}$ getrocknet und das Lösungsmittel unter vermindertem Druck entfernt. Säulenchromatographische Reinigung an Kieselgel (Petrolether/EtOAc 4:1) lieferte das Phenol 246 (562 mg, 1.51 mmol, 83\%) in Form eines farblosen Öls.

UV $\left(\mathrm{CH}_{3} \mathrm{CN}\right): \lambda_{\max }[\mathrm{nm}](\mathrm{lg} \varepsilon)=194$ (5.297), 205 (4.751), 275 (3.264).

IR (ATR): $\tilde{v}\left[\mathrm{~cm}^{-1}\right]=3339,3086,3065,3030,2991,2934,2883,2858,2841,1650,1615$, 1596, 1513, 1497, 1451, 1426, 1379, 1362, 1318, 1268, 1242, 1211, 1147, 1093, 1041, 989, 901, 824, 737, 696, 668, 606, 565, 533.

${ }^{1} \mathrm{H}-\mathrm{NMR}\left(500 \mathrm{MHz}, \mathrm{CDCl}_{3}\right): \delta[\mathrm{ppm}]=2.30$ (ddd, $\left.J=8.1,7.0,1.0 \mathrm{~Hz}, 2 \mathrm{H}, 2^{\prime}-\mathrm{H}_{2}\right), 2.80$ (dd, $\left.J=7.9,6.9 \mathrm{~Hz}, 2 \mathrm{H}, 1^{\prime}-\mathrm{H}_{2}\right), 3.40$ (s, $\left.3 \mathrm{H}, \mathrm{OCH}_{2} \mathrm{OCH}_{3}\right), 3.78$ (s, $\left.3 \mathrm{H}, 3-\mathrm{OCH}_{3}\right), 4.02$ (d, $J=$ $\left.1.0 \mathrm{~Hz}, 2 \mathrm{H}, 3^{\prime}-\mathrm{CH}_{2}\right), 4.49$ (d, $\left.J=0.6 \mathrm{~Hz}, 2 \mathrm{H}, \mathrm{OCH}_{2} \mathrm{OCH}_{3}\right), 4.58$ (s, $2 \mathrm{H}, \mathrm{OCH}_{2} \mathrm{Ph}$ ), 4.68 (s, $2 \mathrm{H}, 5-\mathrm{CH}_{2}$ ), 4.76 (dd, $J=1.8,1.1 \mathrm{~Hz}, 1 \mathrm{H}$ ), 4.94 (dt, $\left.J=2.1,1.2 \mathrm{~Hz}, 1 \mathrm{H}\right)\left(4^{\prime}-\mathrm{H}_{2}\right), 6.28$ (s, $1 \mathrm{H}, \mathrm{OH}), 6.44(\mathrm{~d}, J=1.4 \mathrm{~Hz}, 1 \mathrm{H}), 6.46(\mathrm{dd}, J=1.5,0.7 \mathrm{~Hz}, 1 \mathrm{H})(4-\mathrm{H}, 6-\mathrm{H}), 7.26-$ $7.31\left(\mathrm{~m}, 1 \mathrm{H}, \mathrm{Ph}-\mathrm{H}_{p}\right), 7.32-7.38\left(\mathrm{~m}, 4 \mathrm{H}, 2 \times \mathrm{Ph}-\mathrm{H}_{0}, 2 \times \mathrm{Ph}^{-\mathrm{H}_{m}}\right)$.

${ }^{13} \mathrm{C}-\mathrm{NMR}\left(126 \mathrm{MHz}, \mathrm{CDCl}_{3}\right): \delta[\mathrm{ppm}]=22.4\left(\mathrm{C}-1^{\prime}\right), 33.9\left(\mathrm{C}-2^{\prime}\right), 55.4\left(\mathrm{OCH}_{2} \mathrm{O}^{\mathrm{C}} \mathrm{H}_{3}\right), 55.6$ $\left(3-\mathrm{OCH}_{3}\right), 69.2\left(5-\mathrm{CH}_{2}\right), 72.8\left(\mathrm{OCH}_{2} \mathrm{Ph}\right), 74.6\left(3{ }^{\prime}-\mathrm{CH}_{2}\right), 95.6\left(\mathrm{O}_{\underline{C}} \mathrm{OCH}_{3}\right), 102.1,108.5$

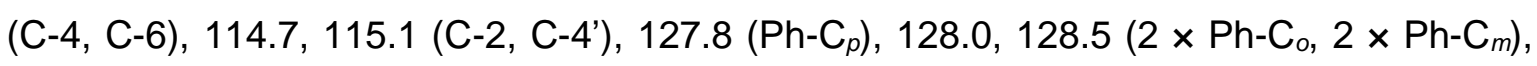
137.0 (C-5), $137.6\left(\mathrm{Ph}^{-\mathrm{C}_{i}}\right), 144.6$ (C-3'), 155.2 (C-1), 158.4 (C-3).

MS (ESI): $m / z(\%)=395.2(100)\left[\mathrm{M}+\mathrm{Na}^{+}, 411.2(88)[\mathrm{M}+\mathrm{K}]^{+} ; 371.2(100)[\mathrm{M}-\mathrm{H}]^{-}\right.$, $743.4(36)[2 \times \mathrm{M}-\mathrm{H}]^{-}$.

$\mathrm{C}_{22} \mathrm{H}_{28} \mathrm{O}_{5}$ (372.46)

ber.: 371.1864

395.1829

411.1568 
gef.: $371.1857[\mathrm{M}-\mathrm{H}]^{-}$

$395.1838[\mathrm{M}+\mathrm{Na}]^{+}$

$411.1578[\mathrm{M}+\mathrm{K}]^{+}$(ESI-HRMS). 


\subsection{7 (S)-2-(2-((Benzyloxy)methyl)-5-methoxy-7-((methoxymethoxy)- methyl)chroman-2-yl)essigsäuremethylester ((S)-250)}

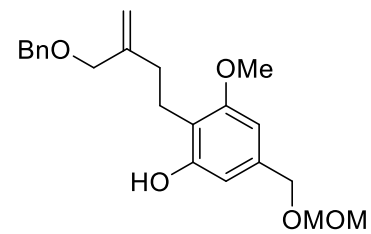

246

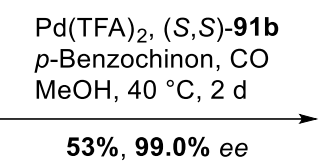

$53 \%, 99.0 \%$ ee

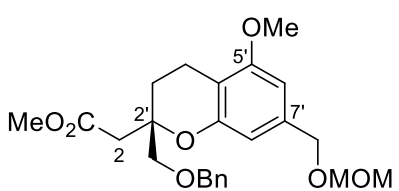

(S)-250

Eine Suspension von $\mathrm{Pd}(\mathrm{TFA})_{2}(2.23 \mathrm{mg}, 6.71 \mu \mathrm{mol}, 5 \mathrm{~mol} \%)$ sowie des $(S, S)$ - $\mathrm{Pr}$ BOXAX-Liganden (S,S)-91b (12.8 mg, $26.8 \mu \mathrm{mol}, 20 \mathrm{~mol} \%$ ) in $\mathrm{MeOH}$ (1 ml) wurde $30 \mathrm{~min}$ bei Raumtemperatur gerührt, anschließend eine Lösung des Phenols 246 (50.0 mg, $134 \mu \mathrm{mol}, 1.00 \mathrm{Äq}$.) in $\mathrm{MeOH}(1 \mathrm{ml})$ sowie $p$-Benzochinon (58.0 mg, $537 \mu \mathrm{mol}, 4.00 \mathrm{Äq}$.) bei dieser Temperatur zugegeben, $5 \mathrm{~min} \mathrm{CO}$ durch die resultierende Reaktionslösung geleitet und daraufhin $2 \mathrm{~d}$ bei $40^{\circ} \mathrm{C}$ unter CO-Atmosphäre (1 atm) gerührt. Nach Abkühlen auf Raumtemperatur wurde das Reaktionsgemisch in verd. $\mathrm{HCl}(1 \mathrm{M}, 5 \mathrm{ml})$ gegeben, mit MTBE $(3 \times 5 \mathrm{ml})$ extrahiert und die vereinigten org. Phasen mit verd. $\mathrm{NaOH}$ Lsg. ( $1 \mathrm{M}, 3 \times 10 \mathrm{ml}$ ) gewaschen. Nach Trocknen über $\mathrm{MgSO}_{4}$, Entfernen des Lösungsmittels unter vermindertem Druck und Säulenchromatographie an Kieselgel (Petrolether/EtOAc 4:1) wurde der Methylester (S)-250 (30.6 mg, $71.1 \mu \mathrm{mol}, 53 \%, 99.0 \%$ ee) als farbloses Öl erhalten.

HPLC (analytisch): $\quad$ Säule: $\quad$ CHIRALPAK ${ }^{\circledR} \mathrm{IA}, 4.6 \times 250 \mathrm{~mm}, 5 \mu \mathrm{m}$

Eluent: $\quad n$-Hexan/PrOH 99:1

Fluß: $\quad 0.8 \mathrm{ml} / \mathrm{min}$

$\lambda$ : $\quad 207 \mathrm{~nm}$

$t_{\mathrm{R}}: \quad 19.2 \min (+)-(R)-250,0.5 \%$

$20.7 \min (-)-(S)-250,99.5 \%, 99.0 \%$ ee.

Drehwert: $[\alpha]_{D}^{22}\left[^{\circ}\right]=-325.1\left(c=0.30, \mathrm{CHCl}_{3}\right)$.

UV $\left(\mathrm{CH}_{3} \mathrm{CN}\right): \lambda_{\max }[\mathrm{nm}](\mathrm{lg} \varepsilon)=208$ (4.708), 228 (4.094).

IR (ATR): $\tilde{v}\left[\mathrm{~cm}^{-1}\right]=3087,3061,3029,2993,2947,2880,1735,1679,1659,1650,1640$, 1619, 1586, 1552, 1530, 1512, 1497, 1452, 1436, 1424, 1377, 1355, 1322, 1296, 1213, 1179, 1147, 1100, 1048, 1019, 958, 917, 889, 825, 739, 699, 675, 646, 609, 572, 547, 524.

${ }^{1} \mathrm{H}-\mathrm{NMR}\left(300 \mathrm{MHz}, \mathrm{CDCl}_{3}\right): \delta[\mathrm{ppm}]=2.02\left(\mathrm{td}, J=6.8,1.7 \mathrm{~Hz}, 2 \mathrm{H}, 3^{\prime}-\mathrm{H}_{2}\right), 2.61$ (td, $J=$ 6.8, 2.7 Hz, $2 \mathrm{H}, 4^{\prime}-\mathrm{H}_{2}$ ), 2.70 (d, J = 14.5 Hz, $1 \mathrm{H}$ ), 2.82 (d, J = 14.5 Hz, $\left.1 \mathrm{H}\right)\left(2-\mathrm{H}_{2}\right), 3.40$ (s, $3 \mathrm{H}, \mathrm{OCH}_{2} \mathrm{OCH}_{3}$ ), 3.61 (s, $3 \mathrm{H}, \mathrm{CO}_{2} \mathrm{CH}_{3}$ ), 3.64 (d, J = $\left.0.7 \mathrm{~Hz}, 2 \mathrm{H}, 2^{\prime}-\mathrm{CH}_{2}\right), 3.80(\mathrm{~s}, 3 \mathrm{H}$, 
5' $\left.-\mathrm{OCH}_{3}\right), 4.48\left(\mathrm{~s}, 2 \mathrm{H}, \mathrm{OC}_{2} \mathrm{OCH}_{3}\right), 4.52(\mathrm{~d}, J=12.0 \mathrm{~Hz}, 1 \mathrm{H}), 4.60(\mathrm{~d}, J=12.1 \mathrm{~Hz}, 1 \mathrm{H})$ $\left(\mathrm{OCH}_{2} \mathrm{Ph}\right), 4.68\left(\mathrm{~s}, 2 \mathrm{H}, 7^{\prime}-\mathrm{CH}_{2}\right), 6.41(\mathrm{~d}, J=1.5 \mathrm{~Hz}, 1 \mathrm{H}), 6.48(\mathrm{dd}, J=1.4,0.7 \mathrm{~Hz}, 1 \mathrm{H})$ (6'-H, 8'-H), 7.22-7.33 (m, $5 \mathrm{H}, 5 \times \mathrm{Ph}-\mathrm{H})$.

${ }^{13} \mathrm{C}-N M R\left(126 \mathrm{MHz}, \mathrm{CDCl}_{3}\right): \delta[\mathrm{ppm}]=16.0\left(\mathrm{C}-4^{\prime}\right), 26.1$ (C-3'), $39.3(\mathrm{C}-2), 51.5\left(\mathrm{CO}_{2} \mathrm{CH}_{3}\right)$, 55.3, $55.4\left(5^{\prime}-\mathrm{OCH}_{3}, \mathrm{OCH}_{2} \mathrm{O}_{\mathrm{C}} \mathrm{H}_{3}\right), 69.2\left(7^{\prime}-\mathrm{CH}_{2}\right), 72.6\left(2^{\prime}-\mathrm{CH}_{2}\right), 73.6\left(\mathrm{OCH}_{2} \mathrm{Ph}\right), 76.4$ (C-2'), $95.6\left(\mathrm{O}_{\mathrm{C}} \mathrm{H}_{2} \mathrm{OCH}_{3}\right), 101.4,109.4,109.5$ (C-4a', C-6', C-8'), $127.5\left(\mathrm{Ph}-\mathrm{C}_{p}\right), 127.6$, $128.3\left(2 \times \mathrm{Ph}^{-\mathrm{C}_{o}, 2} \times \mathrm{Ph}_{\mathrm{C}}\right), 137.2$ (C-7'), $138.2\left(\mathrm{Ph}-\mathrm{C}_{i}\right), 153.4,157.8$ (C-5', C-8a'), $170.6\left(\underline{\mathrm{CO}_{2}} \mathrm{CH}_{3}\right)$.

MS (ESI): $m / z(\%)=453.2(100)[\mathrm{M}+\mathrm{Na}]^{+}, 883.4(26)[2 \times \mathrm{M}+\mathrm{Na}]^{+} ; 429.2(62)\left[\mathrm{M}-\mathrm{H}^{-}\right.$.

$\mathrm{C}_{24} \mathrm{H}_{30} \mathrm{O}_{7}(430.50)$

ber.: 429.1919

453.1884

gef.: $429.1913[\mathrm{M}-\mathrm{H}]^{-}$

$453.1885[\mathrm{M}+\mathrm{Na}]^{+}(\mathrm{ESI}-\mathrm{HRMS})$. 


\subsection{8 (S)-2-(2-((Benzyloxy)methyl)-5-methoxy-7-((methoxymethoxy)- methyl)chroman-2-yl)ethan-1-ol ((S)-251)}

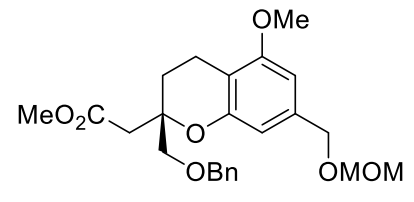

(S)-250

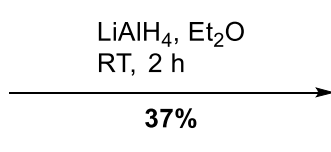

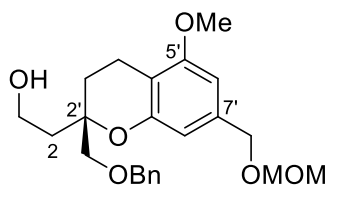

$(S)-\mathbf{2 5 1}$

Eine Lösung des Methylesters (S)-250 (90.0 mg, 209 mol, 1.00 Äq.) in $\mathrm{Et}_{2} \mathrm{O}$ (5 ml) wurde bei $0^{\circ} \mathrm{C}$ mit $\mathrm{LiAlH}_{4} \quad(8.73 \mathrm{mg}, 230 \mu \mathrm{mol}, 1.10$ Äq. $)$ versetzt, die resultierende Reaktionslösung auf Raumtemperatur erwärmt und $2 \mathrm{~h}$ bei dieser Temperatur gerührt. Die Reaktion wurde durch vorsichtige Zugabe von $\mathrm{H}_{2} \mathrm{O}(15 \mathrm{ml})$ bei $0^{\circ} \mathrm{C}$ beendet, die Phasen getrennt und die wässr. Phase mit EtOAc $(3 \times 10 \mathrm{ml})$ extrahiert. Die vereinigten org. Phasen wurden über $\mathrm{MgSO}_{4}$ getrocknet, das Lösungsmittel unter vermindertem Druck entfernt und nach Säulenchromatographie an Kieselgel (Petrolether/EtOAc 4:1 $\rightarrow$ EtOAc) der Alkohol (S)-251 (31.3 mg, $77.8 \mu \mathrm{mol}, 37 \%$ ) als farbloses Öl isoliert.

UV $\left(\mathrm{CH}_{3} \mathrm{CN}\right): \lambda_{\max }[\mathrm{nm}](\mathrm{lg} \varepsilon)=208$ (4.747), 228 (4.022), 281 (3.326).

IR (ATR): $\tilde{v}\left[\mathrm{~cm}^{-1}\right]=3421,3087,3062,3030,2933,2882,1618,1586,1497,1452,1423$, $1377,1354,1325,1295,1270,1245,1211,1147,1100,1047,1021,960,918,883,846$, 824, 739, 699, 669, 611, 592, 576, 568, 552, 531, 517, 509.

${ }^{1} \mathrm{H}-\mathrm{NMR}\left(300 \mathrm{MHz}, \mathrm{CDCl}_{3}\right): \delta[\mathrm{ppm}]=1.83\left(\mathrm{ddd}, J=14.2,8.4,6.5 \mathrm{~Hz}, 1 \mathrm{H}, 3\right.$ '- $\mathrm{H}_{\mathrm{a}}$ ), $1.93-$ $2.06\left(\mathrm{~m}, 3 \mathrm{H}, 2-\mathrm{H}_{2}, 3^{\prime}-\mathrm{H}_{\mathrm{b}}\right), 2.45-2.69\left(\mathrm{~m}, 3 \mathrm{H}, 4^{\prime}-\mathrm{H}_{2}, \mathrm{OH}\right), 3.40\left(\mathrm{~s}, 3 \mathrm{H}, \mathrm{OCH}_{2} \mathrm{OC}_{3}\right), 3.50$ (d, $J=9.6 \mathrm{~Hz}, 1 \mathrm{H}), 3.55$ (d, J = 9.6 Hz, $1 \mathrm{H})\left(2 \mathrm{C}-\mathrm{CH}_{2}\right), 3.74-3.79\left(\mathrm{~m}, 2 \mathrm{H}, 1-\mathrm{H}_{2}\right), 3.80$ (s, $\left.3 \mathrm{H}, 5^{\prime}-\mathrm{OCH}_{3}\right), 4.48\left(\mathrm{~s}, 2 \mathrm{H}, \mathrm{OCH}_{2} \mathrm{OCH}_{3}\right), 4.51(\mathrm{~d}, J=12.2 \mathrm{~Hz}, 1 \mathrm{H}), 4.56(\mathrm{~d}, J=12.0 \mathrm{~Hz}$, $1 \mathrm{H})\left(\mathrm{OCH}_{2} \mathrm{Ph}\right), 4.68\left(\mathrm{~s}, 2 \mathrm{H}, 7^{\prime}-\mathrm{CH}_{2}\right), 6.41(\mathrm{~d}, J=1.5 \mathrm{~Hz}, 1 \mathrm{H}), 6.45(\mathrm{dd}, J=1.4,0.7 \mathrm{~Hz}$, $1 \mathrm{H})\left(6^{\prime}-\mathrm{H}, 8^{\prime}-\mathrm{H}\right), 7.26-7.36(\mathrm{~m}, 5 \mathrm{H}, 5 \times \mathrm{Ph}-\mathrm{H})$.

${ }^{13} \mathrm{C}-N M R\left(126 \mathrm{MHz}, \mathrm{CDCl}_{3}\right): \delta[\mathrm{ppm}]=16.0$ (C-4'), 26.9 (C-3'), 38.7 (C-2), 55.3, 55.4 (5'- $\left.\mathrm{OCH}_{3}, \mathrm{OCH}_{2} \mathrm{O}^{-} \mathrm{H}_{3}\right), 58.5$ (C-1), $69.1\left(7^{\prime}-\mathrm{CH}_{2}\right), 72.3\left(2^{\prime}-\mathrm{CH}_{2}\right), 73.8\left(\mathrm{OCH}_{2} \mathrm{Ph}\right), 77.5$ (C-2'), $95.6\left(\mathrm{O}_{\mathrm{CH}} \mathrm{OCH}_{3}\right), 101.4,109.2,109.6$ (C-4a', C-6', C-8'), 127.7, 127.8, 128.4 (2 × $\left.\mathrm{Ph}-\mathrm{C}_{o}, 2 \times \mathrm{Ph} \mathrm{C}_{m}, \mathrm{Ph}-\mathrm{C}_{p}\right), 137.3,137.6$ (C-7', Ph- $\left.\mathrm{C}_{i}\right), 153.5,157.8$ (C-5', C-8a').

MS (ESI): $m / z(\%)=403.2(9)[\mathrm{M}+\mathrm{H}]^{+}, 425.2(100)[\mathrm{M}+\mathrm{Na}]^{+}, 441.2(11)[\mathrm{M}+\mathrm{K}]^{+}, 827.4$ (23) $[2 \times \mathrm{M}+\mathrm{Na}]^{+} ; 401.2(100)[\mathrm{M}-\mathrm{H}]^{-}, 803.4(11)[2 \times \mathrm{M}-\mathrm{H}]^{-}$.

$\mathrm{C}_{23} \mathrm{H}_{30} \mathrm{O}_{6}(402.49)$

ber.: 401.1970

403.2115

425.1935

gef.: $401.1960[\mathrm{M}-\mathrm{H}]^{-}$ 
403.2110 [M + H $]^{+}$

$425.1936[\mathrm{M}+\mathrm{Na}]^{+}(\mathrm{ESI}-\mathrm{HRMS})$. 


\subsection{9 (S)-2-((Benzyloxy)methyl)-5-methoxy-7-((methoxymethoxy)- methyl)-2-vinylchroman ((S)-252)}

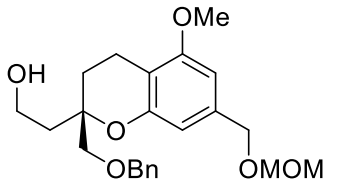

(S)-251

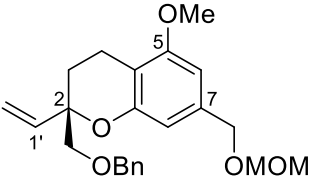

(S)-252

Eine Lösung des Alkohols (S)-251 (25.0 mg, $62.1 \mu \mathrm{mol}, 1.00$ Äq.) in THF (2 ml) wurde bei $0{ }^{\circ} \mathrm{C}$ mit 1-Nitro-2-selenocyanatbenzol (254) (35.3 mg, $155 \mu \mathrm{mol}, 2.50$ Äq.) sowie $\mathrm{PnBu}_{3}$ (36.9 $\mu$ l, 29.9 mg, $148 \mu \mathrm{mol}$, 2.38 Äq.) versetzt und $4 \mathrm{~h}$ bei dieser Temperatur gerührt. Die Reaktion wurde bei $0^{\circ} \mathrm{C}$ durch Zugabe von ges. $\mathrm{NaHCO}_{3}$-Lsg. $(5 \mathrm{ml})$ beendet, mit MTBE $(5 \times 5 \mathrm{ml})$ extrahiert, die vereinigten org. Phasen über $\mathrm{MgSO}_{4}$ getrocknet und das Lösungsmittel unter vermindertem Druck entfernt.

Der Rückstand wurde in $\mathrm{CH}_{2} \mathrm{Cl}_{2}(2 \mathrm{ml})$ aufgenommen, auf $-40^{\circ} \mathrm{C}$ gekühlt, mit $\mathrm{Na}_{2} \mathrm{HPO}_{4} \cdot 2 \mathrm{H}_{2} \mathrm{O}$ (55.3 mg, $311 \mu \mathrm{mol}, 5.00$ Äq.) sowie mCPBA (26.8 mg, $155 \mu \mathrm{mol}$, 2.50 Äq.) versetzt und anschließend $1 \mathrm{~h}$ bei dieser Temperatur gerührt. Daraufhin wurde $\operatorname{Pr}_{2} \mathrm{NH} \quad(43.5 \mu \mathrm{l}, 31.4 \mathrm{mg}, 311 \mu \mathrm{mol}, 5.00$ Äq.) hinzugegeben, auf Raumtemperatur erwärmt und weitere $15 \mathrm{~h}$ bei dieser Temperatur gerührt. Abschließend wurde das Reaktionsgemisch auf Kieselgel adsorbiert, das Lösungsmittel unter vermindertem Druck entfernt und nach Säulenchromatographie an Kieselgel (Petrolether/EtOAc 9:1 $\rightarrow$ 4:1) das Vinylchroman (S)-252 (15.8 mg, $41.1 \mu \mathrm{mol}, 66 \%, 99.6 \%$ ee) als farbloses Öl erhalten.

HPLC (analytisch): $\quad$ Säule: $\quad$ CHIRALPAK ${ }^{\circledR} / \mathrm{A}, 4.6 \times 250 \mathrm{~mm}, 5 \mu \mathrm{m}$

Eluent: $\quad n$-Hexan/ $\mathrm{PrOH}$ 99:1

Fluß: $\quad 0.8 \mathrm{ml} / \mathrm{min}$

$\lambda: \quad 207 \mathrm{~nm}$

$t_{\mathrm{R}}: \quad 11.2 \min (+)-(R)-252,0.2 \%$

$13.9 \min (-)-(S)-252,99.8 \%, 99.6 \%$ ee.

Drehwert: $[\alpha]_{D}^{21}\left[^{\circ}\right]=-59.6\left(c=0.65, \mathrm{CHCl}_{3}\right)$.

UV $\left(\mathrm{CH}_{3} \mathrm{CN}\right): \lambda_{\max }[\mathrm{nm}](\lg \varepsilon)=208$ (4.724), 229 (4.026), 275 (3.256).

IR (ATR): $\widetilde{v}\left[\mathrm{~cm}^{-1}\right]=3088,3062,3029,2986,2926,2884,2858,2789,1717,1698,1685$, $1671,1646,1618,1586,1541,1522,1497,1452,1424,1378,1355,1321,1294,1272$, $1245,1212,1196,1147,1136,1100,1049,1026,1001,958,925,824,739,698,679$, $609,590,571,553,526,512,504$. 
${ }^{1} \mathrm{H}-\mathrm{NMR}\left(500 \mathrm{MHz}, \mathrm{CDCl}_{3}\right): \delta[\mathrm{ppm}]=1.90$ (ddd, $\left.J=13.6,6.2,4.0 \mathrm{~Hz}, 1 \mathrm{H}\right), 1.99$ (ddd, $J=$ 13.6, 10.9, $5.6 \mathrm{~Hz}, 1 \mathrm{H}$ ) (3- $\mathrm{H}_{2}$ ), 2.40 (ddd, $J=17.1,10.9,6.2 \mathrm{~Hz}, 1 \mathrm{H}$ ), 2.69 (ddd, $J=17.0$, 5.6, $3.9 \mathrm{~Hz}, 1 \mathrm{H})\left(4-\mathrm{H}_{2}\right), 3.40\left(\mathrm{~s}, 3 \mathrm{H}, \mathrm{OCH}_{2} \mathrm{OC}_{3}\right), 3.51(\mathrm{~d}, J=10.0 \mathrm{~Hz}, 1 \mathrm{H}), 3.56(\mathrm{~d}, J=$ $10.0 \mathrm{~Hz}, 1 \mathrm{H})\left(2-\mathrm{CH}_{2}\right), 3.79\left(\mathrm{~s}, 3 \mathrm{H}, 5-\mathrm{OCH}_{3}\right), 4.50\left(\mathrm{~s}, 2 \mathrm{H}, \mathrm{OCH}_{2} \mathrm{OCH}_{3}\right), 4.58(\mathrm{~d}, J=$ $12.2 \mathrm{~Hz}, 1 \mathrm{H}), 4.62(\mathrm{~d}, J=12.2 \mathrm{~Hz}, 1 \mathrm{H})\left(\mathrm{OCH}_{2} \mathrm{Ph}\right), 4.68\left(\mathrm{~s}, 2 \mathrm{H}, 7-\mathrm{CH}_{2}\right), 5.14(\mathrm{dd}, J=$ 10.9, $1.3 \mathrm{~Hz}, 1 \mathrm{H}, 2^{\prime}-\mathrm{H}_{c i s}$ ), 5.23 (dd, $J=17.3,1.3 \mathrm{~Hz}, 1 \mathrm{H}, 2^{\prime}-\mathrm{H}_{\text {trans }}$ ), 5.82 (dd, $J=17.3$, $\left.10.9 \mathrm{~Hz}, 1 \mathrm{H}, 1^{\prime}-\mathrm{H}\right), 6.40$ (d, $\left.J=1.4 \mathrm{~Hz}, 1 \mathrm{H}\right), 6.56(\mathrm{dd}, J=1.5,0.7 \mathrm{~Hz}, 1 \mathrm{H})(6-\mathrm{H}, 8-\mathrm{H})$, 7.23-7.28 (m, $\left.1 \mathrm{H}, \mathrm{Ph}-\mathrm{H}_{p}\right), 7.31-7.32\left(\mathrm{~m}, 4 \mathrm{H}, 2 \times \mathrm{Ph}-\mathrm{H}_{o}, 2 \times \mathrm{Ph}-\mathrm{H}_{m}\right)$.

${ }^{13} \mathrm{C}-N M R\left(126 \mathrm{MHz}, \mathrm{CDCl}_{3}\right): \delta[\mathrm{ppm}]=16.2 \quad(\mathrm{C}-4), 26.4$ (C-3), 55.3, $55.4 \quad\left(5-\mathrm{OCH}_{3}\right.$, $\left.\mathrm{OCH}_{2} \mathrm{O}_{\mathrm{CH}}\right), 69.2\left(7-\mathrm{CH}_{2}\right), 73.6\left(\mathrm{OCH}_{2} \mathrm{Ph}\right), 75.5\left(2-\mathrm{CH}_{2}\right), 78.8(\mathrm{C}-2), 95.6\left(\mathrm{O}_{2} \mathrm{H}_{2} \mathrm{OCH}_{3}\right)$, 101.2, 109.2, 110.1 (C-4a, C-6, C-8), 116.2 (C-2'), $127.5\left(\mathrm{Ph}_{-} \mathrm{C}_{p}\right), 127.6,128.3$ (2 × Ph-Co, $\left.2 \times \mathrm{Ph}^{-\mathrm{C}_{m}}\right), 137.0$ (C-7), 137.8 (C-1'), $138.3\left(\mathrm{Ph}-\mathrm{C}_{i}\right), 154.3,157.7$ (C-5, C-8a).

MS (ESI): $m / z(\%)=385.2(9)[\mathrm{M}+\mathrm{H}]^{+}, 407.2(100)[\mathrm{M}+\mathrm{Na}]^{+}, 791.4(11)[2 \times \mathrm{M}+\mathrm{Na}]^{+}$.

$\mathrm{C}_{23} \mathrm{H}_{28} \mathrm{O}_{5}(384.47)$

ber.: 407.1829

gef.: $407.1830[\mathrm{M}+\mathrm{Na}]^{+}(\mathrm{ESI}-\mathrm{HRMS})$. 


\subsubsection{0 (E/Z)-2-(3-((Benzyloxy)methyl)pent-3-en-1-yl)-1,3-dimethoxy-5- ((methoxymethoxy)methyl)benzol ((E/Z)-255)}

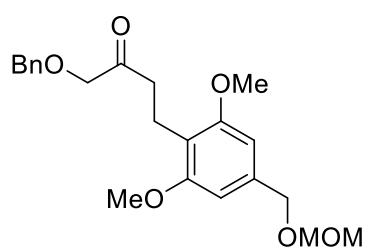

$244 a$

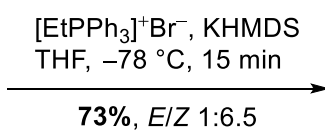

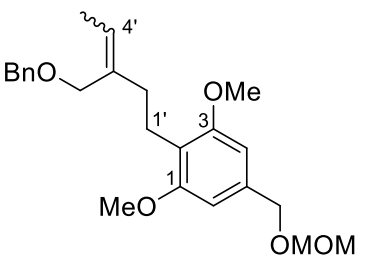

$(E / Z)-\mathbf{2 5 5}$

Zu einer Suspension von Ethyltriphenylphosphoniumbromid (19.1 g, 51.5 mmol, 10.0 Äq.) in THF (20 ml) wurde bei $0^{\circ} \mathrm{C}$ KHMDS $(9.24 \mathrm{~g}, 46.3 \mathrm{mmol}, 9.00 \mathrm{Äq}$.) gegeben, das resultierende Reaktionsgemisch auf $66^{\circ} \mathrm{C}$ erwärmt und $1 \mathrm{~h}$ bei dieser Temperatur unter Rückfluss gerührt. Anschließend wurde die Reaktionslösung auf $-78^{\circ} \mathrm{C}$ gekühlt, eine Lösung des Ketons 244a (2.00 g, 5.15 mmol, 1.00 Äq.) in THF (30 ml) zugegeben und 15 min bei dieser Temperatur gerührt. Daraufhin wurde auf Raumtemperatur erwärmt, die Reaktion durch vorsichtige Zugabe von ges. $\mathrm{NH}_{4} \mathrm{Cl}$-Lsg. $(100 \mathrm{ml})$ sowie $\mathrm{H}_{2} \mathrm{O}(100 \mathrm{ml})$ beendet und die wässr. Phase mit MTBE $(3 \times 200 \mathrm{ml})$ extrahiert. Die vereinigten org. Phasen wurden über $\mathrm{MgSO}_{4}$ getrocknet, das Lösungsmittel unter vermindertem Druck entfernt und nach säulenchromatographischer Reinigung an Kieselgel (Petrolether/EtOAc 4:1) das Alken (E/Z)-255 (1.50 g, $3.75 \mathrm{mmol}, 73 \%, E / Z 1: 6.5)$ als farbloses Öl isoliert.

UV $\left(\mathrm{CH}_{3} \mathrm{CN}\right): \lambda_{\max }[\mathrm{nm}](\lg \varepsilon)=207$ (4.725), 235 (3.911), 272 (3.142).

IR (ATR): $\tilde{v}\left[\mathrm{~cm}^{-1}\right]=3087,3061,3028,2932,2880,2857,2839,1721,1692,1677,1665$, $1640,1608,1588,1553,1535,1512,1496,1454,1420,1376,1332,1312,1268,1236$, $1210,1183,1166,1147,1119,1055,1039,984,942,918,823,736,697,599,578,542$, $525,513,505$.

${ }^{1} \mathrm{H}-\mathrm{NMR}\left(300 \mathrm{MHz}, \mathrm{CDCl}_{3}\right.$, Diastereomerengemisch E/Z1:6.5): $\delta[\mathrm{ppm}]=1.56 \quad(\mathrm{~d}, \quad J=$ $\left.6.8 \mathrm{~Hz}, 3 \mathrm{H}, 5^{\prime}-\mathrm{H}_{3, E}\right), 1.58-1.65\left(\mathrm{~m}, 3 \mathrm{H}, 5^{\prime}-\mathrm{H}_{3, Z}\right), 2.22-2.31$ (m, $\left.4 \mathrm{H}, 2^{\prime}-\mathrm{H}_{2, E+Z}\right), 2.73$ (ddd, $\left.J=9.5,7.2,3.9 \mathrm{~Hz}, 4 \mathrm{H}, 1^{\prime}-\mathrm{H}_{2, E+Z}\right), 3.41\left(\mathrm{~d}, J=0.4 \mathrm{~Hz}, 6 \mathrm{H}, \mathrm{OCH}_{2} \mathrm{OC}_{3}, E+Z\right), 3.77(\mathrm{~s}, 6 \mathrm{H}$, $\left.1-\mathrm{OCH}_{3, Z}, 3-\mathrm{OCH}_{3, Z}\right), 3.78\left(\mathrm{~s}, 6 \mathrm{H}, 1-\mathrm{OCH}_{3, E}, 3-\mathrm{OCH}_{3, E}\right), 4.09-4.11\left(\mathrm{~m}, 4 \mathrm{H}, 3\right.$ '- $\left.\mathrm{CH}_{2, E+Z}\right)$, 4.48 (s, $2 \mathrm{H}, \mathrm{OCH}_{2} \mathrm{Ph}_{E}$ ), 4.48-4.49 (m, $\left.2 \mathrm{H}, \mathrm{OCH}_{2} \mathrm{Ph}_{z}\right), 4.54$ (s. $4 \mathrm{H}, \mathrm{OC}_{2} \mathrm{OCH}_{3, E+Z)}, 4.70$ (s, $\left.4 \mathrm{H}, 5-\mathrm{H}_{2, E+Z}\right), 5.46$ (q, J = 6.8 Hz, $\left.2 \mathrm{H}, 4^{\prime}-\mathrm{H}_{E+Z}\right), 6.51$ (s, $\left.4 \mathrm{H}, 4-\mathrm{H}_{E+Z}, 6-\mathrm{H}_{E+Z}\right), 7.21-7.39$ $\left(\mathrm{m}, 10 \mathrm{H}, 5 \times \mathrm{Ph}-\mathrm{H}_{E+Z}\right)$.

${ }^{13} \mathrm{C}-\mathrm{NMR}\left(126 \mathrm{MHz}, \mathrm{CDCl}_{3},(Z)-255\right): \delta[\mathrm{ppm}]=13.3$ (C-5'), 22.0 (C-1'), 34.6 (C-2'), 55.4 $\left(\mathrm{OCH}_{2} \mathrm{O}_{\mathrm{CH}} \mathrm{H}_{3}\right), 55.6\left(1-\mathrm{OCH}_{3}, 3-\mathrm{OCH}_{3}\right), 67.0\left(\mathrm{OCH}_{2} \mathrm{Ph}\right), 69.6\left(5-\mathrm{CH}_{2}\right), 71.9\left(3^{\prime}-\mathrm{CH}_{2}\right), 95.6$ $\left(\mathrm{O}_{\mathrm{CH}} \mathrm{OCH}_{3}\right), 103.3$ (C-4, C-6), 118.3 (C-2), 123.4 (C-4'), $127.3\left(\mathrm{Ph}-\mathrm{C}_{p}\right), 127.7,128.2$ (2 × $\left.\mathrm{Ph}-\mathrm{C}_{o}, 2 \times \mathrm{Ph}^{-\mathrm{C}_{m}}\right), 136.5$ (C-3'), 137.1 (C-5), $138.9\left(\mathrm{Ph}-\mathrm{C}_{i}\right), 158.2$ (C-1, C-3). 
MS (ESI): $m / z(\%)=423.2(51)[\mathrm{M}+\mathrm{Na}]^{+}, 439.2(100)[\mathrm{M}+\mathrm{K}]^{+}$.

$\mathrm{C}_{24} \mathrm{H}_{32} \mathrm{O}_{5}$ (400.52)

ber.: 423.2142

439.1881

gef.: $423.2144[\mathrm{M}+\mathrm{Na}]^{+}$

$439.1889[\mathrm{M}+\mathrm{K}]^{+}(\mathrm{ESI}-\mathrm{HRMS})$. 


\subsubsection{1 (E/Z)-2-(3-((Benzyloxy)methyl)pent-3-en-1-yl)-3-methoxy-5- ((methoxymethoxy)methyl)phenol ((E/Z)-256)}

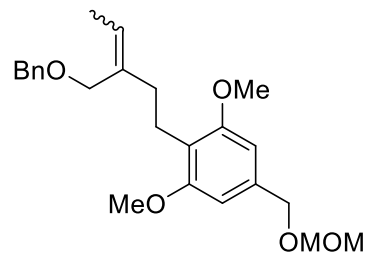

$(E / Z)-\mathbf{2 5 5}$

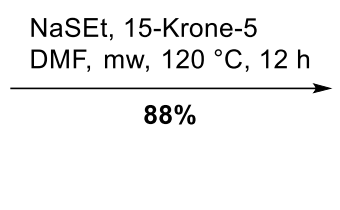

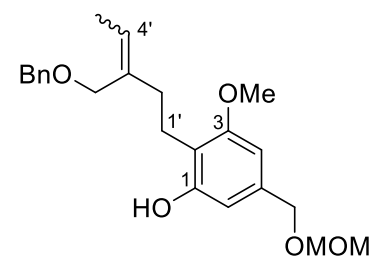

$(E / Z)-256$

Eine Lösung des Alkens (E/Z)-255 (1.50 g, 3.75 mmol, 1.00 Äq.), NaSEt (90\%ig, $1.05 \mathrm{~g}$, $11.2 \mathrm{mmol}, 3.00$ Äq.) sowie $15-\mathrm{Krone}-5$ (1.48 ml, $1.65 \mathrm{~g}, 7.49 \mathrm{mmol}, 2.00$ Äq.) in DMF $(22.5 \mathrm{ml})$ wurde $12 \mathrm{~h}$ bei $120^{\circ} \mathrm{C}$ in der Mikrowelle gerührt. Nach Abkühlen auf Raumtemperatur wurde $\mathrm{H}_{2} \mathrm{O}(40 \mathrm{ml})$ zugegeben und mit EtOAc $(3 \times 30 \mathrm{ml})$ extrahiert. Die vereinigten org. Phasen wurden mit $\mathrm{H}_{2} \mathrm{O}(50 \mathrm{ml})$ sowie ges. NaCl-Lsg. $(2 \times 50 \mathrm{ml})$ gewaschen, über $\mathrm{MgSO}_{4}$ getrocknet und das Lösungsmittel unter vermindertem Druck entfernt. Säulenchromatographische Reinigung an Kieselgel (Petrolether/EtOAc 3:1) lieferte das Phenol (E/Z)-256 (1.27 g, 3.29 mmol, 88\%) in Form eines farblosen Öls.

UV $\left(\mathrm{CH}_{3} \mathrm{CN}\right): \lambda_{\max }[\mathrm{nm}](\lg \varepsilon)=206$ (4.728), 233 (3.935).

IR (ATR): $\widetilde{v}\left[\mathrm{~cm}^{-1}\right]=3336,3088,3062,3030,2934,2881,2860,1737,1710,1692,1665$, 1640, 1614, 1595, 1551, 1514, 1497, 1452, 1426, 1378, 1366, 1318, 1267, 1243, 1211, 1193, 1164, 1147, 1098, 1040, 992, 920, 823, 737, 697, 652, 605, 564, 533.

${ }^{1} \mathrm{H}-\mathrm{NMR}\left(500 \mathrm{MHz}, \mathrm{CDCl}_{3}\right.$, Diastereomerengemisch): $\delta[\mathrm{ppm}]=1.17(\mathrm{~d}, J=6.8 \mathrm{~Hz}, 3 \mathrm{H}$, $\left.5^{\prime}-\mathrm{H}_{3, E}\right), 1.48$ (dt, J =6.9, 0.7 Hz, 3 H, 5'- $\left.\mathrm{H}_{3, z}\right), 2.25$ (tt, $\left.J=6.8,1.0 \mathrm{~Hz}, 2 \mathrm{H}, 2^{\prime}-\mathrm{H}_{2, z}\right), 2.37$ (t, $\left.J=7.0 \mathrm{~Hz}, 2 \mathrm{H}, 2^{\prime}-\mathrm{H}_{2, E}\right), 2.74\left(\mathrm{t}, J=7.0 \mathrm{~Hz}, 2 \mathrm{H}, 1^{\prime}-\mathrm{H}_{2, z}\right), 2.81$ (t, $J=6.9 \mathrm{~Hz}, 2 \mathrm{H}$, $\left.1^{\prime}-\mathrm{H}_{2, E}\right), 3.39\left(\mathrm{~s}, 3 \mathrm{H}, \mathrm{OCH}_{2} \mathrm{OC}_{3}, E\right), 3.40\left(\mathrm{~s}, 3 \mathrm{H}, \mathrm{OCH}_{2} \mathrm{OC}_{3, z}\right), 3.78\left(\mathrm{~s}, 3 \mathrm{H}, 3-\mathrm{OCH}_{3, Z}\right)$, 3.81 (s, $\left.3 \mathrm{H}, 3-\mathrm{OCH}_{3, E}\right), 3.94-3.96\left(\mathrm{~m}, 2 \mathrm{H}, 3\right.$ '- $\left.\mathrm{CH}_{2, E}\right), 4.07$ (t, J = 0.7 Hz, $2 \mathrm{H}, 3$ '- $-\mathrm{CH}_{2, \mathrm{z}}$ ), $4.48\left(\mathrm{~s}, 2 \mathrm{H}, \mathrm{OC}_{2} \mathrm{OCH}_{3, E}\right), 4.49\left(\mathrm{t}, J=0.6 \mathrm{~Hz}, 2 \mathrm{H}, \mathrm{OC}_{2} \mathrm{OCH}_{3, Z}\right), 4.60(\mathrm{~s}, 2 \mathrm{H}$, $\mathrm{OCH}_{2} \mathrm{Ph}_{E}$ ), 4.62 (s, $2 \mathrm{H}, \mathrm{OCH}_{2} \mathrm{Ph}_{z}$ ), 4.66 (s, $2 \mathrm{H}, 5-\mathrm{CH}_{2, E}$ ), 4.68 (s, $\left.2 \mathrm{H}, 5-\mathrm{CH}_{2, z}\right), 5.18$ (qt, $\left.J=6.9,0.9 \mathrm{~Hz}, 1 \mathrm{H}, 4^{\prime}-\mathrm{H}_{z}\right), 5.48\left(\mathrm{q}, J=6.8 \mathrm{~Hz}, 1 \mathrm{H}, 4^{\prime}-\mathrm{H}_{E}\right), 6.40(\mathrm{~d}, J=1.5 \mathrm{~Hz}, 1 \mathrm{H}$, $\left.4-\mathrm{H}_{E} / 6-\mathrm{H}_{E}\right), 6.43\left(\mathrm{~d}, J=1.4 \mathrm{~Hz}, 1 \mathrm{H}, 4-\mathrm{H}_{Z} / 6-\mathrm{H}_{z}\right), 6.45-6.47\left(\mathrm{~m}, 1 \mathrm{H}, 4-\mathrm{H}_{E} / 6-\mathrm{H}_{E}\right), 6.48$ (dt, $\left.J=1.3,0.6 \mathrm{~Hz}, 1 \mathrm{H}, 4-\mathrm{H}_{z} / 6-\mathrm{H}_{z}\right), 6.92\left(\mathrm{~s}, 1 \mathrm{H}, \mathrm{OH}_{z}\right), 6.94\left(\mathrm{~s}, 1 \mathrm{H}, \mathrm{OH}_{E}\right), 7.27-7.41(\mathrm{~m}$, $\left.10 \mathrm{H}, 5 \times \mathrm{Ph}-\mathrm{H}_{E+Z}\right)$.

${ }^{13} \mathrm{C}-\mathrm{NMR}\left(126 \mathrm{MHz}, \mathrm{CDCl}_{3},(Z)-256\right): \delta[\mathrm{ppm}]=13.3$ (C-5'), 23.0 (C-1'), 36.7 (C-2'), 55.3, 55.6 (3-OCH3, $\left.\quad \mathrm{OCH}_{2} \mathrm{O}_{\mathrm{CH}}\right), \quad 68.7\left(\mathrm{OCH}_{2} \mathrm{Ph}\right), \quad 69.3 \quad\left(5-\mathrm{CH}_{2}\right), \quad 73.1 \quad\left(3^{\prime}-\mathrm{CH}_{2}\right), \quad 95.6$ $\left(\mathrm{O}_{\mathrm{CH}} \mathrm{OCH}_{3}\right), 101.8$ (C-4), 108.9 (C-6), 115.1 (C-2), 126.1 (C-4'), $128.0\left(\mathrm{Ph}-\mathrm{C}_{p}\right), 128.2$, 


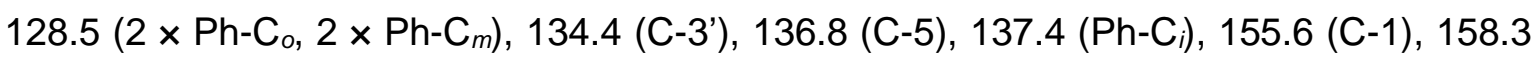
(C-3).

MS (ESI): $m / z(\%)=387.2(5)[\mathrm{M}+\mathrm{H}]^{+}, 409.2(100)[\mathrm{M}+\mathrm{Na}]^{+}, 425.2(86)[\mathrm{M}+\mathrm{K}]^{+} ; 385.2$ (100) $[\mathrm{M}-\mathrm{H}]^{-}, 771.4(28)[2 \times \mathrm{M}-\mathrm{H}]^{-}$.

$\mathrm{C}_{23} \mathrm{H}_{30} \mathrm{O}_{5}(386.49)$

ber.: 385.2020

409.1985

425.1725

gef.: $385.2008[\mathrm{M}-\mathrm{H}]^{-}$

$409.1992[\mathrm{M}+\mathrm{Na}]^{+}$

$425.1730[\mathrm{M}+\mathrm{K}]^{+}$(ESI-HRMS). 


\subsubsection{2 (S)-2-((Benzyloxy)methyl)-5-methoxy-7-((methoxymethoxy)- methyl)-2-vinylchroman ((S)-252)}

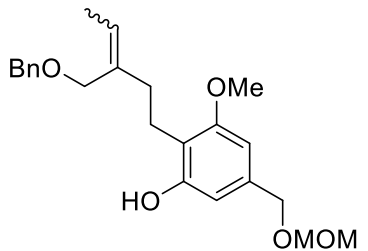

$(E / Z)-\mathbf{2 5 6}$

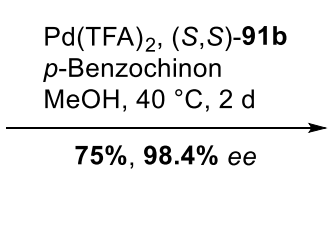

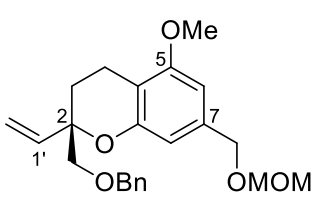

(S)-252

Eine Suspension von $\mathrm{Pd}(\mathrm{TFA})_{2}(10.3 \mathrm{mg}, 310 \mu \mathrm{mol}, 10 \mathrm{~mol} \%)$ sowie des $(S, S)$-iPrBOXAX-Liganden (S,S)-91b (296 mg, $621 \mu \mathrm{mol}, 20 \mathrm{~mol} \%$ ) in MeOH (25 ml) wurde $30 \mathrm{~min}$ bei Raumtemperatur gerührt, anschließend eine Lösung des Phenols (E/Z)-256 (1.20 g, $3.10 \mathrm{mmol}, 1.00$ Äq.) in $\mathrm{MeOH}$ (25 ml) sowie $p$-Benzochinon (1.34 g, $12.4 \mathrm{mmol}, 4.00$ Äq.) bei dieser Temperatur zugegeben, die resultierende Reaktionslösung auf $40^{\circ} \mathrm{C}$ erwärmt und $2 \mathrm{~d}$ bei dieser Temperatur gerührt. Nach Abkühlen auf Raumtemperatur wurde das Reaktionsgemisch in verd. $\mathrm{HCl}(1 \mathrm{M}, 50 \mathrm{ml})$ gegeben, mit MTBE $(3 \times 50 \mathrm{ml})$ extrahiert und die vereinigten org. Phasen mit verd. NaOH-Lsg. $(1 \mathrm{M}, 3 \times 100 \mathrm{ml})$ gewaschen. Nach Trocknen über $\mathrm{MgSO}_{4}$, Entfernen des Lösungsmittels unter vermindertem Druck und Säulenchromatographie an Kieselgel (Petrolether/EtOAc 9:1 $\rightarrow$ 4:1) wurde das Vinylchroman (S)-252 (897 mg, 2.33 mmol, 75\%, 98.4\% ee) als farbloses Öl erhalten.

HPLC (analytisch): Säule: $\quad$ CHIRALPAK ${ }^{\circledR} \mathrm{A}, 4.6 \times 250 \mathrm{~mm}, 5 \mu \mathrm{m}$

Eluent: $\quad n$-Hexan/ $/ \mathrm{PrOH}$ 99:1

Fluß: $\quad 0.8 \mathrm{ml} / \mathrm{min}$

$\lambda$ : $\quad 207 \mathrm{~nm}$

$t_{\mathrm{R}}: \quad 10.5 \min (+)-(R)-252,0.8 \%$

$13.0 \min (-)-(S)-252,99.2 \%, 98.4 \%$ ee.

Drehwert: $[\alpha]_{D}^{21}\left[^{\circ}\right]=-59.6\left(c=0.65, \mathrm{CHCl}_{3}\right)$.

UV $\left(\mathrm{CH}_{3} \mathrm{CN}\right): \lambda_{\max }[\mathrm{nm}](\mathrm{lg} \varepsilon)=208$ (4.724), 229 (4.026), 275 (3.256).

IR (ATR): $\tilde{v}\left[\mathrm{~cm}^{-1}\right]=3088,3062,3029,2986,2926,2884,2858,2789,1717,1698,1685$, $1671,1646,1618,1586,1541,1522,1497,1452,1424,1378,1355,1321,1294,1272$, 1245, 1212, 1196, 1147, 1136, 1100, 1049, 1026, 1001, 958, 925, 824, 739, 698, 679, 609, 590, 571, 553, 526, 512, 504.

${ }^{1} \mathrm{H}-\mathrm{NMR}\left(500 \mathrm{MHz}, \mathrm{CDCl}_{3}\right): \delta[\mathrm{ppm}]=1.90$ (ddd, $\left.J=13.6,6.2,4.0 \mathrm{~Hz}, 1 \mathrm{H}\right), 1.99$ (ddd, $J=$ 13.6, 10.9, $5.6 \mathrm{~Hz}, 1 \mathrm{H}$ ) (3- $\left.\mathrm{H}_{2}\right), 2.40$ (ddd, $J=17.1,10.9,6.2 \mathrm{~Hz}, 1 \mathrm{H}$ ), 2.69 (ddd, $J=17.0$, 5.6, $3.9 \mathrm{~Hz}, 1 \mathrm{H})\left(4-\mathrm{H}_{2}\right), 3.40\left(\mathrm{~s}, 3 \mathrm{H}, \mathrm{OCH}_{2} \mathrm{OC}_{3}\right), 3.51$ (d, $\left.J=10.0 \mathrm{~Hz}, 1 \mathrm{H}\right), 3.56(\mathrm{~d}, J=$ $10.0 \mathrm{~Hz}, 1 \mathrm{H})\left(2-\mathrm{CH}_{2}\right), 3.79\left(\mathrm{~s}, 3 \mathrm{H}, 5-\mathrm{OCH}_{3}\right), 4.50\left(\mathrm{~s}, 2 \mathrm{H}, \mathrm{OCH}_{2} \mathrm{OCH}_{3}\right), 4.58(\mathrm{~d}, J=$ 
$12.2 \mathrm{~Hz}, 1 \mathrm{H}), 4.62(\mathrm{~d}, J=12.2 \mathrm{~Hz}, 1 \mathrm{H})\left(\mathrm{OCH}_{2} \mathrm{Ph}\right), 4.68\left(\mathrm{~s}, 2 \mathrm{H}, 7-\mathrm{CH}_{2}\right), 5.14(\mathrm{dd}, J=$ 10.9, $1.3 \mathrm{~Hz}, 1 \mathrm{H}, 2^{\prime}-\mathrm{H}_{\text {cis }}$ ), 5.23 (dd, $J=17.3,1.3 \mathrm{~Hz}, 1 \mathrm{H}, 2^{\prime}-\mathrm{H}_{\text {trans }}$ ), 5.82 (dd, $J=17.3$, $10.9 \mathrm{~Hz}, 1 \mathrm{H}, 1$ '-H), 6.40 (d, J = 1.4 Hz, $1 \mathrm{H}), 6.56(\mathrm{dd}, J=1.5,0.7 \mathrm{~Hz}, 1 \mathrm{H})(6-\mathrm{H}, 8-\mathrm{H})$, 7.23-7.28 (m, $\left.1 \mathrm{H}, \mathrm{Ph}-\mathrm{H}_{p}\right), 7.31-7.32\left(\mathrm{~m}, 4 \mathrm{H}, 2 \times \mathrm{Ph}-\mathrm{H}_{o}, 2 \times \mathrm{Ph}-\mathrm{H}_{m}\right)$.

${ }^{13} \mathrm{C}-\mathrm{NMR}\left(126 \mathrm{MHz}, \mathrm{CDCl}_{3}\right): \delta[\mathrm{ppm}]=16.2(\mathrm{C}-4), 26.4$ (C-3), 55.3, $55.4 \quad\left(5-\mathrm{OCH}_{3}\right.$, $\left.\mathrm{OCH}_{2} \mathrm{O}_{\mathrm{CH}}\right), 69.2\left(7-\mathrm{CH}_{2}\right), 73.6\left(\mathrm{OCH}_{2} \mathrm{Ph}\right), 75.5\left(2-\mathrm{CH}_{2}\right), 78.8(\mathrm{C}-2), 95.6\left(\mathrm{O}_{2} \mathrm{H}_{2} \mathrm{OCH}_{3}\right)$, 101.2, 109.2, 110.1 (C-4a, C-6, C-8), 116.2 (C-2'), $127.5\left(\mathrm{Ph}_{-} \mathrm{C}_{p}\right), 127.6,128.3$ (2 × Ph-Co,

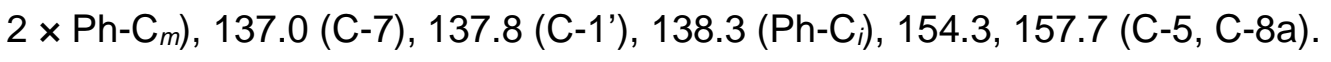

MS (ESI): $m / z(\%)=385.2(9)[\mathrm{M}+\mathrm{H}]^{+}, 407.2(100)[\mathrm{M}+\mathrm{Na}]^{+}, 791.4(11)[2 \times \mathrm{M}+\mathrm{Na}]^{+}$.

$\mathrm{C}_{23} \mathrm{H}_{28} \mathrm{O}_{5}$ (384.47)

ber.: 407.1829

gef.: $407.1830[\mathrm{M}+\mathrm{Na}]^{+}(\mathrm{ESI}-\mathrm{HRMS})$. 


\subsubsection{3 (2'R)-1-(2-((Benzyloxy)methyl)-5-methoxy-7-((methoxymethoxy)- methyl)chroman-2-yl)ethan-1,2-diol (anti/syn-257)}

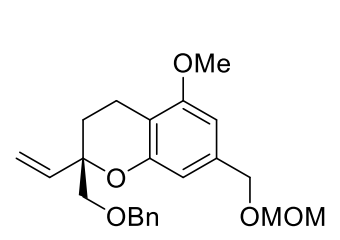

(S)-252

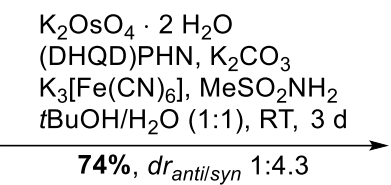

$\mathbf{7 4 \%}, d r_{\text {anti/syn }} 1: 4.3 \longrightarrow$

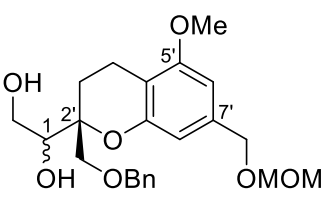

antilsyn-257

$\mathrm{Zu}$ einer Lösung von $\mathrm{K}_{2} \mathrm{OsO}_{4} \cdot 2 \mathrm{H}_{2} \mathrm{O}$ (31.1 mg, $84.5 \mu \mathrm{mol}, 5 \mathrm{~mol} \%$ ), (DHQD)PHN (85.0 mg, $169 \mu \mathrm{mol}, 10 \mathrm{~mol} \%), \mathrm{K}_{3}\left[\mathrm{Fe}(\mathrm{CN})_{6}\right]\left(3.34 \mathrm{~g}, 10.1 \mathrm{mmol}, 6.00 \mathrm{Äq}\right.$.) sowie $\mathrm{K}_{2} \mathrm{CO}_{3}$ (1.40 g, $10.1 \mathrm{mmol}, 6.00 \mathrm{Äq}$.) in $\mathrm{tBuOH} / \mathrm{H}_{2} \mathrm{O}(1: 1,10 \mathrm{ml})$ wurde bei Raumtemperatur eine Lösung des Vinylchromans (S)-252 (650 mg, 1.69 mmol, 1.00 Äq.) sowie $\mathrm{MeSO}_{2} \mathrm{NH}_{2}$ (161 mg, $1.69 \mathrm{mmol}, 1.00$ Äq.) in $\mathrm{tBuOH} / \mathrm{H}_{2} \mathrm{O}(1: 1,10 \mathrm{ml})$ gegeben und $3 \mathrm{~d}$ bei dieser Temperatur gerührt. Anschließend wurde auf $0^{\circ} \mathrm{C}$ gekühlt, ges. $\mathrm{NaHSO}_{3}$-Lsg. (40 ml) zugegeben, auf Raumtemperatur erwärmt und weitere $30 \mathrm{~min}$ bei dieser Temperatur gerührt. Die wässr. Phase wurde mit EtOAc $(3 \times 30 \mathrm{ml})$ extrahiert, die vereinigten org. Phasen über $\mathrm{MgSO}_{4}$ getrocknet und das Lösungsmittel unter vermindertem Druck entfernt. Säulenchromatographie an Kieselgel (Petrolether/EtOAc 1:1 $\rightarrow 1: 3$ ) lieferte das Diol anti/syn-257 (521 mg, 1.24 mmol, 74\%, dr anti/syn 1:4.3) in Form eines farblosen Öls.

HPLC (analytisch): Säule: $\quad$ CHIRALPAK ${ }^{\circledR} \mathrm{IB}, 4.6 \times 250 \mathrm{~mm}, 5 \mu \mathrm{m}$

Eluent: $\quad n$-Hexan/PrOH 90:10

Fluß: $\quad 0.8 \mathrm{ml} / \mathrm{min}$

$\lambda: \quad 210 \mathrm{~nm}$

$t_{\mathrm{R}}: \quad 17.3 \mathrm{~min}$ anti-257, $18.8 \%$

20.5 min syn-257, 81.2\%, dr $r_{\text {anti/syn }} 1: 4.3$.

UV $\left(\mathrm{CH}_{3} \mathrm{CN}\right): \lambda_{\max }[\mathrm{nm}](\lg \varepsilon)=207$ (4.631), 231 (3.850).

IR (ATR): $\tilde{v}\left[\mathrm{~cm}^{-1}\right]=3586,3566,3545,3522,3445,3420,3392,3064,3031,2937,2882$, 1698, 1684, 1653, 1647, 1635, 1618, 1587, 1558, 1541, 1522, 1507, 1497, 1454, 1424, 1378, 1354, 1325, 1298, 1246, 1213, 1196, 1148, 1099, 1046, 1028, 958, 918, 883, 825, 793, 741, 699, 634, 601, 571, 552, 526, 508.

${ }^{1} \mathrm{H}-\mathrm{NMR}\left(300 \mathrm{MHz}, \mathrm{CDCl}_{3}\right.$, Diastereomerengemisch anti/syn 1:4.3): $\delta[\mathrm{ppm}]=1.64 \quad\left(\mathrm{~s}_{\mathrm{br}}\right.$, $2 \mathrm{H}, 2-\mathrm{OH}_{\text {anti }+ \text { syn }}$ ), 1.84 (ddd, $\left.J=13.9,11.0,6.2 \mathrm{~Hz}, 1 \mathrm{H}, 3{ }^{\prime}-\mathrm{H}_{\mathrm{a}, \text { syn }}\right), 1.97-2.04(\mathrm{~m}, 2 \mathrm{H}$, 3'- $\mathrm{H}_{2, \text { anti) }}, 2.14$ (ddd, $J=13.9,6.3,3.7 \mathrm{~Hz}, 1 \mathrm{H}, 3$ '- $\mathrm{H}_{\mathrm{b}, \text { syn }}$ ), 2.42 (ddd, $J=17.5,11.0,6.4 \mathrm{~Hz}$, $2 \mathrm{H}$ ), 2.72 (ddd, $J=17.6,6.3,3.5 \mathrm{~Hz}, 2 \mathrm{H})\left(4^{\prime}-\mathrm{H}_{2, \text { anti+syn }}\right), 2.94$ ( $\left.\mathrm{sbr}_{\mathrm{b}}, 2 \mathrm{H}, 1-\mathrm{OH}_{\text {anti-syn }}\right), 3.40$ (s, $6 \mathrm{H}, \mathrm{OCH}_{2} \mathrm{OC}_{3}$,antitsyn), $3.51\left(\mathrm{~d}, J=9.7 \mathrm{~Hz}, 1 \mathrm{H}, 2{ }^{\prime}-\mathrm{C}_{\mathrm{a}} \mathrm{H}_{\mathrm{b}, \text { syn }}\right), 3.56(\mathrm{~d}, J=10.0 \mathrm{~Hz}$, 
$\left.1 \mathrm{H}, 2^{\prime}-\underline{\mathrm{H}}_{\mathrm{a}} \mathrm{H}_{\mathrm{b}, \text { anti }}\right), 3.63\left(\mathrm{~d}, J=10.0 \mathrm{~Hz}, 1 \mathrm{H}, 2^{\prime}-\mathrm{CH}_{\mathrm{a}} \underline{\mathrm{H}}_{\mathrm{b}, \text { anti }}\right), 3.65$ (d, $J=9.7 \mathrm{~Hz}, 1 \mathrm{H}$,

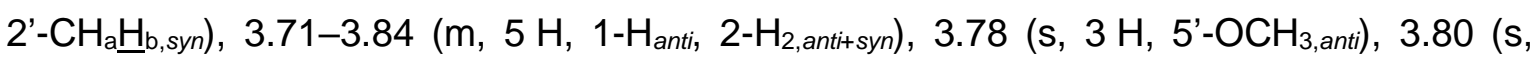
$3 \mathrm{H}, 5$ '- $\left.\mathrm{OCH}_{3, \text { syn }}\right), 3.90\left(\mathrm{t}, \mathrm{J}=5.0 \mathrm{~Hz}, 1 \mathrm{H}, 1-\mathrm{H}_{\text {syn }}\right), 4.42-4.58\left(\mathrm{~m}, 4 \mathrm{H}, \mathrm{OCH}_{2} \mathrm{Ph}_{\text {anti-syn }}\right), 4.47$

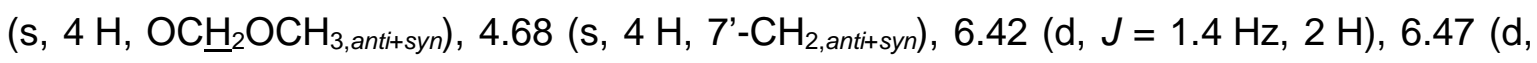
$J=1.4 \mathrm{~Hz}, 2 \mathrm{H})\left(6^{\prime}-\mathrm{H}_{\text {anti+syn }}, 8^{\prime}-\mathrm{H}_{\text {anti+syn }}\right), 7.22-7.36$ (m, $\left.10 \mathrm{H}, 5 \times \mathrm{Ph}-\mathrm{H}_{\text {anti+syn }}\right)$.

${ }^{13} \mathrm{C}-N M R\left(126 \mathrm{MHz}, \mathrm{CDCl}_{3}\right.$, syn-257): $\delta[\mathrm{ppm}]=15.6 \quad\left(\mathrm{C}-4^{\prime}\right), \quad 23.0 \quad$ (C-3), 55.4, 55.5 (5'- $\left.\mathrm{OCH}_{3}, \mathrm{OCH}_{2} \mathrm{OCH}_{3}\right), 62.2$ (C-2), 68.9, $69.1\left(2^{\prime}-\mathrm{CH}_{2}, 7{ }^{\prime}-\mathrm{CH}_{2}\right), 73.9\left(\mathrm{OCH}_{2} \mathrm{Ph}\right), 75.3$ (C-1), 78.3 (C-2'), $95.6\left(\mathrm{O}_{\mathrm{C}} \mathrm{H}_{2} \mathrm{OCH}_{3}\right), 101.7,109.1,109.4$ (C-4a', C-6', C-8'), 127.7, 128.0, $128.5\left(2 \times \mathrm{Ph}_{-} \mathrm{C}_{o}, 2 \times \mathrm{Ph}-\mathrm{C}_{m}, \mathrm{Ph}-\mathrm{C}_{p}\right), 137.2,137.4\left(\mathrm{C}-7^{\prime}, \mathrm{Ph}-\mathrm{C}_{i}\right), 153.2,157.7$ (C-5', C-8a').

MS (ESI): $m / z(\%)=419.2(9)[\mathrm{M}+\mathrm{H}]^{+}, 436.2(9)\left[\mathrm{M}+\mathrm{NH}_{4}\right]^{+}, 441.2(94)[\mathrm{M}+\mathrm{Na}]^{+}, 859.4$ (100) $[2 \times \mathrm{M}+\mathrm{Na}]^{+} ; 417.2(100)[\mathrm{M}-\mathrm{H}]^{-}, 835.4(41)[2 \times \mathrm{M}-\mathrm{H}]^{-}$.

$\mathrm{C}_{23} \mathrm{H}_{30} \mathrm{O}_{7}(418.49)$

ber.: 417.1919

441.1884

gef.: $417.1905[\mathrm{M}-\mathrm{H}]^{-}$

$441.1884[\mathrm{M}+\mathrm{Na}]^{+}(\mathrm{ESI}-\mathrm{HRMS})$. 


\subsubsection{4 (2' R)-1-(2-(Benzyloxy)methyl-5-methoxy-7-((methoxymethoxy)- methyl)chroman-2-yl)-1,2-bis((tert-butyldimethylsilyl)oxy)ethan (anti/syn-263)}

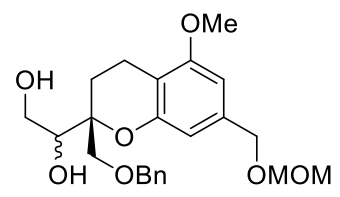

anti/syn-257
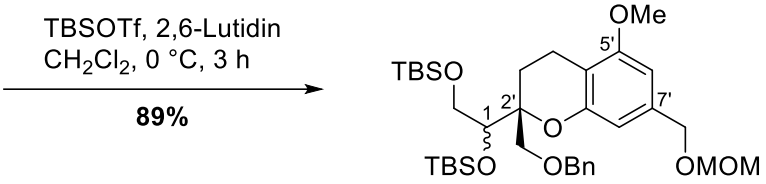

antilsyn-263

Eine Lösung des Diols antilsyn-257 (500 mg, 1.19 mmol, 1.00 Äq.) in $\mathrm{CH}_{2} \mathrm{Cl}_{2}$ (30 ml) wurde bei $0^{\circ} \mathrm{C}$ mit 2,6-Lutidin $(696 \mu \mathrm{l}, 640 \mathrm{mg}, 5.97 \mathrm{mmol}, 5.00$ Äq.) sowie TBSOTf (1.10 ml, $1.26 \mathrm{~g}, 4.78 \mathrm{mmol}, 4.00$ Äq.) versetzt und $3 \mathrm{~h}$ bei dieser Temperatur gerührt. Die Reaktion wurde durch Zugabe von ges. $\mathrm{NaHCO}_{3}$-Lsg. $(50 \mathrm{ml})$ bei $0^{\circ} \mathrm{C}$ beendet, die Phasen getrennt und die wässr. Phase mit EtOAc $(3 \times 25 \mathrm{ml})$ extrahiert. Die vereinigten org. Phasen wurden über $\mathrm{MgSO}_{4}$ getrocknet, das Lösungsmittel unter vermindertem Druck entfernt und nach Säulenchromatographie an Kieselgel (Petrolether/EtOAc 19:1 $\rightarrow$ 9:1) der Silylether anti/syn-263 (691 mg, 1.07 mmol, 89\%) in Form eines farblosen Öls erhalten.

UV $\left(\mathrm{CH}_{3} \mathrm{CN}\right): \lambda_{\max }[\mathrm{nm}](\lg \varepsilon)=208$ (4.755), 230 (4.036), 275 (3.344).

IR (ATR): $\tilde{v}\left[\mathrm{~cm}^{-1}\right]=3064,3032,2951,2927,2883,2854,1727,1709,1692,1640,1619$, 1587, 1549, 1512, 1497, 1462, 1453, 1425, 1378, 1361, 1324, 1298, 1251, 1213, 1196, $1148,1100,1045,1005,963,939,920,829,815,775,735,696,680,664,617,597,569$, $554,540,514$.

${ }^{1} \mathrm{H}-\mathrm{NMR}$ (300 MHz, $\mathrm{CDCl}_{3}$, Diastereomerengemisch): $\delta[\mathrm{ppm}]=-0.03(\mathrm{~d}, J=3.3 \mathrm{~Hz}, 3 \mathrm{H}$ ), $0.03(\mathrm{~d}, J=2.0 \mathrm{~Hz}, 3 \mathrm{H}), 0.06(\mathrm{~d}, J=0.7 \mathrm{~Hz}, 3 \mathrm{H}), 0.09(\mathrm{~d}, J=2.2 \mathrm{~Hz}, 3 \mathrm{H})(2 \times$ $\left.\mathrm{Si}\left(\mathrm{CH}_{3}\right)_{2, \text { anti+syn }}\right), 0.84$ (d, J = $\left.1.3 \mathrm{~Hz}, 18 \mathrm{H}, 2 \times \mathrm{SiC}\left(\mathrm{CH}_{3}\right)_{3, a n t i)}\right), 0.88(\mathrm{~d}, J=3.5 \mathrm{~Hz}, 18 \mathrm{H}, 2 \times$ $\mathrm{SiC}\left(\mathrm{CH}_{3}\right)_{3, \text { syn }}$ ), 1.74 (ddd, $\left.J=13.9,10.1,6.2 \mathrm{~Hz}, 1 \mathrm{H}, 3^{\prime}-\mathrm{H}_{\mathrm{a}, \text { syn }}\right), 1.99$ (tq, $J=13.8,6.8 \mathrm{~Hz}$, $1 \mathrm{H}, 3^{\prime}-\mathrm{H}_{\mathrm{a}, \text { anti }}$ ), 2.12 (ddd, $\left.J=13.8,6.1,4.6 \mathrm{~Hz}, 1 \mathrm{H}, 3^{\prime}-\mathrm{H}_{\mathrm{b}, \text { syn }}\right), 2.38-2.71\left(\mathrm{~m}, 5 \mathrm{H}, 3^{\prime}-\mathrm{H}_{\mathrm{b}, \text { anti, }}\right.$

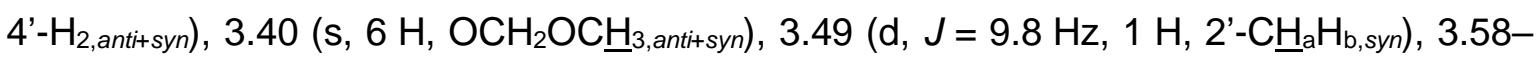

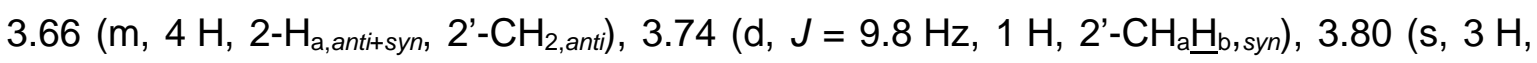
5'- $-\mathrm{OCH}_{3, \text { syn }}$ ), 3.80 (s, $3 \mathrm{H}, 5$ '- $-\mathrm{OCH}_{3, \text { anti }}$ ), 3.90 (dd, $J=10.6,3.2 \mathrm{~Hz}, 2 \mathrm{H}, 1-\mathrm{H}_{\text {anti, }} 2-\mathrm{H}_{\mathrm{b}, \text { syn }}$ ), 3.97 (dd, $J=6.4,3.0 \mathrm{~Hz}, 2 \mathrm{H}, 1-\mathrm{H}_{\text {syn }}, 2-\mathrm{H}_{\mathrm{b}, \text { anti }}$ ), 4.47 (d, $J=0.8 \mathrm{~Hz}, 4 \mathrm{H}, \mathrm{OCH}_{2} \mathrm{Ph}_{\text {anti+syn }}$ ), 4.49 (s, $\left.4 \mathrm{H}, \mathrm{OCH}_{2} \mathrm{OCH}_{3, \text { anti }+ \text { syn }}\right), 4.67$ (s, $2 \mathrm{H}, 7^{\prime}-\mathrm{CH}_{2, \text { anti) }}, 4.68$ (s, $\left.2 \mathrm{H}, 7^{\prime}-\mathrm{CH}_{2, \text { syn }}\right), 6.40$ (d, $J=1.5 \mathrm{~Hz}, 2 \mathrm{H}), 6.45-6.47(\mathrm{~m}, 2 \mathrm{H})\left(6^{\prime}-\mathrm{H}_{\text {anti }}\right.$ syn, 8'- $\left.\mathrm{H}_{\text {anti+syn }}\right), 7.20-7.31(\mathrm{~m}, 10 \mathrm{H}, 5 \times$ $\mathrm{Ph}-\mathrm{H}_{\text {anti+syn) }}$. 
${ }^{13} \mathrm{C}-\mathrm{NMR}\left(126 \mathrm{MHz}, \mathrm{CDCl}_{3}\right.$, syn-263): $\delta[\mathrm{ppm}]=-5.4,-4.9,-4.2\left(2 \times \mathrm{Si}\left(\mathrm{CH}_{3}\right)_{2}\right), \quad 16.0$ (C-4'), 18.3, $18.5\left(2 \times \operatorname{Si} \underline{C}\left(\mathrm{CH}_{3}\right)_{3}\right), 23.2\left(\mathrm{C}-3^{\prime}\right), 26.1,26.1\left(2 \times \operatorname{SiC}\left(\underline{C}_{3}\right)_{3}\right), 55.3,55.4$ (5'- $\left.\mathrm{OCH}_{3}, \mathrm{OCH}_{2} \mathrm{OCH}_{3}\right), 65.3$ (C-2), 69.0, $69.2\left(2^{\prime}-\mathrm{CH}_{2}, 7^{\prime}-\mathrm{CH}_{2}\right), 73.4\left(\mathrm{OCH}_{2} \mathrm{Ph}\right), 76.9$ (C-1), 79.5 (C-2'), $95.5\left(\mathrm{O}_{\mathrm{C}} \mathrm{H}_{2} \mathrm{OCH}_{3}\right), 101.0,109.5,110.0$ (C-4a', C-6', C-8'), 127.3, 128.2 (2 × Ph-C $\left., 2 \times \mathrm{Ph}_{-} \mathrm{C}_{m}, \mathrm{Ph}-\mathrm{C}_{p}\right), 136.9,138.5$ (C-7', Ph- $\left.\mathrm{C}_{i}\right)$, 154.0, 157.7 (C-5', C-8a').

MS (ESI): $m / z(\%)=647.4(50)[\mathrm{M}+\mathrm{H}]^{+}, 664.4(33)\left[\mathrm{M}+\mathrm{NH}_{4}\right]^{+}, 669.4(100)[\mathrm{M}+\mathrm{Na}]^{+}$, 1315.8 (35) $[2 \times \mathrm{M}+\mathrm{Na}]^{+}$.

$\mathrm{C}_{35} \mathrm{H}_{58} \mathrm{O}_{7} \mathrm{Si}_{2}$ (647.01) ber.: 647.3794 669.3613

gef.: $647.3794[\mathrm{M}+\mathrm{H}]^{+}$ $669.3615[\mathrm{M}+\mathrm{Na}]^{+}(\mathrm{ESI}-\mathrm{HRMS})$. 


\subsubsection{5 (2'R)-2-(2-((Benzyloxy)methyl)-5-methoxy-7-((methoxymethoxy)- methyl)chroman-2-yl)-2-((tert-butyldimethylsilyl)oxy)ethan-1-ol (anti/syn-264)}

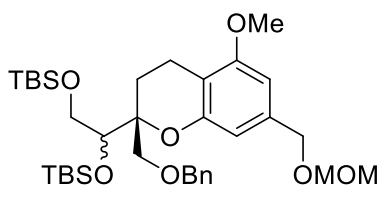

antilsyn-263

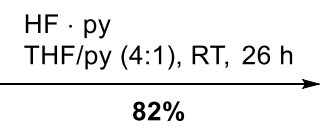

antilsyn-264

Eine Lösung des Silylethers anti/syn-263 (650 mg, 1.00 mmol, 1.00 Äq.) in THF/Pyridin (4:1, $25 \mathrm{ml})$ wurde bei $0{ }^{\circ} \mathrm{C}$ mit $\mathrm{HF} \cdot$ py (70\% HF, $1.04 \mathrm{ml}, 1.15 \mathrm{~g}, 40.2 \mathrm{mmol}, 40.0 \mathrm{Äq}$.) versetzt, auf Raumtemperatur erwärmt und $26 \mathrm{~h}$ bei dieser Temperatur gerührt. Die Reaktion wurde bei $0^{\circ} \mathrm{C}$ durch Zugabe von ges. $\mathrm{NaHCO}_{3}$-Lsg. $(20 \mathrm{ml})$ sowie $\mathrm{H}_{2} \mathrm{O}(40 \mathrm{ml})$ beendet, mit EtOAc $(3 \times 30 \mathrm{ml})$ extrahiert, die vereinigten org. Phasen über $\mathrm{MgSO}_{4}$ getrocknet und das Lösungsmittel unter vermindertem Druck entfernt. Nach säulenchromatographischer Reinigung an Kieselgel (Petrolether/EtOAc 4:1) wurde der Alkohol anti/syn-264 (440 mg, $825 \mu \mathrm{mol}, 82 \%$ ) als farbloses Öl isoliert.

UV $\left(\mathrm{CH}_{3} \mathrm{CN}\right): \lambda_{\max }[\mathrm{nm}](\lg \varepsilon)=208$ (4.741), 231 (3.920).

IR (ATR): $\tilde{v}\left[\mathrm{~cm}^{-1}\right]=3460,3063,3031,2949,2928,2882,2854,1619,1587,1530,1497$, $1461,1453,1424,1378,1359,1322,1297,1249,1213,1196,1147,1101,1046,1029$, 947, 919, 830, 776, 736, 697, 666, 598, 575, 554, 546, 519.

${ }^{1} \mathrm{H}-\mathrm{NMR}\left(300 \mathrm{MHz}, \mathrm{CDCl}_{3}\right.$, Diastereomerengemisch): $\delta[\mathrm{ppm}]=0.04 \quad(\mathrm{~s}, 3 \mathrm{H}), 0.08$ (s, $3 \mathrm{H}), 0.12(\mathrm{~s}, 3 \mathrm{H}), 0.14(\mathrm{~s}, 3 \mathrm{H})\left(\mathrm{Si}\left(\mathrm{CH}_{3}\right)_{2, \text { anti }+ \text { syn }}\right), 0.85\left(\mathrm{~s}, 9 \mathrm{H}, \mathrm{SiC}\left(\mathrm{CH}_{3}\right)_{3, \text { anti }}\right), 0.91(\mathrm{~s}, 9 \mathrm{H}$, $\mathrm{SiC}\left(\mathrm{CH}_{3}\right)_{3, \text { syn }}$ ), 1.78 (ddd, $J=13.8,10.5,6.2 \mathrm{~Hz}, 1 \mathrm{H}, 3^{\prime}-\mathrm{H}_{\mathrm{a}, \text { syn }}$ ), 1.98 (dd, $J=8.4,5.4 \mathrm{~Hz}$, $2 \mathrm{H}, 3^{\prime}-\mathrm{H}_{2, \text { anti }}$ ), 2.09 (ddd, $J=13.9,6.3,4.2 \mathrm{~Hz}, 1 \mathrm{H}, 3$ '- $\left.\mathrm{H}_{\mathrm{b}, \text { syn }}\right), 2.38-2.58$ (m, $\left.2 \mathrm{H}\right), 2.68$ (ddd, $J=17.5,6.2,4.1 \mathrm{~Hz}, 2 \mathrm{H})\left(4^{\prime}-\mathrm{H}_{2, \text { anti-syn) }}, 3.40\right.$ (d, $J=0.3 \mathrm{~Hz}, 6 \mathrm{H}, \mathrm{OCH}_{2} \mathrm{OC}_{3}$,anti+syn), 3.41 (d, J = 9.7 Hz, $\left.1 \mathrm{H}, 2^{\prime}-\mathrm{C}_{\mathrm{H}} \mathrm{H}_{\mathrm{b}, \text { syn }}\right), 3.53$ (d, J=10.7 Hz, $1 \mathrm{H}, 2^{\prime}-\underline{\mathrm{C}}_{\mathrm{a}} \mathrm{H}_{\mathrm{b}, \text { anti }}$ ), 3.58 (d, J = $10.6 \mathrm{~Hz}, 1 \mathrm{H}, 2$ '- $\left.\mathrm{CH}_{a} \underline{\mathrm{H}}_{\mathrm{b}, a n t i}\right), 3.63-3.81\left(\mathrm{~m}, 4 \mathrm{H}, 1-\mathrm{H}_{2, \text { anti syn }}\right), 3.77(\mathrm{~d}, J=9.7 \mathrm{~Hz}, 1 \mathrm{H}$, 2'- $\left.\mathrm{CH}_{\mathrm{a}} \underline{\mathrm{H}}_{\mathrm{b}, \text { syn }}\right), 3.80\left(\mathrm{~d}, \mathrm{~J}=1.2 \mathrm{~Hz}, 6 \mathrm{H}, 5\right.$ '- $\left.\mathrm{OCH}_{3, \text { anti-syn }}\right), 3.96-4.02\left(\mathrm{~m}, 2 \mathrm{H}, 2-\mathrm{H}_{\text {anti-syn }}\right), 4.48$ (d, $\left.J=2.3 \mathrm{~Hz}, 4 \mathrm{H}, \mathrm{OCH}_{2} \mathrm{OCH}_{3, \text { anti+syn }}\right), 4.68$ (s, $\left.4 \mathrm{H}, 7^{\prime}-\mathrm{CH}_{2, \text { anti syn }}\right), 6.41$ (d, J = $1.4 \mathrm{~Hz}$, $2 \mathrm{H}), 6.43-6.45(\mathrm{~m}, 1 \mathrm{H}), 6.46(\mathrm{~d}, J=1.4 \mathrm{~Hz}, 1 \mathrm{H})\left(6^{\prime}-\mathrm{H}_{\text {anti }+ \text { syn, }}\right.$ 8'- $\left._{\text {anti }+ \text { syn }}\right), 7.22-7.33$ (m, $\left.10 \mathrm{H}, 5 \times \mathrm{Ph}-\mathrm{H}_{\text {antitsyn }}\right)$.

${ }^{13} \mathrm{C}-\mathrm{NMR}\left(126 \mathrm{MHz}, \mathrm{CDCl}_{3}\right.$, syn-264): $\delta[\mathrm{ppm}]=-4.7,-4.5\left(\mathrm{Si}\left(\mathrm{CH}_{3}\right)_{2}\right), 15.8\left(\mathrm{C}-4{ }^{\prime}\right), 18.3$ $\left(\mathrm{Si} \underline{\mathrm{C}}\left(\mathrm{CH}_{3}\right)_{3}\right), 23.2\left(\mathrm{C}-3^{\prime}\right), 26.0\left(\mathrm{SiC}\left(\underline{\mathrm{C}} \mathrm{H}_{3}\right)_{3}\right), 55.3,55.4\left(5^{\prime}-\mathrm{OCH}_{3}, \mathrm{OCH}_{2} \mathrm{O}_{\underline{C}} H_{3}\right), 63.1$ (C-1), $67.9\left(2^{\prime}-\mathrm{CH}_{2}\right), 69.2\left(7^{\prime}-\mathrm{CH}_{2}\right), 73.6\left(\mathrm{OCH}_{2} \mathrm{Ph}\right), 75.8(\mathrm{C}-2), 79.7\left(\mathrm{C}-2\right.$ '), $95.6\left(\mathrm{O}^{\mathrm{C}} \mathrm{H}_{2} \mathrm{OCH}_{3}\right)$, 
101.3, 109.2, 109.7 (C-4a', C-6', C-8'), 127.5, 127.6, 128.3 (2 × Ph-C $\left., 2 \times \mathrm{Ph}^{\prime} \mathrm{C}_{m}, \mathrm{Ph}-\mathrm{C}_{p}\right)$, 137.2, 137.7 (C-7', Ph- $\left.C_{i}\right), 153.5,157.7$ (C-5', C-8a').

MS (ESI): $m / z(\%)=533.3(58)[\mathrm{M}+\mathrm{H}]^{+}, 550.3(18)\left[\mathrm{M}+\mathrm{NH}_{4}\right]^{+}, 555.3(100)[\mathrm{M}+\mathrm{Na}]^{+}$, $1087.6(80)[2 \times \mathrm{M}+\mathrm{Na}]^{+} ; 531.3(100)[\mathrm{M}-\mathrm{H}]^{-}$.

$\mathrm{C}_{29} \mathrm{H}_{44} \mathrm{O}_{7} \mathrm{Si}(532.75)$

ber.: 531.2784

533.2929

555.2749

gef.: $531.2770[\mathrm{M}-\mathrm{H}]^{-}$

$533.2925[\mathrm{M}+\mathrm{H}]^{+}$

$555.2754[\mathrm{M}+\mathrm{Na}]^{+}(\mathrm{ESI}-\mathrm{HRMS})$. 


\subsubsection{6 (2R,2'R)-2-(2-(Benzyloxy)methyl)-5-methoxy-7-((methoxymethoxy)- methyl)chroman-2-yl)-2-((tert-butyldimethylsilyl)oxy)acetaldehyd (syn-265)}

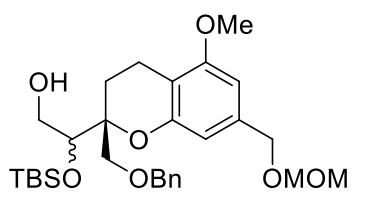

anti/syn-264

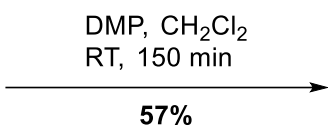

DMP (276) (637 mg, $1.50 \mathrm{mmol}, 2.00$ Äq.) wurde bei $0{ }^{\circ} \mathrm{C}$ zu einer Lösung des Alkohols antilsyn-265 (400 mg, $751 \mu \mathrm{mol}, 1.00 \mathrm{Aq}$.) in $\mathrm{CH}_{2} \mathrm{Cl}_{2}(20 \mathrm{ml})$ gegeben, die resultierende Reaktionslösung auf Raumtemperatur erwärmt und $2 \mathrm{~h}$ bei dieser Temperatur gerührt. Daraufhin wurde weiteres DMP (276) (318 mg, $751 \mu \mathrm{mol}, 1.00$ Äq.) zum Reaktionsgemisch gegeben und zusätzliche $30 \mathrm{~min}$ bei Raumtemperatur gerührt. Die Reaktion wurde bei $0^{\circ} \mathrm{C}$ durch Zugabe von ges. $\mathrm{NaHCO}_{3}$-Lsg. $(25 \mathrm{ml})$ beendet, die Phasen getrennt und die wässr. Phase mit $\mathrm{CH}_{2} \mathrm{Cl}_{2}(3 \times 20 \mathrm{ml})$ extrahiert. Die vereinigten org. Phasen wurden über $\mathrm{MgSO}_{4}$ getrocknet und das Lösungsmittel unter vermindertem Druck entfernt. Trennung der beiden Diastereomere via Säulenchromatographie an Kieselgel (Petrolether/EtOAc 9:1) lieferte die Aldehyde syn-265 (228 mg, $429 \mu \mathrm{mol}, 57 \%$ ) und anti-265 (46.5 mg, $87.6 \mu \mathrm{mol}, 12 \%)$ in Form leicht gelber Öle.

Analytische Daten für syn-265:

Drehwert: $[\alpha]_{D}^{23}\left[^{\circ}\right]=-12.5\left(c=0.28, \mathrm{CHCl}_{3}\right)$.

UV $\left(\mathrm{CH}_{3} \mathrm{CN}\right): \lambda_{\max }[\mathrm{nm}](\lg \varepsilon)=207$ (4.713), 230 (4.017), 268 (3.172).

IR (ATR): $\widetilde{v}\left[\mathrm{~cm}^{-1}\right]=3447,3088,3064,3031,2950,2930,2883,2856,1792,1732,1698$, $1647,1619,1588,1559,1541,1498,1454,1424,1378,1359,1328,1298,1252,1212$, 1195, 1147, 1102, 1047, 1019, 1006, 960, 937, 917, 863, 837, 780, 738, 698, 674, 599, $572,550,538,518,508,502$.

${ }^{1} \mathrm{H}-\mathrm{NMR}\left(300 \mathrm{MHz}, \mathrm{CDCl}_{3}\right): \delta[\mathrm{ppm}]=0.01(\mathrm{~s}, 3 \mathrm{H}), 0.04(\mathrm{~s}, 3 \mathrm{H})\left(\mathrm{Si}\left(\mathrm{CH}_{3}\right)_{2}\right), 0.92(\mathrm{~s}, 9 \mathrm{H}$, $\left.\mathrm{SiC}\left(\mathrm{CH}_{3}\right)_{3}\right), 1.96-2.05\left(\mathrm{~m}, 2 \mathrm{H}, 3^{\prime}-\mathrm{H}_{2}\right), 2.43-2.56(\mathrm{~m}, 1 \mathrm{H}), 2.73(\mathrm{dt}, J=17.6,6.2 \mathrm{~Hz}, 1 \mathrm{H})$ (4'- $\mathrm{H}_{2}$ ), 3.38 (d, J=9.0 Hz, $\left.1 \mathrm{H}, 2^{\prime}-\underline{\mathrm{H}}_{\mathrm{a}} \mathrm{H}_{\mathrm{b}}\right), 3.40$ (s, $\left.3 \mathrm{H}, \mathrm{OCH}_{2} \mathrm{OC}_{3}\right), 3.80$ (d, J = $9.0 \mathrm{~Hz}$, $\left.1 \mathrm{H}, 2^{\prime}-\mathrm{CH}_{\mathrm{a}} \underline{\mathrm{H}}_{\mathrm{b}}\right), 3.80\left(\mathrm{~s}, 3 \mathrm{H}, 5^{\prime}-\mathrm{OCH}_{3}\right), 4.15(\mathrm{~d}, J=1.8 \mathrm{~Hz}, 1 \mathrm{H}, 2-\mathrm{H}), 4.49(\mathrm{~s}, 2 \mathrm{H}$, $\mathrm{OCH}_{2} \mathrm{OCH}_{3}$ ), 4.51 (s, $\left.2 \mathrm{H}, \mathrm{OCH}_{2} \mathrm{Ph}\right), 4.67\left(\mathrm{~s}, 2 \mathrm{H}, 7^{\prime}-\mathrm{CH}_{2}\right), 6.42(\mathrm{~d}, J=1.5 \mathrm{~Hz}, 1 \mathrm{H}), 6.43$ (d, $J=1.4 \mathrm{~Hz}, 1 \mathrm{H})\left(6^{\prime}-\mathrm{H}, 8^{\prime}-\mathrm{H}\right), 7.21-7.35(\mathrm{~m}, 5 \mathrm{H}, 5 \times \mathrm{Ph}-\mathrm{H}), 9.58(\mathrm{~d}, J=1.9 \mathrm{~Hz}, 1 \mathrm{H}$, $\mathrm{CHO})$.

${ }^{13} \mathrm{C}-\mathrm{NMR}\left(126 \mathrm{MHz}, \mathrm{CDCl}_{3}\right): \delta[\mathrm{ppm}]=-4.9\left(\mathrm{Si}\left(\mathrm{CH}_{3}\right)_{2}\right), 15.7\left(\mathrm{C}-4^{\prime}\right), 18.2\left(\mathrm{SiC}\left(\mathrm{CH}_{3}\right)_{3}\right), 23.6$ (C-3'), $25.7\left(\mathrm{SiC}\left(\underline{\mathrm{CH}}_{3}\right)_{3}\right), 55.3\left(\mathrm{OCH}_{2} \mathrm{O}_{\mathrm{C}} \mathrm{H}_{3}\right), 55.4\left(5^{\prime}-\mathrm{OCH}_{3}\right), 68.4\left(2^{\prime}-\mathrm{CH}_{2}\right), 69.1\left(7^{\prime}-\mathrm{CH}_{2}\right)$, 
$73.6\left(\mathrm{OCH}_{2} \mathrm{Ph}\right), 79.4$ (C-2), 79.7 (C-2'), $95.9\left(\mathrm{O}_{\underline{C}} \mathrm{H}_{2} \mathrm{OCH}_{3}\right), 101.5,109.2,109.8$ (C-4a', C-6', C-8'), 127.4, 127.6, 128.3 (2 × $\left.\mathrm{Ph}^{\prime} \mathrm{C}_{o}, 2 \times \mathrm{Ph}-\mathrm{C}_{m}, \mathrm{Ph}-\mathrm{C}_{p}\right), 137.2,137.7$ (C-7', Ph- $\left.\mathrm{C}_{i}\right)$, 153.2, 157.8 (C-5', C-8a'), 201.9 (CHO).

MS (ESI): $m / z(\%)=531.3(64)[\mathrm{M}+\mathrm{H}]^{+}, 553.3(86)[\mathrm{M}+\mathrm{Na}]^{+} ; 529.3(100)[\mathrm{M}-\mathrm{H}]^{-}$.

$\mathrm{C}_{29} \mathrm{H}_{42} \mathrm{O}_{7} \mathrm{Si}(530.73)$

ber.: 529.2627

531.2773

553.2592

gef.: $529.2611[\mathrm{M}-\mathrm{H}]^{-}$

$531.2764[\mathrm{M}+\mathrm{H}]^{+}$

$553.2590[\mathrm{M}+\mathrm{Na}]^{+}(\mathrm{ESI}-\mathrm{HRMS})$. 


\subsubsection{7 (4S,2'R)-(E)-4-(2-(Benzyloxy)methyl)-5-methoxy-7-((methoxy- methoxy)methyl)chroman-2-yl)-4-((tert-butyldimethylsilyl)oxy)but- 2-ensäuremethylester (syn-266)}

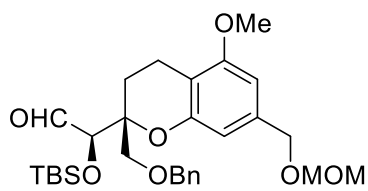

syn-265

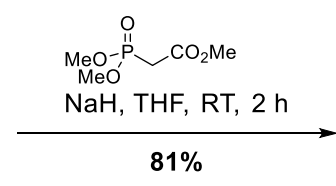

syn-266

Eine Lösung von Trimethylphosphonoacetat $(111 \mu \mathrm{l}, 125 \mathrm{mg}, 685 \mu \mathrm{mol}, 1.73$ Äq.) in THF $(5 \mathrm{ml})$ wurde bei $0^{\circ} \mathrm{C}$ mit NaH (60\%ig in Mineralöl, $20.6 \mathrm{mg}, 514 \mu \mathrm{mol}, 1.30$ Äq.) versetzt und 30 min bei dieser Temperatur gerührt. Anschließend wurde ebenfalls bei $0{ }^{\circ} \mathrm{C}$ eine Lösung des Aldehyds syn-265 (210 mg, 396 umol, 1.00 Äq.) in THF (5 ml) tropfenweise hinzugegeben, die resultierende Reaktionslösung auf Raumtemperatur erwärmt und $2 \mathrm{~h}$ bei dieser Temperatur gerührt. Die Reaktion wurde bei $0^{\circ} \mathrm{C}$ durch Zugabe von ges. $\mathrm{NH}_{4} \mathrm{Cl}$-Lsg. $(15 \mathrm{ml})$ beendet, mit EtOAc $(3 \times 15 \mathrm{ml})$ extrahiert, die vereinigten org. Phasen über $\mathrm{MgSO}_{4}$ getrocknet und das Lösungsmittel unter vermindertem Druck entfernt. Säulenchromatographie an Kieselgel (Petrolether/EtOAc 9:1 $\rightarrow$ 4:1) lieferte den Methylester syn-266 (188 mg, $321 \mu \mathrm{mol}, 81 \%$ ) als farbloses Öl.

UV $\left(\mathrm{CH}_{3} \mathrm{CN}\right): \lambda_{\max }[\mathrm{nm}](\lg \varepsilon)=208(4.761)$.

IR (ATR): $\tilde{v}\left[\mathrm{~cm}^{-1}\right]=3064,3030,2951,2929,2884,2856,1724,1659,1619,1587,1552$, 1532, 1497, 1462, 1453, 1435, 1425, 1378, 1360, 1273, 1259, 1213, 1193, 1166, 1148, 1101, 1047, 1007, 988, 940, 919, 836, 778, 736, 698, 672, 603, 555, 544, 523, 513.

${ }^{1} \mathrm{H}-\mathrm{NMR}\left(500 \mathrm{MHz}, \mathrm{CDCl}_{3}\right): \delta[\mathrm{ppm}]=-0.02(\mathrm{~s}, 3 \mathrm{H}), 0.05(\mathrm{~d}, J=1.5 \mathrm{~Hz}, 3 \mathrm{H})\left(\mathrm{Si}\left(\mathrm{CH}_{3}\right)_{2}\right)$, $0.90\left(\mathrm{~s}, 9 \mathrm{H}, \mathrm{SiC}\left(\mathrm{CH}_{3}\right)_{3}\right), 1.72$ (ddd, $\left.J=13.9,10.5,6.3 \mathrm{~Hz}, 1 \mathrm{H}\right), 1.99-2.06(\mathrm{~m}, 1 \mathrm{H})$ $\left(3^{\prime}-\mathrm{H}_{2}\right.$ ), 2.42 (ddd, $J=17.3,10.5,6.5 \mathrm{~Hz}, 1 \mathrm{H}$ ), 2.64 (ddd, $J=17.4,6.3,4.3 \mathrm{~Hz}, 1 \mathrm{H}$ ) (4'- $\left.\mathrm{H}_{2}\right), 3.40$ (s, $\left.3 \mathrm{H}, \mathrm{OCH}_{2} \mathrm{OCH}_{3}\right), 3.52(\mathrm{~d}, J=10.2 \mathrm{~Hz}, 1 \mathrm{H}), 3.60(\mathrm{~d}, J=10.2 \mathrm{~Hz}, 1 \mathrm{H})$ (2'- $\mathrm{CH}_{2}$ ), 3.69 (d, $J=0.6 \mathrm{~Hz}, 3 \mathrm{H}, \mathrm{CO}_{2} \mathrm{CH}_{3}$ ), 3.79 (s, $3 \mathrm{H}, 5$ ' $\left.-\mathrm{OCH}_{3}\right), 4.43(\mathrm{~d}, J=12.1 \mathrm{~Hz}$, $1 \mathrm{H}), 4.47(\mathrm{~d}, J=12.2 \mathrm{~Hz}, 1 \mathrm{H})\left(\mathrm{OCH}_{2} \mathrm{Ph}\right), 4.49\left(\mathrm{~s}, 2 \mathrm{H}, \mathrm{OCH}_{2} \mathrm{OCH}_{3}\right), 4.62(\mathrm{dd}, J=5.5$, $1.6 \mathrm{~Hz}, 1 \mathrm{H}, 4-\mathrm{H}), 4.68$ (s, $2 \mathrm{H}, 7$ '- $\mathrm{CH}_{2}$ ), 6.02 (dd, J = 15.7, $\left.1.6 \mathrm{~Hz}, 1 \mathrm{H}, 2-\mathrm{H}\right), 6.40$ (d, J = $1.5 \mathrm{~Hz}, 1 \mathrm{H}), 6.48$ (dd, $J=1.5,0.7 \mathrm{~Hz}, 1 \mathrm{H})\left(6^{\prime}-\mathrm{H}, 8\right.$ '-H), 7.09 (dd, $J=15.7,5.5 \mathrm{~Hz}, 1 \mathrm{H}$, 3-H), 7.19-7.28 (m, $5 \mathrm{H}, 5 \times \mathrm{Ph}-\mathrm{H})$.

$\left.{ }^{13} \mathrm{C}-\mathrm{NMR}(126 \mathrm{MHz}, \mathrm{CDCl})_{3}\right): \delta[\mathrm{ppm}]=-5.0,-4.4\left(\mathrm{Si}\left(\mathrm{CH}_{3}\right)_{2}\right), 15.8(\mathrm{C}-4), 18.2\left(\mathrm{SiC}\left(\mathrm{CH}_{3}\right)_{3}\right)$, 22.6 (C-3'), $25.9\left(\mathrm{SiC}\left(\underline{\mathrm{C}} \mathrm{H}_{3}\right)_{3}\right), 51.5\left(\mathrm{CO}_{2} \underline{\mathrm{C}} \mathrm{H}_{3}\right), 55.4,55.4\left(5^{\prime}-\mathrm{OCH}_{3}, \mathrm{OCH}_{2} \mathrm{O}^{-} \mathrm{H}_{3}\right), 68.6$ (2'- $\left.\mathrm{CH}_{2}\right), 69.2\left(7^{\prime}-\mathrm{CH}_{2}\right), 73.4,73.7\left(\mathrm{C}-4, \mathrm{OCH}_{2} \mathrm{Ph}\right), 80.0$ (C-2'), $95.6\left(\mathrm{O}^{\mathrm{C}} \mathrm{H}_{2} \mathrm{OCH}_{3}\right), 101.3$, 109.5, 109.7 (C-4a', C-6', C-8'), 121.8 (C-2), 127.3, 127.4, 128.2 (2 × Ph-C, $2 \times \mathrm{Ph}_{0} \mathrm{C}_{m}$, 
Ph-C $\left.{ }_{p}\right)$, 137.2, 138.2 (C-7', Ph-C 147.6 (C-3), 153.7, 157.7 (C-5', C-8a'), 166.7 $\left(\underline{\mathrm{CO}_{2}} \mathrm{CH}_{3}\right)$.

MS (ESI): $m / z(\%)=587.3(12)[\mathrm{M}+\mathrm{H}]^{+}, 604.3(42)\left[\mathrm{M}+\mathrm{NH}_{4}\right]^{+}, 609.3(100)[\mathrm{M}+\mathrm{Na}]^{+}$, $1195.6(43)[2 \times \mathrm{M}+\mathrm{Na}]^{+} ; 585.3(36)[\mathrm{M}-\mathrm{H}]^{-}$.

$\mathrm{C}_{32} \mathrm{H}_{46} \mathrm{O}_{8} \mathrm{Si}(586.80)$

ber.: 604.3300

609.2854

gef.: $604.3293\left[\mathrm{M}+\mathrm{NH}_{4}\right]^{+}$

$609.2850[\mathrm{M}+\mathrm{Na}]^{+}(\mathrm{ESI}-\mathrm{HRMS})$. 


\subsubsection{1-Benzyloxy-4-(3-iod-2,6-dimethoxy-4-((methoxymethoxy)- methyl)phenyl)butan-2-on (269)}

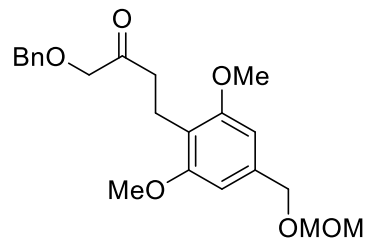

244a
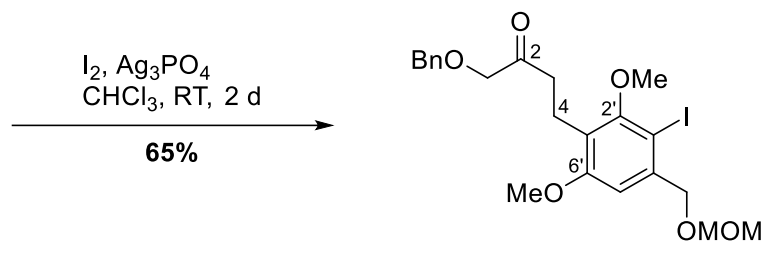

269

Zu einer Lösung des Ketons 244a (500 mg, 1.29 mmol, 1.00 Äq.) in $\mathrm{CHCl}_{3}$ (10 ml) wurde bei Raumtemperatur eine Lösung von $\mathrm{I}_{2}$ (327 mg, $1.29 \mathrm{mmol}, 1.00 \mathrm{Äq}$.) in $\mathrm{CHCl}_{3}$ (10 ml) gegeben und nach erfolgter Zugabe die Reaktionslösung mit $\mathrm{Ag}_{3} \mathrm{PO}_{4}$ (198 mg, $472 \mu \mathrm{mol}$, 0.37 Äq.) versetzt. Das Reaktionsgemisch wurde $2 \mathrm{~d}$ bei dieser Temperatur gerührt. Daraufhin wurde der Feststoff abfiltriert, mit $\mathrm{CH}_{2} \mathrm{Cl}_{2}(10 \mathrm{ml})$ nachgewaschen, das Filtrat mit ges. $\mathrm{Na}_{2} \mathrm{~S}_{2} \mathrm{O}_{3}$-Lsg. $(3 \times 10 \mathrm{ml})$ sowie ges. $\mathrm{NaCl}$-Lsg $(10 \mathrm{ml})$ gewaschen, die org. Phase über $\mathrm{MgSO}_{4}$ getrocknet und das Lösungsmittel unter vermindertem Druck entfernt. Säulenchromatographie an Kieselgel (Petrolether/EtOAc 4:1) lieferte das Aryliodid 269 (429 mg, $835 \mu \mathrm{mol}, 65 \%$ ) in Form eines leicht gelben Öls.

UV $\left(\mathrm{CH}_{3} \mathrm{CN}\right): \lambda_{\max }[\mathrm{nm}](\mathrm{lg} \varepsilon)=209$ (4.643), 231 (4.231), 273 (3.476).

IR (ATR): $\tilde{v}\left[\mathrm{~cm}^{-1}\right]=3446,3085,3061,3030,2935,2888,2846,1717,1655,1637,1588$, 1558, 1497, 1454, 1419, 1389, 1372, 1312, 1276, 1204, 1191, 1150, 1097, 1061, 1026, 997, 979, 967, 921, 846, 829, 741, 716, 697, 650, 577, 541, 505.

${ }^{1} \mathrm{H}-\mathrm{NMR}\left(300 \mathrm{MHz}, \mathrm{CDCl}_{3}\right): \delta[\mathrm{ppm}]=2.63-2.71\left(\mathrm{~m}, 2 \mathrm{H}, 3-\mathrm{H}_{2}\right), 2.90-2.99\left(\mathrm{~m}, 2 \mathrm{H}, 4-\mathrm{H}_{2}\right)$, $3.43\left(\mathrm{~s}, 3 \mathrm{H}, \mathrm{OCH}_{2} \mathrm{OCH}_{3}\right), 3.74\left(\mathrm{~s}, 3 \mathrm{H}, 2^{\prime}-\mathrm{OCH}_{3}\right), 3.79$ (s, $\left.3 \mathrm{H}, 6{ }^{\prime}-\mathrm{OCH}_{3}\right), 4.05$ (s, $2 \mathrm{H}$, 1- $\left.\mathrm{H}_{2}\right), 4.56(\mathrm{~s}, 2 \mathrm{H}), 4.57(\mathrm{~d}, J=0.6 \mathrm{~Hz}, 2 \mathrm{H})\left(\mathrm{OCH}_{2} \mathrm{Ph}, \mathrm{OC}_{2} \mathrm{OCH}_{3}\right), 4.76\left(\mathrm{~s}, 2 \mathrm{H}, 4^{\prime}-\mathrm{CH}_{2}\right)$, $6.84(\mathrm{~s}, 1 \mathrm{H}, 5$ '-H) $7.25-7.35(\mathrm{~m}, 5 \mathrm{H}, 5 \times \mathrm{Ph}-\mathrm{H})$.

${ }^{13} \mathrm{C}-\mathrm{NMR}\left(126 \mathrm{MHz}, \mathrm{CDCl}_{3}\right): \delta[\mathrm{ppm}]=18.9$ (C-4), 38.6 (C-3), 55.6, $55.7 \quad\left(6{ }^{\prime}-\mathrm{OCH}_{3}\right.$, $\left.\mathrm{OCH}_{2} \mathrm{O}_{\mathrm{CH}} \mathrm{H}_{3}\right), 61.3\left(2^{\prime}-\mathrm{OCH}_{3}\right), 73.3\left(\mathrm{OCH}_{2} \mathrm{Ph}\right), 73.7\left(4^{\prime}-\mathrm{CH}_{2}\right), 74.9$ (C-1), $84.9\left(\mathrm{C}-3^{\prime}\right), 96.2$ $\left(\mathrm{O}_{\mathrm{CH}} \mathrm{OCH}_{3}\right), 107.4\left(\mathrm{C}-5^{\prime}\right), 122.7\left(\mathrm{C}-1^{\prime}\right), 127.9,127.9,128.4$ (2 $\times \mathrm{Ph}_{-} \mathrm{C}_{o}, 2 \times \mathrm{Ph}-\mathrm{C}_{m}$, Ph- $\mathrm{C}_{p}$ ), $137.2\left(\mathrm{Ph}-\mathrm{C}_{i}\right), 140.0$ (C-4'), 158.1 (C-2'), 158.9 (C-6'), 208.2 (C-2).

MS (ESI): $m / z(\%)=537.1(74)[\mathrm{M}+\mathrm{Na}]^{+}, 553.1(100)[\mathrm{M}+\mathrm{K}]^{+}, 1051.2(7)[2 \times \mathrm{M}+\mathrm{Na}]^{+}$.

$\mathrm{C}_{22} \mathrm{H}_{27} \mathrm{IO}_{6}(514.36)$

ber.: 537.0745

553.0484

gef.: $537.0749[\mathrm{M}+\mathrm{Na}]^{+}$

$553.0491[\mathrm{M}+\mathrm{K}]^{+}(\mathrm{ESI}-\mathrm{HRMS})$. 


\subsubsection{1-Benzyloxy-4-(2,6-dimethoxy-4-((methoxymethoxy)methyl)-3- (4,4,5,5-tetramethyl-1,3,2-dioxaborolan-2-yl)phenyl)butan-2-on (270)}

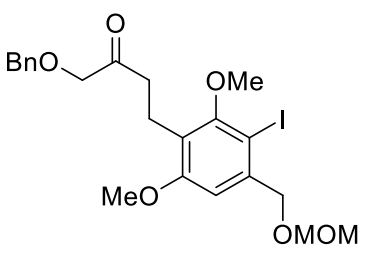

269
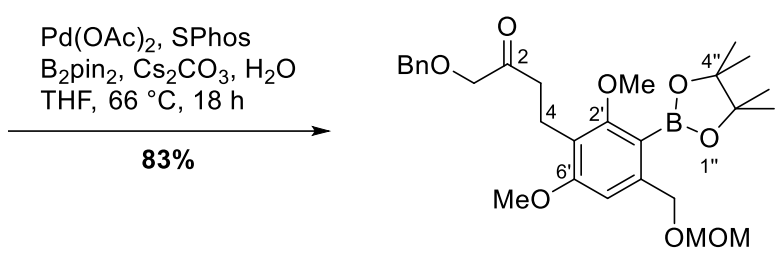

270

Zu einem Gemisch aus $\mathrm{Pd}(\mathrm{OAc})_{2}(17.5 \mathrm{mg}, 77.8 \mu \mathrm{mol}, 10 \mathrm{~mol} \%)$, SPhos $(79.8 \mathrm{mg}$, $194 \mu \mathrm{mol}, 25 \mathrm{~mol} \%$ ), B $\mathrm{pin}_{2}$ (395 mg, $1.56 \mathrm{mmol}, 2.00$ Äq.) sowie $\mathrm{Cs}_{2} \mathrm{CO}_{3}$ (507 mg, $1.56 \mathrm{mmol}, 2.00 \mathrm{Äq}$.) wurde bei Raumtemperatur eine Lösung des Aryliodids 269 (400 mg, $778 \mu \mathrm{mol}, 1.00$ Äq.) in sorgfältig entgastem THF $(25 \mathrm{ml})$ und $\mathrm{H}_{2} \mathrm{O}(56.0 \mu \mathrm{l}$, $56.0 \mathrm{mg}, 3.11 \mathrm{mmol}, 4.00 \mathrm{Äq}$.) gegeben, die resultierende Reaktionslösung auf $66^{\circ} \mathrm{C}$ erwärmt und $18 \mathrm{~h}$ bei dieser Temperatur unter Rückfluss gerührt. Anschließend wurde die Reaktionslösung auf Raumtemperatur abgekühlt, durch Celite ${ }^{\circledR}$ filtriert, mit EtOAc $(3 \times$ $25 \mathrm{ml}$ ) nachgewaschen, das Lösungsmittel unter vermindertem Druck entfernt und nach Säulenchromatographie an Kieselgel (Petrolether/EtOAc 3:1) der Pinakolboronsäureester 270 (333 mg, $648 \mu \mathrm{mol}, 83 \%$ ) als leicht braunes Öl erhalten.

UV $\left(\mathrm{CH}_{3} \mathrm{CN}\right): \lambda_{\max }[\mathrm{nm}](\lg \varepsilon)=208$ (4.741), 235 (4.001), 274 (3.387).

IR (ATR): $\widetilde{v}\left[\mathrm{~cm}^{-1}\right]=3449,3088,3063,3030,2977,2938,2886,2838,1719,1593,1569$, 1497, 1454, 1420, 1402, 1371, 1334, 1306, 1273, 1235, 1211, 1189, 1139, 1100, 1066, 1037, 1005, 985, 962, 919, 851, 784, 739, 698, 672, 578, 544, 505.

${ }^{1} \mathrm{H}-\mathrm{NMR}\left(300 \mathrm{MHz}, \mathrm{CDCl}_{3}\right): \delta[\mathrm{ppm}]=1.35\left(\mathrm{~s}, 12 \mathrm{H}, 4 \times \mathrm{CH}_{3}\right), 2.60-2.68\left(\mathrm{~m}, 2 \mathrm{H}, 3-\mathrm{H}_{2}\right)$, 2.82-2.91 (m, $\left.2 \mathrm{H}, 4-\mathrm{H}_{2}\right), 3.38\left(\mathrm{~s}, 3 \mathrm{H}, \mathrm{OCH}_{2} \mathrm{OCH}_{3}\right), 3.72\left(\mathrm{~s}, 3 \mathrm{H}, 2\right.$ '- $\left.\mathrm{OCH}_{3}\right), 3.76(\mathrm{~d}, J=$ $0.9 \mathrm{~Hz}, 3 \mathrm{H}, 6$ ' $\left.-\mathrm{OCH}_{3}\right), 4.05$ (s, $\left.2 \mathrm{H}, 1-\mathrm{H}_{2}\right), 4.56\left(\mathrm{~s}, 2 \mathrm{H}, \mathrm{OCH}_{2} \mathrm{Ph}\right), 4.62$ (d, J = $0.5 \mathrm{~Hz}$, $\mathrm{OC}_{2} \mathrm{OCH}_{3}$ ), 4.65 (s, $\left.2 \mathrm{H}, 4^{\prime}-\mathrm{CH}_{2}\right), 6.67$ (s, $\left.1 \mathrm{H}, 5^{\prime}-\mathrm{H}\right), 7.24-7.34(\mathrm{~m}, 5 \mathrm{H}, 5 \times \mathrm{Ph}-\mathrm{H})$.

${ }^{13} \mathrm{C}-\mathrm{NMR}\left(126 \mathrm{MHz}, \mathrm{CDCl}_{3}\right): \delta[\mathrm{ppm}]=17.6(\mathrm{C}-4), 24.9\left(4 \times \mathrm{CH}_{3}\right), 38.8(\mathrm{C}-3), 55.4,55.5$ (6'- $\left.\mathrm{OCH}_{3}, \mathrm{OCH}_{2} \mathrm{O}^{\mathrm{C}} \mathrm{H}_{3}\right), 63.0\left(2^{\prime}-\mathrm{OCH}_{3}\right), 68.9\left(4^{\prime}-\mathrm{CH}_{2}\right), 73.3\left(\mathrm{OCH}_{2} \mathrm{Ph}\right), 74.9$ (C-1), 83.5 (C-3', C-4", C-5"), $95.5\left(\mathrm{O}_{\mathrm{C}} \mathrm{H}_{2} \mathrm{OCH}_{3}\right), 106.5$ (C-5'), 121.0 (C-1'), $127.9\left(\mathrm{Ph}-\mathrm{C}_{p}\right), 127.9$,

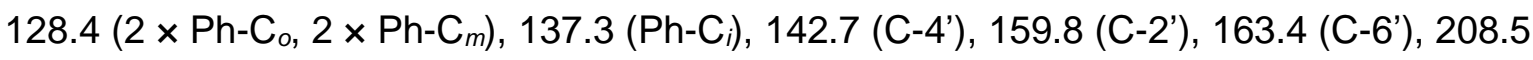
(C-2).

MS (ESI): $m / z(\%)=532.3(28)\left[\mathrm{M}+\mathrm{NH}_{4}\right]^{+}, 537.3(100)[\mathrm{M}+\mathrm{Na}]^{+}, 1051.5(51)[2 \times \mathrm{M}+$ $\mathrm{Na}]^{+}$.

$\mathrm{C}_{28} \mathrm{H}_{39} \mathrm{BO}_{8}(514.42)$

ber.: 532.3081 
gef.: $532.3074\left[\mathrm{M}+\mathrm{NH}_{4}\right]^{+}$

$537.2635[\mathrm{M}+\mathrm{Na}]^{+}(\mathrm{ESI}-\mathrm{HRMS})$. 


\subsubsection{3'-(4-Benzyloxy-3-oxobutyl)-2',4'-dimethoxy-6'-((methoxy- methoxy)methyl)-2-nitro-[1,1'-biphenyl]-3-carbonsäuremethylester} (271)

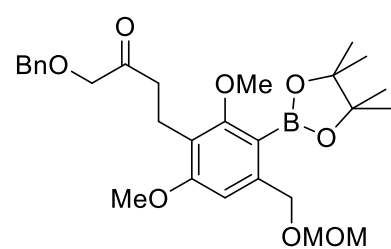

270

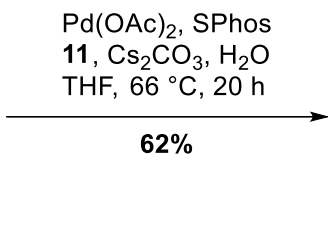

11, $\mathrm{Cs}_{2} \mathrm{CO}_{3}, \mathrm{H}_{2} \mathrm{O}$

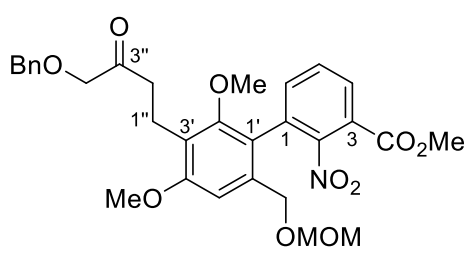

271

Zu einem Gemisch des Chlorarens 11 (126 mg, $583 \mu \mathrm{mol}, 1.00$ Äq.), Pd(OAc) 2 (13.1 mg, $58.3 \mu \mathrm{mol}, 10 \mathrm{~mol} \%$ ), SPhos $\left(60.0 \mathrm{mg}, 146 \mu \mathrm{mol}, 25 \mathrm{~mol} \%\right.$ ) sowie $\mathrm{Cs}_{2} \mathrm{CO}_{3}$ (380 mg, $1.17 \mathrm{mmol}, 2.00$ Äq.) wurde bei Raumtemperatur eine Lösung des Pinakolboronsäureesters 270 (300 mg, $583 \mu \mathrm{mol}, 1.00$ Äq.) in sorgfältig entgastem THF (20 ml) und $\mathrm{H}_{2} \mathrm{O}$ (42.0 $\mu \mathrm{l}, 42.0 \mathrm{mg}, 2.33 \mathrm{mmol}, 4.00$ Äq.) gegeben, die resultierende Reaktionslösung auf $66^{\circ} \mathrm{C}$ erwärmt und $20 \mathrm{~h}$ bei dieser Temperatur unter Rückfluss gerührt. Anschließend wurde die Reaktionslösung auf Raumtemperatur abgekühlt, durch Celite ${ }^{\circledR}$ filtriert, mit EtOAc $(3 \times 20 \mathrm{ml})$ nachgewaschen, das Lösungsmittel unter vermindertem Druck entfernt und nach Säulenchromatographie an Kieselgel (Petrolether/EtOAc 4:1 $\rightarrow$ 1:1 $\rightarrow$ EtOAc) das Biaryl 271 (204 mg, 359 umol, 62\%) als leicht braunes Öl isoliert.

UV $\left(\mathrm{CH}_{3} \mathrm{CN}\right): \lambda_{\max }[\mathrm{nm}](\lg \varepsilon)=204$ (4.834).

IR (ATR): $\tilde{v}\left[\mathrm{~cm}^{-1}\right]=3530,3065,3031,2942,2886,2841,1730,1601,1575,1542,1490$, 1453, 1440, 1405, 1369, 1323, 1287, 1264, 1210, 1148, 1120, 1097, 1066, 1035, 998, $964,918,886,852,826,772,740,700,647,602,577,546,529,506$.

${ }^{1} \mathrm{H}-\mathrm{NMR}\left(300 \mathrm{MHz}, \mathrm{CDCl}_{3}\right): \delta[\mathrm{ppm}]=2.63-2.71\left(\mathrm{~m}, 2 \mathrm{H}, 2\right.$ '"- $\left.\mathrm{H}_{2}\right), 2.80-2.95(\mathrm{~m}, 2 \mathrm{H}$, 1'- $-\mathrm{H}_{2}$ ), 3.23 (d, J = 0.4 Hz, $3 \mathrm{H}, 2$ '- $\mathrm{OCH}_{3}$ ), 3.36 (s, $\left.3 \mathrm{H}, \mathrm{OCH}_{2} \mathrm{OCH}_{3}\right), 3.81$ (s, $3 \mathrm{H}$, 4'- $\mathrm{OCH}_{3}$ ), $3.88\left(\mathrm{~s}, 3 \mathrm{H}, \mathrm{CO}_{2} \mathrm{CH}_{3}\right), 4.06\left(\mathrm{~d}, J=1.0 \mathrm{~Hz}, 2 \mathrm{H}, 4\right.$ '"- $\left.\mathrm{H}_{2}\right), 4.16(\mathrm{~d}, J=12.1 \mathrm{~Hz}$, $1 \mathrm{H}), 4.30(\mathrm{dd}, J=12.2,0.6 \mathrm{~Hz}, 1 \mathrm{H})\left(6^{\prime}-\mathrm{CH}_{2}\right), 4.48(\mathrm{~d}, J=6.5 \mathrm{~Hz}, 1 \mathrm{H}), 4.54(\mathrm{~d}, J=$ $\left.6.6 \mathrm{~Hz}, 1 \mathrm{H})\left(\mathrm{OCH}_{2} \mathrm{OCH}_{3}\right), 4.56\left(\mathrm{~s}, 2 \mathrm{H}, \mathrm{OCH}_{2} \mathrm{Ph}\right), 6.79(\mathrm{~s}, 1 \mathrm{H}, 5)-\mathrm{H}\right), 7.24-7.36(\mathrm{~m}, 5 \mathrm{H}$, $5 \times \mathrm{Ph}-\mathrm{H}), 7.54(\mathrm{dd}, J=7.7,1.9 \mathrm{~Hz}, 1 \mathrm{H}, 6-\mathrm{H}), 7.56-7.62(\mathrm{~m}, 1 \mathrm{H}, 5-\mathrm{H}), 8.01$ (dd, $J=7.3$, $1.9 \mathrm{~Hz}, 1 \mathrm{H}, 4-\mathrm{H})$.

${ }^{13} \mathrm{C}-\mathrm{NMR}\left(126 \mathrm{MHz}, \mathrm{CDCl}_{3}\right): \delta[\mathrm{ppm}]=18.0$ (C-1"), 38.5 (C-2"), $53.0\left(\mathrm{CO}_{2} \underline{\mathrm{CH}}_{3}\right), 55.3,55.6$ (4'- $\left.\mathrm{OCH}_{3}, \mathrm{OCH}_{2} \mathrm{O}^{\mathrm{C}} \mathrm{H}_{3}\right), 61.5\left(2^{\prime}-\mathrm{OCH}_{3}\right), 67.0\left(4^{\prime}-\mathrm{CH}_{2}\right), 73.3\left(\mathrm{OCH}_{2} \mathrm{Ph}\right), 74.8(\mathrm{C}-4$ '”), 95.9 $\left(\mathrm{O}_{\mathrm{CH}} \mathrm{OCH}_{3}\right), 106.6$ (C-5'), 120.4 (C-1'), 122.1 (C-3'), 123.8 (C-3), $127.9\left(\mathrm{Ph}-\mathrm{C}_{p}\right), 127.9$, $128.4\left(2 \times \mathrm{Ph}^{-\mathrm{C}_{o},} 2 \times \mathrm{Ph}_{\mathrm{C}}\right.$ ), 129.6 (C-5), 130.4 (C-4), 130.6 (C-1), 136.5, 136.7 (C-6, C-6'), 137.3 (Ph-Ci), 150.3 (C-2), 156.8 (C-2'), 159.1 (C-4'), $164.0\left(\underline{\mathrm{CO}}_{2} \mathrm{CH}_{3}\right), 208.2$ (C-3'). 
MS (ESI): $m / z(\%)=585.2(32)\left[\mathrm{M}+\mathrm{NH}_{4}\right]^{+}, 590.2(52)[\mathrm{M}+\mathrm{Na}]^{+}, 1157.4(27)[2 \times \mathrm{M}+$ $\mathrm{Na}]^{+} ; 566.2(22)[\mathrm{M}-\mathrm{H}]^{-}$.

$\mathrm{C}_{30} \mathrm{H}_{33} \mathrm{NO}_{10}(567.59)$

ber.: 566.2032

590.1997

gef.: $566.2031[\mathrm{M}-\mathrm{H}]^{-}$

$590.1997[\mathrm{M}+\mathrm{Na}]^{+}(\mathrm{ESI}-\mathrm{HRMS})$. 


\subsubsection{1 (Z)-3'-(3-((Benzyloxy)methyl)pent-3-en-1-yl)-2',4'-dimethoxy-6'- ((methoxymethoxy)methyl)-2-nitro-[1,1'-biphenyl]-3-carbonsäure- methylester ((Z)-272)}

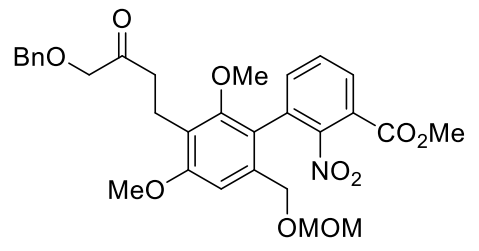

271

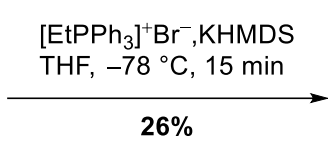

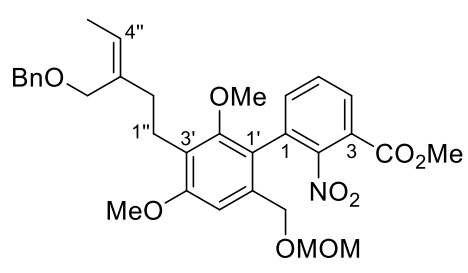

(Z)-272

$\mathrm{Zu}$ einer Suspension von Ethyltriphenylphosphoniumbromid $(654 \mathrm{mg}, 1.76 \mathrm{mmol}$, 10.0 Äq.) in THF (1 ml) wurde bei $0^{\circ} \mathrm{C}$ KHMDS (316 mg, $1.59 \mathrm{mmol}, 9.00 \mathrm{Äq}$.) gegeben, das resultierende Reaktionsgemisch auf $66^{\circ} \mathrm{C}$ erwärmt und $1 \mathrm{~h}$ bei dieser Temperatur unter Rückfluss gerührt. Anschließend wurde die Reaktionslösung auf $-78^{\circ} \mathrm{C}$ gekühlt, eine Lösung des Biaryls 271 (100 mg, $176 \mu$ mol, 1.00 Äq.) in THF (2 ml) zugegeben und 15 min bei dieser Temperatur gerührt. Daraufhin wurde auf Raumtemperatur erwärmt, die Reaktion durch vorsichtige Zugabe von ges. $\mathrm{NH}_{4} \mathrm{Cl}$-Lsg. $(5 \mathrm{ml})$ sowie $\mathrm{H}_{2} \mathrm{O}(5 \mathrm{ml})$ beendet und die wässr. Phase mit MTBE $(3 \times 5 \mathrm{ml})$ extrahiert. Die vereinigten org. Phasen wurden über $\mathrm{MgSO}_{4}$ getrocknet, das Lösungsmittel unter vermindertem Druck entfernt und nach säulenchromatographischer Reinigung an Kieselgel (Petrolether/EtOAc 3:1 $\rightarrow$ 1:1) das Alken (Z)-272 (26.9 mg, 46.4 umol, 26\%) als leicht gelbes Öl isoliert.

UV $\left(\mathrm{CH}_{3} \mathrm{CN}\right): \lambda_{\max }[\mathrm{nm}](\lg \varepsilon)=204$ (4.909).

IR (ATR): $\tilde{v}\left[\mathrm{~cm}^{-1}\right]=3448,3063,3029,2936,2884,2859,1732,1600,1575,1542,1489$, 1452, 1440, 1405, 1368, 1322, 1287, 1265, 1208, 1192, 1148, 1120, 1098, 1066, 1035, 999, 964, 941, 917, 851, 828, 771, 736, 699, 647, 634, 575, 523.

'H-NMR (300 MHz, CDCl $): \delta[p p m]=1.62\left(\mathrm{~d}, J=6.9 \mathrm{~Hz}, 3 \mathrm{H}, 5\right.$ '- $\left.\mathrm{H}_{3}\right), 2.33(\mathrm{q}, J=6.7 \mathrm{~Hz}$, $2 \mathrm{H}, 2$ '"- $\mathrm{H}_{2}$ ), 2.69-2.78 (m, $2 \mathrm{H}, 1$ ''- $\mathrm{H}_{2}$ ), 3.24 (d, J = 0.3 Hz, $3 \mathrm{H}, 2$ '- $\left.-\mathrm{OCH}_{3}\right), 3.37$ (s, $3 \mathrm{H}$, $\mathrm{OCH}_{2} \mathrm{OCH}_{3}$ ), 3.81 (s, $3 \mathrm{H}, 4^{\prime}-\mathrm{OCH}_{3}$ ), 3.89 (s, $3 \mathrm{H}, \mathrm{CO}_{2} \mathrm{CH}_{3}$ ), 4.09 (s, $2 \mathrm{H}, 3$ '”- $\mathrm{CH}_{2}$ ), 4.17 (d, $J=12.1 \mathrm{~Hz}, 1 \mathrm{H}), 4.31(\mathrm{~d}, J=12.0 \mathrm{~Hz}, 1 \mathrm{H})\left(6^{\prime}-\mathrm{CH}_{2}\right), 4.47\left(\mathrm{~s}, 2 \mathrm{H}, \mathrm{OCH}_{2} \mathrm{Ph}\right), 4.50$ (d, J = $6.5 \mathrm{~Hz}, 1 \mathrm{H}), 4.55(\mathrm{~d}, J=6.5 \mathrm{~Hz}, 1 \mathrm{H})\left(\mathrm{OCH}_{2} \mathrm{OCH}_{3}\right), 5.47(\mathrm{q}, J=6.8 \mathrm{~Hz}, 1 \mathrm{H}, 4$ "'- $\mathrm{H}), 6.79$ (s, $1 \mathrm{H}, 5$ '-H) $7.21-7.37(\mathrm{~m}, 5 \mathrm{H}, 5 \times \mathrm{Ph}-\mathrm{H}), 7.54(\mathrm{~d}, J=5.5 \mathrm{~Hz}, 1 \mathrm{H}, 6-\mathrm{H}), 7.55-7.61(\mathrm{~m}$, $1 \mathrm{H}, 5-\mathrm{H}$ ), 8.00 (dd, $J=7.1,2.2 \mathrm{~Hz}, 1 \mathrm{H}, 4-\mathrm{H}$ ).

${ }^{13} \mathrm{C}-\mathrm{NMR}\left(126 \mathrm{MHz}, \mathrm{CDCl}_{3}\right): \delta[\mathrm{ppm}]=13.3$ (C-5"), 23.4 (C-1"), 34.9 (C-2"), 53.1 $\left(\mathrm{CO}_{2} \underline{\mathrm{CH}}_{3}\right), 55.3,55.5\left(4^{\prime}-\mathrm{OCH}_{3}, \mathrm{OCH}_{2} \mathrm{O} \mathrm{H}_{3}\right), 61.5\left(2^{\prime}-\mathrm{OCH}_{3}\right), 66.9\left(3^{\prime \prime}-\mathrm{CH}_{2}\right), 67.2$ (6'- $\left.\mathrm{CH}_{2}\right), 71.9\left(\mathrm{OCH}_{2} \mathrm{Ph}\right), 95.9\left(\mathrm{O}_{\mathrm{CH}} \mathrm{OCH}_{3}\right), 106.5$ (C-5'), 120.3 (C-1'), 123.7, 123.8,

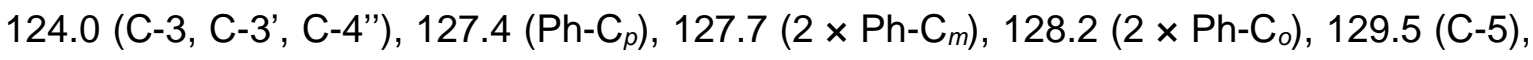


130.3 (C-4), 131.0 (C-1), 135.8, 136.6, 136.9 (C-6, C-6', C-3"), 138.8 (Ph-Ci), 150.3 (C-2), 156.8 (C-2'), 159.4 (C-4'), $164.2\left(\mathrm{CO}_{2} \mathrm{CH}_{3}\right)$.

MS (ESI): $m / z(\%)=602.2(100)[\mathrm{M}+\mathrm{Na}]^{+}, 1181.5(9)[2 \times \mathrm{M}+\mathrm{Na}]^{+}$.

$\mathrm{C}_{32} \mathrm{H}_{37} \mathrm{NO}_{9}(579.65)$ ber.: 602.2361

gef.: $602.2365[\mathrm{M}+\mathrm{Na}]^{+}$(ESI-HRMS). 
IV Anhang 


\subsection{Abkürzungsverzeichnis}

$\delta$

$\varepsilon$

$\circ$

$\mu$

$[\alpha]_{D}^{\vartheta}$

$\vartheta$

$\lambda$

$\tilde{v}$

9-BBN

$\mathrm{A}$

$\AA$

Ac

$\mathrm{Ad}$

AIBN

APT

aq.

Äq.

AQN

$\mathrm{Ar}$

atm

ATR

B

BDP

ber.

$\mathrm{Bn}$

Boc

BOXAX

brsm

$\mathrm{Bu}$

$\mathrm{Bz}$

bzw.

C

C

ca.

$\mathrm{CaFT}$

CAN

CLB

$\mathrm{cm}^{-1}$ chemische Verschiebung

Extinktionskoeffizient

Grad

mikro-

spezifischer Drehwinkel

Temperatur

Wellenlänge

Wellenzahl

9-Borabicyclo[3.3.1]nonan

Adenosin

Ångström

Acetyl-

Adamantyl-

2,2'-Azobis(2-methylpropionitril)

attached proton test

wässrig

Äquivalente

Anthrachinon

Aryl-

Atmosphäre

attenuated total reflection, abgeschwächte Totalreflexion

Base

1,2-Bis(diphenylphosphin)benzol

berechnet

Benzyl-

tert-Butyloxycarbonyl-

2,2'-Bis(oxazolyl)-1,1'-binaphthyl based on recovered starting material, bezogen auf reisoliertes Startmaterial

Butyl-

Benzoyl

beziehungsweise

Konzentration

Celsius

circa

Candida albicans Fitness Test

Ammoniumcer(IV)-nitrat

$p$-Chlorbenzoat

Wellenzahl 
Co.

cod

COSY

Cy

d

$\mathrm{dba}$

DC

DCE

DFT

$\mathrm{DHQ}$

DHQD

DIBAL-H

div. Bed.

DMAP

DME

DMF

DMP

DMSO

DNA

dppf

$d r$

E

$\mathrm{e}^{-}$

ee

El

endo-

ent-

ESI

Et

et al.

EtOAc

$\mathrm{eV}$

ff.

$\mathrm{g}$

gef.

ges.

$\mathrm{h}$

Het

HIV
Compagnie

1,5-Cyclooctadien

correlated spectroscopy, $(\mathrm{H}, \mathrm{H})$-Korrelationsspektroskopie

Cyclohexyl-

$\operatorname{Tag}(\mathrm{e})$

trans, trans-Dibenzylidenaceton

Dünnschichtchromatographie

1,2-Dichlorethen

diskrete Fourier-Transformation

Dihydrochinin

Dihydrochinidin

Di-iso-butylaluminiumhydrid

diverse Bedingungen

$\mathrm{N}, \mathrm{N}$-Dimethylpyridin-4-amin

$N, N$-Dimethylethylamin

Dimethylformamid

Dess-Martin-Periodinan

Dimethylsulfoxid

Desoxyribonukleinsäure

1,1'-Bis(diphenylphosphano)ferrocen

diastereomeric ratio,

Diastereomerenverhältnis

entgegen

Elektron

enantiomeric excess,

Enantiomerenüberschuss

Elektronenstoß-Ionisation

griechisch endon, innen

enantio-

Elektronspray-Ionisation

Ethyl-

et alumni, und Mitarbeiter

Essigsäureethylester

Elektronenvolt

folgende

Gramm

gefunden

gesucht

Stunde(n)

Heterozyklus

Humane Immundefizienz-Virus 
HMBC

HMDS

HMPA

HRMS

HPLC

HSQC

$\mathrm{Hz}$

i

$i$, iso

IBX

I-Effekt

Inc.

IR

$J$

Kap.

kat.

Kat.

kcal

konz.

L

I

$\mathrm{L}$

LDA

LG

LM

Lsg.

Ltd.

$\mathrm{M}$

$\mathrm{m}$

$M$

$m$

$\mathrm{M}$

$\mathrm{m}$

$\mathrm{m}$

$m C P B A$

$\mathrm{Me}$

MEQ

MIC heteronuclear multiple bond correlation, $(\mathrm{C}, \mathrm{H})$-Korrelationsspektroskopie (über mehrere Bindungen)

Bis(trimethylsilyl)amid

Hexamethylphosphorsäuretriamid

high resolution mass spectrometry, hochaufgelöste Massenspektrometrie high performance liquid chromatography, Hochleistungsflüssigkeitschromatographie heteronuclear single quantum correlation, $(\mathrm{C}, \mathrm{H})$-Korrelationsspektroskopie Hertz

ipso

isomer, verzweigt

2-Iodoxybenzoesäure

induktiver Effekt

incorporated

Infrarotspektroskopie

Kopplungskonstante

Kapitel

katalytisch

Katalysator

Kilokalorie

konzentriert

lat. laevus, links

Liter

Ligand

Lithiumdi-iso-propylamid leaving group, Abgangsgruppe

Lösungsmittel

Lösung

limited

Molarität, molar

Masse

mega-

meta

Metall

Meter

milli-

meta-Chlorperbenzoesäure

Methyl-

4-Methyl-2-chinolyl-

minimal inhibitory concentration 
$\min$

Mio.

MOM

Mrd.

mRNA

Ms

MS

MTBE

mw

$\mathrm{n}$

$n$

$\mathrm{n}$

NBS

NE

NMM

NMO

NMR

NOESY

NW

O

org.

OTf

$p$

p.a.

PAP

$P G$

$\mathrm{Ph}$

PHAL

PhMe

PHN

pin

Piv

PMB

PMHS

ppm

$\mathrm{Pr}$

py

P-Ylid
Minute(n)

Million(en)

Methoxymethyl-

Milliarde(n)

messenger ribonucleic acid, Boten-

Ribonukleinsäure

Methansulfonyl-

Massenspektrometrie

Methyl-tert-butylether

Mikrowelle

nano

normal, unverzweigt

Variable

$N$-Bromsuccinimid

north east, nordost

$\mathrm{N}$-Methylmorpholin

$\mathrm{N}$-Methylmorpholin- $\mathrm{N}$-oxid

nuclear magnetic resonance,

Kernresonanz

nuclear-Overhauser-effect spectroscopy,

$(\mathrm{H}, \mathrm{H})$-Korrelationsspektroskopie (durch den Raum)

north west, nordwest

ortho

organisch

Triflyl-, Trifluormethansulfonyl-

para

pro analysi

Polyadenosin-Polymerase

protecting group, Schutzgruppe

Phenyl-

Phthalazin

Toluol

Phenanthryl-

Pinakol

Pivaloyl-

p-Methoxybenzyl-

Poly(methylhydrosiloxan)

parts per million

Propyl-

Pyridin

Phosphor-Ylid 


\section{PYR}

quant.

(

$\mathrm{R}$

(R)

$R_{f}$

RT

$s, \sec$

$\mathrm{S}$

S.

$\mathrm{S}$

(S)

Sdp.

SE

$\mathrm{S}_{\mathrm{E}} \mathrm{Ar}$

SEM

SMC

Smp.

$\mathrm{S}_{\mathrm{N}} 2$

SOMO

SPhos

SPRIX

SW

$t$, tert

TBDPS

TBHP

TBS

TC

Teoc

TES

TFA

THF

TIPS

TMEDA

TMS

TPAP

$t_{\mathrm{R}}$

trig

Ts

UV
Pyrimidin

quantitativ

registered trademark

Rest

lat. rectus, rechts

Retentionswert

Raumtemperatur

sekundär

Sekunde(n)

siehe

solid, fest

lat. sinister, links

Siedepunkt

south east, südost

elektrophile aromatische Substitution

$\beta$-(Trimethylsilyl)ethoxymethyl-

Suzuki-Miyaura-Kreuzkupplung

Schmelzpunkt

nukleophile Substitution zweiter Ordnung

single occupied molecular orbital

2-Dicyclohexylphosphin-2',6'-dimethoxybiphenyl

Spiro(isoxazol-isoxazolin)

south west, südwest

tertiär

tert-Butyldiphenylsilyl-

tert-Butylhydroperoxid

tert-Butyldimethylsilyl-

Thiophen-2-carboxylat

2-(Trimethylsilyl)ethoxycarbonyl-

Triethylsilyl-

Trifluoracetat/-essigsäure

Tetrahydrofuran

Tri-iso-propylsilyl-

$N, N, N, N$-Tetramethylethylendiamin

Trimethylsilyl-

Tetrapropylammoniumperruthenat

Retentionszeit

trigonal-planar

$p$-Toluolsulfonyl-

Ultraviolett 
v. a.

verd.

Vis

wässr.

$X$

XPhos

z

$Z$

z. B. vor allem

verdünnt

Visueller Bereich (380-750 nm)

wässrig

Halogenid

2-Cicyclohexylphosphin-2',4',6'-tri-isopropylbiphenyl

Ladung

zusammen

zum Beispiel 


\subsection{Literatur}

[1] a) T. Vos, A. D. Flaxman, M. Naghavi, R. Lozano, C. Michaud, M. Ezzati, K. Shibuya, J. A. Salomon, S. Abdalla, V. Aboyans et al., The Lancet 2012, 380, $2163-$ 2196; b) G. D. Brown, D. W. Denning, N. A. R. Gow, S. M. Levitz, M. G. Netea, T. C. White, Science translational medicine 2012, 4, 165rv13.

[2] "Gaffi - Global Action Fund for Fungal Infections", zu finden unter http://www.gaffi.org/.

[3] a) G. Aperis, N. Myriounis, E. K. Spanakis, E. Mylonakis, Expert opinion on investigational drugs 2006, 15, 1319-1336; b) H. Hof, Pharmazie in unserer Zeit 2003, 32, 96-103.

[4] H. A. Gallis, Richard H. Drew, William W. Pickard, Reviews of Infectious Diseases 1990, 12, 308-329.

[5] a) J. M. Valdez, P. Scheinberg, O. Nunez, C. O. Wu, N. S. Young, T. J. Walsh, Clinical infectious diseases : an official publication of the Infectious Diseases Society of America 2011, 52, 726-735; b) S. Perkhofer, C. Lass-Flörl, M. Hell, G. Russ, R. Krause, M. Hönigl, C. Geltner, J. Auberger, G. Gastl, M. Mitterbauer et al., International journal of antimicrobial agents 2010, 36, 531-536.

[6] N. A. Kartsonis, J. Nielsen, C. M. Douglas, Drug Resistance Updates 2003, 6, 197218.

[7] N. P. Wiederhold, J. S. Lewis, Expert opinion on pharmacotherapy 2007, 8, 11551166.

[8] J. A. Vazquez, J. D. Sobel, Clinical infectious diseases : an official publication of the Infectious Diseases Society of America 2006, 43, 215-222.

[9] E. Snelders, S. M. T. Camps, A. Karawajczyk, G. Schaftenaar, G. H. J. Kema, van der Lee, Henrich A, C. H. Klaassen, W. J. G. Melchers, P. E. Verweij, PloS one 2012, 7, e31801.

[10] M. Slavin, S. van Hal, T. C. Sorrell, A. Lee, D. J. Marriott, K. Daveson, K. Kennedy, K. Hajkowicz, C. Halliday, E. Athan et al., Clinical microbiology and infection : the official publication of the European Society of Clinical Microbiology and Infectious Diseases 2015, 21, 490.e1-490.e10.

[11] D. W. Denning, M. J. Bromley, Science (New York, N.Y.) 2015, 347, 1414-1416.

[12] a) D. J. Newman, G. M. Cragg, Journal of natural products 2007, 70, 461-477; b) D. J. Newman, G. M. Cragg, K. M. Snader, Journal of natural products 2003, 66, 1022 1037; c) M. S. Butler, D. J. Newman in Progress in Drug Research, Vol. 65 (Hrsg.: F. Petersen, R. Amstutz), Birkhäuser Verlag AG, Basel, 2008; d) J. J. Bowling, A. J. Kochanowska, N. Kasanah, M. T. Hamann, Expert opinion on drug discovery 2007, 
2, 1505-1522; e) M. T. Hamann, R. Hill, S. Roggo, CHIMIA 2007, 61, 313-321; f) M. S. Butler, Journal of natural products 2004, 67, 2141-2153; g) S. M. Colegate, R. J. Molyneux (Hrsg.) Bioactive natural products. Detection, isolation, and structural determination, CRC Press/Taylor \& Francis, Boca Raton, Fla., 2008; h) F. Petersen, R. Amstutz (Hrsg.) Progress in Drug Research, Vol. 65, Birkhäuser Verlag AG, Basel, 2008.

[13] a) Govi-Verlag Pharmazeutischer Verlag GmbH, "Pharmazeutische Zeitung online: Die bessere Hälfte bewährter Arzneistoffe", zu finden unter http://www.pharmazeutische-zeitung.de/index.php?id=28058; b) L. A. Sorbera, A. Graul, J. Castañer, Drugs Fut 1998, 23, 838.

[14] C. A. Parish, S. K. Smith, K. Calati, D. Zink, K. Wilson, T. Roemer, B. Jiang, D. Xu, G. Bills, G. Platas et al., J. Am. Chem. Soc. 2008, 130, 7060-7066.

[15] D. Overy, K. Calati, J. N. Kahn, M.-J. Hsu, J. Martín, J. Collado, T. Roemer, G. Harris, C. A. Parish, Bioorg. Med. Chem. Lett. 2009, 19, 1224-1227.

[16] G. F. Bills, G. Platas, D. P. Overy, J. Collado, A. Fillola, M. R. Jimenez, J. Martin, A. G. del Val, F. Vicente, J. R. Tormo et al., Mycologia 2009, 101, 449-472.

[17] a) I. KUROBANE, L. C. VINING, A. G. MCINNES, J. Antibiot. 1979, 32, 1256-1266; b) B. Franck, G. Bringmann, G. Flohr, Angew. Chem. Int. Ed. Engl. 1980, 19, 460461.

[18] Q. Zhou, B. B. Snider, Org. Lett. 2009, 11, 2936-2939.

[19] B. Jiang, D. Xu, J. Allocco, C. Parish, J. Davison, K. Veillette, S. Sillaots, W. Hu, R. Rodriguez-Suarez, S. Trosok et al., Chem. Biol. 2008, 15, 363-374.

[20] G. C. Adam, C. A. Parish, D. Wisniewski, J. Meng, M. Liu, K. Calati, B. D. Stein, J. Athanasopoulos, P. Liberator, T. Roemer et al., J. Am. Chem. Soc. 2008, 130, 16704-16710.

[21] Q. Zhou, B. B. Snider, J. Org. Chem. 2010, 75, 8224-8233.

[22] a) M. Schlosser (Hrsg.) Organometallics in synthesis. A manual, Wiley, Chichester, 2004; b) J. Tsuji, Palladium reagents and catalysts. Innovations in organic synthesis, Wiley, Chichester, 1995; c) R. F. Heck, Palladium reagents in Organic Syntheses, 2. Aufl., Academic Press, London, 1987; d) L. S. Hegedus, H.-G. Schmalz, Organische Synthese mit Übergangsmetallen, VCH, Weinheim, 1995.

[23] a) A. d. Meijere, F. Diederich (Hrsg.) Metal-catalyzed cross-coupling reactions, Wiley-VCH, Weinheim, 2004; b) F. Bellina, A. Carpita, R. Rossi, Synthesis 2004, 2004, 2419-2440; c) A. Suzuki, Journal of Organometallic Chemistry 2002, 653, 8390; d) N. Miyaura (Hrsg.) Topics in Current Chemistry, Vol. 219, Springer, Berlin, 2002; e) A. Suzuki, Journal of Organometallic Chemistry 1999, 576, 147-168; f) L. S. Liebeskind, Advances in metal-organic chemistry, JAI Press, Greenwich, Conn., 
London, 1998; g) S. P. Stanforth, Tetrahedron 1998, 54, 263-303; h) N. Miyaura, K. Yamada, A. Suzuki, Tetrahedron Letters 1979, 20, 3437-3440; i) N. Miyaura, A. Suzuki, J. Chem. Soc., Chem. Commun. 1979, 866.

[24] N. Miyaura, Journal of Organometallic Chemistry 2002, 653, 54-57.

[25] N. Miyaura, A. Suzuki, Chem. Rev. 1995, 95, 2457-2483.

[26] a) Johansson Seechurn, Carin C. C., M. O. Kitching, T. J. Colacot, V. Snieckus, Angewandte Chemie International Edition 2012, 51, 5062-5085; b) A. Suzuki, Angewandte Chemie (International ed. in English) 2011, 50, 6722-6737; c) X.-F. Wu, P. Anbarasan, H. Neumann, M. Beller, Angewandte Chemie (International ed. in English) 2010, 49, 9047-9050; d) "The Nobel Prize in Chemistry 2010", zu finden unter http://www.nobelprize.org/nobel_prizes/chemistry/laureates/2010/.

[27] D. G. Hall (Hrsg.) Boronic acids. Preparation and applications in organic synthesis, medicine and materials, Wiley-VCH, Weinheim, 2011.

[28] J. H. Kirchhoff, M. R. Netherton, I. D. Hills, G. C. Fu, J. Am. Chem. Soc. 2002, 124, 13662-13663.

[29] a) G. C. Fu, Acc. Chem. Res. 2008, 41, 1555-1564; b) A. F. Littke, G. C. Fu, Angewandte Chemie International Edition 2002, 41, 4176-4211; c) A. F. Littke, G. C. Fu, J. Am. Chem. Soc. 2001, 123, 6989-7000; d) A. F. Littke, L. Schwarz, G. C. Fu, J. Am. Chem. Soc. 2002, 124, 6343-6348; e) M. R. Netherton, G. C. Fu, Org. Lett. 2001, 3, 4295-4298; f) C. Dai, G. C. Fu, J. Am. Chem. Soc. 2001, 123, 27192724; g) A. F. Littke, G. C. Fu, Angewandte Chemie 1999, 111, 2568-2570; h) A. F. Littke, G. C. Fu, Angewandte Chemie 1998, 110, 3586-3587.

[30] A. F. Littke, G. C. Fu, J. Org. Chem. 1999, 64, 10-11.

[31] A. F. Littke, C. Dai, G. C. Fu, J. Am. Chem. Soc. 2000, 122, 4020-4028.

[32] a) A. Zapf, M. Beller, Chemistry - A European Journal 2001, 7, 2908-2915; b) M. Beller, T. H. Riermeier, European Journal of Inorganic Chemistry 1998, 1998, 29 35; c) M. Beller, H. Fischer, W. A. Herrmann, K. öfele, C. Broßmer, Angewandte Chemie 1995, 107, 1992-1993; d) W. A. Herrmann, V. P. Böhm, C.-P. Reisinger, Journal of Organometallic Chemistry 1999, 576, 23-41; e) W. A. Herrmann, C. Broßmer, K. öfele, C. Reisinger, T. Priermeier, M. Beller, H. Fischer, Angewandte Chemie 1995, 107, 1989-1992; f) W. A. Herrmann, C. Brossmer, C. Reisinger, T. H. Riermeier, K. öfele, M. Beller, Chemistry - A European Journal 1997, 3, 1357-1364; g) W. A. Herrmann, M. Elison, J. Fischer, C. Köcher, G. R. J. Artus, Angewandte Chemie 1995, 107, 2602-2605; h) W. A. Herrmann, M. Elison, J. Fischer, C. Köcher, G. R. J. Artus, Chemistry - A European Journal 1996, 2, 772-780; i) W. A. Herrmann, J. Fischer, K. öfele, G. R. Artus, Journal of Organometallic Chemistry 
1997, 530, 259-262; j) W. A. Herrmann, C. Köcher, Angewandte Chemie 1997, 109, 2256-2282.

[33] a) R. Martin, S. L. Buchwald, Acc. Chem. Res. 2008, 41, 1461-1473; b) T. E. Barder, S. D. Walker, J. R. Martinelli, S. L. Buchwald, J. Am. Chem. Soc. 2005, 127, 4685-4696; c) S. D. Walker, T. E. Barder, J. R. Martinelli, S. L. Buchwald, Angew. Chem. Int. Ed. 2004, 43, 1871-1876; d) J. P. Wolfe, S. L. Buchwald, Angewandte Chemie 1999, 111, 2570-2573; e) J. P. Wolfe, R. A. Singer, B. H. Yang, S. L. Buchwald, J. Am. Chem. Soc. 1999, 121, 9550-9561; f) David W. Old, John P. Wolfe, and Stephen L. Buchwald, J. Am. Chem. Soc. 1998, 120, 9722-9723.

[34] S. R. Chemler, D. Trauner, S. J. Danishefsky, Angewandte Chemie 2001, 113, 4676-4701.

[35] M. F. Lipton, M. A. Mauragis, M. T. Maloney, M. F. Veley, D. W. VanderBor, J. J. Newby, R. B. Appell, E. D. Daugs, Org. Process Res. Dev. 2003, 7, 385-392.

[36] L. S. Santos, G. B. Rosso, R. A. Pilli, M. N. Eberlin, J. Org. Chem. 2007, 72, 58095812.

[37] a) J. F. Hartwig, F. Paul, J. Am. Chem. Soc. 1995, 117, 5373-5374; b) M. R. Biscoe, B. P. Fors, S. L. Buchwald, J. Am. Chem. Soc. 2008, 130, 6686-6687; c) M. W. Hooper, M. Utsunomiya, J. F. Hartwig, J. Org. Chem. 2003, 68, 2861-2873; d) E. R. Strieter, D. G. Blackmond, S. L. Buchwald, J. Am. Chem. Soc. 2003, 125, 1397813980.

[38] F. Ozawa, A. Kubo, T. Hayashi, Chem. Lett. 1992, 2177-2180.

[39] C. Amatore, A. Jutand, Acc. Chem. Res. 2000, 33, 314-321.

[40] C. Amatore, G. Le Duc, A. Jutand, Chemistry - A European Journal 2013, 19, 10082-10093.

[41] C. Amatore, A. Jutand, G. Le Duc, Chem. Eur. J. 2011, 17, 2492-2503.

[42] C. Amatore, A. Jutand, G. Le Duc, Chem. Eur. J. 2012, 18, 6616-6625.

[43] a) C. Amatore, A. Jutand, G. Le Duc, Angew. Chem. Int. Ed. 2012, 51, 1379-1382;

b) C. Amatore, A. Jutand, G. Le Duc, Angew. Chem. 2012, 124, 1408-1411; c) B. P. Carrow, J. F. Hartwig, J. Am. Chem. Soc. 2011, 133, 2116-2119.

[44] a) R. Giovannini, P. Knochel, J. Am. Chem. Soc. 1998, 120, 11186-11187; b) F. d'Orlyé, A. Jutand, Tetrahedron 2005, 61, 9670-9678; c) A. G. Jarvis, I. J. S. Fairlamb, COC 2011, 15, 3175-3196; d) C. Amatore, A. Jutand, A. Suarez, J. Am. Chem. Soc. 1993, 115, 9531-9541; e) C. Amatore, E. Carré, A. Jutand, H. Tanaka, Q. Ren, S. Torii, Chem. Eur. J. 1996, 2, 957-966.

[45] K. Matos, J. A. Soderquist, J. Org. Chem. 1998, 63, 461-470.

[46] B. H. Ridgway, K. A. Woerpel, J. Org. Chem. 1998, 63, 458-460. 
[47] a) H. Nakamura, M. Fujiwara, Y. Yamamoto, J. Org. Chem. 1998, 63, 7529-7530; b) M. Murata, S. Watanabe, Y. Masuda, J. Org. Chem. 1997, 62, 6458-6459; c) T. Ishiyama, Y. Itoh, T. Kitano, N. Miyaura, Tetrahedron Letters 1997, 38, 3447-3450.

[48] a) T. Ishiyama, J. Takagi, K. Ishida, N. Miyaura, N. R. Anastasi, J. F. Hartwig, J. Am. Chem. Soc. 2002, 124, 390-391; b) J.-Y. Cho, Science 2001, 295, 305-308.

[49] a) K. C. Nicolaou, S. Natarajan, H. Li, N. F. Jain, R. Hughes, M. E. Solomon, J. M. Ramanjulu, C. N. C. Boddy, M. Takayanagi, Angewandte Chemie International Edition 1998, 37, 2708-2714; b) K. C. Nicolaou, N. F. Jain, S. Natarajan, R. Hughes, M. E. Solomon, H. Li, J. M. Ramanjulu, M. Takayanagi, A. E. Koumbis, T. Bando, Angewandte Chemie International Edition 1998, 37, 2714-2716; c) K. C. Nicolaou, M. Takayanagi, N. F. Jain, S. Natarajan, A. E. Koumbis, T. Bando, J. M. Ramanjulu, Angewandte Chemie International Edition 1998, 37, 2717-2719.

[50] a) E. M. Suh, Y. Kishi, J. Am. Chem. Soc. 1994, 116, 11205-11206; b) R. W. Armstrong, J. M. Beau, S. H. Cheon, W. J. Christ, H. Fujioka, W. H. Ham, L. D. Hawkins, H. Jin, S. H. Kang, J. Am. Chem. Soc. 1989, 111, 7525-7530.

[51] J. Uenishi, J. M. Beau, R. W. Armstrong, Y. Kishi, J. Am. Chem. Soc. 1987, 109, 4756-4758.

[52] a) J. Smidt, W. Hafner, R. Jira, J. Sedlmeier, R. Sieber, R. Rüttinger, H. Kojer, Angewandte Chemie 1959, 71, 176-182; b) J. Smidt, W. Hafner, R. Jira, R. Sieber, J. Sedlmeier, A. Sabel, Angewandte Chemie 1962, 74, 93-102; c) R. Jira, Angewandte Chemie 2009, 121, 9196-9199; d) R. Jira, Angewandte Chemie (International ed. in English) 2009, 48, 9034-9037.

[53] F. C. Phillips, Zeitschrift für anorganische Chemie 1894, 6, 213-228.

[54] A. Haynes in Topics in Organometallic Chemistry, Vol. 18 (Hrsg.: M. Beller), Springer, Berlin, Heidelberg, 2006.

[55] R. Franke, D. Selent, A. Börner, Chem. Rev. 2012, 112, 5675-5732.

[56] R. I. McDonald, G. Liu, S. S. Stahl, Chem. Rev. 2011, 111, 2981-3019.

[57] J. P. Wolfe, Eur. J. Org. Chem. 2007, 2007, 571-582.

[58] J. Keith, P. Henry, Angew. Chem. Int. Ed. 2009, 48, 9038-9049.

[59] J. Keith, P. Henry, Angew. Chem. 2009, 121, 9200-9212.

[60] a) P. M. Henry, J. Am. Chem. Soc. 1964, 86, 3246-3250; b) J. E. Baeckvall, B. Akermark, S. O. Ljunggren, J. Am. Chem. Soc. 1979, 101, 2411-2416; c) J. A. Keith, J. Oxgaard, W. A. Goddard, J. Am. Chem. Soc. 2006, 128, 3132-3133; d) J. A. Keith, R. J. Nielsen, J. Oxgaard, W. A. Goddard, P. M. Henry, Organometallics 2009, 28, 1618-1619; e) O. Hamed, C. Thompson, P. M. Henry, J. Org. Chem. 1997, 62, 7082-7083; f) N. Gregor, K. Zaw, P. M. Henry, Organometallics 1984, 3, 
1251-1256; g) J. W. Francis, P. M. Henry, Journal of Molecular Catalysis A: Chemical 1996, 112, 317-326.

[61] J. A. Keith, R. J. Nielsen, J. Oxgaard, W. A. Goddard, J. Am. Chem. Soc. 2007, 129, 12342-12343.

[62] J. J. Cruywagen, R. J. Kriek†, Journal of Coordination Chemistry 2007, 60, 439447.

[63] T. Hosokawa, T. Uno, S. Inui, S. Murahashi, J. Am. Chem. Soc. 1981, 103, 23182323.

[64] T. Hosokawa, C. Okuda, S. Murahashi, J. Org. Chem. 1985, 50, 1282-1287.

[65] T. Hosokawa, T. Nomura, S.-I. Murahashi, Journal of Organometallic Chemistry 1998, 551, 387-389.

[66] B. J. Anderson, J. A. Keith, M. S. Sigman, J. Am. Chem. Soc. 2010, 132, 11872 11874.

[67] a) A. Comas-Vives, A. Stirling, A. Lledós, G. Ujaque, Chem. Eur. J. 2010, 16, 87388747 ; b) V. Imandi, S. Kunnikuruvan, N. N. Nair, Chem. Eur. J. 2013, 19, 47244731; c) A. Stirling, N. N. Nair, A. Lledós, G. Ujaque, Chem. Soc. Rev. 2014, 43, 4940.

[68] a) T. Hosokawa, H. Ohkata, I. Moritani, Bull. Chem. Soc. Jpn. 1975, 48, 1533-1536;

b) T. Hosokawa, M. Hirata, S.-I. Murahashi, A. Sonoda, Tetrahedron Letters 1976, $17,1821-1824$.

[69] a) I. I. Moiseev, M. N. Vargaftik, Coordination Chemistry Reviews 2004, 248, 23812391 ; b) L. S. Hegedus, G. F. Allen, J. J. Bozell, E. L. Waterman, J. Am. Chem. Soc. 1978, 100, 5800-5807.

[70] a) R. C. Larock, L. Wei, T. R. Hightower, Synlett 1998, 1998, 522-524; b) M. Rönn, J.-E. Bäckvall, P. G. Andersson, Tetrahedron Letters 1995, 36, 7749-7752; c) R. C. Larock, T. R. Hightower, J. Org. Chem. 1993, 58, 5298-5300.

[71] B. W. Michel, M. S. Sigman, ChemInform 2012, 43.

[72] a) B. Morandi, Z. K. Wickens, R. H. Grubbs, Angew. Chem. Int. Ed. 2013, 52, 2944 2948; b) B. Morandi, Z. K. Wickens, R. H. Grubbs, Angew. Chem. 2013, 125, 30163020.

[73] a) K. C. Nicolaou, G. S. Tria, D. J. Edmonds, Angew. Chem. Int. Ed. 2008, 47, 1780-1783; b) K. C. Nicolaou, G. S. Tria, D. J. Edmonds, Angew. Chem. 2008, 120, 1804-1807.

[74] a) E. M. Beccalli, G. Broggini, M. Martinelli, S. Sottocornola, Chem. Rev. 2007, 107, 5318-5365; b) J. Muzart, Tetrahedron 2005, 61, 5955-6008; c) A. Minatti, K. Muñiz, Chem. Soc. Rev. 2007, 36, 1142-1152; d) I. Nakamura, Y. Yamamoto, Chem. Rev. 2004, 104, 2127-2198. 
[75] A. Kuramochi, H. Usuda, K. Yamatsugu, M. Kanai, M. Shibasaki, J. Am. Chem. Soc. 2005, 127, 14200-14201.

[76] E. J. Alexanian, C. Lee, E. J. Sorensen, J. Am. Chem. Soc. 2005, 127, 7690-7691.

[77] Y. Li, D. Song, V. M. Dong, J. Am. Chem. Soc. 2008, 130, 2962-2964.

[78] J. Streuff, C. H. Hövelmann, M. Nieger, K. Muñiz, J. Am. Chem. Soc. 2005, 127, $14586-14587$.

[79] a) S. Nicolai, S. Erard, D. F. González, J. Waser, Org. Lett. 2010, 12, 384-387; b) S. Nicolai, C. Piemontesi, J. Waser, Angew. Chem. Int. Ed. 2011, 50, 4680-4683; c) S. Nicolai, C. Piemontesi, J. Waser, Angew. Chem. 2011, 123, 4776-4779.

[80] a) C. F. Rosewall, P. A. Sibbald, D. V. Liskin, F. E. Michael, J. Am. Chem. Soc. 2009, 131, 9488-9489; b) K.-T. Yip, D. Yang, Org. Lett. 2011, 13, 2134-2137; c) R. Zhu, S. L. Buchwald, Angew. Chem. Int. Ed. 2012, 51, 1926-1929; d) R. Zhu, S. L. Buchwald, Angew. Chem. 2012, 124, 1962-1965.

[81] T. P. Pathak, K. M. Gligorich, B. E. Welm, M. S. Sigman, J. Am. Chem. Soc. 2010, 132, 7870-7871.

[82] M. F. Semmelhack, W. R. Epa, Tetrahedron Letters 1993, 34, 7205-7208.

[83] L. F. Tietze, F. Stecker, J. Zinngrebe, K. M. Sommer, Chem. Eur. J. 2006, 12, 87708776.

[84] F. Silva, M. Reiter, R. Mills-Webb, M. Sawicki, D. Klär, N. Bensel, A. Wagner, V. Gouverneur, J. Org. Chem. 2006, 71, 8390-8394.

[85] M. F. Semmelhack, C. Bodurow, J. Am. Chem. Soc. 1984, 106, 1496-1498.

[86] a) J. D. White, P. Kuntiyong, T. H. Lee, Org. Lett. 2006, 8, 6039-6042; b) J. D. White, T. H. Lee, P. Kuntiyong, Org. Lett. 2006, 8, 6043-6046.

[87] a) Q. Xiao, W.-W. Ren, Z.-X. Chen, T.-W. Sun, Y. Li, Q.-D. Ye, J.-X. Gong, F.-K. Meng, L. You, Y.-F. Liu et al., Angew. Chem. Int. Ed. 2011, 50, 7373-7377; b) Q. Xiao, W.-W. Ren, Z.-X. Chen, T.-W. Sun, Y. Li, Q.-D. Ye, J.-X. Gong, F.-K. Meng, L. You, Y.-F. Liu et al., Angew. Chem. 2011, 123, 7511-7515.

[88] a) Y. Bai, D. C. Davis, M. Dai, Angew. Chem. Int. Ed. 2014, 53, 6519-6522; b) Y. Bai, D. C. Davis, M. Dai, Angew. Chem. 2014, 126, 6637-6640.

[89] T. Hosokawa, S. Miyagi, S.-I. Murahashi, A. Sonoda, J. Chem. Soc., Chem. Commun. 1978, 687-688.

[90] Y. Uozumi, K. Kato, T. Hayashi, J. Am. Chem. Soc. 1997, 119, 5063-5064.

[91] Y. Uozumi, K. Kato, T. Hayashi, J. Org. Chem. 1998, 63, 5071-5075.

[92] a) L. F. Tietze, K. M. Sommer, J. Zinngrebe, F. Stecker, Angew. Chem. Int. Ed. 2005, 44, 257-259; b) L. F. Tietze, K. M. Sommer, J. Zinngrebe, F. Stecker, Angew. Chem. 2005, 117, 262-264; c) L. F. Tietze, K. F. Wilckens, S. Yilmaz, F. Stecker, J. Zinngrebe, HETEROCYCLES 2006, 70, 309; d) L. F. Tietze, J. Zinngrebe, D. A. 
Spiegl, F. Stecker, HETEROCYCLES 2007, 74, 473; e) L. F. Tietze, A. Heins, M. Soleiman-Beigi, C. Raith, HETEROCYCLES 2009, 77, 1123.

[93] L. F. Tietze, D. A. Spiegl, F. Stecker, J. Major, C. Raith, C. Große, Chem. Eur. J. 2008, 14, 8956-8963.

[94] a) F. Wang, Y. J. Zhang, G. Yang, W. Zhang, Tetrahedron Letters 2007, 48, 41794182; b) Y. J. Zhang, F. Wang, W. Zhang, J. Org. Chem. 2007, 72, 9208-9213; c) F. Wang, G. Yang, Y. J. Zhang, W. Zhang, Tetrahedron 2008, 64, 9413-9416; d) Q. Liu, K. Wen, Z. Zhang, Z. Wu, Y. J. Zhang, W. Zhang, Tetrahedron 2012, 68, 52095215.

[95] a) S. S. Stahl, Angew. Chem. Int. Ed. 2004, 43, 3400-3420; b) S. S. Stahl, Angew. Chem. 2004, 116, 3480-3501; c) K. M. Gligorich, M. S. Sigman, Chem. Commun. 2009, 3854.

[96] a) R. M. Trend, Y. K. Ramtohul, E. M. Ferreira, B. M. Stoltz, Angew. Chem. Int. Ed. 2003, 42, 2892-2895; b) R. M. Trend, Y. K. Ramtohul, B. M. Stoltz, J. Am. Chem. Soc. 2005, 127, 17778-17788; c) R. M. Trend, Y. K. Ramtohul, E. M. Ferreira, B. M. Stoltz, Angew. Chem. 2003, 115, 2998-3001.

[97] a) M. A. Arai, M. Kuraishi, T. Arai, H. Sasai, J. Am. Chem. Soc. 2001, 123, 29072908; b) P. S. Koranne, T. Tsujihara, M. A. Arai, G. B. Bajracharya, T. Suzuki, K. Onitsuka, H. Sasai, Tetrahedron: Asymmetry 2007, 18, 919-923.

[98] a) L.-F. Tietze (Hrsg.) Domino reactions. Concepts for efficient organic synthesis, Wiley-VCH, Weinheim, Germany, 2014; b) H. Pellissier, Chem. Rev. 2013, 113, 442-524; c) L. F. Tietze, M. A. Düfert, S. C. Schild in Comprehensive Chirality (Hrsg.: E. M. Carreira, H. Yamamoto), Elsevier Science, Amsterdam., 2012; d) J. Cossy, S. Arseniyadis (Hrsg.) Modern tools for the synthesis of complex bioactive molecules, Wiley, Hoboken, N.J, 2012; e) H. Pellissier, Adv. Synth. Catal. 2012, 354, 237-294; f) S. Giboulot, F. Liron, G. Prestat, B. Wahl, M. Sauthier, Y. Castanet, A. Mortreux, G. Poli, Chem. Commun. 2012, 48, 5889; g) M. Platon, R. Amardeil, L. Djakovitch, J.-C. Hierso, Chem. Soc. Rev. 2012, 41, 3929; h) L. F. Tietze, A. Düfert, Pure and Applied Chemistry 2010, 82, 1375-1392; i) A. Córdova (Hrsg.) Catalytic asymmetric conjugate reactions, Wiley-VCH, Weinheim, 2010; j) C. Grondal, M. Jeanty, D. Enders, Nature Chem 2010, 2, 167-178; k) L. F. Tietze, Chem. Rev. 1996, 96, 115-136.

[99] a) L. F. Tietze, U. Beifuss, Angew. Chem. Int. Ed. Engl. 1993, 32, 131-163; b) L. F. Tietze, U. Beifuss, Angew. Chem. 1993, 105, 137-170.

[100] a) K. C. Nicolaou, D. J. Edmonds, P. G. Bulger, Angew. Chem. Int. Ed. 2006, 45, 7134-7186; b) K. C. Nicolaou, D. J. Edmonds, P. G. Bulger, Angew. Chem. 2006, 
118, 7292-7344; c) S. E. Denmark, A. Thorarensen, Chem. Rev. 1996, 96, 137166.

[101] a) C. Hulme, V. Gore, CMC 2003, 10, 51-80; b) M. Colombo, I. Peretto, Drug Discovery Today 2008, 13, 677-684; c) A. Padwa, S. K. Bur, Tetrahedron 2007, 63, 5341-5378.

[102] a) E. J. Corey, W. E. Russey, de Montellano, Paul R. Ortiz, J. Am. Chem. Soc. 1966, 88, 4750-4751; b) E. J. Corey, S. C. Virgil, J. Am. Chem. Soc. 1991, 113, 4025-4026; c) E. J. Corey, S. C. Virgil, S. Sarshar, J. Am. Chem. Soc. 1991, 113, 8171-8172; d) E. J. Corey, S. C. Virgil, D. R. Liu, S. Sarshar, J. Am. Chem. Soc. 1992, 114, 1524-1525; e) R. A. Yoder, J. N. Johnston, Chem. Rev. 2005, 105 , 4730-4756; f) K. U. Wendt, G. E. Schulz, E. J. Corey, D. R. Liu, Angewandte Chemie International Edition 2000, 39, 2812-2833; g) K. U. Wendt, G. E. Schulz, E. J. Corey, D. R. Liu, Angewandte Chemie 2000, 112, 2930-2952.

[103] S. Rendler, D. W. C. MacMillan, J. Am. Chem. Soc. 2010, 132, 5027-5029.

[104] H. Wei, T. Itoh, M. Kinoshita, N. Kotoku, S. Aoki, M. Kobayashi, Tetrahedron 2005, 61, 8054-8058.

[105] V. Sofiyev, G. Navarro, D. Trauner, Org. Lett. 2008, 10, 149-152.

[106] a) H. Ishikawa, T. Suzuki, Y. Hayashi, Angew. Chem. Int. Ed. 2009, 48, 1304-1307;

b) H. Ishikawa, T. Suzuki, H. Orita, T. Uchimaru, Y. Hayashi, Chem. Eur. J. 2010, 16, 12616-12626.

[107] L. F. Tietze, B. Waldecker, D. Ganapathy, C. Eichhorst, T. Lenzer, K. Oum, S. O. Reichmann, D. Stalke, Angew. Chem. Int. Ed. 2015, 54, 10317-10321.

[108] E. N. Jacobsen, I. Marko, W. S. Mungall, G. Schroeder, K. B. Sharpless, J. Am. Chem. Soc. 1988, 110, 1968-1970.

[109] K. B. Sharpless, W. Amberg, Y. L. Bennani, G. A. Crispino, J. Hartung, K. S. Jeong, H. L. Kwong, K. Morikawa, Z. M. Wang, J. Org. Chem. 1992, 57, 2768-2771.

[110] H. C. Kolb, M. S. VanNieuwenhze, K. B. Sharpless, Chem. Rev. 1994, 94, 24832547.

[111] H. C. Kolb, P. G. Andersson, K. B. Sharpless, J. Am. Chem. Soc. 1994, 116, 12781291.

[112] a) D. J. Berrisford, C. Bolm, K. B. Sharpless, Angew. Chem. Int. Ed. Engl. 1995, 34, 1059-1070; b) D. J. Berrisford, C. Bolm, K. B. Sharpless, Angew. Chem. 1995, 107, 1159-1171.

[113] J. S. M. Wai, I. Marko, J. S. Svendsen, M. G. Finn, E. N. Jacobsen, K. B. Sharpless, J. Am. Chem. Soc. 1989, 111, 1123-1125.

[114] K. Hoi-Lun, C. Sorato, Y. Ogino, C. Hou, K. Barry Sharpless, Tetrahedron Letters 1990, 31, 2999-3002. 
[115] K. B. Sharpless, A. Y. Teranishi, J. E. Backvall, J. Am. Chem. Soc. 1977, 99, 31203128

[116] E. J. Corey, P. D. Jardine, S. Virgil, P. W. Yuen, R. D. Connell, J. Am. Chem. Soc. 1989, 111, 9243-9244.

[117] D. V. Deubel, G. Frenking, Acc. Chem. Res. 2003, 36, 645-651.

[118] a) U. Pidun, C. Boehme, G. Frenking, Angew. Chem. 1996, 108, 3008-3011; b) U. Pidun, C. Boehme, G. Frenking, Angew. Chem. Int. Ed. Engl. 1996, 35, 2817-2820; c) S. Dapprich, G. Ujaque, F. Maseras, A. Lledós, D. G. Musaev, K. Morokuma, J. Am. Chem. Soc. 1996, 118, 11660-11661; d) M. Torrent, L. Deng, M. Duran, M. Sola, T. Ziegler, Organometallics 1997, 16, 13-19.

[119] A. Armstrong, P. A. Barsanti, L. H. Jones, G. Ahmed, J. Org. Chem. 2000, 65, 7020-7032.

[120] K. C. Fortner, D. Kato, Y. Tanaka, M. D. Shair, J. Am. Chem. Soc. 2010, 132, 275280.

[121] B. Goericke, Strategien zur Stabilisierung des Parnafungin Grundgerüstes, Göttingen, 2014.

[122] S. Heidemann, Untersuchungen zur enantioselektiven Totalsynthese von Parnafungin C, Göttingen, 2011.

[123] L. F. Tietze, S. Jackenkroll, C. Raith, D. A. Spiegl, J. R. Reiner, M. C. Ochoa Campos, Chemistry (Weinheim an der Bergstrasse, Germany) 2013, 19, 48764882.

[124] L. F. Tietze, L. Ma, S. Jackenkroll, J. R. Reiner, J. Hierold, B. Gnanaprakasam, S. Heidemann, HETEROCYCLES 2014, 88, 1101.

[125] L. F. Tietze, L. Ma, J. R. Reiner, S. Jackenkroll, S. Heidemann, Chem. Eur. J. 2013, 19, 8610-8614.

[126] L. F. Tietze, S. Jackenkroll, J. Hierold, L. Ma, B. Waldecker, Chem. Eur. J. 2014, 20, 8628-8635.

[127] L. Ma, Domino-Reaktion zum Aufbau von Tetrahydroxanthenonen. Zur Totalsynthese der Secalonsäure. Univ., Diss.--Göttingen, 2013, 1. Aufl., Sierke, Göttingen, 2013.

[128] S. Jackenkroll, Domino Reactions for the Syntheses of Chiral Chromanes. Enantioselective Total Syntheses of (-)-Diversonol,(-)-Blennolide C, (-)-Gonytolide $C$ and Formal Synthesis of Siccanin, Göttingen, 2014.

[129] T. D. Nelson, A. I. Meyers, J. Org. Chem. 1994, 59, 2655-2658.

[130] K. F. Wilckens, Domino-Wacker-Heck-Reaktionen zur effizienten Synthese von 2,3Dihydrobenzo[1,4]dioxinen und verwandten Verbindungen, Göttingen, 2006. 
[131] K. Sommer, Enantioselektive Synthese von Vitamin E durch monoklonale Antikörper und einen neuen Palladium-katalysierten Domino-Wacker-Heck-Prozeß, Göttingen, 2002.

[132] Y. Uozumi, H. Kyota, E. Kishi, K. Kitayama, T. Hayashi, Tetrahedron: Asymmetry 1996, 7, 1603-1606.

[133] Y. Uozumi, H. Kyota, K. Kato, M. Ogasawara, T. Hayashi, J. Org. Chem. 1999, 64, $1620-1625$.

[134] K. R. West, K. D. Bake, S. Otto, Organic letters 2005, 7, 2615-2618.

[135] a) M. Butters, J. N. Harvey, J. Jover, A. J. J. Lennox, G. C. Lloyd-Jones, P. M. Murray, Angewandte Chemie International Edition 2010, 49, 5156-5160; b) T. E. Barder, S. L. Buchwald, Organic letters 2004, 6, 2649-2652; c) P.-P. Fang, A. Jutand, Z.-Q. Tian, C. Amatore, Angew. Chem. 2011, 123, 12392-12396; d) P.-P. Fang, A. Jutand, Z.-Q. Tian, C. Amatore, Angewandte Chemie (International ed. in English) 2011, 50, 12184-12188; e) G. B. Smith, G. C. Dezeny, D. L. Hughes, A. O. King, T. R. Verhoeven, J. Org. Chem. 1994, 59, 8151-8156.

[136] a) J.-M. Weibel, A. Blanc, P. Pale, Chemical reviews 2008, 108, 3149-3173; b) M. Harmata (Hrsg.) Silver in organic chemistry, John Wiley \& Sons, Hoboken, NJ, 2010; c) H. Chen, M.-Z. Deng, J. Org. Chem. 2000, 65, 4444-4446.

[137] a) R. E. Mulvey, F. Mongin, M. Uchiyama, Y. Kondo, Angewandte Chemie (International ed. in English) 2007, 46, 3802-3824; b) L. C. McCann, M. G. Organ, Angewandte Chemie International Edition 2014, 53; c) J. A. Garden, A. R. Kennedy, R. E. Mulvey, S. D. Robertson, Dalton transactions (Cambridge, England : 2003) 2011, 40, 11945-11954; d) W. J. Scott, J. K. Stille, J. Am. Chem. Soc. 1986, 108, 3033-3040; e) H. Ochiai, M. Jang, K. Hirano, H. Yorimitsu, K. Oshima, Organic letters 2008, 10, 2681-2683; f) R. E. Mulvey, F. Mongin, M. Uchiyama, Y. Kondo, Angew. Chem. 2007, 119, 3876-3899.

[138] P. G. M. Wuts, T. W. Greene, Greene's protective groups in organic synthesis, 5. Aufl., Wiley, Hoboken, NJ, 2014.

[139] G. N. Varseev, M. E. Maier, Angewandte Chemie (International ed. in English) 2006, 45, 4767-4771.

[140] a) B. M. Trost, H. C. Shen, J.-P. Surivet, Journal of the American Chemical Society 2004, 126, 12565-12579; b) B. M. Trost, H. C. Shen, J.-P. Surivet, Angew. Chem. 2003, 115, 4073-4077; c) B. M. Trost, H. C. Shen, J.-P. Surivet, Angewandte Chemie (International ed. in English) 2003, 42, 3943-3947.

[141] B. M. Trost, H. C. Shen, L. Dong, J.-P. Surivet, C. Sylvain, Journal of the American Chemical Society 2004, 126, 11966-11983. 
[142] a) T. Mukaiyama, I. Shiina, H. Iwadare, M. Saitoh, T. Nishimura, N. Ohkawa, H. Sakoh, K. Nishimura, Y. Tani, M. Hasegawa et al., Chemistry - A European Journal 1999, 5, 121-161; b) M. Desage-El Murr, S. Nowaczyk, T. Le Gall, C. Mioskowski, Eur. J. Org. Chem. 2006, 2006, 1489-1498; c) D. M. Bradley, R. Mapitse, N. M. Thomson, C. J. Hayes, J. Org. Chem. 2002, 67, 7613-7617; d) M. Ollivault-Shiflett, D. B. Kimball, L. A. P. Silks, The Journal of organic chemistry 2004, 69, 5150-5152. [143] a) D. Ma, X. Pu, J. Wang, Tetrahedron: Asymmetry 2002, 13, 2257-2260; b) T. Persigand, F. Laure, D. Blondet, J. Pascal, Synthetic Communications 1992, 22 , 2877-2882; c) J. Bonjoch, N. Casamitjana, J. Quirante, C. Garriga, J. Bosch, Tetrahedron 1992, 48, 3131-3138.

[144] J. D. More, N. S. Finney, Org. Lett. 2002, 4, 3001-3003.

[145] a) R. Ahmad, J. M. Saa, M. P. Cava, J. Org. Chem. 1977, 42, 1228-1230; b) J. A. Dodge, M. G. Stocksdale, K. J. Fahey, C. D. Jones, J. Org. Chem. 1995, 60, 739 741.

[146] a) W. S. Mahoney, J. M. Stryker, J. Am. Chem. Soc. 1989, 111, 8818-8823; b) W. S. Mahoney, D. M. Brestensky, J. M. Stryker, J. Am. Chem. Soc. 1988, 110, $291-$ 293.

[147] a) A. Shirai, O. Miyata, N. Tohnai, M. Miyata, D. J. Procter, D. Sucunza, T. Naito, The Journal of organic chemistry 2008, 73, 4464-4475; b) J.-B. Bongui, A. Elomri, D. Cahard, F. Tillequin, B. Pfeiffer, A. Pierré, E. Seguin, Chem. Pharm. Bull. 2005, 53, 1540-1546.

[148] a) Y. Zhang, G. Song, G. Ma, J. Zhao, C.-L. Pan, X. Li, Organometallics 2009, 28, 3233-3238; b) R. G. Harvey, Q. Dai, C. Ran, T. M. Penning, The Journal of organic chemistry 2004, 69, 2024-2032.

[149] a) B. O. Lindgren, T. Nilsson, S. Husebye, Ø. Mikalsen, K. Leander, C.-G. Swahn, Acta Chem. Scand. 1973, 27, 888-890; b) G. A. Kraus, M. J. Taschner, J. Org. Chem. 1980, 45, 1175-1176.

[150] L. F. Tietze, T. Eicher, U. Diederichsen, A. Speicher, N. Schützenmeister, Reactions and syntheses. In the organic chemistry laboratory, 2. Aufl., Wiley-VCH, Weinheim, 2015.

[151] L. F. Tietze, J. K. Lohmann, Synlett 2002, 2083-2085.

[152] M. B. Andrus, D. Asgari, J. A. Sclafani, J. Org. Chem. 1997, 62, 9365-9368.

[153] A. M. Linsenmeier, Parnafungine und Phenanthridine - Leitstrukturen für die Wirkstoffsynthese. Karlsruher Inst. für Technologie (KIT), Diss.--Karlsruhe, 2012, Logos-Verl., Berlin, 2012.

[154] D. Koch, S. Maechling, S. Blechert, Tetrahedron 2007, 63, 7112-7119. 
[155] A. Bonet, H. Gulyas, I. O. Koshevoy, F. Estevan, M. Sanau, M. A. Ubeda, E. Fernandez, Chemistry (Weinheim an der Bergstrasse, Germany) 2010, 16, 63826390.

[156] J. Hierold, Untersuchungen zur Synthese von Parnafungin-Derivaten. unveröffentlichte Arbeiten, Göttingen, 2015.

[157] a) P. Wipf, C. R. J. Stephenson, Organic letters 2005, 7, 1137-1140; b) P. A. Grieco, S. Gilman, M. Nishizawa, J. Org. Chem. 1976, 41, 1485-1486.

[158] a) H. Özkan, S. Yavuz, A. Dişli, Y. Yıldırır, L. Türker, Heteroatom Chem. 2007, 18, 255-258; b) H. Özkan, A. Disli, Y. Yildirir, Organic Preparations and Procedures International 2004, 36, 161-163; c) Z. Časar, I. Leban, A. M.-L. Maréchal, D. Lorcy, J. Chem. Soc., Perkin Trans. 1 2002, 1568-1573.

[159] F. Stecker, Palladium(II)-katalysierte Domino-Reaktionen zur enantioselektiven Synthese von Chromanen - Totalsynthese von Vitamin E und anderen biologisch aktiven Naturstoffen. Dissertation, Göttingen, 2008.

[160] a) Z.-M. Wang, X.-L. Zhang, K. Sharpless, Tetrahedron Letters 1993, 34, 22672270; b) R. Oi, K. Sharpless, Tetrahedron Letters 1992, 33, 2095-2098.

[161] a) J. Kim, Y. N. Weledji, M. M. Greenberg, The Journal of organic chemistry 2004, 69, 6100-6104; b) T. Hu, N. Takenaka, J. S. Panek, J. Am. Chem. Soc. 2002, 124, $12806-12815$.

[162] N. Schützenmeister, Totalsynthese von Spinosynanaloga. Univ., Diss.--Göttingen, 2012, 1. Aufl., Cuvillier, Göttingen, 2012. 


\subsection{Danksagung}

Zum Abschluss dieser Arbeit möchte ich mich bei allen bedanken, die auf die eine oder andere Weise ihren Anteil an dem Gelingen dieser Arbeit beigetragen haben.

Zuallererst gilt mein Dank den Mitarbeitern der analytischen Abteilungen der Fakultät für Chemie. Ich danke Reinhard Machinek, Michael John, Christiane Siebert, Martin Weitemeyer, Carola Zolke und Ralf Schöne für das überaus schnelle und zuverlässige Messen von unzähligen NMR-Spektren. Ganz besonders möchte ich Reinhard Machinek und Michael John danken, die auch bei schwierigsten NMR-Problemen mit Rat und Tat zur Seite standen. Des Weiteren danke ich Holm Frauendorf, Frank Hambloch, Györgi Sommer-Udvarnoki, Ulrike Bode und Gabriele Krökel für das zügige Messen der Massenspektren. Besonderer Dank gilt dabei Holm Frauendorf für seine wichtigen Ratschläge, seine Unterstützung bei Problemen und die interessanten und lehrreichen Gespräche. Evelyn Pfeil und Katja Grube danke ich für das unverzügliche Messen von UV/Vis- und IR-Spektren sowie das Messen der Drehwerte.

Mein Dank gilt außerdem Martina Pretor für ihre schnelle und kompetente Hilfe bei EDVProblemen jeglicher Art. Sabine Schacht möchte ich für die unermüdliche Verwaltungsarbeit danken, die den Arbeitskreis am Laufen hielt. Gleiches gilt für Isabel Trzeciok, Berta Hampel und Carolin Ständer, die im Dekanat bei Problemen mit etwaigen bürokratischen Hürden stets hilfsbereit waren.

Außerdem danke ich Holger Tucholla und Rupert Schrommek für die vorbildliche Versorgung mit Chemikalien und anderen Laborutensilien sowie die kleinen Scherze zwischendurch. Ein Dank gebührt auch den Hausmeistern Frank Peters und Wolfram Matthies sowie den Mitarbeitern der Werkstätten für die schnelle Reparatur jeglicher Laborgeräte.

Weiterhin möchte ich den Materialwärten der Abteilung Tietze, Galina Pestel, Johannes Reiner, Judith Hierold und Simon Biller für die unproblematische und schnelle Bereitstellung von Chemikalien und Verbrauchsmaterialien danken.

Meinen Mitstreitern auf dem Parnafungin-Thema, namentlich Judith Hierold und Björn Göricke danke ich für die gute Zusammenarbeit und dem regen Wissensaustausch, auch und vor allem in Zeiten in denen die praktisch erzielten Ergebnisse nicht mit der theoretischen Planung vereinbar waren. Des Weiteren sollen an dieser Stelle meine Laborpraktikanten, Andi Reim, Julian Koeller und Markus Schön ihren hart verdienten Dank erhalten, die mir durch ihre Arbeit ebenjene erspart haben.

Ich danke außerdem allen Mitarbeitern der Abteilung Tietze, die auch die frustrierenden und ermüdenden Episoden dieser Arbeit zu einem Vergnügen gemacht haben. Ein besonderer Dank gebührt dabei Jérôme Clerc, Christoph Eichhorst, Simon Biller, Tobias 
Nack, Judith Hierold, Stefan Jackenkroll, Timo Scheffer, Kianga Schmuck und Bernd Waldecker vor allem für die Zeit, die man abseits des Laboralltags, sei es im „Casino“, im Göttinger Nachtleben, beim Golfen, Pokern und sonstigen Aktivitäten miteinander verbracht hat. Nina Schützenmeister, Judith Hierold und Simon Biller möchte ich für die unterhaltsame gemeinsame Zeit sowie für die entspannte und angenehme Atmosphäre im Labor danken.

Ein besonderer Dank gilt Judith Hierold, Christoph Eichhorst, Simon Biller und Sebastian Horn für das zügige und gewissenhafte Korrekturlesen dieser Arbeit. Alle verbliebenen Fehler sind allein mir zuzuschreiben und selbstverständlich gewollt, um die Aufmerksamkeit des Lesers zu testen.

Verschiedene Menschen haben meinen Weg in Göttingen begleitet und diesen zu einer angenehmen Episode in meinem Leben gemacht. Hierfür möchte ich mich besonders bei Andi Reim, Eibe Dücker, Ulrike Rost, Peter Stollberg, Jérôme Clerc, Christoph Eichhorst, Stefan Jackenkroll, Simon Biller, Tobias Nack, Timo Scheffer, Kianga Schmuck, Judith Hierold und Bernd Waldecker bedanken. Sebastian Horn, Daniel März, Christian Plate, Felix Breckau und Andreas Finze danke ich an dieser Stelle für die teilweise fast lebenslange Freundschaft und Unterstützung.

Zu guter Letzt gilt der größte Dank meiner Familie, allen voran meinen Eltern und meiner Großmutter für die vorbehaltslose Unterstützung in allen Lebenslagen, die diese Arbeit erst ermöglichte.

Herzlichsten Dank!

Meine akademischen Lehrer waren unter anderem:

D. Stalke, G. M. Sheldrick, S. Schmatz, J. Schroeder, G. Beuermann, J. Magull, A. de Meijere, P. Botschwina, K. Samwer, P. von Zezschwitz, U. Klingebiel, C. Schulzke, R. Machinek, U. Diederichsen, D. B. Werz, L. Ackermann, L. F. Tietze, C. Steinem, H.-J. Troe, D. Schwarzer, F. Meyer, C. Ducho, K. Luther, H. Frauendorf, M. Suhm, W.-H. Zimmermann. 


\subsection{Lebenslauf}

Persönliche Daten

Name:

Sven Heidemann

Geburtsdatum:

21.08 .1984

Geburtsort:

Burg

Staatsangehörigkeit:

deutsch

Familienstand:

ledig

\section{Schulausbildung}

07/1991-06/1995

Grundschule II, Burg

07/1995-07/2004

Gymnasium Burg, Burg

07/2004

Allgemeine Hochschulreife, Note „1.4“

\section{Hochschulausbildung}

$10 / 2005-08 / 2016$

Studium der Chemie an der Georg-August-Universität Göttingen

$10 / 2007$

Diplomvorprüfung, Note „sehr gut“

07/2010-01/2011

Diplomarbeit am Institut für Organische und Biomolekulare Chemie unter der Leitung von Prof. Dr. Dr. h.c. Lutz F. Tietze mit dem Thema: „Untersuchungen zur enantioselektiven Totalsynthese von Parnafungin C"

$04 / 2011$

Diplomprüfung, Note „sehr gut“

$06 / 2011-06 / 2015$

Promotion am Institut für Organische und Biomolekulare Chemie unter der Leitung von Prof. Dr. Dr. h.c. Lutz F. Tietze mit dem Thema: „Untersuchungen zur enantioselektiven Totalsynthese von Parnafungin C"

$08 / 2016$

Disputation 